Federal Reserve Bank of Dallas

Globalization and Monetary Policy Institute

Working Paper No. 16

http://www.dallasfed.org/assets/documents/institute/wpapers/2008/0016.pdf

\title{
Technical Note on 'The Real Exchange Rate in Sticky Price Models: Does Investment Matter?"*
}

\author{
Enrique Martínez-García \\ Federal Reserve Bank of Dallas \\ Jens Søndergaard \\ Bank of England \\ July 2008 \\ Revised: November 2010
}

\begin{abstract}
This technical note is developed in part as a mathematical companion to the paper 'The Real Exchange Rate in Sticky Price Models: Does Investment Matter?' (GMPI working paper no. 17). Our two-country model incorporates capital accumulation with adjustment costs, variable capital utilization and investment-specific technological shocks. Nominal rigidities and monopolistic competition distort the goods markets of each country and allow monetary policy to have real effects. We investigate two different international pricing scenarios, localcurrency pricing (where the law of one price fails) and producer-currency pricing (where the law of one price holds). This technical note contains three basic calculations. First, we derive the equilibrium conditions of the open economy model under local-currency pricing and producer-currency pricing. Second, we compute the zero-inflation, zero-trade balance (deterministic) steady state. Third, we describe the log-linearization of the equilibrium conditions around the deterministic steady state. Simultaneously, commentary is provided whenever necessary to enhance the model description and to place the assumptions embedded in our DSGE framework into context.
\end{abstract}

JEL codes: F31, F37, F41

\footnotetext{
* Enrique Martínez-García, Research Department, Federal Reserve Bank of Dallas. 2200 N. Pearl Street, Dallas, TX 75201. 214-922-5262. enrique.martinez-garcia@dal.frb.org. Jens Søndergaard, Monetary Instruments and Markets Division, Monetary Analysis, Bank of England. Threadneedle Street, London EC2R 8AH, UK. +44 (0)20-7601-4869. jens.sondergaard@bankofengland.co.uk. We would like to thank Mark Astley, Charles Engel, Alberto Musso, Roman Sustek, Jian Wang, Mark Wynne, Wenjuan Xie, and many seminar and conference participants for helpful discussions. We also acknowledge the support of the Federal Reserve Bank of Dallas and the Bank of England. All remaining errors are ours alone. The views in this paper are those of the authors and do not necessarily reflect the views of the Bank of England, the Federal Reserve Bank of Dallas or the Federal Reserve System.
} 


\section{The Benchmark Monetary Open Economy}

Here, we briefly describe the structure of the benchmark monetary open economy model.

\subsection{The Intertemporal Consumption and Savings Problem}

We specify a stochastic, two-country general equilibrium model. Each country is populated by a continuum of infinitely lived (and identical) households in the interval $[0,1]$. In each period, the domestic households' utility function is additively separable in consumption, $C_{t}$, and labor, $L_{t}$. Domestic households maximize,

$$
\sum_{\tau=0}^{+\infty} \beta^{\tau} \mathbb{E}_{t}\left[\frac{1}{1-\sigma^{-1}}\left(C_{t+\tau}\right)^{1-\sigma^{-1}}-\frac{1}{1+\varphi}\left(L_{t+\tau}\right)^{1+\varphi}\right]
$$

where $0<\beta<1$ is the subjective intertemporal discount factor. The elasticity of intertemporal substitution satisfies that $\sigma>0(\sigma \neq 1)$ while the inverse of the Frisch elasticity of labor supply satisfies that $\varphi>0$.

We assume that both countries have unrestricted access to a complete set of contingent claims, traded internationally. The domestic household maximizes its lifetime utility in (1) subject to the sequence of budget constraints described by,

$$
P_{t}\left(C_{t}+X_{t}\right)+T_{t}+\int P^{b}\left(s^{t+1} \mid s^{t}\right) B\left(s^{t+1}\right) d s_{t+1} \leq B\left(s^{t}\right)+W_{t} L_{t}+Z_{t} K_{t}+P r_{t},
$$

and the law of motion for capital,

$$
K_{t+1} \leq(1-\delta) K_{t}+V_{t} \Phi\left(X_{t}, X_{t-1}, K_{t}\right) X_{t}
$$

where $W_{t}$ is the domestic nominal wage, $P_{t}$ is the domestic consumption price index (CPI), $P r_{t}$ are the nominal profits generated by the domestic firms, and $T_{t}$ is a lump-sum nominal tax levied on the domestic households. ${ }^{1}$ Moreover, $X_{t}$ is domestic real investment, $K_{t}$ stands for domestic real capital, $Z_{t}$ defines the nominal rental rate of capital, and $V_{t}$ is an exogenous, investment-specific technological (IST) shock. IST shocks affect the efficiency in producing investment goods.

We denote $s_{t+1}$ the event that occurs at time $t+1$ and $s^{t+1}=\left(s^{t}, s_{t+1}\right)$ the history of events up to that point. The households' portfolio includes a complete set of one-period contingent claims (Arrow-Debreu securities), traded internationally and quoted in units of the domestic currency. Households have unrestricted access to all contingent claims, $P^{b}\left(s^{t} \mid s^{t-1}\right)$ is the domestic price at time $t-1$ of the contingent claim that pays off after $s_{t}$ is realized at time $t$, and $\frac{P^{b}\left(s^{t} \mid s^{t-1}\right)}{S_{t}}$ is the corresponding price in foreign currency units. $S_{t}$ denotes the nominal exchange rate, $B\left(s^{t}\right)$ is the nominal pay-off received by the domestic households after the event $s_{t}$ occurs at time $t$ on a contingent claim purchased at time $t-1$ (which would have paid nothing if event $s_{t}$ had not happened). The foreign households maximize their lifetime utility subject to an analogous sequence of budget constraints and the same law of motion for capital.

We assume that there is no trade in either domestic or foreign firm shares imposing de facto a strict home bias in stock portfolios. Sole ownership of the local firms rests in the hands of the local households.

\footnotetext{
${ }^{1}$ We do not fully incorporate fiscal policy in our model. However, we include lump-sum taxes in order to finance a subsidy for firms that can neutralize the mark-up distortion associated with our assumption that companies operate under monopolistic competition. In case no subsidy is introduced, then the lump-sum tax would be equal to zero, i.e. $T_{t}=0$.
} 
However, this strong assumption on share-holdings does not undo our postulate of complete international asset markets because households in both countries do have access to a full set of contingent claims with which they can pool and share risks efficiently. Therefore, households can replicate any stream of payoffs that trade in firm shares would afford them by using the available Arrow-Debreu securities only. Money is purely a unit of account, although monetary policy has an impact by regulating short-term nominal interest rates in the presence of nominal rigidities. Embedded in the specification of the budget constraint lies also the assumption that both factor markets (for labor and capital) are homogenous and perfectly competitive within a country, but segregated across countries. In other words, factors can be used for production purposes in any firm within the same country, but they are immobile across borders.

Capital accumulation may be subject to adjustment costs. We consider three special cases: the capital adjustment cost (CAC) case, the investment adjustment cost (IAC) case, and the case with no adjustment costs (NAC). The (NAC) function is simply,

$$
\Phi\left(X_{t}, X_{t-1}, K_{t}\right)=1
$$

This implies that in steady state $\Phi(\bar{X}, \bar{X}, \bar{K})=1, \Phi^{\prime}(\bar{X}, \bar{X}, \bar{K})=0$, and $\Phi^{\prime \prime}(\bar{X}, \bar{X}, \bar{K})=0$. The CAC and IAC adjustment cost functions, however, require a more detailed description.

Capital Adjustment Cost (CAC) Function. We adopt the same specification for the capital adjustment cost (CAC) function as Chari, et al. (2002), which implies that the function $\Phi(\cdot)$ in (3) takes the following form,

$$
\Phi\left(\frac{X_{t}}{K_{t}}\right)=1-\frac{1}{2} \chi \frac{\left(\frac{X_{t}}{K_{t}}-\delta\right)^{2}}{\frac{X_{t}}{K_{t}}},
$$

where $\frac{X_{t}}{K_{t}}$ is the corresponding investment-to-capital ratio and $\delta$ is the depreciation rate coming from the law of motion for capital. Among the properties of this adjustment cost function that are relevant for our model, we note that,

$$
\begin{aligned}
\Phi^{\prime}\left(\frac{X_{t}}{K_{t}}\right) & =-\frac{1}{2} \chi\left[\frac{2 \frac{X_{t}}{K_{t}}\left(\frac{X_{t}}{K_{t}}-\delta\right)-\left(\frac{X_{t}}{K_{t}}-\delta\right)^{2}}{\left(\frac{X_{t}}{K_{t}}\right)^{2}}\right] \\
\Phi^{\prime \prime}\left(\frac{X_{t}}{K_{t}}\right) & =-\frac{1}{2} \chi\left[\frac{2\left(\frac{X_{t}}{K_{t}}\right)^{3}-2\left(2 \frac{X_{t}}{K_{t}}\left(\frac{X_{t}}{K_{t}}-\delta\right)-\left(\frac{X_{t}}{K_{t}}-\delta\right)^{2}\right) \frac{X_{t}}{K_{t}}}{\left(\frac{X_{t}}{K_{t}}\right)^{4}}\right] .
\end{aligned}
$$

We assume that in steady state the investment-specific shock is at its unconditional mean, and $\bar{V}=1$. Hence, in steady state the adjustment costs dissipate and the investment-to-capital ratio is equal to the depreciation rate (i.e., $\bar{X}=\delta \bar{K}$ ) as in the standard neoclassical model. This implies that $\Phi(\delta)=1$, $\Phi^{\prime}(\delta)=0$, and $\Phi^{\prime \prime}(\delta)=-\frac{\chi}{\delta}$. The same adjustment cost formula applies to the foreign households' problem.

Investment Adjustment Cost (IAC) Function. We also explore the investment adjustment cost function (IAC) used among others by Smets and Wouters (2003) and Christiano, et al. (2005). We conjecture 
that the IAC function takes the following form,

$$
\Phi\left(\frac{X_{t}}{X_{t-1}}\right)=1-\frac{1}{2} \kappa \frac{\left(\frac{X_{t}}{X_{t-1}}-1\right)^{2}}{\frac{X_{t}}{X_{t-1}}}
$$

Among the properties of the IAC function that are relevant for our model, we note that,

$$
\begin{aligned}
\Phi^{\prime}\left(\frac{X_{t}}{X_{t-1}}\right) & =-\frac{1}{2} \kappa\left[\frac{2 \frac{X_{t}}{X_{t-1}}\left(\frac{X_{t}}{X_{t-1}}-1\right)-\left(\frac{X_{t}}{X_{t-1}}-1\right)^{2}}{\left(\frac{X_{t}}{X_{t-1}}\right)^{2}}\right] \\
\Phi^{\prime \prime}\left(\frac{X_{t}}{X_{t-1}}\right) & =-\frac{1}{2} \kappa\left[\frac{2\left(\frac{X_{t}}{X_{t-1}}\right)^{3}-2\left(2 \frac{X_{t}}{X_{t-1}}\left(\frac{X_{t}}{X_{t-1}}-1\right)-\left(\frac{X_{t}}{X_{t-1}}-1\right)^{2}\right)\left(\frac{X_{t}}{X_{t-1}}\right)}{\left(\frac{X_{t}}{X_{t-1}}\right)^{4}}\right] .
\end{aligned}
$$

In steady state, the adjustment costs dissipate again and the net investment growth is zero. Hence, the law of motion for capital is the same as in the standard neoclassical model in steady state. This also implies that $\Phi(1)=1, \Phi^{\prime}(1)=0$, and $\Phi^{\prime \prime}(1)=-\kappa$. It is costly to change the level of investment and the cost is increasing in the size of the change, but there are no adjustment costs in steady state. The same adjustment cost formula applies to the foreign households' problem.

Aggregation Rules and the Price Indexes. We assume that investment, like consumption, is a composite index of domestic and imported foreign varieties. The home and foreign consumption bundles of the domestic household, $C_{t}^{H}$ and $C_{t}^{F}$, as well as the investment bundles, $X_{t}^{H}$ and $X_{t}^{F}$, are aggregated by means of a CES index as,

$$
\begin{aligned}
& C_{t}^{H}=\left[\int_{0}^{1} C_{t}(h)^{\frac{\theta-1}{\theta}} d h\right]^{\frac{\theta}{\theta-1}}, C_{t}^{F}=\left[\int_{0}^{1} C_{t}(f)^{\frac{\theta-1}{\theta}} d f\right]^{\frac{\theta}{\theta-1}} \\
& X_{t}^{H}=\left[\int_{0}^{1} X_{t}(h)^{\frac{\theta-1}{\theta}} d h\right]^{\frac{\theta}{\theta-1}}, X_{t}^{F}=\left[\int_{0}^{1} X_{t}(f)^{\frac{\theta-1}{\theta}} d f\right]^{\frac{\theta}{\theta-1}}
\end{aligned}
$$

while aggregate consumption and investment, $C_{t}$ and $X_{t}$, are defined with another CES index as,

$$
\begin{aligned}
& C_{t}=\left[\phi_{H}^{\frac{1}{\eta}}\left(C_{t}^{H}\right)^{\frac{\eta-1}{\eta}}+\phi_{F}^{\frac{1}{\eta}}\left(C_{t}^{F}\right)^{\frac{\eta-1}{\eta}}\right]^{\frac{\eta}{\eta-1}} \\
& X_{t}=\left[\phi_{H}^{\frac{1}{\eta}}\left(X_{t}^{H}\right)^{\frac{\eta-1}{\eta}}+\phi_{F}^{\frac{1}{\eta}}\left(X_{t}^{F}\right)^{\frac{\eta-1}{\eta}}\right]^{\frac{\eta}{\eta-1}}
\end{aligned}
$$

The elasticity of substitution across varieties produced within a country is $\theta>1$, and the elasticity of intratemporal substitution between the home and foreign bundles of varieties is $\eta>0$. The share of the home goods in the domestic aggregators is $\phi_{H}$, while the share of foreign goods is $\phi_{F}$. We assume the shares are homogeneous, i.e. $\phi_{H}+\phi_{F}=1$. Similarly, we can define the aggregators for the foreign household. The only difference being that the share of the home goods in the foreign aggregators is $\phi_{H}^{*}=\phi_{F}$, while the share of foreign goods in the foreign aggregator is $\phi_{F}^{*}=\phi_{H}$. 
The model introduces home-product bias in preferences (Warnock, 2003) as well as in the composition of investment. By assumption, investment goods can only be used for local production after aggregation. This is also the case because of compositional differences across countries. However, it must be noted that all local and foreign varieties can be traded internationally for either consumption or investment purposes. Moreover, the symmetry of the aggregators implies that the corresponding price indexes are identical for the investment and consumption bundles. Hence, the price of consumption and investment is the same as reflected in the budget constraint (that is, in equation (2)).

Under standard results on functional separability, the CPI indexes which correspond to our specification of the domestic aggregators in (9) - (10) and their foreign counterparts are,

$$
\begin{aligned}
P_{t} & =\left[\phi_{H}\left(P_{t}^{H}\right)^{1-\eta}+\phi_{F}\left(P_{t}^{F}\right)^{1-\eta}\right]^{\frac{1}{1-\eta}}, \\
P_{t}^{*} & =\left[\phi_{H}^{*}\left(P_{t}^{H *}\right)^{1-\eta}+\phi_{F}^{*}\left(P_{t}^{F *}\right)^{1-\eta}\right]^{\frac{1}{1-\eta}},
\end{aligned}
$$

and the price sub-indexes are,

$$
\begin{aligned}
P_{t}^{H} & =\left[\int_{0}^{1} P_{t}(h)^{1-\theta} d h\right]^{\frac{1}{1-\theta}}, P_{t}^{F}=\left[\int_{0}^{1} P_{t}(f)^{1-\theta} d f\right]^{\frac{1}{1-\theta}}, \\
P_{t}^{H *} & =\left[\int_{0}^{1} P_{t}^{*}(h)^{1-\theta} d h\right]^{\frac{1}{1-\theta}}, P_{t}^{F *}=\left[\int_{0}^{1} P_{t}^{*}(f)^{1-\theta} d f\right]^{\frac{1}{1-\theta}},
\end{aligned}
$$

where $P_{t}^{H}$ and $P_{t}^{F}$ are the price sub-indexes for the home- and foreign-produced bundle of goods in units of the home currency. Similarly for $P_{t}^{H *}$ and $P_{t}^{F *}$ in units of the foreign currency. We define the real exchange rate as,

$$
R S_{t} \equiv \frac{S_{t} P_{t}^{*}}{P_{t}}
$$

where $S_{t}$ denotes the nominal exchange rate.

\subsection{The Firms' Problem}

Each firm supplies the home and foreign market, and sets prices in the local currency (henceforth, localcurrency pricing or LCP). Firms engage in third-degree price discrimination across markets (re-selling is infeasible) and, furthermore, enjoy monopolistic power in their own variety. Frictions in the goods market are modelled with nominal price stickiness à la Calvo (1983). At time $t$ any firm (whether domestic or foreign) is forced to maintain its previous period prices in the domestic and foreign markets with probability $0<\alpha<1$. Instead, with probability $(1-\alpha)$, the firm receives a signal to optimally reset each price.

We assume that production employs a (homogeneous of degree one) Cobb-Douglas technology, i.e.

$$
\begin{aligned}
Y_{t}(h) & =A_{t}\left(K_{t}(h)\right)^{1-\psi}\left(L_{t}(h)\right)^{\psi}, \forall h \in[0,1], \\
Y_{t}^{*}(f) & =A_{t}^{*}\left(K_{t}^{*}(f)\right)^{1-\psi}\left(L_{t}^{*}(f)\right)^{\psi}, \forall f \in[0,1],
\end{aligned}
$$

where $A_{t}$ is the domestic productivity shock and $A_{t}^{*}$ is the foreign productivity shock. The labor share in 
the production function is represented by $0<\psi \leq 1 .^{2}$ Obviously, by consistency it should follow that the aggregate capital accumulated by households in each country is $K_{t}=\int_{0}^{1} K_{t}(h) d h$ and $K_{t}^{*}=\int_{0}^{1} K_{t}(f) d f$ respectively, while aggregate labor is $L_{t}=\int_{0}^{1} L_{t}(h) d h$ and $L_{t}^{*}=\int_{0}^{1} L_{t}(f) d f$. Solving the cost-minimization problem of each individual firm yields an efficiency condition linking the capital-to-labor ratios to factor price ratios as follows,

$$
\begin{aligned}
\frac{K_{t}}{L_{t}} & =\frac{K_{t}(h)}{L_{t}(h)}=\frac{1-\psi}{\psi} \frac{W_{t}}{Z_{t}}, \forall h \in[0,1], \\
\frac{K_{t}^{*}}{L_{t}^{*}} & =\frac{K_{t}^{*}(f)}{L_{t}^{*}(f)}=\frac{1-\psi}{\psi} \frac{W_{t}^{*}}{Z_{t}^{*}}, \forall f \in[0,1],
\end{aligned}
$$

as well as a characterization for the (pre-subsidy) nominal marginal costs,

$$
\begin{aligned}
M C_{t} & =\frac{1}{A_{t}} \frac{1}{\psi^{\psi}(1-\psi)^{1-\psi}}\left(W_{t}\right)^{\psi}\left(Z_{t}\right)^{1-\psi}, \\
M C_{t}^{*} & =\frac{1}{A_{t}^{*}} \frac{1}{\psi^{\psi}(1-\psi)^{1-\psi}}\left(W_{t}^{*}\right)^{\psi}\left(Z_{t}^{*}\right)^{1-\psi} .
\end{aligned}
$$

The labor force is homogenous within a country and immobile across borders, and the national labor markets are perfectly competitive. Wages equalize in each country (but not necessarily across countries), i.e. $W_{t}(h)=$ $W_{t}$ for all $h \in[0,1]$ and $W_{t}^{*}(f)=W_{t}^{*}$ for all $f \in[0,1]$, and so does the rental rate on capital, i.e. $Z_{t}(h)=Z_{t}$ for all $h \in[0,1]$ and $Z_{t}^{*}(f)=Z_{t}^{*}$ for all $f \in[0,1]$. Then, since the production function is homogeneous of degree one (constant returns-to-scale), this implies that all local firms choose the same capital-to-labor ratio (even though they may produce different quantities in every period). Moreover, the factors of production are compensated according to their marginal product in all firms. ${ }^{3}$

We introduce a government subsidy in each country that is proportional to the local firms' production costs, i.e. $\xi_{t} M C_{t} Y_{t}(h)$ and $\xi_{t}^{*} M C_{t}^{*} Y_{t}^{*}(f)$ respectively in the domestic and foreign countries. The pre-subsidy production costs of a firm are simply a fraction of the (pre-subsidy) nominal marginal costs, $M C_{t}$ and $M C_{t}^{*}$, in (20) - (21) times the output of that firm. Governments only subsidize the production of firms located in their own country, independently of whether the goods are sold locally or exported. Governments set no

\footnotetext{
${ }^{2}$ These expressions reduce to the standard case of linear-in-labor technologies if $\psi=1$.

${ }^{3}$ The production functions in $(16)-(17)$ can be re-written as

$$
\begin{aligned}
Y_{t}(h) & =A_{t}\left(\frac{K_{t}}{L_{t}}\right)^{1-\psi} L_{t}(h), \forall h \in[0,1], \\
Y_{t}^{*}(f) & =A_{t}^{*}\left(\frac{K_{t}^{*}}{L_{t}^{*}}\right)^{1-\psi} L_{t}^{*}(f), \forall f \in[0,1],
\end{aligned}
$$
}

since capital-to-labor ratios are equated across all firms within a country. All households located in one country supply the same amount of labor and capital, given that factor prices are equalized whenever the factors of production of each country are homogeneous (though immobile across borders) and factor markets are perfectly competitive. However, since pricing decisions are not synchronized, the amounts of labor and capital assigned to each firm will differ in every period. On aggregate, by the market clearing conditions, the capital and labor demands equal their respective supplies and a measure of aggregate output can be expressed as a function of aggregate capital and labor, i.e.

$$
\begin{aligned}
Y_{t} & =\int_{0}^{1} Y_{t}(h) d h=A_{t}\left(K_{t}\right)^{1-\psi}\left(L_{t}\right)^{\psi}, \\
Y_{t}^{*} & =\int_{0}^{1} Y_{t}(f) d f=A_{t}^{*}\left(K_{t}^{*}\right)^{1-\psi}\left(L_{t}^{*}\right)^{\psi} .
\end{aligned}
$$


import tariffs or subsidize the local demand over the export demand, hence not distorting the international relative prices (e.g., the terms of trade and the real exchange rate). Using the pre-subsidy marginal costs described in (20) - (21), we can write the post-subsidy marginal costs that enter into the pricing decisions of firms of both countries as follows,

$$
\begin{aligned}
\left(1-\xi_{t}\right) M C_{t} & =\frac{1}{A_{t}} \frac{1}{\psi^{\psi}(1-\psi)^{1-\psi}}\left(\left(1-\xi_{t}\right) W_{t}\right)^{\psi}\left(\left(1-\xi_{t}\right) Z_{t}\right)^{1-\psi} \\
\left(1-\xi_{t}^{*}\right) M C_{t}^{*} & =\frac{1}{A_{t}^{*}} \frac{1}{\psi^{\psi}(1-\psi)^{1-\psi}}\left(\left(1-\xi_{t}^{*}\right) W_{t}^{*}\right)^{\psi}\left(\left(1-\xi_{t}^{*}\right) Z_{t}^{*}\right)^{1-\psi} .
\end{aligned}
$$

This characterization of the subsidies suffices for the purpose of either reducing or eliminating the mark-up distortions associated with monopolistic competition in the goods markets. However, we must point out that any given subsidy that the government desires to set can be implemented with any possible combination of wage subsidies, $\xi_{t}^{L}$ and $\xi_{t}^{L *}$, and capital rental subsidies, $\xi_{t}^{K}$ and $\xi_{t}^{K *}$, which satisfies that,

$$
\begin{aligned}
\left(1-\xi_{t}\right) & =\left(1-\xi_{t}^{L}\right)^{\psi}\left(1-\xi_{t}^{K}\right)^{1-\psi} \\
\left(1-\xi_{t}^{*}\right) & =\left(1-\xi_{t}^{L *}\right)^{\psi}\left(1-\xi_{t}^{K *}\right)^{1-\psi}
\end{aligned}
$$

Under these conditions it immediately follows that,

$$
\begin{aligned}
\frac{1}{A_{t}} \frac{1}{\psi^{\psi}(1-\psi)^{1-\psi}}\left(\left(1-\xi_{t}^{L}\right) W_{t}\right)^{\psi}\left(\left(1-\xi_{t}^{K}\right) Z_{t}\right)^{1-\psi} & =\left(1-\xi_{t}\right) M C_{t}, \\
\frac{1}{A_{t}^{*}} \frac{1}{\psi^{\psi}(1-\psi)^{1-\psi}}\left(\left(1-\xi_{t}^{L *}\right) W_{t}^{*}\right)^{\psi}\left(\left(1-\xi_{t}^{K *}\right) Z_{t}^{*}\right)^{1-\psi} & =\left(1-\xi_{t}^{*}\right) M C_{t}^{*},
\end{aligned}
$$

which show that the post-subsidy marginal costs are the same as before. However, the efficiency conditions in (18) - (19) would not be the same with or without subsidies unless the wage and capital rental subsidies are assumed to be the same, i.e. unless $\xi_{t}=\xi_{t}^{L}=\xi_{t}^{K}$ and $\xi_{t}^{*}=\xi_{t}^{L *}=\xi_{t}^{K *}$.

In other words, we impose the assumption that both factors of production are subsidized in the same proportion in order not to distort the allocation of capital and labor while trying to correct for the mark-up distortions with the introduction of these subsidies. Hence, the wage $W_{t}$ and the capital rental rate $Z_{t}$ denote the amounts perceived by the domestic households in the supply of each factor of production, while the wage $W_{t}^{*}$ and the capital rental rate $Z_{t}^{*}$ are the amounts perceived by the foreign households. In turn, the cost of a unit of labor and the cost of renting a unit of capital are $\left(1-\xi_{t}\right) W_{t}$ and $\left(1-\xi_{t}\right) Z_{t}$ for the domestic firms, and similarly $\left(1-\xi_{t}^{*}\right) W_{t}^{*}$ and $\left(1-\xi_{t}^{*}\right) Z_{t}^{*}$ are the corresponding costs for the foreign firms.

The Optimal Pricing Problem. A re-optimizing domestic firm $h$ under LCP pricing chooses a domestic and a foreign price, $\widetilde{P}_{t}(h)$ and $\widetilde{P}_{t}^{*}(h)$, to maximize the expected discounted value of its net profits,

$$
\sum_{\tau=0}^{+\infty} \mathbb{E}_{t}\left\{\alpha^{\tau} M_{t, t+\tau}\left[\begin{array}{l}
\left(\widetilde{C}_{t, t+\tau}(h)+\widetilde{X}_{t, t+\tau}(h)\right)\left(\widetilde{P}_{t}(h)-\left(1-\xi_{t+\tau}\right) M C_{t+\tau}\right)+\ldots \\
\left(\widetilde{C}_{t, t+\tau}^{*}(h)+\widetilde{X}_{t, t+\tau}^{*}(h)\right)\left(S_{t+\tau} \widetilde{P}_{t}^{*}(h)-\left(1-\xi_{t+\tau}\right) M C_{t+\tau}\right)
\end{array}\right]\right\}
$$


where $M_{t, t+\tau} \equiv \beta^{\tau}\left(\frac{C_{t+\tau}}{C_{t}}\right)^{-\sigma^{-1}} \frac{P_{t}}{P_{t+\tau}}$ is the stochastic discount factor (SDF) for $\tau$-periods ahead nominal payoffs (corresponding to the domestic household), subject to a pair of demand constraints in each goods market,

$$
\begin{aligned}
\widetilde{C}_{t, t+\tau}(h)+\widetilde{X}_{t, t+\tau}(h) & =\left(\frac{\widetilde{P}_{t}(h)}{P_{t+\tau}^{H}}\right)^{-\theta}\left(C_{t+\tau}^{H}+X_{t+\tau}^{H}\right), \\
\widetilde{C}_{t, t+\tau}^{*}(h)+\widetilde{X}_{t, t+\tau}^{*}(h) & =\left(\frac{\widetilde{P}_{t}^{*}(h)}{P_{t+\tau}^{H *}}\right)^{-\theta}\left(C_{t+\tau}^{H *}+X_{t+\tau}^{H *}\right) .
\end{aligned}
$$

$\widetilde{C}_{t, t+\tau}(h)$ and $\widetilde{C}_{t, t+\tau}^{*}(h)$ indicate the consumption demand for any variety $h$ at home and abroad respectively, given that prices $\widetilde{P}_{t}(h)$ and $\widetilde{P}_{t}^{*}(h)$ remain unchanged between time $t$ and $t+\tau$. Similarly, $\widetilde{X}_{t, t+\tau}(h)$ and $\widetilde{X}_{t, t+\tau}^{*}(h)$ indicate the households' investment demand. ${ }^{4}$ Similarly, we characterize the objective of the foreign firm as,

$$
\sum_{\tau=0}^{+\infty} \mathbb{E}_{t}\left\{\alpha^{\tau} M_{t, t+\tau}^{*}\left[\begin{array}{c}
\left(\widetilde{C}_{t, t+\tau}(f)+\widetilde{X}_{t, t+\tau}(f)\right)\left(\frac{\widetilde{P}_{t}(f)}{S_{t+\tau}}-\left(1-\xi_{t+\tau}^{*}\right) M C_{t+\tau}^{*}\right)+\ldots \\
\left(\widetilde{C}_{t, t+\tau}^{*}(f)+\widetilde{X}_{t, t+\tau}^{*}(f)\right)\left(\widetilde{P}_{t}^{*}(f)-\left(1-\xi_{t+\tau}^{*}\right) M C_{t+\tau}^{*}\right)
\end{array}\right]\right\}
$$

where $M_{t, t+\tau}^{*} \equiv \beta\left(\frac{C_{t+\tau}^{*}}{C_{t}^{*}}\right)^{-\sigma^{-1}} \frac{P_{t}^{*}}{P_{t+\tau}^{*}}$ is the foreign SDF. The demand constraints of the foreign firm are,

$$
\begin{aligned}
& \widetilde{C}_{t, t+\tau}(f)+\widetilde{X}_{t, t+\tau}(f)=\left(\frac{\widetilde{P}_{t}(f)}{P_{t+\tau}^{F}}\right)^{-\theta}\left(C_{t+\tau}^{F}+X_{t+\tau}^{F}\right), \\
& \widetilde{C}_{t, t+\tau}^{*}(f)+\widetilde{X}_{t, t+\tau}^{*}(f)=\left(\frac{\widetilde{P}_{t}^{*}(f)}{P_{t+\tau}^{F *}}\right)^{-\theta}\left(C_{t+\tau}^{F *}+X_{t+\tau}^{F *}\right),
\end{aligned}
$$

given that prices $\widetilde{P}_{t}(h)$ and $\widetilde{P}_{t}^{*}(h)$ remain unchanged between time $t$ and $t+\tau$.

The Optimal Pricing Problem: The PCP Case. Alternatively, we keep the optimization problem of the firms as before but we replace the assumption of LCP pricing with producer-currency pricing (henceforth, PCP pricing). This special case implies that deviations of the law of one price would not occur even if prices are sticky in either country or in both countries. A re-optimizing domestic firm $h$ under PCP pricing chooses a unique price expressed in the domestic currency for its own variety, $\widetilde{P}_{t}(h)$, irrespective of whether the variety is to be sold in the domestic market or exported to the foreign market, in order to maximize the expected discounted value of its net profits,

$\sum_{\tau=0}^{+\infty} \mathbb{E}_{t}\left\{\alpha^{\tau} M_{t, t+\tau}\left[\left(\left(\widetilde{C}_{t, t+\tau}(h)+\widetilde{X}_{t, t+\tau}(h)\right)+\left(\widetilde{C}_{t, t+\tau}^{*}(h)+\widetilde{X}_{t, t+\tau}^{*}(h)\right)\right)\left(\widetilde{P}_{t}(h)-\left(1-\xi_{t+\tau}\right) M C_{t+\tau}\right)\right]\right\}$,

where $M_{t, t+\tau} \equiv \beta^{\tau}\left(\frac{C_{t+\tau}}{C_{t}}\right)^{-\sigma^{-1}} \frac{P_{t}}{P_{t+\tau}}$ is the stochastic discount factor (SDF) for $\tau$-periods ahead nominal payoffs (corresponding to the domestic household), subject to a pair of demand constraints in each goods

\footnotetext{
${ }^{4}$ We derive the demand for variety $h$ in the home and foreign markets by combining the first-order conditions in (49) - (50), section 2 .
} 
market,

$$
\begin{aligned}
\widetilde{C}_{t, t+\tau}(h)+\widetilde{X}_{t, t+\tau}(h) & =\left(\frac{\widetilde{P}_{t}(h)}{P_{t+\tau}^{H}}\right)^{-\theta}\left(C_{t+\tau}^{H}+X_{t+\tau}^{H}\right), \\
\widetilde{C}_{t, t+\tau}^{*}(h)+\widetilde{X}_{t, t+\tau}^{*}(h) & =\left(\frac{\widetilde{P}_{t}^{*}(h)}{P_{t+\tau}^{H *}}\right)^{-\theta}\left(C_{t+\tau}^{H *}+X_{t+\tau}^{H *}\right) .
\end{aligned}
$$

$\widetilde{C}_{t, t+\tau}(h)$ and $\widetilde{C}_{t, t+\tau}^{*}(h)$ indicate the consumption demand for any variety $h$ at home and abroad respectively, given that prices $\widetilde{P}_{t}(h)$ and $\widetilde{P}_{t}^{*}(h)$ remain unchanged between time $t$ and $t+\tau$. Similarly, $\widetilde{X}_{t, t+\tau}(h)$ and $\widetilde{X}_{t, t+\tau}^{*}(h)$ indicate the households' investment demand. ${ }^{5}$ The price paid for the domestic variety in the foreign market expressed in units of the foreign currency, $\widetilde{P}_{t}^{*}(h)$, must satisfy under PCP that $P_{t+\tau}(h)=$ $S_{t+\tau} P_{t+\tau}^{*}(h)$. Moreover, that has to be the case also when prices are sticky, i.e. $\widetilde{P}_{t}(h)=S_{t+\tau} \widetilde{P}_{t}^{*}(h)$. Given the aggregation rules in (13) - (14), then it can easily be shown that,

$$
P_{t}^{H}=S_{t} P_{t}^{H *}
$$

In other words, the law of one price holds at the variety level and so it does when those varieties are aggregated in a bundle of domestic goods. However, this result holds true because the bundle of home goods is exactly the same in the domestic and foreign country. PPP can still fail to hold at the CPI level if the aggregation between domestic and foreign bundles of goods differs across countries (that is, if their consumption baskets are different) as in our model. Using the law of one price, we can re-write the demand constraints of the domestic firm jointly as follows,

$$
\left(\widetilde{C}_{t, t+\tau}(h)+\widetilde{X}_{t, t+\tau}(h)\right)+\left(\widetilde{C}_{t, t+\tau}^{*}(h)+\widetilde{X}_{t, t+\tau}^{*}(h)\right)=\left(\frac{\widetilde{P}_{t}(h)}{P_{t+\tau}^{H}}\right)^{-\theta}\left(\left(C_{t+\tau}^{H}+X_{t+\tau}^{H}\right)+\left(C_{t+\tau}^{H *}+X_{t+\tau}^{H *}\right)\right) .
$$

Similarly, we characterize the objective of the foreign firm as,

$\sum_{\tau=0}^{+\infty} \mathbb{E}_{t}\left\{\alpha^{\tau} M_{t, t+\tau}^{*}\left[\left(\left(\widetilde{C}_{t, t+\tau}(f)+\widetilde{X}_{t, t+\tau}(h)\right)+\left(\widetilde{C}_{t, t+\tau}^{*}(f)+\widetilde{X}_{t, t+\tau}^{*}(h)\right)\right)\left(\widetilde{P}_{t}^{*}(f)-\left(1-\xi_{t+\tau}^{*}\right) M C_{t+\tau}^{*}\right)\right]\right\}$

where $M_{t, t+\tau}^{*} \equiv \beta\left(\frac{C_{t+\tau}^{*}}{C_{t}^{*}}\right)^{-\sigma^{-1}} \frac{P_{t}^{*}}{P_{t+\tau}^{*}}$ is the foreign SDF. The demand constraints of the foreign firm are,

$$
\begin{aligned}
& \widetilde{C}_{t, t+\tau}(f)+\widetilde{X}_{t, t+\tau}(f)=\left(\frac{\widetilde{P}_{t}(f)}{P_{t+\tau}^{F}}\right)^{-\theta}\left(C_{t+\tau}^{F}+X_{t+\tau}^{F}\right), \\
& \widetilde{C}_{t, t+\tau}^{*}(f)+\widetilde{X}_{t, t+\tau}^{*}(f)=\left(\frac{\widetilde{P}_{t}^{*}(f)}{P_{t+\tau}^{F *}}\right)^{-\theta}\left(C_{t+\tau}^{F *}+X_{t+\tau}^{F *}\right),
\end{aligned}
$$

given that prices $\widetilde{P}_{t}(h)$ and $\widetilde{P}_{t}^{*}(h)$ remain unchanged between time $t$ and $t+\tau$. The price paid for the foreign variety in the domestic market expressed in units of the domestic currency, $\widetilde{P}_{t}(f)$, must satisfy under PCP

\footnotetext{
${ }^{5}$ We derive the demand for variety $h$ in the home and foreign markets by combining the first-order conditions in (49) - (50), section 2 .
} 
that $\frac{P_{t+\tau}(f)}{S_{t+\tau}}=P_{t+\tau}^{*}(f)$. Moreover, that has to be the case also when prices are sticky, i.e. $\frac{\widetilde{P}_{t}(f)}{S_{t+\tau}}=\widetilde{P}_{t}^{*}(f)$. Given the aggregation rules in (13) - (14), then it can easily be shown that,

$$
\frac{P_{t}^{F}}{S_{t}}=P_{t}^{F *}
$$

Using the law of one price, we can re-write the demand constraints of the foreign firm jointly as follows,

$$
\left(\widetilde{C}_{t, t+\tau}(f)+\widetilde{X}_{t, t+\tau}(f)\right)+\left(\widetilde{C}_{t, t+\tau}^{*}(f)+\widetilde{X}_{t, t+\tau}^{*}(f)\right)=\left(\frac{\widetilde{P}_{t}^{*}(f)}{P_{t+\tau}^{F *}}\right)^{-\theta}\left(\left(C_{t+\tau}^{F}+X_{t+\tau}^{F}\right)+\left(C_{t+\tau}^{F *}+X_{t+\tau}^{F *}\right)\right) .
$$

\subsection{The Monetary Policy Rules}

The Taylor rule is often defined as the trademark of modern monetary policy. In that case the policy instrument of the domestic and foreign monetary authorities are the short-term rates $I_{t}$ and $I_{t}^{*}$ respectively, while $\bar{I}$ and $\bar{I}^{*}$ are their corresponding steady state values. We assume that the monetary authorities set short-term nominal interest rates according to Taylor (1993) type rules,

$$
\begin{aligned}
I_{t} & =M_{t}\left(I_{t-1}\right)^{\rho_{i}}\left[\bar{I}\left(\Pi_{t}\right)^{\psi_{\pi}}\left(\frac{Y_{t}}{\bar{Y}}\right)^{\psi_{y}}\right]^{1-\rho_{i}}, \\
I_{t}^{*} & =M_{t}^{*}\left(I_{t-1}^{*}\right)^{\rho_{i}}\left[\bar{I}^{*}\left(\Pi_{t}^{*}\right)^{\psi_{\pi}}\left(\frac{Y_{t}^{*}}{\bar{Y}^{*}}\right)^{\psi_{y}}\right]^{1-\rho_{i}},
\end{aligned}
$$

where $M_{t}$ and $M_{t}^{*}$ are the (domestic and foreign) monetary policy shocks or the shocks to the interest rate feedback rule, $\Pi_{t} \equiv \frac{P_{t}}{P_{t-1}}$ and $\Pi_{t}^{*} \equiv \frac{P_{t}^{*}}{P_{t-1}^{*}}$ are the (gross) CPI inflation rates, and $\frac{Y_{t}}{\bar{Y}}$ and $\frac{Y_{t}^{*}}{\bar{Y}^{*}}$ are the output levels in deviations from their steady state. The index captures both a smoothing term and a systematic policy component. This index specification of the Taylor rule takes a more standard form once it is loglinearized.

The fiscal policy in each country is characterized by balanced budgets in every period, and a subsidy to the production costs of the local producers that is fully financed by a lump-sum tax on households. We summarize the government's budget constraints as,

$$
\begin{aligned}
T_{t} & =\xi_{t} M C_{t} \int_{0}^{1} Y_{t}(h) d h, \\
T_{t}^{*} & =\xi_{t}^{*} M C_{t}^{*} \int_{0}^{1} Y_{t}^{*}(f) d f,
\end{aligned}
$$

where $T_{t}$ and $T_{t}^{*}$ are the domestic and foreign lump-sum taxes on households, and $\xi_{t}$ and $\xi_{t}^{*}$ are the domestic and foreign subsidies expressed as a fraction of production costs. Given the production functions in (16)-(17), the pre-subsidy marginal cost equations in (20) - (21) and the fact that capital-to-labor ratios in (18) - (19) are equalized across firms within each country, it is possible to write the government budget constraints as 
follows,

$$
\begin{aligned}
T_{t} & =\xi_{t} M C_{t} A_{t}\left(\frac{K_{t}}{L_{t}}\right)^{1-\psi} \int_{0}^{1} L_{t}(h) d h \\
& =\xi_{t} M C_{t} A_{t}\left(K_{t}\right)^{1-\psi}\left(L_{t}\right)^{\psi} \\
& =\xi_{t} \frac{1}{\psi^{\psi}(1-\psi)^{1-\psi}}\left(W_{t} L_{t}\right)^{\psi}\left(Z_{t} K_{t}\right)^{1-\psi}, \\
T_{t}^{*} & =\xi_{t}^{*} M C_{t}^{*} A_{t}^{*}\left(\frac{K_{t}^{*}}{L_{t}^{*}}\right)^{1-\psi} \int_{0}^{1} L_{t}^{*}(f) d f \\
& =\xi_{t}^{*} M C_{t}^{*} A_{t}^{*}\left(K_{t}^{*}\right)^{1-\psi}\left(L_{t}^{*}\right)^{\psi} \\
& =\xi_{t}^{*} \frac{1}{\psi^{\psi}(1-\psi)^{1-\psi}}\left(W_{t}^{*} L_{t}^{*}\right)^{\psi}\left(Z_{t}^{*} K_{t}^{*}\right)^{1-\psi},
\end{aligned}
$$

where the second equality follows also from the labor market clearing conditions (i.e. $L_{t}=\int_{0}^{1} L_{t}(h) d h$ and $\left.L_{t}^{*}=\int_{0}^{1} L_{t}^{*}(f) d f\right)$. As these government budget constraints illustrate, the value of the subsidy can be expressed as a share of an aggregate function of the labor income, $W_{t} L_{t}$ and $W_{t}^{*} L_{t}^{*}$ respectively, and the capital rental income, $Z_{t} K_{t}$ and $Z_{t}^{*} K_{t}^{*}$ respectively. As expected, in the limit whenever the labor share converges to one, i.e. $\psi \rightarrow 1$, the technology becomes linear-in-labor, and the subsidy becomes equal to a fraction of the labor income for each country.

In Martínez-García and Søndergaard (2008) this tax subsidy is completely ignored, so the implicit assumption is that $\xi_{t}=\xi_{t}^{*}=0$ and $T_{t}=T_{t}^{*}=0$. Often, however, these subsidies are used to neutralize the mark-up distortion introduced by the assumption that firms produce and sell their varieties under monopolistic competition. In order to eliminate this distortion, suffices to set the subsidy in each country to satisfy the following pair of conditions,

$$
\begin{aligned}
& \frac{\theta}{\theta-1}\left(1-\xi_{t}\right)=1 \\
& \frac{\theta}{\theta-1}\left(1-\xi_{t}^{*}\right)=1 .
\end{aligned}
$$

From here it follows that the subsidy for both countries is characterized as,

$$
\xi_{t}=\xi_{t}^{*}=\frac{1}{\theta}<1
$$

which is a function of the elasticity of substitution across varieties produced within a country, i.e. $\theta>1$. The mark-up is also a function solely of the elasticity of substitution across varieties. Since the elasticity is time-invariant, so are the mark-ups and the subsidies (expressed as a share of the production costs) needed.

For simplicity, we treat the subsidies as a secondary policy instrument intended exclusively to deal with the mark-up distortion and, therefore, we assume from now on that the subsidy as a fraction of the production costs will be invariant over time and identical across countries, i.e.

$$
\xi_{t}=\xi_{t}^{*}=\bar{\xi} \in\left[0, \frac{1}{\theta}\right]
$$


where $\bar{\xi}$ also denotes the steady state subsidy in both countries. In turn, the mark-up and the subsidyindependently of whether the subsidy is set to zero or neutralizes partially or totally the mark-up distortiononly affect the dynamics of the model up to a first-order approximation because those terms enter into the computations of the steady state investment share. Rather than choosing a specific value for the subsidy, we view its share over the production costs as another structural parameter of the model.

\section{The Optimality Conditions}

Here, we present the relevant equilibrium conditions of the model. Since the model is built around two mostly symmetric countries, all the first-order conditions reported correspond to the home country unless otherwise noted.

The Optimality Conditions from the Households' Problem. Given the structure described in (7) (8), the solution to the sub-utility maximization problem implies that the home and foreign households' demands for each variety are given by,

$$
\begin{aligned}
& C_{t}(h)=\left(\frac{P_{t}(h)}{P_{t}^{H}}\right)^{-\theta} C_{t}^{H}, X_{t}(h)=\left(\frac{P_{t}(h)}{P_{t}^{H}}\right)^{-\theta} X_{t}^{H}, \forall h \in[0,1], \\
& C_{t}(f)=\left(\frac{P_{t}(f)}{P_{t}^{F}}\right)^{-\theta} C_{t}^{F}, X_{t}(f)=\left(\frac{P_{t}(f)}{P_{t}^{F}}\right)^{-\theta} X_{t}^{F}, \forall f \in[0,1],
\end{aligned}
$$

while the demands for the bundles of home and foreign goods are simply equal to,

$$
\begin{aligned}
C_{t}^{H} & =\phi_{H}\left(\frac{P_{t}^{H}}{P_{t}}\right)^{-\eta} C_{t}, X_{t}^{H}=\phi_{H}\left(\frac{P_{t}^{H}}{P_{t}}\right)^{-\eta} X_{t}, \\
C_{t}^{F} & =\phi_{F}\left(\frac{P_{t}^{F}}{P_{t}}\right)^{-\eta} C_{t}, X_{t}^{F}=\phi_{F}\left(\frac{P_{t}^{F}}{P_{t}}\right)^{-\eta} X_{t} .
\end{aligned}
$$

These equations, combined with the analogous counterparts for the foreign country, determine the demand functions in the model.

Under complete international asset markets, the intertemporal first-order conditions for an interior solution result in the following (well-known) equilibrium condition on perfect international risk-sharing (derived by backward induction),

$$
R S_{t}=\nu\left(\frac{C_{t}^{*}}{C_{t}}\right)^{-\gamma}
$$

where $\nu \equiv \frac{S_{0} P_{0}^{*}}{P_{0}}\left(\frac{C_{0}^{*}}{C_{0}}\right)^{\gamma}$ is a constant that depends on the initial conditions. The intertemporal conditions also pin down the price of any given Arrow-Debreu security. Let $I_{t}$ be the (gross) one-period riskless nominal interest rate in terms of the domestic currency, and $I_{t}^{*}$ be the corresponding rate in terms of the foreign currency. Under complete asset markets, we can price a one-period nominal (uncontingent) bond using the 
price of the contingent claims available and obtain that,

$$
\begin{aligned}
\frac{1}{I_{t}} & =\beta \mathbb{E}_{t}\left[\left(\frac{C_{t+1}}{C_{t}}\right)^{-\sigma^{-1}} \frac{P_{t}}{P_{t+1}}\right], \\
\frac{1}{I_{t}^{*}} & =\beta \mathbb{E}_{t}\left[\left(\frac{C_{t+1}^{*}}{C_{t}^{*}}\right)^{-\sigma^{-1}} \frac{P_{t}^{*}}{P_{t+1}^{*}}\right] .
\end{aligned}
$$

The equilibrium conditions of the households' problem also include a pair of labor supply functions (the intratemporal first-order conditions) which can be expressed as,

$$
\begin{aligned}
\frac{W_{t}}{P_{t}} & =\left(C_{t}\right)^{\sigma^{-1}}\left(L_{t}^{s}\right)^{\varphi}, \\
\frac{W_{t}^{*}}{P_{t}^{*}} & =\left(C_{t}^{*}\right)^{\sigma^{-1}}\left(L_{t}^{s *}\right)^{\varphi},
\end{aligned}
$$

plus the appropriate no-Ponzi games, transversality conditions, the budget constraints and the law of motions for capital in both countries. Finally, the equilibrium conditions are completed with a pair of equations that account for the capital-investment decisions of households. The capital-investment conditions, however, depend on the choice of the adjustment cost function $\Phi(\cdot)$.

The domestic households' maximization problem can be summarized generically in the following Lagrangian,

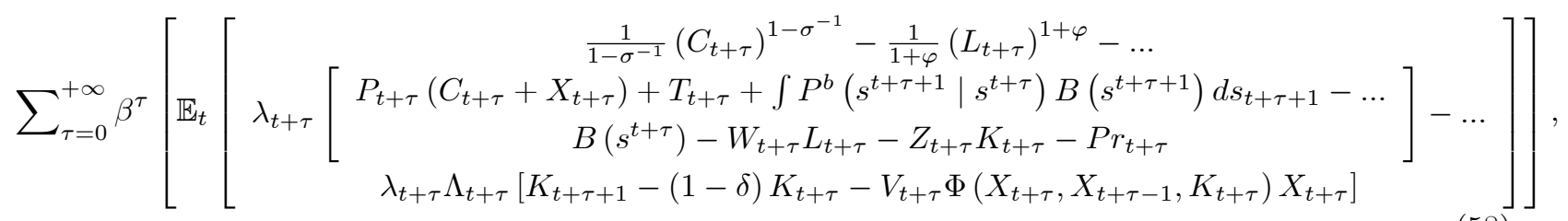

and the capital-investment decisions can be represented with the following set of equilibrium conditions,

$$
\begin{aligned}
C_{t}: & \lambda_{t} P_{t}=\left(C_{t}\right)^{-\sigma^{-1}} \\
K_{t+1} & : \quad \Lambda_{t}=\beta \mathbb{E}_{t}\left[\frac{\lambda_{t+1}}{\lambda_{t}} Z_{t+1}+\frac{\lambda_{t+1}}{\lambda_{t}} \Lambda_{t+1}\left((1-\delta)+V_{t+1} \frac{\partial \Phi\left(X_{t+1}, X_{t}, K_{t+1}\right)}{\partial K_{t+1}} X_{t+1}\right)\right] \\
X_{t} & : \quad 1=\frac{\Lambda_{t}}{P_{t}} V_{t}\left[\Phi\left(X_{t}, X_{t-1}, K_{t}\right)+\frac{\partial \Phi\left(X_{t}, X_{t-1}, K_{t}\right)}{\partial X_{t}} X_{t}\right]+\beta \mathbb{E}_{t}\left[\frac{\lambda_{t+1} \Lambda_{t+1}}{\lambda_{t} P_{t}} V_{t+1} \frac{\partial \Phi\left(X_{t+1}, X_{t}, K_{t+1}\right)}{\partial X_{t}} X_{t+1}\right] .
\end{aligned}
$$

Let us define Tobin's q as $Q_{t} \equiv \frac{\Lambda_{t}}{P_{t}}$. Then, after further manipulation, it is possible to re-write the equilibrium conditions as,

$$
\begin{aligned}
Q_{t} & =\beta \mathbb{E}_{t}\left\{\left(\frac{C_{t+1}}{C_{t}}\right)^{-\sigma^{-1}}\left[\frac{Z_{t+1}}{P_{t+1}}+Q_{t+1}\left((1-\delta)+V_{t+1} \frac{\partial \Phi\left(X_{t+1}, X_{t}, K_{t+1}\right)}{\partial K_{t+1}} X_{t+1}\right)\right]\right\} \\
Q_{t} V_{t} & {\left[\Phi\left(X_{t}, X_{t-1}, K_{t}\right)+\frac{\partial \Phi\left(X_{t}, X_{t-1}, K_{t}\right)}{\partial X_{t}} X_{t}\right] } \\
& =1-\beta \mathbb{E}_{t}\left\{\left(\frac{C_{t+1}}{C_{t}}\right)^{-\sigma^{-1}}\left[Q_{t+1} V_{t+1} \frac{\partial \Phi\left(X_{t+1}, X_{t}, K_{t+1}\right)}{\partial X_{t}} X_{t+1}\right]\right\} .
\end{aligned}
$$


Under no adjustment costs (NAC), the pair of conditions added to account for the capital-investment decisions of the domestic households are summarized as,

$$
\begin{aligned}
Q_{t} & =\beta \mathbb{E}_{t}\left\{\left(\frac{C_{t+1}}{C_{t}}\right)^{-\sigma^{-1}}\left[\frac{Z_{t+1}}{P_{t+1}}+Q_{t+1}(1-\delta)\right]\right\}, \\
Q_{t} & =\frac{1}{V_{t}} .
\end{aligned}
$$

A similar set of derivations allows us to write the following system of equations for Tobin's q in the foreign country,

$$
\begin{aligned}
& Q_{t}^{*}=\beta \mathbb{E}_{t}\left\{\left(\frac{C_{t+1}^{*}}{C_{t}^{*}}\right)^{-\sigma^{-1}}\left[\frac{Z_{t+1}^{*}}{P_{t+1}^{*}}+Q_{t+1}^{*}(1-\delta)\right]\right\}, \\
& Q_{t}^{*}=\frac{1}{V_{t}^{*}} .
\end{aligned}
$$

The Lagrange multiplier on the law of motion relative to the Lagrange multiplier on the budget constraint expressed in real terms, denoted $Q_{t}$ and $Q_{t}^{*}$ respectively, has the interpretation of being the real shadow value of an additional unit of capital (or Tobin's q). In the neoclassical case is well-known that Tobin's q is exactly equal to one, as these equations prove, without adjustment costs and IST shocks.

Under capital adjustment costs (CAC), the pair of conditions added to account for the capital-investment decisions of the domestic households are,

$$
\begin{aligned}
Q_{t} & =\beta \mathbb{E}_{t}\left\{\left(\frac{C_{t+1}}{C_{t}}\right)^{-\sigma^{-1}}\left[\frac{Z_{t+1}}{P_{t+1}}+Q_{t+1}\left((1-\delta)-V_{t+1} \Phi^{\prime}\left(\frac{X_{t+1}}{K_{t+1}}\right)\left(\frac{X_{t+1}}{K_{t+1}}\right)^{2}\right)\right]\right\}, \\
Q_{t} & =\frac{1}{V_{t}}\left[\Phi\left(\frac{X_{t}}{K_{t}}\right)+\Phi^{\prime}\left(\frac{X_{t}}{K_{t}}\right) \frac{X_{t}}{K_{t}}\right]^{-1} .
\end{aligned}
$$

A similar set of derivations allows us to write the following system of equations for Tobin's q in the foreign country,

$$
\begin{aligned}
Q_{t}^{*} & =\beta \mathbb{E}_{t}\left\{\left(\frac{C_{t+1}^{*}}{C_{t}^{*}}\right)^{-\sigma^{-1}}\left[\frac{Z_{t+1}^{*}}{P_{t+1}^{*}}+Q_{t+1}^{*}\left((1-\delta)-V_{t+1}^{*} \Phi^{\prime}\left(\frac{X_{t+1}^{*}}{K_{t+1}^{*}}\right)\left(\frac{X_{t+1}^{*}}{K_{t+1}^{*}}\right)^{2}\right)\right]\right\}, \\
Q_{t}^{*} & =\frac{1}{V_{t}^{*}}\left[\Phi\left(\frac{X_{t}^{*}}{K_{t}^{*}}\right)+\Phi^{\prime}\left(\frac{X_{t}^{*}}{K_{t}^{*}}\right) \frac{X_{t}^{*}}{K_{t}^{*}}\right]^{-1} .
\end{aligned}
$$

The Lagrange multiplier on the law of motion relative to the Lagrange multiplier on the budget constraint expressed in real terms, denoted $Q_{t}$ and $Q_{t}^{*}$ respectively, has the interpretation of being the real shadow value of an additional unit of capital (or Tobin's q). Under investment adjustment costs (IAC), the pair of 
conditions added to account for the capital-investment decisions of the domestic households are,

$$
\begin{aligned}
Q_{t} & =\beta \mathbb{E}_{t}\left\{\left(\frac{C_{t+1}}{C_{t}}\right)^{-\sigma^{-1}}\left[\frac{Z_{t+1}}{P_{t+1}}+Q_{t+1}(1-\delta)\right]\right\}, \\
Q_{t} & =\frac{1}{V_{t}}\left[\Phi\left(\frac{X_{t}}{X_{t-1}}\right)+\Phi^{\prime}\left(\frac{X_{t}}{X_{t-1}}\right) \frac{X_{t}}{X_{t-1}}\right]^{-1}\left[\beta \mathbb{E}_{t}\left\{\left(\frac{C_{t+1}}{C_{t}}\right)^{-\sigma^{-1}}\left[Q_{t+1} V_{t+1} \Phi^{\prime}\left(\frac{X_{t+1}}{X_{t}}\right)\left(\frac{X_{t+1}}{X_{t}}\right)^{2}\right]\right\}(68)\right.
\end{aligned}
$$

A similar set of derivations allows us to write the following system of equations for Tobin's q in the foreign country,

$$
\begin{aligned}
Q_{t}^{*} & =\beta \mathbb{E}_{t}\left\{\left(\frac{C_{t+1}^{*}}{C_{t}^{*}}\right)^{-\sigma^{-1}}\left[\frac{Z_{t+1}^{*}}{P_{t+1}^{*}}+Q_{t+1}^{*}(1-\delta)\right]\right\} \\
Q_{t}^{*} & =\frac{1}{V_{t}^{*}}\left[\Phi\left(\frac{X_{t}^{*}}{X_{t-1}^{*}}\right)+\Phi^{\prime}\left(\frac{X_{t}^{*}}{X_{t-1}^{*}}\right) \frac{X_{t}^{*}}{X_{t-1}^{*}}\right]^{-1}\left[\beta \mathbb{E}_{t}\left\{\left(\frac{C_{t+1}^{*}}{C_{t}^{*}}\right)^{-\sigma^{-1}}\left[Q_{t+1}^{*} V_{t+1}^{*} \Phi^{\prime}\left(\frac{X_{t+1}^{*}}{X_{t}^{*}}\right)\left(\frac{X_{t+1}^{*}}{X_{t}^{*}}\right)^{2}\right]\right\}\right. \text { (to.) }
\end{aligned}
$$

Once again, the Lagrange multiplier on the law of motion relative to the Lagrange multiplier on the budget constraint expressed in real terms, denoted $Q_{t}$ and $Q_{t}^{*}$ respectively, has the interpretation of being the real shadow value of an additional unit of capital (or Tobin's q).

The Optimality Conditions from the Firms' Problem. The necessary and sufficient first-order conditions for the domestic firm producing variety $h$ give us the following pair of price-setting formulas,

$$
\begin{aligned}
\sum_{\tau=0}^{+\infty} \alpha^{\tau} \mathbb{E}_{t}\left[M_{t, t+\tau}\left(\widetilde{C}_{t, t+\tau}(h)+\widetilde{X}_{t, t+\tau}(h)\right)\left(\widetilde{P}_{t}(h)-\frac{\theta}{\theta-1}\left(1-\xi_{t+\tau}\right) M C_{t+\tau}\right)\right] & =0 \\
\sum_{\tau=0}^{+\infty} \alpha^{\tau} \mathbb{E}_{t}\left[M_{t, t+\tau}\left(\widetilde{C}_{t, t+\tau}^{*}(h)+\widetilde{X}_{t, t+\tau}^{*}(h)\right)\left(S_{t+\tau} \widetilde{P}_{t}^{*}(h)-\frac{\theta}{\theta-1}\left(1-\xi_{t+\tau}\right) M C_{t+\tau}\right)\right] & =0 .
\end{aligned}
$$

Similarly, the first-order conditions for the foreign firm producing variety $f$ give us the following price-setting formulas,

$$
\begin{aligned}
& \sum_{\tau=0}^{+\infty} \alpha^{\tau} \mathbb{E}_{t}\left[M_{t, t+\tau}^{*}\left(\widetilde{C}_{t, t+\tau}(f)+\widetilde{X}_{t, t+\tau}(f)\right)\left(\frac{\widetilde{P}_{t}(f)}{S_{t+\tau}}-\frac{\theta}{\theta-1}\left(1-\xi_{t+\tau}^{*}\right) M C_{t+\tau}^{*}\right)\right]=0 \\
& \sum_{\tau=0}^{+\infty} \alpha^{\tau} \mathbb{E}_{t}\left[M_{t, t+\tau}^{*}\left(\widetilde{C}_{t, t+\tau}^{*}(f)+\widetilde{X}_{t, t+\tau}^{*}(f)\right)\left(\widetilde{P}_{t}^{*}(f)-\frac{\theta}{\theta-1}\left(1-\xi_{t+\tau}^{*}\right) M C_{t+\tau}^{*}\right)\right]=0 .
\end{aligned}
$$

Using the law of large numbers and the inherent symmetry of the firms' problem, the price sub-indexes on domestic varieties, $P_{t}^{H}$ and $P_{t}^{H *}$, become,

$$
\begin{aligned}
P_{t}^{H} & =\left[\alpha\left(P_{t-1}^{H}\right)^{1-\theta}+(1-\alpha)\left(\widetilde{P}_{t}(h)\right)^{1-\theta}\right]^{\frac{1}{1-\theta}}, \\
P_{t}^{H *} & =\left[\alpha\left(P_{t-1}^{H *}\right)^{1-\theta}+(1-\alpha)\left(\widetilde{P}_{t}^{*}(h)\right)^{1-\theta}\right]^{\frac{1}{1-\theta}},
\end{aligned}
$$


while the price sub-indexes on foreign varieties, $P_{t}^{F}$ and $P_{t}^{F *}$, are computed as,

$$
\begin{aligned}
P_{t}^{F} & =\left[\alpha\left(P_{t-1}^{F}\right)^{1-\theta}+(1-\alpha)\left(\widetilde{P}_{t}(f)\right)^{1-\theta}\right]^{\frac{1}{1-\theta}}, \\
P_{t}^{F *} & =\left[\alpha\left(P_{t-1}^{F *}\right)^{1-\theta}+(1-\alpha)\left(\widetilde{P}_{t}^{*}(f)\right)^{1-\theta}\right]^{\frac{1}{1-\theta}} .
\end{aligned}
$$

Equations $(75)-(78)$ are a convenient way to reformulate (13) - (14).

The Optimality Conditions from the Firms' Problem: The PCP Case. The necessary and sufficient first-order conditions for the domestic firm producing variety $h$ under PCP give us the following pair of price-setting formulas,

$$
\begin{aligned}
& \sum_{\tau=0}^{+\infty} \alpha^{\tau} \mathbb{E}_{t}\left[M_{t, t+\tau}\left(\begin{array}{c}
\left(\widetilde{C}_{t, t+\tau}(h)+\widetilde{X}_{t, t+\tau}(h)\right)+\ldots \\
\left(\widetilde{C}_{t, t+\tau}^{*}(h)+\widetilde{X}_{t, t+\tau}^{*}(h)\right)
\end{array}\right)\left(\begin{array}{c}
\widetilde{P}_{t}(h)-\ldots \\
\frac{\theta}{\theta-1}\left(1-\xi_{t+\tau}\right) M C_{t+\tau}
\end{array}\right)\right]=0, \\
& \widetilde{P}_{t}(h)=S_{t+\tau} \widetilde{P}_{t}^{*}(h) .
\end{aligned}
$$

Similarly, the first-order conditions for the foreign firm producing variety $f$ under PCP give us the following price-setting formulas,

$$
\begin{aligned}
& \frac{\widetilde{P}_{t}(f)}{S_{t+\tau}}=\widetilde{P}_{t}^{*}(f) \\
& \sum_{\tau=0}^{+\infty} \alpha^{\tau} \mathbb{E}_{t}\left[M_{t, t+\tau}^{*}\left(\begin{array}{c}
\left(\widetilde{C}_{t, t+\tau}(f)+\widetilde{X}_{t, t+\tau}(f)\right)+\ldots \\
\left(\widetilde{C}_{t, t+\tau}^{*}(f)+\widetilde{X}_{t, t+\tau}^{*}(f)\right)
\end{array}\right)\left(\begin{array}{c}
\widetilde{P}_{t}^{*}(f)-\ldots \\
\frac{\theta}{\theta-1}\left(1-\xi_{t+\tau}^{*}\right) M C_{t+\tau}^{*}
\end{array}\right)\right]=0 .
\end{aligned}
$$

Using the law of large numbers and the inherent symmetry of the firms' problem, the price sub-indexes on domestic varieties, $P_{t}^{H}$ and $P_{t}^{H *}$, become,

$$
\begin{aligned}
P_{t}^{H} & =\left[\alpha\left(P_{t-1}^{H}\right)^{1-\theta}+(1-\alpha)\left(\widetilde{P}_{t}(h)\right)^{1-\theta}\right]^{\frac{1}{1-\theta}}=S_{t} P_{t}^{H *} \text { since } P_{t}(h)=S_{t} P_{t}^{*}(h), \\
P_{t}^{H *} & =\left[\alpha\left(P_{t-1}^{H *}\right)^{1-\theta}+(1-\alpha)\left(\widetilde{P}_{t}^{*}(h)\right)^{1-\theta}\right]^{\frac{1}{1-\theta}},
\end{aligned}
$$

while the price sub-indexes on foreign varieties, $P_{t}^{F}$ and $P_{t}^{F *}$, are computed as,

$$
\begin{aligned}
P_{t}^{F} & =\left[\alpha\left(P_{t-1}^{F}\right)^{1-\theta}+(1-\alpha)\left(\widetilde{P}_{t}(f)\right)^{1-\theta}\right]^{\frac{1}{1-\theta}} \\
P_{t}^{F *} & =\left[\alpha\left(P_{t-1}^{F *}\right)^{1-\theta}+(1-\alpha)\left(\widetilde{P}_{t}^{*}(f)\right)^{1-\theta}\right]^{\frac{1}{1-\theta}}=\frac{P_{t}^{F}}{S_{t}} \text { since } \frac{P_{t}(f)}{S_{t}}=P_{t}^{*}(f) .
\end{aligned}
$$

Equations $(83)-(86)$ are a convenient way to reformulate (13) - (14).

Aggregate Output and Real Rental Rates on Capital. Equations (49) - (52) determine the demand function for each variety. Those demand functions coupled with the market clearing conditions at the variety 
level allows us to aggregate output from the demand-side as follows,

$$
\begin{aligned}
Y_{t} & =\int_{0}^{1}\left[C_{t}(h)+X_{t}(h)+C_{t}^{*}(h)+X_{t}^{*}(h)\right] d h \\
& =\left[\int_{0}^{1}\left(\frac{P_{t}(h)}{P_{t}^{H}}\right)^{-\theta} d h\right] \phi_{H}\left(\frac{P_{t}^{H}}{P_{t}}\right)^{-\eta}\left(C_{t}+X_{t}\right)+\left[\int_{0}^{1}\left(\frac{P_{t}^{*}(h)}{P_{t}^{H *}}\right)^{-\theta} d h\right] \phi_{H}^{*}\left(\frac{P_{t}^{H *}}{P_{t}^{*}}\right)^{-\eta}\left(C_{t}^{*}+X_{t}^{*}\right), \\
Y_{t}^{*} & =\int_{0}^{1}\left[C_{t}(f)+X_{t}(f)+C_{t}^{*}(f)+X_{t}^{*}(f)\right] d f \\
& =\left[\int_{0}^{1}\left(\frac{P_{t}(f)}{P_{t}^{F}}\right)^{-\theta} d f\right] \phi_{F}\left(\frac{P_{t}^{F}}{P_{t}}\right)^{-\eta}\left(C_{t}+X_{t}\right)+\left[\int_{0}^{1}\left(\frac{P_{t}^{*}(f)}{P_{t}^{F *}}\right)^{-\theta} d f\right] \phi_{F}^{*}\left(\frac{P_{t}^{F *}}{P_{t}^{*}}\right)^{-\eta}\left(C_{t}^{*}+X_{t}^{*}\right)
\end{aligned}
$$

Equations (87) - (88) tie the aggregate output demand in both countries to aggregate consumption, aggregate investment as well as to optimal prices. In the PCP case where the law of one price holds at the variety level, we can re-express these aggregate output equations as,

$$
\begin{aligned}
Y_{t} & =\int_{0}^{1}\left[C_{t}(h)+X_{t}(h)+C_{t}^{*}(h)+X_{t}^{*}(h)\right] d h \\
& =\left[\int_{0}^{1}\left(\frac{P_{t}(h)}{P_{t}^{H}}\right)^{-\theta} d h\right]\left(\frac{P_{t}^{H}}{P_{t}}\right)^{-\eta}\left[\phi_{H}\left(C_{t}+X_{t}\right)+\phi_{H}^{*}\left(R S_{t}\right)^{\eta}\left(C_{t}^{*}+X_{t}^{*}\right)\right], \\
Y_{t}^{*} & =\int_{0}^{1}\left[C_{t}(f)+X_{t}(f)+C_{t}^{*}(f)+X_{t}^{*}(f)\right] d f \\
& =\left[\int_{0}^{1}\left(\frac{P_{t}^{*}(f)}{P_{t}^{F *}}\right)^{-\theta} d f\right]\left(\frac{P_{t}^{F *}}{P_{t}^{*}}\right)^{-\eta}\left[\phi_{F}\left(R S_{t}\right)^{-\eta}\left(C_{t}+X_{t}\right)+\phi_{F}^{*}\left(C_{t}^{*}+X_{t}^{*}\right)\right] .
\end{aligned}
$$

Given the production functions in (16) - (17) and the fact that capital-to-labor ratios are equalized across firms within each country, it is possible to write the following aggregate output equations,

$$
\begin{aligned}
Y_{t} & =A_{t}\left(K_{t}\right)^{1-\psi}\left(L_{t}\right)^{\psi}, \\
Y_{t}^{*} & =A_{t}^{*}\left(K_{t}^{*}\right)^{1-\psi}\left(L_{t}^{*}\right)^{\psi} .
\end{aligned}
$$

Combining these aggregate production functions with the efficiency conditions in (18) - (19) and the labor supply equations from the households' problem (as in equations (56) - (57)), we can express the real rental rate of capital in terms of productivity shocks, consumption, output and the stock of capital,

$$
\begin{aligned}
\frac{Z_{t}}{P_{t}} & =\frac{1-\psi}{\psi} \frac{W_{t}}{P_{t}} \frac{L_{t}}{K_{t}}=\frac{1-\psi}{\psi}\left(A_{t}\right)^{-\frac{1+\varphi}{\psi}}\left(C_{t}\right)^{\sigma^{-1}}\left(Y_{t}\right)^{\frac{1+\varphi}{\psi}}\left(K_{t}\right)^{-\left(\frac{1+(1-\psi) \varphi}{\psi}\right)} \\
\frac{Z_{t}^{*}}{P_{t}^{*}} & =\frac{1-\psi}{\psi} \frac{W_{t}^{*}}{P_{t}^{*}} \frac{L_{t}^{*}}{K_{t}^{*}}=\frac{1-\psi}{\psi}\left(A_{t}^{*}\right)^{-\frac{1+\varphi}{\psi}}\left(C_{t}^{*}\right)^{\sigma^{-1}}\left(Y_{t}^{*}\right)^{\frac{1+\varphi}{\psi}}\left(K_{t}^{*}\right)^{-\left(\frac{1+(1-\psi) \varphi}{\psi}\right)} .
\end{aligned}
$$

These two equations summarize the efficiency condition that requires the capital-to-labor ratios to be proportional to the factor price ratios. This characterization is also convenient because it implies that we do not need to keep track of wages or labor in the simulation of this equilibrium. Manipulating the same set of conditions a little bit more also allows us to re-write the real wages in terms of the real rental rate of capital 
as well as productivity shocks, consumption, output and the stock of capital, i.e.

$$
\begin{aligned}
\frac{W_{t}}{P_{t}} & =\left(\frac{1-\psi}{\psi}\right)^{-\frac{(1-\psi) \varphi}{1+(1-\psi) \varphi}}\left(A_{t}\right)^{-\frac{\varphi}{1+(1-\psi) \varphi}}\left(C_{t}\right)^{\frac{\sigma^{-1}}{1+(1-\psi) \varphi}}\left(Y_{t}\right)^{\frac{\varphi}{1+(1-\psi) \varphi}}\left(\frac{Z_{t}}{P_{t}}\right)^{\frac{(1-\psi) \varphi}{1+(1-\psi) \varphi}}, \\
\frac{W_{t}^{*}}{P_{t}^{*}} & =\left(\frac{1-\psi}{\psi}\right)^{-\frac{(1-\psi)}{1+(1-\psi) \varphi} \varphi}\left(A_{t}^{*}\right)^{-\frac{\varphi}{1+(1-\psi) \varphi}}\left(C_{t}^{*}\right)^{\frac{\sigma^{-1}}{1+(1-\psi) \varphi}}\left(Y_{t}^{*}\right)^{\frac{\varphi}{1+(1-\psi) \varphi}}\left(\frac{Z_{t}^{*}}{P_{t}^{*}}\right)^{\frac{(1-\psi) \varphi}{1+(1-\psi) \varphi}} .
\end{aligned}
$$

These two equations suffice for the purpose of replacing real wages out of the marginal cost equations.

\section{The Steady State}

There is a zero-inflation steady state that implies consumption gets equalized across countries, $\bar{C}=\bar{C}^{*}$, the nominal exchange rate equals one, $\bar{S}=1$, and prices are identical for all goods, $\bar{P}=\bar{P}^{H}=\bar{P}^{F}$ and

$\bar{P}^{*}=\bar{P}^{H *}=\bar{P}^{F *}$. This normalization is customary in the literature, and often used in the background to derive the log-linearization of the equilibrium conditions of the open-economy model with nominal rigidities. We set the unconditional expectation of the shocks to be equal to one, i.e. $\bar{A}=\bar{A}^{*}=1, \bar{V}=\bar{V}^{*}=1$, and $\bar{M}=\bar{M}^{*}=1$. For the purpose of this paper, suffices to consider the implications of adding capital in the steady state.

First, we look at the steady state investment-to-capital ratio. The capital accumulation equation in (3) (and its foreign counterpart) require that in steady state the following equalities must hold,

$$
\begin{aligned}
\bar{K} & =(1-\delta) \bar{K}+\bar{V} \Phi(\bar{X}, \bar{X}, \bar{K}) \bar{X} \\
\bar{K}^{*} & =(1-\delta) \bar{K}^{*}+\bar{V}^{*} \Phi\left(\bar{X}^{*}, \bar{X}^{*}, \bar{K}^{*}\right) \bar{X}^{*}
\end{aligned}
$$

Operating on the capital accumulation equations it follows that,

$$
\delta=\bar{V} \Phi(\bar{X}, \bar{X}, \bar{K}) \frac{\bar{X}}{\bar{K}}=\bar{V}^{*} \Phi\left(\bar{X}^{*}, \bar{X}^{*}, \bar{K}^{*}\right) \frac{\bar{X}^{*}}{\bar{K}^{*}} .
$$

These two equalities are satisfied whenever $\frac{\bar{X}}{\bar{K}}=\frac{\bar{X}^{*}}{\bar{K}^{*}}=\delta$ because we normalize the unconditional mean of the IST shocks to be one, i.e. $\bar{V}=\bar{V}^{*}=1$, and because: (a) under our specification of the capital adjustment cost (CAC) function we know that $\Phi\left(\frac{\bar{X}}{\bar{K}}\right)=\Phi\left(\frac{\bar{X}^{*}}{\bar{K}^{*}}\right)=\Phi(\delta)=1$ in steady state, and (b) under our specification of the investment adjustment cost function we know that $\Phi\left(\frac{\bar{X}}{\bar{X}}\right)=\Phi\left(\frac{\bar{X}^{*}}{\bar{X}^{*}}\right)=\Phi(1)=1$ in steady state. Therefore, in steady state these adjustment costs have no effect (independently of the specification that we choose), and investment purely replaces the depreciated capital.

Second, using the fact that the investment-to-capital ratio is pined down by the depreciation rate and the properties of the adjustment cost function $\Phi(\cdot)$, we can write the steady state household efficiency conditions that summarize the behavior of Tobin's q and the real rental rate on capital in either $(63)-(66)$ or $(67)-(70)$ 
as follows,

$$
\begin{aligned}
\bar{Q} & =\beta\left[\frac{\bar{Z}}{\bar{P}}+\bar{Q}(1-\delta)\right], \\
\bar{Q}^{*} & =\beta\left[\frac{\bar{Z}^{*}}{\bar{P}^{*}}+\bar{Q}^{*}(1-\delta)\right], \\
\bar{Q} & =\bar{Q}^{*}=1,
\end{aligned}
$$

where we also the following assumptions: $\Phi^{\prime}\left(\frac{\bar{X}}{\bar{K}}\right)=\Phi^{\prime}\left(\frac{\bar{X}^{*}}{\bar{K}^{*}}\right)=\Phi^{\prime}(\delta)=0, \Phi^{\prime}\left(\frac{\bar{X}}{\bar{X}}\right)=\Phi^{\prime}\left(\frac{\bar{X}^{*}}{\bar{X}^{*}}\right)=\Phi^{\prime}(1)=0$, and $\bar{V}=\bar{V}^{*}=1$. Naturally, the steady state real rental rate on capital (which corresponds to the aggregate marginal product of capital) must be equal to,

$$
\frac{\bar{Z}}{\bar{P}}=\frac{\bar{Z}^{*}}{\bar{P}^{*}}=\beta^{-1}-(1-\delta) .
$$

This result is quite standard in the neoclassical literature. It says that Tobin's q must be equal to 1 in steady state, and that the real rental rate on capital must be equal to the real returns on bonds (since based on (54) - (55) the steady state interest rate is $\bar{I}=\bar{I}^{*}=\beta^{-1}$ ) after accounting for the effect of capital depreciation.

Third, we look at the steady state consumption and investment shares defined as $\gamma_{c} \equiv \frac{\bar{C}}{\bar{C}+\bar{X}}$ and $\gamma_{x} \equiv$ $\frac{\bar{X}}{\bar{C}+\bar{X}}=1-\gamma_{c}$, respectively. The standard normalizations not only require that consumption equalizes in steady state, but also that the real trade balance becomes zero. Therefore, it must follow from the goods market clearing conditions that,

$$
\begin{aligned}
\bar{Y} & =\bar{C}+\bar{X}, \\
\bar{Y}^{*} & =\bar{C}^{*}+\bar{X}^{*} .
\end{aligned}
$$

Using these goods market clearing conditions and the fact that in steady state the investment-to-capital ratio is determined by the depreciation rate, we can write the investment share as proportional to the capital-to-output ratio,

$$
\gamma_{x} \equiv \delta \frac{\bar{K}}{\bar{Y}}
$$

On one hand, we know from the Cobb-Douglas specification of the production function in (16) and the efficiency condition on the firms' problem in (18) that the capital-to-output ratio can be expressed as a function of productivity shocks and the factor price ratio. Hence, it follows that,

$$
\gamma_{x}=\delta\left(\frac{1-\psi}{\psi}\right)^{\psi} \frac{1}{\bar{A}}\left(\frac{\bar{W}}{\bar{Z}}\right)^{\psi}=\delta\left(\frac{1-\psi}{\psi}\right)^{\psi}\left(\frac{\bar{Z}}{\bar{P}}\right)^{-\psi}\left[\frac{1}{\bar{A}}\left(\frac{\bar{W}}{\bar{P}}\right)^{\psi}\right]
$$

where $\bar{A}$ is the steady state domestic productivity shock. On the other hand, we also know that in steady state all firms charge the same price (the Calvo parameter is irrelevant) and the standard pricing formula under monopolistic competition applies. The assumptions of LCP or PCP pricing are also irrelevant for the determination of the steady state allocation, and have no bearing on the steady state. Hence, prices are 
equal to a mark-up over marginal costs, and the pricing equation in (71) and the marginal cost function in (20) reduce to,

$$
\bar{P}^{H}=\frac{\theta(1-\bar{\xi})}{\theta-1} \frac{1}{\bar{A}} \frac{1}{\psi^{\psi}(1-\psi)^{1-\psi}}(\bar{W})^{\psi}(\bar{Z})^{1-\psi} .
$$

Dividing this expression by $\bar{P}$ and re-arranging terms, we infer that real wages are linked to the real rental rates on capital by,

$$
\frac{1}{\bar{A}}\left(\frac{\bar{W}}{\bar{P}}\right)^{\psi}=\frac{\theta-1}{\theta(1-\bar{\xi})}\left[\psi^{\psi}(1-\psi)^{1-\psi}\left(\frac{\bar{Z}}{\bar{P}}\right)^{\psi-1}\right] .
$$

If we replace this expression inside the formula for the investment share $\gamma_{x}$ obtained before we get that,

$$
\begin{aligned}
\gamma_{x} & =\delta\left(\frac{1-\psi}{\psi}\right)^{\psi}\left(\frac{\bar{Z}}{\bar{P}}\right)^{-\psi}\left[\frac{\theta-1}{\theta(1-\bar{\xi})}\left(\psi^{\psi}(1-\psi)^{1-\psi}\left(\frac{\bar{Z}}{\bar{P}}\right)^{\psi-1}\right)\right] \\
& =\delta(1-\psi)\left[\left(\frac{\theta(1-\bar{\xi})}{\theta-1}\right)\left(\frac{\bar{Z}}{\bar{P}}\right)\right]^{-1} .
\end{aligned}
$$

Then, using the steady state real rental rate on capital, we can say that,

$$
\gamma_{x} \equiv \delta(1-\psi)\left[\left(\frac{\theta(1-\bar{\xi})}{\theta-1}\right)\left(\beta^{-1}-(1-\delta)\right)\right]^{-1}
$$

In other words, the investment share in steady state depends directly on the depreciation rate of capital, $\delta$, and the capital share on the production function, $1-\psi$, and indirectly on the mark-up, $\frac{\theta}{\theta-1}$, the firms' subsidy in steady state, $\bar{\xi}$, and the real rental rate on capital, $\beta^{-1}-(1-\delta)$. An analogous set of derivations allows us to obtain the same expression for the investment share in the foreign country - under the assumption that the steady state subsidy is the same in both countries, i.e. $\bar{\xi}=\bar{\xi}^{*}$.

Finally, in the deterministic steady state of our model, it follows easily from equation (52) and its foreign counterpart that,

$$
\begin{aligned}
\bar{C}^{F} & =\phi_{F}\left(\frac{\bar{P}^{F}}{\bar{P}}\right)^{-\eta} \bar{C}=\phi_{F} \bar{C}, \bar{X}^{F}=\phi_{F}\left(\frac{\bar{P}^{F}}{\bar{P}}\right)^{-\eta} \bar{X}=\phi_{F} \bar{X}, \\
\bar{C}^{H *} & =\phi_{H}^{*}\left(\frac{\bar{P}^{H *}}{\bar{P}^{*}}\right)^{-\eta} \bar{C}^{*}=\phi_{H}^{*} \bar{C}^{*}, \bar{X}^{H *}=\phi_{H}^{*}\left(\frac{\bar{P}^{H *}}{\bar{P}^{*}}\right)^{-\eta} \bar{X}^{*}=\phi_{H}^{*} \bar{X}^{*},
\end{aligned}
$$

since $\bar{P}=\bar{P}^{H}=\bar{P}^{F}$ and $\bar{P}^{*}=\bar{P}^{H *}=\bar{P}^{F *}$. Under the (symmetric) home-product bias assumption (i.e., $\phi_{H}^{*}=\phi_{F}$ ), given that $\bar{C}=\bar{C}^{*}$ and $\bar{X}=\bar{X}^{*}$, we therefore conclude that,

$$
\begin{aligned}
\bar{C}^{F}+\bar{X}^{F} & =\phi_{F}(\bar{C}+\bar{X})=\phi_{F} \bar{Y}, \\
\bar{C}^{H *}+\bar{X}^{H *} & =\phi_{H}^{*}\left(\bar{C}^{*}+\bar{X}^{*}\right)=\phi_{F}(\bar{C}+\bar{X})=\phi_{F} \bar{Y},
\end{aligned}
$$

where the last equality follows from the steady state market clearing condition in both countries. In steady state, the trade is balanced in real terms. However, because households have preferences for domestic as well as foreign goods, exchanges do occur between the two countries. The parameter $\phi_{F}$ denotes the share of real 
domestic imports (and real foreign exports) for consumption and investment purposes relative to aggregate output in steady state.

As noted earlier, the deterministic steady state is the same independently of whether we assume LCP or PCP pricing because price stickiness becomes irrelevant in the steady state anyway. In the special case in which the firms' subsidy is set at $\bar{\xi}=\bar{\xi}^{*}=\frac{1}{\theta}$, then this fiscal policy instrument suffices to fully neutralize the mark-up distortion caused by monopolistic competition. Therefore, the steady state of the model becomes observationally identical to the steady state of a frictionless model under flexible prices and perfect competition. Such scenario is often appealing in the literature because it implies that the frictions due to price stickiness, monopolistic competition (and LCP pricing whenever applicable) have a first-order effect on the short-run dynamics of the economy but no effect in the long-run (in its steady state).

\section{The Log-Linearized Equilibrium Conditions}

Here, we log-linearize the equilibrium conditions around the deterministic, zero-inflation steady state. We denote generically $\widehat{n}_{t} \equiv \ln N_{t}-\ln \bar{N}$ the deviation of a variable $N_{t}$ in logs from its steady state.

\subsection{The Households' Equilibrium Conditions}

The log-linearization of the Euler equations in (54) - (55) is quite standard, and characterizes the consumptionsavings decisions of the households as follows,

$$
\begin{aligned}
& \widehat{c}_{t} \approx \mathbb{E}_{t}\left[\widehat{c}_{t+1}\right]-\sigma\left(\widehat{i}_{t}-\mathbb{E}_{t}\left[\widehat{\pi}_{t+1}\right]\right), \\
& \widehat{c}_{t}^{*} \approx \mathbb{E}_{t}\left[\widehat{c}_{t+1}^{*}\right]-\sigma\left(\hat{i}_{t}^{*}-\mathbb{E}_{t}\left[\widehat{\pi}_{t+1}^{*}\right]\right) .
\end{aligned}
$$

The perfect international risk-sharing condition comes from the log-linearization of (53), i.e.

$$
\widehat{c}_{t}-\widehat{c}_{t}^{*} \approx \sigma \widehat{r s} t
$$

As expected, we obtain that relative consumption must be proportional to the real exchange rate.

The log-linearization of the domestic capital accumulation formula in (3) and its foreign counterpart in the case without adjustment costs (NAC) allows us to obtain the following set of equations,

$$
\begin{aligned}
\widehat{k}_{t+1} & \approx(1-\delta) \widehat{k}_{t}+(\bar{V} \overline{\bar{X}})\left(\widehat{x}_{t}+\widehat{v}_{t}\right) \\
& =(1-\delta) \widehat{k}_{t}+\delta\left(\widehat{x}_{t}+\widehat{v}_{t}\right), \\
\widehat{k}_{t+1}^{*} & \approx(1-\delta) \widehat{k}_{t}^{*}+\left(\bar{V}^{*} \frac{\bar{X}^{*}}{\bar{K}^{*}}\right)\left(\widehat{x}_{t}^{*}+\widehat{v}_{t}^{*}\right) \\
& =(1-\delta) \widehat{k}_{t}^{*}+\delta\left(\widehat{x}_{t}^{*}+\widehat{v}_{t}^{*}\right),
\end{aligned}
$$

where the second-equality follows from the steady state investment-to-capital ratio being pinned down by the depreciation rate. Notice that $\widehat{v}_{t}$ and $\widehat{v}_{t}^{*}$ are the investment-specific technological (IST) shocks in this model, and that their unconditional mean is normalized to one (i.e. $\bar{V}=\bar{V}^{*}=1$ ). 
The log-linearization of the domestic capital accumulation formula in (3) and its foreign counterpart under capital adjustment costs (CAC) allows us to obtain the following set of equations,

$$
\begin{aligned}
& \widehat{k}_{t+1} \approx\left[(1-\delta)-\bar{V} \Phi^{\prime}\left(\frac{\bar{X}}{\bar{K}}\right)\left(\frac{\bar{X}}{\bar{K}}\right)^{2}\right] \widehat{k}_{t}+\left[\bar{V} \Phi\left(\frac{\bar{X}}{\bar{K}}\right) \frac{\bar{X}}{\bar{K}}+\bar{V} \Phi^{\prime}\left(\frac{\bar{X}}{\bar{K}}\right)\left(\frac{\bar{X}}{\bar{K}}\right)^{2}\right] \widehat{x}_{t}+\ldots \\
& \quad \bar{V} \Phi\left(\frac{\bar{X}}{\bar{K}}\right) \frac{\bar{X}}{\bar{K}} \widehat{v}_{t} \\
& \quad=(1-\delta) \widehat{k}_{t}+\delta\left(\widehat{x}_{t}+\widehat{v}_{t}\right), \\
& \widehat{k}_{t+1}^{*} \approx\left[(1-\delta)-\bar{V}^{*} \Phi^{\prime}\left(\frac{\bar{X}^{*}}{\bar{K}^{*}}\right)\left(\frac{\bar{X}^{*}}{\bar{K}^{*}}\right)^{2}\right] \widehat{k}_{t}^{*}+\left[\bar{V}^{*} \Phi\left(\frac{\bar{X}^{*}}{\bar{K}^{*}}\right) \frac{\bar{X}^{*}}{\bar{K}^{*}}+\bar{V}^{*} \Phi^{\prime}\left(\frac{\bar{X}^{*}}{\bar{K}^{*}}\right)\left(\frac{\bar{X}^{*}}{\bar{K}^{*}}\right)^{2}\right] \widehat{x}_{t}^{*}+\ldots \\
& \bar{V}^{*} \Phi\left(\frac{\bar{X}^{*}}{\bar{K}^{*}}\right) \bar{X}^{*} \widehat{\bar{K}}_{t}^{*} \\
& \quad=(1-\delta) \widehat{k}_{t}^{*}+\delta\left(\widehat{x}_{t}^{*}+\widehat{v}_{t}^{*}\right),
\end{aligned}
$$

where the second-equality follows from the steady state properties of the CAC function, and the fact that $\bar{X}=\delta \bar{K}$ and $\bar{X}^{*}=\delta \bar{K}^{*}$ and $\bar{V}=\bar{V}^{*}=1$. The log-linearization of the capital accumulation formula in (3) and its foreign counterpart under investment adjustment costs (IAC) allows us to obtain the following set of equations,

$$
\begin{aligned}
& \widehat{k}_{t+1} \approx(1-\delta) \widehat{k}_{t}+\left[\bar{V} \Phi\left(\frac{\bar{X}}{\bar{X}}\right) \frac{\bar{X}}{\bar{K}}+\bar{V} \Phi^{\prime}\left(\frac{\bar{X}}{\bar{X}}\right)\left(\frac{\bar{X}}{\bar{X}}\right) \frac{\bar{X}}{\bar{K}}\right] \widehat{x}_{t}-\ldots \\
& \quad\left[\bar{V} \Phi^{\prime}\left(\frac{\bar{X}}{\bar{X}}\right)\left(\frac{\bar{X}}{\bar{X}}\right)^{2} \frac{\bar{X}}{\bar{K}}\right] \widehat{x}_{t}+\bar{V} \Phi\left(\frac{\bar{X}}{\bar{X}}\right) \frac{\bar{X}}{\bar{K}} \widehat{v}_{t} \\
& \quad=(1-\delta) \widehat{k}_{t}+\delta\left(\widehat{x}_{t}+\widehat{v}_{t}\right), \\
& \widehat{k}_{t+1}^{*} \approx(1-\delta) \widehat{k}_{t}^{*}+\left[\bar{V}^{*} \Phi\left(\frac{\bar{X}^{*}}{\bar{X}^{*}}\right) \frac{\bar{X}^{*}}{\bar{K}^{*}}+\bar{V}^{*} \Phi^{\prime}\left(\frac{\bar{X}^{*}}{\bar{X}^{*}}\right)\left(\frac{\bar{X}^{*}}{\bar{X}^{*}}\right) \bar{X}^{*} \overline{\bar{K}}^{*}\right] \widehat{x}_{t}^{*}-\ldots \\
& {\left[\bar{V}^{*} \Phi^{\prime}\left(\frac{\bar{X}^{*}}{\bar{X}^{*}}\right)\left(\frac{\bar{X}^{*}}{\bar{X}^{*}}\right)^{2} \bar{X}^{*} \overline{\bar{K}}^{*}\right.} \\
& \widehat{x}_{t}^{*}+\bar{V}^{*} \Phi\left(\frac{\bar{X}^{*}}{\bar{X}^{*}}\right) \frac{\bar{X}^{*}}{\bar{K}^{*}} \widehat{v}_{t}^{*} \\
& =(1-\delta) \widehat{k}_{t}^{*}+\delta\left(\widehat{x}_{t}^{*}+\widehat{v}_{t}^{*}\right),
\end{aligned}
$$

where the second-equality follows from the steady state properties of the IAC function, and the fact that $\bar{X}=\delta \bar{K}$ and $\bar{X}^{*}=\delta \bar{K}^{*}$ and $\bar{V}=\bar{V}^{*}=1$. It is interesting to note that in spite of the fact that we are using three different specifications for the adjustment cost function, the log-linearized law of motion for capital is the same in all cases. However, unlike what happens with the law of motion, the log-linearization of the equilibrium conditions on capital-investment is not independent of the choice of the adjustment cost function.

The Capital-Investment Decision under NAC. The log-linearization of the capital-investment conditions coming from the domestic households' problem in (59) - (60) are,

$$
\begin{aligned}
& \widehat{q}_{t} \approx \mathbb{E}_{t}\left\{-\frac{1}{\sigma}\left(\widehat{c}_{t+1}-\widehat{c}_{t}\right)+(1-(1-\delta) \beta) \widehat{r}_{t+1}^{z}+(1-\delta) \beta \widehat{q}_{t+1}\right\}, \\
& \widehat{q}_{t} \approx-\widehat{v}_{t}
\end{aligned}
$$


and, analogously for the foreign counterparts in (61) - (62), the log-linearizations give us that,

$$
\begin{aligned}
& \widehat{q}_{t}^{*} \approx \mathbb{E}_{t}\left\{-\frac{1}{\sigma}\left(\widehat{c}_{t+1}^{*}-\widehat{c}_{t}^{*}\right)+(1-(1-\delta) \beta) \widehat{r}_{t+1}^{z *}+(1-\delta) \beta \widehat{q}_{t+1}^{*}\right\} \\
& \widehat{q}_{t}^{*} \approx-\widehat{v}_{t}^{*}
\end{aligned}
$$

where naturally $\widehat{q}_{t}$ and $\widehat{q}_{t}^{*}$ are the real shadow values of an additional unit of capital (or Tobin's q) in each country, and $\widehat{r}_{t+1}^{z} \equiv \widehat{z}_{t+1}-\widehat{p}_{t+1}$ and $\widehat{r}_{t+1}^{z *} \equiv \widehat{z}_{t+1}^{*}-\widehat{p}_{t+1}^{*}$ denote the real rental rates on capital in the domestic and foreign countries, respectively. This pair of equations can be re-arranged to show that,

$$
\begin{aligned}
(1-(1-\delta) \beta) \mathbb{E}_{t}\left(\widehat{r}_{t+1}^{z}\right) & \approx\left(\widehat{i}_{t}-\mathbb{E}_{t}\left(\widehat{\pi}_{t+1}\right)\right)+(1-\delta) \beta \mathbb{E}_{t}\left(\widehat{v}_{t+1}\right)-\widehat{v}_{t} \\
(1-(1-\delta) \beta) \mathbb{E}_{t}\left(\widehat{r}_{t+1}^{z *}\right) & \approx\left(\hat{i}_{t}^{*}-\mathbb{E}_{t}\left(\widehat{\pi}_{t+1}^{*}\right)\right)+(1-\delta) \beta \mathbb{E}_{t}\left(\widehat{v}_{t+1}^{*}\right)-\widehat{v}_{t}^{*}
\end{aligned}
$$

by adding the Euler equations in (97) - (98). We could interpret this pair of equations as indicating that the real rental rate on capital (the aggregate marginal product of capital) is proportional to the real interest rate. The two rates are not equal, however, because capital depreciates over time, while borrowing and lending in the bond markets is not subject to the same physical depreciation. They also differ because of the IST shocks. In other words, the real interest rate should be proportional to the aggregate marginal product of capital only if there are no adjustment costs and IST shocks.

The Capital-Investment Decision under CAC. The log-linearization of the capital-investment conditions coming from the domestic households' problem in (63) - (64) are,

$$
\begin{aligned}
\widehat{q}_{t} & \approx \mathbb{E}_{t}\left\{\frac{\bar{Q}\left(\bar{V} \Phi^{\prime \prime}\left(\frac{\bar{X}}{\bar{K}}\right)\left(\frac{\bar{X}}{\bar{K}}\right)^{2}+2 \bar{V} \Phi^{\prime}\left(\frac{\bar{X}}{\bar{K}}\right)\left(\frac{\bar{X}}{K}\right)\right) \frac{\bar{X}}{\bar{K}}}{\frac{\bar{Z}}{\bar{P}}+\bar{Q}\left((1-\delta)-\bar{V} \Phi^{\prime}\left(\frac{\bar{X}}{\bar{K}}\right)\left(\frac{\bar{X}}{\bar{K}}\right)^{2}\right)}\left(\widehat{x}_{t+1}-\widehat{k}_{t+1}\right)-\frac{\bar{Q} \overline{Q V} \Phi^{\prime}\left(\frac{\bar{X}}{\bar{K}}\right)\left(\frac{\bar{X}}{\bar{K}}\right)^{2}}{\frac{\bar{Z}}{\bar{P}}+\bar{Q}\left((1-\delta)-\bar{V} \Phi^{\prime}\left(\frac{\bar{X}}{\bar{K}}\right)\left(\frac{\bar{X}}{\bar{K}}\right)^{2}\right)} \widehat{v}_{t+1}\right\} \\
& =\mathbb{E}_{t}\left\{-\frac{1}{\sigma}\left(\widehat{c}_{t+1}-\widehat{c}_{t}\right)+(1-(1-\delta) \beta) \widehat{r}_{t+1}^{z}+(1-\delta) \beta \widehat{q}_{t+1}+\chi \delta^{2} \beta\left(\widehat{x}_{t+1}-\widehat{k}_{t+1}\right)\right\} \\
\widehat{q}_{t} & \approx-\left[\frac{\left(\Phi^{\prime \prime}\left(\frac{\bar{X}}{\bar{K}}\right) \frac{\bar{X}}{\bar{K}}+2 \Phi^{\prime}\left(\frac{\bar{X}}{\bar{K}}\right)\right) \frac{\bar{X}}{\bar{K}}}{\Phi\left(\frac{\bar{X}}{\bar{K}}\right)+\Phi^{\prime}\left(\frac{\bar{X}}{\bar{K}}\right) \frac{\bar{X}}{\bar{K}}}\right]\left(\widehat{x}_{t}-\widehat{k}_{t}\right)-\widehat{v}_{t} \\
& =\chi \delta\left(\widehat{x}_{t}-\widehat{k}_{t}\right)-\widehat{v}_{t},
\end{aligned}
$$

and, analogously for the foreign counterparts in $(65)-(66)$, we obtain that,

$$
\begin{aligned}
& \widehat{q}_{t}^{*} \approx \mathbb{E}_{t}\left\{-\frac{1}{\sigma}\left(\widehat{c}_{t+1}^{*}-\widehat{c}_{t}^{*}\right)+(1-(1-\delta) \beta) \widehat{r}_{t+1}^{z *}+(1-\delta) \beta \widehat{q}_{t+1}^{*}+\chi \delta^{2} \beta\left(\widehat{x}_{t+1}^{*}-\widehat{k}_{t+1}^{*}\right)\right\}, \\
& \widehat{q}_{t}^{*} \approx \chi \delta\left(\widehat{x}_{t}^{*}-\widehat{k}_{t}^{*}\right)-\widehat{v}_{t}^{*} .
\end{aligned}
$$

This pair of equations describes $\widehat{q}_{t}$ and $\widehat{q}_{t}^{*}$ as the real shadow values of an additional unit of capital (or Tobin's q) in each country, $\widehat{r}_{t+1}^{z} \equiv \widehat{z}_{t+1}-\widehat{p}_{t+1}$ and $\widehat{r}_{t+1}^{z *} \equiv \widehat{z}_{t+1}^{*}-\widehat{p}_{t+1}^{*}$ denote the real rental rates on capital in the domestic and foreign countries, while $\chi$ regulates the degree of concavity of the capital adjustment cost (CAC) function around the steady state. 
The pair of equations that relate the current and expected Tobin's q to the real rental rate on capital and the real interest rate can be re-arranged as,

$$
\begin{aligned}
& \widehat{q}_{t} \approx \beta \mathbb{E}_{t}\left[\widehat{q}_{t+1}\right]+\left[(1-(1-\delta) \beta) \mathbb{E}_{t}\left(\widehat{r}_{t+1}^{z}\right)-\left(\widehat{i}_{t}-\mathbb{E}_{t}\left(\widehat{\pi}_{t+1}\right)\right)\right], \\
& \widehat{q}_{t}^{*} \approx \beta \mathbb{E}_{t}\left[\widehat{q}_{t+1}^{*}\right]+\left[(1-(1-\delta) \beta) \mathbb{E}_{t}\left(\widehat{r}_{t+1}^{z *}\right)-\left(\widehat{i}_{t}^{*}-\mathbb{E}_{t}\left(\widehat{\pi}_{t+1}^{*}\right)\right)\right],
\end{aligned}
$$

by adding the Euler equations in (97) - (98). We could interpret this pair of equations as indicating that the differences between the real interest rate and the real rental rate on capital (the aggregate marginal product of capital) are the result of fluctuations in Tobin's q. Notice that in the extreme case where there are no adjustment costs and IST shocks (i.e., when $\chi=0$ and $\widehat{v}_{t}=\widehat{v}_{t}^{*}=0$ ), then $\widehat{q}_{t}=\widehat{q}_{t}^{*}=0$ for all $t$.

The Capital-Investment Decision under IAC. The log-linearization of the capital-investment conditions coming from the first-order conditions of the households' problem in (67) - (68) are,

$$
\begin{aligned}
& \widehat{q}_{t} \approx \mathbb{E}_{t}\left\{-\frac{1}{\sigma}\left(\widehat{c}_{t+1}-\widehat{c}_{t}\right)+\frac{\frac{\bar{Z}}{\bar{P}}}{\frac{\bar{Z}}{\bar{P}}+(1-\delta)} \widehat{r}_{t+1}^{z}+\frac{(1-\delta)}{\frac{\bar{Z}}{\bar{P}}+(1-\delta)} \widehat{q}_{t+1}\right\} \\
& =\mathbb{E}_{t}\left\{-\frac{1}{\sigma}\left(\widehat{c}_{t+1}-\widehat{c}_{t}\right)+(1-(1-\delta) \beta) \widehat{r}_{t+1}^{z}+(1-\delta) \beta \widehat{q}_{t+1}\right\} \text {, } \\
& \widehat{q}_{t} \approx-\widehat{v}_{t}-\frac{\left(2 \bar{V} \Phi^{\prime}\left(\frac{\bar{X}}{\bar{X}}\right)+\bar{V} \Phi^{\prime \prime}\left(\frac{\bar{X}}{\bar{X}}\right)\left(\frac{\bar{X}}{\bar{X}}\right)\right)\left(\frac{\bar{X}}{\bar{X}}\right)}{\bar{V} \Phi\left(\frac{\bar{X}}{\bar{X}}\right)+\bar{V} \Phi^{\prime}\left(\frac{\bar{X}}{\bar{X}}\right)\left(\frac{\bar{X}}{\bar{X}}\right)}\left(\widehat{x}_{t}-\widehat{x}_{t-1}\right)+\ldots
\end{aligned}
$$

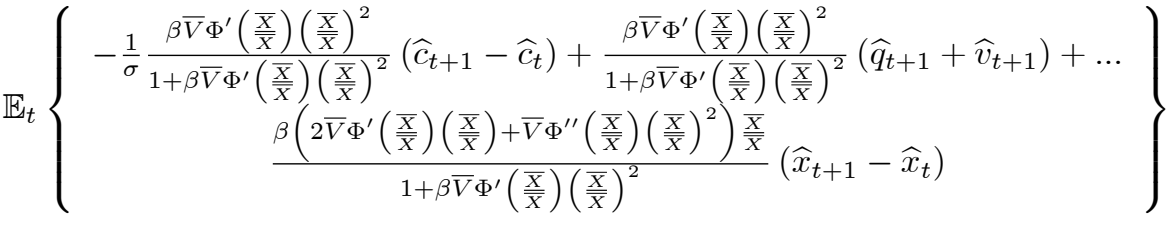

$$
\begin{aligned}
& =\kappa\left[\left(\widehat{x}_{t}-\widehat{x}_{t-1}\right)-\beta \mathbb{E}_{t}\left(\widehat{x}_{t+1}-\widehat{x}_{t}\right)\right]-\widehat{v}_{t},
\end{aligned}
$$

and, analogously for the foreign counterparts in (69) - (70), we obtain that,

$$
\begin{aligned}
& \widehat{q}_{t}^{*} \approx \mathbb{E}_{t}\left\{-\frac{1}{\sigma}\left(\widehat{c}_{t+1}^{*}-\widehat{c}_{t}^{*}\right)+(1-(1-\delta) \beta) \widehat{r}_{t+1}^{* *}+(1-\delta) \beta \widehat{q}_{t+1}^{*}\right\}, \\
& \widehat{q}_{t}^{*} \approx \kappa\left[\left(\widehat{x}_{t}^{*}-\widehat{x}_{t-1}^{*}\right)-\beta \mathbb{E}_{t}\left(\widehat{x}_{t+1}^{*}-\widehat{x}_{t}^{*}\right)\right]-\widehat{v}_{t}^{*} .
\end{aligned}
$$

This pair of equations summarizes $\widehat{q}_{t}$ and $\widehat{q}_{t}^{*}$ as the real shadow values of an additional unit of capital (or Tobin's q) in each country, $\widehat{r}_{t+1}^{z} \equiv \widehat{z}_{t+1}-\widehat{p}_{t+1}$ and $\widehat{r}_{t+1}^{z *} \equiv \widehat{z}_{t+1}^{*}-\widehat{p}_{t+1}^{*}$ denote the real rental rates on capital in the domestic and foreign countries, and $\kappa$ regulates the degree of concavity of the IAC adjustment cost function around the steady state.

The pair of equations that relate the current and expected Tobin's q to the real rental rates on capital and the real interest rate can be re-arranged as,

$$
\begin{aligned}
\widehat{q}_{t} & \approx(1-\delta) \beta \mathbb{E}_{t}\left[\widehat{q}_{t+1}\right]+\left[(1-(1-\delta) \beta) \mathbb{E}_{t}\left(\widehat{r}_{t+1}^{z}\right)-\left(\widehat{i}_{t}-\mathbb{E}_{t}\left[\widehat{\pi}_{t+1}\right]\right)\right], \\
\widehat{q}_{t}^{*} & \approx(1-\delta) \beta \mathbb{E}_{t}\left[\widehat{q}_{t+1}^{*}\right]+\left[(1-(1-\delta) \beta) \mathbb{E}_{t}\left(\widehat{r}_{t+1}^{z *}\right)-\left(\widehat{i}_{t}^{*}-\mathbb{E}_{t}\left[\widehat{\pi}_{t+1}^{*}\right]\right)\right],
\end{aligned}
$$


by adding the Euler equations in (97) - (98). We could interpret this pair of equations as indicating that the differences between the real interest rate and the real rental rate on capital are the result of fluctuations in Tobin's q. These equations are almost identical to (116) and (117) except for the fact that the expectation term on the right-hand side is diminished by $(1-\delta)$. Hence, we conjecture that expectations about the future play a potentially 'smaller' role in the dynamics of Tobin's q under the IAC specification. Notice that in the extreme case where there are no adjustment costs and IST shocks (i.e., when $\kappa=0$ and $\widehat{v}_{t}=\widehat{v}_{t}^{*}=0$ ), then $\widehat{q}_{t}=\widehat{q}_{t}^{*}=0$ for all $t$.

Finally, we can re-write equations (119) and (121) in a more compact form as follows,

$$
\begin{aligned}
& \widehat{x}_{t} \approx \frac{1}{1+\beta} \widehat{x}_{t-1}+\frac{\beta}{1+\beta} \mathbb{E}_{t}\left[\widehat{x}_{t+1}\right]+\frac{1}{\kappa(1+\beta)}\left(\widehat{q}_{t}+\widehat{v}_{t}\right), \\
& \widehat{x}_{t}^{*} \approx \frac{1}{1+\beta} \widehat{x}_{t-1}^{*}+\frac{\beta}{1+\beta} \mathbb{E}_{t}\left[\widehat{x}_{t+1}^{*}\right]+\frac{1}{\kappa(1+\beta)}\left(\widehat{q}_{t}^{*}+\widehat{v}_{t}^{*}\right) .
\end{aligned}
$$

The presence of investment adjustment costs (IAC) changes equations (119) and (121) completely. First, it introduces an element of inertia in investment captured by the lagged terms in (124) and (125). Second, the investment decision also becomes forward-looking, captured by the expectation term, because it becomes costly to adjust the level of investment. The elasticity of investment with respect to Tobin's q (the shadow value of an additional unit of capital) is inversely related to the curvature of the IAC function (regulated by $\kappa)$. By contrast, investment under the assumption of capital adjustment costs (CAC) responds immediately to movements in Tobin's q as can be seen from equations (113) and (115), while the elasticity of investment with respect to Tobin's q is inversely related to the curvature of the CAC function (regulated by $\chi$ ) and the depreciation rate $(\delta)$.

\subsection{The Monetary Policy Rules}

A simple log-linearization of the Taylor indexes described in equations (42) - (43) gives us the following monetary policy rules,

$$
\begin{aligned}
\hat{i}_{t} & \approx \rho_{i} \widehat{i}_{t-1}+\left(1-\rho_{i}\right)\left[\psi_{\pi} \widehat{\pi}_{t}+\psi_{y} \widehat{y}_{t}\right]+\widehat{m}_{t}, \\
\widehat{i}_{t}^{*} & \approx \rho_{i} \hat{i}_{t-1}^{*}+\left(1-\rho_{i}\right)\left[\psi_{\pi} \widehat{\pi}_{t}^{*}+\psi_{y} \widehat{y}_{t}^{*}\right]+\widehat{m}_{t}^{*},
\end{aligned}
$$

where $\widehat{m}_{t}$ and $\widehat{m}_{t}^{*}$ denote a pair of monetary policy shocks (expressed in logs and relative to their unconditional expectations). The Taylor rule for each country is symmetric, has a smoothing component, and it also responds to fluctuations in output and inflation.

\subsection{The Firms' Equilibrium Conditions}

\subsubsection{The Open Economy Phillips Curves}

Efficiency Conditions and Aggregate Output. The efficiency conditions can be summarized by equations $(93)-(94)$, as reported before. The log-linearization of these conditions implies that the real rental 
rate on capital must be approximately equal to,

$$
\begin{aligned}
\widehat{r}_{t}^{z} & \approx \frac{1}{\sigma} \widehat{c}_{t}+\frac{1+\varphi}{\psi} \widehat{y}_{t}-\left(\frac{1+(1-\psi) \varphi}{\psi}\right) \widehat{k}_{t}-\frac{1+\varphi}{\psi} \widehat{a}_{t} \\
\widehat{r}_{t}^{z *} & \approx \frac{1}{\sigma} \widehat{c}_{t}^{*}+\frac{1+\varphi}{\psi} \widehat{y}_{t}^{*}-\left(\frac{1+(1-\psi) \varphi}{\psi}\right) \widehat{k}_{t}^{*}-\frac{1+\varphi}{\psi} \widehat{a}_{t}^{*}
\end{aligned}
$$

If we define the world consumption as $\widehat{c}_{t}^{W} \equiv \phi_{H} \widehat{c}_{t}+\phi_{F} \widehat{c}_{t}^{*}$ and $\widehat{c}_{t}^{W *} \equiv \phi_{F} \widehat{c}_{t}+\phi_{H} \widehat{c}_{t}^{*}$ and the relative consumption as $\widehat{c}_{t}^{R} \equiv \widehat{c}_{t}-\widehat{c}_{t}^{*}$, then we can write that,

$$
\begin{aligned}
\widehat{c}_{t} & =\widehat{c}_{t}^{W}+\phi_{F} \widehat{c}_{t}^{R} \approx \widehat{c}_{t}^{W}+\phi_{F} \sigma \widehat{r s}_{t}, \\
\widehat{c}_{t}^{*} & =\widehat{c}_{t}^{W *}-\phi_{F} \widehat{c}_{t}^{R} \approx \widehat{c}_{t}^{W *}-\phi_{F} \sigma \widehat{r s}_{t},
\end{aligned}
$$

where the second approximation follows from the perfect international risk-sharing condition in (99). Then, we can express the efficiency conditions in (128) - (129) as,

$$
\begin{aligned}
\widehat{r}_{t}^{z} & \approx-\frac{1+\varphi}{\psi} \widehat{a}_{t}+\frac{1}{\sigma} \widehat{c}_{t}^{W}+\phi_{F} \widehat{r}_{t}+\frac{1+\varphi}{\psi} \widehat{y}_{t}-\left(\frac{1+(1-\psi) \varphi}{\psi}\right) \widehat{k}_{t}, \\
\widehat{r}_{t}^{z *} & \approx-\frac{1+\varphi}{\psi} \widehat{a}_{t}^{*}+\frac{1}{\sigma} \widehat{c}_{t}^{W *}-\phi_{F} \widehat{r}_{t}+\frac{1+\varphi}{\psi} \widehat{y}_{t}^{*}-\left(\frac{1+(1-\psi) \varphi}{\psi}\right) \widehat{k}_{t}^{*} .
\end{aligned}
$$

These equations are necessary to close down the model without having to keep track of either labor or wages explicitly.

Using the demand constraints of the domestic firm in equations (27) - (28), the demand constraints of the foreign firm in equations (30) - (31), complemented by $(51)-(52)$ and the corresponding foreign counterparts, it follows that the log-linearization around the steady state of the output demand for a given firm, i.e. $\widehat{y}_{t}(h)$ for a domestic firm $h$ and $\widehat{y}_{t}^{*}(f)$ for a foreign firm $f$, takes the following form,

$$
\begin{aligned}
& \widehat{y}_{t}(h) \approx-\theta\left(\widehat{p}_{t}^{W}(h)-\widehat{p}_{t}^{W}\right)+(\theta-\eta)\left(\widehat{p}_{t}^{H, W}-\widehat{p}_{t}^{W}\right)+\left(1-\gamma_{x}\right) \widehat{c}_{t}^{W}+\gamma_{x} \widehat{x}_{t}^{W}, \\
& \widehat{y}_{t}^{*}(f) \approx-\theta\left(\widehat{p}_{t}^{W *}(f)-\widehat{p}_{t}^{W *}\right)+(\theta-\eta)\left(\widehat{p}_{t}^{F, W *}-\widehat{p}_{t}^{W *}\right)+\left(1-\gamma_{x}\right) \widehat{c}_{t}^{W *}+\gamma_{x} \widehat{x}_{t}^{W *},
\end{aligned}
$$

where the weighted variables are,

$$
\begin{aligned}
\widehat{c}_{t}^{W} & \equiv \phi_{H} \widehat{c}_{t}+\phi_{F} \widehat{c}_{t}^{*}, \widehat{c}_{t}^{W *} \equiv \phi_{F} \widehat{c}_{t}+\phi_{H} \widehat{c}_{t}^{*} \\
\widehat{x}_{t}^{W} & \equiv \phi_{H} \widehat{x}_{t}+\phi_{F} \widehat{x}_{t}^{*}, \widehat{x}_{t}^{W *} \equiv \phi_{F} \widehat{x}_{t}+\phi_{H} \widehat{x}_{t}^{*} \\
\widehat{p}_{t}^{W}(h) & \equiv \phi_{H} \widehat{p}_{t}(h)+\phi_{F} \widehat{p}_{t}^{*}(h), \widehat{p}_{t}^{W *}(f) \equiv \phi_{F} \widehat{p}_{t}(f)+\phi_{H} \widehat{p}_{t}^{*}(f), \\
\widehat{p}_{t}^{H, W} & \equiv \phi_{H} \widehat{p}_{t}^{H}+\phi_{F} \widehat{p}_{t}^{H *}, \widehat{p}_{t}^{F, W *} \equiv \phi_{F} \widehat{p}_{t}^{F}+\phi_{H} \widehat{p}_{t}^{F *} \\
\widehat{p}_{t}^{W} & \equiv \phi_{H} \widehat{p}_{t}+\phi_{F} \widehat{p}_{t}^{*}, \widehat{p}_{t}^{W *} \equiv \phi_{F} \widehat{p}_{t}+\phi_{H} \widehat{p}_{t}^{*} .
\end{aligned}
$$

We define the steady state consumption and investment shares as $\gamma_{c} \equiv \frac{\bar{C}}{\bar{C}+\bar{X}}=1-\gamma_{x}$ and $\gamma_{x} \equiv \frac{\bar{X}}{\bar{C}+\bar{X}}$, 
respectively. We can log-linearize the price sub-indexes in (13) - (14) as,

$$
\begin{aligned}
\widehat{p}_{t}^{H} & \approx \int_{0}^{1} \widehat{p}_{t}(h) d h, \widehat{p}_{t}^{F} \approx \int_{0}^{1} \widehat{p}_{t}(f) d f \\
\widehat{p}_{t}^{H *} & \approx \int_{0}^{1} \widehat{p}_{t}^{*}(h) d h, \widehat{p}_{t}^{F *} \approx \int_{0}^{1} \widehat{p}_{t}^{*}(f) d f,
\end{aligned}
$$

so it follows that the weighted prices of all domestic and all foreign firms are $\widehat{p}_{t}^{H, W} \approx \int_{0}^{1} \widehat{p}_{t}^{W}(h) d h$ and $\widehat{p}_{t}^{F, W *} \approx \int_{0}^{1} \widehat{p}_{t}^{W *}(f) d f$, respectively. Adding up the output market clearing conditions for all varieties (firms) within each country, we obtain an expression for aggregate output in these terms,

$$
\begin{aligned}
& \widehat{y}_{t} \approx-\eta\left(\widehat{p}_{t}^{H, W}-\widehat{p}_{t}^{W}\right)+\left(1-\gamma_{x}\right) \widehat{c}_{t}^{W}+\gamma_{x} \widehat{x}_{t}^{W} \\
& \widehat{y}_{t}^{*} \approx-\eta\left(\widehat{p}_{t}^{F, W *}-\widehat{p}_{t}^{W *}\right)+\left(1-\gamma_{x}\right) \widehat{c}_{t}^{W *}+\gamma_{x} \widehat{x}_{t}^{W *}
\end{aligned}
$$

These equations become very important in our posterior derivations of the Phillips curves. Furthermore, if we combine the efficiency conditions in (130) - (131) with the output equations derived in (132) - (133), it follows that,

$$
\begin{aligned}
& \widehat{r}_{t}^{z} \approx\left(\frac{1}{\sigma}+\left(1-\gamma_{x}\right) \frac{1+\varphi}{\psi}\right) \widehat{c}_{t}^{W}+\gamma_{x} \frac{1+\varphi}{\psi} \widehat{x}_{t}^{W}+\phi_{F} \widehat{r s}_{t}-\eta \frac{1+\varphi}{\psi}\left(\widehat{p}_{t}^{H, W}-\widehat{p}_{t}^{W}\right)-\ldots \\
& \quad\left(\frac{1+(1-\psi) \varphi}{\psi}\right) \widehat{k}_{t}-\frac{1+\varphi}{\psi} \widehat{a}_{t}, \\
& \widehat{r}_{t}^{z *} \approx\left(\frac{1}{\sigma}+\left(1-\gamma_{x}\right) \frac{1+\varphi}{\psi}\right) \widehat{c}_{t}^{W *}+\gamma_{x} \frac{1+\varphi}{\psi} \widehat{x}_{t}^{W *}-\phi_{F} \widehat{r s}_{t}-\eta \frac{1+\varphi}{\psi}\left(\widehat{p}_{t}^{F, W *}-\widehat{p}_{t}^{W *}\right)-\ldots \\
& \quad\left(\frac{1+(1-\psi) \varphi}{\psi}\right) \widehat{k}_{t}^{*}-\frac{1+\varphi}{\psi} \widehat{a}_{t}^{*},
\end{aligned}
$$

These conditions can be appropriately used to simplify the description of the inflation dynamics.

The Optimal Pricing Equations. In steady state the standard pricing rule under monopolistic competition holds. Accordingly, the log-linearization of the optimal pricing equations in (71), (72), (73) and (74) can be compactly expressed as follows,

$$
\begin{aligned}
& \widehat{\widetilde{p}}_{t}(h)-\widehat{p}_{t} \approx \mathbb{E}_{t}\left[\sum_{\tau=1}^{+\infty}(\beta \alpha)^{\tau} \widehat{\pi}_{t+\tau}\right]+(1-\beta \alpha) \mathbb{E}_{t}\left[\sum_{\tau=0}^{+\infty}(\beta \alpha)^{\tau}\left(\widehat{m c}_{t+\tau}-\widehat{p}_{t+\tau}\right)\right], \\
& \widehat{\widetilde{p}}_{t}^{*}(h)-\widehat{p}_{t}^{*} \approx \mathbb{E}_{t}\left[\sum_{\tau=1}^{+\infty}(\beta \alpha)^{\tau} \widehat{\pi}_{t+\tau}^{*}\right]+(1-\beta \alpha) \mathbb{E}_{t}\left[\sum_{\tau=0}^{+\infty}(\beta \alpha)^{\tau}\left(\widehat{m c}_{t+\tau}-\widehat{p}_{t+\tau}-\widehat{r s}_{t+\tau}\right)\right], \\
& \widehat{\widetilde{p}}_{t}(f)-\widehat{p}_{t} \approx \mathbb{E}_{t}\left[\sum_{\tau=1}^{+\infty}(\beta \alpha)^{\tau} \widehat{\pi}_{t+\tau}\right]+(1-\beta \alpha) \mathbb{E}_{t}\left[\sum_{\tau=0}^{+\infty}(\beta \alpha)^{\tau}\left(\widehat{m c}_{t+\tau}^{*}-\widehat{p}_{t+\tau}^{*}+\widehat{r s}_{t+\tau}\right)\right], \\
& \widehat{\widetilde{p}}_{t}^{*}(f)-\widehat{p}_{t}^{*} \approx \mathbb{E}_{t}\left[\sum_{\tau=1}^{+\infty}(\beta \alpha)^{\tau} \widehat{\pi}_{t+\tau}^{*}\right]+(1-\beta \alpha) \mathbb{E}_{t}\left[\sum_{\tau=0}^{+\infty}(\beta \alpha)^{\tau}\left(\widehat{m c}_{t+\tau}^{*}-\widehat{p}_{t+\tau}^{*}\right)\right],
\end{aligned}
$$

which defines the distance between the optimal price decision of a given re-optimizing firm and the CPI level prevailing in each market as a weighted function of current and expected future CPI inflation and real marginal costs. Here I must recall the assumption that the government subsidy is time-invariant and equal to its steady state value in every period, which explains why the government subsidies do not appear in the log-linearized pricing equations. We derive the (pre-subsidy) marginal cost equations in (20) - (21), and 
they can be log-linearized as,

$$
\begin{aligned}
& \widehat{m c}_{t+\tau} \approx \psi \widehat{w}_{t+\tau}+(1-\psi)\left(\widehat{r}_{t+\tau}^{z}+\widehat{p}_{t+\tau}\right)-\widehat{a}_{t+\tau}, \\
& \widehat{m c}_{t+\tau}^{*} \approx \psi \widehat{w}_{t+\tau}^{*}+(1-\psi)\left(\widehat{r}_{t+\tau}^{* *}+\widehat{p}_{t+\tau}^{*}\right)-\widehat{a}_{t+\tau}^{*},
\end{aligned}
$$

while the labor market clearing conditions, which are implicit in (95) - (96), can be approximated as,

$$
\begin{aligned}
& \widehat{w}_{t+\tau} \approx-\frac{\varphi}{1+(1-\psi) \varphi} \widehat{a}_{t+\tau}+\frac{1}{1+(1-\psi) \varphi} \frac{1}{\sigma} \widehat{c}_{t+\tau}+\frac{\varphi}{1+(1-\psi) \varphi} \widehat{y}_{t+\tau}+\frac{(1-\psi) \varphi}{1+(1-\psi) \varphi}\left(\widehat{r}_{t+\tau}^{z}+\widehat{p}_{t+\tau}\right)+\frac{1}{1+(1-\psi) \varphi} \widehat{p}_{t+\tau}, \\
& \widehat{w}_{t+\tau}^{*} \approx-\frac{\varphi}{1+(1-\psi) \varphi} \widehat{a}_{t+\tau}^{*}+\frac{1}{1+(1-\psi) \varphi} \frac{1}{\sigma} \widehat{c}_{t+\tau}^{*}+\frac{\varphi}{1+(1-\psi) \varphi} \widehat{y}_{t+\tau}^{*}+\frac{(1-\psi) \varphi}{1+(1-\psi) \varphi}\left(\widehat{r}_{t+\tau}^{* *}+\widehat{p}_{t+\tau}^{*}\right)+\frac{1}{1+(1-\psi) \varphi} \widehat{p}_{t+\tau}^{*} .
\end{aligned}
$$

The labor market clearing conditions and the marginal costs reduce to the standard linear-in-labor case without capital if the labor share in the production function goes to one (i.e., $\psi \rightarrow 1$ ), as expected. If we combine these two log-linearized equations, it follows that the marginal costs can be expressed as,

$$
\begin{aligned}
& \widehat{m c}_{t+\tau} \approx \frac{\psi}{1+(1-\psi) \varphi} \frac{1}{\sigma} \widehat{c}_{t+\tau}+\frac{\varphi \psi}{1+(1-\psi) \varphi} \widehat{y}_{t+\tau}-\frac{1+\varphi}{1+(1-\psi) \varphi}\left(\widehat{a}_{t+\tau}-(1-\psi)\left(\widehat{r}_{t+\tau}^{z}+\widehat{p}_{t+\tau}\right)\right)+\frac{\psi}{1+(1-\psi) \varphi} \widehat{p}_{t+\tau}, \\
& \widehat{m c}_{t+\tau}^{*} \approx \frac{\psi}{1+(1-\psi) \varphi} \frac{1}{\sigma} \widehat{c}_{t+\tau}^{*}+\frac{\varphi \psi}{1+(1-\psi) \varphi} \widehat{y}_{t+\tau}^{*}-\frac{1+\varphi}{1+(1-\psi) \varphi}\left(\widehat{a}_{t+\tau}^{*}-(1-\psi)\left(\widehat{r}_{t+\tau}^{z *}+\widehat{p}_{t+\tau}^{*}\right)\right)+\frac{\psi}{1+(1-\psi) \varphi} \widehat{p}_{t+\tau}^{*},
\end{aligned}
$$

where $\widehat{y}_{t+\tau}$ and $\widehat{y}_{t+\tau}^{*}$ reflect domestic and foreign aggregate output. Finally, if we combine the marginal cost equations with the output equations derived before in (132) - (133), it follows that,

$$
\begin{aligned}
& \widehat{m c}_{t+\tau}-\widehat{p}_{t+\tau} \approx \frac{\psi}{1+(1-\psi) \varphi} \frac{1}{\sigma} \widehat{c}_{t+\tau}-\frac{\varphi \psi}{1+(1-\psi) \varphi} \eta\left(\widehat{p}_{t+\tau}^{H, W}-\widehat{p}_{t+\tau}^{W}\right)+\frac{\varphi \psi}{1+(1-\psi) \varphi}\left(\left(1-\gamma_{x}\right) \widehat{c}_{t+\tau}^{W}+\gamma_{x} \widehat{x}_{t+\tau}^{W}\right)-\ldots \\
& \quad \frac{1+\varphi}{1+(1-\psi) \varphi}\left(\widehat{a}_{t+\tau}-(1-\psi) \widehat{r}_{t+\tau}^{z}\right), \\
& \widehat{m c}_{t+\tau}^{*}-\widehat{p}_{t+\tau}^{*} \approx \frac{\psi}{1+(1-\psi) \varphi} \frac{1}{\sigma} \widehat{c}_{t+\tau}^{*}-\frac{\varphi \psi}{1+(1-\psi) \varphi} \eta\left(\widehat{p}_{t+\tau}^{F, W *}-\widehat{p}_{t+\tau}^{W *}\right)+\frac{\varphi \psi}{1+(1-\psi) \varphi}\left(\left(1-\gamma_{x}\right) \widehat{c}_{t+\tau}^{W *}+\gamma_{x} \widehat{x}_{t+\tau}^{W *}\right)-\ldots \\
& \quad \frac{1+\varphi}{1+(1-\psi) \varphi}\left(\widehat{a}_{t+\tau}^{*}-(1-\psi) \widehat{r}_{t+\tau}^{z *}\right) .
\end{aligned}
$$

We can use our characterization of the real marginal costs with the pricing formulas log-linearized before 
to write that,

$$
\begin{aligned}
& \widehat{\widetilde{p}}_{t}(h)-\widehat{p}_{t} \approx \\
& \quad(1-\beta \alpha)\left(\frac{\psi}{1+(1-\psi) \varphi}\right) \sum_{\tau=0}^{+\infty}(\beta \alpha)^{\tau} \mathbb{E}_{t}\left[\begin{array}{c}
\frac{1}{\sigma} \widehat{c}_{t+\tau}-\varphi \eta\left(\widehat{p}_{t+\tau}^{H, W}-\widehat{p}_{t+\tau}^{W}\right)+\varphi\left(\left(1-\gamma_{x}\right) \widehat{c}_{t+\tau}^{W}+\gamma_{x} \widehat{x}_{t+\tau}^{W}\right)-\ldots \\
\left(\frac{1+\varphi}{\psi}\right)\left(\widehat{a}_{t+\tau}-(1-\psi) \widehat{r}_{t+\tau}^{z}\right)
\end{array}\right]+\ldots \\
& \quad \sum_{\tau=1}^{+\infty}(\beta \alpha)^{\tau} \mathbb{E}_{t}\left(\widehat{\pi}_{t+\tau}\right), \\
& \widehat{\widetilde{p}}_{t}^{*}(h)-\widehat{p}_{t}^{*} \approx \\
& \quad(1-\beta \alpha)\left(\frac{\psi}{1+(1-\psi) \varphi}\right) \sum_{\tau=0}^{+\infty}(\beta \alpha)^{\tau} \mathbb{E}_{t}\left[\begin{array}{c}
\frac{1}{\sigma} \widehat{c}_{t+\tau}-\varphi \eta\left(\widehat{p}_{t+\tau}^{H, W}-\widehat{p}_{t+\tau}^{W}\right)+\varphi\left(\left(1-\gamma_{x}\right) \widehat{c}_{t+\tau}^{W}+\gamma_{x} \widehat{x}_{t+\tau}^{W}\right)-\ldots \\
\left(\frac{1+\varphi}{\psi}\right)\left(\widehat{a}_{t+\tau}-(1-\psi) \widehat{r}_{t+\tau}^{z}\right)-\left(\frac{1+(1-\psi) \varphi}{\psi}\right) \widehat{r s}_{t+\tau}
\end{array}\right]+\ldots \\
& \quad \sum_{\tau=1}^{+\infty}(\beta \alpha)^{\tau} \mathbb{E}_{t}\left(\widehat{\pi}_{t+\tau}^{*}\right), \\
& \widehat{\widetilde{p}}_{t}(f)-\widehat{p}_{t} \approx \\
& \quad(1-\beta \alpha)\left(\frac{\psi}{1+(1-\psi) \varphi}\right) \sum_{\tau=0}^{+\infty}(\beta \alpha)^{\tau} \mathbb{E}_{t}\left[\begin{array}{c}
\frac{1}{\sigma} \widehat{c}_{t+\tau}^{*}-\varphi \eta\left(\widehat{p}_{t+\tau}^{F, W *}-\widehat{p}_{t+\tau}^{W *}\right)+\varphi\left(\left(1-\gamma_{x}\right) \widehat{c}_{t+\tau}^{W *}+\gamma_{x} \widehat{x}_{t+\tau}^{W *}\right)-\ldots \\
\left(\frac{1+\varphi}{\psi}\right)\left(\widehat{a}_{t+\tau}^{*}-(1-\psi) \widehat{r}_{t+\tau}^{z *}\right)+\left(\frac{1+(1-\psi) \varphi}{\psi}\right) \widehat{r s}_{t+\tau}
\end{array}\right]+\ldots \\
& \sum_{\tau=1}^{+\infty}(\beta \alpha)^{\tau} \mathbb{E}_{t}\left(\widehat{\pi}_{t+\tau}\right), \\
& \widehat{\widetilde{p}}_{t}^{*}(f)-\widehat{p}_{t}^{*} \approx \\
& \quad(1-\beta \alpha)\left(\frac{\psi}{1+(1-\psi) \varphi}\right) \sum_{\tau=0}^{+\infty}(\beta \alpha)^{\tau} \mathbb{E}_{t}\left[\begin{array}{c}
\frac{1}{\sigma} \widehat{c}_{t+\tau}^{*}-\varphi \eta\left(\widehat{p}_{t+\tau}^{F, W *}-\widehat{p}_{t+\tau}^{W *}\right)+\varphi\left(\left(1-\gamma_{x}\right) \widehat{c}_{t+\tau}^{W *}+\gamma_{x} \widehat{x}_{t+\tau}^{W *}\right)-\ldots \\
\left(\frac{1+\varphi}{\psi}\right)\left(\widehat{a}_{t+\tau}^{*}-(1-\psi) \widehat{r}_{t+\tau}^{z *}\right)
\end{array}\right]+\ldots \\
& \quad \sum_{\tau=1}^{+\infty}(\beta \alpha)^{\tau} \mathbb{E}_{t}\left(\widehat{\pi}_{t+\tau}^{*}\right) .
\end{aligned}
$$

We log-linearize the price sub-indexes in (75) - (76) and (77) - (78) and re-arrange them to obtain that,

$$
\begin{aligned}
& \widehat{\widetilde{p}}_{t}(h)-\widehat{p}_{t} \approx\left(\widehat{p}_{t}^{H}-\widehat{p}_{t}\right)+\left(\frac{\alpha}{1-\alpha}\right) \widehat{\pi}_{t}^{H}, \\
& \widehat{\widetilde{p}}_{t}^{*}(h)-\widehat{p}_{t}^{*} \approx\left(\widehat{p}_{t}^{H *}-\widehat{p}_{t}^{*}\right)+\left(\frac{\alpha}{1-\alpha}\right) \widehat{\pi}_{t}^{H *}, \\
& \widehat{\widetilde{p}}_{t}(f)-\widehat{p}_{t} \approx\left(\widehat{p}_{t}^{F}-\widehat{p}_{t}\right)+\left(\frac{\alpha}{1-\alpha}\right) \widehat{\pi}_{t}^{F}, \\
& \widehat{\widetilde{p}}_{t}^{*}(f)-\widehat{p}_{t}^{*} \approx\left(\widehat{p}_{t}^{F *}-\widehat{p}_{t}^{*}\right)+\left(\frac{\alpha}{1-\alpha}\right) \widehat{\pi}_{t}^{F *},
\end{aligned}
$$

which is quite convenient for our purposes. We replace the isolated terms $\frac{1}{\sigma} \widehat{c}_{t}$ and $\frac{1}{\sigma} \widehat{c}_{t}^{*}$ out of the marginal cost. If we define the world consumption as $\widehat{c}_{t}^{W} \equiv \phi_{H} \widehat{c}_{t}+\phi_{F} \widehat{c}_{t}^{*}$ and $\widehat{c}_{t}^{W *} \equiv \phi_{F} \widehat{c}_{t}+\phi_{H} \widehat{c}_{t}^{*}$ and the relative consumption as $\widehat{c}_{t}^{R} \equiv \widehat{c}_{t}-\widehat{c}_{t}^{*}$, then we can write that,

$$
\begin{aligned}
\widehat{c}_{t} & =\widehat{c}_{t}^{W}+\phi_{F} \widehat{c}_{t}^{R} \approx \widehat{c}_{t}^{W}+\phi_{F} \sigma \widehat{r s}_{t}, \\
\widehat{c}_{t}^{*} & =\widehat{c}_{t}^{W *}-\phi_{F} \widehat{c}_{t}^{R} \approx \widehat{c}_{t}^{W *}-\phi_{F} \sigma \widehat{r} s_{t},
\end{aligned}
$$

where the second approximation follows from the perfect international risk-sharing condition in (99). Hence, 
the pricing equations can be expressed more compactly as,

$$
\begin{aligned}
& \widehat{\pi}_{t}^{H}+\left(\frac{1-\alpha}{\alpha}\right)\left(\widehat{p}_{t}^{H}-\widehat{p}_{t}\right) \approx \\
& \Psi\left(\frac{\psi}{1+(1-\psi) \varphi}\right) \sum_{\tau=0}^{+\infty}(\beta \alpha)^{\tau} \mathbb{E}_{t}\left[\begin{array}{c}
\left(\sigma^{-1}+\left(1-\gamma_{x}\right) \varphi\right) \widehat{c}_{t+\tau}^{W}+\gamma_{x} \varphi \widehat{x}_{t+\tau}^{W}+\ldots \\
\phi_{F} \widehat{r s s}_{t+\tau}-\varphi \eta\left(\widehat{p}_{t+\tau}^{H, W}-\widehat{p}_{t+\tau}^{W}\right)-\ldots \\
\left(\frac{1+\varphi}{\psi}\right)\left(\widehat{a}_{t+\tau}-(1-\psi) \widehat{r}_{t+\tau}^{z}\right)
\end{array}\right]+\ldots \\
& \left(\frac{1-\alpha}{\alpha}\right) \sum_{\tau=1}^{+\infty}(\beta \alpha)^{\tau} \mathbb{E}_{t}\left(\widehat{\pi}_{t+\tau}\right), \\
& \widehat{\pi}_{t}^{H *}+\left(\frac{1-\alpha}{\alpha}\right)\left(\widehat{p}_{t}^{H *}-\widehat{p}_{t}^{*}\right) \approx \\
& \Psi\left(\frac{\psi}{1+(1-\psi) \varphi}\right) \sum_{\tau=0}^{+\infty}(\beta \alpha)^{\tau} \mathbb{E}_{t}\left[\begin{array}{c}
\left(\sigma^{-1}+\left(1-\gamma_{x}\right) \varphi\right) \widehat{c}_{t+\tau}^{W}+\gamma_{x} \varphi \widehat{x}_{t+\tau}^{W}-\ldots \\
\left(\phi_{H}+\frac{1-\psi}{\psi}(1+\varphi)\right) \widehat{r s}_{t+\tau}-\varphi \eta\left(\widehat{p}_{t+\tau}^{H, W}-\widehat{p}_{t+\tau}^{W}\right)-\ldots \\
\left(\frac{1+\varphi}{\psi}\right)\left(\widehat{a}_{t+\tau}-(1-\psi) \widehat{r}_{t+\tau}^{z}\right)
\end{array}\right]+\ldots \\
& \left(\frac{1-\alpha}{\alpha}\right) \sum_{\tau=1}^{+\infty}(\beta \alpha)^{\tau} \mathbb{E}_{t}\left(\widehat{\pi}_{t+\tau}^{*}\right), \\
& \widehat{\pi}_{t}^{F}+\left(\frac{1-\alpha}{\alpha}\right)\left(\widehat{p}_{t}^{F}-\widehat{p}_{t}\right) \approx \\
& \Psi\left(\frac{\psi}{1+(1-\psi) \varphi}\right) \sum_{\tau=0}^{+\infty}(\beta \alpha)^{\tau} \mathbb{E}_{t}\left[\begin{array}{c}
\left(\sigma^{-1}+\left(1-\gamma_{x}\right) \varphi\right) \widehat{c}_{t+\tau}^{W *}+\gamma_{x} \varphi \widehat{x}_{t+\tau}^{W *}+\ldots \\
\left(\phi_{H}+\frac{1-\psi}{\psi}(1+\varphi)\right) \widehat{r s}_{t+\tau}-\varphi \eta\left(\widehat{p}_{t+\tau}^{F, W *}-\widehat{p}_{t+\tau}^{W *}\right)-\ldots \\
\left(\frac{1+\varphi}{\psi}\right)\left(\widehat{a}_{t+\tau}^{*}-(1-\psi) \widehat{r}_{t+\tau}^{z *}\right)
\end{array}\right]+\ldots \\
& \left(\frac{1-\alpha}{\alpha}\right) \sum_{\tau=1}^{+\infty}(\beta \alpha)^{\tau} \mathbb{E}_{t}\left(\widehat{\pi}_{t+\tau}\right), \\
& \widehat{\pi}_{t}^{F *}+\left(\frac{1-\alpha}{\alpha}\right)\left(\widehat{p}_{t}^{F *}-\widehat{p}_{t}^{*}\right) \approx \\
& \Psi\left(\frac{\psi}{1+(1-\psi) \varphi}\right) \sum_{\tau=0}^{+\infty}(\beta \alpha)^{\tau} \mathbb{E}_{t}\left[\begin{array}{c}
\left(\sigma^{-1}+\left(1-\gamma_{x}\right) \varphi\right) \widehat{c}_{t+\tau}^{W *}+\gamma_{x} \varphi \widehat{x}_{t+\tau}^{W *}-\ldots \\
\phi_{F} \widehat{r s}_{t+\tau}-\varphi \eta\left(\widehat{p}_{t+\tau}^{F, W *}-\widehat{p}_{t+\tau}^{W *}\right)-\ldots \\
\left(\frac{1+\varphi}{\psi}\right)\left(\widehat{a}_{t+\tau}^{*}-(1-\psi) \widehat{r}_{t+\tau}^{z *}\right)
\end{array}\right]+\ldots \\
& \left(\frac{1-\alpha}{\alpha}\right) \sum_{\tau=1}^{+\infty}(\beta \alpha)^{\tau} \mathbb{E}_{t}\left(\widehat{\pi}_{t+\tau}^{*}\right),
\end{aligned}
$$

where $\Psi \equiv \frac{(1-\alpha)(1-\beta \alpha)}{\alpha}$.

Furthermore, these pricing equations can be expressed in the form of a system of expectational difference equations. Let us focus on the first equation as an example. If we re-write the equation at time $t+1$ and take conditional expectations on information up to time $t$, it should follow that,

$$
\begin{aligned}
& \mathbb{E}_{t}\left[\widehat{\pi}_{t+1}^{H}+\left(\frac{1-\alpha}{\alpha}\right)\left(\widehat{p}_{t+1}^{H}-\widehat{p}_{t+1}\right)\right] \approx \\
& \Psi\left(\frac{\psi}{1+(1-\psi) \varphi}\right) \sum_{\tau=0}^{+\infty}(\beta \alpha)^{\tau} \mathbb{E}_{t}\left[\begin{array}{c}
\left(\sigma^{-1}+\left(1-\gamma_{x}\right) \varphi\right) \widehat{c}_{t+1+\tau}^{W}+\gamma_{x} \varphi \widehat{x}_{t+1+\tau}^{W}+\ldots \\
\phi_{F} \widehat{r s}_{t+1+\tau}-\varphi \eta\left(\widehat{p}_{t+1+\tau}^{H, W}-\widehat{p}_{t+1+\tau}^{W}\right)-\ldots \\
\left(\frac{1+\varphi}{\psi}\right)\left(\widehat{a}_{t+1+\tau}-(1-\psi) \widehat{r}_{t+1+\tau}^{z}\right)
\end{array}\right]+\ldots \\
& \left(\frac{1-\alpha}{\alpha}\right) \sum_{\tau=1}^{+\infty}(\beta \alpha)^{\tau} \mathbb{E}_{t}\left(\widehat{\pi}_{t+1+\tau}\right) .
\end{aligned}
$$


Hence, using this conditional expectation, the pricing equation can easily be decomposed in two terms as,

$$
\begin{aligned}
& \widehat{\pi}_{t}^{H}+\left(\frac{1-\alpha}{\alpha}\right)\left(\widehat{p}_{t}^{H}-\widehat{p}_{t}\right) \approx \\
& \quad\left(\frac{\psi}{1+(1-\psi) \varphi}\right) \Psi\left[\begin{array}{c}
\left.\left(\sigma^{-1}+\left(1-\gamma_{x}\right) \varphi\right) \widehat{c}_{t}^{W}+\gamma_{x} \varphi \widehat{x}_{t}^{W}+\phi_{F} \widehat{r s}_{t}-\varphi \eta\left(\widehat{p}_{t}^{H, W}-\widehat{p}_{t}^{W}\right)-\ldots\right] \\
\left(\frac{1+\varphi}{\psi}\right)\left(\widehat{a}_{t}-(1-\psi) \widehat{r}_{t}^{z}\right)
\end{array}\right]+\ldots \\
& \quad(1-\alpha) \beta \mathbb{E}_{t}\left(\widehat{\pi}_{t+1}\right)+\alpha \beta \mathbb{E}_{t}\left[\widehat{\pi}_{t+1}^{H}+\left(\frac{1-\alpha}{\alpha}\right)\left(\widehat{p}_{t+1}^{H}-\widehat{p}_{t+1}\right)\right] .
\end{aligned}
$$

Further re-arranging allows us to express the expectational difference equation as,

$$
\begin{aligned}
& \widehat{\pi}_{t}^{H}-\beta \mathbb{E}_{t}\left(\widehat{\pi}_{t+1}^{H}\right)+\Psi\left(\widehat{p}_{t}^{H}-\widehat{p}_{t}\right) \\
& \quad \approx\left(\frac{\psi}{1+(1-\psi) \varphi}\right) \Psi\left[\begin{array}{c}
\left(\sigma^{-1}+\left(1-\gamma_{x}\right) \varphi\right) \widehat{c}_{t}^{W}+\gamma_{x} \varphi \widehat{x}_{t}^{W}+\phi_{F} \widehat{r s}_{t}-\varphi \eta\left(\widehat{p}_{t}^{H, W}-\widehat{p}_{t}^{W}\right)-\ldots \\
\left(\frac{1+\varphi}{\psi}\right)\left(\widehat{a}_{t}-(1-\psi) \widehat{r}_{t}^{z}\right)
\end{array}\right] .
\end{aligned}
$$

We can apply the same approach (and algebraic steps) to re-write all other pricing equations as expectational difference equations, i.e.

$$
\begin{aligned}
& \widehat{\pi}_{t}^{H *}-\beta \mathbb{E}_{t}\left(\widehat{\pi}_{t+1}^{H *}\right)+\Psi\left(\widehat{p}_{t}^{H *}-\widehat{p}_{t}^{*}\right) \\
& \quad \approx\left(\frac{\psi}{1+(1-\psi) \varphi}\right) \Psi\left[\begin{array}{c}
\left(\sigma^{-1}+\left(1-\gamma_{x}\right) \varphi\right) \widehat{c}_{t}^{W}+\gamma_{x} \varphi \widehat{x}_{t}^{W}-\left(\phi_{H}+\frac{1-\psi}{\psi}(1+\varphi)\right) \widehat{r s}_{t}-\varphi \eta\left(\widehat{p}_{t}^{H, W}-\widehat{p}_{t}^{W}\right)-\ldots \\
\left(\frac{1+\varphi}{\psi}\right)\left(\widehat{a}_{t}-(1-\psi) \widehat{r}_{t}^{z}\right)
\end{array}\right], \\
& \widehat{\pi}_{t}^{F}-\beta \mathbb{E}_{t}\left(\widehat{\pi}_{t+1}^{F}\right)+\Psi\left(\widehat{p}_{t}^{F}-\widehat{p}_{t}\right) \\
& \quad \approx\left(\frac{\psi}{1+(1-\psi) \varphi}\right) \Psi\left[\begin{array}{c}
\left(\sigma^{-1}+\left(1-\gamma_{x}\right) \varphi\right) \widehat{c}_{t}^{W *}+\gamma_{x} \varphi \widehat{x}_{t}^{W *}+\left(\phi_{H}+\frac{1-\psi}{\psi}(1+\varphi)\right) \widehat{r s}_{t}-\varphi \eta\left(\widehat{p}_{t}^{F, W *}-\widehat{p}_{t}^{W *}\right)-\ldots \\
\left(\frac{1+\varphi}{\psi}\right)\left(\widehat{a}_{t}^{*}-(1-\psi) \widehat{r}_{t}^{z *}\right)
\end{array}\right], \\
& \widehat{\pi}_{t}^{F *}-\beta \mathbb{E}_{t}\left(\widehat{\pi}_{t+1}^{F *}\right)+\Psi\left(\widehat{p}_{t}^{F *}-\widehat{p}_{t}^{*}\right) \\
& \quad \approx\left(\frac{\psi}{1+(1-\psi) \varphi}\right) \Psi\left[\begin{array}{c}
\left.\left(\sigma^{-1}+\left(1-\gamma_{x}\right) \varphi\right) \widehat{c}_{t}^{W *}+\gamma_{x} \varphi \widehat{x}_{t}^{W *}-\phi_{F} \widehat{r s}_{t}-\varphi \eta\left(\widehat{p}_{t}^{F, W *}-\widehat{p}_{t}^{W *}\right)-\ldots\right] \\
\left(\frac{1+\varphi}{\psi}\right)\left(\widehat{a}_{t}^{*}-(1-\psi) \widehat{r}_{t}^{z *}\right)
\end{array}\right] .
\end{aligned}
$$

These equations provide a very simple characterization of the price dynamics at the price sub-index level.

Now, we use the pricing equations described above to infer the dynamics of the relative price sub-indexes $\widehat{\pi}_{t}^{H, R} \equiv \widehat{\pi}_{t}^{H}-\widehat{\pi}_{t}^{H *}\left(\widehat{p}_{t}^{H, R} \equiv \widehat{p}_{t}^{H}-\widehat{p}_{t}^{H *}\right)$ and $\widehat{\pi}_{t}^{F, R} \equiv \widehat{\pi}_{t}^{F}-\widehat{\pi}_{t}^{F *}\left(\widehat{p}_{t}^{F, R} \equiv \widehat{p}_{t}^{F}-\widehat{p}_{t}^{F *}\right)$ as follows,

$$
\begin{aligned}
\widehat{\pi}_{t}^{H, R}-\beta \mathbb{E}_{t}\left(\widehat{\pi}_{t+1}^{H, R}\right)+\Psi\left(\widehat{p}_{t}^{H, R}-\widehat{p}_{t}^{R}\right) & \approx \Psi \widehat{r s}_{t}, \\
\widehat{\pi}_{t}^{F, R}-\beta \mathbb{E}_{t}\left(\widehat{\pi}_{t+1}^{F, R}\right)+\Psi\left(\widehat{p}_{t}^{F, R}-\widehat{p}_{t}^{R}\right) & \approx \Psi \widehat{r s}_{t},
\end{aligned}
$$


where $\widehat{p}_{t}^{R} \equiv \widehat{p}_{t}-\widehat{p}_{t}^{*}$ is the relative CPI. We can re-write the price sub-index equations further as follows,

$$
\begin{aligned}
& \widehat{\pi}_{t}^{H} \approx \beta \mathbb{E}_{t}\left(\widehat{\pi}_{t+1}^{H}\right)+\ldots \\
& \left(\frac{\psi}{1+(1-\psi) \varphi}\right) \Psi\left[\begin{array}{c}
\left(\sigma^{-1}+\left(1-\gamma_{x}\right) \varphi\right) \widehat{c}_{t}^{W}+\gamma_{x} \varphi \widehat{x}_{t}^{W}+\ldots \\
\phi_{F} \widehat{r}_{t}-\varphi \eta\left(\widehat{p}_{t}^{H, W}-\widehat{p}_{t}^{W}\right)-\ldots \\
\left(\frac{1+(1-\psi) \varphi}{\psi}\right)\left(\widehat{p}_{t}^{H}-\widehat{p}_{t}\right)-\left(\frac{1+\varphi}{\psi}\right)\left(\widehat{a}_{t}-(1-\psi) \widehat{r}_{t}^{Z}\right)
\end{array}\right], \\
& \widehat{\pi}_{t}^{H *} \approx \beta \mathbb{E}_{t}\left(\widehat{\pi}_{t+1}^{H *}\right)+\ldots \\
& \left(\frac{\psi}{1+(1-\psi) \varphi}\right) \Psi\left[\begin{array}{c}
\left(\sigma^{-1}+\left(1-\gamma_{x}\right) \varphi\right) \widehat{c}_{t}^{W}+\gamma_{x} \varphi \widehat{x}_{t}^{W}-\ldots \\
\left(\phi_{H}+\frac{1-\psi}{\psi}(1+\varphi)\right) \widehat{r}_{t}-\varphi \eta\left(\widehat{p}_{t}^{H, W}-\widehat{p}_{t}^{W}\right)-\ldots \\
\left(\frac{1+(1-\psi) \varphi}{\psi}\right)\left(\widehat{p}_{t}^{H *}-\widehat{p}_{t}^{*}\right)-\left(\frac{1+\varphi}{\psi}\right)\left(\widehat{a}_{t}-(1-\psi) \widehat{r}_{t}^{z}\right)
\end{array}\right], \\
& \widehat{\pi}_{t}^{F} \approx \beta \mathbb{E}_{t}\left(\widehat{\pi}_{t+1}^{F}\right)+\ldots \\
& \left(\frac{\psi}{1+(1-\psi) \varphi}\right) \Psi\left[\begin{array}{c}
\left(\sigma^{-1}+\left(1-\gamma_{x}\right) \varphi\right) \widehat{c}_{t}^{W *}+\gamma_{x} \varphi \widehat{x}_{t}^{W *}+\ldots \\
\left(\phi_{H}+\frac{1-\psi}{\psi}(1+\varphi)\right) \widehat{r s}_{t}-\varphi \eta\left(\widehat{p}_{t}^{F, W *}-\widehat{p}_{t}^{W *}\right)-\ldots \\
\left(\frac{1+(1-\psi) \varphi}{\psi}\right)\left(\widehat{p}_{t}^{F}-\widehat{p}_{t}\right)-\left(\frac{1+\varphi}{\psi}\right)\left(\widehat{a}_{t}^{*}-(1-\psi) \widehat{r}_{t}^{z *}\right)
\end{array}\right] \\
& \widehat{\pi}_{t}^{F *} \approx \beta \mathbb{E}_{t}\left(\widehat{\pi}_{t+1}^{F *}\right)+\ldots \\
& \left(\frac{\psi}{1+(1-\psi) \varphi}\right) \Psi\left[\begin{array}{c}
\left(\sigma^{-1}+\left(1-\gamma_{x}\right) \varphi\right) \widehat{c}_{t}^{W *}+\gamma_{x} \varphi \widehat{x}_{t}^{W *}-\ldots \\
\phi_{F} \widehat{r s}_{t}-\varphi \eta\left(\widehat{p}_{t}^{F, W *}-\widehat{p}_{t}^{W *}\right)-\ldots \\
\left(\frac{1+(1-\psi) \varphi}{\psi}\right)\left(\widehat{p}_{t}^{F *}-\widehat{p}_{t}^{*}\right)-\left(\frac{1+\varphi}{\psi}\right)\left(\widehat{a}_{t}^{*}-(1-\psi) \widehat{r}_{t}^{2 *}\right)
\end{array}\right] .
\end{aligned}
$$

We have defined the world price sub-indexes as $\widehat{p}_{t}^{H, W} \equiv \phi_{H} \widehat{p}_{t}^{H}+\phi_{F} \widehat{p}_{t}^{H *}$ and $\widehat{p}_{t}^{F, W *} \equiv \phi_{F} \widehat{p}_{t}^{F}+\phi_{H} \widehat{p}_{t}^{F *}$, and the relative price sub-indexes as $\widehat{p}_{t}^{H, R} \equiv \widehat{p}_{t}^{H}-\widehat{p}_{t}^{H *}$ and $\widehat{p}_{t}^{F, R} \equiv \widehat{p}_{t}^{F}-\widehat{p}_{t}^{F *}$. Then, naturally, we can write that,

$$
\begin{aligned}
\widehat{p}_{t}^{H} & =\widehat{p}_{t}^{H, W}+\phi_{F} \widehat{p}_{t}^{H, R}, \widehat{p}_{t}^{H *}=\widehat{p}_{t}^{H, W}-\phi_{H} \widehat{p}_{t}^{H, R}, \\
\widehat{p}_{t}^{F} & =\widehat{p}_{t}^{F, W *}+\phi_{H} \widehat{p}_{t}^{F, R}, \widehat{p}_{t}^{F *}=\widehat{p}_{t}^{F, W *}-\phi_{F} \widehat{p}_{t}^{F, R} .
\end{aligned}
$$

Analogously, we have defined the world CPI as $\widehat{p}_{t}^{W} \equiv \phi_{H} \widehat{p}_{t}+\phi_{F} \widehat{p}_{t}^{*}$ and $\widehat{p}_{t}^{W *} \equiv \phi_{F} \widehat{p}_{t}+\phi_{H} \widehat{p}_{t}^{*}$, and the relative CPI as $\widehat{p}_{t}^{R} \equiv \widehat{p}_{t}-\widehat{p}_{t}^{*}$. Then, we can write that,

$$
\begin{aligned}
& \widehat{p}_{t}=\widehat{p}_{t}^{W}+\phi_{F} \widehat{p}_{t}^{R}, \widehat{p}_{t}^{*}=\widehat{p}_{t}^{W}-\phi_{H} \widehat{p}_{t}^{R}, \\
& \widehat{p}_{t}=\widehat{p}_{t}^{W *}+\phi_{H} \widehat{p}_{t}^{R}, \widehat{p}_{t}^{*}=\widehat{p}_{t}^{W *}-\phi_{F} \widehat{p}_{t}^{R} .
\end{aligned}
$$


Therefore, we can re-arrange the pricing equations as follows,

$$
\begin{aligned}
& \widehat{\pi}_{t}^{H} \approx \beta \mathbb{E}_{t}\left(\widehat{\pi}_{t+1}^{H}\right)+\ldots \\
& \left(\frac{\psi}{1+(1-\psi) \varphi}\right) \Psi\left[\begin{array}{c}
\left(\sigma^{-1}+\left(1-\gamma_{x}\right) \varphi\right) \widehat{c}_{t}^{W}+\gamma_{x} \varphi \widehat{x}_{t}^{W}+\ldots \\
\phi_{F} \widehat{r}_{t}-\left(\frac{1+(1-\psi) \varphi}{\psi}+\varphi \eta\right)\left(\widehat{p}_{t}^{H, W}-\widehat{p}_{t}^{W}\right)-\ldots \\
\left(\frac{1+(1-\psi) \varphi}{\psi}\right) \phi_{F}\left(\widehat{p}_{t}^{H, R}-\widehat{p}_{t}^{R}\right)-\left(\frac{1+\varphi}{\psi}\right)\left(\widehat{a}_{t}-(1-\psi) \widehat{r}_{t}^{z}\right)
\end{array}\right], \\
& \widehat{\pi}_{t}^{H *} \approx \beta \mathbb{E}_{t}\left(\widehat{\pi}_{t+1}^{H *}\right)+\ldots
\end{aligned}
$$

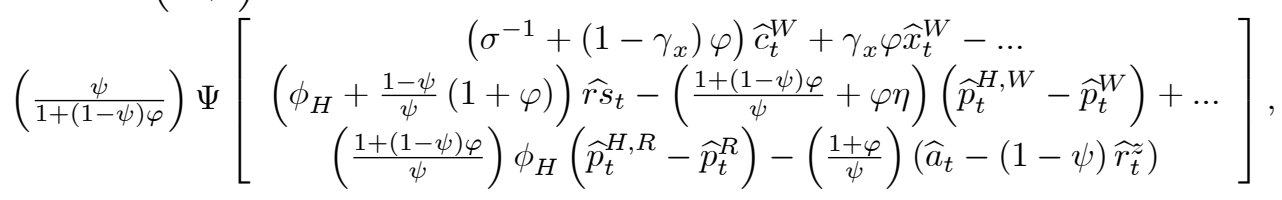

$$
\begin{aligned}
& \widehat{\pi}_{t}^{F} \approx \beta \mathbb{E}_{t}\left(\widehat{\pi}_{t+1}^{F}\right)+\ldots \\
& \left(\frac{\psi}{1+(1-\psi) \varphi}\right) \Psi\left[\begin{array}{c}
\left(\sigma^{-1}+\left(1-\gamma_{x}\right) \varphi\right) \widehat{c}_{t}^{W *}+\gamma_{x} \varphi \widehat{x}_{t}^{W *}+\ldots \\
\left(\phi_{H}+\frac{1-\psi}{\psi}(1+\varphi)\right) \widehat{r}_{t}-\left(\frac{1+(1-\psi) \varphi}{\psi}+\varphi \eta\right)\left(\widehat{p}_{t}^{F, W *}-\widehat{p}_{t}^{W *}\right)-\ldots \\
\left(\frac{1+(1-\psi) \varphi}{\psi}\right) \phi_{H}\left(\widehat{p}_{t}^{F, R}-\widehat{p}_{t}^{R}\right)-\left(\frac{1+\varphi}{\psi}\right)\left(\widehat{a}_{t}^{*}-(1-\psi) \widehat{r}_{t}^{z *}\right)
\end{array}\right], \\
& \widehat{\pi}_{t}^{F *} \approx \beta \mathbb{E}_{t}\left(\widehat{\pi}_{t+1}^{F *}\right)+\ldots \\
& \left(\frac{\psi}{1+(1-\psi) \varphi}\right) \Psi\left[\begin{array}{c}
\left(\sigma^{-1}+\left(1-\gamma_{x}\right) \varphi\right) \widehat{c}_{t}^{W *}+\gamma_{x} \varphi \widehat{x}_{t}^{W *}-\ldots \\
\phi_{F} \widehat{r s}_{t}-\left(\frac{1+(1-\psi) \varphi}{\psi}+\varphi \eta\right)\left(\widehat{p}_{t}^{F, W *}-\widehat{p}_{t}^{W *}\right)+\ldots \\
\left(\frac{1+(1-\psi) \varphi}{\psi}\right) \phi_{F}\left(\widehat{p}_{t}^{F, R}-\widehat{p}_{t}^{R}\right)-\left(\frac{1+\varphi}{\psi}\right)\left(\widehat{a}_{t}^{*}-(1-\psi) \widehat{r}_{t}^{z *}\right)
\end{array}\right] .
\end{aligned}
$$

By appropriately replacing the efficiency conditions in (134) - (135), and after a little bit of algebra, we can 
now re-arrange the pricing equations for the sub-indexes in (138) - (141) as follows,

$$
\begin{aligned}
& \widehat{\pi}_{t}^{H} \approx \beta \mathbb{E}_{t}\left(\widehat{\pi}_{t+1}^{H}\right)+\ldots \\
& \Psi\left[\begin{array}{c}
\left(\sigma^{-1}+\left(1-\gamma_{x}\right)\left(\frac{\psi}{1+(1-\psi) \varphi}\right)\left(\varphi+(1-\psi)\left(\frac{1+\varphi}{\psi}\right)^{2}\right)\right) \widehat{c}_{t}^{W}+\ldots \\
\gamma_{x}\left(\frac{\psi}{1+(1-\psi) \varphi}\right)\left(\varphi+(1-\psi)\left(\frac{1+\varphi}{\psi}\right)^{2}\right) \widehat{x}_{t}^{W}+\ldots \\
\phi_{F} \widehat{r s}_{t}-\left(1+\eta\left(\frac{\psi}{1+(1-\psi) \varphi}\right)\left(\varphi+(1-\psi)\left(\frac{1+\varphi}{\psi}\right)^{2}\right)\right)\left(\widehat{p}_{t}^{H, W}-\widehat{p}_{t}^{W}\right)-\ldots \\
\phi_{F}\left(\widehat{p}_{t}^{H, R}-\widehat{p}_{t}^{R}\right)-(1-\psi)\left(\frac{1+\varphi}{\psi}\right) \widehat{k}_{t}-\left(\frac{1+\varphi}{\psi}\right) \widehat{a}_{t}
\end{array}\right], \\
& \widehat{\pi}_{t}^{H *} \approx \beta \mathbb{E}_{t}\left(\widehat{\pi}_{t+1}^{H *}\right)+\ldots \\
& \Psi\left[\begin{array}{c}
\left(\sigma^{-1}+\left(1-\gamma_{x}\right)\left(\frac{\psi}{1+(1-\psi) \varphi}\right)\left(\varphi+(1-\psi)\left(\frac{1+\varphi}{\psi}\right)^{2}\right)\right) \widehat{c}_{t}^{W}+\ldots \\
\gamma_{x}\left(\frac{\psi}{1+(1-\psi) \varphi}\right)\left(\varphi+(1-\psi)\left(\frac{1+\varphi}{\psi}\right)^{2}\right) \widehat{x}_{t}^{W}-\ldots \\
\phi_{H} \widehat{r s s}_{t}-\left(1+\eta\left(\frac{\psi}{1+(1-\psi) \varphi}\right)\left(\varphi+(1-\psi)\left(\frac{1+\varphi}{\psi}\right)^{2}\right)\right)\left(\widehat{p}_{t}^{H, W}-\widehat{p}_{t}^{W}\right)+\ldots \\
\phi_{H}\left(\widehat{p}_{t}^{H, R}-\widehat{p}_{t}^{R}\right)-(1-\psi)\left(\frac{1+\varphi}{\psi}\right) \widehat{k}_{t}-\left(\frac{1+\varphi}{\psi}\right) \widehat{a}_{t}
\end{array}\right], \\
& \widehat{\pi}_{t}^{F} \approx \beta \mathbb{E}_{t}\left(\widehat{\pi}_{t+1}^{F}\right)+\ldots \\
& \Psi\left[\begin{array}{c}
\left(\sigma^{-1}+\left(1-\gamma_{x}\right)\left(\frac{\psi}{1+(1-\psi) \varphi}\right)\left(\varphi+(1-\psi)\left(\frac{1+\varphi}{\psi}\right)^{2}\right)\right) \widehat{c}_{t}^{W *}+\ldots \\
\gamma_{x}\left(\frac{\psi}{1+(1-\psi) \varphi}\right)\left(\varphi+(1-\psi)\left(\frac{1+\varphi}{\psi}\right)^{2}\right) \widehat{x}_{t}^{W *}+\ldots \\
\phi_{H} \widehat{r s}_{t}-\left(1+\eta\left(\frac{\psi}{1+(1-\psi) \varphi}\right)\left(\varphi+(1-\psi)\left(\frac{1+\varphi}{\psi}\right)^{2}\right)\right)\left(\widehat{p}_{t}^{F, W *}-\widehat{p}_{t}^{W *}\right)-\ldots \\
\phi_{H}\left(\widehat{p}_{t}^{F, R}-\widehat{p}_{t}^{R}\right)-(1-\psi)\left(\frac{1+\varphi}{\psi}\right) \widehat{k}_{t}^{*}-\left(\frac{1+\varphi}{\psi}\right) \widehat{a}_{t}^{*}
\end{array}\right], \\
& \widehat{\pi}_{t}^{F *} \approx \beta \mathbb{E}_{t}\left(\widehat{\pi}_{t+1}^{F *}\right)+\ldots \\
& \Psi\left[\begin{array}{c}
\left(\sigma^{-1}+\left(1-\gamma_{x}\right)\left(\frac{\psi}{1+(1-\psi) \varphi}\right)\left(\varphi+(1-\psi)\left(\frac{1+\varphi}{\psi}\right)^{2}\right)\right) \widehat{c}_{t}^{W *}+\ldots \\
\gamma_{x}\left(\frac{\psi}{1+(1-\psi) \varphi}\right)\left(\varphi+(1-\psi)\left(\frac{1+\varphi}{\psi}\right)^{2}\right) \widehat{x}_{t}^{W *}-\ldots \\
\phi_{F} \widehat{r s}_{t}-\left(1+\eta\left(\frac{\psi}{1+(1-\psi) \varphi}\right)\left(\varphi+(1-\psi)\left(\frac{1+\varphi}{\psi}\right)^{2}\right)\right)\left(\widehat{p}_{t}^{F, W *}-\widehat{p}_{t}^{W *}\right)+\ldots \\
\phi_{F}\left(\widehat{p}_{t}^{F, R}-\widehat{p}_{t}^{R}\right)-(1-\psi)\left(\frac{1+\varphi}{\psi}\right) \widehat{k}_{t}^{*}-\left(\frac{1+\varphi}{\psi}\right) \widehat{a}_{t}^{*}
\end{array}\right] .
\end{aligned}
$$

We define the world price sub-indexes as $\widehat{p}_{t}^{H, W} \equiv \phi_{H} \widehat{p}_{t}^{H}+\phi_{F} \widehat{p}_{t}^{H *}$ and $\widehat{p}_{t}^{F, W *} \equiv \phi_{F} \widehat{p}_{t}^{F}+\phi_{H} \widehat{p}_{t}^{F *}$. Therefore, we easily derive the dynamics of $\widehat{\pi}_{t}^{H, W} \equiv \widehat{p}_{t}^{H, W}-\widehat{p}_{t-1}^{H, W}$ and $\widehat{\pi}_{t}^{F, W *} \equiv \widehat{p}_{t}^{F, W *}-\widehat{p}_{t-1}^{F, W *}$ from the equations 
above as,

$$
\begin{aligned}
& \widehat{\pi}_{t}^{H, W} \approx \beta \mathbb{E}_{t}\left(\widehat{\pi}_{t+1}^{H, W}\right)+\ldots \\
& \Psi\left[\begin{array}{c}
\left(\sigma^{-1}+\left(1-\gamma_{x}\right)\left(\frac{\psi}{1+(1-\psi) \varphi}\right)\left(\varphi+(1-\psi)\left(\frac{1+\varphi}{\psi}\right)^{2}\right)\right) \widehat{c}_{t}^{W}+\ldots \\
\gamma_{x}\left(\frac{\psi}{1+(1-\psi) \varphi}\right)\left(\varphi+(1-\psi)\left(\frac{1+\varphi}{\psi}\right)^{2}\right) \widehat{x}_{t}^{W}-\ldots \\
\left(1+\eta\left(\frac{\psi}{1+(1-\psi) \varphi}\right)\left(\varphi+(1-\psi)\left(\frac{1+\varphi}{\psi}\right)^{2}\right)\right)\left(\widehat{p}_{t}^{H, W}-\widehat{p}_{t}^{W}\right)-\ldots \\
(1-\psi)\left(\frac{1+\varphi}{\psi}\right) \widehat{k}_{t}-\left(\frac{1+\varphi}{\psi}\right) \widehat{a}_{t}
\end{array}\right], \\
& \widehat{\pi}_{t}^{F, W *} \approx \beta \mathbb{E}_{t}\left(\widehat{\pi}_{t+1}^{F, W *}\right)+\ldots \\
& {\left[\begin{array}{c}
\left(\sigma^{-1}+\left(1-\gamma_{x}\right)\left(\frac{\psi}{1+(1-\psi) \varphi}\right)\left(\varphi+(1-\psi)\left(\frac{1+\varphi}{\psi}\right)^{2}\right)\right) \widehat{c}_{t}^{W *}+\ldots \\
\gamma_{x}\left(\frac{\psi}{1+(1-\psi) \varphi}\right)\left(\varphi+(1-\psi)\left(\frac{1+\varphi}{\psi}\right)^{2}\right) \widehat{x}_{t}^{W *}-\ldots \\
\left(1+\eta\left(\frac{\psi}{1+(1-\psi) \varphi}\right)\left(\varphi+(1-\psi)\left(\frac{1+\varphi}{\psi}\right)^{2}\right)\right)\left(\widehat{p}_{t}^{F, W *}-\widehat{p}_{t}^{W *}\right)-\ldots \\
(1-\psi)\left(\frac{1+\varphi}{\psi}\right) \widehat{k}_{t}^{*}-\left(\frac{1+\varphi}{\psi}\right) \widehat{a}_{t}^{*}
\end{array}\right] .}
\end{aligned}
$$

We define the domestic and foreign CPI indexes as $\widehat{p}_{t} \approx \phi_{H} \widehat{p}_{t}^{H}+\phi_{F} \widehat{p}_{t}^{F}$ and $\widehat{p}_{t}^{*} \approx \phi_{F} \widehat{p}_{t}^{H *}+\phi_{H} \widehat{p}_{t}^{F *}$, respectively. Therefore, it is easy to derive the dynamics of $\widehat{\pi}_{t} \equiv \widehat{p}_{t}-\widehat{p}_{t-1}$ and $\widehat{\pi}_{t}^{*} \equiv \widehat{p}_{t}^{*}-\widehat{p}_{t-1}^{*}$ from the equations above as follows,

$$
\begin{aligned}
& \widehat{\pi}_{t} \approx \beta \mathbb{E}_{t}\left(\widehat{\pi}_{t+1}\right)+\ldots \\
& \Psi\left[\begin{array}{c}
\left(\sigma^{-1}+\left(1-\gamma_{x}\right)\left(\frac{\psi}{1+(1-\psi) \varphi}\right)\left(\varphi+(1-\psi)\left(\frac{1+\varphi}{\psi}\right)^{2}\right)\right)\left[\phi_{H} \widehat{c}_{t}^{W}+\phi_{F} \widehat{c}_{t}^{W *}\right]+\ldots \\
\gamma_{x}\left(\frac{\psi}{1+(1-\psi) \varphi}\right)\left(\varphi+(1-\psi)\left(\frac{1+\varphi}{\psi}\right)^{2}\right)\left[\phi_{H} \widehat{x}_{t}^{W}+\phi_{F} \widehat{x}_{t}^{W *}\right]+2 \phi_{H} \phi_{F} \widehat{r}_{t}-\ldots \\
\left(1+\eta\left(\frac{\psi}{1+(1-\psi) \varphi}\right)\left(\varphi+(1-\psi)\left(\frac{1+\varphi}{\psi}\right)^{2}\right)\right)\left[\phi_{H}\left(\widehat{p}_{t}^{H, W}-\widehat{p}_{t}^{W}\right)+\phi_{F}\left(\widehat{p}_{t}^{F, W *}-\widehat{p}_{t}^{W *}\right)\right]-\ldots \\
\phi_{H} \phi_{F}\left[\left(\widehat{p}_{t}^{H, R}-\widehat{p}_{t}^{R}\right)+\left(\widehat{p}_{t}^{F, R}-\widehat{p}_{t}^{R}\right)\right]-(1-\psi)\left(\frac{1+\varphi}{\psi}\right) \widehat{k}_{t}^{W}-\ldots \\
\left(\frac{1+\varphi}{\psi}\right)\left[\phi_{H} \widehat{a}_{t}+\phi_{F} \widehat{a}_{t}^{*}\right]
\end{array}\right], \\
& \widehat{\pi}_{t}^{*} \approx \beta \mathbb{E}_{t}\left(\widehat{\pi}_{t+1}^{*}\right)+\ldots \\
& \Psi\left[\begin{array}{c}
\left(\sigma^{-1}+\left(1-\gamma_{x}\right)\left(\frac{\psi}{1+(1-\psi) \varphi}\right)\left(\varphi+(1-\psi)\left(\frac{1+\varphi}{\psi}\right)^{2}\right)\right)\left[\phi_{F} \widehat{c}_{t}^{W}+\phi_{H} \widehat{c}_{t}^{W *}\right]+\ldots \\
\gamma_{x}\left(\frac{\psi}{1+(1-\psi) \varphi}\right)\left(\varphi+(1-\psi)\left(\frac{1+\varphi}{\psi}\right)^{2}\right)\left[\phi_{F} \widehat{x}_{t}^{W}+\phi_{H} \widehat{x}_{t}^{W *}\right]-2 \phi_{F} \phi_{H} \widehat{r s s}_{t}-\ldots \\
\left(1+\eta\left(\frac{\psi}{1+(1-\psi) \varphi}\right)\left(\varphi+(1-\psi)\left(\frac{1+\varphi}{\psi}\right)^{2}\right)\right)\left[\phi_{F}\left(\widehat{p}_{t}^{H, W}-\widehat{p}_{t}^{W}\right)+\phi_{H}\left(\widehat{p}_{t}^{F, W *}-\widehat{p}_{t}^{W *}\right)\right]+\ldots \\
\phi_{F} \phi_{H}\left[\left(\widehat{p}_{t}^{H, R}-\widehat{p}_{t}^{R}\right)+\left(\widehat{p}_{t}^{F, R}-\widehat{p}_{t}^{R}\right)\right]-(1-\psi)\left(\frac{1+\varphi}{\psi}\right) \widehat{k}_{t}^{W *}-\ldots \\
\left(\frac{1+\varphi}{\psi}\right)\left[\phi_{F} \widehat{a}_{t}+\phi_{H} \widehat{a}_{t}^{*}\right]
\end{array}\right]
\end{aligned}
$$

where world aggregate capital is defined as $\widehat{k}_{t}^{W} \equiv \phi_{H} \widehat{k}_{t}+\phi_{F} \widehat{k}_{t}^{*}$ and $\widehat{k}_{t}^{W *} \equiv \phi_{F} \widehat{k}_{t}+\phi_{H} \widehat{k}_{t}^{*}$. We can also write 
certain terms inside the brackets of the inflation dynamics in a more compact form as,

$$
\begin{aligned}
- & \left(1+\eta\left(\frac{\psi}{1+(1-\psi) \varphi}\right)\left(\varphi+(1-\psi)\left(\frac{1+\varphi}{\psi}\right)^{2}\right)\right)\left[\phi_{H}\left(\widehat{p}_{t}^{H, W}-\widehat{p}_{t}^{W}\right)+\phi_{F}\left(\widehat{p}_{t}^{F, W *}-\widehat{p}_{t}^{W *}\right)\right]-\ldots \\
& \phi_{H} \phi_{F}\left[\left(\widehat{p}_{t}^{H, R}-\widehat{p}_{t}^{R}\right)+\left(\widehat{p}_{t}^{F, R}-\widehat{p}_{t}^{R}\right)\right] \\
& =\eta\left(\frac{\psi}{1+(1-\psi) \varphi}\right)\left(\varphi+(1-\psi)\left(\frac{1+\varphi}{\psi}\right)^{2}\right)\left[\phi_{H}-\phi_{F}\right]\left(\widehat{p}_{t}^{F, W *}-\widehat{p}_{t}^{W *}\right)-\ldots \\
& \eta\left(\frac{\psi}{1+(1-\psi) \varphi}\right)\left(\varphi+(1-\psi)\left(\frac{1+\varphi}{\psi}\right)^{2}\right) \phi_{H}\left[\left(\widehat{p}_{t}^{H, W}-\widehat{p}_{t}^{W}\right)+\left(\widehat{p}_{t}^{F, W *}-\widehat{p}_{t}^{W *}\right)\right]-\ldots \\
& \phi_{H}\left[\left(\widehat{p}_{t}^{H, W}-\widehat{p}_{t}^{W}\right)+\phi_{F}\left(\widehat{p}_{t}^{H, R}-\widehat{p}_{t}^{R}\right)\right]-\phi_{F}\left[\left(\widehat{p}_{t}^{F, W *}-\widehat{p}_{t}^{W *}\right)+\phi_{H}\left(\widehat{p}_{t}^{F, R}-\widehat{p}_{t}^{R}\right)\right], \\
- & \left(1+\eta\left(\frac{\psi}{1+(1-\psi) \varphi}\right)\left(\varphi+(1-\psi)\left(\frac{1+\varphi}{\psi}\right)^{2}\right)\right)\left[\phi_{F}\left(\widehat{p}_{t}^{H, W}-\widehat{p}_{t}^{W}\right)+\phi_{H}\left(\widehat{p}_{t}^{F, W *}-\widehat{p}_{t}^{W *}\right)\right]+\ldots \\
& \phi_{F} \phi_{H}\left[\left(\widehat{p}_{t}^{H, R}-\widehat{p}_{t}^{R}\right)+\left(\widehat{p}_{t}^{F, R}-\widehat{p}_{t}^{R}\right)\right] \\
& =-\eta\left(\frac{\psi}{1+(1-\psi) \varphi}\right)\left(\varphi+(1-\psi)\left(\frac{1+\varphi}{\psi}\right)^{2}\right)\left[\phi_{H}-\phi_{F}\right]\left(\widehat{p}_{t}^{F, W *}-\widehat{p}_{t}^{W *}\right)-\ldots \\
& \eta\left(\frac{\psi}{1+(1-\psi) \varphi}\right)\left(\varphi+(1-\psi)\left(\frac{1+\varphi}{\psi}\right)^{2}\right) \phi_{F}\left[\left(\widehat{p}_{t}^{H, W}-\widehat{p}_{t}^{W}\right)+\left(\widehat{p}_{t}^{F, W *}-\widehat{p}_{t}^{W *}\right)\right]-\ldots \\
& -\phi_{F}\left[\left(\widehat{p}_{t}^{H, W}-\widehat{p}_{t}^{W}\right)-\phi_{H}\left(\widehat{p}_{t}^{H, R}-\widehat{p}_{t}^{R}\right)\right]-\phi_{H}\left[\left(\widehat{p}_{t}^{F, W *}-\widehat{p}_{t}^{W *}\right)-\phi_{F}\left(\widehat{p}_{t}^{F, R}-\widehat{p}_{t}^{R}\right)\right] .
\end{aligned}
$$

Based on our definitions of the world weighted price indexes, denoted with the superscripts $W$ and $W^{*}$, it is possible for us to argue that,

$$
\begin{aligned}
& \phi_{H}\left[\left(\widehat{p}_{t}^{H, W}-\widehat{p}_{t}^{W}\right)+\phi_{F}\left(\widehat{p}_{t}^{H, R}-\widehat{p}_{t}^{R}\right)\right]+\phi_{F}\left[\left(\widehat{p}_{t}^{F, W *}-\widehat{p}_{t}^{W *}\right)+\phi_{H}\left(\widehat{p}_{t}^{F, R}-\widehat{p}_{t}^{R}\right)\right] \\
& \quad=\phi_{H}\left(\widehat{p}_{t}^{H}-\widehat{p}_{t}\right)+\phi_{F}\left(\widehat{p}_{t}^{F}-\widehat{p}_{t}\right)=0, \\
& \phi_{F}\left[\left(\widehat{p}_{t}^{H, W}-\widehat{p}_{t}^{W}\right)-\phi_{H}\left(\widehat{p}_{t}^{H, R}-\widehat{p}_{t}^{R}\right)\right]+\phi_{H}\left[\left(\widehat{p}_{t}^{F, W *}-\widehat{p}_{t}^{W *}\right)-\phi_{F}\left(\widehat{p}_{t}^{F, R}-\widehat{p}_{t}^{R}\right)\right] \\
& \quad=\phi_{F}\left(\widehat{p}_{t}^{H *}-\widehat{p}_{t}^{*}\right)+\phi_{H}\left(\widehat{p}_{t}^{F *}-\widehat{p}_{t}^{*}\right)=0,
\end{aligned}
$$

since $\widehat{p}_{t} \approx \phi_{H} \widehat{p}_{t}^{H}+\phi_{F} \widehat{p}_{t}^{F}$ and $\widehat{p}_{t}^{*} \approx \phi_{F} \widehat{p}_{t}^{H *}+\phi_{H} \widehat{p}_{t}^{F *}$. Furthermore, we also know based on those same definitions, that the following result must hold true,

$$
\begin{aligned}
& \left(\widehat{p}_{t}^{H, W}-\widehat{p}_{t}^{W}\right)+\left(\widehat{p}_{t}^{F, W *}-\widehat{p}_{t}^{W *}\right)=\widehat{p}_{t}^{H, W}+\widehat{p}_{t}^{F, W *}-\left(\widehat{p}_{t}^{W}+\widehat{p}_{t}^{W *}\right) \\
& \quad=\phi_{H}\left(\widehat{p}_{t}^{H}-\widehat{p}_{t}\right)+\phi_{F}\left(\widehat{p}_{t}^{H *}-\widehat{p}_{t}^{*}\right)+\phi_{F}\left(\widehat{p}_{t}^{F}-\widehat{p}_{t}\right)+\phi_{H}\left(\widehat{p}_{t}^{F *}-\widehat{p}_{t}^{*}\right) \\
& \quad=\left[\phi_{H}\left(\widehat{p}_{t}^{H}-\widehat{p}_{t}\right)+\phi_{F}\left(\widehat{p}_{t}^{F}-\widehat{p}_{t}\right)\right]+\left[\phi_{F}\left(\widehat{p}_{t}^{H *}-\widehat{p}_{t}^{*}\right)+\phi_{H}\left(\widehat{p}_{t}^{F *}-\widehat{p}_{t}^{*}\right)\right]=0 .
\end{aligned}
$$

Hence, we argue that those terms inside the brackets of the Phillips curves involving prices can be simplified as,

$$
\begin{aligned}
- & \left(1+\eta\left(\frac{\psi}{1+(1-\psi) \varphi}\right)\left(\varphi+(1-\psi)\left(\frac{1+\varphi}{\psi}\right)^{2}\right)\right)\left[\phi_{H}\left(\widehat{p}_{t}^{H, W}-\widehat{p}_{t}^{W}\right)+\phi_{F}\left(\widehat{p}_{t}^{F, W *}-\widehat{p}_{t}^{W *}\right)\right]-\ldots \\
& \phi_{H} \phi_{F}\left[\left(\widehat{p}_{t}^{H, R}-\widehat{p}_{t}^{R}\right)+\left(\widehat{p}_{t}^{F, R}-\widehat{p}_{t}^{R}\right)\right]=\eta\left(\frac{\psi}{1+(1-\psi) \varphi}\right)\left(\varphi+(1-\psi)\left(\frac{1+\varphi}{\psi}\right)^{2}\right)\left[\phi_{H}-\phi_{F}\right]\left(\widehat{p}_{t}^{F, W *}-\widehat{p}_{t}^{W *}\right), \\
- & \left(1+\eta\left(\frac{\psi}{1+(1-\psi) \varphi}\right)\left(\varphi+(1-\psi)\left(\frac{1+\varphi}{\psi}\right)^{2}\right)\right)\left[\phi_{F}\left(\widehat{p}_{t}^{H, W}-\widehat{p}_{t}^{W}\right)+\phi_{H}\left(\widehat{p}_{t}^{F, W *}-\widehat{p}_{t}^{W *}\right)\right]+\ldots \\
& \phi_{F} \phi_{H}\left[\left(\widehat{p}_{t}^{H, R}-\widehat{p}_{t}^{R}\right)+\left(\widehat{p}_{t}^{F, R}-\widehat{p}_{t}^{R}\right)\right]=-\eta\left(\frac{\psi}{1+(1-\psi) \varphi}\right)\left(\varphi+(1-\psi)\left(\frac{1+\varphi}{\psi}\right)^{2}\right)\left[\phi_{H}-\phi_{F}\right]\left(\widehat{p}_{t}^{F, W *}-\widehat{p}_{t}^{W *}\right) .
\end{aligned}
$$


We conclude that both Phillips curves in the model take the following form,

$$
\begin{gathered}
\widehat{\pi}_{t} \approx \beta \mathbb{E}_{t}\left(\widehat{\pi}_{t+1}\right)+\ldots \\
\Psi\left[\begin{array}{c}
\left(\sigma^{-1}+\left(1-\gamma_{x}\right)\left(\frac{\psi}{1+(1-\psi) \varphi}\right)\left(\varphi+(1-\psi)\left(\frac{1+\varphi}{\psi}\right)^{2}\right)\right)\left[\phi_{H} \widehat{c}_{t}^{W}+\phi_{F} \widehat{c}_{t}^{W *}\right]+\ldots \\
\gamma_{x}\left(\frac{\psi}{1+(1-\psi) \varphi}\right)\left(\varphi+(1-\psi)\left(\frac{1+\varphi}{\psi}\right)^{2}\right)\left[\phi_{H} \widehat{x}_{t}^{W}+\phi_{F} \widehat{x}_{t}^{W *}\right]+2 \phi_{H} \phi_{F} \widehat{r s}_{t}+\ldots \\
\left(\phi_{H}-\phi_{F}\right) \eta\left(\frac{\psi}{1+(1-\psi) \varphi}\right)\left(\varphi+(1-\psi)\left(\frac{1+\varphi}{\psi}\right)^{2}\right)\left(\widehat{p}_{t}^{F, W *}-\widehat{p}_{t}^{W *}\right)-\ldots \\
(1-\psi)\left(\frac{1+\varphi}{\psi}\right) \widehat{k}_{t}^{W}-\left(\frac{1+\varphi}{\psi}\right)\left[\phi_{H} \widehat{a}_{t}+\phi_{F} \widehat{a}_{t}^{*}\right]
\end{array}\right], \\
\widehat{\pi}_{t}^{*} \approx \beta \mathbb{E}_{t}\left(\widehat{\pi}_{t+1}^{*}\right)+\ldots \\
{\left[\begin{array}{c}
\left(\sigma^{-1}+\left(1-\gamma_{x}\right)\left(\frac{\psi}{1+(1-\psi) \varphi}\right)\left(\varphi+(1-\psi)\left(\frac{1+\varphi}{\psi}\right)^{2}\right)\right)\left[\phi_{F} \widehat{c}_{t}^{W}+\phi_{H} \widehat{c}_{t}^{W *}\right]+\ldots \\
\gamma_{x}\left(\frac{\psi}{1+(1-\psi) \varphi}\right)\left(\varphi+(1-\psi)\left(\frac{1+\varphi}{\psi}\right)^{2}\right)\left[\phi_{F} \widehat{x}_{t}^{W}+\phi_{H} \widehat{x}_{t}^{W *}\right]-2 \phi_{F} \phi_{H} \widehat{r s}_{t}-\ldots \\
\left(\phi_{H}-\phi_{F}\right) \eta\left(\frac{\psi}{1+(1-\psi) \varphi}\right)\left(\varphi+(1-\psi)\left(\frac{1+\varphi}{\psi}\right)^{2}\right)\left(\widehat{p}_{t}^{F, W *}-\widehat{p}_{t}^{W *}\right)-\ldots \\
(1-\psi)\left(\frac{1+\varphi}{\psi}\right) \widehat{k}_{t}^{W *}-\left(\frac{1+\varphi}{\psi}\right)\left[\phi_{F} \widehat{a}_{t}+\phi_{H} \widehat{a}_{t}^{*}\right]
\end{array}\right],}
\end{gathered}
$$

which extends the characterization of the inflation dynamics in models like those of Steinsson (2008) by adding capital and investment. However, even though we start with an equivalent structure, our model does not replicate exactly the Phillips curves discussed in Steinsson's (2008) homogeneous labor market model. Unlike him, whenever the labor share in the production function goes to one, we cannot eliminate the impact of relative prices through $\left(\widehat{p}_{t}^{F, W *}-\widehat{p}_{t}^{W *}\right)$ on CPI inflation except in the special case when there is no home-product bias, i.e. $\phi_{H}=\phi_{F}$.

With a little bit of additional algebra, it is possible to obtain simply that,

$$
\begin{aligned}
& \widehat{\pi}_{t}^{H, W} \approx \beta \mathbb{E}_{t}\left(\widehat{\pi}_{t+1}^{H, W}\right)+\ldots \\
& \Psi\left[\begin{array}{c}
\left(\sigma^{-1}+\left(1-\gamma_{x}\right) \varphi\left(\frac{\varphi \psi^{2}+(1-\psi)(1+\varphi)^{2}}{\varphi \psi+(1-\psi) \psi \varphi^{2}}\right)\right) \widehat{c}_{t}^{W}+\gamma_{x} \varphi\left(\frac{\varphi \psi^{2}+(1-\psi)(1+\varphi)^{2}}{\varphi \psi+(1-\psi) \psi \varphi^{2}}\right) \widehat{x}_{t}^{W}-\ldots \\
\left(1+\eta \varphi\left(\frac{\varphi \psi^{2}+(1-\psi)(1+\varphi)^{2}}{\varphi \psi+(1-\psi) \psi \varphi^{2}}\right)\right)\left(\widehat{p}_{t}^{H, W}-\widehat{p}_{t}^{W}\right)-\ldots \\
\left(\frac{(1-\psi)(1+\varphi)}{\psi}\right) \widehat{k}_{t}-\left(\frac{1+\varphi}{\psi}\right) \widehat{a}_{t}
\end{array}\right] \\
& \widehat{\pi}_{t}^{F, W *} \approx \beta \mathbb{E}_{t}\left(\widehat{\pi}_{t+1}^{F, W *}\right)+\ldots \\
& \Psi\left[\begin{array}{c}
\left(\sigma^{-1}+\left(1-\gamma_{x}\right) \varphi\left(\frac{\varphi \psi^{2}+(1-\psi)(1+\varphi)^{2}}{\varphi \psi+(1-\psi) \psi \varphi^{2}}\right)\right) \widehat{c}_{t}^{W *}+\gamma_{x} \varphi\left(\frac{\varphi \psi^{2}+(1-\psi)(1+\varphi)^{2}}{\varphi \psi+(1-\psi) \psi \varphi^{2}}\right) \widehat{x}_{t}^{W *}-\ldots \\
\left(1+\eta \varphi\left(\frac{\varphi \psi^{2}+(1-\psi)(1+\varphi)^{2}}{\varphi \psi+(1-\psi) \psi \varphi^{2}}\right)\right)\left(\widehat{p}_{t}^{F, W *}-\widehat{p}_{t}^{W *}\right)-\ldots \\
\left(\frac{(1-\psi)(1+\varphi)}{\psi}\right) \widehat{k}_{t}^{*}-\left(\frac{1+\varphi}{\psi}\right) \widehat{a}_{t}^{*}
\end{array}\right]
\end{aligned}
$$


and, naturally,

$$
\begin{gathered}
\widehat{\pi}_{t} \approx \beta \mathbb{E}_{t}\left(\widehat{\pi}_{t+1}\right)+\ldots \\
\Psi\left[\begin{array}{c}
\left(\sigma^{-1}+\left(1-\gamma_{x}\right) \varphi\left(\frac{\varphi \psi^{2}+(1-\psi)(1+\varphi)^{2}}{\varphi \psi+(1-\psi) \psi \varphi^{2}}\right)\right)\left[\phi_{H} \widehat{c}_{t}^{W}+\phi_{F} \widehat{c}_{t}^{W *}\right]+ \\
+\gamma_{x} \varphi\left(\frac{\varphi \psi^{2}+(1-\psi)(1+\varphi)^{2}}{\varphi \psi+(1-\psi) \psi \varphi^{2}}\right)\left[\phi_{H} \widehat{x}_{t}^{W}+\phi_{F} \widehat{x}_{t}^{W *}\right]+ \\
+2 \phi_{H} \phi_{F} \widehat{r s}_{t}+\left(\phi_{H}-\phi_{F}\right) \eta \varphi\left(\frac{\varphi \psi^{2}+(1-\psi)(1+\varphi)^{2}}{\varphi \psi+(1-\psi) \psi \varphi^{2}}\right)\left(\widehat{p}_{t}^{F, W *}-\widehat{p}_{t}^{W *}\right)- \\
-\left(\frac{(1-\psi)(1+\varphi)}{\psi}\right) \widehat{k}_{t}^{W}-\left(\frac{1+\varphi}{\psi}\right)\left[\phi_{H} \widehat{a}_{t}+\phi_{F} \widehat{a}_{t}^{*}\right]
\end{array}\right], \\
\widehat{\pi}_{t}^{*} \approx \beta \mathbb{E}_{t}\left(\widehat{\pi}_{t+1}^{*}\right)+\ldots \\
\Psi\left[\begin{array}{c}
\left(\sigma^{-1}+\left(1-\gamma_{x}\right) \varphi\left(\frac{\varphi \psi^{2}+(1-\psi)(1+\varphi)^{2}}{\varphi \psi+(1-\psi) \psi \varphi^{2}}\right)\right)\left[\phi_{F} \widehat{c}_{t}^{W}+\phi_{H} \widehat{c}_{t}^{W *}\right]+\ldots \\
\gamma_{x} \varphi\left(\frac{\varphi \psi^{2}+(1-\psi)(1+\varphi)^{2}}{\varphi \psi+(1-\psi) \psi \varphi^{2}}\right)\left[\phi_{F} \widehat{x}_{t}^{W}+\phi_{H} \widehat{x}_{t}^{W *}\right]-\ldots \\
2 \phi_{F} \phi_{H} \widehat{r s s}_{t}-\left(\phi_{H}-\phi_{F}\right) \eta \varphi\left(\frac{\varphi \psi^{2}+(1-\psi)(1+\varphi)^{2}}{\varphi \psi+(1-\psi) \psi \varphi^{2}}\right)\left(\widehat{p}_{t}^{F, W *}-\widehat{p}_{t}^{W *}\right)-\ldots \\
\left(\frac{(1-\psi)(1+\varphi)}{\psi}\right) \widehat{k}_{t}^{W *}-\left(\frac{1+\varphi}{\psi}\right)\left[\phi_{F} \widehat{a}_{t}+\phi_{H} \widehat{a}_{t}^{*}\right]
\end{array}\right] .
\end{gathered}
$$

Capital appears in the equation dynamics because it reflects the impact of the efficiency conditions on the marginal costs of firms.

Let us define $\widehat{t}_{t}^{W} \equiv \widehat{p}_{t}^{F, W *}-\widehat{p}_{t}^{W *}$ as the world measure of terms of trade in the model. Then, the Phillips curves under LCP pricing can be re-expressed as,

$$
\begin{gathered}
\widehat{\pi}_{t} \approx \beta \mathbb{E}_{t}\left(\widehat{\pi}_{t+1}\right)+\ldots \\
\Psi\left[\begin{array}{c}
\left(\sigma^{-1}+\left(1-\gamma_{x}\right) \varphi\left(\frac{\varphi \psi^{2}+(1-\psi)(1+\varphi)^{2}}{\varphi \psi+(1-\psi) \psi \varphi^{2}}\right)\right)\left[\phi_{H} \widehat{c}_{t}^{W}+\phi_{F} \widehat{c}_{t}^{W *}\right]+\ldots \\
\gamma_{x} \varphi\left(\frac{\varphi \psi^{2}+(1-\psi)(1+\varphi)^{2}}{\varphi \psi+(1-\psi) \psi \varphi^{2}}\right)\left[\phi_{H} \widehat{x}_{t}^{W}+\phi_{F} \widehat{x}_{t}^{W *}\right]+\ldots \\
2 \phi_{H} \phi_{F} \widehat{r s}_{t}+\left(\phi_{H}-\phi_{F}\right) \eta \varphi\left(\frac{\varphi \psi^{2}+(1-\psi)(1+\varphi)^{2}}{\varphi \psi+(1-\psi) \psi \varphi^{2}}\right) \widehat{t}_{t}^{W}-\ldots \\
\left(\frac{(1-\psi)(1+\varphi)}{\psi}\right) \widehat{k}_{t}^{W}-\left(\frac{1+\varphi}{\psi}\right)\left[\phi_{H} \widehat{a}_{t}+\phi_{F} \widehat{a}_{t}^{*}\right]
\end{array}\right], \\
\widehat{\pi}_{t}^{*} \approx \beta \mathbb{E}_{t}\left(\widehat{\pi}_{t+1}^{*}\right)+\ldots \\
\Psi\left[\begin{array}{c}
\left(\sigma^{-1}+\left(1-\gamma_{x}\right) \varphi\left(\frac{\varphi \psi^{2}+(1-\psi)(1+\varphi)^{2}}{\varphi \psi+(1-\psi) \psi \varphi^{2}}\right)\right)\left[\phi_{F} \widehat{c}_{t}^{W}+\phi_{H} \widehat{c}_{t}^{W *}\right]+\ldots \\
\gamma_{x} \varphi\left(\frac{\varphi \psi^{2}+(1-\psi)(1+\varphi)^{2}}{\varphi \psi+(1-\psi) \psi \varphi^{2}}\right)\left[\phi_{F} \widehat{x}_{t}^{W}+\phi_{H} \widehat{x}_{t}^{W *}\right]-\ldots \\
2 \phi_{F} \phi_{H} \widehat{r s s}_{t}-\left(\phi_{H}-\phi_{F}\right) \eta \varphi\left(\frac{\varphi \psi^{2}+(1-\psi)(1+\varphi)^{2}}{\varphi \psi+(1-\psi) \psi \varphi^{2}}\right) \widehat{t}_{t}^{W}-\ldots \\
\left(\frac{(1-\psi)(1+\varphi)}{\psi}\right) \widehat{k}_{t}^{W *}-\left(\frac{1+\varphi}{\psi}\right)\left[\phi_{F} \widehat{a}_{t}+\phi_{H} \widehat{a}_{t}^{*}\right]
\end{array}\right] .
\end{gathered}
$$

These equations constitute the aggregate supply block in this economy under LCP pricing.

The International Relative Prices. We have defined the world price sub-indexes as $\widehat{p}_{t}^{H, W} \equiv \phi_{H} \widehat{p}_{t}^{H}+$ $\phi_{F} \widehat{p}_{t}^{H *}$ and $\widehat{p}_{t}^{F, W *} \equiv \phi_{F} \widehat{p}_{t}^{F}+\phi_{H} \widehat{p}_{t}^{F *}$, and the relative price sub-indexes as $\widehat{p}_{t}^{H, R} \equiv \widehat{p}_{t}^{H}-\widehat{p}_{t}^{H *}$ and $\widehat{p}_{t}^{F, R} \equiv$ $\widehat{p}_{t}^{F}-\widehat{p}_{t}^{F *}$. Then, naturally, we can write that,

$$
\begin{aligned}
\widehat{p}_{t}^{H} & =\widehat{p}_{t}^{H, W}+\phi_{F} \widehat{p}_{t}^{H, R}, \widehat{p}_{t}^{H *}=\widehat{p}_{t}^{H, W}-\phi_{H} \widehat{p}_{t}^{H, R} \\
\widehat{p}_{t}^{F} & =\widehat{p}_{t}^{F, W *}+\phi_{H} \widehat{p}_{t}^{F, R}, \widehat{p}_{t}^{F *}=\widehat{p}_{t}^{F, W *}-\phi_{F} \widehat{p}_{t}^{F, R}
\end{aligned}
$$


Analogously, we have defined the world CPI as $\widehat{p}_{t}^{W} \equiv \phi_{H} \widehat{p}_{t}+\phi_{F} \widehat{p}_{t}^{*}$ and $\widehat{p}_{t}^{W *} \equiv \phi_{F} \widehat{p}_{t}+\phi_{H} \widehat{p}_{t}^{*}$, and the relative CPI as $\widehat{p}_{t}^{R} \equiv \widehat{p}_{t}-\widehat{p}_{t}^{*}$. Then, we can write that,

$$
\begin{aligned}
& \widehat{p}_{t}=\widehat{p}_{t}^{W}+\phi_{F} \widehat{p}_{t}^{R}, \widehat{p}_{t}^{*}=\widehat{p}_{t}^{W}-\phi_{H} \widehat{p}_{t}^{R} \\
& \widehat{p}_{t}=\widehat{p}_{t}^{W *}+\phi_{H} \widehat{p}_{t}^{R}, \widehat{p}_{t}^{*}=\widehat{p}_{t}^{W *}-\phi_{F} \widehat{p}_{t}^{R}
\end{aligned}
$$

The definition of CPI in both countries, i.e. $\widehat{p}_{t} \approx \phi_{H} \widehat{p}_{t}^{H}+\phi_{F} \widehat{p}_{t}^{F}$ and $\widehat{p}_{t}^{*} \approx \phi_{F} \widehat{p}_{t}^{H *}+\phi_{H} \widehat{p}_{t}^{F *}$, can be written as,

$$
\begin{aligned}
\phi_{H}\left[\widehat{p}_{t}^{H}-\widehat{p}_{t}\right]+\phi_{F}\left[\widehat{p}_{t}^{F}-\widehat{p}_{t}\right] & \approx 0, \\
\phi_{F}\left[\widehat{p}_{t}^{H *}-\widehat{p}_{t}^{*}\right]+\phi_{H}\left[\widehat{p}_{t}^{F *}-\widehat{p}_{t}^{*}\right] & \approx 0 .
\end{aligned}
$$

Then, based on the relationships described before, we can write the definitions of the CPI indexes as,

$$
\begin{aligned}
& \phi_{H}\left[\left(\widehat{p}_{t}^{H, W}-\widehat{p}_{t}^{W}\right)+\phi_{F}\left(\widehat{p}_{t}^{H, R}-\widehat{p}_{t}^{R}\right)\right]+\phi_{F}\left[\left(\widehat{p}_{t}^{F, W *}-\widehat{p}_{t}^{W *}\right)+\phi_{H}\left(\widehat{p}_{t}^{F, R}-\widehat{p}_{t}^{R}\right)\right] \approx 0, \\
& \phi_{F}\left[\left(\widehat{p}_{t}^{H, W}-\widehat{p}_{t}^{W}\right)-\phi_{H}\left(\widehat{p}_{t}^{H, R}-\widehat{p}_{t}^{R}\right)\right]+\phi_{H}\left[\left(\widehat{p}_{t}^{F, W *}-\widehat{p}_{t}^{W *}\right)-\phi_{F}\left(\widehat{p}_{t}^{F, R}-\widehat{p}_{t}^{R}\right)\right] \approx 0 .
\end{aligned}
$$

Let us define $\widehat{t}_{t}^{W} \equiv \widehat{p}_{t}^{F, W *}-\widehat{p}_{t}^{W *}$ as the world measure of terms of trade in this model. World terms of trade are implicitly characterized by the previous pair of equations.

We already know that by construction $\left(\widehat{p}_{t}^{H, W}-\widehat{p}_{t}^{W}\right)+\left(\widehat{p}_{t}^{F, W *}-\widehat{p}_{t}^{W *}\right) \approx 0$ (see equation (146) for a demonstration), hence the two expressions above become simply,

$$
\begin{aligned}
\left(\phi_{H}-\phi_{F}\right)\left(\widehat{p}_{t}^{F, W *}-\widehat{p}_{t}^{W *}\right) & \approx \phi_{H} \phi_{F}\left[\left(\widehat{p}_{t}^{H, R}-\widehat{p}_{t}^{R}\right)+\left(\widehat{p}_{t}^{F, R}-\widehat{p}_{t}^{R}\right)\right], \\
\left(\phi_{H}-\phi_{F}\right)\left(\widehat{p}_{t}^{F, W *}-\widehat{p}_{t}^{W *}\right) & \approx \phi_{H} \phi_{F}\left[\left(\widehat{p}_{t}^{H, R}-\widehat{p}_{t}^{R}\right)+\left(\widehat{p}_{t}^{F, R}-\widehat{p}_{t}^{R}\right)\right] .
\end{aligned}
$$

In summary, the only constraint that pins down the world terms of trade is given by,

$$
\left(\phi_{H}-\phi_{F}\right)\left(\widehat{p}_{t}^{F, W *}-\widehat{p}_{t}^{W *}\right) \approx \phi_{H} \phi_{F}\left[\left(\widehat{p}_{t}^{H, R}-\widehat{p}_{t}^{R}\right)+\left(\widehat{p}_{t}^{F, R}-\widehat{p}_{t}^{R}\right)\right] .
$$

If the model has no home-product bias in consumption, i.e. if $\phi_{H}=\phi_{F}$, then $\left(\widehat{p}_{t}^{F, W *}-\widehat{p}_{t}^{W *}\right)$ only matters because it affects output and output enters into the specification of the Taylor rules in (126) - (127). Therefore, it must follow from (155) that $\left(\widehat{p}_{t}^{H, R}-\widehat{p}_{t}^{R}\right)+\left(\widehat{p}_{t}^{F, R}-\widehat{p}_{t}^{R}\right) \approx 0$. In that case, this constraint imposes no restriction on the world terms of trade $\widehat{t}_{t}^{W} \equiv \widehat{p}_{t}^{F, W *}-\widehat{p}_{t}^{W *}$, and we would need to keep track of the price sub-indexes in order to close down the model.

If the model has a home-product bias in consumption, i.e. if $\phi_{H} \neq \phi_{F}$, then $\widehat{t}_{t}^{W} \equiv\left(\widehat{p}_{t}^{F, W *}-\widehat{p}_{t}^{W *}\right)$ matters because it affects output in both countries and it also matters because it affects the inflation dynamics through the Phillips curves. Moreover, we can write the world terms of trade as follows,

$$
\widehat{t}_{t}^{W} \equiv \widehat{p}_{t}^{F, W *}-\widehat{p}_{t}^{W *}=\frac{\phi_{H} \phi_{F}}{\phi_{H}-\phi_{F}}\left[\left(\widehat{p}_{t}^{H, R}-\widehat{p}_{t}^{R}\right)+\left(\widehat{p}_{t}^{F, R}-\widehat{p}_{t}^{R}\right)\right]
$$

In equations (136) and (137) we derive a simple characterization for the relative price sub-indexes $\widehat{\pi}_{t}^{H, R}$ and 
$\widehat{\pi}_{t}^{F, R}$ under LCP, i.e.,

$$
\begin{aligned}
\widehat{\pi}_{t}^{H, R}-\beta \mathbb{E}_{t}\left(\widehat{\pi}_{t+1}^{H, R}\right)+\Psi\left(\widehat{p}_{t}^{H, R}-\widehat{p}_{t}^{R}\right) & \approx \Psi \widehat{r s}_{t}, \\
\widehat{\pi}_{t}^{F, R}-\beta \mathbb{E}_{t}\left(\widehat{\pi}_{t+1}^{F, R}\right)+\Psi\left(\widehat{p}_{t}^{F, R}-\widehat{p}_{t}^{R}\right) & \approx \Psi \widehat{r s}_{t} .
\end{aligned}
$$

Simple manipulations allow us to write this pair of equations as,

$$
\begin{aligned}
\left(\widehat{\pi}_{t}^{H, R}-\widehat{\pi}_{t}^{R}\right)-\beta \mathbb{E}_{t}\left(\widehat{\pi}_{t+1}^{H, R}-\widehat{\pi}_{t+1}^{R}\right)+\Psi\left(\widehat{p}_{t}^{H, R}-\widehat{p}_{t}^{R}\right) & \approx \Psi \widehat{r s}_{t}-\widehat{\pi}_{t}^{R}+\beta \mathbb{E}_{t}\left(\widehat{\pi}_{t+1}^{R}\right), \\
\left(\widehat{\pi}_{t}^{F, R}-\widehat{\pi}_{t}^{R}\right)-\beta \mathbb{E}_{t}\left(\widehat{\pi}_{t+1}^{F, R}-\widehat{\pi}_{t+1}^{R}\right)+\Psi\left(\widehat{p}_{t}^{F, R}-\widehat{p}_{t}^{R}\right) & \approx \Psi \widehat{r}_{t}-\widehat{\pi}_{t}^{R}+\beta \mathbb{E}_{t}\left(\widehat{\pi}_{t+1}^{R}\right),
\end{aligned}
$$

where the relative CPI is defined as $\widehat{p}_{t}^{R} \equiv \widehat{p}_{t}-\widehat{p}_{t}^{*}$. If we use the definition of world terms of trade and we combine it with these two equations, we can write the dynamics of $\widehat{t}_{t}^{W}$ as,

$$
\Delta \widehat{t}_{t}^{W}-\beta \mathbb{E}_{t}\left(\Delta \widehat{t}_{t+1}^{W}\right)+\Psi \widehat{t}_{t}^{W} \approx \frac{\phi_{H} \phi_{F}}{\phi_{H}-\phi_{F}}\left[\Psi \widehat{r}_{t}-\widehat{\pi}_{t}^{R}+\beta \mathbb{E}_{t}\left(\widehat{\pi}_{t+1}^{R}\right)\right]
$$

where we define the first-difference of world terms of trade as $\Delta \widehat{t}_{t}^{W} \equiv \widehat{t}_{t}^{W}-\widehat{t}_{t-1}^{W}$. This suffices to close down our model under LCP pricing. Closing the model under PCP pricing is - as we shall see later - a much simpler proposition.

Following on Engel (forthcoming), we can show that when the degree of price stickiness is the same across firms and markets then the relative prices in each country must be equalized even if the law of one price fails to hold, i.e. $\left(\widehat{p}_{t}^{F}-\widehat{p}_{t}^{H}\right) \approx\left(\widehat{p}_{t}^{F *}-\widehat{p}_{t}^{H *}\right)$. To show this, we start by computing the inflation for the relative prices in each country $\left(\widehat{\pi}_{t}^{F}-\widehat{\pi}_{t}^{H}\right)$ and $\left(\widehat{\pi}_{t}^{F *}-\widehat{\pi}_{t}^{H *}\right)$ from the dynamics of the price sub-indexes in $(142)-(145)$ as follows,

$$
\begin{aligned}
& \widehat{\pi}_{t}^{F}-\widehat{\pi}_{t}^{H} \approx \beta \mathbb{E}_{t}\left(\widehat{\pi}_{t+1}^{F}-\widehat{\pi}_{t+1}^{H}\right)+\ldots \\
& \Psi\left[\begin{array}{c}
\left(\sigma^{-1}+\left(1-\gamma_{x}\right)\left(\frac{\psi}{1+(1-\psi) \varphi}\right)\left(\varphi+(1-\psi)\left(\frac{1+\varphi}{\psi}\right)^{2}\right)\right)\left(\widehat{c}_{t}^{W *}-\widehat{c}_{t}^{W}\right)+\ldots \\
\gamma_{x}\left(\frac{\psi}{1+(1-\psi) \varphi}\right)\left(\varphi+(1-\psi)\left(\frac{1+\varphi}{\psi}\right)^{2}\right)\left(\widehat{x}_{t}^{W *}-\widehat{x}_{t}^{W}\right)+\ldots \\
\left(\phi_{H}-\phi_{F}\right) \widehat{r s}_{t}-\left(1+\eta\left(\frac{\psi}{1+(1-\psi) \varphi}\right)\left(\varphi+(1-\psi)\left(\frac{1+\varphi}{\psi}\right)^{2}\right)\right)\left(\left(\widehat{p}_{t}^{F, W *}-\widehat{p}_{t}^{W *}\right)-\left(\widehat{p}_{t}^{H, W}-\widehat{p}_{t}^{W}\right)\right)-\ldots \\
\left(\phi_{H}\left(\widehat{p}_{t}^{F, R}-\widehat{p}_{t}^{R}\right)-\phi_{F}\left(\widehat{p}_{t}^{H, R}-\widehat{p}_{t}^{R}\right)\right)-(1-\psi)\left(\frac{1+\varphi}{\psi}\right)\left(\widehat{k}_{t}^{*}-\widehat{k}_{t}\right)-\left(\frac{1+\varphi}{\psi}\right)\left(\widehat{a}_{t}^{*}-\widehat{a}_{t}\right)
\end{array}\right], \\
& \widehat{\pi}_{t}^{F *}-\widehat{\pi}_{t}^{H *} \approx \beta \mathbb{E}_{t}\left(\widehat{\pi}_{t+1}^{F *}-\widehat{\pi}_{t+1}^{H *}\right)+\ldots \\
& \Psi\left[\begin{array}{c}
\left(\sigma^{-1}+\left(1-\gamma_{x}\right)\left(\frac{\psi}{1+(1-\psi) \varphi}\right)\left(\varphi+(1-\psi)\left(\frac{1+\varphi}{\psi}\right)^{2}\right)\right)\left(\widehat{c}_{t}^{W *}-\widehat{c}_{t}^{W}\right)+\ldots \\
\gamma_{x}\left(\frac{\psi}{1+(1-\psi) \varphi}\right)\left(\varphi+(1-\psi)\left(\frac{1+\varphi}{\psi}\right)^{2}\right)\left(\widehat{x}_{t}^{W *}-\widehat{x}_{t}^{W}\right)-\ldots \\
\left(\phi_{F}-\phi_{H}\right) \widehat{r s}_{t}-\left(1+\eta\left(\frac{\psi}{1+(1-\psi) \varphi}\right)\left(\varphi+(1-\psi)\left(\frac{1+\varphi}{\psi}\right)^{2}\right)\right)\left(\left(\widehat{p}_{t}^{F, W *}-\widehat{p}_{t}^{W *}\right)-\left(\widehat{p}_{t}^{H, W}-\widehat{p}_{t}^{W}\right)\right)+\ldots \\
\left(\phi_{F}\left(\widehat{p}_{t}^{F, R}-\widehat{p}_{t}^{R}\right)-\phi_{H}\left(\widehat{p}_{t}^{H, R}-\widehat{p}_{t}^{R}\right)\right)-(1-\psi)\left(\frac{1+\varphi}{\psi}\right)\left(\widehat{k}_{t}^{*}-\widehat{k}_{t}\right)-\left(\frac{1+\varphi}{\psi}\right)\left(\widehat{a}_{t}^{*}-\widehat{a}_{t}\right)
\end{array}\right] .
\end{aligned}
$$

We noted already that by construction $\left(\widehat{p}_{t}^{H, W}-\widehat{p}_{t}^{W}\right)+\left(\widehat{p}_{t}^{F, W *}-\widehat{p}_{t}^{W *}\right) \approx 0$, hence the two expressions for 
the relative prices above become simply,

$$
\begin{aligned}
& \widehat{\pi}_{t}^{F}-\widehat{\pi}_{t}^{H} \approx \beta \mathbb{E}_{t}\left(\widehat{\pi}_{t+1}^{F}-\widehat{\pi}_{t+1}^{H}\right)+\ldots \\
& \Psi\left[\begin{array}{c}
\left(\sigma^{-1}+\left(1-\gamma_{x}\right)\left(\frac{\psi}{1+(1-\psi) \varphi}\right)\left(\varphi+(1-\psi)\left(\frac{1+\varphi}{\psi}\right)^{2}\right)\right)\left(\widehat{c}_{t}^{W *}-\widehat{c}_{t}^{W}\right)+\ldots \\
\gamma_{x}\left(\frac{\psi}{1+(1-\psi) \varphi}\right)\left(\varphi+(1-\psi)\left(\frac{1+\varphi}{\psi}\right)^{2}\right)\left(\widehat{x}_{t}^{W *}-\widehat{x}_{t}^{W}\right)+\ldots \\
\left(\phi_{H}-\phi_{F}\right) \widehat{r}_{t}-2\left(1+\eta\left(\frac{\psi}{1+(1-\psi) \varphi}\right)\left(\varphi+(1-\psi)\left(\frac{1+\varphi}{\psi}\right)^{2}\right)\right)\left(\widehat{p}_{t}^{F, W *}-\widehat{p}_{t}^{W *}\right)-\ldots \\
\left(\phi_{H}\left(\widehat{p}_{t}^{F, R}-\widehat{p}_{t}^{R}\right)-\phi_{F}\left(\widehat{p}_{t}^{H, R}-\widehat{p}_{t}^{R}\right)\right)-(1-\psi)\left(\frac{1+\varphi}{\psi}\right)\left(\widehat{k}_{t}^{*}-\widehat{k}_{t}\right)-\left(\frac{1+\varphi}{\psi}\right)\left(\widehat{a}_{t}^{*}-\widehat{a}_{t}\right)
\end{array}\right], \\
& \widehat{\pi}_{t}^{F *}-\widehat{\pi}_{t}^{H *} \approx \beta \mathbb{E}_{t}\left(\widehat{\pi}_{t+1}^{F *}-\widehat{\pi}_{t+1}^{H *}\right)+\ldots \\
& \Psi\left[\begin{array}{c}
\left(\sigma^{-1}+\left(1-\gamma_{x}\right)\left(\frac{\psi}{1+(1-\psi) \varphi}\right)\left(\varphi+(1-\psi)\left(\frac{1+\varphi}{\psi}\right)^{2}\right)\right)\left(\widehat{c}_{t}^{W *}-\widehat{c}_{t}^{W}\right)+\ldots \\
\gamma_{x}\left(\frac{\psi}{1+(1-\psi) \varphi}\right)\left(\varphi+(1-\psi)\left(\frac{1+\varphi}{\psi}\right)^{2}\right)\left(\widehat{x}_{t}^{W *}-\widehat{x}_{t}^{W}\right)-\ldots \\
\left(\phi_{F}-\phi_{H}\right) \widehat{r s}_{t}-2\left(1+\eta\left(\frac{\psi}{1+(1-\psi) \varphi}\right)\left(\varphi+(1-\psi)\left(\frac{1+\varphi}{\psi}\right)^{2}\right)\right)\left(\widehat{p}_{t}^{F, W *}-\widehat{p}_{t}^{W *}\right)+\ldots \\
\left(\phi_{F}\left(\widehat{p}_{t}^{F, R}-\widehat{p}_{t}^{R}\right)-\phi_{H}\left(\widehat{p}_{t}^{H, R}-\widehat{p}_{t}^{R}\right)\right)-(1-\psi)\left(\frac{1+\varphi}{\psi}\right)\left(\widehat{k}_{t}^{*}-\widehat{k}_{t}\right)-\left(\frac{1+\varphi}{\psi}\right)\left(\widehat{a}_{t}^{*}-\widehat{a}_{t}\right)
\end{array}\right]
\end{aligned}
$$

Let us define the variable $\widehat{z_{t}}$ as the difference between the relative prices in both countries, i.e. $\widehat{z}_{t} \equiv$ $\left(\widehat{p}_{t}^{F}-\widehat{p}_{t}^{H}\right)-\left(\widehat{p}_{t}^{F *}-\widehat{p}_{t}^{H *}\right)$, and the first-difference of $\widehat{z}_{t}$ as $\Delta \widehat{z}_{t} \equiv\left(\widehat{\pi}_{t}^{F}-\widehat{\pi}_{t}^{H}\right)-\left(\widehat{\pi}_{t}^{F *}-\widehat{\pi}_{t}^{H *}\right)$. Using the two equations we derived previously for $\left(\widehat{\pi}_{t}^{F}-\widehat{\pi}_{t}^{H}\right)$ and $\left(\widehat{\pi}_{t}^{F *}-\widehat{\pi}_{t}^{H *}\right)$, it immediately follows that,

$$
\begin{aligned}
& \left(\widehat{\pi}_{t}^{F}-\widehat{\pi}_{t}^{H}\right)-\left(\widehat{\pi}_{t}^{F *}-\widehat{\pi}_{t}^{H *}\right) \approx \beta \mathbb{E}_{t}\left(\left(\widehat{\pi}_{t+1}^{F}-\widehat{\pi}_{t+1}^{H}\right)-\left(\widehat{\pi}_{t+1}^{F *}-\widehat{\pi}_{t+1}^{H *}\right)\right)-\ldots \\
& \Psi\left[\phi_{H}\left(\widehat{p}_{t}^{F, R}-\widehat{p}_{t}^{R}\right)-\phi_{F}\left(\widehat{p}_{t}^{H, R}-\widehat{p}_{t}^{R}\right)+\phi_{F}\left(\widehat{p}_{t}^{F, R}-\widehat{p}_{t}^{R}\right)-\phi_{H}\left(\widehat{p}_{t}^{H, R}-\widehat{p}_{t}^{R}\right)\right] \\
& \approx \beta \mathbb{E}_{t}\left(\left(\widehat{\pi}_{t+1}^{F}-\widehat{\pi}_{t+1}^{H}\right)-\left(\widehat{\pi}_{t+1}^{F *}-\widehat{\pi}_{t+1}^{H *}\right)\right)-\Psi\left[\left(\widehat{p}_{t}^{F, R}-\widehat{p}_{t}^{R}\right)-\left(\widehat{p}_{t}^{H, R}-\widehat{p}_{t}^{R}\right)\right] \\
& \quad \approx \beta \mathbb{E}_{t}\left(\left(\widehat{\pi}_{t+1}^{F}-\widehat{\pi}_{t+1}^{H}\right)-\left(\widehat{\pi}_{t+1}^{F *}-\widehat{\pi}_{t+1}^{H *}\right)\right)-\Psi\left[\widehat{p}_{t}^{F, R}-\widehat{p}_{t}^{H, R}\right] .
\end{aligned}
$$

Finally, since we have already defined $\widehat{p}_{t}^{H, R} \equiv\left(\widehat{p}_{t}^{H}-\widehat{p}_{t}^{H *}\right)$ and $\widehat{p}_{t}^{F, R} \equiv\left(\widehat{p}_{t}^{F}-\widehat{p}_{t}^{F *}\right)$, we can easily infer that,

$$
\left[\widehat{p}_{t}^{F, R}-\widehat{p}_{t}^{H, R}\right]=\left(\widehat{p}_{t}^{F}-\widehat{p}_{t}^{F *}\right)-\left(\widehat{p}_{t}^{H}-\widehat{p}_{t}^{H *}\right)=\left(\widehat{p}_{t}^{F}-\widehat{p}_{t}^{H}\right)-\left(\widehat{p}_{t}^{F *}-\widehat{p}_{t}^{H *}\right)=\widehat{z}_{t},
$$

and, accordingly, we can re-write the expression above as,

$$
\Delta \widehat{z}_{t} \approx \beta \mathbb{E}_{t}\left(\Delta \widehat{z}_{t+1}\right)-\Psi \widehat{z}_{t}
$$

Naturally, as Engel (forthcoming) emphasizes, if we combine equation (159) with the initial condition $\widehat{z}_{0}=0$, then it has to be the case that the solution implies that the relative prices in both countries ought to equalize as we postulated initially,

$$
\widehat{z}_{t} \equiv\left(\widehat{p}_{t}^{F}-\widehat{p}_{t}^{H}\right)-\left(\widehat{p}_{t}^{F *}-\widehat{p}_{t}^{H *}\right) \approx 0
$$

or simply that,

$$
\left(\widehat{p}_{t}^{F}-\widehat{p}_{t}^{H}\right) \approx\left(\widehat{p}_{t}^{F *}-\widehat{p}_{t}^{H *}\right) .
$$


In steady state, the relative prices of both countries do equalize as well.

Furthermore, if we combine equations (158) with the solution in (160), we obtain the following result,

$$
\left(\widehat{p}_{t}^{F, R}-\widehat{p}_{t}^{R}\right)-\left(\widehat{p}_{t}^{H, R}-\widehat{p}_{t}^{R}\right) \approx 0
$$

or simply,

$$
\left(\widehat{p}_{t}^{F, R}-\widehat{p}_{t}^{R}\right) \approx\left(\widehat{p}_{t}^{H, R}-\widehat{p}_{t}^{R}\right) .
$$

Therefore, we can re-write the world terms of trade in (156) as follows,

$$
\widehat{t}_{t}^{W} \equiv \widehat{p}_{t}^{F, W *}-\widehat{p}_{t}^{W *}=2 \frac{\phi_{H} \phi_{F}}{\phi_{H}-\phi_{F}}\left(\widehat{p}_{t}^{F, R}-\widehat{p}_{t}^{R}\right) .
$$

This expression is going to be particularly helpful to simplify the equations for the real exports and real imports later.

\subsubsection{The Open Economy Phillips Curves: The PCP Case}

Efficiency Conditions and Aggregate Output. Under PCP pricing it follows that the law of one price holds, i.e.

$$
\begin{aligned}
\widehat{p}_{t}(h) & =\widehat{s}_{t}+\widehat{p}_{t}^{*}(h), \widehat{p}_{t}(f)=\widehat{s}_{t}+\widehat{p}_{t}^{*}(f), \\
\widehat{p}_{t}^{H} & \approx \int_{0}^{1} \widehat{p}_{t}(h) d h=\widehat{s}_{t}+\int_{0}^{1} \widehat{p}_{t}^{*}(h) d h=\widehat{s}_{t}+\widehat{p}_{t}^{H *}, \\
\widehat{p}_{t}^{F} & \approx \int_{0}^{1} \widehat{p}_{t}(f) d f=\widehat{s}_{t}+\int_{0}^{1} \widehat{p}_{t}^{*}(f) d f=\widehat{s}_{t}+\widehat{p}_{t}^{F *},
\end{aligned}
$$

so the output equations in (11) - (12) can be easily simplified. We notice that the weighted prices can be re-expressed as,

$$
\begin{aligned}
\widehat{p}_{t}^{W}(h) & \equiv \widehat{p}_{t}(h)-\phi_{F} \widehat{s}_{t}, \widehat{p}_{t}^{W *}(f) \equiv \widehat{p}_{t}^{*}(f)+\phi_{F} \widehat{s}_{t}, \\
\widehat{p}_{t}^{H, W} & \equiv \widehat{p}_{t}^{H}-\phi_{F} \widehat{s}_{t}, \widehat{p}_{t}^{F, W *} \equiv \widehat{p}_{t}^{F *}+\phi_{F} \widehat{s}_{t}, \\
\widehat{p}_{t}^{W} & \equiv \phi_{H} \widehat{p}_{t}+\phi_{F} \widehat{p}_{t}^{*}, \widehat{p}_{t}^{W *} \equiv \phi_{F} \widehat{p}_{t}+\phi_{H} \widehat{p}_{t}^{*}
\end{aligned}
$$

while it follows from equations (11) - (12) that the log-linearized CPIs are,

$$
\begin{aligned}
\widehat{p}_{t} & \approx \phi_{H} \widehat{p}_{t}^{H}+\phi_{F} \widehat{p}_{t}^{F}, \\
\widehat{p}_{t}^{*} & \approx \phi_{H}^{*} \widehat{p}_{t}^{H *}+\phi_{F}^{*} \widehat{p}_{t}^{F *}=\phi_{F} \widehat{p}_{t}^{H *}+\phi_{H} \widehat{p}_{t}^{F *} .
\end{aligned}
$$

The second equality comes from the assumption that the share of the home goods in the foreign aggregator is $\phi_{H}^{*}=\phi_{F}$, while the share of the foreign goods in the foreign aggregator is $\phi_{F}^{*}=\phi_{H}$. Moreover, it follows 
that,

$$
\begin{aligned}
\widehat{p}_{t}^{*}-\widehat{p}_{t} & \approx \phi_{F} \widehat{p}_{t}^{H *}+\phi_{H} \widehat{p}_{t}^{F *}-\phi_{H} \widehat{p}_{t}^{H}-\phi_{F} \widehat{p}_{t}^{F} \\
& =\phi_{F}\left(\widehat{p}_{t}^{H}-\widehat{s}_{t}\right)+\phi_{H}\left(\widehat{p}_{t}^{F}-\widehat{s}_{t}\right)-\phi_{H} \widehat{p}_{t}^{H}-\phi_{F} \widehat{p}_{t}^{F} \\
& =-\widehat{s}_{t}+\phi_{F} \widehat{p}_{t}^{H}+\phi_{H} \widehat{p}_{t}^{F}-\phi_{H} \widehat{p}_{t}^{H}-\phi_{F} \widehat{p}_{t}^{F} \\
& =-\widehat{s}_{t}+\left(\phi_{F}-\phi_{H}\right)\left(\widehat{p}_{t}^{H}-\widehat{p}_{t}^{F}\right),
\end{aligned}
$$

which tells us that the real exchange rate ought to be proportional to terms of trade, i.e.

$$
\widehat{r s}_{t}=\widehat{s}_{t}+\widehat{p}_{t}^{*}-\widehat{p}_{t} \approx\left(\phi_{H}-\phi_{F}\right)\left(\widehat{p}_{t}^{F}-\widehat{p}_{t}^{H}\right)
$$

Domestic terms of trade are defined as the price of imports relative to the price of exports, and in the PCP case they are computed as $\widehat{t o t}_{t} \equiv\left(\widehat{p}_{t}^{F}-\widehat{p}_{t}^{H}\right) .{ }^{6}$ Notice that the real exchange rate does not become constant even though the law of one price holds at the variety level in the PCP case. That is so because we also assume that the shares of domestic and foreign goods differ in the consumption baskets of each country (as in Warnock, 2003). To close down this channel that ties real exchange rate fluctuations to movements in the terms of trade we must assume, in turn, that the consumption baskets are identical in all countries, i.e. $\phi_{H}=\phi_{H}^{*}$ and $\phi_{F}=\phi_{F}^{*}$.

Therefore, the aggregate output equations in (132) - (133) can be re-written under PCP as,

$$
\begin{aligned}
\widehat{y}_{t} & \approx-\eta\left(\widehat{p}_{t}^{H}-\phi_{F} \widehat{s}_{t}-\left(\phi_{H} \widehat{p}_{t}+\phi_{F} \widehat{p}_{t}^{*}\right)\right)+\left(1-\gamma_{x}\right) \widehat{c}_{t}^{W}+\gamma_{x} \widehat{x}_{t}^{W} \\
& \approx-\eta\left(\widehat{p}_{t}^{H}-\left(\phi_{H} \widehat{p}_{t}^{H}+\phi_{F} \widehat{p}_{t}^{F}\right)-\phi_{F}\left(\widehat{s}_{t}+\widehat{p}_{t}^{*}-\widehat{p}_{t}\right)\right)+\left(1-\gamma_{x}\right) \widehat{c}_{t}^{W}+\gamma_{x} \widehat{x}_{t}^{W} \\
& \approx 2 \eta \phi_{F} \phi_{H}\left(\widehat{p}_{t}^{F}-\widehat{p}_{t}^{H}\right)+\left(1-\gamma_{x}\right) \widehat{c}_{t}^{W}+\gamma_{x} \widehat{x}_{t}^{W}, \\
\widehat{y}_{t}^{*} & \approx-\eta\left(\widehat{p}_{t}^{F *}+\phi_{F} \widehat{s}_{t}-\left(\phi_{F} \widehat{p}_{t}+\phi_{H} \widehat{p}_{t}^{*}\right)\right)+\left(1-\gamma_{x}\right) \widehat{c}_{t}^{W *}+\gamma_{x} \widehat{x}_{t}^{W *} \\
& \approx-\eta\left(\widehat{p}_{t}^{F *}-\left(\phi_{F} \widehat{p}_{t}^{H *}+\phi_{H} \widehat{p}_{t}^{F *}\right)+\phi_{F}\left(\widehat{s}_{t}+\widehat{p}_{t}^{*}-\widehat{p}_{t}\right)\right)+\left(1-\gamma_{x}\right) \widehat{c}_{t}^{W *}+\gamma_{x} \widehat{x}_{t}^{W *} \\
& \approx-\eta \phi_{F}\left(\left(\widehat{p}_{t}^{F *}-\widehat{p}_{t}^{H *}\right)+\left(\phi_{H}-\phi_{F}\right)\left(\widehat{p}_{t}^{F}-\widehat{p}_{t}^{H}\right)\right)+\left(1-\gamma_{x}\right) \widehat{c}_{t}^{W *}+\gamma_{x} \widehat{x}_{t}^{W *} \\
& =-2 \eta \phi_{F} \phi_{H}\left(\widehat{p}_{t}^{F}-\widehat{p}_{t}^{H}\right)+\left(1-\gamma_{x}\right) \widehat{c}_{t}^{W *}+\gamma_{x} \widehat{x}_{t}^{W *} .
\end{aligned}
$$

In other words, aggregate output depends on country-weighted consumption and investment and it also depends on the domestic terms of trade, i.e. $\widehat{\operatorname{tot}}_{t} \equiv\left(\widehat{p}_{t}^{F}-\widehat{p}_{t}^{H}\right)$. Given that in our model terms of trade are proportional to the real exchange rate under PCP, we can easily re-write these equations in terms of the real exchange rate as well.

We note that if we define the world consumption as $\widehat{c}_{t}^{W} \equiv \phi_{H} \widehat{c}_{t}+\phi_{F} \widehat{c}_{t}^{*}$ and $\widehat{c}_{t}^{W *} \equiv \phi_{F} \widehat{c}_{t}+\phi_{H} \widehat{c}_{t}^{*}$ and the relative consumption as $\widehat{c}_{t}^{R} \equiv \widehat{c}_{t}-\widehat{c}_{t}^{*}$, then we can write that,

$$
\begin{aligned}
\widehat{c}_{t} & =\widehat{c}_{t}^{W}+\phi_{F} \widehat{c}_{t}^{R} \approx \widehat{c}_{t}^{W}+\phi_{F} \sigma \widehat{r s}_{t}, \\
\widehat{c}_{t}^{*} & =\widehat{c}_{t}^{W *}-\phi_{F} \widehat{c}_{t}^{R} \approx \widehat{c}_{t}^{W *}-\phi_{F} \sigma \widehat{r s}_{t},
\end{aligned}
$$

\footnotetext{
${ }^{6}$ Under LCP pricing the law of one price does not hold, so we cannot use the local price of the domestic good $\left(\widehat{p}_{t}^{H}\right)$ in our definition of terms of trade and must replace it with the price of exports expressed in units of the domestic currency $\left(\widehat{s}_{t}+\widehat{p}_{t}^{H *}\right)$. Hence, terms of trade is computed in the LCP case as $\widehat{t o t}_{t} \equiv\left(\widehat{p}_{t}^{F}-\widehat{s}_{t}-\widehat{p}_{t}^{H *}\right)$.
} 
where the second approximation follows from the perfect international risk-sharing condition in (99). This is a substitution trick that we have used before. Then, we can express the efficiency conditions in (128) - (129) as,

$$
\begin{aligned}
\widehat{r}_{t}^{z} & \approx-\frac{1+\varphi}{\psi} \widehat{a}_{t}+\frac{1}{\sigma} \widehat{c}_{t}^{W}+\phi_{F} \widehat{r}_{t}+\frac{1+\varphi}{\psi} \widehat{y}_{t}-\left(\frac{1+(1-\psi) \varphi}{\psi}\right) \widehat{k}_{t}, \\
\widehat{r}_{t}^{z *} & \approx-\frac{1+\varphi}{\psi} \widehat{a}_{t}^{*}+\frac{1}{\sigma} \widehat{c}_{t}^{W *}-\phi_{F} \widehat{r}_{t}+\frac{1+\varphi}{\psi} \widehat{y}_{t}^{*}-\left(\frac{1+(1-\psi) \varphi}{\psi}\right) \widehat{k}_{t}^{*} .
\end{aligned}
$$

Furthermore, if we combine the efficiency conditions with the output equations derived in (132) - (133), it follows that,

$$
\begin{aligned}
& \widehat{r}_{t}^{z} \approx\left(\sigma^{-1}+\left(1-\gamma_{x}\right) \frac{1+\varphi}{\psi}\right) \widehat{c}_{t}^{W}+\gamma_{x} \frac{1+\varphi}{\psi} \widehat{x}_{t}^{W}+\phi_{F} \widehat{r s}_{t}-\eta \frac{1+\varphi}{\psi}\left(\widehat{p}_{t}^{H, W}-\widehat{p}_{t}^{W}\right)-\ldots \\
& \quad\left(\frac{1+(1-\psi) \varphi}{\psi}\right) \widehat{k}_{t}-\frac{1+\varphi}{\psi} \widehat{a}_{t}, \\
& \widehat{r}_{t}^{z *} \approx\left(\frac{1}{\sigma}+\left(1-\gamma_{x}\right) \frac{1+\varphi}{\psi}\right) \widehat{c}_{t}^{W *}+\gamma_{x} \frac{1+\varphi}{\psi} \widehat{x}_{t}^{W *}-\phi_{F} \widehat{r}_{t}-\eta \frac{1+\varphi}{\psi}\left(\widehat{p}_{t}^{F, W *}-\widehat{p}_{t}^{W *}\right)-\ldots \\
& \quad\left(\frac{1+(1-\psi) \varphi}{\psi}\right) \widehat{k}_{t}^{*}-\frac{1+\varphi}{\psi} \widehat{a}_{t}^{*} .
\end{aligned}
$$

These conditions can be appropriately used to simplify the description of the inflation dynamics. The terms $\left(\widehat{p}_{t}^{H, W}-\widehat{p}_{t}^{W}\right)$ and $\left(\widehat{p}_{t}^{F, W *}-\widehat{p}_{t}^{W *}\right)$ can be further simplified using the implications of the law of one price as we have done with the aggregate output equations. However, the efficiency conditions are used only to replace out the real rental rates on capital in our characterization of the Phillips curves. The simplification under PCP does not become very useful until we have derived the Phillips curves, so we leave it for later.

The Optimal Pricing Equations. In steady state the standard pricing rule under monopolistic competition holds. Accordingly, the log-linearization of the optimal pricing equations in (79), (80), (81) and (82) can be compactly expressed as follows,

$$
\begin{aligned}
& \widehat{\widetilde{p}}_{t}(h)-\widehat{p}_{t} \approx \mathbb{E}_{t}\left[\sum_{\tau=1}^{+\infty}(\beta \alpha)^{\tau} \widehat{\pi}_{t+\tau}\right]+(1-\beta \alpha) \mathbb{E}_{t}\left[\sum_{\tau=0}^{+\infty}(\beta \alpha)^{\tau}\left(\widehat{m c}_{t+\tau}-\widehat{p}_{t+\tau}\right)\right], \\
& \widehat{\widetilde{p}}_{t}^{*}(f)-\widehat{p}_{t}^{*} \approx \mathbb{E}_{t}\left[\sum_{\tau=1}^{+\infty}(\beta \alpha)^{\tau} \widehat{\pi}_{t+\tau}^{*}\right]+(1-\beta \alpha) \mathbb{E}_{t}\left[\sum_{\tau=0}^{+\infty}(\beta \alpha)^{\tau}\left(\widehat{m c}_{t+\tau}^{*}-\widehat{p}_{t+\tau}^{*}\right)\right],
\end{aligned}
$$

which defines the distance between the optimal price decision of a given re-optimizing firm and the CPI level prevailing in each market as a weighted function of current and expected future CPI inflation and real marginal costs. Unlike in the LCP pricing case, there are only two prices formally set instead of four. The prices of the exported goods of each variety from each country are determined by the price set in the local market expressed in units of the currency of the importing country by means of the nominal exchange rate. Hence, exchange rate pass-through is complete. Here I must recall the assumption that the government subsidy is time-invariant and equal to its steady state value in every period, which explains why the government subsidies do not appear in the log-linearized pricing equations. We derive the (pre-subsidy) marginal cost equations in (20) - (21), and they can be log-linearized as,

$$
\begin{aligned}
& \widehat{m c}_{t+\tau} \approx \psi \widehat{w}_{t+\tau}+(1-\psi)\left(\widehat{r}_{t+\tau}^{z}+\widehat{p}_{t+\tau}\right)-\widehat{a}_{t+\tau} \\
& \widehat{m c}_{t+\tau}^{*} \approx \psi \widehat{w}_{t+\tau}^{*}+(1-\psi)\left(\widehat{r}_{t+\tau}^{* *}+\widehat{p}_{t+\tau}^{*}\right)-\widehat{a}_{t+\tau}^{*},
\end{aligned}
$$


while the labor market clearing conditions, which are implicit in (95) - (96), can be approximated as,

$$
\begin{aligned}
& \widehat{w}_{t+\tau} \approx-\frac{\varphi}{1+(1-\psi) \varphi} \widehat{a}_{t+\tau}+\frac{1}{1+(1-\psi) \varphi} \frac{1}{\sigma} \widehat{c}_{t+\tau}+\frac{\varphi}{1+(1-\psi) \varphi} \widehat{y}_{t+\tau}+\frac{(1-\psi) \varphi}{1+(1-\psi) \varphi}\left(\widehat{r}_{t+\tau}^{z}+\widehat{p}_{t+\tau}\right)+\frac{1}{1+(1-\psi) \varphi} \widehat{p}_{t+\tau}, \\
& \widehat{w}_{t+\tau}^{*} \approx-\frac{\varphi}{1+(1-\psi) \varphi} \widehat{a}_{t+\tau}^{*}+\frac{1}{1+(1-\psi) \varphi} \frac{1}{\sigma} \widehat{c}_{t+\tau}^{*}+\frac{\varphi}{1+(1-\psi) \varphi} \widehat{y}_{t+\tau}^{*}+\frac{(1-\psi) \varphi}{1+(1-\psi) \varphi}\left(\widehat{r}_{t+\tau}^{z *}+\widehat{p}_{t+\tau}^{*}\right)+\frac{1}{1+(1-\psi) \varphi} \widehat{p}_{t+\tau}^{*} .
\end{aligned}
$$

The labor market clearing conditions and the marginal costs reduce to the standard linear-in-labor case without capital if the labor share in the production function goes to one (i.e., $\psi \rightarrow 1$ ), as expected.

If we combine these log-linearized equations, it follows that the marginal costs can be expressed as,

$$
\begin{aligned}
& \widehat{m c}_{t+\tau} \approx \frac{\psi}{1+(1-\psi) \varphi} \frac{1}{\sigma} \widehat{c}_{t+\tau}+\frac{\varphi \psi}{1+(1-\psi) \varphi} \widehat{y}_{t+\tau}-\frac{1+\varphi}{1+(1-\psi) \varphi}\left(\widehat{a}_{t+\tau}-(1-\psi)\left(\widehat{r}_{t+\tau}^{z}+\widehat{p}_{t+\tau}\right)\right)+\frac{\psi}{1+(1-\psi) \varphi} \widehat{p}_{t+\tau}, \\
& \widehat{m c}_{t+\tau}^{*} \approx \frac{\psi}{1+(1-\psi) \varphi} \frac{1}{\sigma} \widehat{c}_{t+\tau}^{*}+\frac{\varphi \psi}{1+(1-\psi) \varphi} \widehat{y}_{t+\tau}^{*}-\frac{1+\varphi}{1+(1-\psi) \varphi}\left(\widehat{a}_{t+\tau}^{*}-(1-\psi)\left(\widehat{r}_{t+\tau}^{z *}+\widehat{p}_{t+\tau}^{*}\right)\right)+\frac{\psi}{1+(1-\psi) \varphi} \widehat{p}_{t+\tau}^{*},
\end{aligned}
$$

where $\widehat{y}_{t+\tau}$ and $\widehat{y}_{t+\tau}^{*}$ reflect domestic and foreign aggregate output. Finally, if we combine the marginal cost equations with the output equations derived before in (132) - (133), it follows that,

$$
\begin{aligned}
& \widehat{m c}_{t+\tau}-\widehat{p}_{t+\tau} \approx \frac{\psi}{1+(1-\psi) \varphi} \frac{1}{\sigma} \widehat{c}_{t+\tau}-\frac{\varphi \psi}{1+(1-\psi) \varphi} \eta\left(\widehat{p}_{t+\tau}^{H, W}-\widehat{p}_{t+\tau}^{W}\right)+\frac{\varphi \psi}{1+(1-\psi) \varphi}\left(\left(1-\gamma_{x}\right) \widehat{c}_{t+\tau}^{W}+\gamma_{x} \widehat{x}_{t+\tau}^{W}\right)-\ldots \\
& \quad \frac{1+\varphi}{1+(1-\psi) \varphi}\left(\widehat{a}_{t+\tau}-(1-\psi) \widehat{r}_{t+\tau}^{z}\right), \\
& \widehat{m c}_{t+\tau}^{*}-\widehat{p}_{t+\tau}^{*} \approx \frac{\psi}{1+(1-\psi) \varphi} \frac{1}{\sigma} \widehat{c}_{t+\tau}^{*}-\frac{\varphi \psi}{1+(1-\psi) \varphi} \eta\left(\widehat{p}_{t+\tau}^{F, W *}-\widehat{p}_{t+\tau}^{W *}\right)+\frac{\varphi \psi}{1+(1-\psi) \varphi}\left(\left(1-\gamma_{x}\right) \widehat{c}_{t+\tau}^{W *}+\gamma_{x} \widehat{x}_{t+\tau}^{W *}\right)-\ldots \\
& \quad \frac{1+\varphi}{1+(1-\psi) \varphi}\left(\widehat{a}_{t+\tau}^{*}-(1-\psi) \widehat{r}_{t+\tau}^{* *}\right) .
\end{aligned}
$$

At this stage the marginal costs have not been simplified to take into account the fact that the law of one price holds in the PCP case. However, this will be crucial when we simplify the Phillips Curve equations later on.

We can use our characterization of the real marginal costs with the pricing formulas log-linearized before to write that,

$$
\begin{aligned}
& \widehat{\widetilde{p}}_{t}(h)-\widehat{p}_{t} \approx \\
& \quad(1-\beta \alpha)\left(\frac{\psi}{1+(1-\psi) \varphi}\right) \sum_{\tau=0}^{+\infty}(\beta \alpha)^{\tau} \mathbb{E}_{t}\left[\begin{array}{c}
\frac{1}{\sigma} \widehat{c}_{t+\tau}-\varphi \eta\left(\widehat{p}_{t+\tau}^{H, W}-\widehat{p}_{t+\tau}^{W}\right)+\varphi\left(\left(1-\gamma_{x}\right) \widehat{c}_{t+\tau}^{W}+\gamma_{x} \widehat{x}_{t+\tau}^{W}\right)-\ldots \\
\left(\frac{1+\varphi}{\psi}\right)\left(\widehat{a}_{t+\tau}-(1-\psi) \widehat{r}_{t+\tau}^{z}\right)
\end{array}\right]+\ldots \\
& \sum_{\tau=1}^{+\infty}(\beta \alpha)^{\tau} \mathbb{E}_{t}\left(\widehat{\pi}_{t+\tau}\right), \\
& \widehat{\widetilde{p}}_{t}^{*}(f)-\widehat{p}_{t}^{*} \approx \\
& \quad(1-\beta \alpha)\left(\frac{\psi}{1+(1-\psi) \varphi}\right) \sum_{\tau=0}^{+\infty}(\beta \alpha)^{\tau} \mathbb{E}_{t}\left[\begin{array}{c}
\frac{1}{\sigma} \widehat{c}_{t+\tau}^{*}-\varphi \eta\left(\widehat{p}_{t+\tau}^{F, W *}-\widehat{p}_{t+\tau}^{W *}\right)+\varphi\left(\left(1-\gamma_{x}\right) \widehat{c}_{t+\tau}^{W *}+\gamma_{x} \widehat{x}_{t+\tau}^{W *}\right)-\ldots \\
\left(\frac{1+\varphi}{\psi}\right)\left(\widehat{a}_{t+\tau}^{*}-(1-\psi) \widehat{r}_{t+\tau}^{z *}\right)
\end{array}\right]+\ldots \\
& \sum_{\tau=1}^{+\infty}(\beta \alpha)^{\tau} \mathbb{E}_{t}\left(\widehat{\pi}_{t+\tau}^{*}\right) .
\end{aligned}
$$

We log-linearize the price sub-indexes in (75) - (76) and (77) - (78) and re-arrange them to obtain that,

$$
\begin{aligned}
& \widehat{\widetilde{p}}_{t}(h)-\widehat{p}_{t} \approx\left(\widehat{p}_{t}^{H}-\widehat{p}_{t}\right)+\left(\frac{\alpha}{1-\alpha}\right) \widehat{\pi}_{t}^{H}, \\
& \widehat{\widetilde{p}}_{t}^{*}(f)-\widehat{p}_{t}^{*} \approx\left(\widehat{p}_{t}^{F *}-\widehat{p}_{t}^{*}\right)+\left(\frac{\alpha}{1-\alpha}\right) \widehat{\pi}_{t}^{F *},
\end{aligned}
$$


which is quite convenient for our purposes. We replace the isolated terms $\frac{1}{\sigma} \widehat{c}_{t}$ and $\frac{1}{\sigma} \widehat{c}_{t}^{*}$ out of the marginal cost. If we define the world consumption as $\widehat{c}_{t}^{W} \equiv \phi_{H} \widehat{c}_{t}+\phi_{F} \widehat{c}_{t}^{*}$ and $\widehat{c}_{t}^{W *} \equiv \phi_{F} \widehat{c}_{t}+\phi_{H} \widehat{c}_{t}^{*}$ and the relative consumption as $\widehat{c}_{t}^{R} \equiv \widehat{c}_{t}-\widehat{c}_{t}^{*}$, then we can write that,

$$
\begin{aligned}
& \widehat{c}_{t}=\widehat{c}_{t}^{W}+\phi_{F} \widehat{c}_{t}^{R} \approx \widehat{c}_{t}^{W}+\phi_{F} \sigma \widehat{r s}_{t}, \\
& \widehat{c}_{t}^{*}=\widehat{c}_{t}^{W *}-\phi_{F} \widehat{c}_{t}^{R} \approx \widehat{c}_{t}^{W *}-\phi_{F} \sigma \widehat{r s}_{t},
\end{aligned}
$$

where the second approximation follows from the perfect international risk-sharing condition in (99). Hence, the pricing equations can be expressed more compactly as,

$$
\begin{aligned}
& \widehat{\pi}_{t}^{H}+\left(\frac{1-\alpha}{\alpha}\right)\left(\widehat{p}_{t}^{H}-\widehat{p}_{t}\right) \approx \\
& \Psi\left(\frac{\psi}{1+(1-\psi) \varphi}\right) \sum_{\tau=0}^{+\infty}(\beta \alpha)^{\tau} \mathbb{E}_{t}\left[\begin{array}{c}
\left(\sigma^{-1}+\left(1-\gamma_{x}\right) \varphi\right) \widehat{c}_{t+\tau}^{W}+\gamma_{x} \varphi \widehat{x}_{t+\tau}^{W}+\ldots \\
\phi_{F} \widehat{r s}_{t+\tau}-\varphi \eta\left(\widehat{p}_{t+\tau}^{H, W}-\widehat{p}_{t+\tau}^{W}\right)-\ldots \\
\left(\frac{1+\varphi}{\psi}\right)\left(\widehat{a}_{t+\tau}-(1-\psi) \widehat{r}_{t+\tau}^{z}\right)
\end{array}\right]+\ldots \\
& \left(\frac{1-\alpha}{\alpha}\right) \sum_{\tau=1}^{+\infty}(\beta \alpha)^{\tau} \mathbb{E}_{t}\left(\widehat{\pi}_{t+\tau}\right), \\
& \widehat{\pi}_{t}^{F *}+\left(\frac{1-\alpha}{\alpha}\right)\left(\widehat{p}_{t}^{F *}-\widehat{p}_{t}^{*}\right) \approx \\
& \Psi\left(\frac{\psi}{1+(1-\psi) \varphi}\right) \sum_{\tau=0}^{+\infty}(\beta \alpha)^{\tau} \mathbb{E}_{t}\left[\begin{array}{c}
\left(\sigma^{-1}+\left(1-\gamma_{x}\right) \varphi\right) \widehat{c}_{t+\tau}^{W *}+\gamma_{x} \varphi \widehat{x}_{t+\tau}^{W *}-\ldots \\
\phi_{F} \widehat{r s}_{t+\tau}-\varphi \eta\left(\widehat{p}_{t+\tau}^{F, W *}-\widehat{p}_{t+\tau}^{W *}\right)-\ldots \\
\left(\frac{1+\varphi}{\psi}\right)\left(\widehat{a}_{t+\tau}^{*}-(1-\psi) \widehat{r}_{t+\tau}^{z *}\right)
\end{array}\right]+\ldots \\
& \left(\frac{1-\alpha}{\alpha}\right) \sum_{\tau=1}^{+\infty}(\beta \alpha)^{\tau} \mathbb{E}_{t}\left(\widehat{\pi}_{t+\tau}^{*}\right),
\end{aligned}
$$

where $\Psi \equiv \frac{(1-\alpha)(1-\beta \alpha)}{\alpha}$.

Furthermore, these inflation equations can be expressed in the form of a system of expectational difference equations. Let us focus on the first equation as an example. If we re-write the equation at time $t+1$ and take conditional expectations with information available up to time $t$, it should follow that,

$$
\begin{aligned}
& \mathbb{E}_{t}\left[\widehat{\pi}_{t+1}^{H}+\left(\frac{1-\alpha}{\alpha}\right)\left(\widehat{p}_{t+1}^{H}-\widehat{p}_{t+1}\right)\right] \approx \\
& \Psi\left(\frac{\psi}{1+(1-\psi) \varphi}\right) \sum_{\tau=0}^{+\infty}(\beta \alpha)^{\tau} \mathbb{E}_{t}\left[\begin{array}{c}
\left(\sigma^{-1}+\left(1-\gamma_{x}\right) \varphi\right) \widehat{c}_{t+1+\tau}^{W}+\gamma_{x} \varphi \widehat{x}_{t+1+\tau}^{W}+\ldots \\
\phi_{F} \widehat{r s}_{t+1+\tau}-\varphi \eta\left(\widehat{p}_{t+1+\tau}^{H, W}-\widehat{p}_{t+1+\tau}^{W}\right)-\ldots \\
\left(\frac{1+\varphi}{\psi}\right)\left(\widehat{a}_{t+1+\tau}-(1-\psi) \widehat{r}_{t+1+\tau}^{z}\right)
\end{array}\right]+\ldots \\
& \left(\frac{1-\alpha}{\alpha}\right) \sum_{\tau=1}^{+\infty}(\beta \alpha)^{\tau} \mathbb{E}_{t}\left(\widehat{\pi}_{t+1+\tau}\right) .
\end{aligned}
$$

Hence, using the computation of the conditional expectation, the pricing equation can easily be decomposed in two terms as,

$$
\begin{aligned}
& \widehat{\pi}_{t}^{H}+\left(\frac{1-\alpha}{\alpha}\right)\left(\widehat{p}_{t}^{H}-\widehat{p}_{t}\right) \approx \\
& \quad\left(\frac{\psi}{1+(1-\psi) \varphi}\right) \Psi\left[\begin{array}{c}
\left.\left(\sigma^{-1}+\left(1-\gamma_{x}\right) \varphi\right) \widehat{c}_{t}^{W}+\gamma_{x} \varphi \widehat{x}_{t}^{W}+\phi_{F} \widehat{r s}_{t}-\varphi \eta\left(\widehat{p}_{t}^{H, W}-\widehat{p}_{t}^{W}\right)-\ldots\right] \\
\left(\frac{1+\varphi}{\psi}\right)\left(\widehat{a}_{t}-(1-\psi) \widehat{r}_{t}^{z}\right)
\end{array}\right]+\ldots \\
& \quad(1-\alpha) \beta \mathbb{E}_{t}\left(\widehat{\pi}_{t+1}\right)+\alpha \beta \mathbb{E}_{t}\left[\widehat{\pi}_{t+1}^{H}+\left(\frac{1-\alpha}{\alpha}\right)\left(\widehat{p}_{t+1}^{H}-\widehat{p}_{t+1}\right)\right] .
\end{aligned}
$$


Further re-arranging allows us to express the expectational difference equation as,

$$
\begin{aligned}
& \widehat{\pi}_{t}^{H}-\beta \mathbb{E}_{t}\left(\widehat{\pi}_{t+1}^{H}\right)+\Psi\left(\widehat{p}_{t}^{H}-\widehat{p}_{t}\right) \\
& \quad \approx\left(\frac{\psi}{1+(1-\psi) \varphi}\right) \Psi\left[\begin{array}{c}
\left(\sigma^{-1}+\left(1-\gamma_{x}\right) \varphi\right) \widehat{c}_{t}^{W}+\gamma_{x} \varphi \widehat{x}_{t}^{W}+\phi_{F} \widehat{r s}_{t}-\varphi \eta\left(\widehat{p}_{t}^{H, W}-\widehat{p}_{t}^{W}\right)-\ldots \\
\left(\frac{1+\varphi}{\psi}\right)\left(\widehat{a}_{t}-(1-\psi) \widehat{r}_{t}^{z}\right)
\end{array}\right] .
\end{aligned}
$$

We can apply the same approach (and algebraic steps) to re-write the other pricing equation as an expectational difference equation, i.e.

$$
\begin{aligned}
& \widehat{\pi}_{t}^{F *}+\left(\frac{1-\alpha}{\alpha}\right)\left(\widehat{p}_{t}^{F *}-\widehat{p}_{t}^{*}\right) \\
& \quad \approx\left(\frac{\psi}{1+(1-\psi) \varphi}\right) \Psi\left[\begin{array}{c}
\left(\sigma^{-1}+\left(1-\gamma_{x}\right) \varphi\right) \widehat{c}_{t}^{W *}+\gamma\left(\widehat{x}_{t}^{W *}-\phi_{F} \widehat{r s}_{t}-\varphi \eta\left(\widehat{p}_{t}^{F, W *}-\widehat{p}_{t}^{W *}\right)-\ldots\right. \\
\left(\frac{1+\varphi}{\psi}\right)\left(\widehat{a}_{t}^{*}-(1-\psi) \widehat{r}_{t}^{z *}\right)
\end{array}\right]+\ldots \\
& \quad(1-\alpha) \beta \mathbb{E}_{t}\left(\widehat{\pi}_{t+1}^{*}\right)+\alpha \beta \mathbb{E}_{t}\left[\widehat{\pi}_{t+1}^{F *}+\left(\frac{1-\alpha}{\alpha}\right)\left(\widehat{p}_{t+1}^{F *}-\widehat{p}_{t+1}^{*}\right)\right],
\end{aligned}
$$

and,

$$
\begin{aligned}
& \widehat{\pi}_{t}^{F *}-\beta \mathbb{E}_{t}\left(\widehat{\pi}_{t+1}^{F *}\right)+\Psi\left(\widehat{p}_{t}^{F *}-\widehat{p}_{t}^{*}\right) \\
& \quad \approx\left(\frac{\psi}{1+(1-\psi) \varphi}\right) \Psi\left[\begin{array}{c}
\left(\sigma^{-1}+\left(1-\gamma_{x}\right) \varphi\right) \widehat{c}_{t}^{W *}+\gamma_{x} \varphi \widehat{x}_{t}^{W *}-\phi_{F} \widehat{r s}_{t}-\varphi \eta\left(\widehat{p}_{t}^{F, W *}-\widehat{p}_{t}^{W *}\right)-\ldots \\
\left(\frac{1+\varphi}{\psi}\right)\left(\widehat{a}_{t}^{*}-(1-\psi) \widehat{r}_{t}^{z *}\right)
\end{array}\right] .
\end{aligned}
$$

These equations provide a very simple characterization of the price dynamics at the price sub-index level. The price sub-index for imports in each country is entirely determined by the nominal exchange rate and the price sub-index of the same bundle of goods in its local market. Naturally, the dynamics of the relative price sub-indexes $\widehat{\pi}_{t}^{H, R} \equiv \widehat{\pi}_{t}^{H}-\widehat{\pi}_{t}^{H *}\left(\widehat{p}_{t}^{H, R} \equiv \widehat{p}_{t}^{H}-\widehat{p}_{t}^{H *}\right)$ and $\widehat{\pi}_{t}^{F, R} \equiv \widehat{\pi}_{t}^{F}-\widehat{\pi}_{t}^{F *}\left(\widehat{p}_{t}^{F, R} \equiv \widehat{p}_{t}^{F}-\widehat{p}_{t}^{F *}\right)$ can be derived as follows,

$$
\begin{aligned}
\widehat{\pi}_{t}^{H, R} & \equiv \widehat{\pi}_{t}^{H}-\widehat{\pi}_{t}^{H *} \approx \widehat{s}_{t}-\widehat{s}_{t-1}=\left(\widehat{\pi}_{t}-\widehat{\pi}_{t}^{*}\right)+\widehat{r s}_{t}-\widehat{r s}_{t-1}, \\
\widehat{p}_{t}^{H, R} & \equiv \widehat{p}_{t}^{H}-\widehat{p}_{t}^{H *} \approx \widehat{s}_{t}=\left(\widehat{p}_{t}-\widehat{p}_{t}^{*}\right)+\widehat{r s}_{t}, \\
\widehat{\pi}_{t}^{F, R} & \equiv \widehat{\pi}_{t}^{F}-\widehat{\pi}_{t}^{F *} \approx \widehat{s}_{t}-\widehat{s}_{t-1}=\left(\widehat{\pi}_{t}-\widehat{\pi}_{t}^{*}\right)+\widehat{r s}_{t}-\widehat{r s}_{t-1}, \\
\widehat{p}_{t}^{F, R} & \equiv \widehat{p}_{t}^{F}-\widehat{p}_{t}^{F *} \approx \widehat{s}_{t}=\left(\widehat{p}_{t}-\widehat{p}_{t}^{*}\right)+\widehat{r s}_{t},
\end{aligned}
$$

where $\widehat{p}_{t}^{R} \equiv \widehat{p}_{t}-\widehat{p}_{t}^{*}$ and $\widehat{\pi}_{t}^{R} \equiv \widehat{\pi}_{t}-\widehat{\pi}_{t}^{*}$ are the relative CPI and the relative CPI inflation, respectively. 
We can re-write the pricing equations after further simplification as follows,

$$
\begin{aligned}
& \widehat{\pi}_{t}^{H} \approx \beta \mathbb{E}_{t}\left(\widehat{\pi}_{t+1}^{H}\right)+\ldots \\
& \left(\frac{\psi}{1+(1-\psi) \varphi}\right) \Psi\left[\begin{array}{c}
\left(\sigma^{-1}+\left(1-\gamma_{x}\right) \varphi\right) \widehat{c}_{t}^{W}+\gamma_{x} \varphi \widehat{x}_{t}^{W}+\ldots \\
\phi_{F} \widehat{r s}_{t}-\varphi \eta\left(\widehat{p}_{t}^{H, W}-\widehat{p}_{t}^{W}\right)-\ldots \\
\left(\frac{1+(1-\psi) \varphi}{\psi}\right)\left(\widehat{p}_{t}^{H}-\widehat{p}_{t}\right)-\left(\frac{1+\varphi}{\psi}\right)\left(\widehat{a}_{t}-(1-\psi) \widehat{r}_{t}^{z}\right)
\end{array}\right], \\
& \widehat{\pi}_{t}^{F *} \approx \beta \mathbb{E}_{t}\left(\widehat{\pi}_{t+1}^{F *}\right)+\ldots \quad \\
& \left(\frac{\psi}{1+(1-\psi) \varphi}\right) \Psi\left[\begin{array}{c}
\left(\sigma^{-1}+\left(1-\gamma_{x}\right) \varphi\right) \widehat{c}_{t}^{W *}+\gamma_{x} \varphi \widehat{x}_{t}^{W *}-\ldots \\
\phi_{F} \widehat{r s}_{t}-\varphi \eta\left(\widehat{p}_{t}^{F, W *}-\widehat{p}_{t}^{W *}\right)-\ldots \\
\left(\frac{1+(1-\psi) \varphi}{\psi}\right)\left(\widehat{p}_{t}^{F *}-\widehat{p}_{t}^{*}\right)-\left(\frac{1+\varphi}{\psi}\right)\left(\widehat{a}_{t}^{*}-(1-\psi) \widehat{r}_{t}^{z *}\right)
\end{array}\right] .
\end{aligned}
$$

We have defined the world price sub-indexes as $\widehat{p}_{t}^{H, W} \equiv \phi_{H} \widehat{p}_{t}^{H}+\phi_{F} \widehat{p}_{t}^{H *}$ and $\widehat{p}_{t}^{F, W *} \equiv \phi_{F} \widehat{p}_{t}^{F}+\phi_{H} \widehat{p}_{t}^{F *}$, and the relative price sub-indexes as $\widehat{p}_{t}^{H, R} \equiv \widehat{p}_{t}^{H}-\widehat{p}_{t}^{H *}$ and $\widehat{p}_{t}^{F, R} \equiv \widehat{p}_{t}^{F}-\widehat{p}_{t}^{F *}$. Then, naturally, we can write that,

$$
\begin{aligned}
\widehat{p}_{t}^{H} & =\widehat{p}_{t}^{H, W}+\phi_{F} \widehat{p}_{t}^{H, R}, \widehat{p}_{t}^{H *}=\widehat{p}_{t}^{H, W}-\phi_{H} \widehat{p}_{t}^{H, R}, \\
\widehat{p}_{t}^{F} & =\widehat{p}_{t}^{F, W *}+\phi_{H} \widehat{p}_{t}^{F, R}, \widehat{p}_{t}^{F *}=\widehat{p}_{t}^{F, W *}-\phi_{F} \widehat{p}_{t}^{F, R} .
\end{aligned}
$$

Analogously, we have defined the world CPI as $\widehat{p}_{t}^{W} \equiv \phi_{H} \widehat{p}_{t}+\phi_{F} \widehat{p}_{t}^{*}$ and $\widehat{p}_{t}^{W *} \equiv \phi_{F} \widehat{p}_{t}+\phi_{H} \widehat{p}_{t}^{*}$, and the relative CPI as $\widehat{p}_{t}^{R} \equiv \widehat{p}_{t}-\widehat{p}_{t}^{*}$. Then, we can write that,

$$
\begin{aligned}
& \widehat{p}_{t}=\widehat{p}_{t}^{W}+\phi_{F} \widehat{p}_{t}^{R}, \widehat{p}_{t}^{*}=\widehat{p}_{t}^{W}-\phi_{H} \widehat{p}_{t}^{R} \\
& \widehat{p}_{t}=\widehat{p}_{t}^{W *}+\phi_{H} \widehat{p}_{t}^{R}, \widehat{p}_{t}^{*}=\widehat{p}_{t}^{W *}-\phi_{F} \widehat{p}_{t}^{R}
\end{aligned}
$$

Therefore, we can re-arrange the pricing equations as follows,

$$
\begin{aligned}
& \widehat{\pi}_{t}^{H} \approx \beta \mathbb{E}_{t}\left(\widehat{\pi}_{t+1}^{H}\right)+\ldots \\
& \left(\frac{\psi}{1+(1-\psi) \varphi}\right) \Psi\left[\begin{array}{c}
\left(\sigma^{-1}+\left(1-\gamma_{x}\right) \varphi\right) \widehat{c}_{t}^{W}+\gamma_{x} \varphi \widehat{x}_{t}^{W}+\ldots \\
\phi_{F} \widehat{r s}_{t}-\left(\frac{1+(1-\psi) \varphi}{\psi}+\varphi \eta\right)\left(\widehat{p}_{t}^{H, W}-\widehat{p}_{t}^{W}\right)-\ldots \\
\left(\frac{1+(1-\psi) \varphi}{\psi}\right) \phi_{F}\left(\widehat{p}_{t}^{H, R}-\widehat{p}_{t}^{R}\right)-\left(\frac{1+\varphi}{\psi}\right)\left(\widehat{a}_{t}-(1-\psi) \widehat{r}_{t}^{z}\right)
\end{array}\right], \\
& \widehat{\pi}_{t}^{F *} \approx \beta \mathbb{E}_{t}\left(\widehat{\pi}_{t+1}^{F *}\right)+\ldots \quad \\
& \left(\frac{\psi}{1+(1-\psi) \varphi}\right) \Psi\left[\begin{array}{c}
\left(\sigma^{-1}+\left(1-\gamma_{x}\right) \varphi\right) \widehat{c}_{t}^{W *}+\gamma_{x} \varphi \widehat{x}_{t}^{W *}-\ldots \\
\phi_{F} \widehat{r s s}_{t}-\left(\frac{1+(1-\psi) \varphi}{\psi}+\varphi \eta\right)\left(\widehat{p}_{t}^{F, W *}-\widehat{p}_{t}^{W *}\right)+\ldots \\
\left(\frac{1+(1-\psi) \varphi}{\psi}\right) \phi_{F}\left(\widehat{p}_{t}^{F, R}-\widehat{p}_{t}^{R}\right)-\left(\frac{1+\varphi}{\psi}\right)\left(\widehat{a}_{t}^{*}-(1-\psi) \widehat{r}_{t}^{z *}\right)
\end{array}\right] .
\end{aligned}
$$

By appropriately replacing the efficiency conditions in (166) - (167), and after a little bit of algebra, we 
can now re-arrange the pricing equations for the sub-indexes in (172) - (173) as follows,

$$
\begin{aligned}
& \widehat{\pi}_{t}^{H} \approx \beta \mathbb{E}_{t}\left(\widehat{\pi}_{t+1}^{H}\right)+\ldots \\
& \Psi\left[\begin{array}{c}
\left(\sigma^{-1}+\left(1-\gamma_{x}\right)\left(\frac{\psi}{1+(1-\psi) \varphi}\right)\left(\varphi+(1-\psi)\left(\frac{1+\varphi}{\psi}\right)^{2}\right)\right) \widehat{c}_{t}^{W}+\ldots \\
\gamma_{x}\left(\frac{\psi}{1+(1-\psi) \varphi}\right)\left(\varphi+(1-\psi)\left(\frac{1+\varphi}{\psi}\right)^{2}\right) \widehat{x}_{t}^{W}+\ldots \\
\phi_{F} \widehat{r s}_{t}-\left(1+\eta\left(\frac{\psi}{1+(1-\psi) \varphi}\right)\left(\varphi+(1-\psi)\left(\frac{1+\varphi}{\psi}\right)^{2}\right)\right)\left(\widehat{p}_{t}^{H, W}-\widehat{p}_{t}^{W}\right)-\ldots \\
\phi_{F}\left(\widehat{p}_{t}^{H, R}-\widehat{p}_{t}^{R}\right)-(1-\psi)\left(\frac{1+\varphi}{\psi}\right) \widehat{k}_{t}-\left(\frac{1+\varphi}{\psi}\right) \widehat{a}_{t}
\end{array}\right], \\
& \widehat{\pi}_{t}^{F *} \approx \beta \mathbb{E}_{t}\left(\widehat{\pi}_{t+1}^{F *}\right)+\ldots \\
& \Psi\left[\begin{array}{c}
\left(\sigma^{-1}+\left(1-\gamma_{x}\right)\left(\frac{\psi}{1+(1-\psi) \varphi}\right)\left(\varphi+(1-\psi)\left(\frac{1+\varphi}{\psi}\right)^{2}\right)\right) \widehat{c}_{t}^{W *}+\ldots \\
\gamma_{x}\left(\frac{\psi}{1+(1-\psi) \varphi}\right)\left(\varphi+(1-\psi)\left(\frac{1+\varphi}{\psi}\right)^{2}\right) \widehat{x}_{t}^{W *}-\ldots \\
\phi_{F} \widehat{r s}_{t}-\left(1+\eta\left(\frac{\psi}{1+(1-\psi) \varphi}\right)\left(\varphi+(1-\psi)\left(\frac{1+\varphi}{\psi}\right)^{2}\right)\right)\left(\widehat{p}_{t}^{F, W *}-\widehat{p}_{t}^{W *}\right)+\ldots \\
\phi_{F}\left(\widehat{p}_{t}^{F, R}-\widehat{p}_{t}^{R}\right)-(1-\psi)\left(\frac{1+\varphi}{\psi}\right) \widehat{k}_{t}^{*}-\left(\frac{1+\varphi}{\psi}\right) \widehat{a}_{t}^{*}
\end{array}\right] .
\end{aligned}
$$

Furthermore, given that under PCP the law of one price holds at the variety level, then it must be the case that $\widehat{p}_{t}^{H} \approx \widehat{s}_{t}+\widehat{p}_{t}^{H *}$ and $\widehat{p}_{t}^{F} \approx \widehat{s}_{t}+\widehat{p}_{t}^{F *}$. Hence, we can express the dynamics of the other implied price sub-indexes as follows,

$$
\begin{aligned}
& \left(\widehat{\pi}_{t}^{H}-\left(\widehat{s}_{t}-\widehat{s}_{t-1}\right)\right)+\left(\widehat{s}_{t}-\widehat{s}_{t-1}\right) \approx \beta \mathbb{E}_{t}\left(\left(\widehat{\pi}_{t+1}^{H}-\left(\widehat{s}_{t+1}-\widehat{s}_{t}\right)\right)+\left(\widehat{s}_{t+1}-\widehat{s}_{t}\right)\right)+\ldots \\
& \Psi\left[\begin{array}{c}
\left(\sigma^{-1}+\left(1-\gamma_{x}\right)\left(\frac{\psi}{1+(1-\psi) \varphi}\right)\left(\varphi+(1-\psi)\left(\frac{1+\varphi}{\psi}\right)^{2}\right)\right) \widehat{c}_{t}^{W}+\ldots \\
\gamma_{x}\left(\frac{\psi}{1+(1-\psi) \varphi}\right)\left(\varphi+(1-\psi)\left(\frac{1+\varphi}{\psi}\right)^{2}\right) \widehat{x}_{t}^{W}+\ldots \\
\phi_{F} \widehat{r s s}_{t}-\left(1+\eta\left(\frac{\psi}{1+(1-\psi) \varphi}\right)\left(\varphi+(1-\psi)\left(\frac{1+\varphi}{\psi}\right)^{2}\right)\right)\left(\widehat{p}_{t}^{H, W}-\widehat{p}_{t}^{W}\right)-\ldots \\
\phi_{F}\left(\widehat{p}_{t}^{H, R}-\widehat{p}_{t}^{R}\right)-(1-\psi)\left(\frac{1+\varphi}{\psi}\right) \widehat{k}_{t}-\left(\frac{1+\varphi}{\psi}\right) \widehat{a}_{t}
\end{array}\right], \\
& \left(\widehat{\pi}_{t}^{F *}+\left(\widehat{s}_{t}-\widehat{s}_{t-1}\right)\right)-\left(\widehat{s}_{t}-\widehat{s}_{t-1}\right) \approx \beta \mathbb{E}_{t}\left(\left(\widehat{\pi}_{t+1}^{F *}+\left(\widehat{s}_{t+1}-\widehat{s}_{t}\right)\right)-\left(\widehat{s}_{t+1}-\widehat{s}_{t}\right)\right)+\ldots \\
& \Psi\left[\begin{array}{c}
\left(\sigma^{-1}+\left(1-\gamma_{x}\right)\left(\frac{\psi}{1+(1-\psi) \varphi}\right)\left(\varphi+(1-\psi)\left(\frac{1+\varphi}{\psi}\right)^{2}\right)\right) \widehat{c}_{t}^{W *}+\ldots \\
\gamma_{x}\left(\frac{\psi}{1+(1-\psi) \varphi}\right)\left(\varphi+(1-\psi)\left(\frac{1+\varphi}{\psi}\right)^{2}\right) \widehat{x}_{t}^{W *}-\ldots \\
\phi_{F} \widehat{r s}_{t}-\left(1+\eta\left(\frac{\psi}{1+(1-\psi) \varphi}\right)\left(\varphi+(1-\psi)\left(\frac{1+\varphi}{\psi}\right)^{2}\right)\right)\left(\widehat{p}_{t}^{F, W *}-\widehat{p}_{t}^{W *}\right)+\ldots \\
\phi_{F}\left(\widehat{p}_{t}^{F, R}-\widehat{p}_{t}^{R}\right)-(1-\psi)\left(\frac{1+\varphi}{\psi}\right) \widehat{k}_{t}^{*}-\left(\frac{1+\varphi}{\psi}\right) \widehat{a}_{t}^{*}
\end{array}\right],
\end{aligned}
$$


which implies that,

$$
\begin{aligned}
& \widehat{\pi}_{t}^{H *} \approx \beta \mathbb{E}_{t}\left(\widehat{\pi}_{t+1}^{H *}\right)-\left(\widehat{s}_{t}-\widehat{s}_{t-1}\right)+\beta \mathbb{E}_{t}\left(\widehat{s}_{t+1}-\widehat{s}_{t}\right)+\ldots \\
& \Psi\left[\begin{array}{c}
\left(\sigma^{-1}+\left(1-\gamma_{x}\right)\left(\frac{\psi}{1+(1-\psi) \varphi}\right)\left(\varphi+(1-\psi)\left(\frac{1+\varphi}{\psi}\right)^{2}\right)\right) \widehat{c}_{t}^{W}+\ldots \\
\gamma_{x}\left(\frac{\psi}{1+(1-\psi) \varphi}\right)\left(\varphi+(1-\psi)\left(\frac{1+\varphi}{\psi}\right)^{2}\right) \widehat{x}_{t}^{W}+\ldots \\
\phi_{F} \widehat{r s}_{t}-\left(1+\eta\left(\frac{\psi}{1+(1-\psi) \varphi}\right)\left(\varphi+(1-\psi)\left(\frac{1+\varphi}{\psi}\right)^{2}\right)\right)\left(\widehat{p}_{t}^{H, W}-\widehat{p}_{t}^{W}\right)-\ldots \\
\phi_{F}\left(\widehat{p}_{t}^{H, R}-\widehat{p}_{t}^{R}\right)-(1-\psi)\left(\frac{1+\varphi}{\psi}\right) \widehat{k}_{t}-\left(\frac{1+\varphi}{\psi}\right) \widehat{a}_{t}
\end{array}\right], \\
& \widehat{\pi}_{t}^{F} \approx \beta \mathbb{E}_{t}\left(\widehat{\pi}_{t+1}^{F}\right)+\left(\widehat{s}_{t}-\widehat{s}_{t-1}\right)-\beta \mathbb{E}_{t}\left(\widehat{s}_{t+1}-\widehat{s}_{t}\right)+\ldots \\
& \Psi\left[\begin{array}{c}
\left(\sigma^{-1}+\left(1-\gamma_{x}\right)\left(\frac{\psi}{1+(1-\psi) \varphi}\right)\left(\varphi+(1-\psi)\left(\frac{1+\varphi}{\psi}\right)^{2}\right)\right) \widehat{c}_{t}^{W *}+\ldots \\
\gamma_{x}\left(\frac{\psi}{1+(1-\psi) \varphi}\right)\left(\varphi+(1-\psi)\left(\frac{1+\varphi}{\psi}\right)^{2}\right) \widehat{x}_{t}^{W *}-\ldots \\
\phi_{F} \widehat{r s}_{t}-\left(1+\eta\left(\frac{\psi}{1+(1-\psi) \varphi}\right)\left(\varphi+(1-\psi)\left(\frac{1+\varphi}{\psi}\right)^{2}\right)\right)\left(\widehat{p}_{t}^{F, W *}-\widehat{p}_{t}^{W *}\right)+\ldots \\
\phi_{F}\left(\widehat{p}_{t}^{F, R}-\widehat{p}_{t}^{R}\right)-(1-\psi)\left(\frac{1+\varphi}{\psi}\right) \widehat{k}_{t}^{*}-\left(\frac{1+\varphi}{\psi}\right) \widehat{a}_{t}^{*}
\end{array}\right] .
\end{aligned}
$$

We define the log-linearized CPI indexes of both countries as $\widehat{p}_{t} \approx \phi_{H} \widehat{p}_{t}^{H}+\phi_{F} \widehat{p}_{t}^{F}$ and $\widehat{p}_{t}^{*} \approx \phi_{F} \widehat{p}_{t}^{H *}+\phi_{H} \widehat{p}_{t}^{F *}$. In turn, it follows that CPI inflation can be calculated as $\widehat{\pi}_{t} \approx \phi_{H} \widehat{\pi}_{t}^{H}+\phi_{F} \widehat{\pi}_{t}^{F}$ and $\widehat{\pi}_{t}^{*} \approx \phi_{F} \widehat{\pi}_{t}^{H *}+\phi_{H} \widehat{\pi}_{t}^{F *}$. Therefore, it is easy to derive the dynamics of $\widehat{\pi}_{t}$ and $\widehat{\pi}_{t}^{*}$ from the equations above. For domestic CPI inflation, $\widehat{\pi}_{t}$, it follows that,

$$
\begin{aligned}
& \widehat{\pi}_{t} \approx \beta \mathbb{E}_{t}\left(\phi_{H} \widehat{\pi}_{t+1}^{H}\right)+\Psi\left[\begin{array}{c}
\left(\sigma^{-1}+\left(1-\gamma_{x}\right)\left(\frac{\psi}{1+(1-\psi) \varphi}\right)\left(\varphi+(1-\psi)\left(\frac{1+\varphi}{\psi}\right)^{2}\right)\right) \phi_{H} \widehat{c}_{t}^{W}+\ldots \\
\gamma_{x}\left(\frac{\psi}{1+(1-\psi) \varphi}\right)\left(\varphi+(1-\psi)\left(\frac{1+\varphi}{\psi}\right)^{2}\right) \phi_{H} \widehat{x}_{t}^{W}+\ldots \\
\phi_{H} \phi_{F} \widehat{r}_{t}-\left(1+\eta\left(\frac{\psi}{1+(1-\psi) \varphi}\right)\left(\varphi+(1-\psi)\left(\frac{1+\varphi}{\psi}\right)^{2}\right)\right) \phi_{H}\left(\widehat{p}_{t}^{H, W}-\widehat{p}_{t}^{W}\right)-\ldots \\
\phi_{H} \phi_{F}\left(\widehat{p}_{t}^{H, R}-\widehat{p}_{t}^{R}\right)-(1-\psi)\left(\frac{1+\varphi}{\psi}\right) \phi_{H} \widehat{k}_{t}-\left(\frac{1+\varphi}{\psi}\right) \phi_{H} \widehat{a}_{t}
\end{array}\right]+\ldots \\
& \beta \mathbb{E}_{t}\left(\phi_{F} \widehat{\pi}_{t+1}^{F}\right)+\phi_{F}\left(\widehat{s}_{t}-\widehat{s}_{t-1}\right)-\phi_{F} \beta \mathbb{E}_{t}\left(\widehat{s}_{t+1}-\widehat{s}_{t}\right)+\ldots \\
& \Psi\left[\begin{array}{c}
\left(\sigma^{-1}+\left(1-\gamma_{x}\right)\left(\frac{\psi}{1+(1-\psi) \varphi}\right)\left(\varphi+(1-\psi)\left(\frac{1+\varphi}{\psi}\right)^{2}\right)\right) \phi_{F} \widehat{c}_{t}^{W *}+\ldots \\
\gamma_{x}\left(\frac{\psi}{1+(1-\psi) \varphi}\right)\left(\varphi+(1-\psi)\left(\frac{1+\varphi}{\psi}\right)^{2}\right) \phi_{F} \widehat{x}_{t}^{W *}-\ldots \\
\phi_{F} \phi_{F} \widehat{r s}_{t}-\left(1+\eta\left(\frac{\psi}{1+(1-\psi) \varphi}\right)\left(\varphi+(1-\psi)\left(\frac{1+\varphi}{\psi}\right)^{2}\right)\right) \phi_{F}\left(\widehat{p}_{t}^{F, W *}-\widehat{p}_{t}^{W *}\right)+\ldots \\
\phi_{F} \phi_{F}\left(\widehat{p}_{t}^{F, R}-\widehat{p}_{t}^{R}\right)-(1-\psi)\left(\frac{1+\varphi}{\psi}\right) \phi_{F} \widehat{k}_{t}^{*}-\left(\frac{1+\varphi}{\psi}\right) \phi_{F} \widehat{a}_{t}^{*}
\end{array}\right]
\end{aligned}
$$


and, after further re-arranging, it follows that,

$$
\begin{aligned}
& \widehat{\pi}_{t} \approx \beta \mathbb{E}_{t}\left(\widehat{\pi}_{t+1}\right)+\phi_{F}\left(\widehat{s}_{t}-\widehat{s}_{t-1}\right)-\phi_{F} \beta \mathbb{E}_{t}\left(\widehat{s}_{t+1}-\widehat{s}_{t}\right)+\ldots \\
& \Psi\left[\begin{array}{c}
\left(\sigma^{-1}+\left(1-\gamma_{x}\right)\left(\frac{\psi}{1+(1-\psi) \varphi}\right)\left(\varphi+(1-\psi)\left(\frac{1+\varphi}{\psi}\right)^{2}\right)\right)\left[\phi_{H} \widehat{c}_{t}^{W}+\phi_{F} \widehat{c}_{t}^{W *}\right]+\ldots \\
\gamma_{x}\left(\frac{\psi}{1+(1-\psi) \varphi}\right)\left(\varphi+(1-\psi)\left(\frac{1+\varphi}{\psi}\right)^{2}\right)\left[\phi_{H} \widehat{x}_{t}^{W}+\phi_{F} \widehat{x}_{t}^{W *}\right]+\left(\phi_{H}-\phi_{F}\right) \phi_{F} \widehat{r s}_{t}-\ldots \\
\left(1+\eta\left(\frac{\psi}{1+(1-\psi) \varphi}\right)\left(\varphi+(1-\psi)\left(\frac{1+\varphi}{\psi}\right)^{2}\right)\right)\left[\phi_{H}\left(\widehat{p}_{t}^{H, W}-\widehat{p}_{t}^{W}\right)+\phi_{F}\left(\widehat{p}_{t}^{F, W *}-\widehat{p}_{t}^{W *}\right)\right]-\ldots \\
\phi_{H} \phi_{F}\left(\widehat{p}_{t}^{H, R}-\widehat{p}_{t}^{R}\right)+\phi_{F} \phi_{F}\left(\widehat{p}_{t}^{F, R}-\widehat{p}_{t}^{R}\right)-(1-\psi)\left(\frac{1+\varphi}{\psi}\right) \widehat{k}_{t}^{W}-\left(\frac{1+\varphi}{\psi}\right)\left[\phi_{H} \widehat{a}_{t}+\phi_{F} \widehat{a}_{t}^{*}\right]
\end{array}\right],
\end{aligned}
$$

and,

$$
\begin{aligned}
\widehat{\pi}_{t} & \approx \beta \mathbb{E}_{t}\left(\widehat{\pi}_{t+1}\right)+\phi_{F}\left[\left(\widehat{s}_{t}-\widehat{s}_{t-1}\right)-\beta \mathbb{E}_{t}\left(\widehat{s}_{t+1}-\widehat{s}_{t}\right)-\Psi\left(\widehat{r s}_{t}-\left(\widehat{p}_{t}^{F, R}-\widehat{p}_{t}^{R}\right)\right)\right]+\ldots \\
& {\left[\begin{array}{c}
\left(\sigma^{-1}+\left(1-\gamma_{x}\right)\left(\frac{\psi}{1+(1-\psi) \varphi}\right)\left(\varphi+(1-\psi)\left(\frac{1+\varphi}{\psi}\right)^{2}\right)\right)\left[\phi_{H} \widehat{c}_{t}^{W}+\phi_{F} \widehat{c}_{t}^{W *}\right]+\ldots \\
\gamma_{x}\left(\frac{\psi}{1+(1-\psi) \varphi}\right)\left(\varphi+(1-\psi)\left(\frac{1+\varphi}{\psi}\right)^{2}\right)\left[\phi_{H} \widehat{x}_{t}^{W}+\phi_{F} \widehat{x}_{t}^{W *}\right]+2 \phi_{H} \phi_{F} \widehat{r s}_{t}-\ldots \\
\left(1+\eta\left(\frac{\psi}{1+(1-\psi) \varphi}\right)\left(\varphi+(1-\psi)\left(\frac{1+\varphi}{\psi}\right)^{2}\right)\right)\left[\phi_{H}\left(\widehat{p}_{t}^{H, W}-\widehat{p}_{t}^{W}\right)+\phi_{F}\left(\widehat{p}_{t}^{F, W *}-\widehat{p}_{t}^{W *}\right)\right]-\ldots \\
\phi_{H} \phi_{F}\left[\left(\widehat{p}_{t}^{H, R}-\widehat{p}_{t}^{R}\right)+\left(\widehat{p}_{t}^{F, R}-\widehat{p}_{t}^{R}\right)\right]-(1-\psi)\left(\frac{1+\varphi}{\psi}\right) \widehat{k}_{t}^{W}-\left(\frac{1+\varphi}{\psi}\right)\left[\phi_{H} \widehat{a}_{t}+\phi_{F} \widehat{a}_{t}^{*}\right]
\end{array}\right], }
\end{aligned}
$$

where world aggregate capital is defined as $\widehat{k}_{t}^{W} \equiv \phi_{H} \widehat{k}_{t}+\phi_{F} \widehat{k}_{t}^{*}$. For foreign CPI inflation, $\widehat{\pi}_{t}^{*}$, it follows that,

$$
\begin{aligned}
& \widehat{\pi}_{t}^{*} \approx \beta \mathbb{E}_{t}\left(\phi_{F} \widehat{\pi}_{t+1}^{H *}\right)-\phi_{F}\left(\widehat{s}_{t}-\widehat{s}_{t-1}\right)+\phi_{F} \beta \mathbb{E}_{t}\left(\widehat{s}_{t+1}-\widehat{s}_{t}\right)+\ldots \\
& \Psi\left[\begin{array}{c}
\left(\sigma^{-1}+\left(1-\gamma_{x}\right)\left(\frac{\psi}{1+(1-\psi) \varphi}\right)\left(\varphi+(1-\psi)\left(\frac{1+\varphi}{\psi}\right)^{2}\right)\right) \phi_{F} \widehat{c}_{t}^{W}+\ldots \\
\gamma_{x}\left(\frac{\psi}{1+(1-\psi) \varphi}\right)\left(\varphi+(1-\psi)\left(\frac{1+\varphi}{\psi}\right)^{2}\right) \phi_{F} \widehat{x}_{t}^{W}+\ldots \\
\phi_{F} \phi_{F} \widehat{r}_{t}-\left(1+\eta\left(\frac{\psi}{1+(1-\psi) \varphi}\right)\left(\varphi+(1-\psi)\left(\frac{1+\varphi}{\psi}\right)^{2}\right)\right) \phi_{F}\left(\widehat{p}_{t}^{H, W}-\widehat{p}_{t}^{W}\right)-\ldots \\
\phi_{F} \phi_{F}\left(\widehat{p}_{t}^{H, R}-\widehat{p}_{t}^{R}\right)-(1-\psi)\left(\frac{1+\varphi}{\psi}\right) \phi_{F} \widehat{k}_{t}-\left(\frac{1+\varphi}{\psi}\right) \phi_{F} \widehat{a}_{t}
\end{array}\right]+\ldots \\
& \beta \mathbb{E}_{t}\left(\phi_{H} \widehat{\pi}_{t+1}^{F *}\right)+\Psi\left[\begin{array}{c}
\left(\sigma^{-1}+\left(1-\gamma_{x}\right)\left(\frac{\psi}{1+(1-\psi) \varphi}\right)\left(\varphi+(1-\psi)\left(\frac{1+\varphi}{\psi}\right)^{2}\right)\right) \phi_{H} \widehat{c}_{t}^{W *}+\ldots \\
\gamma_{x}\left(\frac{\psi}{1+(1-\psi) \varphi}\right)\left(\varphi+(1-\psi)\left(\frac{1+\varphi}{\psi}\right)^{2}\right) \phi_{H} \widehat{x}_{t}^{W *}-\ldots \\
\phi_{H} \phi_{F} \widehat{r}_{t}-\left(1+\eta\left(\frac{\psi}{1+(1-\psi) \varphi}\right)\left(\varphi+(1-\psi)\left(\frac{1+\varphi}{\psi}\right)^{2}\right)\right) \phi_{H}\left(\widehat{p}_{t}^{F, W *}-\widehat{p}_{t}^{W *}\right)+\ldots \\
\phi_{H} \phi_{F}\left(\widehat{p}_{t}^{F, R}-\widehat{p}_{t}^{R}\right)-(1-\psi)\left(\frac{1+\varphi}{\psi}\right) \phi_{H} \widehat{k}_{t}^{*}-\left(\frac{1+\varphi}{\psi}\right) \phi_{H} \widehat{a}_{t}^{*}
\end{array}\right],
\end{aligned}
$$


and, after a little bit of algebra,

$$
\begin{aligned}
& \widehat{\pi}_{t}^{*} \approx \beta \mathbb{E}_{t}\left(\widehat{\pi}_{t+1}^{*}\right)-\phi_{F}\left(\widehat{s}_{t}-\widehat{s}_{t-1}\right)+\phi_{F} \beta \mathbb{E}_{t}\left(\widehat{s}_{t+1}-\widehat{s}_{t}\right)+\ldots \\
& \Psi\left[\begin{array}{c}
\left(\sigma^{-1}+\left(1-\gamma_{x}\right)\left(\frac{\psi}{1+(1-\psi) \varphi}\right)\left(\varphi+(1-\psi)\left(\frac{1+\varphi}{\psi}\right)^{2}\right)\right) \phi_{F} \widehat{c}_{t}^{W}+\ldots \\
\gamma_{x}\left(\frac{\psi}{1+(1-\psi) \varphi}\right)\left(\varphi+(1-\psi)\left(\frac{1+\varphi}{\psi}\right)^{2}\right) \phi_{F} \widehat{x}_{t}^{W}+\ldots \\
\phi_{F} \phi_{F} \widehat{r s}_{t}-\left(1+\eta\left(\frac{\psi}{1+(1-\psi) \varphi}\right)\left(\varphi+(1-\psi)\left(\frac{1+\varphi}{\psi}\right)^{2}\right)\right) \phi_{F}\left(\widehat{p}_{t}^{H, W}-\widehat{p}_{t}^{W}\right)-\ldots \\
\phi_{F} \phi_{F}\left(\widehat{p}_{t}^{H, R}-\widehat{p}_{t}^{R}\right)-(1-\psi)\left(\frac{1+\varphi}{\psi}\right) \phi_{F} \widehat{k}_{t}-\left(\frac{1+\varphi}{\psi}\right) \phi_{F} \widehat{a}_{t} \\
\left(\sigma^{-1}+\left(1-\gamma_{x}\right)\left(\frac{\psi}{1+(1-\psi) \varphi}\right)\left(\varphi+(1-\psi)\left(\frac{1+\varphi}{\psi}\right)^{2}\right)\right) \phi_{H} \widehat{c}_{t}^{W *}+\ldots \\
\gamma_{x}\left(\frac{\psi}{1+(1-\psi) \varphi}\right)\left(\varphi+(1-\psi)\left(\frac{1+\varphi}{\psi}\right)^{2}\right) \phi_{H} \widehat{x}_{t}^{W *}-\ldots \\
\phi_{H} \phi_{F} \widehat{r s}_{t}-\left(1+\eta\left(\frac{\psi}{1+(1-\psi) \varphi}\right)\left(\varphi+(1-\psi)\left(\frac{1+\varphi}{\psi}\right)^{2}\right)\right) \phi_{H}\left(\widehat{p}_{t}^{F, W *}-\widehat{p}_{t}^{W *}\right)+\ldots \\
\phi_{H} \phi_{F}\left(\widehat{p}_{t}^{F, R}-\widehat{p}_{t}^{R}\right)-(1-\psi)\left(\frac{1+\varphi}{\psi}\right) \phi_{H} \widehat{k}_{t}^{*}-\left(\frac{1+\varphi}{\psi}\right) \phi_{H} \widehat{a}_{t}^{*}
\end{array}\right],
\end{aligned}
$$

and,

$$
\begin{aligned}
& \widehat{\pi}_{t}^{*} \approx \beta \mathbb{E}_{t}\left(\widehat{\pi}_{t+1}^{*}\right)-\phi_{F}\left(\widehat{s}_{t}-\widehat{s}_{t-1}\right)+\phi_{F} \beta \mathbb{E}_{t}\left(\widehat{s}_{t+1}-\widehat{s}_{t}\right)+\ldots \\
& \Psi\left[\begin{array}{c}
\left(\sigma^{-1}+\left(1-\gamma_{x}\right)\left(\frac{\psi}{1+(1-\psi) \varphi}\right)\left(\varphi+(1-\psi)\left(\frac{1+\varphi}{\psi}\right)^{2}\right)\right)\left[\phi_{F} \widehat{c}_{t}^{W}+\phi_{H} \widehat{c}_{t}^{W *}\right]+\ldots \\
\gamma_{x}\left(\frac{\psi}{1+(1-\psi) \varphi}\right)\left(\varphi+(1-\psi)\left(\frac{1+\varphi}{\psi}\right)^{2}\right)\left[\phi_{F} \widehat{x}_{t}^{W}+\phi_{H} \widehat{x}_{t}^{W *}\right]+\ldots \\
\phi_{F} \phi_{F} \widehat{r s}_{t}-\phi_{H} \phi_{F} \widehat{r s}_{t}-\ldots \\
\left(1+\eta\left(\frac{\psi}{1+(1-\psi) \varphi}\right)\left(\varphi+(1-\psi)\left(\frac{1+\varphi}{\psi}\right)^{2}\right)\right)\left[\phi_{F}\left(\widehat{p}_{t}^{H, W}-\widehat{p}_{t}^{W}\right)+\phi_{H}\left(\widehat{p}_{t}^{F, W *}-\widehat{p}_{t}^{W *}\right)\right]-\ldots \\
\phi_{F} \phi_{F}\left(\widehat{p}_{t}^{H, R}-\widehat{p}_{t}^{R}\right)+\phi_{H} \phi_{F}\left(\widehat{p}_{t}^{F, R}-\widehat{p}_{t}^{R}\right)-(1-\psi)\left(\frac{1+\varphi}{\psi}\right)\left[\phi_{F} \widehat{k}_{t}+\phi_{H} \widehat{k}_{t}^{*}\right]-\left(\frac{1+\varphi}{\psi}\right)\left[\phi_{F} \widehat{a}_{t}+\phi_{H} \widehat{a}_{t}^{*}\right]
\end{array}\right],
\end{aligned}
$$

where world aggregate capital is defined as $\widehat{k}_{t}^{W *} \equiv \phi_{F} \widehat{k}_{t}+\phi_{H} \widehat{k}_{t}^{*}$. Moreover, foreign CPI inflation can be expressed alternatively as,

$$
\begin{aligned}
& \widehat{\pi}_{t}^{*} \approx \beta \mathbb{E}_{t}\left(\widehat{\pi}_{t+1}^{*}\right)-\phi_{F}\left[\left(\widehat{s}_{t}-\widehat{s}_{t-1}\right)-\beta \mathbb{E}_{t}\left(\widehat{s}_{t+1}-\widehat{s}_{t}\right)-\Psi\left(\widehat{r}_{t}-\left(\widehat{p}_{t}^{H, R}-\widehat{p}_{t}^{R}\right)\right)\right]+\ldots \\
& \Psi\left[\begin{array}{c}
\left(\sigma^{-1}+\left(1-\gamma_{x}\right)\left(\frac{\psi}{1+(1-\psi) \varphi}\right)\left(\varphi+(1-\psi)\left(\frac{1+\varphi}{\psi}\right)^{2}\right)\right)\left[\phi_{F} \widehat{c}_{t}^{W}+\phi_{H} \widehat{c}_{t}^{W *}\right]+\ldots \\
\gamma_{x}\left(\frac{\psi}{1+(1-\psi) \varphi}\right)\left(\varphi+(1-\psi)\left(\frac{1+\varphi}{\psi}\right)^{2}\right)\left[\phi_{F} \widehat{x}_{t}^{W}+\phi_{H} \widehat{x}_{t}^{W *}\right]-\ldots \\
2 \phi_{H} \phi_{F} \widehat{r s}_{t}-\left(1+\eta\left(\frac{\psi}{1+(1-\psi) \varphi}\right)\left(\varphi+(1-\psi)\left(\frac{1+\varphi}{\psi}\right)^{2}\right)\right)\left[\phi_{F}\left(\widehat{p}_{t}^{H, W}-\widehat{p}_{t}^{W}\right)+\phi_{H}\left(\widehat{p}_{t}^{F, W *}-\widehat{p}_{t}^{W *}\right)\right]+\ldots \\
\phi_{H} \phi_{F}\left[\left(\widehat{p}_{t}^{H, R}-\widehat{p}_{t}^{R}\right)+\left(\widehat{p}_{t}^{F, R}-\widehat{p}_{t}^{R}\right)\right]-(1-\psi)\left(\frac{1+\varphi}{\psi}\right) \widehat{k}_{t}^{W *}-\left(\frac{1+\varphi}{\psi}\right)\left[\phi_{F} \widehat{a}_{t}+\phi_{H} \widehat{a}_{t}^{*}\right]
\end{array}\right] .
\end{aligned}
$$


In summary, it follows that the Phillips curves under PCP can be expressed as,

$$
\begin{aligned}
& \widehat{\pi}_{t} \approx \beta \mathbb{E}_{t}\left(\widehat{\pi}_{t+1}\right)+\phi_{F}\left[\left(\widehat{s}_{t}-\widehat{s}_{t-1}\right)-\beta \mathbb{E}_{t}\left(\widehat{s}_{t+1}-\widehat{s}_{t}\right)+\Psi\left(\widehat{p}_{t}^{F, R}-\widehat{s}_{t}\right)\right]+\ldots \\
& \Psi\left[\begin{array}{c}
\left(\sigma^{-1}+\left(1-\gamma_{x}\right)\left(\frac{\psi}{1+(1-\psi) \varphi}\right)\left(\varphi+(1-\psi)\left(\frac{1+\varphi}{\psi}\right)^{2}\right)\right)\left[\phi_{H} \widehat{c}_{t}^{W}+\phi_{F} \widehat{c}_{t}^{W *}\right]+\ldots \\
\gamma_{x}\left(\frac{\psi}{1+(1-\psi) \varphi}\right)\left(\varphi+(1-\psi)\left(\frac{1+\varphi}{\psi}\right)^{2}\right)\left[\phi_{H} \widehat{x}_{t}^{W}+\phi_{F} \widehat{x}_{t}^{W *}\right]+2 \phi_{H} \phi_{F} \widehat{r}_{t}-\ldots \\
\left(1+\eta\left(\frac{\psi}{1+(1-\psi) \varphi}\right)\left(\varphi+(1-\psi)\left(\frac{1+\varphi}{\psi}\right)^{2}\right)\right)\left[\phi_{H}\left(\widehat{p}_{t}^{H, W}-\widehat{p}_{t}^{W}\right)+\phi_{F}\left(\widehat{p}_{t}^{F, W *}-\widehat{p}_{t}^{W *}\right)\right]-\ldots \\
\phi_{H} \phi_{F}\left[\left(\widehat{p}_{t}^{H, R}-\widehat{p}_{t}^{R}\right)+\left(\widehat{p}_{t}^{F, R}-\widehat{p}_{t}^{R}\right)\right]-(1-\psi)\left(\frac{1+\varphi}{\psi}\right) \widehat{k}_{t}^{W}-\left(\frac{1+\varphi}{\psi}\right)\left[\phi_{H} \widehat{a}_{t}+\phi_{F} \widehat{a}_{t}^{*}\right]
\end{array}\right], \\
& \widehat{\pi}_{t}^{*} \approx \beta \mathbb{E}_{t}\left(\widehat{\pi}_{t+1}^{*}\right)-\phi_{F}\left[\left(\widehat{s}_{t}-\widehat{s}_{t-1}\right)-\beta \mathbb{E}_{t}\left(\widehat{s}_{t+1}-\widehat{s}_{t}\right)+\Psi\left(\widehat{p}_{t}^{H, R}-\widehat{s}_{t}\right)\right]+\ldots \\
& \Psi\left[\begin{array}{c}
\left(\sigma^{-1}+\left(1-\gamma_{x}\right)\left(\frac{\psi}{1+(1-\psi) \varphi}\right)\left(\varphi+(1-\psi)\left(\frac{1+\varphi}{\psi}\right)^{2}\right)\right)\left[\phi_{F} \widehat{c}_{t}^{W}+\phi_{H} \widehat{c}_{t}^{W *}\right]+\ldots \\
\gamma_{x}\left(\frac{\psi}{1+(1-\psi) \varphi}\right)\left(\varphi+(1-\psi)\left(\frac{1+\varphi}{\psi}\right)^{2}\right)\left[\phi_{F} \widehat{x}_{t}^{W}+\phi_{H} \widehat{x}_{t}^{W *}\right]-2 \phi_{H} \phi_{F} \widehat{r}_{t}-\ldots \\
\left(1+\eta\left(\frac{\psi}{1+(1-\psi) \varphi}\right)\left(\varphi+(1-\psi)\left(\frac{1+\varphi}{\psi}\right)^{2}\right)\right)\left[\phi_{F}\left(\widehat{p}_{t}^{H, W}-\widehat{p}_{t}^{W}\right)+\phi_{H}\left(\widehat{p}_{t}^{F, W *}-\widehat{p}_{t}^{W *}\right)\right]+\ldots \\
\phi_{H} \phi_{F}\left[\left(\widehat{p}_{t}^{H, R}-\widehat{p}_{t}^{R}\right)+\left(\widehat{p}_{t}^{F, R}-\widehat{p}_{t}^{R}\right)\right]-(1-\psi)\left(\frac{1+\varphi}{\psi}\right) \widehat{k}_{t}^{W *}-\left(\frac{1+\varphi}{\psi}\right)\left[\phi_{F} \widehat{a}_{t}+\phi_{H} \widehat{a}_{t}^{*}\right]
\end{array}\right],
\end{aligned}
$$

where world aggregate capital is defined as $\widehat{k}_{t}^{W} \equiv \phi_{H} \widehat{k}_{t}+\phi_{F} \widehat{k}_{t}^{*}$ and $\widehat{k}_{t}^{W *} \equiv \phi_{F} \widehat{k}_{t}+\phi_{H} \widehat{k}_{t}^{*}$. We can also write certain terms inside the brackets of the inflation equations more compactly as,

$$
\begin{aligned}
- & \left(1+\eta\left(\frac{\psi}{1+(1-\psi) \varphi}\right)\left(\varphi+(1-\psi)\left(\frac{1+\varphi}{\psi}\right)^{2}\right)\right)\left[\phi_{H}\left(\widehat{p}_{t}^{H, W}-\widehat{p}_{t}^{W}\right)+\phi_{F}\left(\widehat{p}_{t}^{F, W *}-\widehat{p}_{t}^{W *}\right)\right]-\ldots \\
& \phi_{H} \phi_{F}\left[\left(\widehat{p}_{t}^{H, R}-\widehat{p}_{t}^{R}\right)+\left(\widehat{p}_{t}^{F, R}-\widehat{p}_{t}^{R}\right)\right] \\
& =\eta\left(\frac{\psi}{1+(1-\psi) \varphi}\right)\left(\varphi+(1-\psi)\left(\frac{1+\varphi}{\psi}\right)^{2}\right)\left[\phi_{H}-\phi_{F}\right]\left(\widehat{p}_{t}^{F, W *}-\widehat{p}_{t}^{W *}\right)-\ldots \\
& \eta\left(\frac{\psi}{1+(1-\psi) \varphi}\right)\left(\varphi+(1-\psi)\left(\frac{1+\varphi}{\psi}\right)^{2}\right) \phi_{H}\left[\left(\widehat{p}_{t}^{H, W}-\widehat{p}_{t}^{W}\right)+\left(\widehat{p}_{t}^{F, W *}-\widehat{p}_{t}^{W *}\right)\right]-\ldots \\
& \phi_{H}\left[\left(\widehat{p}_{t}^{H, W}-\widehat{p}_{t}^{W}\right)+\phi_{F}\left(\widehat{p}_{t}^{H, R}-\widehat{p}_{t}^{R}\right)\right]-\phi_{F}\left[\left(\widehat{p}_{t}^{F, W *}-\widehat{p}_{t}^{W *}\right)+\phi_{H}\left(\widehat{p}_{t}^{F, R}-\widehat{p}_{t}^{R}\right)\right], \\
- & \left(1+\eta\left(\frac{\psi}{1+(1-\psi) \varphi}\right)\left(\varphi+(1-\psi)\left(\frac{1+\varphi}{\psi}\right)^{2}\right)\right)\left[\phi_{F}\left(\widehat{p}_{t}^{H, W}-\widehat{p}_{t}^{W}\right)+\phi_{H}\left(\widehat{p}_{t}^{F, W *}-\widehat{p}_{t}^{W *}\right)\right]+\ldots \\
& \phi_{F} \phi_{H}\left[\left(\widehat{p}_{t}^{H, R}-\widehat{p}_{t}^{R}\right)+\left(\widehat{p}_{t}^{F, R}-\widehat{p}_{t}^{R}\right)\right] \\
& =-\eta\left(\frac{\psi}{1+(1-\psi) \varphi}\right)\left(\varphi+(1-\psi)\left(\frac{1+\varphi}{\psi}\right)^{2}\right)\left[\phi_{H}-\phi_{F}\right]\left(\widehat{p}_{t}^{F, W *}-\widehat{p}_{t}^{W *}\right)-\ldots \\
& \eta\left(\frac{\psi}{1+(1-\psi) \varphi}\right)\left(\varphi+(1-\psi)\left(\frac{1+\varphi}{\psi}\right)^{2}\right) \phi_{F}\left[\left(\widehat{p}_{t}^{H, W}-\widehat{p}_{t}^{W}\right)+\left(\widehat{p}_{t}^{F, W *}-\widehat{p}_{t}^{W *}\right)\right]-\ldots \\
& \phi_{F}\left[\left(\widehat{p}_{t}^{H, W}-\widehat{p}_{t}^{W}\right)-\phi_{H}\left(\widehat{p}_{t}^{H, R}-\widehat{p}_{t}^{R}\right)\right]-\phi_{H}\left[\left(\widehat{p}_{t}^{F, W *}-\widehat{p}_{t}^{W *}\right)-\phi_{F}\left(\widehat{p}_{t}^{F, R}-\widehat{p}_{t}^{R}\right)\right] .
\end{aligned}
$$

Based on our definitions of the world weighted price indexes, denoted with the superscripts $W$ and $W^{*}$, it 
is possible for us to argue that,

$$
\begin{aligned}
& \phi_{H}\left[\left(\widehat{p}_{t}^{H, W}-\widehat{p}_{t}^{W}\right)+\phi_{F}\left(\widehat{p}_{t}^{H, R}-\widehat{p}_{t}^{R}\right)\right]+\phi_{F}\left[\left(\widehat{p}_{t}^{F, W *}-\widehat{p}_{t}^{W *}\right)+\phi_{H}\left(\widehat{p}_{t}^{F, R}-\widehat{p}_{t}^{R}\right)\right] \\
& \quad=\phi_{H}\left(\widehat{p}_{t}^{H}-\widehat{p}_{t}\right)+\phi_{F}\left(\widehat{p}_{t}^{F}-\widehat{p}_{t}\right)=0, \\
& \phi_{F}\left[\left(\widehat{p}_{t}^{H, W}-\widehat{p}_{t}^{W}\right)-\phi_{H}\left(\widehat{p}_{t}^{H, R}-\widehat{p}_{t}^{R}\right)\right]+\phi_{H}\left[\left(\widehat{p}_{t}^{F, W *}-\widehat{p}_{t}^{W *}\right)-\phi_{F}\left(\widehat{p}_{t}^{F, R}-\widehat{p}_{t}^{R}\right)\right] \\
& \quad=\phi_{F}\left(\widehat{p}_{t}^{H *}-\widehat{p}_{t}^{*}\right)+\phi_{H}\left(\widehat{p}_{t}^{F *}-\widehat{p}_{t}^{*}\right)=0,
\end{aligned}
$$

since $\widehat{p}_{t}=\phi_{H} \widehat{p}_{t}^{H}+\phi_{F} \widehat{p}_{t}^{F}$ and $\widehat{p}_{t}^{*}=\phi_{F} \widehat{p}_{t}^{H *}+\phi_{H} \widehat{p}_{t}^{F *}$. Furthermore, we also know based on those same definitions, that the following result must hold true,

$$
\begin{aligned}
& \left(\widehat{p}_{t}^{H, W}-\widehat{p}_{t}^{W}\right)+\left(\widehat{p}_{t}^{F, W *}-\widehat{p}_{t}^{W *}\right)=\widehat{p}_{t}^{H, W}+\widehat{p}_{t}^{F, W *}-\left(\widehat{p}_{t}^{W}+\widehat{p}_{t}^{W *}\right) \\
& \quad=\phi_{H}\left(\widehat{p}_{t}^{H}-\widehat{p}_{t}\right)+\phi_{F}\left(\widehat{p}_{t}^{H *}-\widehat{p}_{t}^{*}\right)+\phi_{F}\left(\widehat{p}_{t}^{F}-\widehat{p}_{t}\right)+\phi_{H}\left(\widehat{p}_{t}^{F *}-\widehat{p}_{t}^{*}\right) \\
& \quad=\left[\phi_{H}\left(\widehat{p}_{t}^{H}-\widehat{p}_{t}\right)+\phi_{F}\left(\widehat{p}_{t}^{F}-\widehat{p}_{t}\right)\right]+\left[\phi_{F}\left(\widehat{p}_{t}^{H *}-\widehat{p}_{t}^{*}\right)+\phi_{H}\left(\widehat{p}_{t}^{F *}-\widehat{p}_{t}^{*}\right)\right]=0 .
\end{aligned}
$$

Hence, we argue that those terms inside the brackets of the Phillips curves can be simplified as follows,

$$
\begin{aligned}
- & \left(1+\eta\left(\frac{\psi}{1+(1-\psi) \varphi}\right)\left(\varphi+(1-\psi)\left(\frac{1+\varphi}{\psi}\right)^{2}\right)\right)\left[\phi_{H}\left(\widehat{p}_{t}^{H, W}-\widehat{p}_{t}^{W}\right)+\phi_{F}\left(\widehat{p}_{t}^{F, W *}-\widehat{p}_{t}^{W *}\right)\right]-\ldots \\
& \phi_{H} \phi_{F}\left[\left(\widehat{p}_{t}^{H, R}-\widehat{p}_{t}^{R}\right)+\left(\widehat{p}_{t}^{F, R}-\widehat{p}_{t}^{R}\right)\right]=\eta\left(\frac{\psi}{1+(1-\psi) \varphi}\right)\left(\varphi+(1-\psi)\left(\frac{1+\varphi}{\psi}\right)^{2}\right)\left[\phi_{H}-\phi_{F}\right]\left(\widehat{p}_{t}^{F, W *}-\widehat{p}_{t}^{W *}\right), \\
- & \left(1+\eta\left(\frac{\psi}{1+(1-\psi) \varphi}\right)\left(\varphi+(1-\psi)\left(\frac{1+\varphi}{\psi}\right)^{2}\right)\right)\left[\phi_{F}\left(\widehat{p}_{t}^{H, W}-\widehat{p}_{t}^{W}\right)+\phi_{H}\left(\widehat{p}_{t}^{F, W *}-\widehat{p}_{t}^{W *}\right)\right]+\ldots \\
& \phi_{F} \phi_{H}\left[\left(\widehat{p}_{t}^{H, R}-\widehat{p}_{t}^{R}\right)+\left(\widehat{p}_{t}^{F, R}-\widehat{p}_{t}^{R}\right)\right]=-\eta\left(\frac{\psi}{1+(1-\psi) \varphi}\right)\left(\varphi+(1-\psi)\left(\frac{1+\varphi}{\psi}\right)^{2}\right)\left[\phi_{H}-\phi_{F}\right]\left(\widehat{p}_{t}^{F, W *}-\widehat{p}_{t}^{W *}\right) .
\end{aligned}
$$

These derivations hold true irrespective of whether the law of one price holds or not.

Finally, we conclude that both Phillips curves in the PCP model take the following form,

$$
\begin{gathered}
\widehat{\pi}_{t} \approx \beta \mathbb{E}_{t}\left(\widehat{\pi}_{t+1}\right)+\phi_{F}\left[\left(\widehat{s}_{t}-\widehat{s}_{t-1}\right)-\beta \mathbb{E}_{t}\left(\widehat{s}_{t+1}-\widehat{s}_{t}\right)+\Psi\left(\widehat{p}_{t}^{F, R}-\widehat{s}_{t}\right)\right]+\ldots \\
\Psi\left[\begin{array}{c}
\left(\sigma^{-1}+\left(1-\gamma_{x}\right)\left(\frac{\psi}{1+(1-\psi) \varphi}\right)\left(\varphi+(1-\psi)\left(\frac{1+\varphi}{\psi}\right)^{2}\right)\right)\left[\phi_{H} \widehat{c}_{t}^{W}+\phi_{F} \widehat{c}_{t}^{W *}\right]+\ldots \\
\gamma_{x}\left(\frac{\psi}{1+(1-\psi) \varphi}\right)\left(\varphi+(1-\psi)\left(\frac{1+\varphi}{\psi}\right)^{2}\right)\left[\phi_{H} \widehat{x}_{t}^{W}+\phi_{F} \widehat{x}_{t}^{W *}\right]+2 \phi_{H} \phi_{F} \widehat{r s}_{t}+\ldots \\
\left(\phi_{H}-\phi_{F}\right) \eta\left(\frac{\psi}{1+(1-\psi) \varphi}\right)\left(\varphi+(1-\psi)\left(\frac{1+\varphi}{\psi}\right)^{2}\right)\left(\widehat{p}_{t}^{F, W *}-\widehat{p}_{t}^{W *}\right)-\ldots \\
(1-\psi)\left(\frac{1+\varphi}{\psi}\right) \widehat{k}_{t}^{W}-\left(\frac{1+\varphi}{\psi}\right)\left[\phi_{H} \widehat{a}_{t}+\phi_{F} \widehat{a}_{t}^{*}\right]
\end{array}\right], \\
\widehat{\pi}_{t}^{*} \approx \beta \mathbb{E}_{t}\left(\widehat{\pi}_{t+1}^{*}\right)-\phi_{F}\left[\left(\widehat{s}_{t}-\widehat{s}_{t-1}\right)-\beta \mathbb{E}_{t}\left(\widehat{s}_{t+1}-\widehat{s}_{t}\right)+\Psi\left(\widehat{p}_{t}^{H, R}-\widehat{s}_{t}\right)\right]+\ldots \\
\Psi\left[\begin{array}{c}
\left(\sigma^{-1}+\left(1-\gamma_{x}\right)\left(\frac{\psi}{1+(1-\psi) \varphi}\right)\left(\varphi+(1-\psi)\left(\frac{1+\varphi}{\psi}\right)^{2}\right)\right)\left[\phi_{F} \widehat{c}_{t}^{W}+\phi_{H} \widehat{c}_{t}^{W *}\right]+\ldots \\
\gamma_{x}\left(\frac{\psi}{1+(1-\psi) \varphi}\right)\left(\varphi+(1-\psi)\left(\frac{1+\varphi}{\psi}\right)^{2}\right)\left[\phi_{F} \widehat{x}_{t}^{W}+\phi_{H} \widehat{x}_{t}^{W *}\right]-2 \phi_{F} \phi_{H} \widehat{r s}_{t}-\ldots \\
\left(\phi_{H}-\phi_{F}\right) \eta\left(\frac{\psi}{1+(1-\psi) \varphi}\right)\left(\varphi+(1-\psi)\left(\frac{1+\varphi}{\psi}\right)^{2}\right)\left(\widehat{p}_{t}^{F, W *}-\widehat{p}_{t}^{W *}\right)-\ldots \\
(1-\psi)\left(\frac{1+\varphi}{\psi}\right) \widehat{k}_{t}^{W *}-\left(\frac{1+\varphi}{\psi}\right)\left[\phi_{F} \widehat{a}_{t}+\phi_{H} \widehat{a}_{t}^{*}\right]
\end{array}\right],
\end{gathered}
$$


or,

$$
\begin{gathered}
\widehat{\pi}_{t} \approx \beta \mathbb{E}_{t}\left(\widehat{\pi}_{t+1}\right)+\phi_{F}\left[\left(\widehat{s}_{t}-\widehat{s}_{t-1}\right)-\beta \mathbb{E}_{t}\left(\widehat{s}_{t+1}-\widehat{s}_{t}\right)+\Psi\left(\widehat{p}_{t}^{F, R}-\widehat{s}_{t}\right)\right]+\ldots \\
\Psi\left[\begin{array}{c}
\left(\sigma^{-1}+\left(1-\gamma_{x}\right) \varphi\left(\frac{\varphi \psi^{2}+(1-\psi)(1+\varphi)^{2}}{\varphi \psi+(1-\psi) \psi \varphi^{2}}\right)\right)\left[\phi_{H} \widehat{c}_{t}^{W}+\phi_{F} \widehat{c}_{t}^{W *}\right]+\ldots \\
\gamma_{x} \varphi\left(\frac{\varphi \psi^{2}+(1-\psi)(1+\varphi)^{2}}{\varphi \psi+(1-\psi) \psi \varphi^{2}}\right)\left[\phi_{H} \widehat{x}_{t}^{W}+\phi_{F} \widehat{x}_{t}^{W *}\right]+\ldots \\
2 \phi_{H} \phi_{F} \widehat{r s}_{t}+\left(\phi_{H}-\phi_{F}\right) \eta \varphi\left(\frac{\varphi \psi^{2}+(1-\psi)(1+\varphi)^{2}}{\varphi \psi+(1-\psi) \psi \varphi^{2}}\right)\left(\widehat{p}_{t}^{F, W *}-\widehat{p}_{t}^{W *}\right)-\ldots \\
\left(\frac{(1-\psi)(1+\varphi)}{\psi}\right) \widehat{k}_{t}^{W}-\left(\frac{1+\varphi}{\psi}\right)\left[\phi_{H} \widehat{a}_{t}+\phi_{F} \widehat{a}_{t}^{*}\right]
\end{array}\right] \\
\widehat{\pi}_{t}^{*} \approx \beta \mathbb{E}_{t}\left(\widehat{\pi}_{t+1}^{*}\right)-\phi_{F}\left[\left(\widehat{s}_{t}-\widehat{s}_{t-1}\right)-\beta \mathbb{E}_{t}\left(\widehat{s}_{t+1}-\widehat{s}_{t}\right)+\Psi\left(\widehat{p}_{t}^{H, R}-\widehat{s}_{t}\right)\right]+\ldots \\
\Psi\left[\begin{array}{c}
\left(\sigma^{-1}+\left(1-\gamma_{x}\right) \varphi\left(\frac{\varphi \psi^{2}+(1-\psi)(1+\varphi)^{2}}{\varphi \psi+(1-\psi) \psi\left(\varphi^{2}\right.}\right)\right)\left[\phi_{F} \widehat{c}_{t}^{W}+\phi_{H} \widehat{c}_{t}^{W *}\right]+\ldots \\
\gamma_{x} \varphi\left(\frac{\varphi \psi^{2}+(1-\psi)(1+\varphi)^{2}}{\varphi \psi+(1-\psi) \psi \varphi^{2}}\right)\left[\phi_{F} \widehat{x}_{t}^{W}+\phi_{H} \widehat{x}_{t}^{W *}\right]-\ldots \\
2 \phi_{F} \phi_{H} \widehat{r s}_{t}-\left(\phi_{H}-\phi_{F}\right) \eta \varphi\left(\frac{\varphi \psi^{2}+(1-\psi)(1+\varphi)^{2}}{\varphi \psi+(1-\psi) \psi \varphi^{2}}\right)\left(\widehat{p}_{t}^{F, W *}-\widehat{p}_{t}^{W *}\right)-\ldots \\
\left(\frac{(1-\psi)(1+\varphi)}{\psi}\right) \widehat{k}_{t}^{W *}-\left(\frac{1+\varphi}{\psi}\right)\left[\phi_{F} \widehat{a}_{t}+\phi_{H} \widehat{a}_{t}^{*}\right]
\end{array}\right.
\end{gathered}
$$

which extends the model under PCP pricing by adding capital and investment. Let us define $\widehat{t}_{t}^{W} \equiv \widehat{p}_{t}^{F, W *}{ }_{-}$ $\widehat{p}_{t}^{W *}$ as the world measure of terms of trade in the model. Then, the Phillips curves can be re-expressed as,

$$
\begin{gathered}
\widehat{\pi}_{t} \approx \beta \mathbb{E}_{t}\left(\widehat{\pi}_{t+1}\right)+\phi_{F}\left[\left(\widehat{s}_{t}-\widehat{s}_{t-1}\right)-\beta \mathbb{E}_{t}\left(\widehat{s}_{t+1}-\widehat{s}_{t}\right)+\left(\frac{(1-\alpha)(1-\alpha \beta)}{\alpha}\right)\left(\widehat{p}_{t}^{F, R}-\widehat{s}_{t}\right)\right]+\ldots \\
\Psi\left[\begin{array}{c}
\left(\sigma^{-1}+\left(1-\gamma_{x}\right) \varphi\left(\frac{\varphi \psi^{2}+(1-\psi)(1+\varphi)^{2}}{\varphi \psi+(1-\psi) \psi \varphi^{2}}\right)\right)\left[\phi_{H} \widehat{c}_{t}^{W}+\phi_{F} \widehat{c}_{t}^{W *}\right]+\ldots \\
\gamma_{x} \varphi\left(\frac{\varphi \psi^{2}+(1-\psi)(1+\varphi)^{2}}{\varphi \psi+(1-\psi) \psi \varphi^{2}}\right)\left[\phi_{H} \widehat{x}_{t}^{W}+\phi_{F} \widehat{x}_{t}^{W *}\right]+\ldots \\
2 \phi_{H} \phi_{F} \widehat{r s}_{t}+\left(\phi_{H}-\phi_{F}\right) \eta \varphi\left(\frac{\varphi \psi^{2}+(1-\psi)(1+\varphi)^{2}}{\varphi \psi+(1-\psi) \psi \varphi^{2}}\right) \widehat{t}_{t}^{W}-\ldots \\
\left(\frac{(1-\psi)(1+\varphi)}{\psi}\right) \widehat{k}_{t}^{W}-\left(\frac{1+\varphi}{\psi}\right)\left[\phi_{H} \widehat{a}_{t}+\phi_{F} \widehat{a}_{t}^{*}\right]
\end{array}\right], \\
\widehat{\pi}_{t}^{*} \approx \beta \mathbb{E}_{t}\left(\widehat{\pi}_{t+1}^{*}\right)-\phi_{F}\left[\left(\widehat{s}_{t}-\widehat{s}_{t-1}\right)-\beta \mathbb{E}_{t}\left(\widehat{s}_{t+1}-\widehat{s}_{t}\right)+\left(\frac{(1-\alpha)(1-\alpha \beta)}{\alpha}\right)\left(\widehat{p}_{t}^{H, R}-\widehat{s}_{t}\right)\right]+\ldots \\
\Psi\left[\begin{array}{c}
\left(\sigma^{-1}+\left(1-\gamma_{x}\right) \varphi\left(\frac{\varphi \psi^{2}+(1-\psi)(1+\varphi)^{2}}{\varphi \psi+(1-\psi) \psi \varphi^{2}}\right)\right)\left[\phi_{F} \widehat{c}_{t}^{W}+\phi_{H} \widehat{c}_{t}^{W *}\right]+\ldots \\
\gamma_{x} \varphi\left(\frac{\varphi \psi^{2}+(1-\psi)(1+\varphi)^{2}}{\varphi \psi+(1-\psi) \psi \varphi^{2}}\right)\left[\phi_{F} \widehat{x}_{t}^{W}+\phi_{H} \widehat{x}_{t}^{W *}\right]-\ldots \\
2 \phi_{F} \phi_{H} \widehat{r s}_{t}-\left(\phi_{H}-\phi_{F}\right) \eta \varphi\left(\frac{\varphi \psi^{2}+(1-\psi)(1+\varphi)^{2}}{\varphi \psi+(1-\psi) \psi \varphi^{2}}\right) \widehat{t}_{t}^{W}-\ldots \\
\left(\frac{(1-\psi)(1+\varphi)}{\psi}\right) \widehat{k}_{t}^{W *}-\left(\frac{1+\varphi}{\psi}\right)\left[\phi_{F} \widehat{a}_{t}+\phi_{H} \widehat{a}_{t}^{*}\right]
\end{array}\right]
\end{gathered}
$$

Furthermore, since the law of one price holds under PCP, then it must be the case that,

$$
\widehat{p}_{t}^{H, R}-\widehat{s}_{t} \approx 0, \widehat{p}_{t}^{F, R}-\widehat{s}_{t} \approx 0,
$$


so the dynamics of inflation can be further simplified as,

$$
\begin{aligned}
& \widehat{\pi}_{t} \approx \beta \mathbb{E}_{t}\left(\widehat{\pi}_{t+1}\right)+\phi_{F}\left[\Delta \widehat{s}_{t}-\beta \mathbb{E}_{t}\left(\Delta \widehat{s}_{t+1}\right)\right]+\ldots \\
& \Psi\left[\begin{array}{c}
\left(\sigma^{-1}+\left(1-\gamma_{x}\right) \varphi\left(\frac{\varphi \psi^{2}+(1-\psi)(1+\varphi)^{2}}{\varphi \psi+(1-\psi) \psi \varphi^{2}}\right)\right)\left[\phi_{H} \widehat{c}_{t}^{W}+\phi_{F} \widehat{c}_{t}^{W *}\right]+\ldots \\
\gamma_{x} \varphi\left(\frac{\varphi \psi^{2}+(1-\psi)(1+\varphi)^{2}}{\varphi \psi+(1-\psi) \psi \varphi^{2}}\right)\left[\phi_{H} \widehat{x}_{t}^{W}+\phi_{F} \widehat{x}_{t}^{W *}\right]+\ldots \\
2 \phi_{H} \phi_{F} \widehat{r s}_{t}+\left(\phi_{H}-\phi_{F}\right) \eta \varphi\left(\frac{\varphi \psi^{2}+(1-\psi)(1+\varphi)^{2}}{\varphi \psi+(1-\psi) \psi \varphi^{2}}\right) \widehat{t}_{t}^{W}-\ldots \\
\left(\frac{(1-\psi)(1+\varphi)}{\psi}\right) \widehat{k}_{t}^{W}-\left(\frac{1+\varphi}{\psi}\right)\left[\phi_{H} \widehat{a}_{t}+\phi_{F} \widehat{a}_{t}^{*}\right]
\end{array}\right], \\
& \widehat{\pi}_{t}^{*} \approx \beta \mathbb{E}_{t}\left(\widehat{\pi}_{t+1}^{*}\right)-\phi_{F}\left[\Delta \widehat{s}_{t}-\beta \mathbb{E}_{t}\left(\Delta \widehat{s}_{t+1}\right)\right]+\ldots \\
& \Psi\left[\begin{array}{c}
\left(\sigma^{-1}+\left(1-\gamma_{x}\right) \varphi\left(\frac{\varphi \psi^{2}+(1-\psi)(1+\varphi)^{2}}{\varphi \psi+(1-\psi) \psi \varphi^{2}}\right)\right)\left[\phi_{F} \widehat{c}_{t}^{W}+\phi_{H} \widehat{c}_{t}^{W *}\right]+\ldots \\
\gamma_{x} \varphi\left(\frac{\varphi \psi^{2}+(1-\psi)(1+\varphi)^{2}}{\varphi \psi+(1-\psi) \psi \varphi^{2}}\right)\left[\phi_{F} \widehat{x}_{t}^{W}+\phi_{H} \widehat{x}_{t}^{W *}\right]-\ldots \\
2 \phi_{F} \phi_{H} \widehat{r s}_{t}-\left(\phi_{H}-\phi_{F}\right) \eta \varphi\left(\frac{\varphi \psi^{2}+(1-\psi)(1+\varphi)^{2}}{\varphi \psi+(1-\psi) \psi \varphi^{2}}\right) \widehat{t}_{t}^{W}-\ldots \\
\left(\frac{(1-\psi)(1+\varphi)}{\psi}\right) \widehat{k}_{t}^{W *}-\left(\frac{1+\varphi}{\psi}\right)\left[\phi_{F} \widehat{a}_{t}+\phi_{H} \widehat{a}_{t}^{*}\right]
\end{array}\right],
\end{aligned}
$$

where we denote the first-difference on the nominal exchange rate as $\Delta \widehat{s}_{t} \equiv \widehat{s}_{t}-\widehat{s}_{t-1}$. Alternatively, we note that,

$$
\begin{aligned}
& \widehat{\pi}_{t}-\phi_{F} \Delta \widehat{s}_{t} \approx \beta \mathbb{E}_{t}\left(\widehat{\pi}_{t+1}-\phi_{F} \Delta \widehat{s}_{t+1}\right)+\ldots \\
& \Psi\left[\begin{array}{c}
\left(\sigma^{-1}+\left(1-\gamma_{x}\right) \varphi\left(\frac{\varphi \psi^{2}+(1-\psi)(1+\varphi)^{2}}{\varphi \psi+(1-\psi) \psi \varphi^{2}}\right)\right)\left[\phi_{H} \widehat{c}_{t}^{W}+\phi_{F} \widehat{c}_{t}^{W *}\right]+\ldots \\
\gamma_{x} \varphi\left(\frac{\varphi \psi^{2}+(1-\psi)(1+\varphi)^{2}}{\varphi \psi+(1-\psi) \psi \varphi^{2}}\right)\left[\phi_{H} \widehat{x}_{t}^{W}+\phi_{F} \widehat{x}_{t}^{W *}\right]+\ldots \\
2 \phi_{H} \phi_{F} \widehat{r s s}_{t}+\left(\phi_{H}-\phi_{F}\right) \eta \varphi\left(\frac{\varphi \psi^{2}+(1-\psi)(1+\varphi)^{2}}{\varphi \psi+(1-\psi) \psi \varphi^{2}}\right) \widehat{t}_{t}^{W}-\ldots \\
\left(\frac{(1-\psi)(1+\varphi)}{\psi}\right) \widehat{k}_{t}^{W}-\left(\frac{1+\varphi}{\psi}\right)\left[\phi_{H} \widehat{a}_{t}+\phi_{F} \widehat{a}_{t}^{*}\right]
\end{array}\right], \\
& \widehat{\pi}_{t}^{*}+\phi_{F} \Delta \widehat{s}_{t} \approx \beta \mathbb{E}_{t}\left(\widehat{\pi}_{t+1}^{*}+\phi_{F} \Delta \widehat{s}_{t+1}\right)+\ldots \\
& \Psi\left[\begin{array}{c}
\left(\sigma^{-1}+\left(1-\gamma_{x}\right) \varphi\left(\frac{\varphi \psi^{2}+(1-\psi)(1+\varphi)^{2}}{\varphi \psi+(1-\psi) \psi \varphi^{2}}\right)\right)\left[\phi_{F} \widehat{c}_{t}^{W}+\phi_{H} \widehat{c}_{t}^{W *}\right]+\ldots \\
\gamma_{x} \varphi\left(\frac{\varphi \psi^{2}+(1-\psi)(1+\varphi)^{2}}{\varphi \psi+(1-\psi) \psi \varphi^{2}}\right)\left[\phi_{F} \widehat{x}_{t}^{W}+\phi_{H} \widehat{x}_{t}^{W *}\right]-\ldots \\
2 \phi_{F} \phi_{H} \widehat{r s}_{t}-\left(\phi_{H}-\phi_{F}\right) \eta \varphi\left(\frac{\varphi \psi^{2}+(1-\psi)(1+\varphi)^{2}}{\varphi \psi+(1-\psi) \psi \varphi^{2}}\right) \widehat{t}_{t}^{W}-\ldots \\
\left(\frac{(1-\psi)(1+\varphi)}{\psi}\right) \widehat{k}_{t}^{W *}-\left(\frac{1+\varphi}{\psi}\right)\left[\phi_{F} \widehat{a}_{t}+\phi_{H} \widehat{a}_{t}^{*}\right]
\end{array}\right] .
\end{aligned}
$$

The Phillip curves under PCP pricing are very similar to those under LCP pricing in (153) - (154), except for the fact that there is full nominal exchange rate pass-through into import prices under PCP.

CPI inflation in each country can be expressed with the exact same present discounted value of real marginal costs as for the LCP pricing case with an additional term that reflects the direct full pass-through of nominal exchange rate movements into import prices. That is, under LCP pricing current domestic and 
foreign inflation can be expressed as,

$$
\begin{aligned}
\widehat{\pi}_{t} \approx \Psi \sum_{j=0}^{+\infty} \beta^{j} \mathbb{E}_{t}\left[\begin{array}{c}
\left(\sigma^{-1}+\left(1-\gamma_{x}\right) \varphi\left(\frac{\varphi \psi^{2}+(1-\psi)(1+\varphi)^{2}}{\varphi \psi+(1-\psi) \psi \varphi^{2}}\right)\right)\left[\phi_{H} \widehat{c}_{t+j}^{W}+\phi_{F} \widehat{c}_{t+j}^{W *}\right]+\ldots \\
\gamma_{x} \varphi\left(\frac{\varphi \psi^{2}+(1-\psi)(1+\varphi)^{2}}{\varphi \psi+(1-\psi) \psi \varphi^{2}}\right)\left[\phi_{H} \widehat{x}_{t+j}^{W}+\phi_{F} \widehat{x}_{t+j}^{W *}\right]+\ldots \\
2 \phi_{H} \phi_{F} \widehat{r s}_{t+j}+\left(\phi_{H}-\phi_{F}\right) \eta \varphi\left(\frac{\varphi \psi^{2}+(1-\psi)(1+\varphi)^{2}}{\varphi \psi+(1-\psi) \psi \varphi^{2}}\right) \widehat{t}_{t+j}^{W}-\ldots \\
\left(\frac{(1-\psi)(1+\varphi)}{\psi}\right) \widehat{k}_{t+j}^{W}-\left(\frac{1+\varphi}{\psi}\right)\left[\phi_{H} \widehat{a}_{t+j}+\phi_{F} \widehat{a}_{t+j}^{*}\right]
\end{array}\right], \\
\widehat{\pi}_{t}^{*} \approx \Psi \sum_{j=0}^{+\infty} \beta^{j} \mathbb{E}_{t}\left[\begin{array}{c}
\left(\sigma^{-1}+\left(1-\gamma_{x}\right) \varphi\left(\frac{\varphi \psi^{2}+(1-\psi)(1+\varphi)^{2}}{\varphi \psi+(1-\psi) \psi \varphi^{2}}\right)\right)\left[\phi_{F} \widehat{c}_{t+j}^{W}+\phi_{H} \widehat{c}_{t+j}^{W *}\right]+\ldots \\
\gamma_{x} \varphi\left(\frac{\varphi \psi^{2}+(1-\psi)(1+\varphi)^{2}}{\varphi \psi+(1-\psi) \psi \varphi^{2}}\right)\left[\phi_{F} \widehat{x}_{t+j}^{W}+\phi_{H} \widehat{x}_{t+j}^{W *}\right]-\ldots \\
2 \phi_{F} \phi_{H} \widehat{r s}_{t+j}-\left(\phi_{H}-\phi_{F}\right) \eta \varphi\left(\frac{\varphi \psi^{2}+(1-\psi)(1+\varphi)^{2}}{\varphi \psi+(1-\psi) \psi \varphi^{2}}\right) \widehat{t}_{t+j}^{W}-\ldots \\
\left(\frac{(1-\psi)(1+\varphi)}{\psi}\right) \widehat{k}_{t+j}^{W *}-\left(\frac{1+\varphi}{\psi}\right)\left[\phi_{F} \widehat{a}_{t+j}+\phi_{H} \widehat{a}_{t+j}^{*}\right]
\end{array}\right],
\end{aligned}
$$

while under PCP pricing current domestic and foreign inflation become,

$$
\begin{gathered}
\widehat{\pi}_{t} \approx \phi_{F} \Delta \widehat{s}_{t}+\Psi \sum_{j=0}^{+\infty} \beta^{j} \mathbb{E}_{t}\left[\begin{array}{c}
\left(\sigma^{-1}+\left(1-\gamma_{x}\right) \varphi\left(\frac{\varphi \psi^{2}+(1-\psi)(1+\varphi)^{2}}{\varphi \psi+(1-\psi) \psi \varphi^{2}}\right)\right)\left[\phi_{H} \widehat{c}_{t+j}^{W}+\phi_{F} \widehat{c}_{t+j}^{W *}\right]+\ldots \\
\gamma_{x} \varphi\left(\frac{\varphi \psi^{2}+(1-\psi)(1+\varphi)^{2}}{\varphi \psi+(1-\psi) \psi \varphi^{2}}\right)\left[\phi_{H} \widehat{x}_{t+j}^{W}+\phi_{F} \widehat{x}_{t+j}^{W *}\right]+\ldots \\
2 \phi_{H} \phi_{F} \widehat{r s}_{t+j}+\left(\phi_{H}-\phi_{F}\right) \eta \varphi\left(\frac{\varphi \psi^{2}+(1-\psi)(1+\varphi)^{2}}{\varphi \psi+(1-\psi) \psi \varphi^{2}}\right) \widehat{t}_{t+j}^{W}-\ldots \\
\left(\frac{(1-\psi)(1+\varphi)}{\psi}\right) \widehat{k}_{t+j}^{W}-\left(\frac{1+\varphi}{\psi}\right)\left[\phi_{H} \widehat{a}_{t+j}+\phi_{F} \widehat{a}_{t+j}^{*}\right]
\end{array}\right], \\
\widehat{\pi}_{t}^{*} \approx-\phi_{F} \Delta \widehat{s}_{t}+\Psi \sum_{j=0}^{+\infty} \beta^{j} \mathbb{E}_{t}\left[\begin{array}{c}
\left(\sigma^{-1}+\left(1-\gamma_{x}\right) \varphi\left(\frac{\varphi \psi^{2}+(1-\psi)(1+\varphi)^{2}}{\varphi \psi+(1-\psi) \psi \varphi^{2}}\right)\right)\left[\phi_{F} \widehat{c}_{t+j}^{W}+\phi_{H} \widehat{c}_{t+j}^{W *}\right]+\ldots \\
\gamma_{x} \varphi\left(\frac{\varphi \psi^{2}+(1-\psi)(1+\varphi)^{2}}{\varphi \psi+(1-\psi) \psi \varphi^{2}}\right)\left[\phi_{F} \widehat{x}_{t+j}^{W}+\phi_{H} \widehat{x}_{t+j}^{W *}\right]-\ldots \\
2 \phi_{F} \phi_{H} \widehat{r s s}_{t+j}-\left(\phi_{H}-\phi_{F}\right) \eta \varphi\left(\frac{\varphi \psi^{2}+(1-\psi)(1+\varphi)^{2}}{\varphi \psi+(1-\psi) \psi \varphi^{2}}\right) \widehat{t}_{t+j}^{W}-\ldots \\
\left(\frac{(1-\psi)(1+\varphi)}{\psi}\right) \widehat{k}_{t+j}^{W *}-\left(\frac{1+\varphi}{\psi}\right)\left[\phi_{F} \widehat{a}_{t+j}+\phi_{H} \widehat{a}_{t+j}^{*}\right]
\end{array}\right] .
\end{gathered}
$$

These expressions reflect the role played by the LCP and PCP assumptions on the trade-off between nominal and real variables captured by the Phillips curves.

The International Relative Prices. We have defined the world price sub-indexes as $\widehat{p}_{t}^{H, W} \equiv \phi_{H} \widehat{p}_{t}^{H}+$ $\phi_{F} \widehat{p}_{t}^{H *}$ and $\widehat{p}_{t}^{F, W *} \equiv \phi_{F} \widehat{p}_{t}^{F}+\phi_{H} \widehat{p}_{t}^{F *}$, and the relative price sub-indexes as $\widehat{p}_{t}^{H, R} \equiv \widehat{p}_{t}^{H}-\widehat{p}_{t}^{H *}$ and $\widehat{p}_{t}^{F, R} \equiv$ $\widehat{p}_{t}^{F}-\widehat{p}_{t}^{F *}$. Then, naturally, we can write that,

$$
\begin{aligned}
\widehat{p}_{t}^{H} & =\widehat{p}_{t}^{H, W}+\phi_{F} \widehat{p}_{t}^{H, R}, \widehat{p}_{t}^{H *}=\widehat{p}_{t}^{H, W}-\phi_{H} \widehat{p}_{t}^{H, R}, \\
\widehat{p}_{t}^{F} & =\widehat{p}_{t}^{F, W *}+\phi_{H} \widehat{p}_{t}^{F, R}, \widehat{p}_{t}^{F *}=\widehat{p}_{t}^{F, W *}-\phi_{F} \widehat{p}_{t}^{F, R} .
\end{aligned}
$$

Analogously, we have defined the world CPI as $\widehat{p}_{t}^{W} \equiv \phi_{H} \widehat{p}_{t}+\phi_{F} \widehat{p}_{t}^{*}$ and $\widehat{p}_{t}^{W *} \equiv \phi_{F} \widehat{p}_{t}+\phi_{H} \widehat{p}_{t}^{*}$, and the relative CPI as $\widehat{p}_{t}^{R} \equiv \widehat{p}_{t}-\widehat{p}_{t}^{*}$. Then, we can write that,

$$
\begin{aligned}
& \widehat{p}_{t}=\widehat{p}_{t}^{W}+\phi_{F} \widehat{p}_{t}^{R}, \widehat{p}_{t}^{*}=\widehat{p}_{t}^{W}-\phi_{H} \widehat{p}_{t}^{R}, \\
& \widehat{p}_{t}=\widehat{p}_{t}^{W *}+\phi_{H} \widehat{p}_{t}^{R}, \widehat{p}_{t}^{*}=\widehat{p}_{t}^{W *}-\phi_{F} \widehat{p}_{t}^{R} .
\end{aligned}
$$


The definition of CPI in both countries, i.e. $\widehat{p}_{t} \approx \phi_{H} \widehat{p}_{t}^{H}+\phi_{F} \widehat{p}_{t}^{F}$ and $\widehat{p}_{t}^{*} \approx \phi_{F} \widehat{p}_{t}^{H *}+\phi_{H} \widehat{p}_{t}^{F *}$, can be written as,

$$
\begin{aligned}
\phi_{H}\left[\widehat{p}_{t}^{H}-\widehat{p}_{t}\right]+\phi_{F}\left[\widehat{p}_{t}^{F}-\widehat{p}_{t}\right] & \approx 0, \\
\phi_{F}\left[\widehat{p}_{t}^{H *}-\widehat{p}_{t}^{*}\right]+\phi_{H}\left[\widehat{p}_{t}^{F *}-\widehat{p}_{t}^{*}\right] & \approx 0 .
\end{aligned}
$$

Then, based on the relationships described before, we can write the definitions of the CPI indexes as,

$$
\begin{aligned}
& \phi_{H}\left[\left(\widehat{p}_{t}^{H, W}-\widehat{p}_{t}^{W}\right)+\phi_{F}\left(\widehat{p}_{t}^{H, R}-\widehat{p}_{t}^{R}\right)\right]+\phi_{F}\left[\left(\widehat{p}_{t}^{F, W *}-\widehat{p}_{t}^{W *}\right)+\phi_{H}\left(\widehat{p}_{t}^{F, R}-\widehat{p}_{t}^{R}\right)\right] \approx 0, \\
& \phi_{F}\left[\left(\widehat{p}_{t}^{H, W}-\widehat{p}_{t}^{W}\right)-\phi_{H}\left(\widehat{p}_{t}^{H, R}-\widehat{p}_{t}^{R}\right)\right]+\phi_{H}\left[\left(\widehat{p}_{t}^{F, W *}-\widehat{p}_{t}^{W *}\right)-\phi_{F}\left(\widehat{p}_{t}^{F, R}-\widehat{p}_{t}^{R}\right)\right] \approx 0 .
\end{aligned}
$$

Let us define $\widehat{t}_{t}^{W} \equiv \widehat{p}_{t}^{F, W *}-\widehat{p}_{t}^{W *}$ as the world measure of terms of trade in this model. Terms of trade are implicitly characterized by the previous pair of equations.

By construction $\left(\widehat{p}_{t}^{H, W}-\widehat{p}_{t}^{W}\right)+\left(\widehat{p}_{t}^{F, W *}-\widehat{p}_{t}^{W *}\right) \approx 0$ (see equation (146) for a demonstration), hence the two expressions above become simply equal to,

$$
\begin{aligned}
\left(\phi_{H}-\phi_{F}\right)\left(\widehat{p}_{t}^{F, W *}-\widehat{p}_{t}^{W *}\right) & \approx \phi_{H} \phi_{F}\left[\left(\widehat{p}_{t}^{H, R}-\widehat{p}_{t}^{R}\right)+\left(\widehat{p}_{t}^{F, R}-\widehat{p}_{t}^{R}\right)\right], \\
\left(\phi_{H}-\phi_{F}\right)\left(\widehat{p}_{t}^{F, W *}-\widehat{p}_{t}^{W *}\right) & \approx \phi_{H} \phi_{F}\left[\left(\widehat{p}_{t}^{H, R}-\widehat{p}_{t}^{R}\right)+\left(\widehat{p}_{t}^{F, R}-\widehat{p}_{t}^{R}\right)\right] .
\end{aligned}
$$

In summary, the only constraint that determines the world terms of trade is given by,

$$
\left(\phi_{H}-\phi_{F}\right)\left(\widehat{p}_{t}^{F, W *}-\widehat{p}_{t}^{W *}\right) \approx \phi_{H} \phi_{F}\left[\left(\widehat{p}_{t}^{H, R}-\widehat{p}_{t}^{R}\right)+\left(\widehat{p}_{t}^{F, R}-\widehat{p}_{t}^{R}\right)\right] .
$$

If the model has no home-product bias in preferences, i.e. if $\phi_{H}=\phi_{F}$, then $\left(\widehat{p}_{t}^{F, W *}-\widehat{p}_{t}^{W *}\right)$ only matters because it affects output and output enters into the specification of the Taylor rule in (126)-(127). Therefore, it must follow from $(176)$ that $\left(\widehat{p}_{t}^{H, R}-\widehat{p}_{t}^{R}\right)+\left(\widehat{p}_{t}^{F, R}-\widehat{p}_{t}^{R}\right) \approx 0$. In that case, this constraint imposes no restriction on the world terms of trade $\widehat{t}_{t}^{W} \equiv \widehat{p}_{t}^{F, W *}-\widehat{p}_{t}^{W *}$, and we would need to keep track of the price sub-indexes in order to close down the model.

If the model has home-product bias in preferences, i.e. if $\phi_{H} \neq \phi_{F}$, then $\widehat{t}_{t}^{W} \equiv\left(\widehat{p}_{t}^{F, W *}-\widehat{p}_{t}^{W *}\right)$ matters because it affects output in both countries and it also matters because it affects the inflation dynamics. Moreover, we can write the world terms of trade as follows,

$$
\widehat{t}_{t}^{W} \equiv \widehat{p}_{t}^{F, W *}-\widehat{p}_{t}^{W *}=\frac{\phi_{H} \phi_{F}}{\phi_{H}-\phi_{F}}\left[\left(\widehat{p}_{t}^{H, R}-\widehat{p}_{t}^{R}\right)+\left(\widehat{p}_{t}^{F, R}-\widehat{p}_{t}^{R}\right)\right]
$$

This is going to be crucial to derive the dynamics of world terms of trade. We have shown that under PCP 
it must be the case that $\widehat{p}_{t}^{F, R} \approx \widehat{p}_{t}^{H, R} \approx \widehat{s}_{t}$, so it follows that,

$$
\begin{aligned}
\widehat{t}_{t}^{W} & \equiv \widehat{p}_{t}^{F, W *}-\widehat{p}_{t}^{W *}=\frac{\phi_{H} \phi_{F}}{\phi_{H}-\phi_{F}}\left[\left(\widehat{s}_{t}-\widehat{p}_{t}^{R}\right)+\left(\widehat{s}_{t}-\widehat{p}_{t}^{R}\right)\right] \\
& =\frac{\phi_{H} \phi_{F}}{\phi_{H}-\phi_{F}}\left[\left(\widehat{p}_{t}^{R}+\widehat{r s}_{t}-\widehat{p}_{t}^{R}\right)+\left(\widehat{p}_{t}^{R}+\widehat{r s}_{t}-\widehat{p}_{t}^{R}\right)\right] \\
& =\frac{2 \phi_{H} \phi_{F}}{\phi_{H}-\phi_{F}} \widehat{r s}_{t},
\end{aligned}
$$

which is an equation that clearly ties the world terms of trade to the real exchange rate (an endogenous variable already accounted for in the model). This relationship coupled with the definition of the real exchange rate, i.e.

$$
\begin{aligned}
\Delta \widehat{r s}_{t} & =\Delta \widehat{s}_{t}+\widehat{\pi}_{t}^{*}-\widehat{\pi}_{t} \\
\Delta \widehat{r s}_{t} & \equiv \widehat{r s}_{t}-\widehat{r s}_{t-1}, \\
\Delta \widehat{s}_{t} & \equiv \widehat{s}_{t}-\widehat{s}_{t-1}
\end{aligned}
$$

suffices to close the model in the PCP case. The trick here is that the world terms of trade under PCP are tied to another observable variable (the real exchange rate), while in the LCP framework they are tied to the real exchange rate and also to deviations from the law of one price across borders. Therefore, in the LCP case it is necessary to model explicitly those interactions that go beyond the connection with the real exchange rate (which is what we show in equation (157)) while that is not necessary in the PCP case. The PCP case is much simpler because world terms of trade can be tied directly to another observable, endogenous variable in this way.

Given that $\widehat{p}_{t}^{F, W *} \equiv \phi_{F} \widehat{p}_{t}^{F}+\phi_{H} \widehat{p}_{t}^{F *}$ and $\widehat{p}_{t}^{W *} \equiv \phi_{F} \widehat{p}_{t}+\phi_{H} \widehat{p}_{t}^{*}$, so the definition of world terms of trade, $\hat{t}_{t}^{W}$, can be expressed as,

$$
\begin{aligned}
\widehat{t}_{t}^{W} & \equiv \widehat{p}_{t}^{F, W *}-\widehat{p}_{t}^{W *}=\phi_{F} \widehat{p}_{t}^{F}+\phi_{H} \widehat{p}_{t}^{F *}-\phi_{F} \widehat{p}_{t}-\phi_{H} \widehat{p}_{t}^{*} \\
& =\phi_{F}\left(\widehat{p}_{t}^{F}-\widehat{p}_{t}\right)+\phi_{H}\left(\widehat{p}_{t}^{F *}-\widehat{p}_{t}^{*}\right)
\end{aligned}
$$

and,

$$
\begin{aligned}
\widehat{t}_{t}^{W} & =\phi_{F}\left(\widehat{p}_{t}^{F}-\widehat{p}_{t}\right)+\phi_{H}\left(\widehat{p}_{t}^{F *}-\widehat{p}_{t}^{*}\right) \\
& \approx \phi_{F}\left(\widehat{p}_{t}^{F}-\phi_{H} \widehat{p}_{t}^{H}-\phi_{F} \widehat{p}_{t}^{F}\right)+\phi_{H}\left(\widehat{p}_{t}^{F *}-\phi_{F} \widehat{p}_{t}^{H *}-\phi_{H} \widehat{p}_{t}^{F *}\right) \\
& =\phi_{F}\left(\left(1-\phi_{F}\right) \widehat{p}_{t}^{F}-\phi_{H} \widehat{p}_{t}^{H}\right)+\phi_{H}\left(\left(1-\phi_{H}\right) \widehat{p}_{t}^{F *}-\phi_{F} \widehat{p}_{t}^{H *}\right) \\
& =\phi_{F} \phi_{H}\left(\widehat{p}_{t}^{F}-\widehat{p}_{t}^{H}\right)+\phi_{F} \phi_{H}\left(\widehat{p}_{t}^{F *}-\widehat{p}_{t}^{H *}\right),
\end{aligned}
$$

where we make use of the approximation of the CPI indexes $\widehat{p}_{t} \approx \phi_{H} \widehat{p}_{t}^{H}+\phi_{F} \widehat{p}_{t}^{F}$ and $\widehat{p}_{t}^{*} \approx \phi_{F} \widehat{p}_{t}^{H *}+\phi_{H} \widehat{p}_{t}^{F *}$. Then, using the implications of the law of one price, i.e. $\widehat{p}_{t}^{H} \approx \widehat{s}_{t}+\widehat{p}_{t}^{H *}$ and $\widehat{p}_{t}^{F} \approx \widehat{s}_{t}+\widehat{p}_{t}^{F *}$, we can immediately 
obtain the following expression,

$$
\begin{aligned}
\widehat{t}_{t}^{W} & =\phi_{F} \phi_{H}\left(\widehat{p}_{t}^{F}-\widehat{p}_{t}^{H}\right)+\phi_{F} \phi_{H}\left(\widehat{p}_{t}^{F *}-\widehat{p}_{t}^{H *}\right) \\
& =\phi_{F} \phi_{H}\left(\widehat{p}_{t}^{F}-\widehat{p}_{t}^{H}\right)+\phi_{F} \phi_{H}\left(\left(\widehat{p}_{t}^{F}-\widehat{s}_{t}\right)-\left(\widehat{p}_{t}^{H}-\widehat{s}_{t}\right)\right) \\
& =2 \phi_{F} \phi_{H}\left(\widehat{p}_{t}^{F}-\widehat{p}_{t}^{H}\right)
\end{aligned}
$$

where $\widehat{t o t}_{t} \equiv\left(\widehat{p}_{t}^{F}-\widehat{p}_{t}^{H}\right)$ is our definition of domestic terms of trade under PCP. If we put this expression together with the expression that we just derived linking the world terms of trade to the real exchange rate, then it immediately follows that,

$$
\frac{2 \phi_{H} \phi_{F}}{\phi_{H}-\phi_{F}} \widehat{r s}_{t}=\widehat{t}_{t}^{W}=2 \phi_{F} \phi_{H}\left(\widehat{p}_{t}^{F}-\widehat{p}_{t}^{H}\right)
$$

and,

$$
\widehat{r s}_{t}=\left(\phi_{H}-\phi_{F}\right)\left(\widehat{p}_{t}^{F}-\widehat{p}_{t}^{H}\right),
$$

which gives us the conventional finding under PCP implying that the real exchange rate is proportional to domestic terms of trade. Hence, if there is no home-product bias (i.e. $\phi_{H}=\phi_{F}$ ), then the real exchange rate is invariant over time.

\subsection{Other Relationships}

On Aggregate Output. We have shown in equations (132) and (133) that the aggregate output in each country can be expressed as,

$$
\begin{aligned}
\widehat{y}_{t} & \approx-\eta\left(\widehat{p}_{t}^{H, W}-\widehat{p}_{t}^{W}\right)+\left(1-\gamma_{x}\right) \widehat{c}_{t}^{W}+\gamma_{x} \widehat{x}_{t}^{W} \\
& =\eta\left(\widehat{p}_{t}^{F, W *}-\widehat{p}_{t}^{W *}\right)+\left(1-\gamma_{x}\right) \widehat{c}_{t}^{W}+\gamma_{x} \widehat{x}_{t}^{W}, \\
\widehat{y}_{t}^{*} & \approx-\eta\left(\widehat{p}_{t}^{F, W *}-\widehat{p}_{t}^{W *}\right)+\left(1-\gamma_{x}\right) \widehat{c}_{t}^{W *}+\gamma_{x} \widehat{x}_{t}^{W *},
\end{aligned}
$$

since $\left(\widehat{p}_{t}^{H, W}-\widehat{p}_{t}^{W}\right)+\left(\widehat{p}_{t}^{F, W *}-\widehat{p}_{t}^{W *}\right)=0$. Most notably, we can write both output equations as a function of world terms of trade without having to keep track of any other international relative price. Using the world terms of trade definition we can write aggregate output as,

$$
\begin{aligned}
\widehat{y}_{t} & \approx \eta \widehat{t}_{t}^{W}+\left(1-\gamma_{x}\right) \widehat{c}_{t}^{W}+\gamma_{x} \widehat{x}_{t}^{W}, \\
\widehat{y}_{t}^{*} & \approx-\eta \widehat{t}_{t}^{W}+\left(1-\gamma_{x}\right) \widehat{c}_{t}^{W *}+\gamma_{x} \widehat{x}_{t}^{W *},
\end{aligned}
$$

which holds true independently of whether the model assume LCP or PCP pricing. However, under PCP pricing we can replace the world terms of trade with a measure of domestic terms of trade or with the real exchange rate as noted before. 
This also means that world aggregate and relative output must satisfy the following conditions,

$$
\begin{aligned}
& \widehat{y}_{t}^{W} \equiv \phi_{H} \widehat{y}_{t}+\phi_{F} \widehat{y}_{t}^{*} \approx-\eta\left[\phi_{H}\left(\widehat{p}_{t}^{H, W}-\widehat{p}_{t}^{W}\right)+\phi_{F}\left(\widehat{p}_{t}^{F, W *}-\widehat{p}_{t}^{W *}\right)\right]+\ldots \\
& \quad\left(1-\gamma_{x}\right)\left[\phi_{H} \widehat{c}_{t}^{W}+\phi_{F} \widehat{c}_{t}^{W *}\right]+\gamma_{x}\left[\phi_{H} \widehat{x}_{t}^{W}+\phi_{F} \widehat{x}_{t}^{W *}\right] \\
& \quad=\eta\left(\phi_{H}-\phi_{F}\right)\left[\widehat{p}_{t}^{F, W *}-\widehat{p}_{t}^{W *}\right]+\left(1-\gamma_{x}\right)\left[\phi_{H} \widehat{c}_{t}^{W}+\phi_{F} \widehat{c}_{t}^{W *}\right]+\gamma_{x}\left[\phi_{H} \widehat{x}_{t}^{W}+\phi_{F} \widehat{x}_{t}^{W *}\right], \\
& \widehat{y}_{t}^{W *} \equiv \phi_{F} \widehat{y}_{t}+\phi_{H} \widehat{y}_{t}^{*} \approx-\eta\left[\phi_{F}\left(\widehat{p}_{t}^{H, W}-\widehat{p}_{t}^{W}\right)+\phi_{H}\left(\widehat{p}_{t}^{F, W *}-\widehat{p}_{t}^{W *}\right)\right]+\ldots \\
& \quad\left(1-\gamma_{x}\right)\left[\phi_{F} \widehat{c}_{t}^{W}+\phi_{H} \widehat{c}_{t}^{W *}\right]+\gamma_{x}\left[\phi_{F} \widehat{x}_{t}^{W}+\phi_{H} \widehat{x}_{t}^{W *}\right] \\
& \quad=-\eta\left(\phi_{H}-\phi_{F}\right)\left[\widehat{p}_{t}^{F, W *}-\widehat{p}_{t}^{W *}\right]+\left(1-\gamma_{x}\right)\left[\phi_{F} \widehat{c}_{t}^{W}+\phi_{H} \widehat{c}_{t}^{W *}\right]+\gamma_{x}\left[\phi_{F} \widehat{x}_{t}^{W}+\phi_{H} \widehat{x}_{t}^{W *}\right], \\
& \widehat{y}_{t}^{R} \equiv \widehat{y}_{t}-\widehat{y}_{t}^{*} \approx-\eta\left[\left(\widehat{p}_{t}^{H, W}-\widehat{p}_{t}^{W}\right)-\left(\widehat{p}_{t}^{F, W *}-\widehat{p}_{t}^{W *}\right)\right]+\ldots \\
& \quad\left(1-\gamma_{x}\right)\left[\widehat{c}_{t}^{W}-\widehat{c}_{t}^{W *}\right]+\gamma_{x}\left[\widehat{x}_{t}^{W}-\widehat{x}_{t}^{W *}\right] \\
& \quad=2 \eta\left[\widehat{p}_{t}^{F, W *}-\widehat{p}_{t}^{W *}\right]+\left(1-\gamma_{x}\right)\left[\widehat{c}_{t}^{W}-\widehat{c}_{t}^{W *}\right]+\gamma_{x}\left[\widehat{x}_{t}^{W}-\widehat{x}_{t}^{W *}\right] .
\end{aligned}
$$

The perfect international risk-sharing condition derived in equation (99) implies that,

$$
\widehat{c}_{t}^{W}-\widehat{c}_{t}^{W *}=\left(\phi_{H}-\phi_{F}\right)\left(\widehat{c}_{t}-\widehat{c}_{t}^{*}\right) \approx\left(\phi_{H}-\phi_{F}\right) \sigma \widehat{r} s_{t},
$$

so we can re-write relative output as,

$$
\widehat{y}_{t}^{R} \equiv \widehat{y}_{t}-\widehat{y}_{t}^{*} \approx 2 \eta\left[\widehat{p}_{t}^{F, W *}-\widehat{p}_{t}^{W *}\right]+\left(\phi_{H}-\phi_{F}\right) \sigma\left(1-\gamma_{x}\right) \widehat{r s}_{t}+\gamma_{x}\left[\widehat{x}_{t}^{W}-\widehat{x}_{t}^{W *}\right] .
$$

In a model without capital and with identical preferences (i.e., if $\phi_{H}=\phi_{F}$ ) the relative output and the implicit measure of world terms of trade in $\widehat{t}_{t}^{W} \equiv\left(\widehat{p}_{t}^{F, W *}-\widehat{p}_{t}^{W *}\right)$ are proportional. We can use the equations derived before for $\widehat{y}_{t}^{W}$ and $\widehat{y}_{t}^{W *}$ in order to substitute out consumption in the inflation dynamics equations, so we can write everything in terms of output instead of consumption. We can also replace out $\hat{t}_{t}^{W} \equiv$ $\left(\widehat{p}_{t}^{F, W *}-\widehat{p}_{t}^{W *}\right)$ using the equation for relative output.

On the Efficiency Conditions. Using the efficiency conditions in (134) and (135) and, after a little bit of algebra, it follows that the real rental rates on capital can be expressed as,

$$
\begin{aligned}
& \widehat{r}_{t}^{z} \approx\left(\frac{1}{\sigma}+\left(1-\gamma_{x}\right) \frac{1+\varphi}{\psi}\right) \widehat{c}_{t}^{W}+\gamma_{x} \frac{1+\varphi}{\psi} \widehat{x}_{t}^{W}+\phi_{F} \widehat{r s}_{t}+\eta \frac{1+\varphi}{\psi} \widehat{t}_{t}^{W}-\ldots \\
& \quad\left(\frac{1+(1-\psi) \varphi}{\psi}\right) \widehat{k}_{t}-\frac{1+\varphi}{\psi} \widehat{a}_{t}, \\
& \widehat{r}_{t}^{z *} \approx\left(\frac{1}{\sigma}+\left(1-\gamma_{x}\right) \frac{1+\varphi}{\psi}\right) \widehat{c}_{t}^{W *}+\gamma_{x} \frac{1+\varphi}{\psi} \widehat{x}_{t}^{W *}-\phi_{F} \widehat{r}_{t}-\eta \frac{1+\varphi}{\psi} \widehat{t}_{t}^{W}-\ldots \\
& \quad\left(\frac{1+(1-\psi) \varphi}{\psi}\right) \widehat{k}_{t}^{*}-\frac{1+\varphi}{\psi} \widehat{a}_{t}^{*} .
\end{aligned}
$$

This simply re-writes the previous conditions replacing the relative prices with the definition of world terms of trade. However, for the purpose of simulating the model, suffices to use the expressions derived in $(134)-(135)$ or in $(166)-(167)$. 
On Aggregate Employment. The aggregate employment can be easily derived by log-linearizing the aggregate production equations in (91) and (92) as,

$$
\begin{aligned}
\widehat{y}_{t} & \approx \widehat{a}_{t}+(1-\psi) \widehat{k}_{t}+\psi \widehat{l}_{t}, \\
\widehat{y}_{t}^{*} & \approx \widehat{a}_{t}^{*}+(1-\psi) \widehat{k}_{t}^{*}+\psi \widehat{l}_{t}^{*} .
\end{aligned}
$$

Naturally, the linear-in-labor case for employment can be derived as a special case of this log-linearized production function in which the labor share converges to one, i.e. $\psi \rightarrow 1$.

\subsubsection{On Real Exports, Real Imports, and the Net Exports Share}

In a two-country model, suffices to determine the net exports of the domestic country. Let us denote the deviation of net exports / GDP from its steady state as $\widehat{t b}_{t} \cdot{ }^{7}$ Then, because the trade balance is easily computed as the difference between domestic aggregate output and domestic aggregate consumption and investment (or domestic absorption) in real terms (see, e.g., Galí and Monacelli, 2005), we obtain that,

$$
\widehat{t b}_{t} \equiv \widehat{y}_{t}-\left(1-\gamma_{x}\right) \widehat{c}_{t}-\gamma_{x} \widehat{x}_{t}
$$

Using the formula derived above for the domestic aggregate output, we obtain the following expression,

$$
\begin{aligned}
\widehat{t b}_{t} & \approx \eta \widehat{t}_{t}^{W}+\left(1-\gamma_{x}\right)\left[\left(\phi_{H} \widehat{c}_{t}+\phi_{F} \widehat{c}_{t}^{*}\right)-\widehat{c}_{t}\right]+\gamma_{x}\left[\left(\phi_{H} \widehat{x}_{t}+\phi_{F} \widehat{x}_{t}^{*}\right)-\widehat{x}_{t}\right] \\
& =\eta \widehat{t}_{t}^{W}-\left(1-\gamma_{x}\right) \phi_{F}\left[\widehat{c}_{t}-\widehat{c}_{t}^{*}\right]-\gamma_{x} \phi_{F} \widehat{x}_{t}^{R}
\end{aligned}
$$

where $\widehat{c}_{t}^{W} \equiv \phi_{H} \widehat{c}_{t}+\phi_{F} \widehat{c}_{t}^{*}, \widehat{x}_{t}^{W} \equiv \phi_{H} \widehat{x}_{t}+\phi_{F} \widehat{x}_{t}^{*}$, and $\widehat{x}_{t}^{R} \equiv \widehat{x}_{t}-\widehat{x}_{t}^{*}$. Using the perfect international risk-sharing condition in (99), we can express the net exports share as,

$$
\widehat{t b}_{t} \approx \eta \widehat{t}_{t}^{W}-\left(1-\gamma_{x}\right) \phi_{F} \sigma \widehat{r s}_{t}-\gamma_{x} \phi_{F} \widehat{x}_{t}^{R}
$$

In other words, adjustment in the trade balance comes either through movements in the world terms of trade, $\widehat{t}_{t}^{W}$, fluctuations in the real exchange rate, $\widehat{r}_{t}$, for a given relative consumption path, or from relative adjustments in investment, $\widehat{x}_{t}^{R}$.

The real exports and imports of domestic goods in the model can be inferred from equations (49) - (52) and their foreign counterparts as follows,

$$
\begin{aligned}
E X P_{t} & \equiv \int_{0}^{1}\left[C_{t}^{*}(h)+X_{t}^{*}(h)\right] d h=\phi_{H}^{*} \int_{0}^{1}\left(\frac{P_{t}^{*}(h)}{P_{t}^{H *}}\right)^{-\theta}\left(\frac{P_{t}^{H *}}{P_{t}^{*}}\right)^{-\eta}\left[C_{t}^{*}+X_{t}^{*}\right] d h, \\
I M P_{t} & \equiv \int_{0}^{1}\left[C_{t}(f)+X_{t}(f)\right] d f=\phi_{F} \int_{0}^{1}\left(\frac{P_{t}(f)}{P_{t}^{F}}\right)^{-\theta}\left(\frac{P_{t}^{F}}{P_{t}}\right)^{-\eta}\left[C_{t}+X_{t}\right] d f,
\end{aligned}
$$

where $\phi_{H}^{*}=\phi_{F}$ under our assumption of (symmetric) home-product bias in consumption and investment.

\footnotetext{
${ }^{7}$ We use $\widehat{t b}_{t}$ instead of the more conventional $\widehat{n x}_{t}$ notation in order to avoid possible confusion with the investment variable.
} 
A simple log-linearization of both definitions allows us to obtain the following pair of equations,

$$
\begin{aligned}
& \widehat{\exp }_{t} \approx-\theta\left(\int_{0}^{1} \widehat{p}_{t}^{*}(h) d h-\widehat{p}_{t}^{H *}\right)-\eta\left(\widehat{p}_{t}^{H *}-\widehat{p}_{t}^{*}\right)+\left(1-\gamma_{x}\right) \widehat{c}_{t}^{*}+\gamma_{x} \widehat{x}_{t}^{*} \\
& \widehat{i m p}_{t} \approx-\theta\left(\int_{0}^{1} \widehat{p}_{t}(f) d h-\widehat{p}_{t}^{F}\right)-\eta\left(\widehat{p}_{t}^{F}-\widehat{p}_{t}\right)+\left(1-\gamma_{x}\right) \widehat{c}_{t}+\gamma_{x} \widehat{x}_{t} .
\end{aligned}
$$

The log-linearization of the price sub-indexes in $(13)-(14)$ clearly implies that $\int_{0}^{1} \widehat{p}_{t}^{*}(h) d h \approx \widehat{p}_{t}^{H *}$ and $\int_{0}^{1} \widehat{p}_{t}(f) d h \approx \widehat{p}_{t}^{F}$. Therefore, relative price dispersion at the variety level has no first-order effects, and we can re-write the export and import equations as,

$$
\begin{aligned}
& \widehat{\exp }_{t} \approx-\eta\left(\widehat{p}_{t}^{H *}-\widehat{p}_{t}^{*}\right)+\left(1-\gamma_{x}\right) \widehat{c}_{t}^{*}+\gamma_{x} \widehat{x}_{t}^{*} \\
& \widehat{i m p}_{t} \approx-\eta\left(\widehat{p}_{t}^{F}-\widehat{p}_{t}\right)+\left(1-\gamma_{x}\right) \widehat{c}_{t}+\gamma_{x} \widehat{x}_{t} .
\end{aligned}
$$

We have defined the world price sub-indexes as $\widehat{p}_{t}^{H, W} \equiv \phi_{H} \widehat{p}_{t}^{H}+\phi_{F} \widehat{p}_{t}^{H *}$ and $\widehat{p}_{t}^{F, W *} \equiv \phi_{F} \widehat{p}_{t}^{F}+\phi_{H} \widehat{p}_{t}^{F *}$, and the relative price sub-indexes as $\widehat{p}_{t}^{H, R} \equiv \widehat{p}_{t}^{H}-\widehat{p}_{t}^{H *}$ and $\widehat{p}_{t}^{F, R} \equiv \widehat{p}_{t}^{F}-\widehat{p}_{t}^{F *}$. Then, naturally, we can write that,

$$
\begin{aligned}
\widehat{p}_{t}^{H} & =\widehat{p}_{t}^{H, W}+\phi_{F} \widehat{p}_{t}^{H, R}, \widehat{p}_{t}^{H *}=\widehat{p}_{t}^{H, W}-\phi_{H} \widehat{p}_{t}^{H, R}, \\
\widehat{p}_{t}^{F} & =\widehat{p}_{t}^{F, W *}+\phi_{H} \widehat{p}_{t}^{F, R}, \widehat{p}_{t}^{F *}=\widehat{p}_{t}^{F, W *}-\phi_{F} \widehat{p}_{t}^{F, R} .
\end{aligned}
$$

Analogously, we have defined the world CPI as $\widehat{p}_{t}^{W} \equiv \phi_{H} \widehat{p}_{t}+\phi_{F} \widehat{p}_{t}^{*}$ and $\widehat{p}_{t}^{W *} \equiv \phi_{F} \widehat{p}_{t}+\phi_{H} \widehat{p}_{t}^{*}$, and the relative CPI as $\widehat{p}_{t}^{R} \equiv \widehat{p}_{t}-\widehat{p}_{t}^{*}$. Then, we can write that,

$$
\begin{aligned}
& \widehat{p}_{t}=\widehat{p}_{t}^{W}+\phi_{F} \widehat{p}_{t}^{R}, \widehat{p}_{t}^{*}=\widehat{p}_{t}^{W}-\phi_{H} \widehat{p}_{t}^{R} \\
& \widehat{p}_{t}=\widehat{p}_{t}^{W *}+\phi_{H} \widehat{p}_{t}^{R}, \widehat{p}_{t}^{*}=\widehat{p}_{t}^{W *}-\phi_{F} \widehat{p}_{t}^{R}
\end{aligned}
$$

Using these definitions, it is possible to express the relative prices embedded in the definition of real exports and imports in the following terms, i.e.

$$
\begin{aligned}
\widehat{p}_{t}^{H *}-\widehat{p}_{t}^{*} & =\left(\widehat{p}_{t}^{H, W}-\phi_{H} \widehat{p}_{t}^{H, R}\right)-\left(\widehat{p}_{t}^{W}-\phi_{H} \widehat{p}_{t}^{R}\right) \\
& =\widehat{p}_{t}^{H, W}-\widehat{p}_{t}^{W}-\phi_{H}\left(\widehat{p}_{t}^{H, R}-\widehat{p}_{t}^{R}\right), \\
\widehat{p}_{t}^{F}-\widehat{p}_{t} & =\widehat{p}_{t}^{F, W *}+\phi_{H} \widehat{p}_{t}^{F, R}-\left(\widehat{p}_{t}^{W *}+\phi_{H} \widehat{p}_{t}^{R}\right) \\
& =\widehat{p}_{t}^{F, W *}-\widehat{p}_{t}^{W *}+\phi_{H}\left(\widehat{p}_{t}^{F, R}-\widehat{p}_{t}^{R}\right),
\end{aligned}
$$

where the world terms of trade is defined as $\widehat{t}_{t}^{W} \equiv \widehat{p}_{t}^{F, W *}-\widehat{p}_{t}^{W *}$. The definition of CPI in both countries, i.e. $\widehat{p}_{t} \approx \phi_{H} \widehat{p}_{t}^{H}+\phi_{F} \widehat{p}_{t}^{F}$ and $\widehat{p}_{t}^{*} \approx \phi_{F} \widehat{p}_{t}^{H *}+\phi_{H} \widehat{p}_{t}^{F *}$, can be re-written as,

$$
\begin{aligned}
\phi_{H}\left[\widehat{p}_{t}^{H}-\widehat{p}_{t}\right]+\phi_{F}\left[\widehat{p}_{t}^{F}-\widehat{p}_{t}\right] & \approx 0, \\
\phi_{F}\left[\widehat{p}_{t}^{H *}-\widehat{p}_{t}^{*}\right]+\phi_{H}\left[\widehat{p}_{t}^{F *}-\widehat{p}_{t}^{*}\right] & \approx 0 .
\end{aligned}
$$

Then, based on the relationships described before, we can further re-write the definitions of the CPI indexes 
as,

$$
\begin{aligned}
& \phi_{H}\left[\left(\widehat{p}_{t}^{H, W}-\widehat{p}_{t}^{W}\right)+\phi_{F}\left(\widehat{p}_{t}^{H, R}-\widehat{p}_{t}^{R}\right)\right]+\phi_{F}\left[\left(\widehat{p}_{t}^{F, W *}-\widehat{p}_{t}^{W *}\right)+\phi_{H}\left(\widehat{p}_{t}^{F, R}-\widehat{p}_{t}^{R}\right)\right] \approx 0, \\
& \phi_{F}\left[\left(\widehat{p}_{t}^{H, W}-\widehat{p}_{t}^{W}\right)-\phi_{H}\left(\widehat{p}_{t}^{H, R}-\widehat{p}_{t}^{R}\right)\right]+\phi_{H}\left[\left(\widehat{p}_{t}^{F, W *}-\widehat{p}_{t}^{W *}\right)-\phi_{F}\left(\widehat{p}_{t}^{F, R}-\widehat{p}_{t}^{R}\right)\right] \approx 0 .
\end{aligned}
$$

Using the second equality derived above and the definition of the world terms of trade, we can write the relative prices embedded in the definition of real exports and imports in the following terms, i.e.

$$
\begin{aligned}
\widehat{p}_{t}^{H *}-\widehat{p}_{t}^{*} & \approx-\frac{\phi_{H}}{\phi_{F}}\left[\widehat{t}_{t}^{W}-\phi_{F}\left(\widehat{p}_{t}^{F, R}-\widehat{p}_{t}^{R}\right)\right] \\
\widehat{p}_{t}^{F}-\widehat{p}_{t} & \approx \widehat{t}_{t}^{W}+\phi_{H}\left(\widehat{p}_{t}^{F, R}-\widehat{p}_{t}^{R}\right)
\end{aligned}
$$

and,

$$
\begin{aligned}
\phi_{F}\left(\widehat{p}_{t}^{H *}-\widehat{p}_{t}^{*}\right) & \approx-\phi_{H}\left[\widehat{t}_{t}^{W}-\phi_{F}\left(\widehat{p}_{t}^{F, R}-\widehat{p}_{t}^{R}\right)\right], \\
\phi_{F}\left(\widehat{p}_{t}^{F}-\widehat{p}_{t}\right) & \approx \phi_{F}\left[\widehat{t}_{t}^{W}+\phi_{H}\left(\widehat{p}_{t}^{F, R}-\widehat{p}_{t}^{R}\right)\right] .
\end{aligned}
$$

Under these conditions it naturally follows that,

$$
\begin{aligned}
\phi_{F}\left[\left(\widehat{p}_{t}^{H *}-\widehat{p}_{t}^{*}\right)-\left(\widehat{p}_{t}^{F}-\widehat{p}_{t}\right)\right] & \approx-\phi_{H}\left[\widehat{t}_{t}^{W}-\phi_{F}\left(\widehat{p}_{t}^{F, R}-\widehat{p}_{t}^{R}\right)\right]-\phi_{F}\left[\widehat{t}_{t}^{W}+\phi_{H}\left(\widehat{p}_{t}^{F, R}-\widehat{p}_{t}^{R}\right)\right] \\
& =-\left(\phi_{H}+\phi_{F}\right) \widehat{t}_{t}^{W}=-\widehat{t}_{t}^{W}
\end{aligned}
$$

Therefore, we can compute the real trade balance in this model straight from the definitions of real exports and imports as,

$$
\begin{aligned}
\phi_{F}\left(\widehat{\exp }_{t}-\widehat{i m p}_{t}\right) & \approx-\eta \phi_{F}\left(\widehat{p}_{t}^{H *}-\widehat{p}_{t}^{*}\right)+\phi_{F}\left[\left(1-\gamma_{x}\right) \widehat{c}_{t}^{*}+\gamma_{x} \widehat{x}_{t}^{*}\right]+\eta \phi_{F}\left(\widehat{p}_{t}^{F}-\widehat{p}_{t}\right)-\phi_{F}\left[\left(1-\gamma_{x}\right) \widehat{c}_{t}+\gamma_{x} \widehat{x}_{t}\right] \\
& =-\eta \phi_{F}\left[\left(\widehat{p}_{t}^{H *}-\widehat{p}_{t}^{*}\right)-\left(\widehat{p}_{t}^{F}-\widehat{p}_{t}\right)\right]+\phi_{F}\left[\left(1-\gamma_{x}\right)\left(\widehat{c}_{t}^{*}-\widehat{c}_{t}\right)+\gamma_{x}\left(\widehat{x}_{t}^{*}-\widehat{x}_{t}\right)\right] \\
& =\eta \widehat{t}_{t}^{W}-\left(1-\gamma_{x}\right) \phi_{F}\left[\widehat{c}_{t}-\widehat{c}_{t}^{*}\right]-\gamma_{x} \phi_{F} \widehat{x}_{t}^{R} \approx \widehat{t b}_{t}
\end{aligned}
$$

where relative investment is defined as $\widehat{x}_{t}^{R} \equiv \widehat{x}_{t}-\widehat{x}_{t}^{*}$. In other words, our measure of the trade balance is equivalent to the difference between the $\log$ of real exports and imports (in deviations relative to their respective steady states), scaled by the parameter $\phi_{F}$. In the deterministic steady state of our model, it follows easily that the parameter $\phi_{F}$ denotes the share of domestic imports (and foreign exports) for consumption and investment purposes relative to aggregate output.

Real exports and real imports can be re-written in the following form,

$$
\begin{aligned}
& \widehat{\exp }_{t} \approx \eta \frac{\phi_{H}}{\phi_{F}} \widehat{t}_{t}^{W}-\eta \phi_{H}\left(\widehat{p}_{t}^{F, R}-\widehat{p}_{t}^{R}\right)+\left(1-\gamma_{x}\right) \widehat{c}_{t}^{*}+\gamma_{x} \widehat{x}_{t}^{*} \\
& \widehat{i m p}_{t} \approx-\eta \widehat{t}_{t}^{W}-\eta \phi_{H}\left(\widehat{p}_{t}^{F, R}-\widehat{p}_{t}^{R}\right)+\left(1-\gamma_{x}\right) \widehat{c}_{t}+\gamma_{x} \widehat{x}_{t}
\end{aligned}
$$


or, simply, as,

$$
\begin{aligned}
& \widehat{\exp }_{t} \approx \eta \phi_{H}\left[\frac{1}{\phi_{F}} \widehat{t}_{t}^{W}-\left(\widehat{p}_{t}^{F, R}-\widehat{p}_{t}^{R}\right)\right]+\left(1-\gamma_{x}\right) \widehat{c}_{t}^{*}+\gamma_{x} \widehat{x}_{t}^{*} \\
& \widehat{i m p}_{t} \approx-\eta \phi_{H}\left[\frac{1}{\phi_{H}} \widehat{t}_{t}^{W}+\left(\widehat{p}_{t}^{F, R}-\widehat{p}_{t}^{R}\right)\right]+\left(1-\gamma_{x}\right) \widehat{c}_{t}+\gamma_{x} \widehat{x}_{t} .
\end{aligned}
$$

Therefore, we can use equation $(163)$ - which relates $\left(\widehat{p}_{t}^{F, R}-\widehat{p}_{t}^{R}\right)$ to the world terms of trade $\widehat{t}_{t}^{W}$-in order to express the real imports and real exports more compactly as follows,

$$
\begin{aligned}
& \widehat{\exp }_{t} \approx \eta \phi_{H}\left[\frac{1}{\phi_{F}}-\frac{\left(\phi_{H}-\phi_{F}\right)}{2 \phi_{H} \phi_{F}}\right] \widehat{t}_{t}^{W}+\left(1-\gamma_{x}\right) \widehat{c}_{t}^{*}+\gamma_{x} \widehat{x}_{t}^{*} \\
& \widehat{i m p}_{t} \approx-\eta \phi_{H}\left[\frac{1}{\phi_{H}}+\frac{\left(\phi_{H}-\phi_{F}\right)}{2 \phi_{H} \phi_{F}}\right] \widehat{t}_{t}^{W}+\left(1-\gamma_{x}\right) \widehat{c}_{t}+\gamma_{x} \widehat{x}_{t} .
\end{aligned}
$$

or,

$$
\begin{aligned}
\widehat{\exp }_{t} & \approx \eta\left[\frac{2 \phi_{H}-\left(\phi_{H}-\phi_{F}\right)}{2 \phi_{F}}\right] \widehat{t}_{t}^{W}+\left(1-\gamma_{x}\right) \widehat{c}_{t}^{*}+\gamma_{x} \widehat{x}_{t}^{*} \\
& \approx \eta\left(\frac{1}{2 \phi_{F}}\right) \widehat{t}_{t}^{W}+\left(1-\gamma_{x}\right) \widehat{c}_{t}^{*}+\gamma_{x} \widehat{x}_{t}^{*} \\
\widehat{i m p}_{t} & \approx-\eta\left[\frac{2 \phi_{F}+\left(\phi_{H}-\phi_{F}\right)}{2 \phi_{F}}\right] \widehat{t}_{t}^{W}+\left(1-\gamma_{x}\right) \widehat{c}_{t}+\gamma_{x} \widehat{x}_{t} \\
& \approx-\eta\left(\frac{1}{2 \phi_{F}}\right) \widehat{t}_{t}^{W}+\left(1-\gamma_{x}\right) \widehat{c}_{t}+\gamma_{x} \widehat{x}_{t} .
\end{aligned}
$$

These two equations tell us that the strength of the demand for consumption and investment purposes is likely to have a major impact on both exports and imports. However, they also tell us that exports and imports depend on world terms of trade, $\widehat{t}_{t}^{W}$, which is the sole variable that summarizes the impact of international relative prices on both real exports and real imports in the context of our model.

The world terms of trade, $\widehat{t}_{t}^{W}$, can be expressed in terms of the relative price of each country as follows,

$$
\begin{aligned}
\widehat{t}_{t}^{W} & \equiv \widehat{p}_{t}^{F, W *}-\widehat{p}_{t}^{W *}=\phi_{F} \widehat{p}_{t}^{F}+\phi_{H} \widehat{p}_{t}^{F *}-\phi_{F} \widehat{p}_{t}-\phi_{H} \widehat{p}_{t}^{*} \\
& =\phi_{F}\left(\widehat{p}_{t}^{F}-\widehat{p}_{t}\right)+\phi_{H}\left(\widehat{p}_{t}^{F *}-\widehat{p}_{t}^{*}\right) \\
& \approx \phi_{F}\left(\widehat{p}_{t}^{F}-\phi_{H} \widehat{p}_{t}^{H}-\phi_{F} \widehat{p}_{t}^{F}\right)+\phi_{H}\left(\widehat{p}_{t}^{F *}-\phi_{F} \widehat{p}_{t}^{H *}-\phi_{H} \widehat{p}_{t}^{F *}\right) \\
& =\phi_{F} \phi_{H}\left(\widehat{p}_{t}^{F}-\widehat{p}_{t}^{H}\right)+\phi_{F} \phi_{H}\left(\widehat{p}_{t}^{F *}-\widehat{p}_{t}^{H *}\right) \\
& =\phi_{F} \phi_{H}\left[\left(\widehat{p}_{t}^{F}-\widehat{p}_{t}^{H}\right)+\left(\widehat{p}_{t}^{F *}-\widehat{p}_{t}^{H *}\right)\right]
\end{aligned}
$$

while using the definition of the CPI for both countries, i.e. $\widehat{p}_{t} \approx \phi_{H} \widehat{p}_{t}^{H}+\phi_{F} \widehat{p}_{t}^{F}$ and $\widehat{p}_{t}^{*} \approx \phi_{F} \widehat{p}_{t}^{H *}+\phi_{H} \widehat{p}_{t}^{F *}$. 
We can re-write the real exchange rate, $\widehat{r}_{t}$, as,

$$
\begin{aligned}
\widehat{r s}_{t} & =\widehat{s}_{t}+\widehat{p}_{t}^{*}-\widehat{p}_{t} \\
& \approx \widehat{s}_{t}+\left(\phi_{F} \widehat{p}_{t}^{H *}+\phi_{H} \widehat{p}_{t}^{F *}\right)-\left(\phi_{H} \widehat{p}_{t}^{H}+\phi_{F} \widehat{p}_{t}^{F}\right) \\
& =\widehat{s}_{t}+\left(\left(1-\phi_{H}\right) \widehat{p}_{t}^{H *}+\phi_{H} \widehat{p}_{t}^{F *}\right)-\left(\phi_{H} \widehat{p}_{t}^{H}+\left(1-\phi_{H}\right) \widehat{p}_{t}^{F}\right) \\
& =\left(\widehat{s}_{t}+\widehat{p}_{t}^{H *}-\widehat{p}_{t}^{F}\right)+\phi_{H}\left[\left(\widehat{p}_{t}^{F}-\widehat{p}_{t}^{H}\right)+\left(\widehat{p}_{t}^{F *}-\widehat{p}_{t}^{H *}\right)\right] .
\end{aligned}
$$

The international relative price effect on trade can be partly captured by the cost of replacing one unit of the foreign good with one unit of the exported domestic good, i.e. it is partly a function of the domestic terms of trade $\widehat{t o t}_{t} \equiv\left(\widehat{p}_{t}^{F}-\widehat{s}_{t}-\widehat{p}_{t}^{H *}\right)$. If the law of one price holds (as it is the case under PCP pricing), then we can express domestic terms of trade as the opportunity cost of replacing one unit of the foreign good with one unit of the domestic good sold locally, $\widehat{t o t}_{t} \equiv\left(\widehat{p}_{t}^{F}-\widehat{p}_{t}^{H}\right)$, since $\widehat{p}_{t}^{H} \approx \widehat{s}_{t}+\widehat{p}_{t}^{H *}$. However, in the LCP pricing case we have to use the definition of world terms of trade, $\widehat{t}_{t}^{W}$, to re-write the real exchange rate, $\widehat{r s}_{t}$, as a function of domestic and world terms of trade as follows,

$$
\widehat{r s}_{t} \approx-\widehat{t o t}_{t}+\left(\frac{1}{\phi_{F}}\right) \widehat{t}_{t}^{W}
$$

We easily see that world terms of trade, $\widehat{t}_{t}^{W}$, can be expressed now as a function of the domestic terms of trade, $\widehat{t o t}_{t} \equiv\left(\widehat{p}_{t}^{F}-\widehat{s}_{t}-\widehat{p}_{t}^{H *}\right)$, and the real exchange rate, $\widehat{r}_{t}$, as,

$$
\widehat{t}_{t}^{W} \approx \phi_{F}\left(\widehat{t o t}_{t}+\widehat{r s}_{t}\right)
$$

The advantage of this transformation is that the world terms of trade can be expressed as a linear function of domestic terms of trade and the real exchange rate which are both measurable in the data-unlike world terms of trade itself. Hence, the trade balance, the real export and the real import equations can all be re-expressed in terms of international relative prices that are easier to match with the data even in the presence of nominal rigidities and LCP pricing (that is, even when the law of one price does not hold).

\subsubsection{On Real Exports and Real Imports: The PCP Case}

The real exports and imports of domestic goods in the model can be inferred from equations (49) - (52) and their foreign counterparts as follows,

$$
\begin{aligned}
E X P_{t} & \equiv \int_{0}^{1}\left[C_{t}^{*}(h)+X_{t}^{*}(h)\right] d h=\phi_{H}^{*} \int_{0}^{1}\left(\frac{P_{t}^{*}(h)}{P_{t}^{H *}}\right)^{-\theta}\left(\frac{P_{t}^{H *}}{P_{t}^{*}}\right)^{-\eta}\left[C_{t}^{*}+X_{t}^{*}\right] d h, \\
I M P_{t} & \equiv \int_{0}^{1}\left[C_{t}(f)+X_{t}(f)\right] d f=\phi_{F} \int_{0}^{1}\left(\frac{P_{t}(f)}{P_{t}^{F}}\right)^{-\theta}\left(\frac{P_{t}^{F}}{P_{t}}\right)^{-\eta}\left[C_{t}+X_{t}\right] d f,
\end{aligned}
$$

where $\phi_{H}^{*}=\phi_{F}$ under our (symmetric) assumption of home-product bias in consumption and investment. In a two-country model, the real exports of the home country are clearly equal to real imports of the foreign country. Similarly, the real imports of the home country are equal to the real exports of the foreign country. From the background on equations (185) - (186), we know that real exports and imports can be log-linearized 
as,

$$
\begin{aligned}
& \widehat{\exp }_{t} \approx-\eta\left(\widehat{p}_{t}^{H *}-\widehat{p}_{t}^{*}\right)+\left(1-\gamma_{x}\right) \widehat{c}_{t}^{*}+\gamma_{x} \widehat{x}_{t}^{*} \\
& \widehat{i m p}_{t} \approx-\eta\left(\widehat{p}_{t}^{F}-\widehat{p}_{t}\right)+\left(1-\gamma_{x}\right) \widehat{c}_{t}+\gamma_{x} \widehat{x}_{t} .
\end{aligned}
$$

It is evident that this approximation follows from the description of real export and real import functions presented before.

The definition of CPI in both countries, i.e. $\widehat{p}_{t} \approx \phi_{H} \widehat{p}_{t}^{H}+\phi_{F} \widehat{p}_{t}^{F}$ and $\widehat{p}_{t}^{*} \approx \phi_{F} \widehat{p}_{t}^{H *}+\phi_{H} \widehat{p}_{t}^{F *}$, allows us to express these two linearized equations also as,

$$
\begin{aligned}
\widehat{\exp }_{t} & \approx-\eta\left(\left(1-\phi_{F}\right) \widehat{p}_{t}^{H *}-\phi_{H} \widehat{p}_{t}^{F *}\right)+\left(1-\gamma_{x}\right) \widehat{c}_{t}^{*}+\gamma_{x} \widehat{x}_{t}^{*} \\
& =-\eta \phi_{H}\left(\widehat{p}_{t}^{H *}-\widehat{p}_{t}^{F *}\right)+\left(1-\gamma_{x}\right) \widehat{c}_{t}^{*}+\gamma_{x} \widehat{x}_{t}^{*} \\
\widehat{i m p}_{t} & \approx-\eta\left(\left(1-\phi_{F}\right) \widehat{p}_{t}^{F}-\phi_{H} \widehat{p}_{t}^{H}\right)+\left(1-\gamma_{x}\right) \widehat{c}_{t}+\gamma_{x} \widehat{x}_{t} \\
& =-\eta \phi_{H}\left(\widehat{p}_{t}^{F}-\widehat{p}_{t}^{H}\right)+\left(1-\gamma_{x}\right) \widehat{c}_{t}+\gamma_{x} \widehat{x}_{t} .
\end{aligned}
$$

In a model under PCP where the law of one price holds we know that,

$$
\begin{aligned}
& \widehat{p}_{t}^{H} \approx \widehat{s}_{t}+\widehat{p}_{t}^{H *}, \\
& \widehat{p}_{t}^{F} \approx \widehat{s}_{t}+\widehat{p}_{t}^{F *},
\end{aligned}
$$

which in turn allows us to re-write the expressions for exports and imports in the following terms,

$$
\begin{aligned}
\widehat{\exp }_{t} & \approx-\eta \phi_{H}\left(\left(\widehat{s}_{t}+\widehat{p}_{t}^{H *}\right)-\left(\widehat{s}_{t}+\widehat{p}_{t}^{F *}\right)\right)+\left(1-\gamma_{x}\right) \widehat{c}_{t}^{*}+\gamma_{x} \widehat{x}_{t}^{*} \\
& =\eta \phi_{H}\left(\widehat{p}_{t}^{F}-\widehat{p}_{t}^{H}\right)+\left(1-\gamma_{x}\right) \widehat{c}_{t}^{*}+\gamma_{x} \widehat{x}_{t}^{*} \\
\widehat{i m p}_{t} & \approx-\eta \phi_{H}\left(\widehat{p}_{t}^{F}-\widehat{p}_{t}^{H}\right)+\left(1-\gamma_{x}\right) \widehat{c}_{t}+\gamma_{x} \widehat{x}_{t} .
\end{aligned}
$$

If we simplify equations (189) - (190) replacing world terms of trade by its equivalence under PCP, i.e. $\widehat{t}_{t}^{W}=2 \phi_{F} \phi_{H}\left(\widehat{p}_{t}^{F}-\widehat{p}_{t}^{H}\right.$ ) (see for a demonstration equation (181)), then we obtain exactly the same real export and real import equations we have derived here.

The equations for real exports and real imports reflect two well-known forces on the demand-side, the international relative price effects and the income / wealth effects. The income effect is captured by the overall demand for consumption and investment (the absorption), respectively $\left(1-\gamma_{x}\right) \widehat{c}_{t}+\gamma_{x} \widehat{x}_{t}$ and $\left(1-\gamma_{x}\right) \widehat{c}_{t}^{*}+$ $\gamma_{x} \widehat{x}_{t}^{*}$ in each country. The international relative price effect is a function of the cost of replacing one unit of the foreign good with one unit of the domestic good, i.e. it is a function of the domestic terms of trade $\widehat{t o t}_{t} \equiv\left(\widehat{p}_{t}^{F}-\widehat{p}_{t}^{H}\right)$. The impact of domestic terms of trade is symmetric in size but enters with opposite sign for imports and exports. Alternatively, we can express real imports and real exports as follows,

$$
\begin{aligned}
& \widehat{\exp }_{t} \approx \eta\left(\frac{\phi_{H}}{\phi_{H}-\phi_{F}}\right) \widehat{r s}_{t}+\left(1-\gamma_{x}\right) \widehat{c}_{t}^{*}+\gamma_{x} \widehat{x}_{t}^{*}, \\
& \widehat{i m p}_{t} \approx-\eta\left(\frac{\phi_{H}}{\phi_{H}-\phi_{F}}\right) \widehat{r s}_{t}+\left(1-\gamma_{x}\right) \widehat{c}_{t}+\gamma_{x} \widehat{x}_{t},
\end{aligned}
$$

since we already know that the real exchange rate is proportional to domestic terms of trade in the PCP 
case. It is easy to see that the real net exports share can then be expressed as,

$$
\begin{aligned}
\widehat{t b}_{t} & \approx \phi_{F}\left(\widehat{\exp }_{t}-\widehat{i m p_{t}}\right) \approx \eta\left(\frac{2 \phi_{H} \phi_{F}}{\phi_{H}-\phi_{F}}\right) \widehat{r s}_{t}+\phi_{F}\left(\left(1-\gamma_{x}\right) \widehat{c}_{t}^{*}+\gamma_{x} \widehat{x}_{t}^{*}\right)-\phi_{F}\left(\left(1-\gamma_{x}\right) \widehat{c}_{t}+\gamma_{x} \widehat{x}_{t}\right) \\
& =\eta\left(\frac{2 \phi_{H} \phi_{F}}{\phi_{H}-\phi_{F}}\right) \widehat{r s}_{t}-\left(1-\gamma_{x}\right) \phi_{F}\left(\widehat{c}_{t}-\widehat{c}_{t}^{*}\right)-\gamma_{x} \phi_{F} \widehat{x}_{t}^{R} \\
& \approx \eta\left(\frac{2 \phi_{H} \phi_{F}}{\phi_{H}-\phi_{F}}\right) \widehat{r s}_{t}-\left(1-\gamma_{x}\right) \phi_{F} \sigma \widehat{r s}_{t}-\gamma_{x} \phi_{F} \widehat{x}_{t}^{R}
\end{aligned}
$$

where $\widehat{x}_{t}^{R} \equiv \widehat{x}_{t}-\widehat{x}_{t}^{*}$ and the last equality follows from the perfect international risk-sharing condition in (99). This is the standard expression for the trade balance under PCP pricing.

Naturally, if these export and import equations are embedded in an otherwise symmetric model, we can expect exports and imports to have a negative correlation if the international relative price effect is strong but to display otherwise symmetric business cycle properties (i.e., volatility and even persistence).

\section{An Extension: The Role of Capacity Utilization}

For this extension, we follow the definition of variable capacity utilization as presented in Christiano, et al. (2005).

\subsection{The Structure of the Model}

\subsubsection{The Intertemporal Consumption and Savings Problem}

We assume that both countries have unrestricted access to a complete set of contingent claims, traded internationally. The domestic household maximizes its lifetime utility in (1) subject to the sequence of budget constraints described by,

$$
P_{t}\left(C_{t}+X_{t}+A\left(U_{t}\right) \widetilde{K}_{t}\right)+T_{t}+\int P^{b}\left(s^{t+1} \mid s^{t}\right) B\left(s^{t+1}\right) d s_{t+1} \leq B\left(s^{t}\right)+W_{t} L_{t}+Z_{t} U_{t} \widetilde{K}_{t}+P r_{t}
$$

and the law of motion for physical capital,

$$
\widetilde{K}_{t+1} \leq(1-\delta) \widetilde{K}_{t}+V_{t} \Phi\left(X_{t}, X_{t-1}, K_{t}\right) X_{t}
$$

where $W_{t}$ is the domestic nominal wage, $P_{t}$ is the domestic CPI, $P r_{t}$ are the nominal profits generated by the domestic firms, and $T_{t}$ is a lump-sum nominal tax levied on the domestic households. Moreover, $X_{t}$ is domestic real investment, $\widetilde{K}_{t}$ stands for domestic physical capital, $Z_{t}$ defines the nominal rental rate on capital services, $P r_{t}$ are the nominal profits generated by the domestic firms, and $V_{t}$ is the domestic investment-specific technological (IST) shock. Money can be thought as playing the role of a unit of account only.

We denote $s_{t+1}$ the event that occurs at time $t+1$ and $s^{t+1}=\left(s^{t}, s_{t+1}\right)$ the history of events up to that point. The households' portfolio includes a complete set of one-period contingent claims (Arrow-Debreu securities), traded internationally and quoted in units of the domestic currency. Households have unrestricted access to all contingent claims, $P^{b}\left(s^{t} \mid s^{t-1}\right)$ is the domestic price at time $t-1$ of the contingent claim that 
pays off after $s_{t}$ is realized at time $t$, and $\frac{P^{b}\left(s^{t} \mid s^{t-1}\right)}{S_{t}}$ is the corresponding price in foreign currency units. $S_{t}$ denotes the nominal exchange rate, $B\left(s^{t}\right)$ is the nominal pay-off received by the domestic households after the event $s_{t}$ occurs at time $t$ on a contingent claim purchased at time $t-1$ (which would have paid nothing if event $s_{t}$ had not happened).

Capital services, $K_{t}$, are related to physical capital, $\widetilde{K}_{t}$, by,

$$
K_{t}=U_{t} \widetilde{K}_{t}
$$

Here, $U_{t}$ denotes the utilization rate of physical capital, which we assume is set by the households. $Z_{t} U_{t} \widetilde{K}$ represents the domestic households' earnings from supplying capital services. The increasing, convex func-

tion, $A\left(U_{t}\right) \widetilde{K}_{t}$, denotes the cost, in units of consumption goods, of setting the utilization rate $U_{t}$ above its long-run steady state of one (over-utilization). The cost is scaled by the size of the stock of physical capital. In turn, whenever the utilization rate is set below one (under-utilization), the function now indicates the units of consumption goods that the household can re-allocate for other purposes like consumption, investment, or net borrowing. The foreign households maximize their lifetime utility subject to an analogous sequence of budget constraints, the same law of motion for physical capital, and the same linear relationship between physical capital and capital services.

Physical capital accumulation may be subject to adjustment costs too. We consider three special cases: the capital adjustment cost (CAC) case in (5), the investment adjustment cost (IAC) in (6), and the case with no adjustment costs (NAC). We define capital adjustment costs in terms of capital services rather than physical capital because we want to capture the idea that setting utilization rates can also influence how costly it becomes to accumulate physical capital. However, this distinction does not matter under IAC adjustment costs. All other assumptions of the model are maintained in this extension.

The home and foreign consumption bundles of the domestic household, $C_{t}^{H}$ and $C_{t}^{F}$, as well as the investment bundles, $X_{t}^{H}$ and $X_{t}^{F}$, are aggregated by means of the CES indexes in (7) - (8), while aggregate consumption and investment, $C_{t}$ and $X_{t}$, are defined with the CES indexes in (9) - (10). Under standard results on functional separability, the CPI indexes which correspond to our specification of aggregators for consumption and investment are (11) - (12), and the price sub-indexes are (13) - (14). An analogous set of consumption and investment aggregators for the foreign household and price indexes and sub-indexes for the foreign market apply. We still define the real exchange rate as in (15).

\subsubsection{The Firms' Problem and Monetary Policy}

Neither the problem of the firms' nor the simple monetary policy rules proposed by Taylor (1993) do change in this environment with variable capital utilization. The only point that is worth emphasizing is that firms in this model rent capital services rather than physical capital. Therefore, the capital utilization rate set by the households and the physical capital they accumulate will have an impact on the firms' marginal costs by influencing the overall amount of capital services supplied in equilibrium. Everything else is unchanged.

We assume that production employs a (homogeneous of degree one) Cobb-Douglas technology as in (16) - (17). Solving the cost-minimization problem of each individual firm yields an efficiency condition linking the capital-services-to-labor ratios to factor price ratios in each country as in (18) - (19), as well as a characterization for the (pre-subsidy) marginal costs as in (20) - (21). The government subsidizes firms as 
in (22) - (23), and each country fully finances its subsidies with a lump-sum tax on households as specified in the government budget constraints in (44) - (45). The firm subsidy is time-invariant and common across countries as in (48).

A re-optimizing domestic firm $h$ under LCP chooses a domestic and a foreign price, $\widetilde{P}_{t}(h)$ and $\widetilde{P}_{t}^{*}(h)$, to maximize the expected discounted value of its net profits in (26), subject to a pair of demand constraints in each goods market in $(27)-(28)$. Similarly, we characterize the objective of the foreign firm $f$ under LCP pricing as in (29), subject to the demand constraints in (30) - (31). A re-optimizing domestic firm $h$

under PCP chooses a price in domestic currency, $\widetilde{P}_{t}(h)$, to maximize the expected discounted value of its net profits in (32), subject to a joint demand constraint for both goods market as in (36). Similarly, we characterize the objective of the foreign firm $f$ under PCP pricing as in (37), subject to the joint demand constraint of the foreign firm in (41). Naturally, the law of one price still holds under PCP.

The Taylor rule is often defined as the trademark of modern monetary policy. We assume that the monetary authorities set short-term nominal interest rates according to Taylor (1993) type rules as in (42) (43).

\subsection{The Optimality Conditions}

Here, we present the relevant equilibrium conditions of the model only when they differ from those reported before.

The Optimality Conditions from the Households' Problem. Given the structure described in (7) (8), the solution to the sub-utility maximization problem implies that the home and foreign households' demands for each variety are given by $(49)$ - (50), while the demands for the bundles of home and foreign goods are given by $(51)-(52)$. Under complete international asset markets, the intertemporal first-order conditions for an interior solution result in the well-known perfect international risk-sharing condition derived in (53).

Let $I_{t}$ be the (gross) one-period riskless nominal interest rate in terms of the domestic currency, and $I_{t}^{*}$ be the corresponding interest rate in terms of the foreign currency. Under complete international asset markets, we can price a one-period nominal (uncontingent) bond using the price of the contingent claims available and obtain that,

$$
\begin{aligned}
& \frac{1}{I_{t}}=\beta \mathbb{E}_{t}\left[\left(\frac{C_{t+1}}{C_{t}}\right)^{-\sigma^{-1}} \frac{P_{t}}{P_{t+1}}\right], \\
& \frac{1}{I_{t}^{*}}=\beta \mathbb{E}_{t}\left[\left(\frac{C_{t+1}^{*}}{C_{t}^{*}}\right)^{-\sigma^{-1}} \frac{P_{t}^{*}}{P_{t+1}^{*}}\right],
\end{aligned}
$$

which are exactly the same Euler equations that we derived in (54) - (55). The equilibrium conditions of the households' problem also include a pair of labor supply functions (the intratemporal first-order conditions) which can be expressed as in (56) - (57), plus the appropriate no-Ponzi games, transversality conditions, the budget constraints, the laws of motion for physical capital in both countries, and the linear transformation functions between physical capital and capital services. Finally, the equilibrium conditions are completed with a number of equations that account for the capital-investment decisions of households. The capital- 
investment equilibrium conditions, however, depend on the choice of the adjustment cost function $\Phi(\cdot)$.

The domestic households' maximization problem can be summarized generically in the following terms,

$$
\sum_{\tau=0}^{+\infty} \beta^{\tau}\left[\mathbb{E}_{t}\left[\begin{array}{c}
\frac{1}{1-\sigma^{-1}}\left(C_{t+\tau}\right)^{1-\sigma^{-1}}-\frac{1}{1+\varphi}\left(L_{t+\tau}\right)^{1+\varphi}-\ldots \\
\left.\lambda_{t+\tau}\left[\begin{array}{c}
P_{t+\tau}\left(C_{t+\tau}+X_{t+\tau}+A\left(U_{t+\tau}\right) \widetilde{K}_{t+\tau}\right)+T_{t+\tau}+\mathbb{E}_{t}\left[M_{t+\tau, t+\tau+1} B_{t+\tau+1}\right]-\ldots \\
B_{t+\tau}-W_{t+\tau} L_{t+\tau}-Z_{t+\tau} U_{t+\tau} \widetilde{K}_{t+\tau} \\
\lambda_{t+\tau} \Lambda_{t+\tau}\left[\widetilde{K}_{t+\tau+1}-(1-\delta) \widetilde{K}_{t+\tau}-V_{t+\tau} \Phi\left(X_{t+\tau}, X_{t+\tau-1}, U_{t+\tau} \widetilde{K}_{t+\tau}\right)\right.
\end{array}\right] X_{t+\tau}\right]
\end{array}\right]\right]
$$

and with the following set of equilibrium conditions,

$$
\begin{aligned}
& C_{t} \quad: \quad \lambda_{t} P_{t}=\left(C_{t}\right)^{-\sigma^{-1}} \text {, } \\
& \widetilde{K}_{t+1}: \Lambda_{t}=\beta \mathbb{E}_{t}\left[\begin{array}{c}
\frac{\lambda_{t+1}}{\lambda_{t}}\left(Z_{t+1} U_{t+1}-P_{t+1} A\left(U_{t+1}\right)\right)+\ldots \\
\frac{\lambda_{t+1}}{\lambda_{t}} \Lambda_{t+1}\left((1-\delta)+V_{t+1} \frac{\partial \Phi\left(X_{t+1}, X_{t}, U_{t+1} \widetilde{K}_{t+1}\right)}{\partial \widetilde{K}_{t+1}} X_{t+1}\right)
\end{array}\right] \text {, } \\
& X_{t} \quad: \quad 1=\frac{\Lambda_{t}}{P_{t}} V_{t}\left[\Phi\left(X_{t}, X_{t-1}, U_{t} \widetilde{K}_{t}\right)+\frac{\partial \Phi\left(X_{t}, X_{t-1}, U_{t} \widetilde{K}_{t}\right)}{\partial X_{t}} X_{t}\right]+\ldots \\
& \beta \mathbb{E}_{t}\left[\frac{\lambda_{t+1} \Lambda_{t+1}}{\lambda_{t} P_{t}} V_{t+1} \frac{\partial \Phi\left(X_{t+1}, X_{t}, U_{t+1} \widetilde{K}_{t+1}\right)}{\partial X_{t}} X_{t+1}\right], \\
& U_{t+1} \quad: \quad \beta \mathbb{E}_{t}\left[\frac{\lambda_{t+1}}{\lambda_{t} P_{t}}\left(\begin{array}{c}
P_{t+1} A^{\prime}\left(U_{t+1}\right) \widetilde{K}_{t+1}-Z_{t+1} \widetilde{K}_{t+1}-\ldots \\
\Lambda_{t+1} V_{t+1} \frac{\partial \Phi\left(X_{t+1}, X_{t}, U_{t+1} \widetilde{K}_{t+1}\right)}{\partial U_{t+1}} X_{t+1}
\end{array}\right)\right]=0 .
\end{aligned}
$$

Let us define Tobin's q as $Q_{t} \equiv \frac{\Lambda_{t}}{P_{t}}$. Then, after further manipulation, it is possible to re-write the equilibrium conditions as,

$$
\begin{aligned}
& Q_{t}=\beta \mathbb{E}_{t}\left\{\left(\frac{C_{t+1}}{C_{t}}\right)^{-\sigma^{-1}}\left[\begin{array}{c}
\frac{Z_{t+1}}{P_{t+1}} U_{t+1}-A\left(U_{t+1}\right)+\ldots \\
Q_{t+1}\left((1-\delta)+V_{t+1} \frac{\partial \Phi\left(X_{t+1}, X_{t}, U_{t+1} \widetilde{K}_{t+1}\right)}{\partial \widetilde{K}_{t+1}} X_{t+1}\right)
\end{array}\right]\right\}, \\
& Q_{t}=\frac{1-\beta \mathbb{E}_{t}\left\{\left(\frac{C_{t+1}}{C_{t}}\right)^{-\sigma^{-1}}\left[Q_{t+1} V_{t+1} \frac{\partial \Phi\left(X_{t+1}, X_{t}, U_{t+1} \widetilde{K}_{t+1}\right)}{\partial X_{t}} X_{t+1}\right]\right\}}{V_{t}\left[\Phi\left(X_{t}, X_{t-1}, U_{t} \widetilde{K}_{t}\right)+\frac{\partial \Phi\left(X_{t}, X_{t-1}, U_{t} \widetilde{K}_{t}\right)}{\partial X_{t}} X_{t}\right]}, \\
& \beta \mathbb{E}_{t}\left\{\left(\frac{C_{t+1}}{C_{t}}\right)^{-\sigma^{-1}} \widetilde{K}_{t+1}\left[\frac{Z_{t+1}}{P_{t+1}}-A^{\prime}\left(U_{t+1}\right)+Q_{t+1} V_{t+1} \frac{\partial \Phi\left(X_{t+1}, X_{t}, U_{t+1} \widetilde{K}_{t+1}\right)}{\partial U_{t+1}} \frac{X_{t+1}}{\widetilde{K}_{t+1}}\right]\right\}=0 .
\end{aligned}
$$

Under no adjustment costs (NAC), the set of conditions added to account for the capital-investment decisions of the domestic households are,

$$
\begin{aligned}
Q_{t} & =\beta \mathbb{E}_{t}\left\{\left(\frac{C_{t+1}}{C_{t}}\right)^{-\sigma^{-1}}\left[\frac{Z_{t+1}}{P_{t+1}} U_{t+1}-A\left(U_{t+1}\right)+Q_{t+1}(1-\delta)\right]\right\}, \\
Q_{t} & =\frac{1}{V_{t}} \\
0 & =\beta \mathbb{E}_{t}\left\{\left(\frac{C_{t+1}}{C_{t}}\right)^{-\sigma^{-1}} \widetilde{K}_{t+1}\left[\frac{Z_{t+1}}{P_{t+1}}-A^{\prime}\left(U_{t+1}\right)\right]\right\} .
\end{aligned}
$$


A similar set of derivations allows us to write the following system of equations for the foreign country,

$$
\begin{aligned}
Q_{t}^{*} & =\beta \mathbb{E}_{t}\left\{\left(\frac{C_{t+1}^{*}}{C_{t}^{*}}\right)^{-\sigma^{-1}}\left[\frac{Z_{t+1}^{*}}{P_{t+1}^{*}} U_{t+1}^{*}-A\left(U_{t+1}^{*}\right)+Q_{t+1}^{*}(1-\delta)\right]\right\}, \\
Q_{t}^{*} & =\frac{1}{V_{t}^{*}}, \\
0 & =\beta \mathbb{E}_{t}\left\{\left(\frac{C_{t+1}^{*}}{C_{t}^{*}}\right)^{-\sigma^{-1}} \widetilde{K}_{t+1}^{*}\left[\frac{Z_{t+1}^{*}}{P_{t+1}^{*}}-A^{\prime}\left(U_{t+1}^{*}\right)\right]\right\} .
\end{aligned}
$$

The Lagrange multiplier on the law of motion relative to the Lagrange multiplier on the budget constraint expressed in real terms, denoted $Q_{t}$ and $Q_{t}^{*}$ respectively, has the interpretation of being the real shadow value of an additional unit of capital (or Tobin's q). In the case without adjustment costs (NAC) Tobin's q is exactly equal to one, if there are no IST shocks.

Under capital adjustment costs (CAC), the set of conditions added to account for the capital-investment decisions of the domestic households are,

$$
\begin{aligned}
& Q_{t}=\beta \mathbb{E}_{t}\left\{\left(\frac{C_{t+1}}{C_{t}}\right)^{-\sigma^{-1}}\left[\begin{array}{c}
\frac{Z_{t+1}}{P_{t+1}} U_{t+1}-A\left(U_{t+1}\right)+\ldots \\
Q_{t+1}\left((1-\delta)-V_{t+1} \Phi^{\prime}\left(\frac{X_{t+1}}{U_{t+1} \widetilde{K}_{t+1}}\right)\left(\frac{X_{t+1}}{U_{t+1} \widetilde{K}_{t+1}}\right)^{2} U_{t+1}\right)
\end{array}\right]\right\}, \\
& Q_{t}=\frac{1}{V_{t}}\left[\Phi\left(\frac{X_{t}}{U_{t} \widetilde{K}_{t}}\right)+\Phi^{\prime}\left(\frac{X_{t}}{U_{t} \widetilde{K}_{t}}\right) \frac{X_{t}}{U_{t} \widetilde{K}_{t}}\right]^{-1} \\
& 0=\beta \mathbb{E}_{t}\left\{\left(\frac{C_{t+1}}{C_{t}}\right)^{-\sigma^{-1}} \widetilde{K}_{t+1}\left[\begin{array}{c}
\frac{Z_{t+1}}{P_{t+1}}-A^{\prime}\left(U_{t+1}\right)-\ldots \\
Q_{t+1} V_{t+1} \Phi^{\prime}\left(\frac{X_{t+1}}{U_{t+1} \widetilde{K}_{t+1}}\right)\left(\frac{X_{t+1}}{U_{t+1} \widetilde{K}_{t+1}}\right)^{2}
\end{array}\right]\right\} .
\end{aligned}
$$

A similar set of derivations allows us to write the following system of equations for the foreign country,

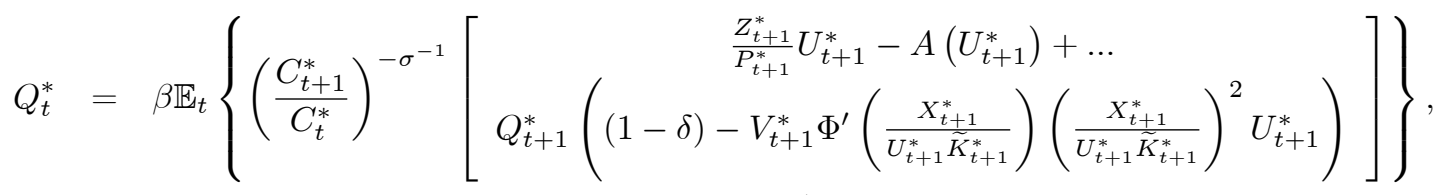

$$
\begin{aligned}
& Q_{t}^{*}=\frac{1}{V_{t}^{*}}\left[\Phi\left(\frac{X_{t}^{*}}{U_{t}^{*} \widetilde{K}_{t}^{*}}\right)+\Phi^{\prime}\left(\frac{X_{t}^{*}}{U_{t}^{*} \widetilde{K}_{t}^{*}}\right) \frac{X_{t}^{*}}{U_{t}^{*} \widetilde{K}_{t}^{*}}\right]^{-1}, \\
& 0=\beta \mathbb{E}_{t}\left\{\left(\frac{C_{t+1}^{*}}{C_{t}^{*}}\right)^{-\sigma^{-1}} \widetilde{K}_{t+1}^{*}\left[\begin{array}{c}
\frac{Z_{t+1}^{*}}{P_{t+1}^{*}}-A^{\prime}\left(U_{t+1}^{*}\right)-\ldots \\
Q_{t+1}^{*} V_{t+1}^{*} \Phi^{\prime}\left(\frac{X_{t+1}^{*}}{U_{t+1}^{*} \widetilde{K}_{t+1}^{*}}\right)\left(\frac{X_{t+1}^{*}}{U_{t+1}^{*} \widetilde{K}_{t+1}^{*}}\right)^{2}
\end{array}\right]\right\} .
\end{aligned}
$$

The Lagrange multiplier on the law of motion relative to the Lagrange multiplier on the budget constraint expressed in real terms, denoted $Q_{t}$ and $Q_{t}^{*}$ respectively, has the interpretation of being the real shadow value of an additional unit of capital (or Tobin's q).

Under investment adjustment costs (IAC), the set of conditions added to account for the capital- 
investment decisions of the domestic households are,

$$
\begin{aligned}
Q_{t} & =\beta \mathbb{E}_{t}\left\{\left(\frac{C_{t+1}}{C_{t}}\right)^{-\sigma^{-1}}\left[\frac{Z_{t+1}}{P_{t+1}} U_{t+1}-A\left(U_{t+1}\right)+Q_{t+1}(1-\delta)\right]\right\} \\
Q_{t} & =\frac{1+\beta \mathbb{E}_{t}\left\{\left(\frac{C_{t+1}}{C_{t}}\right)^{-\sigma^{-1}}\left[Q_{t+1} V_{t+1} \Phi^{\prime}\left(\frac{X_{t+1}}{X_{t}}\right)\left(\frac{X_{t+1}}{X_{t}}\right)^{2}\right]\right\}}{V_{t}\left[\Phi\left(\frac{X_{t}}{X_{t-1}}\right)+\Phi^{\prime}\left(\frac{X_{t}}{X_{t-1}}\right)\left(\frac{X_{t}}{X_{t-1}}\right)\right]} \\
0 & =\beta \mathbb{E}_{t}\left\{\left(\frac{C_{t+1}}{C_{t}}\right)^{-\sigma^{-1}} \widetilde{K}_{t+1}\left[\frac{Z_{t+1}}{P_{t+1}}-A^{\prime}\left(U_{t+1}\right)\right]\right\}
\end{aligned}
$$

A similar set of derivations allows us to write the following system of equations for the foreign country,

$$
\begin{aligned}
Q_{t}^{*} & =\beta \mathbb{E}_{t}\left\{\left(\frac{C_{t+1}^{*}}{C_{t}^{*}}\right)^{-\sigma^{-1}}\left[\frac{Z_{t+1}^{*}}{P_{t+1}^{*}} U_{t+1}^{*}-A\left(U_{t+1}^{*}\right)+Q_{t+1}^{*}(1-\delta)\right]\right\}, \\
Q_{t}^{*} & =\frac{1+\beta \mathbb{E}_{t}\left\{\left(\frac{C_{t+1}^{*}}{C_{t}^{*}}\right)^{-\sigma^{-1}}\left[Q_{t+1}^{*} V_{t+1}^{*} \Phi^{\prime}\left(\frac{X_{t+1}^{*}}{X_{t}^{*}}\right)\left(\frac{X_{t+1}^{*}}{X_{t}^{*}}\right)^{2}\right]\right\}}{V_{t}^{*}\left[\Phi\left(\frac{X^{*}}{X_{t-1}^{*}}\right)+\Phi^{\prime}\left(\frac{X_{t}^{*}}{X_{t-1}^{*}}\right)\left(\frac{X_{t}^{*}}{X_{t-1}^{*}}\right)\right]}, \\
0 & =\beta \mathbb{E}_{t}\left\{\left(\frac{C_{t+1}^{*}}{C_{t}^{*}}\right)^{-\sigma^{-1}} \widetilde{K}_{t+1}^{*}\left[\frac{Z_{t+1}^{*}}{P_{t+1}^{*}}-A^{\prime}\left(U_{t+1}^{*}\right)\right]\right\} .
\end{aligned}
$$

Once again, the Lagrange multiplier on the law of motion relative to the Lagrange multiplier on the budget constraint expressed in real terms, denoted $Q_{t}$ and $Q_{t}^{*}$ respectively, has the interpretation of being the real shadow value of an additional unit of capital (or Tobin's q).

The Optimality Conditions from the Firms' Problem. The necessary and sufficient first-order conditions for the domestic firm producing variety $h$ under LCP pricing give us the pair of price-setting formulas in (71) - (72). Similarly, the first-order conditions for the foreign firm producing variety $f$ under LCP pricing give us the pair of price-setting formulas in $(73)-(74)$. Using the law of large numbers and the inherent symmetry of the firms' problem, the price sub-indexes on domestic and foreign varieties, $P_{t}^{H}, P_{t}^{H *}, P_{t}^{F}$ and $P_{t}^{F *}$, become equal to those reported in equations $(75)-(78)$.

The necessary and sufficient first-order conditions for the domestic firm producing variety $h$ under PCP pricing give us the price-setting formula in (79). Similarly, the first-order conditions for the foreign firm producing variety $f$ under PCP pricing give us the price-setting formula in (82). Using the law of large numbers and the inherent symmetry of the firms' problem, the price sub-indexes on domestic and foreign varieties, $P_{t}^{H}, P_{t}^{H *}, P_{t}^{F}$ and $P_{t}^{F *}$, become equal to those reported in equations $(83)-(86)$.

Aggregate Output and Rental Rates on Capital. Equations (49) - (52) determine the demand function for each variety. Those demand functions coupled with the market clearing conditions at the 
variety level allows us to calculate the aggregate output from the demand-side as follows,

$$
\begin{aligned}
& Y_{t}-A\left(U_{t}\right) \widetilde{K}_{t}=\int_{0}^{1}\left[C_{t}(h)+X_{t}(h)+C_{t}^{*}(h)+X_{t}^{*}(h)\right] d h \\
&=\left[\int_{0}^{1}\left(\frac{P_{t}(h)}{P_{t}^{H}}\right)^{-\theta} d h\right] \phi_{H}\left(\frac{P_{t}^{H}}{P_{t}}\right)^{-\eta}\left(C_{t}+X_{t}\right)+\left[\int_{0}^{1}\left(\frac{P_{t}^{*}(h)}{P_{t}^{H *}}\right)^{-\theta} d h\right] \phi_{H}^{*}\left(\frac{P_{t}^{H *}}{P_{t}^{*}}\right)^{-\eta}\left(C_{t}^{*}+X_{t}^{*}\right), \\
& Y_{t}^{*}-A\left(U_{t}^{*}\right) \widetilde{K}_{t}^{*}=\int_{0}^{1}\left[C_{t}(f)+X_{t}(f)+C_{t}^{*}(f)+X_{t}^{*}(f)\right] d f \\
&=\left[\int_{0}^{1}\left(\frac{P_{t}(f)}{P_{t}^{F}}\right)^{-\theta} d f\right] \phi_{F}\left(\frac{P_{t}^{F}}{P_{t}}\right)^{-\eta}\left(C_{t}+X_{t}\right)+\left[\int_{0}^{1}\left(\frac{P_{t}^{*}(f)}{P_{t}^{F *}}\right)^{-\theta} d f\right] \phi_{F}^{*}\left(\frac{P_{t}^{F *}}{P_{t}^{*}}\right)^{-\eta}\left(C_{t}^{*}+X_{t}^{*}\right)
\end{aligned}
$$

Equations (218) - (219) tie the aggregate output in both countries to consumption as well as to relative prices, after adjusting for the costs of capital utilization. When the utilization costs are set equal to zero, these aggregate output equations correspond exactly to those reported in (87) - (88).

Given the production functions in (16)-(17) and the fact that capital-services-to-labor ratios are equalized across firms within a country, it is possible to write the aggregate output equations as in (91) - (92). Combining these aggregate production functions with the efficiency conditions in (18) - (19) and the labor supply equations from the households' problem (as in equations (56) - (57)), we can express the real rental rates on capital services in terms of productivity shocks, consumption, output and capital services as in equations (93) - (94). Manipulating the same set of conditions a little bit further also allows us to re-write the real wages in terms of real rental rates on capital services as well as productivity shocks, consumption, output and capital services as in equations (95) - (96). Those two efficiency equations suffice for the purpose of replacing real wages out of the marginal cost equations, as before.

\subsection{The Steady State}

We impose two restrictions on the utilization cost function $A(\cdot)$ in steady state. First, we require that the rate of utilization is set at $\bar{U}=\bar{U}^{*}=1$ in steady state. Naturally, this also implies that $\bar{K}=\bar{K}$ and $\widetilde{\widetilde{K}}^{*}=\bar{K}^{*}$. In other words, physical capital and capital services are equated in steady state. Second, we assume that in steady state $A(\bar{U})=A\left(\bar{U}^{*}\right)=0$. Hence, in steady state, the capital utilization cost drops from the first-order conditions (200) and (203), (206) and (209), and (212) and (215). Furthermore, it also drops from the steady state market clearing conditions implied by (218) - (219).

By the first-order conditions in (202) and (205), we can easily derive that,

$$
\begin{aligned}
A^{\prime}(\bar{U}) & =\frac{\bar{Z}}{\bar{P}}, \\
A^{\prime}\left(\bar{U}^{*}\right) & =\frac{\bar{Z}^{*}}{\bar{P}^{*}} .
\end{aligned}
$$

These expressions are derived under the NAC version of the model without capital adjustment costs. For the CAC case, we can derive exactly the same steady state conditions from equations (208) and (211), and the same can be said for the IAC case based on equations (214) and (217). Hence, the steady state with capital utilization is essentially identical to that without capital utilization that we have described earlier. 
The only difference is that the first derivative of the capital utilization cost function must satisfy that,

$$
\begin{aligned}
A^{\prime}(\bar{U}) & =\frac{\bar{Z}}{\bar{P}}=\beta^{-1}-(1-\delta), \\
A^{\prime}\left(\bar{U}^{*}\right) & =\frac{\bar{Z}^{*}}{\bar{P}^{*}}=\beta^{-1}-(1-\delta),
\end{aligned}
$$

where the second equality follows from our derivations of the steady state real rental rates on capital services.

\subsection{The Log-Linearized Equilibrium Conditions}

Here, we log-linearize the equilibrium conditions around the deterministic zero-inflation steady state. We only report those equations that differ from our previous derivations. For instance, we maintain the exact same specification of the Taylor (1993) rules described in (126) - (127) as our benchmark for monetary policy.

\subsubsection{The Households' Equilibrium Conditions}

The log-linearization of the Euler equations and the perfect international risk-sharing condition is the same as reported in equations $(97)-(99)$, i.e.

$$
\begin{aligned}
& \widehat{c}_{t} \approx \mathbb{E}_{t}\left[\widehat{c}_{t+1}\right]-\sigma\left(\widehat{i}_{t}-\mathbb{E}_{t}\left[\widehat{\pi}_{t+1}\right]\right), \\
& \widehat{c}_{t}^{*} \approx \mathbb{E}_{t}\left[\widehat{c}_{t+1}^{*}\right]-\sigma\left(\widehat{i}_{t}^{*}-\mathbb{E}_{t}\left[\widehat{\pi}_{t+1}^{*}\right]\right), \\
& \widehat{c}_{t}-\widehat{c}_{t}^{*} \approx \sigma \widehat{r s}_{t} .
\end{aligned}
$$

The log-linearization of the domestic capital accumulation formula in (195) and its foreign counterpart in the case without adjustment costs (NAC) is unaffected by the addition of variable capital utilization. Hence, simple re-labeling allows us to conclude that,

$$
\begin{aligned}
& \widehat{\widetilde{k}}_{t+1} \approx(1-\delta) \widehat{\widetilde{k}}_{t}+\delta\left(\widehat{x}_{t}+\widehat{v}_{t}\right), \\
& \widehat{\widetilde{k}}_{t+1}^{*} \approx(1-\delta) \widehat{\widetilde{k}}_{t}^{*}+\delta\left(\widehat{x}_{t}^{*}+\widehat{v}_{t}^{*}\right),
\end{aligned}
$$

where $\widehat{\widetilde{k}}_{t}$ and $\widehat{\widetilde{k}}_{t}^{*}$ denote the physical capital, and $\widehat{v}_{t}$ and $\widehat{v}_{t}^{*}$ are the IST shocks.

The log-linearization of the domestic capital accumulation formula in (195) and its foreign counterpart under capital adjustment costs (CAC) allows us to obtain the following set of equations,

$$
\begin{aligned}
& \widehat{\widetilde{k}}_{t+1} \approx\left[(1-\delta)-\bar{V} \Phi^{\prime}\left(\frac{\bar{X}}{\overline{\bar{U}}}\right)\left(\frac{\bar{X}}{\overline{\widetilde{K}}}\right)^{2} \frac{1}{\bar{U}}\right] \widehat{\widetilde{k}}_{t}+\ldots \\
& {\left[\bar{V} \Phi\left(\frac{\bar{X}}{\bar{U} \overline{\widetilde{K}}}\right) \frac{\bar{X}}{\overline{\widetilde{K}}}+\bar{V} \Phi^{\prime}\left(\frac{\bar{X}}{\overline{\bar{U}}}\right)\left(\frac{\bar{X}}{\overline{\widetilde{K}}}\right)^{2} \frac{1}{\bar{U}}\right]\left(\widehat{x}_{t}+\widehat{v}_{t}\right)-\bar{V} \Phi^{\prime}\left(\frac{\bar{X}}{\overline{\bar{U}}}\right)\left(\frac{\bar{X}}{\overline{\widetilde{K}}}\right)^{2} \frac{1}{\bar{U}} \widehat{u}_{t}} \\
& =(1-\delta) \widehat{\widetilde{k}}_{t}+\delta\left(\widehat{x}_{t}+\widehat{v}_{t}\right) \text {, } \\
& \widehat{\widehat{k}}_{t+1}^{*} \approx\left[(1-\delta)-\bar{V}^{*} \Phi^{\prime}\left(\frac{\bar{X}^{*}}{\bar{U}^{*} \bar{K}^{*}}\right)\left(\frac{\bar{X}^{*}}{\bar{K}^{*}}\right)^{2} \frac{1}{\bar{U}^{*}}\right] \widehat{\widetilde{k}}_{t}^{*}+\ldots \\
& {\left[\bar{V}^{*} \Phi\left(\frac{\bar{X}^{*}}{\bar{U}^{*} \bar{K}^{*}}\right) \frac{\bar{X}^{*}}{\bar{K}^{*}}+\bar{V}^{*} \Phi^{\prime}\left(\frac{\bar{X}^{*}}{\bar{U}^{*} \bar{K}^{*}}\right)\left(\frac{\bar{X}^{*}}{\bar{K}^{*}}\right)^{2} \frac{1}{\bar{U}^{*}}\right]\left(\widehat{x}_{t}^{*}+\widehat{v}_{t}^{*}\right)-\bar{V}^{*} \Phi^{\prime}\left(\frac{\bar{X}^{*}}{\bar{U}^{*} \widetilde{K}^{*}}\right)\left(\frac{\bar{X}^{*}}{\widetilde{\widetilde{K}}^{*}}\right)^{2} \frac{1}{\bar{U}^{*}} \widehat{u}_{t}^{*}} \\
& =(1-\delta) \widehat{\widehat{k}}_{t}^{*}+\delta\left(\widehat{x}_{t}^{*}+\widehat{v}_{t}^{*}\right) \text {, }
\end{aligned}
$$


where the second-equality follows from the steady state properties of the CAC function, and the fact that $\bar{X}=\delta \bar{K}, \bar{X}^{*}=\delta \bar{K}^{*}$ and $\bar{V}=\bar{V}^{*}=1$. The log-linearization of the capital accumulation formula in (195) and its foreign counterpart under investment adjustment costs (IAC) allows us to obtain the following set of equations,

$$
\begin{aligned}
& \widehat{\widetilde{k}}_{t+1} \approx(1-\delta) \widehat{\widetilde{k}}_{t}+\left[\bar{V} \Phi\left(\frac{\bar{X}}{\bar{X}}\right) \frac{\bar{X}}{\widetilde{\widetilde{K}}}+\bar{V} \Phi^{\prime}\left(\frac{\bar{X}}{\bar{X}}\right)\left(\frac{\bar{X}}{\bar{X}}\right) \frac{\bar{X}}{\widetilde{\widetilde{K}}}\right] \widehat{x}_{t}-\ldots \\
& {\left[\bar{V} \Phi^{\prime}\left(\frac{\bar{X}}{\bar{X}}\right)\left(\frac{\bar{X}}{\bar{X}}\right)^{2} \frac{\bar{X}}{\overline{\widetilde{K}}}\right] \widehat{x}_{t}+\bar{V} \Phi\left(\frac{\bar{X}}{\bar{X}}\right) \frac{\bar{X}}{\overline{\widetilde{K}}} \widehat{v}_{t}} \\
& =(1-\delta) \widehat{k}_{t}+\delta\left(\widehat{x}_{t}+\widehat{v}_{t}\right) \text {, } \\
& \widehat{k}_{t+1}^{*} \approx(1-\delta) \widehat{k}_{t}^{*}+\left[\bar{V}^{*} \Phi\left(\frac{\bar{X}^{*}}{\bar{X}^{*}}\right) \frac{\bar{X}^{*}}{\widetilde{K}^{*}}+\bar{V}^{*} \Phi^{\prime}\left(\frac{\bar{X}^{*}}{\bar{X}^{*}}\right)\left(\frac{\bar{X}^{*}}{\bar{X}^{*}}\right) \frac{\bar{X}^{*}}{\widetilde{K}^{*}}\right] \widehat{x}_{t}^{*}-\ldots \\
& {\left[\bar{V}^{*} \Phi^{\prime}\left(\frac{\bar{X}^{*}}{\bar{X}^{*}}\right)\left(\frac{\bar{X}^{*}}{\bar{X}^{*}}\right)^{2} \frac{\bar{X}^{*}}{\widetilde{\bar{K}}^{*}}\right] \widehat{x}_{t}^{*}+\bar{V}^{*} \Phi\left(\frac{\bar{X}^{*}}{\bar{X}^{*}}\right) \frac{\bar{X}^{*}}{\widetilde{K}^{*}} \widehat{v}_{t}^{*}} \\
& =(1-\delta) \widehat{k}_{t}^{*}+\delta\left(\widehat{x}_{t}^{*}+\widehat{v}_{t}^{*}\right) \text {, }
\end{aligned}
$$

where the second-equality follows from the steady state properties of the IAC function, and the fact that $\bar{X}=\delta \bar{K}, \bar{X}^{*}=\delta \bar{K}^{*}$ and $\bar{V}=\bar{V}^{*}=1$. In spite of the fact that we are using three different specifications for the adjustment cost function $\Phi(\cdot)$ and that we allow for variable capital utilization, the log-linearized law of motion for physical capital is the same in all cases.

A first-order approximation of the link between capital services and physical capital in (196) also gives us the following relationships between these two variables,

$$
\begin{aligned}
& \widehat{k}_{t} \approx \widehat{u}_{t}+\widehat{\widetilde{k}}_{t}, \\
& \widehat{k}_{t}^{*} \approx \widehat{\widetilde{u}}_{t}^{*}+\widehat{\widetilde{k}}_{t}^{*},
\end{aligned}
$$

where $\widehat{k}_{t}$ and $\widehat{k}_{t}^{*}$ denote the capital services.

The Capital-Investment Decision under NAC. The log-linearization of the capital-investment equilibrium conditions coming from the domestic households' problem in (200) - (201) is as follows,

$$
\begin{aligned}
\widehat{q}_{t} & \approx \mathbb{E}_{t}\left\{-\frac{1}{\sigma}\left(\widehat{c}_{t+1}-\widehat{c}_{t}\right)+\beta\left(\frac{\bar{Z}}{\bar{P}}\right) \widehat{r}_{t+1}^{z}+\beta\left[\frac{\bar{Z}}{\bar{P}}-A^{\prime}(\bar{U})\right] \bar{U} \widehat{u}_{t+1}+\beta(1-\delta) \widehat{q}_{t+1}\right\} \\
& =\mathbb{E}_{t}\left\{-\frac{1}{\sigma}\left(\widehat{c}_{t+1}-\widehat{c}_{t}\right)+(1-\beta(1-\delta)) \widehat{r}_{t+1}^{z}+\beta(1-\delta) \widehat{q}_{t+1}\right\} \\
\widehat{q}_{t} & \approx-\widehat{v}_{t},
\end{aligned}
$$

and, analogously, for the foreign counterparts in (203) - (204),

$$
\begin{aligned}
& \widehat{q}_{t}^{*} \approx \mathbb{E}_{t}\left\{-\frac{1}{\sigma}\left(\widehat{c}_{t+1}^{*}-\widehat{c}_{t}^{*}\right)+(1-\beta(1-\delta)) \widehat{r}_{t+1}^{z *}+\beta(1-\delta) \widehat{q}_{t+1}^{*}\right\} \\
& \widehat{q}_{t}^{*} \approx-\widehat{v}_{t}^{*}
\end{aligned}
$$

where $\widehat{q}_{t}$ and $\widehat{q}_{t}^{*}$ are the real shadow values of an additional unit of capital (or Tobin's q) in each country. These are the same equations derived under no capital utilization in (106) - (109). 
These capital-investment equations can be re-arranged to show that,

$$
\begin{aligned}
(1-\beta(1-\delta)) \mathbb{E}_{t}\left(\widehat{r}_{t+1}^{z}\right) & \approx\left(\widehat{i}_{t}-\mathbb{E}_{t}\left(\widehat{\pi}_{t+1}\right)\right)+\beta(1-\delta) \mathbb{E}_{t}\left[\widehat{v}_{t+1}\right]-\widehat{v}_{t} \\
(1-\beta(1-\delta)) \mathbb{E}_{t}\left(\widehat{r}_{t+1}^{z *}\right) & \approx\left(\widehat{i}_{t}^{*}-\mathbb{E}_{t}\left(\widehat{\pi}_{t+1}^{*}\right)\right)+\beta(1-\delta) \mathbb{E}_{t}\left[\widehat{v}_{t+1}^{*}\right]-\widehat{v}_{t}^{*}
\end{aligned}
$$

by adding the Euler equations in (97) - (98). Finally, we must add the log-linearization of (202) and (205) as,

$$
\begin{aligned}
& \mathbb{E}_{t}\left(\widehat{r}_{t+1}^{z}\right) \approx \lambda \mathbb{E}_{t}\left(\widehat{u}_{t+1}\right), \\
& \mathbb{E}_{t}\left(\widehat{r}_{t+1}^{z *}\right) \approx \lambda \mathbb{E}_{t}\left(\widehat{u}_{t+1}^{*}\right),
\end{aligned}
$$

where $\lambda \equiv \frac{A^{\prime \prime}(\bar{U}) \bar{U}}{A^{\prime}(\bar{U})}=\frac{A^{\prime \prime}\left(\bar{U}^{*}\right) \bar{U}^{*}}{A^{\prime}\left(\bar{U}^{*}\right)}$ and $\bar{U}=\bar{U}^{*}=1$. These two first-order conditions link the real rental rate on capital services to the capital utilization choice made by households.

The Capital-Investment Decision under CAC. The log-linearization of the capital-investment equilibrium conditions coming from the domestic households' problem in (206) - (207) are as follows,

$$
\begin{aligned}
& \widehat{q}_{t} \quad \approx \mathbb{E}_{t}\left\{\begin{array}{c}
-\frac{1}{\sigma}\left(\widehat{c}_{t+1}-\widehat{c}_{t}\right)+(1-(1-\delta) \beta) \widehat{r}_{t+1}^{z}+(1-\delta) \beta \widehat{q}_{t+1}+\chi \delta^{2} \beta\left(\widehat{x}_{t+1}-\widehat{\widetilde{k}}_{t+1}\right)+\ldots \\
{\left[\frac{\bar{z} \bar{U}-A^{\prime}(\bar{U}) \bar{U}+\bar{Q}\left(\bar{V} \Phi^{\prime \prime}\left(\frac{\bar{X}}{\bar{U} \overline{\bar{K}}}\right)\left(\frac{\bar{X}}{\bar{U} \overline{\bar{K}}}\right)^{2}\left(\frac{\bar{X}}{\bar{U} \overline{\bar{K}}}\right)+\bar{V} \Phi^{\prime}\left(\frac{\bar{X}}{\bar{U} \bar{K}}\right)\left(\frac{\bar{X}}{\bar{U} \overline{\bar{K}}}\right)^{2}\right) \bar{U}}{\frac{\bar{z}}{\bar{P}}-A(\bar{U})+\bar{Q}\left((1-\delta)-\bar{V} \Phi^{\prime}\left(\frac{\bar{X}}{\bar{U} \bar{K}}\right)\left(\frac{\bar{x}}{\bar{U} \bar{K}}\right)^{2} \bar{U}\right)}\right] \widehat{u}_{t+1}-\ldots} \\
{\left[\frac{\overline{Q V} \Phi^{\prime}\left(\frac{\bar{x}}{\bar{U} \bar{K}}\right)\left(\frac{\bar{x}}{\bar{U} \bar{K}}\right)^{2} \bar{U}}{\left[\frac{\bar{z}}{\bar{P}} \bar{U}-A(\bar{U})+\bar{Q}\left((1-\delta)-\bar{V} \Phi^{\prime}\left(\frac{\bar{X}}{\bar{U} \bar{K}}\right)\left(\frac{\bar{X}}{\bar{U} \bar{K}}\right)^{2} \bar{U}\right)\right.}\right] \widehat{v}_{t+1}}
\end{array}\right\} \\
& =\mathbb{E}_{t}\left\{-\frac{1}{\sigma}\left(\widehat{c}_{t+1}-\widehat{c}_{t}\right)+(1-(1-\delta) \beta) \widehat{r}_{t+1}^{z}+(1-\delta) \beta \widehat{q}_{t+1}+\chi \delta^{2} \beta\left(\widehat{x}_{t+1}-\widehat{\widetilde{k}}_{t+1}-\widehat{u}_{t+1}\right)\right\},
\end{aligned}
$$

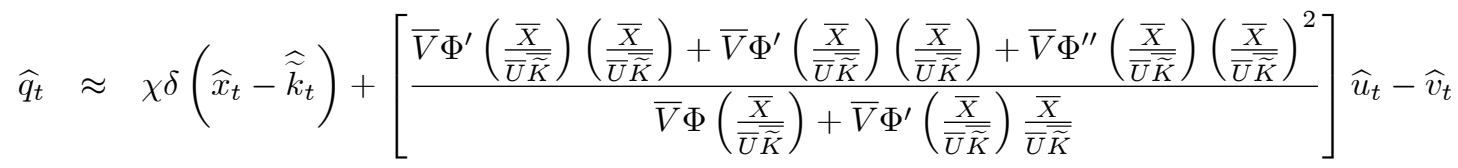

$$
\begin{aligned}
& =\chi \delta\left(\widehat{x}_{t}-\widehat{\widetilde{k}}_{t}-\widehat{u}_{t}\right)-\widehat{v}_{t},
\end{aligned}
$$

and, analogously, for the foreign counterparts in (209) - (210),

$$
\begin{aligned}
& \widehat{q}_{t}^{*} \approx \mathbb{E}_{t}\left\{-\frac{1}{\sigma}\left(\widehat{c}_{t+1}^{*}-\widehat{c}_{t}^{*}\right)+(1-(1-\delta) \beta) \widehat{r}_{t+1}^{z *}+(1-\delta) \beta \widehat{q}_{t+1}^{*}+\chi \delta^{2} \beta\left(\widehat{x}_{t+1}^{*}-\widehat{\widetilde{k}}_{t+1}^{*}-\widehat{u}_{t+1}^{*}\right)\right\} \\
& \widehat{q}_{t}^{*} \approx \chi \delta\left(\widehat{x}_{t}^{*}-\widehat{\widetilde{k}}_{t}^{*}-\widehat{u}_{t}^{*}\right)-\widehat{v}_{t}^{*} .
\end{aligned}
$$

This system of equations describes $\widehat{q}_{t}$ and $\widehat{q}_{t}^{*}$ as the real shadow values of an additional unit of capital (or Tobin's q) in each country, and $\chi$ regulates the degree of concavity of the capital adjustment cost (CAC) function around the steady state.

The pair of equations that relate the current and expected Tobin's q to the real rental rate on capital 
services and the real interest rate can be re-arranged as,

$$
\begin{aligned}
\widehat{q}_{t} & \approx \beta \mathbb{E}_{t}\left[\widehat{q}_{t+1}\right]+\left[(1-(1-\delta) \beta) \mathbb{E}_{t}\left(\widehat{r}_{t+1}^{z}\right)-\left(\widehat{i}_{t}-\mathbb{E}_{t}\left(\widehat{\pi}_{t+1}\right)\right)\right], \\
\widehat{q}_{t}^{*} & \approx \beta \mathbb{E}_{t}\left[\widehat{q}_{t+1}^{*}\right]+\left[(1-(1-\delta) \beta) \mathbb{E}_{t}\left(\widehat{r}_{t+1}^{* *}\right)-\left(\widehat{i}_{t}^{*}-\mathbb{E}_{t}\left(\widehat{\pi}_{t+1}^{*}\right)\right)\right],
\end{aligned}
$$

by adding the Euler equations in (97) - (98). These equations are exactly the same ones that we found in (116) - (117). We can re-write (234) and (236) in terms of capital services as,

$$
\begin{aligned}
& \widehat{q}_{t} \approx \chi \delta\left(\widehat{x}_{t}-\widehat{k}_{t}\right)-\widehat{v}_{t}, \\
& \widehat{q}_{t}^{*} \approx \chi \delta\left(\widehat{x}_{t}^{*}-\widehat{k}_{t}^{*}\right)-\widehat{v}_{t}^{*} .
\end{aligned}
$$

These equations are effectively the same ones that we derived in (113) - (115). Finally, we approximate the first-order conditions on capital utilization in (208) and (211) that complete the model as follows,

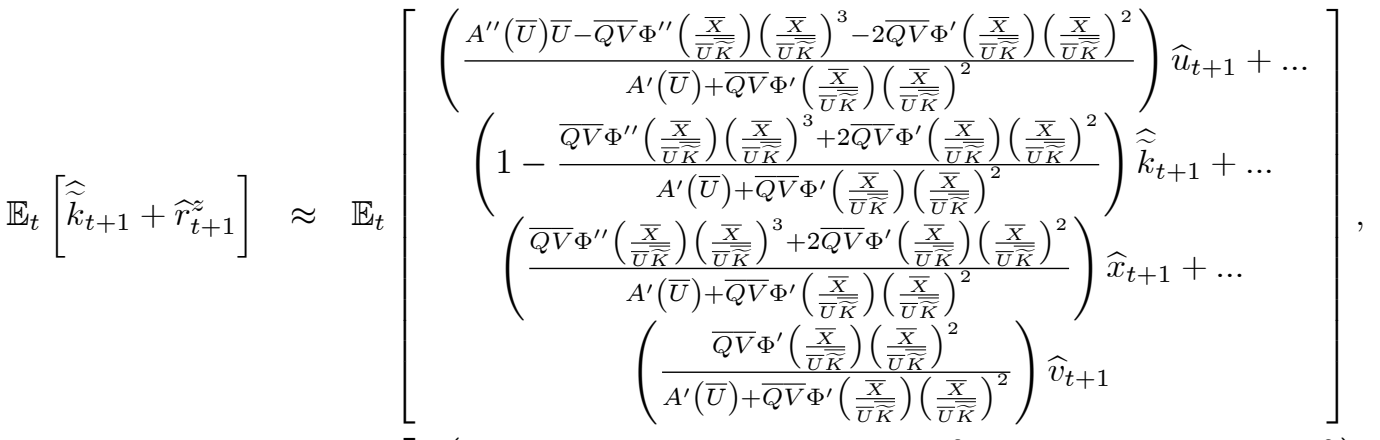

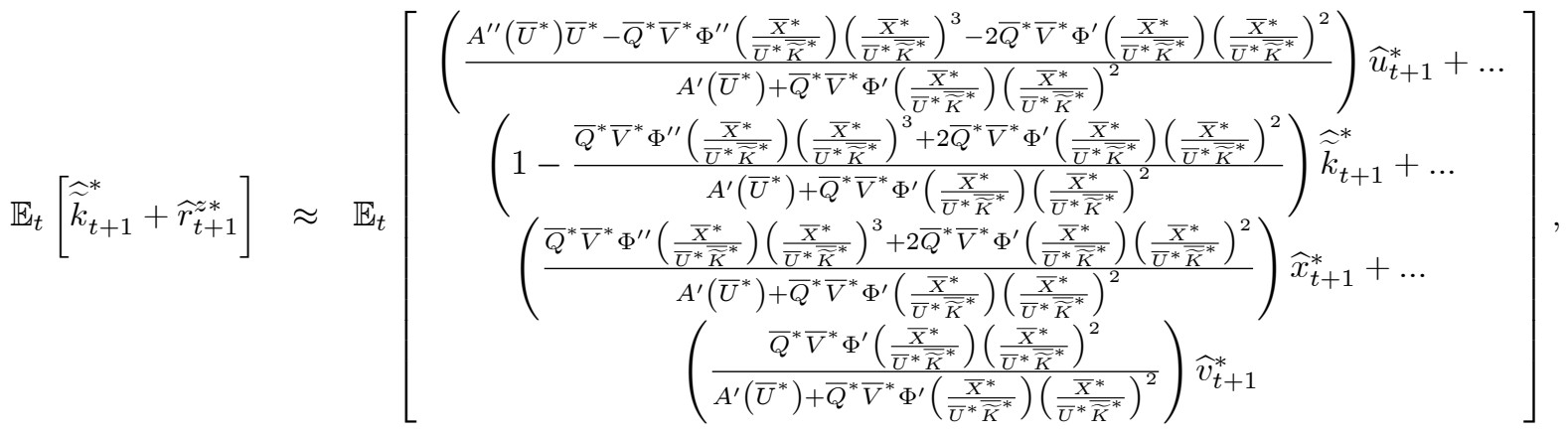


so we obtain that,

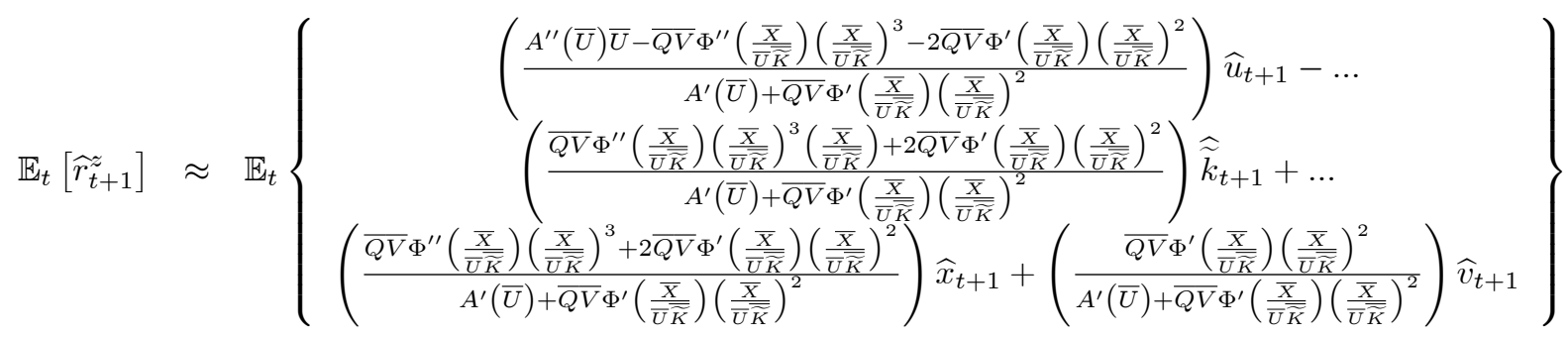

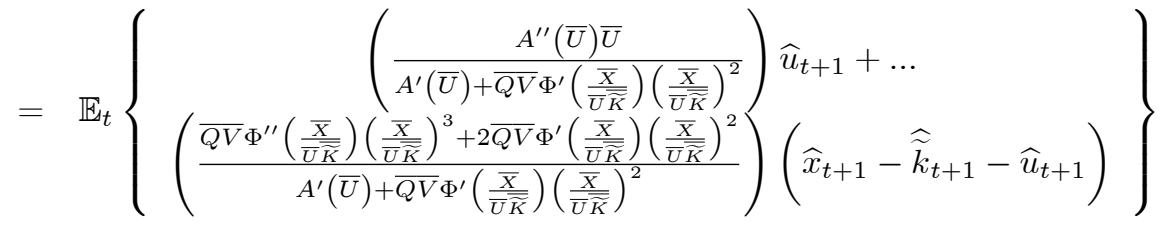

$$
\begin{aligned}
& =\mathbb{E}_{t}\left\{\left(\frac{A^{\prime \prime}(\bar{U}) \bar{U}}{A^{\prime}(\bar{U})}\right) \widehat{u}_{t+1}+\left(\frac{\overline{Q V} \Phi^{\prime \prime}\left(\frac{\bar{X}}{\bar{U} \bar{K}}\right)\left(\frac{\bar{X}}{\bar{U} \bar{K}}\right)^{3}}{A^{\prime}(\bar{U})}\right)\left(\widehat{x}_{t+1}-\widehat{\widetilde{k}}_{t+1}-\widehat{u}_{t+1}\right)\right\} \\
& =\mathbb{E}_{t}\left\{\lambda \widehat{u}_{t+1}+\left(\frac{\Phi^{\prime \prime}(\delta) \delta^{3}}{\beta^{-1}-(1-\delta)}\right)\left(\widehat{x}_{t+1}-\widehat{\widetilde{k}}_{t+1}-\widehat{u}_{t+1}\right)\right\} \\
& =\mathbb{E}_{t}\left\{\lambda \widehat{u}_{t+1}-\left(\frac{\chi \delta^{2} \beta}{1-(1-\delta) \beta}\right)\left(\widehat{x}_{t+1}-\widehat{\widetilde{k}}_{t+1}-\widehat{u}_{t+1}\right)\right\}, \\
& \mathbb{E}_{t}\left(\widehat{r}_{t+1}^{z *}\right) \approx \mathbb{E}_{t}\left\{\lambda \widehat{u}_{t+1}^{*}-\left(\frac{\chi \delta^{2} \beta}{1-(1-\delta) \beta}\right)\left(\widehat{x}_{t+1}^{*}-\widehat{\widetilde{k}}_{t+1}^{*}-\widehat{u}_{t+1}^{*}\right)\right\} .
\end{aligned}
$$

Using equations (234) and (236) we obtain that,

$$
\begin{aligned}
& \mathbb{E}_{t}\left[\widehat{r}_{t+1}^{z}\right] \approx \mathbb{E}_{t}\left\{\lambda \widehat{u}_{t+1}-\left(\frac{\delta \beta}{1-(1-\delta) \beta}\right)\left(\widehat{q}_{t+1}+\widehat{v}_{t+1}\right)\right\} \\
& \mathbb{E}_{t}\left[\widehat{r}_{t+1}^{z *}\right] \approx \mathbb{E}_{t}\left\{\lambda \widehat{u}_{t+1}^{*}-\left(\frac{\delta \beta}{1-(1-\delta) \beta}\right)\left(\widehat{q}_{t+1}^{*}+\widehat{v}_{t+1}^{*}\right)\right\} .
\end{aligned}
$$

These first-order conditions on capital utilization are similar to those derived in the NAC case (equations (231) - (232)), but they show that the real rental rates on capital services are tied to capital utilization, Tobin's q and the IST shocks as well. The extra term on the right-hand side - which is proportional to Tobin's q and the IST shock - is the result of having assumed that the capital adjustment cost function penalizes the size of investment relative to capital services in each period, rather than the size of investment relative to physical capital.

The Capital-Investment Decision under IAC. The log-linearization of the capital-investment equilibrium conditions coming from the first-order conditions of the households' problem in (212) - (213) are as 
follows,

$$
\begin{aligned}
& \widehat{q}_{t} \approx \mathbb{E}_{t}\left\{-\frac{1}{\sigma}\left(\widehat{c}_{t+1}-\widehat{c}_{t}\right)+(1-(1-\delta) \beta) \widehat{r}_{t+1}^{z}+(1-\delta) \beta \widehat{q}_{t+1}+\frac{\left[\overline{\bar{Z}}-A^{\prime}(\bar{U})\right] \bar{U}}{\left[\frac{\bar{Z}}{\bar{P}} \bar{U}-A(\bar{U})+\bar{Q}(1-\delta)\right]} \widehat{u}_{t+1}\right\} \\
& =\mathbb{E}_{t}\left\{-\frac{1}{\sigma}\left(\widehat{c}_{t+1}-\widehat{c}_{t}\right)+(1-(1-\delta) \beta) \widehat{r}_{t+1}^{z}+(1-\delta) \beta \widehat{q}_{t+1}\right\}, \\
& \widehat{q}_{t} \approx-\widehat{v}_{t}-\frac{\left(2 \bar{V} \Phi^{\prime}\left(\frac{\bar{X}}{\bar{X}}\right)+\bar{V} \Phi^{\prime \prime}\left(\frac{\bar{X}}{\bar{X}}\right)\left(\frac{\bar{X}}{\bar{X}}\right)\right)\left(\frac{\bar{X}}{\bar{X}}\right)}{\bar{V} \Phi\left(\frac{\bar{X}}{\bar{X}}\right)+\bar{V} \Phi^{\prime}\left(\frac{\bar{X}}{\bar{X}}\right)\left(\frac{\bar{X}}{\bar{X}}\right)}\left(\widehat{x}_{t}-\widehat{x}_{t-1}\right)+\ldots
\end{aligned}
$$

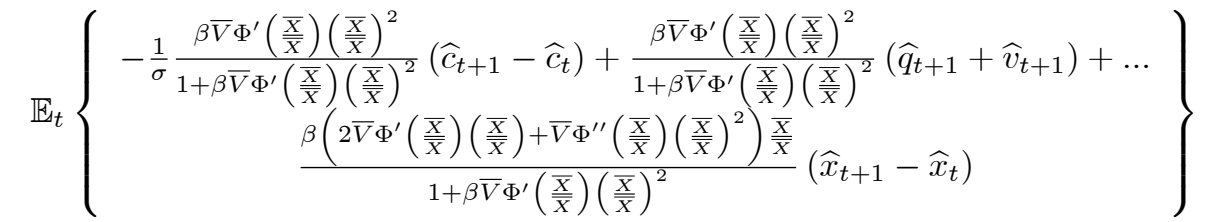

$$
\begin{aligned}
& =\kappa\left[\left(\widehat{x}_{t}-\widehat{x}_{t-1}\right)-\beta \mathbb{E}_{t}\left(\widehat{x}_{t+1}-\widehat{x}_{t}\right)\right]-\widehat{v}_{t},
\end{aligned}
$$

and, analogously, for the foreign counterparts in (215) - (216),

$$
\begin{aligned}
& \widehat{q}_{t}^{*} \approx \mathbb{E}_{t}\left\{-\frac{1}{\sigma}\left(\widehat{c}_{t+1}^{*}-\widehat{c}_{t}^{*}\right)+(1-(1-\delta) \beta) \widehat{r}_{t+1}^{z *}+(1-\delta) \beta \widehat{q}_{t+1}^{*}\right\}, \\
& \widehat{q}_{t}^{*} \approx \kappa\left[\left(\widehat{x}_{t}^{*}-\widehat{x}_{t-1}^{*}\right)-\beta \mathbb{E}_{t}\left(\widehat{x}_{t+1}^{*}-\widehat{x}_{t}^{*}\right)\right]-\widehat{v}_{t}^{*}
\end{aligned}
$$

This system of equations summarizes $\widehat{q}_{t}$ and $\widehat{q}_{t}^{*}$ as the real shadow values of an additional unit of capital (or Tobin's q) in each country, and $\kappa$ regulates the degree of concavity of the investment adjustment cost (IAC) function around the steady state.

The pair of equations that relate the current and expected Tobin's q to the real rental rates on capital and the real interest rate can be re-arranged as,

$$
\begin{aligned}
\widehat{q}_{t} & \approx(1-\delta) \beta \mathbb{E}_{t}\left[\widehat{q}_{t+1}\right]+\left[(1-(1-\delta) \beta) \mathbb{E}_{t}\left(\widehat{r}_{t+1}^{z}\right)-\left(\widehat{i}_{t}-\mathbb{E}_{t}\left[\widehat{\pi}_{t+1}\right]\right)\right] \\
\widehat{q}_{t}^{*} & \approx(1-\delta) \beta \mathbb{E}_{t}\left[\widehat{q}_{t+1}^{*}\right]+\left[(1-(1-\delta) \beta) \mathbb{E}_{t}\left(\widehat{r}_{t+1}^{z *}\right)-\left(\widehat{i}_{t}^{*}-\mathbb{E}_{t}\left[\widehat{\pi}_{t+1}^{*}\right]\right)\right],
\end{aligned}
$$

by adding the Euler equations in (97) - (98). Finally, we re-write equations (242) and (244) in a more compact form as follows,

$$
\begin{aligned}
& \widehat{x}_{t} \approx \frac{1}{1+\beta} \widehat{x}_{t-1}+\frac{\beta}{1+\beta} \mathbb{E}_{t}\left[\widehat{x}_{t+1}\right]+\frac{1}{\kappa(1+\beta)}\left(\widehat{q}_{t}+\widehat{v}_{t}\right), \\
& \widehat{x}_{t}^{*} \approx \frac{1}{1+\beta} \widehat{x}_{t-1}^{*}+\frac{\beta}{1+\beta} \mathbb{E}_{t}\left[\widehat{x}_{t+1}^{*}\right]+\frac{1}{\kappa(1+\beta)}\left(\widehat{q}_{t}^{*}+\widehat{v}_{t}^{*}\right) .
\end{aligned}
$$

The presence of investment adjustment costs (IAC) changes equations (242) and (244) completely. These equations are the same equations derived before without capital utilization in (122) - (125). Capital utiliza- 
tion, however, adds an additional pair of equations to the system of equilibrium conditions,

$$
\begin{aligned}
& \mathbb{E}_{t}\left(\widehat{r}_{t+1}^{z}\right) \approx \lambda \mathbb{E}_{t}\left(\widehat{u}_{t+1}\right), \\
& \mathbb{E}_{t}\left(\widehat{r}_{t+1}^{z *}\right) \approx \lambda \mathbb{E}_{t}\left(\widehat{u}_{t+1}^{*}\right),
\end{aligned}
$$

where $\lambda \equiv \frac{A^{\prime \prime}(\bar{U}) \bar{U}}{A^{\prime}(\bar{U})}=\frac{A^{\prime \prime}\left(\bar{U}^{*}\right) \bar{U}^{*}}{A^{\prime}\left(\bar{U}^{*}\right)}$ and $\bar{U}=\bar{U}^{*}=1$. These conditions link the real rental rates on capital to the capital utilization choice, and they are identical to the equilibrium conditions derived in the case without adjustment costs (NAC).

\subsubsection{The Firms' Equilibrium Conditions: The LCP Case}

Efficiency Conditions and Aggregate Output. The efficiency conditions can be described by the same pair of equations reported in (134) - (135) (or in (166) - (167)). Those efficiency conditions are necessary to close down the model without having to keep track of either labor or wages explicitly. Using the demand constraints of the domestic firm in equations $(27)-(28)$, the demand constraints of the foreign firm in equations (30) - (31), complemented by (51) - (52) and their corresponding foreign counterparts, it follows that the log-linearization around the steady state of output demand for a given re-optimizing firm, i.e. $\widehat{y}_{t}(h)$ for a domestic firm $h$ and $\widehat{y}_{t}^{*}(f)$ for a foreign firm $f$, takes the following form,

$$
\begin{aligned}
& \widehat{y}_{t}(h) \approx-\theta\left(\widehat{p}_{t}^{W}(h)-\widehat{p}_{t}^{W}\right)+(\theta-\eta)\left(\widehat{p}_{t}^{H, W}-\widehat{p}_{t}^{W}\right)+\left(1-\gamma_{x}\right) \widehat{c}_{t}^{W}+\gamma_{x} \widehat{x}_{t}^{W}, \\
& \widehat{y}_{t}^{*}(f) \approx-\theta\left(\widehat{p}_{t}^{W *}(f)-\widehat{p}_{t}^{W *}\right)+(\theta-\eta)\left(\widehat{p}_{t}^{F, W *}-\widehat{p}_{t}^{W *}\right)+\left(1-\gamma_{x}\right) \widehat{c}_{t}^{W *}+\gamma_{x} \widehat{x}_{t}^{W *},
\end{aligned}
$$

where the weighted variables are,

$$
\begin{aligned}
\widehat{c}_{t}^{W} & \equiv \phi_{H} \widehat{c}_{t}+\phi_{F} \widehat{c}_{t}^{*}, \widehat{c}_{t}^{W *} \equiv \phi_{F} \widehat{c}_{t}+\phi_{H} \widehat{c}_{t}^{*} \\
\widehat{x}_{t}^{W} & \equiv \phi_{H} \widehat{x}_{t}+\phi_{F} \widehat{x}_{t}^{*}, \widehat{x}_{t}^{W *} \equiv \phi_{F} \widehat{x}_{t}+\phi_{H} \widehat{x}_{t}^{*} \\
\widehat{p}_{t}^{W}(h) & \equiv \phi_{H} \widehat{p}_{t}(h)+\phi_{F} \widehat{p}_{t}^{*}(h), \widehat{p}_{t}^{W *}(f) \equiv \phi_{F} \widehat{p}_{t}(f)+\phi_{H} \widehat{p}_{t}^{*}(f), \\
\widehat{p}_{t}^{H, W} & \equiv \phi_{H} \widehat{p}_{t}^{H}+\phi_{F} \widehat{p}_{t}^{H *}, \widehat{p}_{t}^{F, W *} \equiv \phi_{F} \widehat{p}_{t}^{F}+\phi_{H} \widehat{p}_{t}^{F *} \\
\widehat{p}_{t}^{W} & \equiv \phi_{H} \widehat{p}_{t}+\phi_{F} \widehat{p}_{t}^{*}, \widehat{p}_{t}^{W *} \equiv \phi_{F} \widehat{p}_{t}+\phi_{H} \widehat{p}_{t}^{*} .
\end{aligned}
$$

We define the steady state consumption and investment shares as $\gamma_{c} \equiv \frac{\bar{C}}{\overline{C+X}}=1-\gamma_{x}$ and $\gamma_{x} \equiv \frac{\bar{X}}{\bar{C}+\bar{X}}$, respectively. These expressions hold true independently of whether we assume LCP pricing or PCP pricing, although the price indexes and sub-indexes can be further simplified in the PCP case (where the law of one price holds).

We can log-linearize the price sub-indexes in (13) - (14) as,

$$
\begin{aligned}
\widehat{p}_{t}^{H} & \approx \int_{0}^{1} \widehat{p}_{t}(h) d h, \widehat{p}_{t}^{F} \approx \int_{0}^{1} \widehat{p}_{t}(f) d f \\
\widehat{p}_{t}^{H *} & \approx \int_{0}^{1} \widehat{p}_{t}^{*}(h) d h, \widehat{p}_{t}^{F *} \approx \int_{0}^{1} \widehat{p}_{t}^{*}(f) d f,
\end{aligned}
$$

so it follows that the weighted prices of all domestic and all foreign firms are $\widehat{p}_{t}^{H, W} \approx \int_{0}^{1} \widehat{p}_{t}^{W}(h) d h$ and 
$\widehat{p}_{t}^{F, W *} \approx \int_{0}^{1} \widehat{p}_{t}^{W *}(f) d f$, respectively. Adding up the output market clearing conditions for all varieties (firms) within each country and adjusting for the capital utilization costs, as per equations (218) - (219), we obtain an expression for aggregate output in these terms,

$$
\begin{gathered}
\widehat{y}_{t}-\left(\frac{A^{\prime}(\bar{U}) \overline{\widetilde{K}} \bar{U}}{\bar{Y}-A(\bar{U}) \overline{\widetilde{K}}}\right) \widehat{u}_{t} \approx-\eta\left(\widehat{p}_{t}^{H, W}-\widehat{p}_{t}^{W}\right)+\left(1-\gamma_{x}\right) \widehat{c}_{t}^{W}+\gamma_{x} \widehat{x}_{t}^{W}, \\
\widehat{y}_{t}^{*}-\left(\frac{A^{\prime}\left(\bar{U}^{*}\right) \overline{\widetilde{K}}^{*} \bar{U}^{*}}{\bar{Y}^{*}-A\left(\bar{U}^{*}\right) \overline{\widetilde{K}}^{*}}\right) \widehat{u}_{t}^{*} \approx-\eta\left(\widehat{p}_{t}^{F, W *}-\widehat{p}_{t}^{W *}\right)+\left(1-\gamma_{x}\right) \widehat{c}_{t}^{W *}+\gamma_{x} \widehat{x}_{t}^{W *},
\end{gathered}
$$

which using our characterization of the steady state can be expressed more compactly as,

$$
\begin{gathered}
\widehat{y}_{t}-\left(\beta^{-1}-(1-\delta)\right)\left(\frac{\bar{K}}{\bar{Y}}\right) \widehat{u}_{t} \approx-\eta\left(\widehat{p}_{t}^{H, W}-\widehat{p}_{t}^{W}\right)+\left(1-\gamma_{x}\right) \widehat{c}_{t}^{W}+\gamma_{x} \widehat{x}_{t}^{W}, \\
\widehat{y}_{t}^{*}-\left(\beta^{-1}-(1-\delta)\right)\left(\frac{\bar{K}^{*}}{\bar{Y}^{*}}\right) \widehat{u}_{t}^{*} \approx-\eta\left(\widehat{p}_{t}^{F, W *}-\widehat{p}_{t}^{W *}\right)+\left(1-\gamma_{x}\right) \widehat{c}_{t}^{W *}+\gamma_{x} \widehat{x}_{t}^{W *} .
\end{gathered}
$$

We know that in steady state we can write the investment share as proportional to the capital-to-output ratio,

$$
\gamma_{x} \equiv \delta \frac{\bar{K}}{\bar{Y}}=\delta \frac{\bar{K}^{*}}{\bar{Y}^{*}}
$$

where physical capital and capital services are equated because the utilization rate is set to $\bar{U}=\bar{U}^{*}=1$. Therefore, we can re-express the aggregate output equations in the following terms,

$$
\begin{aligned}
& \widehat{y}_{t} \approx-\eta\left(\widehat{p}_{t}^{H, W}-\widehat{p}_{t}^{W}\right)+\left(1-\gamma_{x}\right) \widehat{c}_{t}^{W}+\gamma_{x} \widehat{x}_{t}^{W}+\gamma_{x}\left(\frac{1-\beta(1-\delta)}{\beta \delta}\right) \widehat{u}_{t}, \\
& \widehat{y}_{t}^{*} \approx-\eta\left(\widehat{p}_{t}^{F, W *}-\widehat{p}_{t}^{W *}\right)+\left(1-\gamma_{x}\right) \widehat{c}_{t}^{W *}+\gamma_{x} \widehat{x}_{t}^{W *}+\gamma_{x}\left(\frac{1-\beta(1-\delta)}{\beta \delta}\right) \widehat{u}_{t}^{*} .
\end{aligned}
$$

These equations become very important in our posterior derivations of the Phillips curves. Equations (251) (252) are identical to those derived in the model without capital utilization in (132) - (133) only when $\widehat{u}_{t}=\widehat{u}_{t}^{*}=0$. These equations calculate aggregate output from the demand-side by incorporating the capital utilization costs at the same time.

We note that if we define the world weighted consumption as $\widehat{c}_{t}^{W} \equiv \phi_{H} \widehat{c}_{t}+\phi_{F} \widehat{c}_{t}^{*}$ and $\widehat{c}_{t}^{W *} \equiv \phi_{F} \widehat{c}_{t}+\phi_{H} \widehat{c}_{t}^{*}$ and the relative consumption as $\widehat{c}_{t}^{R} \equiv \widehat{c}_{t}-\widehat{c}_{t}^{*}$, then we can write that,

$$
\begin{aligned}
\widehat{c}_{t} & =\widehat{c}_{t}^{W}+\phi_{F} \widehat{c}_{t}^{R} \approx \widehat{c}_{t}^{W}+\phi_{F} \sigma \widehat{r s}_{t}, \\
\widehat{c}_{t}^{*} & =\widehat{c}_{t}^{W *}-\phi_{F} \widehat{c}_{t}^{R} \approx \widehat{c}_{t}^{W *}-\phi_{F} \sigma \widehat{r s}_{t},
\end{aligned}
$$

where the second approximation follows from the perfect international risk-sharing condition in (99). This is a substitution trick that we have used before. Then, we can express the efficiency conditions in (128) - (129) 
as,

$$
\begin{aligned}
\widehat{r}_{t}^{z} & \approx-\frac{1+\varphi}{\psi} \widehat{a}_{t}+\frac{1}{\sigma} \widehat{c}_{t}^{W}+\phi_{F} \widehat{r s} t+\frac{1+\varphi}{\psi} \widehat{y}_{t}-\left(\frac{1+(1-\psi) \varphi}{\psi}\right) \widehat{k}_{t}, \\
\widehat{r}_{t}^{z *} & \approx-\frac{1+\varphi}{\psi} \widehat{a}_{t}^{*}+\frac{1}{\sigma} \widehat{c}_{t}^{W *}-\phi_{F} \widehat{r s} s_{t}+\frac{1+\varphi}{\psi} \widehat{y}_{t}^{*}-\left(\frac{1+(1-\psi) \varphi}{\psi}\right) \widehat{k}_{t}^{*} .
\end{aligned}
$$

Furthermore, if we combine these efficiency conditions with the output equations derived in (251) - (252), it follows that,

$$
\begin{aligned}
& \widehat{r}_{t}^{z} \approx\left(\frac{1}{\sigma}+\left(1-\gamma_{x}\right) \frac{1+\varphi}{\psi}\right) \widehat{c}_{t}^{W}+\gamma_{x} \frac{1+\varphi}{\psi} \widehat{x}_{t}^{W}+\gamma_{x} \frac{1+\varphi}{\psi}\left(\frac{1-\beta(1-\delta)}{\beta \delta}\right) \widehat{u}_{t}+\phi_{F} \widehat{r s}_{t}-\ldots \\
& \quad \eta \frac{1+\varphi}{\psi}\left(\widehat{p}_{t}^{H, W}-\widehat{p}_{t}^{W}\right)-\left(\frac{1+(1-\psi) \varphi}{\psi}\right) \widehat{k}_{t}-\frac{1+\varphi}{\psi} \widehat{a}_{t}, \\
& \widehat{r}_{t}^{z *} \approx\left(\frac{1}{\sigma}+\left(1-\gamma_{x}\right) \frac{1+\varphi}{\psi}\right) \widehat{c}_{t}^{W *}+\gamma_{x} \frac{1+\varphi}{\psi} \widehat{x}_{t}^{W *}+\gamma_{x} \frac{1+\varphi}{\psi}\left(\frac{1-\beta(1-\delta)}{\beta \delta}\right) \widehat{u}_{t}^{*}-\phi_{F} \widehat{r s}_{t}-\ldots \\
& \quad \eta \frac{1+\varphi}{\psi}\left(\widehat{p}_{t}^{F, W *}-\widehat{p}_{t}^{W *}\right)-\left(\frac{1+(1-\psi) \varphi}{\psi}\right) \widehat{k}_{t}^{*}-\frac{1+\varphi}{\psi} \widehat{a}_{t}^{*} .
\end{aligned}
$$

These conditions can be appropriately used to simplify the description of the inflation dynamics.

The Optimal Pricing Equations. In steady state the standard pricing rule under monopolistic competition holds. Accordingly, the log-linearization of the optimal pricing equations in (71), (72), (73) and (74) can be compactly expressed as follows,

$$
\begin{aligned}
& \widehat{\widetilde{p}}_{t}(h)-\widehat{p}_{t} \approx \mathbb{E}_{t}\left[\sum_{\tau=1}^{+\infty}(\beta \alpha)^{\tau} \widehat{\pi}_{t+\tau}\right]+(1-\beta \alpha) \mathbb{E}_{t}\left[\sum_{\tau=0}^{+\infty}(\beta \alpha)^{\tau}\left(\widehat{m c}_{t+\tau}-\widehat{p}_{t+\tau}\right)\right], \\
& \widehat{\widetilde{p}}_{t}^{*}(h)-\widehat{p}_{t}^{*} \approx \mathbb{E}_{t}\left[\sum_{\tau=1}^{+\infty}(\beta \alpha)^{\tau} \widehat{\pi}_{t+\tau}^{*}\right]+(1-\beta \alpha) \mathbb{E}_{t}\left[\sum_{\tau=0}^{+\infty}(\beta \alpha)^{\tau}\left(\widehat{m c}_{t+\tau}-\widehat{p}_{t+\tau}-\widehat{r s}_{t+\tau}\right)\right], \\
& \widehat{\widetilde{p}}_{t}(f)-\widehat{p}_{t} \approx \mathbb{E}_{t}\left[\sum_{\tau=1}^{+\infty}(\beta \alpha)^{\tau} \widehat{\pi}_{t+\tau}\right]+(1-\beta \alpha) \mathbb{E}_{t}\left[\sum_{\tau=0}^{+\infty}(\beta \alpha)^{\tau}\left(\widehat{m c}_{t+\tau}^{*}-\widehat{p}_{t+\tau}^{*}+\widehat{r s}_{t+\tau}\right)\right], \\
& \widetilde{\widetilde{p}}_{t}^{*}(f)-\widehat{p}_{t}^{*} \approx \mathbb{E}_{t}\left[\sum_{\tau=1}^{+\infty}(\beta \alpha)^{\tau} \widehat{\pi}_{t+\tau}^{*}\right]+(1-\beta \alpha) \mathbb{E}_{t}\left[\sum_{\tau=0}^{+\infty}(\beta \alpha)^{\tau}\left(\widehat{m c}_{t+\tau}^{*}-\widehat{p}_{t+\tau}^{*}\right)\right],
\end{aligned}
$$

which gives us the same pricing formulas that we obtained before without capital utilization. Here I must recall the assumption that the government subsidy is time-invariant and equal to its steady state value in every period, which explains why the government subsidies do not appear in the log-linearized pricing equations. We derive the (pre-subsidy) marginal cost equations in (20) - (21), and they can be log-linearized as,

$$
\begin{aligned}
& \widehat{m c}_{t+\tau} \approx \psi \widehat{w}_{t+\tau}+(1-\psi)\left(\widehat{r}_{t+\tau}^{z}+\widehat{p}_{t+\tau}\right)-\widehat{a}_{t+\tau} \\
& \widehat{m c}_{t+\tau}^{*} \approx \psi \widehat{w}_{t+\tau}^{*}+(1-\psi)\left(\widehat{r}_{t+\tau}^{* *}+\widehat{p}_{t+\tau}^{*}\right)-\widehat{a}_{t+\tau}^{*}
\end{aligned}
$$

while the labor market clearing conditions, which are implicit in $(95)-(96)$, can be approximated as,

$$
\begin{aligned}
\widehat{w}_{t+\tau} & \approx-\frac{\varphi}{1+(1-\psi) \varphi} \widehat{a}_{t+\tau}+\frac{1}{1+(1-\psi) \varphi} \frac{1}{\sigma} \widehat{c}_{t+\tau}+\frac{\varphi}{1+(1-\psi) \varphi} \widehat{y}_{t+\tau}+\frac{(1-\psi) \varphi}{1+(1-\psi) \varphi}\left(\widehat{r}_{t+\tau}^{z}+\widehat{p}_{t+\tau}\right)+\frac{1}{1+(1-\psi) \varphi} \widehat{p}_{t+\tau}, \\
\widehat{w}_{t+\tau}^{*} & \approx-\frac{\varphi}{1+(1-\psi) \varphi} \widehat{a}_{t+\tau}^{*}+\frac{1}{1+(1-\psi) \varphi} \frac{1}{\sigma} \widehat{c}_{t+\tau}^{*}+\frac{\varphi}{1+(1-\psi) \varphi} \widehat{y}_{t+\tau}^{*}+\frac{(1-\psi) \varphi}{1+(1-\psi) \varphi}\left(\widehat{r}_{t+\tau}^{z *}+\widehat{p}_{t+\tau}^{*}\right)+\frac{1}{1+(1-\psi) \varphi} \widehat{p}_{t+\tau}^{*} .
\end{aligned}
$$


If we combine these log-linearized equations, it follows that the marginal costs can be expressed as,

$$
\begin{aligned}
& \widehat{m c}_{t+\tau} \approx \frac{\psi}{1+(1-\psi) \varphi} \frac{1}{\sigma} \widehat{c}_{t+\tau}+\frac{\varphi \psi}{1+(1-\psi) \varphi} \widehat{y}_{t+\tau}-\frac{1+\varphi}{1+(1-\psi) \varphi}\left(\widehat{a}_{t+\tau}-(1-\psi)\left(\widehat{r}_{t+\tau}^{z}+\widehat{p}_{t+\tau}\right)\right)+\frac{\psi}{1+(1-\psi) \varphi} \widehat{p}_{t+\tau}, \\
& \widehat{m c}_{t+\tau}^{*} \approx \frac{\psi}{1+(1-\psi) \varphi} \frac{1}{\sigma} \widehat{c}_{t+\tau}^{*}+\frac{\varphi \psi}{1+(1-\psi) \varphi} \widehat{y}_{t+\tau}^{*}-\frac{1+\varphi}{1+(1-\psi) \varphi}\left(\widehat{a}_{t+\tau}^{*}-(1-\psi)\left(\widehat{r}_{t+\tau}^{* *}+\widehat{p}_{t+\tau}^{*}\right)\right)+\frac{\psi}{1+(1-\psi) \varphi} \widehat{p}_{t+\tau}^{*},
\end{aligned}
$$

where $\widehat{y}_{t+\tau}$ and $\widehat{y}_{t+\tau}^{*}$ denote domestic and foreign aggregate output. Up to this point, the derivation of marginal costs is the same independently of whether the model allows for capital utilization or not. If we combine the marginal cost equations with the output equations derived before in (251) - (252), it follows that,

$$
\begin{aligned}
& \widehat{m c}_{t+\tau}-\widehat{p}_{t+\tau} \approx \frac{\psi}{1+(1-\psi) \varphi} \frac{1}{\sigma} \widehat{c}_{t+\tau}-\frac{\varphi \psi}{1+(1-\psi) \varphi} \eta\left(\widehat{p}_{t+\tau}^{H, W}-\widehat{p}_{t+\tau}^{W}\right)+\ldots \\
& \frac{\varphi \psi}{1+(1-\psi) \varphi}\left(\left(1-\gamma_{x}\right) \widehat{c}_{t+\tau}^{W}+\gamma_{x} \widehat{x}_{t+\tau}^{W}+\gamma_{x}\left(\frac{1-\beta(1-\delta)}{\beta \delta}\right) \widehat{u}_{t+\tau}\right)-\ldots \\
& \frac{1+\varphi}{1+(1-\psi) \varphi}\left(\widehat{a}_{t+\tau}-(1-\psi) \widehat{r}_{t+\tau}^{z}\right), \\
& \widehat{m c}_{t+\tau}^{*}-\widehat{p}_{t+\tau}^{*} \approx \frac{\psi}{1+(1-\psi) \varphi} \frac{1}{\sigma} \widehat{c}_{t+\tau}^{*}-\frac{\varphi \psi}{1+(1-\psi) \varphi} \eta\left(\widehat{p}_{t+\tau}^{F, W *}-\widehat{p}_{t+\tau}^{W *}\right)+\ldots \\
& \frac{\varphi \psi}{1+(1-\psi) \varphi}\left(\left(1-\gamma_{x}\right) \widehat{c}_{t+\tau}^{W *}+\gamma_{x} \widehat{x}_{t+\tau}^{W *}+\gamma_{x}\left(\frac{1-\beta(1-\delta)}{\beta \delta}\right) \widehat{u}_{t+\tau}^{*}\right)-\ldots \\
& \quad \frac{1+\varphi}{1+(1-\psi) \varphi}\left(\widehat{a}_{t+\tau}^{*}-(1-\psi) \widehat{r}_{t+\tau}^{* *}\right) .
\end{aligned}
$$

This characterization of the real marginal costs is central to our derivations of the Phillips curve. It naturally shows that now marginal costs have to account for the costs of variable capital utilization.

We can use our characterization of the real marginal costs with the pricing formulas log-linearized before 
to write that,

$$
\begin{aligned}
& \widehat{\widetilde{p}}_{t}(h)-\widehat{p}_{t} \approx \\
& \left(\frac{(1-\beta \alpha) \psi}{1+(1-\psi) \varphi}\right) \sum_{\tau=0}^{+\infty}(\beta \alpha)^{\tau} \mathbb{E}_{t}\left[\begin{array}{c}
\frac{1}{\sigma} \widehat{c}_{t+\tau}-\varphi \eta\left(\widehat{p}_{t+\tau}^{H, W}-\widehat{p}_{t+\tau}^{W}\right)+\ldots \\
\varphi\left(\left(1-\gamma_{x}\right) \widehat{c}_{t+\tau}^{W}+\gamma_{x} \widehat{x}_{t+\tau}^{W}+\gamma_{x}\left(\frac{1-\beta(1-\delta)}{\beta \delta}\right) \widehat{u}_{t+\tau}\right)-\ldots \\
\left(\frac{1+\varphi}{\psi}\right)\left(\widehat{a}_{t+\tau}-(1-\psi) \widehat{r}_{t+\tau}^{z}\right)
\end{array}\right]+\ldots \\
& \sum_{\tau=1}^{+\infty}(\beta \alpha)^{\tau} \mathbb{E}_{t}\left(\widehat{\pi}_{t+\tau}\right), \\
& \widehat{\widetilde{p}}_{t}^{*}(h)-\widehat{p}_{t}^{*} \approx \\
& \left(\frac{(1-\beta \alpha) \psi}{1+(1-\psi) \varphi}\right) \sum_{\tau=0}^{+\infty}(\beta \alpha)^{\tau} \mathbb{E}_{t}\left[\begin{array}{c}
\frac{1}{\sigma} \widehat{c}_{t+\tau}-\varphi \eta\left(\widehat{p}_{t+\tau}^{H, W}-\widehat{p}_{t+\tau}^{W}\right)+\ldots \\
\varphi\left(\left(1-\gamma_{x}\right) \widehat{c}_{t+\tau}^{W}+\gamma_{x} \widehat{x}_{t+\tau}^{W}+\gamma_{x}\left(\frac{1-\beta(1-\delta)}{\beta \delta}\right) \widehat{u}_{t+\tau}\right)-\ldots \\
\left(\frac{1+\varphi}{\psi}\right)\left(\widehat{a}_{t+\tau}-(1-\psi) \widehat{r}_{t+\tau}^{z}\right)-\widehat{r s}_{t+\tau}
\end{array}\right]+\ldots \\
& \sum_{\tau=1}^{+\infty}(\beta \alpha)^{\tau} \mathbb{E}_{t}\left(\widehat{\pi}_{t+\tau}^{*}\right), \\
& \widehat{\widetilde{p}}_{t}(f)-\widehat{p}_{t} \approx \\
& \left(\frac{(1-\beta \alpha) \psi}{1+(1-\psi) \varphi}\right) \sum_{\tau=0}^{+\infty}(\beta \alpha)^{\tau} \mathbb{E}_{t}\left[\begin{array}{c}
\frac{1}{\sigma} \widehat{c}_{t+\tau}^{*}-\varphi \eta\left(\widehat{p}_{t+\tau}^{F, W *}-\widehat{p}_{t+\tau}^{W *}\right)+\ldots \\
\varphi\left(\left(1-\gamma_{x}\right) \widehat{c}_{t+\tau}^{W *}+\gamma_{x} \widehat{x}_{t+\tau}^{W *}+\gamma_{x}\left(\frac{1-\beta(1-\delta)}{\beta \delta}\right) \widehat{u}_{t+\tau}^{*}\right)-\ldots \\
\left(\frac{1+\varphi}{\psi}\right)\left(\widehat{a}_{t+\tau}^{*}-(1-\psi) \widehat{r}_{t+\tau}^{z *}\right)+\widehat{r s}_{t+\tau}
\end{array}\right]+\ldots \\
& \sum_{\tau=1}^{+\infty}(\beta \alpha)^{\tau} \mathbb{E}_{t}\left(\widehat{\pi}_{t+\tau}\right), \\
& \widehat{\widetilde{p}}_{t}^{*}(f)-\widehat{p}_{t}^{*} \approx
\end{aligned}
$$

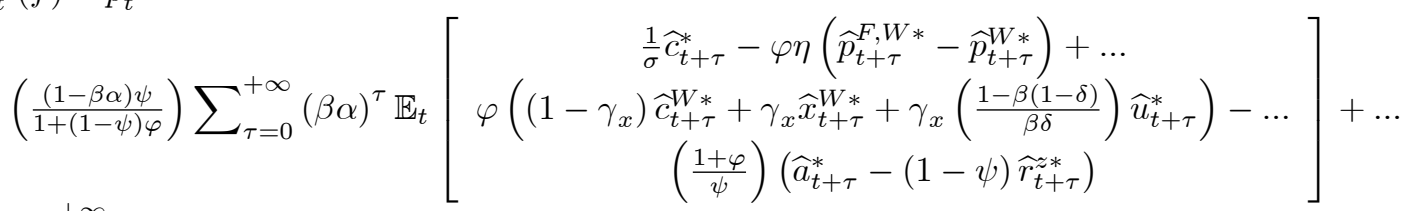

$$
\begin{aligned}
& \sum_{\tau=1}^{+\infty}(\beta \alpha)^{\tau} \mathbb{E}_{t}\left(\hat{\pi}_{t+\tau}^{*}\right) .
\end{aligned}
$$

We log-linearize the price sub-indexes in $(75)$ - (76) and (77) - (78) and re-arrange them to obtain that,

$$
\begin{aligned}
& \widehat{\widetilde{p}}_{t}(h)-\widehat{p}_{t} \approx\left(\widehat{p}_{t}^{H}-\widehat{p}_{t}\right)+\left(\frac{\alpha}{1-\alpha}\right) \widehat{\pi}_{t}^{H}, \\
& \widehat{\widetilde{p}}_{t}^{*}(h)-\widehat{p}_{t}^{*} \approx\left(\widehat{p}_{t}^{H *}-\widehat{p}_{t}^{*}\right)+\left(\frac{\alpha}{1-\alpha}\right) \widehat{\pi}_{t}^{H *}, \\
& \widehat{\widetilde{p}}_{t}(f)-\widehat{p}_{t} \approx\left(\widehat{p}_{t}^{F}-\widehat{p}_{t}\right)+\left(\frac{\alpha}{1-\alpha}\right) \widehat{\pi}_{t}^{F}, \\
& \widehat{\widetilde{p}}_{t}^{*}(f)-\widehat{p}_{t}^{*} \approx\left(\widehat{p}_{t}^{F *}-\widehat{p}_{t}^{*}\right)+\left(\frac{\alpha}{1-\alpha}\right) \widehat{\pi}_{t}^{F *},
\end{aligned}
$$

which is quite convenient to aggregate the optimal pricing decision of firms. We replace the isolated terms $\frac{1}{\sigma} \widehat{c}_{t}$ and $\frac{1}{\sigma} \widehat{c}_{t}^{*}$ out of the marginal cost. If we define the world consumption as $\widehat{c}_{t}^{W} \equiv \phi_{H} \widehat{c}_{t}+\phi_{F} \widehat{c}_{t}^{*}$ and 
$\widehat{c}_{t}^{W *} \equiv \phi_{F} \widehat{c}_{t}+\phi_{H} \widehat{c}_{t}^{*}$ and the relative consumption as $\widehat{c}_{t}^{R} \equiv \widehat{c}_{t}-\widehat{c}_{t}^{*}$, then we can write that,

$$
\begin{aligned}
& \widehat{c}_{t}=\widehat{c}_{t}^{W}+\phi_{F} \widehat{c}_{t}^{R} \approx \widehat{c}_{t}^{W}+\phi_{F} \sigma \widehat{r} s_{t}, \\
& \widehat{c}_{t}^{*}=\widehat{c}_{t}^{W *}-\phi_{F} \widehat{c}_{t}^{R} \approx \widehat{c}_{t}^{W *}-\phi_{F} \sigma \widehat{r s}_{t},
\end{aligned}
$$

where the second approximation follows from the perfect international risk-sharing condition in (99). Hence, the optimal pricing equations can be expressed more compactly as,

$$
\begin{aligned}
& \widehat{\pi}_{t}^{H}+\left(\frac{1-\alpha}{\alpha}\right)\left(\widehat{p}_{t}^{H}-\widehat{p}_{t}\right) \approx \\
& \Psi\left(\frac{\psi}{1+(1-\psi) \varphi}\right) \sum_{\tau=0}^{+\infty}(\beta \alpha)^{\tau} \mathbb{E}_{t}\left[\begin{array}{c}
\left(\sigma^{-1}+\varphi\left(1-\gamma_{x}\right)\right) \widehat{c}_{t+\tau}^{W}+\varphi \gamma_{x} \widehat{x}_{t+\tau}^{W}+\varphi \gamma_{x}\left(\frac{1-\beta(1-\delta)}{\beta \delta}\right) \widehat{u}_{t+\tau}+\ldots \\
\phi_{F} \widehat{r s s}_{t+\tau}-\varphi \eta\left(\widehat{p}_{t+\tau}^{H, W}-\widehat{p}_{t+\tau}^{W}\right)-\ldots \\
\left(\frac{1+\varphi}{\psi}\right)\left(\widehat{a}_{t+\tau}-(1-\psi) \widehat{r}_{t+\tau}^{z}\right)
\end{array}\right]+\ldots \\
& \left(\frac{1-\alpha}{\alpha}\right) \sum_{\tau=1}^{+\infty}(\beta \alpha)^{\tau} \mathbb{E}_{t}\left(\widehat{\pi}_{t+\tau}\right), \\
& \widehat{\pi}_{t}^{H *}+\left(\frac{1-\alpha}{\alpha}\right)\left(\widehat{p}_{t}^{H *}-\widehat{p}_{t}^{*}\right) \approx \\
& \Psi\left(\frac{\psi}{1+(1-\psi) \varphi}\right) \sum_{\tau=0}^{+\infty}(\beta \alpha)^{\tau} \mathbb{E}_{t}\left[\begin{array}{c}
\left(\sigma^{-1}+\left(1-\gamma_{x}\right) \varphi\right) \widehat{c}_{t+\tau}^{W}+\gamma_{x} \varphi \widehat{x}_{t+\tau}^{W}+\varphi \gamma_{x}\left(\frac{1-\beta(1-\delta)}{\beta \delta}\right) \widehat{u}_{t+\tau}-\ldots \\
\left(\phi_{H}+\frac{1-\psi}{\psi}(1+\varphi)\right) \widehat{r s}_{t+\tau}-\varphi \eta\left(\widehat{p}_{t+\tau}^{H, W}-\widehat{p}_{t+\tau}^{W}\right)-\ldots \\
\left(\frac{1+\varphi}{\psi}\right)\left(\widehat{a}_{t+\tau}-(1-\psi) \widehat{r}_{t+\tau}^{z}\right)
\end{array}\right]+\ldots \\
& \left(\frac{1-\alpha}{\alpha}\right) \sum_{\tau=1}^{+\infty}(\beta \alpha)^{\tau} \mathbb{E}_{t}\left(\widehat{\pi}_{t+\tau}^{*}\right), \\
& \widehat{\pi}_{t}^{F}+\left(\frac{1-\alpha}{\alpha}\right)\left(\widehat{p}_{t}^{F}-\widehat{p}_{t}\right) \approx \\
& \Psi\left(\frac{\psi}{1+(1-\psi) \varphi}\right) \sum_{\tau=0}^{+\infty}(\beta \alpha)^{\tau} \mathbb{E}_{t}\left[\begin{array}{c}
\left(\sigma^{-1}+\left(1-\gamma_{x}\right) \varphi\right) \widehat{c}_{t+\tau}^{W *}+\gamma_{x} \varphi \widehat{x}_{t+\tau}^{W *}+\varphi \gamma_{x}\left(\frac{1-\beta(1-\delta)}{\beta \delta}\right) \widehat{u}_{t+\tau}^{*}+\ldots \\
\left(\phi_{H}+\frac{1-\psi}{\psi}(1+\varphi)\right) \widehat{r s}_{t+\tau}-\varphi \eta\left(\widehat{p}_{t+\tau}^{F, W *}-\widehat{p}_{t+\tau}^{W *}\right)-\ldots \\
\left(\frac{1+\varphi}{\psi}\right)\left(\widehat{a}_{t+\tau}^{*}-(1-\psi) \widehat{r}_{t+\tau}^{z *}\right)
\end{array}\right]+\ldots \\
& \left(\frac{1-\alpha}{\alpha}\right) \sum_{\tau=1}^{+\infty}(\beta \alpha)^{\tau} \mathbb{E}_{t}\left(\widehat{\pi}_{t+\tau}\right), \\
& \widehat{\pi}_{t}^{F *}+\left(\frac{1-\alpha}{\alpha}\right)\left(\widehat{p}_{t}^{F *}-\widehat{p}_{t}^{*}\right) \approx \\
& \Psi\left(\frac{\psi}{1+(1-\psi) \varphi}\right) \sum_{\tau=0}^{+\infty}(\beta \alpha)^{\tau} \mathbb{E}_{t}\left[\begin{array}{c}
\left(\sigma^{-1}+\left(1-\gamma_{x}\right) \varphi\right) \widehat{c}_{t+\tau}^{W *}+\gamma_{x} \varphi \widehat{x}_{t+\tau}^{W *}+\varphi \gamma_{x}\left(\frac{1-\beta(1-\delta)}{\beta \delta}\right) \widehat{u}_{t+\tau}^{*}-\ldots \\
\phi_{F} \widehat{r s s}_{t+\tau}-\varphi \eta\left(\widehat{p}_{t+\tau}^{F, W *}-\widehat{p}_{t+\tau}^{W *}\right)-\ldots \\
\left(\frac{1+\varphi}{\psi}\right)\left(\widehat{a}_{t+\tau}^{*}-(1-\psi) \widehat{r}_{t+\tau}^{z *}\right)
\end{array}\right]+\ldots \\
& \left(\frac{1-\alpha}{\alpha}\right) \sum_{\tau=1}^{+\infty}(\beta \alpha)^{\tau} \mathbb{E}_{t}\left(\widehat{\pi}_{t+\tau}^{*}\right),
\end{aligned}
$$

where $\Psi \equiv \frac{(1-\alpha)(1-\beta \alpha)}{\alpha}$.

Furthermore, these pricing equations can be expressed in the form of a system of expectational difference equations. Let us focus on the first equation as an example. If we re-write the equation at time $t+1$ and 
take conditional expectations with all the information available up to time $t$, it should follow that,

$$
\begin{aligned}
& \mathbb{E}_{t}\left[\widehat{\pi}_{t+1}^{H}+\left(\frac{1-\alpha}{\alpha}\right)\left(\widehat{p}_{t+1}^{H}-\widehat{p}_{t+1}\right)\right] \approx \approx \\
& \Psi\left(\frac{\psi}{1+(1-\psi) \varphi}\right) \sum_{\tau=0}^{+\infty}(\beta \alpha)^{\tau} \mathbb{E}_{t}\left[\begin{array}{c}
\left(\sigma^{-1}+\left(1-\gamma_{x}\right) \varphi\right) \widehat{c}_{t+1+\tau}^{W}+\gamma_{x} \varphi \widehat{x}_{t+1+\tau}^{W}+\varphi \gamma_{x}\left(\frac{1-\beta(1-\delta)}{\beta \delta}\right) \widehat{u}_{t+1+\tau}+\ldots \\
\phi_{F} \widehat{r s s}_{t+1+\tau}-\varphi \eta\left(\widehat{p}_{t+1+\tau}^{H, W}-\widehat{p}_{t+1+\tau}^{W}\right)-\ldots \\
\left(\frac{1+\varphi}{\psi}\right)\left(\widehat{a}_{t+1+\tau}-(1-\psi) \widehat{r}_{t+1+\tau}^{z}\right)
\end{array}\right]+\ldots \\
&\left(\frac{1-\alpha}{\alpha}\right) \sum_{\tau=1}^{+\infty}(\beta \alpha)^{\tau} \mathbb{E}_{t}\left(\widehat{\pi}_{t+1+\tau}\right) .
\end{aligned}
$$

Hence, using the properties of the conditional expectation, the pricing equation can easily be decomposed in two terms as,

$$
\begin{aligned}
& \widehat{\pi}_{t}^{H}+\left(\frac{1-\alpha}{\alpha}\right)\left(\widehat{p}_{t}^{H}-\widehat{p}_{t}\right) \approx \\
& \left(\frac{\psi}{1+(1-\psi) \varphi}\right) \Psi\left[\begin{array}{c}
\left(\sigma^{-1}+\left(1-\gamma_{x}\right) \varphi\right) \widehat{c}_{t}^{W}+\gamma_{x} \varphi \widehat{x}_{t}^{W}+\varphi \gamma_{x}\left(\frac{1-\beta(1-\delta)}{\beta \delta}\right) \widehat{u}_{t}+\ldots \\
\phi_{F} \widehat{r s s}_{t}-\varphi \eta\left(\widehat{p}_{t}^{H, W}-\widehat{p}_{t}^{W}\right)-\ldots \\
\left(\frac{1+\varphi}{\psi}\right)\left(\widehat{a}_{t}-(1-\psi) \widehat{r}_{t}^{z}\right)
\end{array}\right]+\ldots \\
& (1-\alpha) \beta \mathbb{E}_{t}\left(\widehat{\pi}_{t+1}\right)+\alpha \beta \mathbb{E}_{t}\left[\widehat{\pi}_{t+1}^{H}+\left(\frac{1-\alpha}{\alpha}\right)\left(\widehat{p}_{t+1}^{H}-\widehat{p}_{t+1}\right)\right] .
\end{aligned}
$$

Further re-arranging allows us to express the expectational difference equation as,

$$
\begin{aligned}
& \widehat{\pi}_{t}^{H}-\beta \mathbb{E}_{t}\left(\widehat{\pi}_{t+1}^{H}\right)+\Psi\left(\widehat{p}_{t}^{H}-\widehat{p}_{t}\right) \\
& \approx\left(\frac{\psi}{1+(1-\psi) \varphi}\right) \Psi\left[\begin{array}{c}
\left(\sigma^{-1}+\left(1-\gamma_{x}\right) \varphi\right) \widehat{c}_{t}^{W}+\gamma_{x} \varphi \widehat{x}_{t}^{W}+\varphi \gamma_{x}\left(\frac{1-\beta(1-\delta)}{\beta \delta}\right) \widehat{u}_{t}+\ldots \\
\phi_{F} \widehat{r s}_{t}-\varphi \eta\left(\widehat{p}_{t}^{H, W}-\widehat{p}_{t}^{W}\right)-\ldots \\
\left(\frac{1+\varphi}{\psi}\right)\left(\widehat{a}_{t}-(1-\psi) \widehat{r}_{t}^{Z}\right)
\end{array}\right] .
\end{aligned}
$$

We can apply the same approach (and algebraic steps) to re-write all other pricing equations as expectational 
difference equations, i.e.,

$$
\begin{aligned}
& \widehat{\pi}_{t}^{H *}-\beta \mathbb{E}_{t}\left(\widehat{\pi}_{t+1}^{H *}\right)+\Psi\left(\widehat{p}_{t}^{H *}-\widehat{p}_{t}^{*}\right) \\
& \approx\left(\frac{\psi}{1+(1-\psi) \varphi}\right) \Psi\left[\begin{array}{c}
\left(\sigma^{-1}+\left(1-\gamma_{x}\right) \varphi\right) \widehat{c}_{t}^{W}+\gamma_{x} \varphi \widehat{x}_{t}^{W}+\varphi \gamma_{x}\left(\frac{1-\beta(1-\delta)}{\beta \delta}\right) \widehat{u}_{t}-\ldots \\
\left(\phi_{H}+\frac{1-\psi}{\psi}(1+\varphi)\right) \widehat{r s}_{t}-\varphi \eta\left(\widehat{p}_{t}^{H, W}-\widehat{p}_{t}^{W}\right)-\ldots \\
\left(\frac{1+\varphi}{\psi}\right)\left(\widehat{a}_{t}-(1-\psi) \widehat{r}_{t}^{Z}\right)
\end{array}\right], \\
& \widehat{\pi}_{t}^{F}-\beta \mathbb{E}_{t}\left(\widehat{\pi}_{t+1}^{F}\right)+\Psi\left(\widehat{p}_{t}^{F}-\widehat{p}_{t}\right) \\
& \approx\left(\frac{\psi}{1+(1-\psi) \varphi}\right) \Psi\left[\begin{array}{c}
\left(\sigma^{-1}+\left(1-\gamma_{x}\right) \varphi\right) \widehat{c}_{t}^{W *}+\gamma_{x} \varphi \widehat{x}_{t}^{W *}+\varphi \gamma_{x}\left(\frac{1-\beta(1-\delta)}{\beta \delta}\right) \widehat{u}_{t}^{*}+\ldots \\
\left(\phi_{H}+\frac{1-\psi}{\psi}(1+\varphi)\right) \widehat{r s}_{t}-\varphi \eta\left(\widehat{p}_{t}^{F, W *}-\widehat{p}_{t}^{W *}\right)-\ldots \\
\left(\frac{1+\varphi}{\psi}\right)\left(\widehat{a}_{t}^{*}-(1-\psi) \widehat{r}_{t}^{z *}\right)
\end{array}\right], \\
& \widehat{\pi}_{t}^{F *}-\beta \mathbb{E}_{t}\left(\widehat{\pi}_{t+1}^{F *}\right)+\Psi\left(\widehat{p}_{t}^{F *}-\widehat{p}_{t}^{*}\right) \\
& \approx\left(\frac{\psi}{1+(1-\psi) \varphi}\right) \Psi\left[\begin{array}{c}
\left(\sigma^{-1}+\left(1-\gamma_{x}\right) \varphi\right) \widehat{c}_{t}^{W *}+\gamma_{x} \varphi \widehat{x}_{t}^{W *}+\varphi \gamma_{x}\left(\frac{1-\beta(1-\delta)}{\beta \delta}\right) \widehat{u}_{t}^{*}-\ldots \\
\phi_{F} \widehat{r s}_{t}-\varphi \eta\left(\widehat{p}_{t}^{F, W *}-\widehat{p}_{t}^{W *}\right)-\ldots \\
\left(\frac{1+\varphi}{\psi}\right)\left(\widehat{a}_{t}^{*}-(1-\psi) \widehat{r}_{t}^{z *}\right)
\end{array}\right] .
\end{aligned}
$$

These equations provide a very simple characterization of the dynamics at the price sub-index level.

Now, we use the pricing equations described above to infer the dynamics of the relative price sub-indexes $\widehat{\pi}_{t}^{H, R} \equiv \widehat{\pi}_{t}^{H}-\widehat{\pi}_{t}^{H *}\left(\widehat{p}_{t}^{H, R} \equiv \widehat{p}_{t}^{H}-\widehat{p}_{t}^{H *}\right)$ and $\widehat{\pi}_{t}^{F, R} \equiv \widehat{\pi}_{t}^{F}-\widehat{\pi}_{t}^{F *}\left(\widehat{p}_{t}^{F, R} \equiv \widehat{p}_{t}^{F}-\widehat{p}_{t}^{F *}\right)$ as follows,

$$
\begin{aligned}
\widehat{\pi}_{t}^{H, R}-\beta \mathbb{E}_{t}\left(\widehat{\pi}_{t+1}^{H, R}\right)+\Psi\left(\widehat{p}_{t}^{H, R}-\widehat{p}_{t}^{R}\right) & \approx \Psi \widehat{r}_{t}, \\
\widehat{\pi}_{t}^{F, R}-\beta \mathbb{E}_{t}\left(\widehat{\pi}_{t+1}^{F, R}\right)+\Psi\left(\widehat{p}_{t}^{F, R}-\widehat{p}_{t}^{R}\right) & \approx \Psi \widehat{r s}_{t},
\end{aligned}
$$

where $\widehat{p}_{t}^{R} \equiv \widehat{p}_{t}-\widehat{p}_{t}^{*}$ is the relative CPI. We can re-write the pricing equations further at the price sub-index 
level as follows,

$$
\begin{aligned}
& \widehat{\pi}_{t}^{H} \approx \beta \mathbb{E}_{t}\left(\widehat{\pi}_{t+1}^{H}\right)+\ldots \\
& \left(\frac{\psi}{1+(1-\psi) \varphi}\right) \Psi\left[\begin{array}{c}
\left(\sigma^{-1}+\left(1-\gamma_{x}\right) \varphi\right) \widehat{c}_{t}^{W}+\gamma_{x} \varphi \widehat{x}_{t}^{W}+\varphi \gamma_{x}\left(\frac{1-\beta(1-\delta)}{\beta \delta}\right) \widehat{u}_{t}+\ldots \\
\phi_{F} \widehat{r s}_{t}-\varphi \eta\left(\widehat{p}_{t}^{H, W}-\widehat{p}_{t}^{W}\right)-\ldots \\
\left(\widehat{p}_{t}^{H}-\widehat{p}_{t}\right)-\left(\frac{1+\varphi}{\psi}\right)\left(\widehat{a}_{t}-(1-\psi) \widehat{r}_{t}^{z}\right)
\end{array}\right], \\
& \widehat{\pi}_{t}^{H *} \approx \beta \mathbb{E}_{t}\left(\widehat{\pi}_{t+1}^{H *}\right)+\ldots \\
& \left(\frac{\psi}{1+(1-\psi) \varphi}\right) \Psi\left[\begin{array}{c}
\left(\sigma^{-1}+\left(1-\gamma_{x}\right) \varphi\right) \widehat{c}_{t}^{W}+\gamma_{x} \varphi \widehat{x}_{t}^{W}+\varphi \gamma_{x}\left(\frac{1-\beta(1-\delta)}{\beta \delta}\right) \widehat{u}_{t}-\ldots \\
\left(\phi_{H}+\frac{1-\psi}{\psi}(1+\varphi)\right) \widehat{r}_{t}-\varphi \eta\left(\widehat{p}_{t}^{H, W}-\widehat{p}_{t}^{W}\right)-\ldots \\
\left(\widehat{p}_{t}^{H *}-\widehat{p}_{t}^{*}\right)-\left(\frac{1+\varphi}{\psi}\right)\left(\widehat{a}_{t}-(1-\psi) \widehat{r}_{t}^{z}\right)
\end{array}\right], \\
& \widehat{\pi}_{t}^{F} \approx \beta \mathbb{E}_{t}\left(\widehat{\pi}_{t+1}^{F}\right)+\ldots \\
& \left(\frac{\psi}{1+(1-\psi) \varphi}\right) \Psi\left[\begin{array}{c}
\left(\sigma^{-1}+\left(1-\gamma_{x}\right) \varphi\right) \widehat{c}_{t}^{W *}+\gamma_{x} \varphi \widehat{x}_{t}^{W *}+\varphi \gamma_{x}\left(\frac{1-\beta(1-\delta)}{\beta \delta}\right) \widehat{u}_{t}^{*}+\ldots \\
\left(\phi_{H}+\frac{1-\psi}{\psi}(1+\varphi)\right) \widehat{r s}_{t}-\varphi \eta\left(\widehat{p}_{t}^{F, W *}-\widehat{p}_{t}^{W *}\right)-\ldots \\
\left(\widehat{p}_{t}^{F}-\widehat{p}_{t}\right)-\left(\frac{1+\varphi}{\psi}\right)\left(\widehat{a}_{t}^{*}-(1-\psi) \widehat{r}_{t}^{z *}\right)
\end{array}\right] \\
& \widehat{\pi}_{t}^{F *} \approx \beta \mathbb{E}_{t}\left(\widehat{\pi}_{t+1}^{F *}\right)+\ldots \\
& \left(\frac{\psi}{1+(1-\psi) \varphi}\right) \Psi\left[\begin{array}{c}
\left(\sigma^{-1}+\left(1-\gamma_{x}\right) \varphi\right) \widehat{c}_{t}^{W *}+\gamma_{x} \varphi \widehat{x}_{t}^{W *}+\varphi \gamma_{x}\left(\frac{1-\beta(1-\delta)}{\beta \delta}\right) \widehat{u}_{t}^{*}-\ldots \\
\phi_{F} \widehat{r s s}_{t}-\varphi \eta\left(\widehat{p}_{t}^{F, W *}-\widehat{p}_{t}^{W *}\right)-\ldots \\
\left(\widehat{p}_{t}^{F *}-\widehat{p}_{t}^{*}\right)-\left(\frac{1+\varphi}{\psi}\right)\left(\widehat{a}_{t}^{*}-(1-\psi) \widehat{r}_{t}^{* *}\right)
\end{array}\right] .
\end{aligned}
$$

We have defined the world weighted price sub-indexes as $\widehat{p}_{t}^{H, W} \equiv \phi_{H} \widehat{p}_{t}^{H}+\phi_{F} \widehat{p}_{t}^{H *}$ and $\widehat{p}_{t}^{F, W *} \equiv \phi_{F} \widehat{p}_{t}^{F}+\phi_{H} \widehat{p}_{t}^{F *}$, and the relative price sub-indexes as $\widehat{p}_{t}^{H, R} \equiv \widehat{p}_{t}^{H}-\widehat{p}_{t}^{H *}$ and $\widehat{p}_{t}^{F, R} \equiv \widehat{p}_{t}^{F}-\widehat{p}_{t}^{F *}$. Then, naturally, we can write that,

$$
\begin{aligned}
\widehat{p}_{t}^{H} & =\widehat{p}_{t}^{H, W}+\phi_{F} \widehat{p}_{t}^{H, R}, \widehat{p}_{t}^{H *}=\widehat{p}_{t}^{H, W}-\phi_{H} \widehat{p}_{t}^{H, R}, \\
\widehat{p}_{t}^{F} & =\widehat{p}_{t}^{F, W *}+\phi_{H} \widehat{p}_{t}^{F, R}, \widehat{p}_{t}^{F *}=\widehat{p}_{t}^{F, W *}-\phi_{F} \widehat{p}_{t}^{F, R} .
\end{aligned}
$$

Analogously, we have defined the world weighted CPI as $\widehat{p}_{t}^{W} \equiv \phi_{H} \widehat{p}_{t}+\phi_{F} \widehat{p}_{t}^{*}$ and $\widehat{p}_{t}^{W *} \equiv \phi_{F} \widehat{p}_{t}+\phi_{H} \widehat{p}_{t}^{*}$, and the relative CPI as $\widehat{p}_{t}^{R} \equiv \widehat{p}_{t}-\widehat{p}_{t}^{*}$. Then, we can write that,

$$
\begin{aligned}
& \widehat{p}_{t}=\widehat{p}_{t}^{W}+\phi_{F} \widehat{p}_{t}^{R}, \widehat{p}_{t}^{*}=\widehat{p}_{t}^{W}-\phi_{H} \widehat{p}_{t}^{R} \\
& \widehat{p}_{t}=\widehat{p}_{t}^{W *}+\phi_{H} \widehat{p}_{t}^{R}, \widehat{p}_{t}^{*}=\widehat{p}_{t}^{W *}-\phi_{F} \widehat{p}_{t}^{R}
\end{aligned}
$$


Therefore, we can re-arrange the pricing equations as follows,

$$
\begin{aligned}
& \widehat{\pi}_{t}^{H} \approx \beta \mathbb{E}_{t}\left(\widehat{\pi}_{t+1}^{H}\right)+\ldots \\
& \left(\frac{\psi}{1+(1-\psi) \varphi}\right) \Psi\left[\begin{array}{c}
\left(\sigma^{-1}+\left(1-\gamma_{x}\right) \varphi\right) \widehat{c}_{t}^{W}+\gamma_{x} \varphi \widehat{x}_{t}^{W}+\varphi \gamma_{x}\left(\frac{1-\beta(1-\delta)}{\beta \delta}\right) \widehat{u}_{t}+\ldots \\
\phi_{F} \widehat{r s s}_{t}-\left(\frac{1+(1-\psi) \varphi}{\psi}+\varphi \eta\right)\left(\widehat{p}_{t}^{H, W}-\widehat{p}_{t}^{W}\right)-\ldots \\
\left(\frac{1+(1-\psi) \varphi}{\psi}\right) \phi_{F}\left(\widehat{p}_{t}^{H, R}-\widehat{p}_{t}^{R}\right)-\left(\frac{1+\varphi}{\psi}\right)\left(\widehat{a}_{t}-(1-\psi) \widehat{r}_{t}^{z}\right)
\end{array}\right], \\
& \widehat{\pi}_{t}^{H *} \approx \beta \mathbb{E}_{t}\left(\widehat{\pi}_{t+1}^{H *}\right)+\ldots \\
& \left(\frac{\psi}{1+(1-\psi) \varphi}\right) \Psi\left[\begin{array}{c}
\left(\sigma^{-1}+\left(1-\gamma_{x}\right) \varphi\right) \widehat{c}_{t}^{W}+\gamma_{x} \varphi \widehat{x}_{t}^{W}+\varphi \gamma_{x}\left(\frac{1-\beta(1-\delta)}{\beta \delta}\right) \widehat{u}_{t}-\ldots \\
\left(\phi_{H}+\frac{1-\psi}{\psi}(1+\varphi)\right) \widehat{r s}_{t}-\left(\frac{1+(1-\psi) \varphi}{\psi}+\varphi \eta\right)\left(\widehat{p}_{t}^{H, W}-\widehat{p}_{t}^{W}\right)+\ldots \\
\left(\frac{1+(1-\psi) \varphi}{\psi}\right) \phi_{H}\left(\widehat{p}_{t}^{H, R}-\widehat{p}_{t}^{R}\right)-\left(\frac{1+\varphi}{\psi}\right)\left(\widehat{a}_{t}-(1-\psi) \widehat{r}_{t}^{z}\right)
\end{array}\right], \\
& \widehat{\pi}_{t}^{F} \approx \beta \mathbb{E}_{t}\left(\widehat{\pi}_{t+1}^{F}\right)+\ldots \\
& \left(\frac{\psi}{1+(1-\psi) \varphi}\right) \Psi\left[\begin{array}{c}
\left(\sigma^{-1}+\left(1-\gamma_{x}\right) \varphi\right) \widehat{c}_{t}^{W *}+\gamma_{x} \varphi \widehat{x}_{t}^{W *}+\varphi \gamma_{x}\left(\frac{1-\beta(1-\delta)}{\beta \delta}\right) \widehat{u}_{t}^{*}+\ldots \\
\left(\phi_{H}+\frac{1-\psi}{\psi}(1+\varphi)\right) \widehat{r s}_{t}-\left(\frac{1+(1-\psi) \varphi}{\psi}+\varphi \eta\right)\left(\widehat{p}_{t}^{F, W *}-\widehat{p}_{t}^{W *}\right)-\ldots \\
\left(\frac{1+(1-\psi) \varphi}{\psi}\right) \phi_{H}\left(\widehat{p}_{t}^{F, R}-\widehat{p}_{t}^{R}\right)-\left(\frac{1+\varphi}{\psi}\right)\left(\widehat{a}_{t}^{*}-(1-\psi) \widehat{r}_{t}^{z *}\right)
\end{array}\right], \\
& \widehat{\pi}_{t}^{F *} \approx \beta \mathbb{E}_{t}\left(\widehat{\pi}_{t+1}^{F *}\right)+\ldots \\
& \left(\frac{\psi}{1+(1-\psi) \varphi}\right) \Psi\left[\begin{array}{c}
\left(\sigma^{-1}+\left(1-\gamma_{x}\right) \varphi\right) \widehat{c}_{t}^{W *}+\gamma_{x} \varphi \widehat{x}_{t}^{W *}+\varphi \gamma_{x}\left(\frac{1-\beta(1-\delta)}{\beta \delta}\right) \widehat{u}_{t}^{*}-\ldots \\
\phi_{F} \widehat{r s}_{t}-\left(\frac{1+(1-\psi) \varphi}{\psi}+\varphi \eta\right)\left(\widehat{p}_{t}^{F, W *}-\widehat{p}_{t}^{W *}\right)+\ldots \\
\left(\frac{1+(1-\psi) \varphi}{\psi}\right) \phi_{F}\left(\widehat{p}_{t}^{F, R}-\widehat{p}_{t}^{R}\right)-\left(\frac{1+\varphi}{\psi}\right)\left(\widehat{a}_{t}^{*}-(1-\psi) \widehat{r}_{t}^{z *}\right)
\end{array}\right] .
\end{aligned}
$$

By appropriately replacing the efficiency conditions in (253) - (254), and after a little bit of algebra, we can 
now re-arrange the pricing equations for the price sub-indexes in $(257)-(260)$ as follows,

$$
\begin{aligned}
& \widehat{\pi}_{t}^{H} \approx \beta \mathbb{E}_{t}\left(\widehat{\pi}_{t+1}^{H}\right)+\ldots \\
& \Psi\left[\begin{array}{c}
\left(\sigma^{-1}+\left(1-\gamma_{x}\right)\left(\frac{\psi}{1+(1-\psi) \varphi}\right)\left(\varphi+(1-\psi)\left(\frac{1+\varphi}{\psi}\right)^{2}\right)\right) \widehat{c}_{t}^{W}+\ldots \\
\gamma_{x}\left(\frac{\psi}{1+(1-\psi) \varphi}\right)\left(\varphi+(1-\psi)\left(\frac{1+\varphi}{\psi}\right)^{2}\right) \widehat{x}_{t}^{W}+\ldots \\
\gamma_{x}\left(\frac{\psi}{1+(1-\psi) \varphi}\right)\left(\varphi+(1-\psi)\left(\frac{1+\varphi}{\psi}\right)^{2}\right)\left(\frac{1-\beta(1-\delta)}{\beta \delta}\right) \widehat{u}_{t}+\ldots \\
\phi_{F} \widehat{r s}_{t}-\left(1+\eta\left(\frac{\psi}{1+(1-\psi) \varphi}\right)\left(\varphi+(1-\psi)\left(\frac{1+\varphi}{\psi}\right)^{2}\right)\right)\left(\widehat{p}_{t}^{H, W}-\widehat{p}_{t}^{W}\right)-\ldots \\
\phi_{F}\left(\widehat{p}_{t}^{H, R}-\widehat{p}_{t}^{R}\right)-(1-\psi)\left(\frac{1+\varphi}{\psi}\right) \widehat{k}_{t}-\left(\frac{1+\varphi}{\psi}\right) \widehat{a}_{t}
\end{array}\right], \\
& \widehat{\pi}_{t}^{H *} \approx \beta \mathbb{E}_{t}\left(\widehat{\pi}_{t+1}^{H *}\right)+\ldots \\
& \Psi\left[\begin{array}{c}
\left(\sigma^{-1}+\left(1-\gamma_{x}\right)\left(\frac{\psi}{1+(1-\psi) \varphi}\right)\left(\varphi+(1-\psi)\left(\frac{1+\varphi}{\psi}\right)^{2}\right)\right) \widehat{c}_{t}^{W}+\ldots \\
\gamma_{x}\left(\frac{\psi}{1+(1-\psi) \varphi}\right)\left(\varphi+(1-\psi)\left(\frac{1+\varphi}{\psi}\right)^{2}\right) \widehat{x}_{t}^{W}+\ldots \\
\gamma_{x}\left(\frac{\psi}{1+(1-\psi) \varphi}\right)\left(\varphi+(1-\psi)\left(\frac{1+\varphi}{\psi}\right)^{2}\right)\left(\frac{1-\beta(1-\delta)}{\beta \delta}\right) \widehat{u}_{t}-\ldots \\
\phi_{H} \widehat{r s}_{t}-\left(1+\eta\left(\frac{\psi}{1+(1-\psi) \varphi}\right)\left(\varphi+(1-\psi)\left(\frac{1+\varphi}{\psi}\right)^{2}\right)\right)\left(\widehat{p}_{t}^{H, W}-\widehat{p}_{t}^{W}\right)+\ldots \\
\phi_{H}\left(\widehat{p}_{t}^{H, R}-\widehat{p}_{t}^{R}\right)-(1-\psi)\left(\frac{1+\varphi}{\psi}\right) \widehat{k}_{t}-\left(\frac{1+\varphi}{\psi}\right) \widehat{a}_{t}
\end{array}\right], \\
& \widehat{\pi}_{t}^{F} \approx \beta \mathbb{E}_{t}\left(\widehat{\pi}_{t+1}^{F}\right)+\ldots \\
& \Psi\left[\begin{array}{c}
\left(\sigma^{-1}+\left(1-\gamma_{x}\right)\left(\frac{\psi}{1+(1-\psi) \varphi}\right)\left(\varphi+(1-\psi)\left(\frac{1+\varphi}{\psi}\right)^{2}\right)\right) \widehat{c}_{t}^{W *}+\ldots \\
\gamma_{x}\left(\frac{\psi}{1+(1-\psi) \varphi}\right)\left(\varphi+(1-\psi)\left(\frac{1+\varphi}{\psi}\right)^{2}\right) \widehat{x}_{t}^{W *}+\ldots \\
\gamma_{x}\left(\frac{\psi}{1+(1-\psi) \varphi}\right)\left(\varphi+(1-\psi)\left(\frac{1+\varphi}{\psi}\right)^{2}\right)\left(\frac{1-\beta(1-\delta)}{\beta \delta}\right) \widehat{u}_{t}^{*}+\ldots \\
\phi_{H} \widehat{r s}_{t}-\left(1+\eta\left(\frac{\psi}{1+(1-\psi) \varphi}\right)\left(\varphi+(1-\psi)\left(\frac{1+\varphi}{\psi}\right)^{2}\right)\right)\left(\widehat{p}_{t}^{F, W *}-\widehat{p}_{t}^{W *}\right)-\ldots \\
\phi_{H}\left(\widehat{p}_{t}^{F, R}-\widehat{p}_{t}^{R}\right)-(1-\psi)\left(\frac{1+\varphi}{\psi}\right) \widehat{k}_{t}^{*}-\left(\frac{1+\varphi}{\psi}\right) \widehat{a}_{t}^{*}
\end{array}\right], \\
& \widehat{\pi}_{t}^{F *} \approx \beta \mathbb{E}_{t}\left(\widehat{\pi}_{t+1}^{F *}\right)+\ldots \\
& \Psi\left[\begin{array}{c}
\left(\sigma^{-1}+\left(1-\gamma_{x}\right)\left(\frac{\psi}{1+(1-\psi) \varphi}\right)\left(\varphi+(1-\psi)\left(\frac{1+\varphi}{\psi}\right)^{2}\right)\right) \widehat{c}_{t}^{W *}+\ldots \\
\gamma_{x}\left(\frac{\psi}{1+(1-\psi) \varphi}\right)\left(\varphi+(1-\psi)\left(\frac{1+\varphi}{\psi}\right)^{2}\right) \widehat{x}_{t}^{W *}+\ldots \\
\gamma_{x}\left(\frac{\psi}{1+(1-\psi) \varphi}\right)\left(\varphi+(1-\psi)\left(\frac{1+\varphi}{\psi}\right)^{2}\right)\left(\frac{1-\beta(1-\delta)}{\beta \delta}\right) \widehat{u}_{t}^{*}-\ldots \\
\phi_{F} \widehat{r s}_{t}-\left(1+\eta\left(\frac{\psi}{1+(1-\psi) \varphi}\right)\left(\varphi+(1-\psi)\left(\frac{1+\varphi}{\psi}\right)^{2}\right)\right)\left(\widehat{p}_{t}^{F, W *}-\widehat{p}_{t}^{W *}\right)+\ldots \\
\phi_{F}\left(\widehat{p}_{t}^{F, R}-\widehat{p}_{t}^{R}\right)-(1-\psi)\left(\frac{1+\varphi}{\psi}\right) \widehat{k}_{t}^{*}-\left(\frac{1+\varphi}{\psi}\right) \widehat{a}_{t}^{*}
\end{array}\right] .
\end{aligned}
$$

We define the world weighted price sub-indexes as $\widehat{p}_{t}^{H, W} \equiv \phi_{H} \widehat{p}_{t}^{H}+\phi_{F} \widehat{p}_{t}^{H *}$ and $\widehat{p}_{t}^{F, W *} \equiv \phi_{F} \widehat{p}_{t}^{F}+\phi_{H} \widehat{p}_{t}^{F *}$. Therefore, we easily derive the dynamics of $\widehat{\pi}_{t}^{H, W} \equiv \widehat{p}_{t}^{H, W}-\widehat{p}_{t-1}^{H, W}$ and $\widehat{\pi}_{t}^{F, W *} \equiv \widehat{p}_{t}^{F, W *}-\widehat{p}_{t-1}^{F, W *}$ from the 
equations above as,

$$
\begin{aligned}
& \widehat{\pi}_{t}^{H, W} \approx \beta \mathbb{E}_{t}\left(\widehat{\pi}_{t+1}^{H, W}\right)+\ldots \\
& \Psi\left[\begin{array}{c}
\left(\sigma^{-1}+\left(1-\gamma_{x}\right)\left(\frac{\psi}{1+(1-\psi) \varphi}\right)\left(\varphi+(1-\psi)\left(\frac{1+\varphi}{\psi}\right)^{2}\right)\right) \widehat{c}_{t}^{W}+\ldots \\
\gamma_{x}\left(\frac{\psi}{1+(1-\psi) \varphi}\right)\left(\varphi+(1-\psi)\left(\frac{1+\varphi}{\psi}\right)^{2}\right) \widehat{x}_{t}^{W}+\ldots \\
\gamma_{x}\left(\frac{\psi}{1+(1-\psi) \varphi}\right)\left(\varphi+(1-\psi)\left(\frac{1+\varphi}{\psi}\right)^{2}\right)\left(\frac{1-\beta(1-\delta)}{\beta \delta}\right) \widehat{u}_{t}-\ldots \\
\left(1+\eta\left(\frac{\psi}{1+(1-\psi) \varphi}\right)\left(\varphi+(1-\psi)\left(\frac{1+\varphi}{\psi}\right)^{2}\right)\right)\left(\widehat{p}_{t}^{H, W}-\widehat{p}_{t}^{W}\right)-\ldots \\
(1-\psi)\left(\frac{1+\varphi}{\psi}\right) \widehat{k}_{t}-\left(\frac{1+\varphi}{\psi}\right) \widehat{a}_{t}
\end{array}\right], \\
& \widehat{\pi}_{t}^{F, W *} \approx \beta \mathbb{E}_{t}\left(\widehat{\pi}_{t+1}^{F, W *}\right)+\ldots \\
& \Psi\left[\begin{array}{c}
\left(\sigma^{-1}+\left(1-\gamma_{x}\right)\left(\frac{\psi}{1+(1-\psi) \varphi}\right)\left(\varphi+(1-\psi)\left(\frac{1+\varphi}{\psi}\right)^{2}\right)\right) \widehat{c}_{t}^{W *}+\ldots \\
\gamma_{x}\left(\frac{\psi}{1+(1-\psi) \varphi}\right)\left(\varphi+(1-\psi)\left(\frac{1+\varphi}{\psi}\right)^{2}\right) \widehat{x}_{t}^{W *}+\ldots \\
\gamma_{x}\left(\frac{\psi}{1+(1-\psi) \varphi}\right)\left(\varphi+(1-\psi)\left(\frac{1+\varphi}{\psi}\right)^{2}\right)\left(\frac{1-\beta(1-\delta)}{\beta \delta}\right) \widehat{u}_{t}^{*}-\ldots \\
\left(1+\eta\left(\frac{\psi}{1+(1-\psi) \varphi}\right)\left(\varphi+(1-\psi)\left(\frac{1+\varphi}{\psi}\right)^{2}\right)\right)\left(\widehat{p}_{t}^{F, W *}-\widehat{p}_{t}^{W *}\right)-\ldots \\
(1-\psi)\left(\frac{1+\varphi}{\psi}\right) \widehat{k}_{t}^{*}-\left(\frac{1+\varphi}{\psi}\right) \widehat{a}_{t}^{*}
\end{array}\right] .
\end{aligned}
$$

We define the domestic and foreign CPI indexes as $\widehat{p}_{t} \approx \phi_{H} \widehat{p}_{t}^{H}+\phi_{F} \widehat{p}_{t}^{F}$ and $\widehat{p}_{t}^{*} \approx \phi_{F} \widehat{p}_{t}^{H *}+\phi_{H} \widehat{p}_{t}^{F *}$, respectively. Therefore, it is easy to derive the dynamics of $\widehat{\pi}_{t} \equiv \widehat{p}_{t}-\widehat{p}_{t-1}$ and $\widehat{\pi}_{t}^{*} \equiv \widehat{p}_{t}^{*}-\widehat{p}_{t-1}^{*}$ from the 
pricing equations above as follows,

$$
\begin{aligned}
& \widehat{\pi}_{t} \approx \beta \mathbb{E}_{t}\left(\widehat{\pi}_{t+1}\right)+\ldots \\
& \Psi\left[\begin{array}{c}
\left(\sigma^{-1}+\left(1-\gamma_{x}\right)\left(\frac{\psi}{1+(1-\psi) \varphi}\right)\left(\varphi+(1-\psi)\left(\frac{1+\varphi}{\psi}\right)^{2}\right)\right)\left[\phi_{H} \widehat{c}_{t}^{W}+\phi_{F} \widehat{c}_{t}^{W *}\right]+\ldots \\
\gamma_{x}\left(\frac{\psi}{1+(1-\psi) \varphi}\right)\left(\varphi+(1-\psi)\left(\frac{1+\varphi}{\psi}\right)^{2}\right)\left[\phi_{H} \widehat{x}_{t}^{W}+\phi_{F} \widehat{x}_{t}^{W *}\right]+\ldots \\
\gamma_{x}\left(\frac{\psi}{1+(1-\psi) \varphi}\right)\left(\varphi+(1-\psi)\left(\frac{1+\varphi}{\psi}\right)^{2}\right)\left(\frac{1-\beta(1-\delta)}{\beta \delta}\right)\left[\phi_{H} \widehat{u}_{t}+\phi_{F} \widehat{u}_{t}^{*}\right]+2 \phi_{H} \phi_{F} \widehat{r s s}_{t}-\ldots \\
\left(1+\eta\left(\frac{\psi}{1+(1-\psi) \varphi}\right)\left(\varphi+(1-\psi)\left(\frac{1+\varphi}{\psi}\right)^{2}\right)\right)\left[\phi_{H}\left(\widehat{p}_{t}^{H, W}-\widehat{p}_{t}^{W}\right)+\phi_{F}\left(\widehat{p}_{t}^{F, W *}-\widehat{p}_{t}^{W *}\right)\right]-\ldots \\
\phi_{H} \phi_{F}\left[\left(\widehat{p}_{t}^{H, R}-\widehat{p}_{t}^{R}\right)+\left(\widehat{p}_{t}^{F, R}-\widehat{p}_{t}^{R}\right)\right]-(1-\psi)\left(\frac{1+\varphi}{\psi}\right) \widehat{k}_{t}^{W}-\ldots \\
\left(\frac{1+\varphi}{\psi}\right)\left[\phi_{H} \widehat{a}_{t}+\phi_{F} \widehat{a}_{t}^{*}\right]
\end{array}\right], \\
& \widehat{\pi}_{t}^{*} \approx \beta \mathbb{E}_{t}\left(\widehat{\pi}_{t+1}^{*}\right)+\ldots \\
& \Psi\left[\begin{array}{c}
\left(\sigma^{-1}+\left(1-\gamma_{x}\right)\left(\frac{\psi}{1+(1-\psi) \varphi}\right)\left(\varphi+(1-\psi)\left(\frac{1+\varphi}{\psi}\right)^{2}\right)\right)\left[\phi_{F} \widehat{c}_{t}^{W}+\phi_{H} \widehat{c}_{t}^{W *}\right]+\ldots \\
\gamma_{x}\left(\frac{\psi}{1+(1-\psi) \varphi}\right)\left(\varphi+(1-\psi)\left(\frac{1+\varphi}{\psi}\right)^{2}\right)\left[\phi_{F} \widehat{x}_{t}^{W}+\phi_{H} \widehat{x}_{t}^{W *}\right]+\ldots \\
\gamma_{x}\left(\frac{\psi}{1+(1-\psi) \varphi}\right)\left(\varphi+(1-\psi)\left(\frac{1+\varphi}{\psi}\right)^{2}\right)\left(\frac{1-\beta(1-\delta)}{\beta \delta}\right)\left[\phi_{F} \widehat{u}_{t}+\phi_{H} \widehat{u}_{t}^{*}\right]-2 \phi_{F} \phi_{H} \widehat{r s}_{t}-\ldots \\
\left(1+\eta\left(\frac{\psi}{1+(1-\psi) \varphi}\right)\left(\varphi+(1-\psi)\left(\frac{1+\varphi}{\psi}\right)^{2}\right)\right)\left[\phi_{F}\left(\widehat{p}_{t}^{H, W}-\widehat{p}_{t}^{W}\right)+\phi_{H}\left(\widehat{p}_{t}^{F, W *}-\widehat{p}_{t}^{W *}\right)\right]+\ldots \\
\phi_{F} \phi_{H}\left[\left(\widehat{p}_{t}^{H, R}-\widehat{p}_{t}^{R}\right)+\left(\widehat{p}_{t}^{F, R}-\widehat{p}_{t}^{R}\right)\right]-(1-\psi)\left(\frac{1+\varphi}{\psi}\right) \widehat{k}_{t}^{W *}-\ldots \\
\left(\frac{1+\varphi}{\psi}\right)\left[\phi_{F} \widehat{a}_{t}+\phi_{H} \widehat{a}_{t}^{*}\right]
\end{array}\right],
\end{aligned}
$$

where world weighted capital is defined as $\widehat{k}_{t}^{W} \equiv \phi_{H} \widehat{k}_{t}+\phi_{F} \widehat{k}_{t}^{*}$ and $\widehat{k}_{t}^{W *} \equiv \phi_{F} \widehat{k}_{t}+\phi_{H} \widehat{k}_{t}^{*}$. We can also write certain terms inside the brackets of the Phillips curves in a more compact form as,

$$
\begin{aligned}
- & \left(1+\eta\left(\frac{\psi}{1+(1-\psi) \varphi}\right)\left(\varphi+(1-\psi)\left(\frac{1+\varphi}{\psi}\right)^{2}\right)\right)\left[\phi_{H}\left(\widehat{p}_{t}^{H, W}-\widehat{p}_{t}^{W}\right)+\phi_{F}\left(\widehat{p}_{t}^{F, W *}-\widehat{p}_{t}^{W *}\right)\right]-\ldots \\
& \phi_{H} \phi_{F}\left[\left(\widehat{p}_{t}^{H, R}-\widehat{p}_{t}^{R}\right)+\left(\widehat{p}_{t}^{F, R}-\widehat{p}_{t}^{R}\right)\right] \\
& =\eta\left(\frac{\psi}{1+(1-\psi) \varphi}\right)\left(\varphi+(1-\psi)\left(\frac{1+\varphi}{\psi}\right)^{2}\right)\left[\phi_{H}-\phi_{F}\right]\left(\widehat{p}_{t}^{F, W *}-\widehat{p}_{t}^{W *}\right)-\ldots \\
& \eta\left(\frac{\psi}{1+(1-\psi) \varphi}\right)\left(\varphi+(1-\psi)\left(\frac{1+\varphi}{\psi}\right)^{2}\right) \phi_{H}\left[\left(\widehat{p}_{t}^{H, W}-\widehat{p}_{t}^{W}\right)+\left(\widehat{p}_{t}^{F, W *}-\widehat{p}_{t}^{W *}\right)\right]-\ldots \\
& \phi_{H}\left[\left(\widehat{p}_{t}^{H, W}-\widehat{p}_{t}^{W}\right)+\phi_{F}\left(\widehat{p}_{t}^{H, R}-\widehat{p}_{t}^{R}\right)\right]-\phi_{F}\left[\left(\widehat{p}_{t}^{F, W *}-\widehat{p}_{t}^{W *}\right)+\phi_{H}\left(\widehat{p}_{t}^{F, R}-\widehat{p}_{t}^{R}\right)\right], \\
- & \left(1+\eta\left(\frac{\psi}{1+(1-\psi) \varphi}\right)\left(\varphi+(1-\psi)\left(\frac{1+\varphi}{\psi}\right)^{2}\right)\right)\left[\phi_{F}\left(\widehat{p}_{t}^{H, W}-\widehat{p}_{t}^{W}\right)+\phi_{H}\left(\widehat{p}_{t}^{F, W *}-\widehat{p}_{t}^{W *}\right)\right]+\ldots \\
& \phi_{F} \phi_{H}\left[\left(\widehat{p}_{t}^{H, R}-\widehat{p}_{t}^{R}\right)+\left(\widehat{p}_{t}^{F, R}-\widehat{p}_{t}^{R}\right)\right] \\
& =-\eta\left(\frac{\psi}{1+(1-\psi) \varphi}\right)\left(\varphi+(1-\psi)\left(\frac{1+\varphi}{\psi}\right)^{2}\right)\left[\phi_{H}-\phi_{F}\right]\left(\widehat{p}_{t}^{F, W *}-\widehat{p}_{t}^{W *}\right)-\ldots \\
& \eta\left(\frac{\psi}{1+(1-\psi) \varphi}\right)\left(\varphi+(1-\psi)\left(\frac{1+\varphi}{\psi}\right)^{2}\right) \phi_{F}\left[\left(\widehat{p}_{t}^{H, W}-\widehat{p}_{t}^{W}\right)+\left(\widehat{p}_{t}^{F, W *}-\widehat{p}_{t}^{W *}\right)\right]-\ldots \\
& \phi_{F}\left[\left(\widehat{p}_{t}^{H, W}-\widehat{p}_{t}^{W}\right)-\phi_{H}\left(\widehat{p}_{t}^{H, R}-\widehat{p}_{t}^{R}\right)\right]-\phi_{H}\left[\left(\widehat{p}_{t}^{F, W *}-\widehat{p}_{t}^{W *}\right)-\phi_{F}\left(\widehat{p}_{t}^{F, R}-\widehat{p}_{t}^{R}\right)\right] .
\end{aligned}
$$

Based on our definitions of the world weighted price indexes and sub-indexes, denoted with the superscripts 
$W$ and $W^{*}$, it is possible for us to argue that,

$$
\begin{aligned}
& \phi_{H}\left[\left(\widehat{p}_{t}^{H, W}-\widehat{p}_{t}^{W}\right)+\phi_{F}\left(\widehat{p}_{t}^{H, R}-\widehat{p}_{t}^{R}\right)\right]+\phi_{F}\left[\left(\widehat{p}_{t}^{F, W *}-\widehat{p}_{t}^{W *}\right)+\phi_{H}\left(\widehat{p}_{t}^{F, R}-\widehat{p}_{t}^{R}\right)\right] \\
& \quad=\phi_{H}\left(\widehat{p}_{t}^{H}-\widehat{p}_{t}\right)+\phi_{F}\left(\widehat{p}_{t}^{F}-\widehat{p}_{t}\right)=0, \\
& \phi_{F}\left[\left(\widehat{p}_{t}^{H, W}-\widehat{p}_{t}^{W}\right)-\phi_{H}\left(\widehat{p}_{t}^{H, R}-\widehat{p}_{t}^{R}\right)\right]+\phi_{H}\left[\left(\widehat{p}_{t}^{F, W *}-\widehat{p}_{t}^{W *}\right)-\phi_{F}\left(\widehat{p}_{t}^{F, R}-\widehat{p}_{t}^{R}\right)\right] \\
& \quad=\phi_{F}\left(\widehat{p}_{t}^{H *}-\widehat{p}_{t}^{*}\right)+\phi_{H}\left(\widehat{p}_{t}^{F *}-\widehat{p}_{t}^{*}\right)=0,
\end{aligned}
$$

since $\widehat{p}_{t} \approx \phi_{H} \widehat{p}_{t}^{H}+\phi_{F} \widehat{p}_{t}^{F}$ and $\widehat{p}_{t}^{*} \approx \phi_{F} \widehat{p}_{t}^{H *}+\phi_{H} \widehat{p}_{t}^{F *}$. Furthermore, we also know based on those same definitions, that the following result must hold true,

$$
\begin{aligned}
& \left(\widehat{p}_{t}^{H, W}-\widehat{p}_{t}^{W}\right)+\left(\widehat{p}_{t}^{F, W *}-\widehat{p}_{t}^{W *}\right)=\widehat{p}_{t}^{H, W}+\widehat{p}_{t}^{F, W *}-\left(\widehat{p}_{t}^{W}+\widehat{p}_{t}^{W *}\right) \\
& \quad=\phi_{H}\left(\widehat{p}_{t}^{H}-\widehat{p}_{t}\right)+\phi_{F}\left(\widehat{p}_{t}^{H *}-\widehat{p}_{t}^{*}\right)+\phi_{F}\left(\widehat{p}_{t}^{F}-\widehat{p}_{t}\right)+\phi_{H}\left(\widehat{p}_{t}^{F *}-\widehat{p}_{t}^{*}\right) \\
& \quad=\left[\phi_{H}\left(\widehat{p}_{t}^{H}-\widehat{p}_{t}\right)+\phi_{F}\left(\widehat{p}_{t}^{F}-\widehat{p}_{t}\right)\right]+\left[\phi_{F}\left(\widehat{p}_{t}^{H *}-\widehat{p}_{t}^{*}\right)+\phi_{H}\left(\widehat{p}_{t}^{F *}-\widehat{p}_{t}^{*}\right)\right]=0 .
\end{aligned}
$$

Hence, we argue that those particular terms inside the brackets of the Phillips curves can be simplified as,

$$
\begin{aligned}
& -\left(1+\eta\left(\frac{\psi}{1+(1-\psi) \varphi}\right)\left(\varphi+(1-\psi)\left(\frac{1+\varphi}{\psi}\right)^{2}\right)\right)\left[\phi_{H}\left(\widehat{p}_{t}^{H, W}-\widehat{p}_{t}^{W}\right)+\phi_{F}\left(\widehat{p}_{t}^{F, W *}-\widehat{p}_{t}^{W *}\right)\right]-\ldots \\
& \phi_{H} \phi_{F}\left[\left(\widehat{p}_{t}^{H, R}-\widehat{p}_{t}^{R}\right)+\left(\widehat{p}_{t}^{F, R}-\widehat{p}_{t}^{R}\right)\right]=\eta\left(\frac{\psi}{1+(1-\psi) \varphi}\right)\left(\varphi+(1-\psi)\left(\frac{1+\varphi}{\psi}\right)^{2}\right)\left[\phi_{H}-\phi_{F}\right]\left(\widehat{p}_{t}^{F, W *}-\widehat{p}_{t}^{W *}\right), \\
& -\left(1+\eta\left(\frac{\psi}{1+(1-\psi) \varphi}\right)\left(\varphi+(1-\psi)\left(\frac{1+\varphi}{\psi}\right)^{2}\right)\right)\left[\phi_{F}\left(\widehat{p}_{t}^{H, W}-\widehat{p}_{t}^{W}\right)+\phi_{H}\left(\widehat{p}_{t}^{F, W *}-\widehat{p}_{t}^{W *}\right)\right]+\ldots \\
& \quad \phi_{F} \phi_{H}\left[\left(\widehat{p}_{t}^{H, R}-\widehat{p}_{t}^{R}\right)+\left(\widehat{p}_{t}^{F, R}-\widehat{p}_{t}^{R}\right)\right]=-\eta\left(\frac{\psi}{1+(1-\psi) \varphi}\right)\left(\varphi+(1-\psi)\left(\frac{1+\varphi}{\psi}\right)^{2}\right)\left[\phi_{H}-\phi_{F}\right]\left(\widehat{p}_{t}^{F, W *}-\widehat{p}_{t}^{W *}\right) .
\end{aligned}
$$

We conclude that both Phillips curves in the model take the following form,

$$
\begin{aligned}
& \widehat{\pi}_{t} \approx \beta \mathbb{E}_{t}\left(\widehat{\pi}_{t+1}\right)+\ldots \\
& \Psi\left[\begin{array}{c}
\left(\sigma^{-1}+\left(1-\gamma_{x}\right)\left(\frac{\psi}{1+(1-\psi) \varphi}\right)\left(\varphi+(1-\psi)\left(\frac{1+\varphi}{\psi}\right)^{2}\right)\right)\left[\phi_{H} \widehat{c}_{t}^{W}+\phi_{F} \widehat{c}_{t}^{W *}\right]+\ldots \\
\gamma_{x}\left(\frac{\psi}{1+(1-\psi) \varphi}\right)\left(\varphi+(1-\psi)\left(\frac{1+\varphi}{\psi}\right)^{2}\right)\left[\phi_{H} \widehat{x}_{t}^{W}+\phi_{F} \widehat{x}_{t}^{W *}\right]+\ldots \\
\gamma_{x}\left(\frac{\psi}{1+(1-\psi) \varphi}\right)\left(\varphi+(1-\psi)\left(\frac{1+\varphi}{\psi}\right)^{2}\right)\left(\frac{1-\beta(1-\delta)}{\beta \delta}\right)\left[\phi_{H} \widehat{u}_{t}+\phi_{F} \widehat{u}_{t}^{*}\right]+2 \phi_{H} \phi_{F} \widehat{r s}_{t}+\ldots \\
\left(\phi_{H}-\phi_{F}\right) \eta\left(\frac{\psi}{1+(1-\psi) \varphi}\right)\left(\varphi+(1-\psi)\left(\frac{1+\varphi}{\psi}\right)^{2}\right)\left(\widehat{p}_{t}^{F, W *}-\widehat{p}_{t}^{W *}\right)-\ldots \\
(1-\psi)\left(\frac{1+\varphi}{\psi}\right) \widehat{k}_{t}^{W}-\left(\frac{1+\varphi}{\psi}\right)\left[\phi_{H} \widehat{a}_{t}+\phi_{F} \widehat{a}_{t}^{*}\right]
\end{array}\right], \\
& \widehat{\pi}_{t}^{*} \approx \beta \mathbb{E}_{t}\left(\widehat{\pi}_{t+1}^{*}\right)+\ldots \\
& \Psi\left[\begin{array}{c}
\left(\sigma^{-1}+\left(1-\gamma_{x}\right)\left(\frac{\psi}{1+(1-\psi) \varphi}\right)\left(\varphi+(1-\psi)\left(\frac{1+\varphi}{\psi}\right)^{2}\right)\right)\left[\phi_{F} \widehat{c}_{t}^{W}+\phi_{H} \widehat{c}_{t}^{W *}\right]+\ldots \\
\gamma_{x}\left(\frac{\psi}{1+(1-\psi) \varphi}\right)\left(\varphi+(1-\psi)\left(\frac{1+\varphi}{\psi}\right)^{2}\right)\left[\phi_{F} \widehat{x}_{t}^{W}+\phi_{H} \widehat{x}_{t}^{W *}\right]+\ldots \\
\gamma_{x}\left(\frac{\psi}{1+(1-\psi) \varphi}\right)\left(\varphi+(1-\psi)\left(\frac{1+\varphi}{\psi}\right)^{2}\right)\left(\frac{1-\beta(1-\delta)}{\beta \delta}\right)\left[\phi_{F} \widehat{u}_{t}+\phi_{H} \widehat{u}_{t}^{*}\right]-2 \phi_{F} \phi_{H} \widehat{r s}_{t}-\ldots \\
\left(\phi_{H}-\phi_{F}\right) \eta\left(\frac{\psi}{1+(1-\psi) \varphi}\right)\left(\varphi+(1-\psi)\left(\frac{1+\varphi}{\psi}\right)^{2}\right)\left(\widehat{p}_{t}^{F, W *}-\widehat{p}_{t}^{W *}\right)-\ldots \\
(1-\psi)\left(\frac{1+\varphi}{\psi}\right) \widehat{k}_{t}^{W *}-\left(\frac{1+\varphi}{\psi}\right)\left[\phi_{F} \widehat{a}_{t}+\phi_{H} \widehat{a}_{t}^{*}\right]
\end{array}\right],
\end{aligned}
$$


which extends the specification in models like those of Steinsson (2008) by adding capital, investment, and variable capital utilization.

With a little bit of additional algebra, it is possible to obtain a simpler characterization of the Phillips curves as,

$$
\left.\begin{array}{l}
\widehat{\pi}_{t}^{H, W} \approx \beta \mathbb{E}_{t}\left(\widehat{\pi}_{t+1}^{H, W}\right)+\ldots \\
\Psi\left[\begin{array}{c}
\left(\sigma^{-1}+\left(1-\gamma_{x}\right) \varphi\left(\frac{\varphi \psi^{2}+(1-\psi)(1+\varphi)^{2}}{\varphi \psi+(1-\psi) \psi \varphi^{2}}\right)\right) \widehat{c}_{t}^{W}+\gamma_{x} \varphi\left(\frac{\varphi \psi^{2}+(1-\psi)(1+\varphi)^{2}}{\varphi \psi+(1-\psi) \psi \varphi^{2}}\right) \widehat{x}_{t}^{W}+\ldots \\
\gamma_{x} \varphi\left(\frac{\varphi \psi^{2}+(1-\psi)(1+\varphi)^{2}}{\varphi \psi+(1-\psi) \psi \varphi^{2}}\right) \\
\left(1+\eta \varphi\left(\frac{\varphi \psi^{2}+(1-\psi)(1+\varphi)^{2}}{\varphi \psi+(1-\psi) \psi \varphi^{2}}\right)\right)\left(\frac{1-\beta(1-\delta)}{\beta \delta}\right) \widehat{u}_{t}-\ldots \\
\left(\frac{(1-\psi)(1+\varphi)}{\psi}\right) \widehat{k}_{t}-\left(\frac{1+\varphi}{\psi}\right) \widehat{a}_{t}
\end{array}\right]
\end{array}\right],
$$

and, naturally,

$$
\begin{aligned}
& \widehat{\pi}_{t} \approx \beta \mathbb{E}_{t}\left(\widehat{\pi}_{t+1}\right)+\ldots \\
& \Psi\left[\begin{array}{c}
\left(\sigma^{-1}+\left(1-\gamma_{x}\right) \varphi\left(\frac{\varphi \psi^{2}+(1-\psi)(1+\varphi)^{2}}{\varphi \psi+(1-\psi) \psi \varphi^{2}}\right)\right)\left[\phi_{H} \widehat{c}_{t}^{W}+\phi_{F} \widehat{c}_{t}^{W *}\right]+\ldots \\
\gamma_{x} \varphi\left(\frac{\varphi \psi^{2}+(1-\psi)(1+\varphi)^{2}}{\varphi \psi+(1-\psi) \psi \varphi^{2}}\right)\left[\phi_{H} \widehat{x}_{t}^{W}+\phi_{F} \widehat{x}_{t}^{W *}\right]+\ldots \\
\gamma_{x} \varphi\left(\frac{\varphi \psi^{2}+(1-\psi)(1+\varphi)^{2}}{\varphi \psi+(1-\psi) \psi \varphi^{2}}\right)\left(\frac{1-\beta(1-\delta)}{\beta \delta}\right)\left[\phi_{H} \widehat{u}_{t}+\phi_{F} \widehat{u}_{t}^{*}\right]+\ldots \\
2 \phi_{H} \phi_{F} \widehat{r s}_{t}+\left(\phi_{H}-\phi_{F}\right) \eta \varphi\left(\frac{\varphi \psi^{2}+(1-\psi)(1+\varphi)^{2}}{\varphi \psi+(1-\psi) \psi \varphi^{2}}\right)\left(\widehat{p}_{t}^{F, W *}-\widehat{p}_{t}^{W *}\right)-\ldots \\
\left(\frac{(1-\psi)(1+\varphi)}{\psi}\right) \widehat{k}_{t}^{W}-\left(\frac{1+\varphi}{\psi}\right)\left[\phi_{H} \widehat{a}_{t}+\phi_{F} \widehat{a}_{t}^{*}\right]
\end{array}\right], \\
& \widehat{\pi}_{t}^{*} \approx \beta \mathbb{E}_{t}\left(\widehat{\pi}_{t+1}^{*}\right)+\ldots \\
& \quad\left[\begin{array}{c}
\left(\sigma^{-1}+\left(1-\gamma_{x}\right) \varphi\left(\frac{\varphi \psi^{2}+(1-\psi)(1+\varphi)^{2}}{\varphi \psi+(1-\psi) \psi \varphi^{2}}\right)\right)\left[\phi_{F} \widehat{c}_{t}^{W}+\phi_{H} \widehat{c}_{t}^{W *}\right]+\ldots \\
\gamma_{x} \varphi\left(\frac{\varphi \psi^{2}+(1-\psi)(1+\varphi)^{2}}{\varphi \psi+(1-\psi) \psi \varphi^{2}}\right)\left[\phi_{F} \widehat{x}_{t}^{W}+\phi_{H} \widehat{x}_{t}^{W *}\right]+\ldots \\
\gamma_{x} \varphi\left(\frac{\varphi \psi^{2}+(1-\psi)(1+\varphi)^{2}}{\varphi \psi+(1-\psi) \psi \varphi^{2}}\right)\left(\frac{1-\beta(1-\delta)}{\beta \delta}\right)\left[\phi_{F} \widehat{u}_{t}+\phi_{H} \widehat{u}_{t}^{*}\right]-\ldots \\
2 \phi_{F} \phi_{H} \widehat{r}_{t}-\left(\phi_{H}-\phi_{F}\right) \eta \varphi\left(\frac{\varphi \psi^{2}+(1-\psi)(1+\varphi)^{2}}{\varphi \psi+(1-\psi) \psi \varphi^{2}}\right)\left(\widehat{p}_{t}^{F, W *}-\widehat{p}_{t}^{W *}\right)-\ldots \\
\left(\frac{(1-\psi)(1+\varphi)}{\psi}\right) \widehat{k}_{t}^{W *}-\left(\frac{1+\varphi}{\psi}\right)\left[\phi_{F} \widehat{a}_{t}+\phi_{H} \widehat{a}_{t}^{*}\right]
\end{array}\right] .
\end{aligned}
$$

Capital appears in the Phillips curves because it reflects the impact of the efficiency conditions on the marginal costs of firms. A similar argument can be made regarding the role of capital utilization on the amount of capital services made available to firms.

Let us define $\widehat{t}_{t}^{W} \equiv \widehat{p}_{t}^{F, W *}-\widehat{p}_{t}^{W *}$ as the world measure of terms of trade in the model. Then, the Phillips 
curves under LCP pricing can be re-expressed as,

$$
\begin{gathered}
\widehat{\pi}_{t} \approx \beta \mathbb{E}_{t}\left(\widehat{\pi}_{t+1}\right)+\ldots \\
\Psi\left[\begin{array}{c}
\left(\sigma^{-1}+\left(1-\gamma_{x}\right) \varphi\left(\frac{\varphi \psi^{2}+(1-\psi)(1+\varphi)^{2}}{\varphi \psi+(1-\psi) \psi \varphi^{2}}\right)\right)\left[\phi_{H} \widehat{c}_{t}^{W}+\phi_{F} \widehat{c}_{t}^{W *}\right]+\ldots \\
\gamma_{x} \varphi\left(\frac{\varphi \psi^{2}+(1-\psi)(1+\varphi)^{2}}{\varphi \psi+(1-\psi) \psi \varphi^{2}}\right)\left[\phi_{H} \widehat{x}_{t}^{W}+\phi_{F} \widehat{x}_{t}^{W *}\right]+\ldots \\
\gamma_{x} \varphi\left(\frac{\varphi \psi^{2}+(1-\psi)(1+\varphi)^{2}}{\varphi \psi+(1-\psi) \psi \varphi^{2}}\right)\left(\frac{1-\beta(1-\delta)}{\beta \delta}\right)\left[\phi_{H} \widehat{u}_{t}+\phi_{F} \widehat{u}_{t}^{*}\right]+\ldots \\
2 \phi_{H} \phi_{F} \widehat{r s}_{t}+\left(\phi_{H}-\phi_{F}\right) \eta \varphi\left(\frac{\varphi \psi^{2}+(1-\psi)(1+\varphi)^{2}}{\varphi \psi+(1-\psi) \psi \varphi^{2}}\right) \widehat{t}_{t}^{W}-\ldots \\
\left(\frac{(1-\psi)(1+\varphi)}{\psi}\right) \widehat{k}_{t}^{W}-\left(\frac{1+\varphi}{\psi}\right)\left[\phi_{H} \widehat{a}_{t}+\phi_{F} \widehat{a}_{t}^{*}\right]
\end{array}\right], \\
\widehat{\pi}_{t}^{*} \approx \beta \mathbb{E}_{t}\left(\widehat{\pi}_{t+1}^{*}\right)+\ldots \\
\Psi\left[\begin{array}{c}
\left(\sigma^{-1}+\left(1-\gamma_{x}\right) \varphi\left(\frac{\varphi \psi^{2}+(1-\psi)(1+\varphi)^{2}}{\varphi \psi+(1-\psi) \psi \varphi^{2}}\right)\right)\left[\phi_{F} \widehat{c}_{t}^{W}+\phi_{H} \widehat{c}_{t}^{W *}\right]+\ldots \\
\gamma_{x} \varphi\left(\frac{\varphi \psi^{2}+(1-\psi)(1+\varphi)^{2}}{\varphi \psi+(1-\psi) \psi \varphi^{2}}\right)\left[\phi_{F} \widehat{x}_{t}^{W}+\phi_{H} \widehat{x}_{t}^{W *}\right]+\ldots \\
\gamma_{x} \varphi\left(\frac{\varphi \psi^{2}+(1-\psi)(1+\varphi)^{2}}{\varphi \psi+(1-\psi) \psi \varphi^{2}}\right)\left(\frac{1-\beta(1-\delta)}{\beta \delta}\right)\left[\phi_{F} \widehat{u}_{t}+\phi_{H} \widehat{u}_{t}^{*}\right]-\ldots \\
2 \phi_{F} \phi_{H} \widehat{r s}_{t}-\left(\phi_{H}-\phi_{F}\right) \eta \varphi\left(\frac{\varphi \psi^{2}+(1-\psi)(1+\varphi)^{2}}{\varphi \psi+(1-\psi) \psi \varphi^{2}}\right) \widehat{t}_{t}^{W}-\ldots \\
\left(\frac{(1-\psi)(1+\varphi)}{\psi}\right) \widehat{k}_{t}^{W *}-\left(\frac{1+\varphi}{\psi}\right)\left[\phi_{F} \widehat{a}_{t}+\phi_{H} \widehat{a}_{t}^{*}\right]
\end{array}\right] .
\end{gathered}
$$

These equations constitute the aggregate supply block in this economy under LCP pricing.

The International Relative Prices. Let us define $\widehat{t}_{t}^{W} \equiv\left(\widehat{p}_{t}^{F, W *}-\widehat{p}_{t}^{W *}\right)$ as the world measure of terms of trade in this model. As in the model under LCP without capital utilization in (155), the only constraint that determines the world terms of trade is given by,

$$
\left(\phi_{H}-\phi_{F}\right)\left(\widehat{p}_{t}^{F, W *}-\widehat{p}_{t}^{W *}\right) \approx \phi_{H} \phi_{F}\left[\left(\widehat{p}_{t}^{H, R}-\widehat{p}_{t}^{R}\right)+\left(\widehat{p}_{t}^{F, R}-\widehat{p}_{t}^{R}\right)\right] .
$$

If the model has no home-product bias in consumption, i.e. $\phi_{H}=\phi_{F}$, then $\widehat{t}_{t}^{W} \equiv\left(\widehat{p}_{t}^{F, W *}-\widehat{p}_{t}^{W *}\right)$ only matters because it affects aggregate output and aggregate output enters into the specification of the monetary policy rules. Therefore, it must follow from $(274)$ that $\left(\widehat{p}_{t}^{H, R}-\widehat{p}_{t}^{R}\right)+\left(\widehat{p}_{t}^{F, R}-\widehat{p}_{t}^{R}\right) \approx 0$. In that case, the constraint imposes no practical restriction on the world terms of trade $\widehat{t}_{t}^{W} \equiv\left(\widehat{p}_{t}^{F, W *}-\widehat{p}_{t}^{W *}\right)$, and we need to keep track of the price sub-indexes in some other way in order to close down the model.

If the model has a home-product bias in consumption, i.e. $\phi_{H} \neq \phi_{F}$, then $\widehat{t}_{t}^{W} \equiv\left(\widehat{p}_{t}^{F, W *}-\widehat{p}_{t}^{W *}\right)$ matters because it affects aggregate output in both countries, and it also matters because it affects the real marginal costs of firms. Moreover, we can write the world terms of trade as follows,

$$
\widehat{t}_{t}^{W} \equiv \widehat{p}_{t}^{F, W *}-\widehat{p}_{t}^{W *}=\frac{\phi_{H} \phi_{F}}{\phi_{H}-\phi_{F}}\left[\left(\widehat{p}_{t}^{H, R}-\widehat{p}_{t}^{R}\right)+\left(\widehat{p}_{t}^{F, R}-\widehat{p}_{t}^{R}\right)\right]
$$

This expression is crucial to derive the dynamics of world terms of trade. In equations (255) and (256) we already derived a simple characterization for the relative price sub-indexes $\widehat{\pi}_{t}^{H, R}$ and $\widehat{\pi}_{t}^{F, R}$ under LCP 
pricing, i.e.

$$
\begin{aligned}
\widehat{\pi}_{t}^{H, R}-\beta \mathbb{E}_{t}\left(\widehat{\pi}_{t+1}^{H, R}\right)+\Psi\left(\widehat{p}_{t}^{H, R}-\widehat{p}_{t}^{R}\right) & \approx \Psi \widehat{r s}_{t}, \\
\widehat{\pi}_{t}^{F, R}-\beta \mathbb{E}_{t}\left(\widehat{\pi}_{t+1}^{F, R}\right)+\Psi\left(\widehat{p}_{t}^{F, R}-\widehat{p}_{t}^{R}\right) & \approx \Psi \widehat{r s}_{t} .
\end{aligned}
$$

Simple manipulations allow us to write this pair of equations as,

$$
\begin{aligned}
\left(\widehat{\pi}_{t}^{H, R}-\widehat{\pi}_{t}^{R}\right)-\beta \mathbb{E}_{t}\left(\widehat{\pi}_{t+1}^{H, R}-\widehat{\pi}_{t+1}^{R}\right)+\Psi\left(\widehat{p}_{t}^{H, R}-\widehat{p}_{t}^{R}\right) & \approx \Psi \widehat{r}_{t}-\widehat{\pi}_{t}^{R}+\beta \mathbb{E}_{t}\left(\widehat{\pi}_{t+1}^{R}\right), \\
\left(\widehat{\pi}_{t}^{F, R}-\widehat{\pi}_{t}^{R}\right)-\beta \mathbb{E}_{t}\left(\widehat{\pi}_{t+1}^{F, R}-\widehat{\pi}_{t+1}^{R}\right)+\Psi\left(\widehat{p}_{t}^{F, R}-\widehat{p}_{t}^{R}\right) & \approx \Psi \widehat{r}_{t}-\widehat{\pi}_{t}^{R}+\beta \mathbb{E}_{t}\left(\widehat{\pi}_{t+1}^{R}\right),
\end{aligned}
$$

where the relative CPI is defined as $\widehat{p}_{t}^{R} \equiv \widehat{p}_{t}-\widehat{p}_{t}^{*}$. If we use the definition of world terms of trade and we combine it with these two equations, we can write the dynamics of $\widehat{t}_{t}^{W} \equiv\left(\widehat{p}_{t}^{F, W *}-\widehat{p}_{t}^{W *}\right)$ as,

$$
\Delta \widehat{t}_{t}^{W}-\beta \mathbb{E}_{t}\left(\Delta \widehat{t}_{t+1}^{W}\right)+\Psi \widehat{t}_{t}^{W} \approx \frac{\phi_{H} \phi_{F}}{\phi_{H}-\phi_{F}}\left[\Psi \widehat{r s}_{t}-\widehat{\pi}_{t}^{R}+\beta \mathbb{E}_{t}\left(\widehat{\pi}_{t+1}^{R}\right)\right]
$$

where we define the first-difference of the world terms of trade as $\Delta \widehat{t}_{t}^{W} \equiv \widehat{t}_{t}^{W}-\widehat{t}_{t-1}^{W}$. This suffices to close down our model under LCP pricing, but it is the same equation as in (157) without variable utilization.

Following on Engel (forthcoming), we can show that when the degree of price stickiness is the same across firms and markets then the relative prices in each country must be equalized even if the law of one price fails to hold, i.e. $\left(\widehat{p}_{t}^{F}-\widehat{p}_{t}^{H}\right) \approx\left(\widehat{p}_{t}^{F *}-\widehat{p}_{t}^{H *}\right)$. To show this, we start by computing the inflation for the relative prices in each country $\left(\widehat{\pi}_{t}^{F}-\widehat{\pi}_{t}^{H}\right)$ and $\left(\widehat{\pi}_{t}^{F *}-\widehat{\pi}_{t}^{H *}\right)$ from the dynamics of the price sub-indexes in (261) - (264) as follows,

$$
\begin{aligned}
& \widehat{\pi}_{t}^{F}-\widehat{\pi}_{t}^{H} \approx \beta \mathbb{E}_{t}\left(\widehat{\pi}_{t+1}^{F}-\widehat{\pi}_{t+1}^{H}\right)+\ldots \\
& \Psi\left[\begin{array}{c}
\left(\sigma^{-1}+\left(1-\gamma_{x}\right)\left(\frac{\psi}{1+(1-\psi) \varphi}\right)\left(\varphi+(1-\psi)\left(\frac{1+\varphi}{\psi}\right)^{2}\right)\right)\left(\widehat{c}_{t}^{W *}-\widehat{c}_{t}^{W}\right)+\ldots \\
\gamma_{x}\left(\frac{\psi}{1+(1-\psi) \varphi}\right)\left(\varphi+(1-\psi)\left(\frac{1+\varphi}{\psi}\right)^{2}\right)\left(\widehat{x}_{t}^{W *}-\widehat{x}_{t}^{W}\right)+\ldots \\
\gamma_{x}\left(\frac{\psi}{1+(1-\psi) \varphi}\right)\left(\varphi+(1-\psi)\left(\frac{1+\varphi}{\psi}\right)^{2}\right)\left(\frac{1-\beta(1-\delta)}{\beta \delta}\right)\left(\widehat{u}_{t}^{*}-\widehat{u}_{t}\right)+\ldots \\
\left(\phi_{H}-\phi_{F}\right) \widehat{r}_{t}-\left(1+\eta\left(\frac{\psi}{1+(1-\psi) \varphi}\right)\left(\varphi+(1-\psi)\left(\frac{1+\varphi}{\psi}\right)^{2}\right)\right)\left(\left(\widehat{p}_{t}^{F, W *}-\widehat{p}_{t}^{W *}\right)-\left(\widehat{p}_{t}^{H, W}-\widehat{p}_{t}^{W}\right)\right)-\ldots \\
\left(\phi_{H}\left(\widehat{p}_{t}^{F, R}-\widehat{p}_{t}^{R}\right)-\phi_{F}\left(\widehat{p}_{t}^{H, R}-\widehat{p}_{t}^{R}\right)\right)-(1-\psi)\left(\frac{1+\varphi}{\psi}\right)\left(\widehat{k}_{t}^{*}-\widehat{k}_{t}\right)-\left(\frac{1+\varphi}{\psi}\right)\left(\widehat{a}_{t}^{*}-\widehat{a}_{t}\right)
\end{array}\right], \\
& \widehat{\pi}_{t}^{F *}-\widehat{\pi}_{t}^{H *} \approx \beta \mathbb{E}_{t}\left(\widehat{\pi}_{t+1}^{F *}-\widehat{\pi}_{t+1}^{H *}\right)+\ldots \\
& \Psi\left[\begin{array}{c}
\left(\sigma^{-1}+\left(1-\gamma_{x}\right)\left(\frac{\psi}{1+(1-\psi) \varphi}\right)\left(\varphi+(1-\psi)\left(\frac{1+\varphi}{\psi}\right)^{2}\right)\right)\left(\widehat{c}_{t}^{W *}-\widehat{c}_{t}^{W}\right)+\ldots \\
\gamma_{x}\left(\frac{\psi}{1+(1-\psi) \varphi}\right)\left(\varphi+(1-\psi)\left(\frac{1+\varphi}{\psi}\right)^{2}\right)\left(\widehat{x}_{t}^{W *}-\widehat{x}_{t}^{W}\right)+\ldots \\
\gamma_{x}\left(\frac{\psi}{1+(1-\psi) \varphi}\right)\left(\varphi+(1-\psi)\left(\frac{1+\varphi}{\psi}\right)^{2}\right)\left(\frac{1-\beta(1-\delta)}{\beta \delta}\right)\left(\widehat{u}_{t}^{*}-\widehat{u}_{t}\right)-\ldots \\
\left(\phi_{F}-\phi_{H}\right) \widehat{r s}_{t}-\left(1+\eta\left(\frac{\psi}{1+(1-\psi) \varphi}\right)\left(\varphi+(1-\psi)\left(\frac{1+\varphi}{\psi}\right)^{2}\right)\right)\left(\left(\widehat{p}_{t}^{F, W *}-\widehat{p}_{t}^{W *}\right)-\left(\widehat{p}_{t}^{H, W}-\widehat{p}_{t}^{W}\right)\right)+\ldots \\
\left(\phi_{F}\left(\widehat{p}_{t}^{F, R}-\widehat{p}_{t}^{R}\right)-\phi_{H}\left(\widehat{p}_{t}^{H, R}-\widehat{p}_{t}^{R}\right)\right)-(1-\psi)\left(\frac{1+\varphi}{\psi}\right)\left(\widehat{k}_{t}^{*}-\widehat{k}_{t}\right)-\left(\frac{1+\varphi}{\psi}\right)\left(\widehat{a}_{t}^{*}-\widehat{a}_{t}\right)
\end{array}\right] .
\end{aligned}
$$


We noted already that by construction $\left(\widehat{p}_{t}^{H, W}-\widehat{p}_{t}^{W}\right)+\left(\widehat{p}_{t}^{F, W *}-\widehat{p}_{t}^{W *}\right) \approx 0$, hence the two expressions for the relative prices above become simply,

$$
\left.\begin{array}{l}
\widehat{\pi}_{t}^{F}-\widehat{\pi}_{t}^{H} \approx \beta \mathbb{E}_{t}\left(\widehat{\pi}_{t+1}^{F}-\widehat{\pi}_{t+1}^{H}\right)+\ldots \\
\left(\sigma^{-1}+\left(1-\gamma_{x}\right)\left(\frac{\psi}{1+(1-\psi) \varphi}\right)\left(\varphi+(1-\psi)\left(\frac{1+\varphi}{\psi}\right)^{2}\right)\right)\left(\widehat{c}_{t}^{W *}-\widehat{c}_{t}^{W}\right)+\ldots \\
\gamma_{x}\left(\frac{\psi}{1+(1-\psi) \varphi}\right)\left(\varphi+(1-\psi)\left(\frac{1+\varphi}{\psi}\right)^{2}\right)\left(\widehat{x}_{t}^{W *}-\widehat{x}_{t}^{W}\right)+\ldots \\
\gamma_{x}\left(\frac{\psi}{1+(1-\psi) \varphi}\right)\left(\varphi+(1-\psi)\left(\frac{1+\varphi}{\psi}\right)^{2}\right)\left(\frac{1-\beta(1-\delta)}{\beta \delta}\right)\left(\widehat{u}_{t}^{*}-\widehat{u}_{t}\right)+\ldots \\
\widehat{\pi}_{t}^{F *}-\widehat{\pi}_{t}^{H *} \approx \beta \mathbb{E}_{t}\left(\widehat{\pi}_{t+1}^{F *}-\widehat{\pi}_{t+1}^{H *}\right)+\ldots \\
\left(\phi_{H}-\phi_{F}\right) \widehat{r s}_{t}-2\left(1+\eta\left(\frac{\psi}{1+(1-\psi) \varphi}\right)\left(\varphi+(1-\psi)\left(\frac{1+\varphi}{\psi}\right)^{2}\right)\right)\left(\widehat{p}_{t}^{F, W *}-\widehat{p}_{t}^{W *}\right)-\ldots \\
\left(\phi_{H}\left(\widehat{p}_{t}^{F, R}-\widehat{p}_{t}^{R}\right)-\phi_{F}\left(\widehat{p}_{t}^{H, R}-\widehat{p}_{t}^{R}\right)\right)-(1-\psi)\left(\frac{1+\varphi}{\psi}\right)\left(\widehat{k}_{t}^{*}-\widehat{k}_{t}\right)-\left(\frac{1+\varphi}{\psi}\right)\left(\widehat{a}_{t}^{*}-\widehat{a}_{t}\right)
\end{array}\right],
$$

Let us define the variable $\widehat{z}_{t}$ as the difference between the relative prices in both countries, i.e. $\widehat{z}_{t} \equiv$ $\left(\widehat{p}_{t}^{F}-\widehat{p}_{t}^{H}\right)-\left(\widehat{p}_{t}^{F *}-\widehat{p}_{t}^{H *}\right)$, and the first difference of $\widehat{z}_{t}$ as $\Delta \widehat{z}_{t} \equiv\left(\widehat{\pi}_{t}^{F}-\widehat{\pi}_{t}^{H}\right)-\left(\widehat{\pi}_{t}^{F *}-\widehat{\pi}_{t}^{H *}\right)$. Using the two equations we derived previously for $\left(\widehat{\pi}_{t}^{F}-\widehat{\pi}_{t}^{H}\right)$ and $\left(\widehat{\pi}_{t}^{F *}-\widehat{\pi}_{t}^{H *}\right)$, it immediately follows that,

$$
\begin{aligned}
& \left(\widehat{\pi}_{t}^{F}-\widehat{\pi}_{t}^{H}\right)-\left(\widehat{\pi}_{t}^{F *}-\widehat{\pi}_{t}^{H *}\right) \approx \beta \mathbb{E}_{t}\left(\left(\widehat{\pi}_{t+1}^{F}-\widehat{\pi}_{t+1}^{H}\right)-\left(\widehat{\pi}_{t+1}^{F *}-\widehat{\pi}_{t+1}^{H *}\right)\right)-\ldots \\
& \Psi\left[\phi_{H}\left(\widehat{p}_{t}^{F, R}-\widehat{p}_{t}^{R}\right)-\phi_{F}\left(\widehat{p}_{t}^{H, R}-\widehat{p}_{t}^{R}\right)+\phi_{F}\left(\widehat{p}_{t}^{F, R}-\widehat{p}_{t}^{R}\right)-\phi_{H}\left(\widehat{p}_{t}^{H, R}-\widehat{p}_{t}^{R}\right)\right] \\
& \approx \beta \mathbb{E}_{t}\left(\left(\widehat{\pi}_{t+1}^{F}-\widehat{\pi}_{t+1}^{H}\right)-\left(\widehat{\pi}_{t+1}^{F *}-\widehat{\pi}_{t+1}^{H *}\right)\right)-\Psi\left[\left(\widehat{p}_{t}^{F, R}-\widehat{p}_{t}^{R}\right)-\left(\widehat{p}_{t}^{H, R}-\widehat{p}_{t}^{R}\right)\right] \\
& \quad \approx \beta \mathbb{E}_{t}\left(\left(\widehat{\pi}_{t+1}^{F}-\widehat{\pi}_{t+1}^{H}\right)-\left(\widehat{\pi}_{t+1}^{F *}-\widehat{\pi}_{t+1}^{H *}\right)\right)-\Psi\left[\widehat{p}_{t}^{F, R}-\widehat{p}_{t}^{H, R}\right] .
\end{aligned}
$$

Finally, since we have already defined $\widehat{p}_{t}^{H, R} \equiv\left(\widehat{p}_{t}^{H}-\widehat{p}_{t}^{H *}\right)$ and $\widehat{p}_{t}^{F, R} \equiv\left(\widehat{p}_{t}^{F}-\widehat{p}_{t}^{F *}\right)$, we can easily infer that,

$$
\left[\widehat{p}_{t}^{F, R}-\widehat{p}_{t}^{H, R}\right]=\left(\widehat{p}_{t}^{F}-\widehat{p}_{t}^{F *}\right)-\left(\widehat{p}_{t}^{H}-\widehat{p}_{t}^{H *}\right)=\left(\widehat{p}_{t}^{F}-\widehat{p}_{t}^{H}\right)-\left(\widehat{p}_{t}^{F *}-\widehat{p}_{t}^{H *}\right)=\widehat{z}_{t},
$$

and, accordingly, we can re-write the expression above as,

$$
\Delta \widehat{z}_{t} \approx \beta \mathbb{E}_{t}\left(\Delta \widehat{z}_{t+1}\right)-\Psi \widehat{z}_{t}
$$

Naturally, as Engel (forthcoming) emphasizes, if we combine equation (278) with the initial condition $\widehat{z}_{0}=0$, then it has to be the case that the solution implies that the relative prices in both countries ought to equalize 
as we postulated initially,

$$
\widehat{z}_{t} \equiv\left(\widehat{p}_{t}^{F}-\widehat{p}_{t}^{H}\right)-\left(\widehat{p}_{t}^{F *}-\widehat{p}_{t}^{H *}\right) \approx 0,
$$

or simply that,

$$
\left(\widehat{p}_{t}^{F}-\widehat{p}_{t}^{H}\right) \approx\left(\widehat{p}_{t}^{F *}-\widehat{p}_{t}^{H *}\right) .
$$

Furthermore, if we combine equations (277) with the solution in (279), we obtain the following result,

$$
\left(\widehat{p}_{t}^{F, R}-\widehat{p}_{t}^{R}\right)-\left(\widehat{p}_{t}^{H, R}-\widehat{p}_{t}^{R}\right) \approx 0
$$

or simply,

$$
\left(\widehat{p}_{t}^{F, R}-\widehat{p}_{t}^{R}\right) \approx\left(\widehat{p}_{t}^{H, R}-\widehat{p}_{t}^{R}\right) .
$$

Therefore, we can re-write the world terms of trade in (275) as follows,

$$
\widehat{t}_{t}^{W} \equiv \widehat{p}_{t}^{F, W *}-\widehat{p}_{t}^{W *}=2 \frac{\phi_{H} \phi_{F}}{\phi_{H}-\phi_{F}}\left(\widehat{p}_{t}^{F, R}-\widehat{p}_{t}^{R}\right) .
$$

This simplification is exactly the same one that we derived in a model without variable capacity utilization in (163), indicating that our result is not sensitive to the addition of utilization into the model.

\subsubsection{The Firms' Equilibrium Conditions: The PCP Case}

Efficiency Conditions and Aggregate Output. The aggregate output equations in (251) - (252) can be simplified under PCP - because in that case the law of one price holds - but will only differ from those

obtained before in (164) - (165) due to the addition of utilization costs. We notice that the weighted prices under PCP can be re-expressed as,

$$
\begin{aligned}
\widehat{p}_{t}^{H, W} & \equiv \widehat{p}_{t}^{H}-\phi_{F} \widehat{s}_{t}, \widehat{p}_{t}^{F, W *} \equiv \widehat{p}_{t}^{F *}+\phi_{F} \widehat{s}_{t} \\
\widehat{p}_{t}^{W} & \equiv \phi_{H} \widehat{p}_{t}+\phi_{F} \widehat{p}_{t}^{*}, \widehat{p}_{t}^{W *} \equiv \phi_{F} \widehat{p}_{t}+\phi_{H} \widehat{p}_{t}^{*}
\end{aligned}
$$

while it follows from equations (11) - (12) that the log-linearized CPIs are,

$$
\begin{aligned}
& \widehat{p}_{t} \approx \phi_{H} \widehat{p}_{t}^{H}+\phi_{F} \widehat{p}_{t}^{F}, \\
& \widehat{p}_{t}^{*} \approx \phi_{H}^{*} \widehat{p}_{t}^{H *}+\phi_{F}^{*} \widehat{p}_{t}^{F *}=\phi_{F} \widehat{p}_{t}^{H *}+\phi_{H} \widehat{p}_{t}^{F *} .
\end{aligned}
$$


Hence, the aggregate output equations can be re-written as follows,

$$
\begin{aligned}
\widehat{y}_{t} & \approx-\eta\left(\widehat{p}_{t}^{H}-\phi_{F} \widehat{s}_{t}-\left(\phi_{H} \widehat{p}_{t}+\phi_{F} \widehat{p}_{t}^{*}\right)\right)+\left(1-\gamma_{x}\right) \widehat{c}_{t}^{W}+\gamma_{x} \widehat{x}_{t}^{W}+\gamma_{x}\left(\frac{1-\beta(1-\delta)}{\beta \delta}\right) \widehat{u}_{t} \\
& \approx-\eta\left(\widehat{p}_{t}^{H}-\left(\phi_{H} \widehat{p}_{t}^{H}+\phi_{F} \widehat{p}_{t}^{F}\right)-\phi_{F}\left(\widehat{s}_{t}+\widehat{p}_{t}^{*}-\widehat{p}_{t}\right)\right)+\left(1-\gamma_{x}\right) \widehat{c}_{t}^{W}+\gamma_{x} \widehat{x}_{t}^{W}+\gamma_{x}\left(\frac{1-\beta(1-\delta)}{\beta \delta}\right) \widehat{u}_{t} \\
& \approx 2 \eta \phi_{F} \phi_{H}\left(\widehat{p}_{t}^{F}-\widehat{p}_{t}^{H}\right)+\left(1-\gamma_{x}\right) \widehat{c}_{t}^{W}+\gamma_{x} \widehat{x}_{t}^{W}+\gamma_{x}\left(\frac{1-\beta(1-\delta)}{\beta \delta}\right) \widehat{u}_{t}, \\
\widehat{y}_{t}^{*} & \approx-\eta\left(\widehat{p}_{t}^{F *}+\phi_{F} \widehat{s}_{t}-\left(\phi_{F} \widehat{p}_{t}+\phi_{H} \widehat{p}_{t}^{*}\right)\right)+\left(1-\gamma_{x}\right) \widehat{c}_{t}^{W *}+\gamma_{x} \widehat{x}_{t}^{W *}+\gamma_{x}\left(\frac{1-\beta(1-\delta)}{\beta \delta}\right) \widehat{u}_{t}^{*} \\
& \approx-\eta\left(\widehat{p}_{t}^{F *}-\left(\phi_{F} \widehat{p}_{t}^{H *}+\phi_{H} \widehat{p}_{t}^{F *}\right)+\phi_{F}\left(\widehat{s}_{t}+\widehat{p}_{t}^{*}-\widehat{p}_{t}\right)\right)+\left(1-\gamma_{x}\right) \widehat{c}_{t}^{W *}+\gamma_{x} \widehat{x}_{t}^{W *}+\gamma_{x}\left(\frac{1-\beta(1-\delta)}{\beta \delta}\right) \widehat{u}_{t}^{*} \\
& \approx-\eta \phi_{F}\left(\left(\widehat{p}_{t}^{F *}-\widehat{p}_{t}^{H *}\right)+\left(\phi_{H}-\phi_{F}\right)\left(\widehat{p}_{t}^{F}-\widehat{p}_{t}^{H}\right)\right)+\left(1-\gamma_{x}\right) \widehat{c}_{t}^{W *}+\gamma_{x} \widehat{x}_{t}^{W *}+\gamma_{x}\left(\frac{1-\beta(1-\delta)}{\beta \delta}\right) \widehat{u}_{t}^{*} \\
& =-2 \eta \phi_{F} \phi_{H}\left(\widehat{p}_{t}^{F}-\widehat{p}_{t}^{H}\right)+\left(1-\gamma_{x}\right) \widehat{c}_{t}^{W *}+\gamma_{x} \widehat{x}_{t}^{W *}+\gamma_{x}\left(\frac{1-\beta(1-\delta)}{\beta \delta}\right) \widehat{u}_{t}^{*} .
\end{aligned}
$$

In other words, aggregate output depends on country-weighted consumption and investment, on the rate of utilization and also depends on the domestic terms of trade, i.e. $\widehat{t o t}_{t} \equiv\left(\widehat{p}_{t}^{F}-\widehat{p}_{t}^{H}\right)$. Given that in our model terms of trade are proportional to the real exchange rate under PCP, we can easily re-write these equations in terms of the real exchange rate as well.

We note that if we define the world consumption as $\widehat{c}_{t}^{W} \equiv \phi_{H} \widehat{c}_{t}+\phi_{F} \widehat{c}_{t}^{*}$ and $\widehat{c}_{t}^{W *} \equiv \phi_{F} \widehat{c}_{t}+\phi_{H} \widehat{c}_{t}^{*}$ and the relative consumption as $\widehat{c}_{t}^{R} \equiv \widehat{c}_{t}-\widehat{c}_{t}^{*}$, then we can write that,

$$
\begin{aligned}
\widehat{c}_{t} & =\widehat{c}_{t}^{W}+\phi_{F} \widehat{c}_{t}^{R} \approx \widehat{c}_{t}^{W}+\phi_{F} \sigma \widehat{r} s_{t}, \\
\widehat{c}_{t}^{*} & =\widehat{c}_{t}^{W *}-\phi_{F} \widehat{c}_{t}^{R} \approx \widehat{c}_{t}^{W *}-\phi_{F} \sigma \widehat{r} s_{t},
\end{aligned}
$$

where the second approximation follows from the perfect international risk-sharing condition in (99). This is a substitution trick that we have used before. Then, we can express the efficiency conditions in (128) - (129) as,

$$
\begin{aligned}
\widehat{r}_{t}^{z} & \approx-\frac{1+\varphi}{\psi} \widehat{a}_{t}+\frac{1}{\sigma} \widehat{c}_{t}^{W}+\phi_{F} \widehat{r} s_{t}+\frac{1+\varphi}{\psi} \widehat{y}_{t}-\left(\frac{1+(1-\psi) \varphi}{\psi}\right) \widehat{k}_{t}, \\
\widehat{r}_{t}^{z *} & \approx-\frac{1+\varphi}{\psi} \widehat{a}_{t}^{*}+\frac{1}{\sigma} \widehat{c}_{t}^{W *}-\phi_{F} \widehat{r}_{t}+\frac{1+\varphi}{\psi} \widehat{y}_{t}^{*}-\left(\frac{1+(1-\psi) \varphi}{\psi}\right) \widehat{k}_{t}^{*} .
\end{aligned}
$$

Furthermore, if we combine the efficiency conditions with the output equations derived in $(251)-(252)$, it follows that,

$$
\begin{aligned}
\widehat{r}_{t}^{z} & \approx\left(\sigma^{-1}+\left(1-\gamma_{x}\right) \frac{1+\varphi}{\psi}\right) \widehat{c}_{t}^{W}+\gamma_{x} \frac{1+\varphi}{\psi} \widehat{x}_{t}^{W}+\gamma_{x} \frac{1+\varphi}{\psi}\left(\frac{1-\beta(1-\delta)}{\beta \delta}\right) \widehat{u}_{t}+\ldots \\
& \phi_{F} \widehat{r r s}_{t}-\eta \frac{1+\varphi}{\psi}\left(\widehat{p}_{t}^{H, W}-\widehat{p}_{t}^{W}\right)-\left(\frac{1+(1-\psi) \varphi}{\psi}\right) \widehat{k}_{t}-\frac{1+\varphi}{\psi} \widehat{a}_{t} \\
\widehat{r}_{t}^{z *} & \approx\left(\frac{1}{\sigma}+\left(1-\gamma_{x}\right) \frac{1+\varphi}{\psi}\right) \widehat{c}_{t}^{W *}+\gamma_{x} \frac{1+\varphi}{\psi} \widehat{x}_{t}^{W *}+\gamma_{x} \frac{1+\varphi}{\psi}\left(\frac{1-\beta(1-\delta)}{\beta \delta}\right) \widehat{u}_{t}^{*}-\ldots \\
& -\phi_{F} \widehat{r s}_{t}-\eta \frac{1+\varphi}{\psi}\left(\widehat{p}_{t}^{F, W *}-\widehat{p}_{t}^{W *}\right)-\left(\frac{1+(1-\psi) \varphi}{\psi}\right) \widehat{k}_{t}^{*}-\frac{1+\varphi}{\psi} \widehat{a}_{t}^{*} .
\end{aligned}
$$


These conditions can be appropriately used to simplify the description of the inflation dynamics. The terms $\left(\widehat{p}_{t}^{H, W}-\widehat{p}_{t}^{W}\right)$ and $\left(\widehat{p}_{t}^{F, W *}-\widehat{p}_{t}^{W *}\right)$ can be further simplified using the implications of the law of one price as we have done with the aggregate output equations. However, the efficiency conditions are used only to replace out the real rental rates on capital in our characterization of the Phillips curves. The simplification under PCP does not become very useful until we have derived the Phillips curves, so we leave it for later.

The Optimal Pricing Equations. In steady state the standard pricing rule under monopolistic competition holds. Accordingly, the log-linearization of the optimal pricing equations in (79), (80), (81) and (82) can be compactly expressed as follows,

$$
\begin{aligned}
& \widehat{\widetilde{p}}_{t}(h)-\widehat{p}_{t} \approx \mathbb{E}_{t}\left[\sum_{\tau=1}^{+\infty}(\beta \alpha)^{\tau} \widehat{\pi}_{t+\tau}\right]+(1-\beta \alpha) \mathbb{E}_{t}\left[\sum_{\tau=0}^{+\infty}(\beta \alpha)^{\tau}\left(\widehat{m c}_{t+\tau}-\widehat{p}_{t+\tau}\right)\right], \\
& \widehat{\widetilde{p}}_{t}^{*}(f)-\widehat{p}_{t}^{*} \approx \mathbb{E}_{t}\left[\sum_{\tau=1}^{+\infty}(\beta \alpha)^{\tau} \widehat{\pi}_{t+\tau}^{*}\right]+(1-\beta \alpha) \mathbb{E}_{t}\left[\sum_{\tau=0}^{+\infty}(\beta \alpha)^{\tau}\left(\widehat{m c}_{t+\tau}^{*}-\widehat{p}_{t+\tau}^{*}\right)\right],
\end{aligned}
$$

which are the same two pricing formulas that we obtained under PCP pricing in the model without capital utilization. Here I must recall the assumption that the government subsidy is time-invariant and equal to its steady state value in every period, which explains why the government subsidies do not appear in the log-linearized pricing equations. We derive the (pre-subsidy) marginal cost equations in (20) - (21), and they can be log-linearized as,

$$
\begin{aligned}
& \widehat{m c}_{t+\tau} \approx \psi \widehat{w}_{t+\tau}+(1-\psi)\left(\widehat{r}_{t+\tau}^{z}+\widehat{p}_{t+\tau}\right)-\widehat{a}_{t+\tau} \\
& \widehat{m c}_{t+\tau}^{*} \approx \psi \widehat{w}_{t+\tau}^{*}+(1-\psi)\left(\widehat{r}_{t+\tau}^{* *}+\widehat{p}_{t+\tau}^{*}\right)-\widehat{a}_{t+\tau}^{*},
\end{aligned}
$$

while the labor market clearing conditions, which are implicit in (95) - (96), can be approximated as,

$$
\begin{aligned}
\widehat{w}_{t+\tau} & \approx-\frac{\varphi}{1+(1-\psi) \varphi} \widehat{a}_{t+\tau}+\frac{1}{1+(1-\psi) \varphi} \frac{1}{\sigma} \widehat{c}_{t+\tau}+\frac{\varphi}{1+(1-\psi) \varphi} \widehat{y}_{t+\tau}+\frac{(1-\psi) \varphi}{1+(1-\psi) \varphi}\left(\widehat{r}_{t+\tau}^{z}+\widehat{p}_{t+\tau}\right)+\frac{1}{1+(1-\psi) \varphi} \widehat{p}_{t+\tau}, \\
\widehat{w}_{t+\tau}^{*} & \approx-\frac{\varphi}{1+(1-\psi) \varphi} \widehat{a}_{t+\tau}^{*}+\frac{1}{1+(1-\psi) \varphi} \frac{1}{\sigma} \widehat{c}_{t+\tau}^{*}+\frac{\varphi}{1+(1-\psi) \varphi} \widehat{y}_{t+\tau}^{*}+\frac{(1-\psi) \varphi}{1+(1-\psi) \varphi}\left(\widehat{r}_{t+\tau}^{*}+\widehat{p}_{t+\tau}^{*}\right)+\frac{1}{1+(1-\psi) \varphi} \widehat{p}_{t+\tau}^{*}
\end{aligned}
$$

If we combine these two log-linearized equations, it follows that the marginal costs can be expressed as,

$$
\begin{aligned}
& \widehat{m c}_{t+\tau} \approx \frac{\psi}{1+(1-\psi) \varphi} \frac{1}{\sigma} \widehat{c}_{t+\tau}+\frac{\varphi \psi}{1+(1-\psi) \varphi} \widehat{y}_{t+\tau}-\frac{1+\varphi}{1+(1-\psi) \varphi}\left(\widehat{a}_{t+\tau}-(1-\psi)\left(\widehat{r}_{t+\tau}^{z}+\widehat{p}_{t+\tau}\right)\right)+\frac{\psi}{1+(1-\psi) \varphi} \widehat{p}_{t+\tau}, \\
& \widehat{m c}_{t+\tau}^{*} \approx \frac{\psi}{1+(1-\psi) \varphi} \frac{1}{\sigma} \widehat{c}_{t+\tau}^{*}+\frac{\varphi \psi}{1+(1-\psi) \varphi} \widehat{y}_{t+\tau}^{*}-\frac{1+\varphi}{1+(1-\psi) \varphi}\left(\widehat{a}_{t+\tau}^{*}-(1-\psi)\left(\widehat{r}_{t+\tau}^{*}+\widehat{p}_{t+\tau}^{*}\right)\right)+\frac{\psi}{1+(1-\psi) \varphi} \widehat{p}_{t+\tau}^{*},
\end{aligned}
$$

where $\widehat{y}_{t+\tau}$ and $\widehat{y}_{t+\tau}^{*}$ define domestic and foreign aggregate output. Up to this point, the derivation of marginal costs is the same as before independently of whether the model allows for capital utilization or not. If we combine the marginal cost equations with the output equations derived before in (251) - (252), 
it follows that,

$$
\begin{aligned}
& \widehat{m c}_{t+\tau}-\widehat{p}_{t+\tau} \approx \frac{\psi}{1+(1-\psi) \varphi} \frac{1}{\sigma} \widehat{c}_{t+\tau}-\frac{\varphi \psi}{1+(1-\psi) \varphi} \eta\left(\widehat{p}_{t+\tau}^{H, W}-\widehat{p}_{t+\tau}^{W}\right)+\ldots \\
& \frac{\varphi \psi}{1+(1-\psi) \varphi}\left(\left(1-\gamma_{x}\right) \widehat{c}_{t+\tau}^{W}+\gamma_{x} \widehat{x}_{t+\tau}^{W}+\gamma_{x}\left(\frac{1-\beta(1-\delta)}{\beta \delta}\right) \widehat{u}_{t+\tau}\right)-\ldots \\
& \frac{1+\varphi}{1+(1-\psi) \varphi}\left(\widehat{a}_{t+\tau}-(1-\psi) \widehat{r}_{t+\tau}^{z}\right) \\
& \widehat{m c}_{t+\tau}^{*}-\widehat{p}_{t+\tau}^{*} \approx \frac{\psi}{1+(1-\psi) \varphi} \frac{1}{\sigma} \widehat{c}_{t+\tau}^{*}-\frac{\varphi \psi}{1+(1-\psi) \varphi} \eta\left(\widehat{p}_{t+\tau}^{F, W *}-\widehat{p}_{t+\tau}^{W *}\right)+\ldots \\
& \frac{\varphi \psi}{1+(1-\psi) \varphi}\left(\left(1-\gamma_{x}\right) \widehat{c}_{t+\tau}^{W *}+\gamma_{x} \widehat{x}_{t+\tau}^{W *}+\gamma_{x}\left(\frac{1-\beta(1-\delta)}{\beta \delta}\right) \widehat{u}_{t+\tau}^{*}\right)-\ldots \\
& \frac{1+\varphi}{1+(1-\psi) \varphi}\left(\widehat{a}_{t+\tau}^{*}-(1-\psi) \widehat{r}_{t+\tau}^{z *}\right) .
\end{aligned}
$$

At this stage the equations have not been simplified to take into account the fact that the law of one price holds in the PCP case. However, we keep that fact on the back of our minds since it will become crucial when we simplify the Phillips Curve equations later on. The expressions now show that real marginal costs have to account for the costs of variable capital utilization.

We can use our characterization of the real marginal costs jointly with the pricing formulas log-linearized before to write that,

$$
\begin{aligned}
& \widehat{\widetilde{p}}_{t}(h)-\widehat{p}_{t} \approx \\
& (1-\beta \alpha)\left(\frac{\psi}{1+(1-\psi) \varphi}\right) \sum_{\tau=0}^{+\infty}(\beta \alpha)^{\tau} \mathbb{E}_{t}\left[\begin{array}{c}
\frac{1}{\sigma} \widehat{c}_{t+\tau}+\varphi\left(\left(1-\gamma_{x}\right) \widehat{c}_{t+\tau}^{W}+\gamma_{x} \widehat{x}_{t+\tau}^{W}+\gamma_{x}\left(\frac{1-\beta(1-\delta)}{\beta \delta}\right) \widehat{u}_{t+\tau}\right)-\ldots \\
\varphi \eta\left(\widehat{p}_{t+\tau}^{H, W}-\widehat{p}_{t+\tau}^{W}\right)-\ldots \\
\left(\frac{1+\varphi}{\psi}\right)\left(\widehat{a}_{t+\tau}-(1-\psi) \widehat{r}_{t+\tau}^{z}\right)
\end{array}\right]+\ldots \\
& \sum_{\tau=1}^{+\infty}(\beta \alpha)^{\tau} \mathbb{E}_{t}\left(\widehat{\pi}_{t+\tau}\right) \\
& \widehat{\widetilde{p}}_{t}^{*}(f)-\widehat{p}_{t}^{*} \approx \\
& (1-\beta \alpha)\left(\frac{\psi}{1+(1-\psi) \varphi}\right) \sum_{\tau=0}^{+\infty}(\beta \alpha)^{\tau} \mathbb{E}_{t}\left[\begin{array}{c}
\frac{1}{\sigma} \widehat{c}_{t+\tau}^{*}+\varphi\left(\left(1-\gamma_{x}\right) \widehat{c}_{t+\tau}^{W *}+\gamma_{x} \widehat{x}_{t+\tau}^{W *}+\gamma_{x}\left(\frac{1-\beta(1-\delta)}{\beta \delta}\right) \widehat{u}_{t+\tau}^{*}\right)-\ldots \\
\varphi \eta\left(\widehat{p}_{t+\tau}^{F, W *}-\widehat{p}_{t+\tau}^{W *}\right)-\ldots \\
\left(\frac{1+\varphi}{\psi}\right)\left(\widehat{a}_{t+\tau}^{*}-(1-\psi) \widehat{r}_{t+\tau}^{z *}\right)
\end{array}\right]+\ldots \\
& \sum_{\tau=1}^{+\infty}(\beta \alpha)^{\tau} \mathbb{E}_{t}\left(\widehat{\pi}_{t+\tau}^{*}\right) .
\end{aligned}
$$

We log-linearize the price sub-indexes in $(75)-(76)$ and $(77)-(78)$ and re-arrange them to obtain that,

$$
\begin{aligned}
& \widehat{\widetilde{p}}_{t}(h)-\widehat{p}_{t} \approx\left(\widehat{p}_{t}^{H}-\widehat{p}_{t}\right)+\left(\frac{\alpha}{1-\alpha}\right) \widehat{\pi}_{t}^{H}, \\
& \widehat{\widetilde{p}}_{t}^{*}(f)-\widehat{p}_{t}^{*} \approx\left(\widehat{p}_{t}^{F *}-\widehat{p}_{t}^{*}\right)+\left(\frac{\alpha}{1-\alpha}\right) \widehat{\pi}_{t}^{F *},
\end{aligned}
$$

which is quite convenient for the purpose of aggregating the optimal pricing equations. We replace the isolated terms $\frac{1}{\sigma} \widehat{c}_{t}$ and $\frac{1}{\sigma} \widehat{c}_{t}^{*}$ out of the marginal cost. If we define the world consumption as $\widehat{c}_{t}^{W} \equiv \phi_{H} \widehat{c}_{t}+\phi_{F} \widehat{c}_{t}^{*}$ and $\widehat{c}_{t}^{W *} \equiv \phi_{F} \widehat{c}_{t}+\phi_{H} \widehat{c}_{t}^{*}$ and the relative consumption as $\widehat{c}_{t}^{R} \equiv \widehat{c}_{t}-\widehat{c}_{t}^{*}$, then we can write that,

$$
\begin{aligned}
\widehat{c}_{t} & =\widehat{c}_{t}^{W}+\phi_{F} \widehat{c}_{t}^{R} \approx \widehat{c}_{t}^{W}+\phi_{F} \sigma \widehat{r s}_{t} \\
\widehat{c}_{t}^{*} & =\widehat{c}_{t}^{W *}-\phi_{F} \widehat{c}_{t}^{R} \approx \widehat{c}_{t}^{W *}-\phi_{F} \sigma \widehat{r s}_{t}
\end{aligned}
$$


where the second approximation follows from the perfect international risk-sharing condition in (99). Hence, the pricing equations can be expressed more compactly as,

$$
\begin{aligned}
& \widehat{\pi}_{t}^{H}+\left(\frac{1-\alpha}{\alpha}\right)\left(\widehat{p}_{t}^{H}-\widehat{p}_{t}\right) \approx \\
& \Psi\left(\frac{\psi}{1+(1-\psi) \varphi}\right) \sum_{\tau=0}^{+\infty}(\beta \alpha)^{\tau} \mathbb{E}_{t}\left[\begin{array}{c}
\left(\sigma^{-1}+\left(1-\gamma_{x}\right) \varphi\right) \widehat{c}_{t+\tau}^{W}+\gamma_{x} \varphi \widehat{x}_{t+\tau}^{W}+\varphi \gamma_{x}\left(\frac{1-\beta(1-\delta)}{\beta \delta}\right) \widehat{u}_{t+\tau}+\ldots \\
\phi_{F} \widehat{r s}_{t+\tau}-\varphi \eta\left(\widehat{p}_{t+\tau}^{H, W}-\widehat{p}_{t+\tau}^{W}\right)-\ldots \\
\left(\frac{1+\varphi}{\psi}\right)\left(\widehat{a}_{t+\tau}-(1-\psi) \widehat{r}_{t+\tau}^{z}\right)
\end{array}\right]+\ldots \\
& \left(\frac{1-\alpha}{\alpha}\right) \sum_{\tau=1}^{+\infty}(\beta \alpha)^{\tau} \mathbb{E}_{t}\left(\widehat{\pi}_{t+\tau}\right), \\
& \widehat{\pi}_{t}^{F *}+\left(\frac{1-\alpha}{\alpha}\right)\left(\widehat{p}_{t}^{F *}-\widehat{p}_{t}^{*}\right) \approx \\
& \Psi\left(\frac{\psi}{1+(1-\psi) \varphi}\right) \sum_{\tau=0}^{+\infty}(\beta \alpha)^{\tau} \mathbb{E}_{t}\left[\begin{array}{c}
\left(\sigma^{-1}+\left(1-\gamma_{x}\right) \varphi\right) \widehat{c}_{t+\tau}^{W *}+\gamma_{x} \varphi \widehat{x}_{t+\tau}^{W *}+\varphi \gamma_{x}\left(\frac{1-\beta(1-\delta)}{\beta \delta}\right) \widehat{u}_{t+\tau}^{*}-\ldots \\
\phi_{F} \widehat{r s s}_{t+\tau}-\varphi \eta\left(\widehat{p}_{t+\tau}^{F, W *}-\widehat{p}_{t+\tau}^{W *}\right)-\ldots \\
\left(\frac{1-\alpha}{\alpha}\right) \sum_{\tau=1}^{+\infty}(\beta \alpha)^{\tau} \mathbb{E}_{t}\left(\widehat{\pi}_{t+\tau}^{*}\right),
\end{array}\right]+\ldots
\end{aligned}
$$

where $\Psi \equiv \frac{(1-\alpha)(1-\beta \alpha)}{\alpha}$.

Furthermore, these pricing equations can be expressed in the form of a system of expectational difference equations. Let us focus on the first equation as an example. If we re-write the equation at time $t+1$ and take conditional expectations with respect to all information available up to time $t$, it should follow that,

$$
\begin{aligned}
& \mathbb{E}_{t}\left[\widehat{\pi}_{t+1}^{H}+\left(\frac{1-\alpha}{\alpha}\right)\left(\widehat{p}_{t+1}^{H}-\widehat{p}_{t+1}\right)\right] \underset{\approx}{\approx} \\
& \Psi\left(\frac{\psi}{1+(1-\psi) \varphi}\right) \sum_{\tau=0}^{+\infty}(\beta \alpha)^{\tau} \mathbb{E}_{t}\left[\begin{array}{c}
\left(\sigma^{-1}+\left(1-\gamma_{x}\right) \varphi\right) \widehat{c}_{t+1+\tau}^{W}+\gamma_{x} \varphi \widehat{x}_{t+1+\tau}^{W}+\varphi \gamma_{x}\left(\frac{1-\beta(1-\delta)}{\beta \delta}\right) \widehat{u}_{t+1+\tau}+\ldots \\
\phi_{F} \widehat{r s s}_{t+1+\tau}-\varphi \eta\left(\widehat{p}_{t+1+\tau}^{H, W}-\widehat{p}_{t+1+\tau}^{W}\right)-\ldots \\
\left(\frac{1+\varphi}{\psi}\right)\left(\widehat{a}_{t+1+\tau}-(1-\psi) \widehat{r}_{t+1+\tau}^{z}\right)
\end{array}\right]+\ldots \\
& \quad\left(\frac{1-\alpha}{\alpha}\right) \sum_{\tau=1}^{+\infty}(\beta \alpha)^{\tau} \mathbb{E}_{t}\left(\widehat{\pi}_{t+1+\tau}\right) .
\end{aligned}
$$

Hence, using the computation of the conditional expectation, the pricing equation can easily be decomposed in two terms as,

$$
\begin{aligned}
& \widehat{\pi}_{t}^{H}+\left(\frac{1-\alpha}{\alpha}\right)\left(\widehat{p}_{t}^{H}-\widehat{p}_{t}\right) \approx \\
& \left(\frac{\psi}{1+(1-\psi) \varphi}\right) \Psi\left[\begin{array}{c}
\left(\sigma^{-1}+\left(1-\gamma_{x}\right) \varphi\right) \widehat{c}_{t}^{W}+\gamma_{x} \varphi \widehat{x}_{t}^{W}+\varphi \gamma_{x}\left(\frac{1-\beta(1-\delta)}{\beta \delta}\right) \widehat{u}_{t}+\ldots \\
\phi_{F} \widehat{r s s}_{t}-\varphi \eta\left(\widehat{p}_{t}^{H, W}-\widehat{p}_{t}^{W}\right)-\ldots \\
\left(\frac{1+\varphi}{\psi}\right)\left(\widehat{a}_{t}-(1-\psi) \widehat{r}_{t}^{z}\right)
\end{array}\right]+\ldots \\
& (1-\alpha) \beta \mathbb{E}_{t}\left(\widehat{\pi}_{t+1}\right)+\alpha \beta \mathbb{E}_{t}\left[\widehat{\pi}_{t+1}^{H}+\left(\frac{1-\alpha}{\alpha}\right)\left(\widehat{p}_{t+1}^{H}-\widehat{p}_{t+1}\right)\right] .
\end{aligned}
$$


Further re-arranging allows us to express the expectational difference equation as,

$$
\begin{aligned}
& \widehat{\pi}_{t}^{H}-\beta \mathbb{E}_{t}\left(\widehat{\pi}_{t+1}^{H}\right)+\Psi\left(\widehat{p}_{t}^{H}-\widehat{p}_{t}\right) \\
& \approx\left(\frac{\psi}{1+(1-\psi) \varphi}\right) \Psi\left[\begin{array}{c}
\left(\sigma^{-1}+\left(1-\gamma_{x}\right) \varphi\right) \widehat{c}_{t}^{W}+\gamma_{x} \varphi \widehat{x}_{t}^{W}+\varphi \gamma_{x}\left(\frac{1-\beta(1-\delta)}{\beta \delta}\right) \widehat{u}_{t}+\ldots \\
\phi_{F} \widehat{r s}_{t}-\varphi \eta\left(\widehat{p}_{t}^{H, W}-\widehat{p}_{t}^{W}\right)-\ldots \\
\left(\frac{1+\varphi}{\psi}\right)\left(\widehat{a}_{t}-(1-\psi) \widehat{r}_{t}^{z}\right)
\end{array}\right] .
\end{aligned}
$$

We can apply the same approach (and algebraic steps) to re-write the other pricing equation as an expectational difference equation, i.e.

$$
\begin{aligned}
& \widehat{\pi}_{t}^{F *}+\left(\frac{1-\alpha}{\alpha}\right)\left(\widehat{p}_{t}^{F *}-\widehat{p}_{t}^{*}\right) \\
& \quad \approx\left(\frac{\psi}{1+(1-\psi) \varphi}\right) \Psi\left[\begin{array}{c}
\left(\sigma^{-1}+\left(1-\gamma_{x}\right) \varphi\right) \widehat{c}_{t}^{W *}+\gamma_{x} \varphi \widehat{x}_{t}^{W *}+\varphi \gamma_{x}\left(\frac{1-\beta(1-\delta)}{\beta \delta}\right) \widehat{u}_{t}^{*}-\ldots \\
\phi_{F} \widehat{r s}_{t}-\varphi \eta\left(\widehat{p}_{t}^{F, W *}-\widehat{p}_{t}^{W *}\right)-\ldots \\
\left(\frac{1+\varphi}{\psi}\right)\left(\widehat{a}_{t}^{*}-(1-\psi) \widehat{r}_{t}^{z *}\right)
\end{array}\right]+\ldots \\
& \quad(1-\alpha) \beta \mathbb{E}_{t}\left(\widehat{\pi}_{t+1}^{*}\right)+\alpha \beta \mathbb{E}_{t}\left[\widehat{\pi}_{t+1}^{F *}+\left(\frac{1-\alpha}{\alpha}\right)\left(\widehat{p}_{t+1}^{F *}-\widehat{p}_{t+1}^{*}\right)\right]
\end{aligned}
$$

and,

$$
\begin{aligned}
& \widehat{\pi}_{t}^{F *}-\beta \mathbb{E}_{t}\left(\widehat{\pi}_{t+1}^{F *}\right)+\Psi\left(\widehat{p}_{t}^{F *}-\widehat{p}_{t}^{*}\right) \\
& \approx\left(\frac{\psi}{1+(1-\psi) \varphi}\right) \Psi\left[\begin{array}{c}
\left(\sigma^{-1}+\left(1-\gamma_{x}\right) \varphi\right) \widehat{c}_{t}^{W *}+\gamma_{x} \varphi \widehat{x}_{t}^{W *}+\varphi \gamma_{x}\left(\frac{1-\beta(1-\delta)}{\beta \delta}\right) \widehat{u}_{t}^{*}-\ldots \\
\phi_{F} \widehat{r s}_{t}-\varphi \eta\left(\widehat{p}_{t}^{F, W *}-\widehat{p}_{t}^{W *}\right)-\ldots \\
\left(\frac{1+\varphi}{\psi}\right)\left(\widehat{a}_{t}^{*}-(1-\psi) \widehat{r}_{t}^{z *}\right)
\end{array}\right] .
\end{aligned}
$$

These equations provide a very simple characterization of the price dynamics at the price sub-index level. By the law of one price, the price sub-index for imports in each country is entirely determined by the nominal exchange rate and the price sub-index of the same bundle of goods in its local market. Naturally, the dynamics of the relative price sub-indexes $\widehat{\pi}_{t}^{H, R} \equiv \widehat{\pi}_{t}^{H}-\widehat{\pi}_{t}^{H *}\left(\widehat{p}_{t}^{H, R} \equiv \widehat{p}_{t}^{H}-\widehat{p}_{t}^{H *}\right)$ and $\widehat{\pi}_{t}^{F, R} \equiv \widehat{\pi}_{t}^{F}-\widehat{\pi}_{t}^{F *}$ $\left(\widehat{p}_{t}^{F, R} \equiv \widehat{p}_{t}^{F}-\widehat{p}_{t}^{F *}\right)$ can be determined as follows,

$$
\begin{aligned}
\widehat{\pi}_{t}^{H, R} & \equiv \widehat{\pi}_{t}^{H}-\widehat{\pi}_{t}^{H *} \approx \widehat{s}_{t}-\widehat{s}_{t-1}=\left(\widehat{\pi}_{t}-\widehat{\pi}_{t}^{*}\right)+\widehat{r s}_{t}-\widehat{r}_{t-1}, \\
\widehat{p}_{t}^{H, R} & \equiv \widehat{p}_{t}^{H}-\widehat{p}_{t}^{H *} \approx \widehat{s}_{t}=\left(\widehat{p}_{t}-\widehat{p}_{t}^{*}\right)+\widehat{r}_{t}, \\
\widehat{\pi}_{t}^{F, R} & \equiv \widehat{\pi}_{t}^{F}-\widehat{\pi}_{t}^{F *} \approx \widehat{s}_{t}-\widehat{s}_{t-1}=\left(\widehat{\pi}_{t}-\widehat{\pi}_{t}^{*}\right)+\widehat{r s}_{t}-\widehat{r s}_{t-1}, \\
\widehat{p}_{t}^{F, R} & \equiv \widehat{p}_{t}^{F}-\widehat{p}_{t}^{F *} \approx \widehat{s}_{t}=\left(\widehat{p}_{t}-\widehat{p}_{t}^{*}\right)+\widehat{r s}_{t},
\end{aligned}
$$

where $\widehat{p}_{t}^{R} \equiv \widehat{p}_{t}-\widehat{p}_{t}^{*}$ and $\widehat{\pi}_{t}^{R} \equiv \widehat{\pi}_{t}-\widehat{\pi}_{t}^{*}$ are the relative CPI and the relative CPI inflation, respectively. 
We can re-write the pricing equations after further simplification as follows,

$$
\begin{aligned}
& \widehat{\pi}_{t}^{H} \approx \beta \mathbb{E}_{t}\left(\widehat{\pi}_{t+1}^{H}\right)+\ldots \\
& \left(\frac{\psi}{1+(1-\psi) \varphi}\right) \Psi\left[\begin{array}{c}
\left(\sigma^{-1}+\left(1-\gamma_{x}\right) \varphi\right) \widehat{c}_{t}^{W}+\gamma_{x} \varphi \widehat{x}_{t}^{W}+\varphi \gamma_{x}\left(\frac{1-\beta(1-\delta)}{\beta \delta}\right) \widehat{u}_{t}+\ldots \\
\phi_{F} \widehat{r s s}_{t}-\varphi \eta\left(\widehat{p}_{t}^{H, W}-\widehat{p}_{t}^{W}\right)-\ldots \\
\left(\frac{1+(1-\psi) \varphi}{\psi}\right)\left(\widehat{p}_{t}^{H}-\widehat{p}_{t}\right)-\left(\frac{1+\varphi}{\psi}\right)\left(\widehat{a}_{t}-(1-\psi) \widehat{r}_{t}^{z}\right)
\end{array}\right], \\
& \widehat{\pi}_{t}^{F *} \approx \beta \mathbb{E}_{t}\left(\widehat{\pi}_{t+1}^{F *}\right)+\ldots \\
& \left(\frac{\psi}{1+(1-\psi) \varphi}\right) \Psi\left[\begin{array}{c}
\left(\sigma^{-1}+\left(1-\gamma_{x}\right) \varphi\right) \widehat{c}_{t}^{W *}+\gamma_{x} \varphi \widehat{x}_{t}^{W *}+\varphi \gamma_{x}\left(\frac{1-\beta(1-\delta)}{\beta \delta}\right) \widehat{u}_{t}^{*}-\ldots \\
\phi_{F} \widehat{r s}_{t}-\varphi \eta\left(\widehat{p}_{t}^{F, W *}-\widehat{p}_{t}^{W *}\right)-\ldots \\
\left(\frac{1+(1-\psi) \varphi}{\psi}\right)\left(\widehat{p}_{t}^{F *}-\widehat{p}_{t}^{*}\right)-\left(\frac{1+\varphi}{\psi}\right)\left(\widehat{a}_{t}^{*}-(1-\psi) \widehat{r}_{t}^{* *}\right)
\end{array}\right] .
\end{aligned}
$$

We have defined the world weighted price sub-indexes as $\widehat{p}_{t}^{H, W} \equiv \phi_{H} \widehat{p}_{t}^{H}+\phi_{F} \widehat{p}_{t}^{H *}$ and $\widehat{p}_{t}^{F, W *} \equiv \phi_{F} \widehat{p}_{t}^{F}+\phi_{H} \widehat{p}_{t}^{F *}$, and the relative price sub-indexes as $\widehat{p}_{t}^{H, R} \equiv \widehat{p}_{t}^{H}-\widehat{p}_{t}^{H *}$ and $\widehat{p}_{t}^{F, R} \equiv \widehat{p}_{t}^{F}-\widehat{p}_{t}^{F *}$. Then, naturally, we can write that,

$$
\begin{aligned}
\widehat{p}_{t}^{H} & =\widehat{p}_{t}^{H, W}+\phi_{F} \widehat{p}_{t}^{H, R}, \widehat{p}_{t}^{H *}=\widehat{p}_{t}^{H, W}-\phi_{H} \widehat{p}_{t}^{H, R}, \\
\widehat{p}_{t}^{F} & =\widehat{p}_{t}^{F, W *}+\phi_{H} \widehat{p}_{t}^{F, R}, \widehat{p}_{t}^{F *}=\widehat{p}_{t}^{F, W *}-\phi_{F} \widehat{p}_{t}^{F, R} .
\end{aligned}
$$

Analogously, we have defined the world CPI as $\widehat{p}_{t}^{W} \equiv \phi_{H} \widehat{p}_{t}+\phi_{F} \widehat{p}_{t}^{*}$ and $\widehat{p}_{t}^{W *} \equiv \phi_{F} \widehat{p}_{t}+\phi_{H} \widehat{p}_{t}^{*}$, and the relative CPI as $\widehat{p}_{t}^{R} \equiv \widehat{p}_{t}-\widehat{p}_{t}^{*}$. Then, we can write that,

$$
\begin{aligned}
& \widehat{p}_{t}=\widehat{p}_{t}^{W}+\phi_{F} \widehat{p}_{t}^{R}, \widehat{p}_{t}^{*}=\widehat{p}_{t}^{W}-\phi_{H} \widehat{p}_{t}^{R} \\
& \widehat{p}_{t}=\widehat{p}_{t}^{W *}+\phi_{H} \widehat{p}_{t}^{R}, \widehat{p}_{t}^{*}=\widehat{p}_{t}^{W *}-\phi_{F} \widehat{p}_{t}^{R}
\end{aligned}
$$

Therefore, we can re-arrange the pricing equations as follows,

$$
\begin{aligned}
& \widehat{\pi}_{t}^{H} \approx \beta \mathbb{E}_{t}\left(\widehat{\pi}_{t+1}^{H}\right)+\ldots \\
& \left(\frac{\psi}{1+(1-\psi) \varphi}\right) \Psi\left[\begin{array}{c}
\left(\sigma^{-1}+\left(1-\gamma_{x}\right) \varphi\right) \widehat{c}_{t}^{W}+\gamma_{x} \varphi \widehat{x}_{t}^{W}+\varphi \gamma_{x}\left(\frac{1-\beta(1-\delta)}{\beta \delta}\right) \widehat{u}_{t}+\ldots \\
\phi_{F} \widehat{r s}_{t}-\left(\frac{1+(1-\psi) \varphi}{\psi}+\varphi \eta\right)\left(\widehat{p}_{t}^{H, W}-\widehat{p}_{t}^{W}\right)-\ldots \\
\left(\frac{1+(1-\psi) \varphi}{\psi}\right) \phi_{F}\left(\widehat{p}_{t}^{H, R}-\widehat{p}_{t}^{R}\right)-\left(\frac{1+\varphi}{\psi}\right)\left(\widehat{a}_{t}-(1-\psi) \widehat{r}_{t}^{z}\right)
\end{array}\right] \\
& \widehat{\pi}_{t}^{F *} \approx \beta \mathbb{E}_{t}\left(\widehat{\pi}_{t+1}^{F *}\right)+\ldots \\
& \left(\frac{\psi}{1+(1-\psi) \varphi}\right) \Psi\left[\begin{array}{c}
\left(\sigma^{-1}+\left(1-\gamma_{x}\right) \varphi\right) \widehat{c}_{t}^{W *}+\gamma_{x} \varphi \widehat{x}_{t}^{W *}+\varphi \gamma_{x}\left(\frac{1-\beta(1-\delta)}{\beta \delta}\right) \widehat{u}_{t}^{*}-\ldots \\
\phi_{F} \widehat{r r s}_{t}-\left(\frac{1+(1-\psi) \varphi}{\psi}+\varphi \eta\right)\left(\widehat{p}_{t}^{F, W *}-\widehat{p}_{t}^{W *}\right)+\ldots \\
\left(\frac{1+(1-\psi) \varphi}{\psi}\right) \phi_{F}\left(\widehat{p}_{t}^{F, R}-\widehat{p}_{t}^{R}\right)-\left(\frac{1+\varphi}{\psi}\right)\left(\widehat{a}_{t}^{*}-(1-\psi) \widehat{r}_{t}^{z *}\right)
\end{array}\right]
\end{aligned}
$$

By appropriately replacing the efficiency conditions in (285) - (286), and after a little bit of algebra, we 
can now re-arrange the pricing equations for the price sub-indexes in (291) - (292) as follows,

$$
\begin{aligned}
& \widehat{\pi}_{t}^{H} \approx \beta \mathbb{E}_{t}\left(\widehat{\pi}_{t+1}^{H}\right)+\ldots \\
& \Psi\left[\begin{array}{c}
\left(\sigma^{-1}+\left(1-\gamma_{x}\right)\left(\frac{\psi}{1+(1-\psi) \varphi}\right)\left(\varphi+(1-\psi)\left(\frac{1+\varphi}{\psi}\right)^{2}\right)\right) \widehat{c}_{t}^{W}+\ldots \\
\gamma_{x}\left(\frac{\psi}{1+(1-\psi) \varphi}\right)\left(\varphi+(1-\psi)\left(\frac{1+\varphi}{\psi}\right)^{2}\right) \widehat{x}_{t}^{W}+\ldots \\
\gamma_{x}\left(\frac{\psi}{1+(1-\psi) \varphi}\right)\left(\varphi+(1-\psi)\left(\frac{1+\varphi}{\psi}\right)^{2}\right)\left(\frac{1-\beta(1-\delta)}{\beta \delta}\right) \widehat{u}_{t}+\ldots \\
\phi_{F} \widehat{r s}_{t}-\left(1+\eta\left(\frac{\psi}{1+(1-\psi) \varphi}\right)\left(\varphi+(1-\psi)\left(\frac{1+\varphi}{\psi}\right)^{2}\right)\right)\left(\widehat{p}_{t}^{H, W}-\widehat{p}_{t}^{W}\right)-\ldots \\
\phi_{F}\left(\widehat{p}_{t}^{H, R}-\widehat{p}_{t}^{R}\right)-(1-\psi)\left(\frac{1+\varphi}{\psi}\right) \widehat{k}_{t}-\left(\frac{1+\varphi}{\psi}\right) \widehat{a}_{t}
\end{array}\right], \\
& \widehat{\pi}_{t}^{F *} \approx \beta \mathbb{E}_{t}\left(\widehat{\pi}_{t+1}^{F *}\right)+\ldots \\
& \Psi\left[\begin{array}{c}
\left(\sigma^{-1}+\left(1-\gamma_{x}\right)\left(\frac{\psi}{1+(1-\psi) \varphi}\right)\left(\varphi+(1-\psi)\left(\frac{1+\varphi}{\psi}\right)^{2}\right)\right) \widehat{c}_{t}^{W *}+\ldots \\
\gamma_{x}\left(\frac{\psi}{1+(1-\psi) \varphi}\right)\left(\varphi+(1-\psi)\left(\frac{1+\varphi}{\psi}\right)^{2}\right) \widehat{x}_{t}^{W *}+\ldots \\
\gamma_{x}\left(\frac{\psi}{1+(1-\psi) \varphi}\right)\left(\varphi+(1-\psi)\left(\frac{1+\varphi}{\psi}\right)^{2}\right)\left(\frac{1-\beta(1-\delta)}{\beta \delta}\right) \widehat{u}_{t}^{*}-\ldots \\
\phi_{F} \widehat{r s}_{t}-\left(1+\eta\left(\frac{\psi}{1+(1-\psi) \varphi}\right)\left(\varphi+(1-\psi)\left(\frac{1+\varphi}{\psi}\right)^{2}\right)\right)\left(\widehat{p}_{t}^{F, W *}-\widehat{p}_{t}^{W *}\right)+\ldots \\
\phi_{F}\left(\widehat{p}_{t}^{F, R}-\widehat{p}_{t}^{R}\right)-(1-\psi)\left(\frac{1+\varphi}{\psi}\right) \widehat{k}_{t}^{*}-\left(\frac{1+\varphi}{\psi}\right) \widehat{a}_{t}^{*}
\end{array}\right] .
\end{aligned}
$$

Furthermore, given that under PCP the law of one price holds at the variety level, then it must be the case that $\widehat{p}_{t}^{H} \approx \widehat{s}_{t}+\widehat{p}_{t}^{H *}$ and $\widehat{p}_{t}^{F} \approx \widehat{s}_{t}+\widehat{p}_{t}^{F *}$. Hence, we can express the dynamics of the other implied price sub-indexes as follows,

$$
\begin{aligned}
& \left(\widehat{\pi}_{t}^{H}-\left(\widehat{s}_{t}-\widehat{s}_{t-1}\right)\right)+\left(\widehat{s}_{t}-\widehat{s}_{t-1}\right) \approx \beta \mathbb{E}_{t}\left(\left(\widehat{\pi}_{t+1}^{H}-\left(\widehat{s}_{t+1}-\widehat{s}_{t}\right)\right)+\left(\widehat{s}_{t+1}-\widehat{s}_{t}\right)\right)+\ldots \\
& \Psi\left[\begin{array}{c}
\left(\sigma^{-1}+\left(1-\gamma_{x}\right)\left(\frac{\psi}{1+(1-\psi) \varphi}\right)\left(\varphi+(1-\psi)\left(\frac{1+\varphi}{\psi}\right)^{2}\right)\right) \widehat{c}_{t}^{W}+\ldots \\
\gamma_{x}\left(\frac{\psi}{1+(1-\psi) \varphi}\right)\left(\varphi+(1-\psi)\left(\frac{1+\varphi}{\psi}\right)^{2}\right) \widehat{x}_{t}^{W}+\ldots \\
\gamma_{x}\left(\frac{\psi}{1+(1-\psi) \varphi}\right)\left(\varphi+(1-\psi)\left(\frac{1+\varphi}{\psi}\right)^{2}\right)\left(\frac{1-\beta(1-\delta)}{\beta \delta}\right) \widehat{u}_{t}+\ldots \\
\phi_{F} \widehat{r s}_{t}-\left(1+\eta\left(\frac{\psi}{1+(1-\psi) \varphi}\right)\left(\varphi+(1-\psi)\left(\frac{1+\varphi}{\psi}\right)^{2}\right)\right)\left(\widehat{p}_{t}^{H, W}-\widehat{p}_{t}^{W}\right)-\ldots \\
\phi_{F}\left(\widehat{p}_{t}^{H, R}-\widehat{p}_{t}^{R}\right)-(1-\psi)\left(\frac{1+\varphi}{\psi}\right) \widehat{k}_{t}-\left(\frac{1+\varphi}{\psi}\right) \widehat{a}_{t}
\end{array}\right], \\
& \left(\widehat{\pi}_{t}^{F *}+\left(\widehat{s}_{t}-\widehat{s}_{t-1}\right)\right)-\left(\widehat{s}_{t}-\widehat{s}_{t-1}\right) \approx \beta \mathbb{E}_{t}\left(\left(\widehat{\pi}_{t+1}^{F *}+\left(\widehat{s}_{t+1}-\widehat{s}_{t}\right)\right)-\left(\widehat{s}_{t+1}-\widehat{s}_{t}\right)\right)+\ldots \\
& \Psi\left[\begin{array}{c}
\left(\sigma^{-1}+\left(1-\gamma_{x}\right)\left(\frac{\psi}{1+(1-\psi) \varphi}\right)\left(\varphi+(1-\psi)\left(\frac{1+\varphi}{\psi}\right)^{2}\right)\right) \widehat{c}_{t}^{W *}+\ldots \\
\gamma_{x}\left(\frac{\psi}{1+(1-\psi) \varphi}\right)\left(\varphi+(1-\psi)\left(\frac{1+\varphi}{\psi}\right)^{2}\right) \widehat{x}_{t}^{W *}+\ldots \\
\gamma_{x}\left(\frac{\psi}{1+(1-\psi) \varphi}\right)\left(\varphi+(1-\psi)\left(\frac{1+\varphi}{\psi}\right)^{2}\right)\left(\frac{1-\beta(1-\delta)}{\beta \delta}\right) \widehat{u}_{t}^{*}-\ldots \\
\phi_{F} \widehat{r s}_{t}-\left(1+\eta\left(\frac{\psi}{1+(1-\psi) \varphi}\right)\left(\varphi+(1-\psi)\left(\frac{1+\varphi}{\psi}\right)^{2}\right)\right)\left(\widehat{p}_{t}^{F, W *}-\widehat{p}_{t}^{W *}\right)+\ldots \\
\phi_{F}\left(\widehat{p}_{t}^{F, R}-\widehat{p}_{t}^{R}\right)-(1-\psi)\left(\frac{1+\varphi}{\psi}\right) \widehat{k}_{t}^{*}-\left(\frac{1+\varphi}{\psi}\right) \widehat{a}_{t}^{*}
\end{array}\right],
\end{aligned}
$$


which implies that,

$$
\begin{aligned}
& \widehat{\pi}_{t}^{H *} \approx \beta \mathbb{E}_{t}\left(\widehat{\pi}_{t+1}^{H *}\right)-\left(\widehat{s}_{t}-\widehat{s}_{t-1}\right)+\beta \mathbb{E}_{t}\left(\widehat{s}_{t+1}-\widehat{s}_{t}\right)+\ldots \\
& \left(\sigma^{-1}+\left(1-\gamma_{x}\right)\left(\frac{\psi}{1+(1-\psi) \varphi}\right)\left(\varphi+(1-\psi)\left(\frac{1+\varphi}{\psi}\right)^{2}\right)\right) \widehat{c}_{t}^{W}+\ldots \\
& \gamma_{x}\left(\frac{\psi}{1+(1-\psi) \varphi}\right)\left(\varphi+(1-\psi)\left(\frac{1+\varphi}{\psi}\right)^{2}\right) \widehat{x}_{t}^{W}+\ldots \\
& \gamma_{x}\left(\frac{\psi}{1+(1-\psi) \varphi}\right)\left(\varphi+(1-\psi)\left(\frac{1+\varphi}{\psi}\right)^{2}\right)\left(\frac{1-\beta(1-\delta)}{\beta \delta}\right) \widehat{u}_{t}+\ldots \\
& \widehat{\pi}_{t} \widehat{r}_{t}-\left(\begin{array}{c}
\left.1+\eta\left(\frac{\psi}{1+(1-\psi) \varphi}\right)\left(\varphi+(1-\psi)\left(\frac{1+\varphi}{\psi}\right)^{2}\right)\right)\left(\widehat{p}_{t}^{H, W}-\widehat{p}_{t}^{W}\right)-\ldots \\
\phi_{F}\left(\widehat{p}_{t}^{H, R}-\widehat{p}_{t}^{R}\right)-(1-\psi)\left(\frac{1+\varphi}{\psi}\right) \widehat{k}_{t}-\left(\frac{1+\varphi}{\psi}\right) \widehat{a}_{t}
\end{array}\right], \\
& \Psi \mathbb{E}_{t}\left(\widehat{\pi}_{t+1}^{F}\right)+\left(\widehat{s}_{t}-\widehat{s}_{t-1}\right)-\beta \mathbb{E}_{t}\left(\widehat{s}_{t+1}-\widehat{s}_{t}\right)+\ldots \\
& \left(\begin{array}{c}
\left.\sigma^{-1}+\left(1-\gamma_{x}\right)\left(\frac{\psi}{1+(1-\psi) \varphi}\right)\left(\varphi+(1-\psi)\left(\frac{1+\varphi}{\psi}\right)^{2}\right)\right) \widehat{c}_{t}^{W *}+\ldots \\
\gamma_{x}\left(\frac{\psi}{1+(1-\psi) \varphi}\right)\left(\varphi+(1-\psi)\left(\frac{1+\varphi}{\psi}\right)^{2}\right) \widehat{x}_{t}^{W *}+\ldots \\
\gamma_{x}\left(\frac{\psi}{1+(1-\psi) \varphi}\right)\left(\varphi+(1-\psi)\left(\frac{1+\varphi}{\psi}\right)^{2}\right)\left(\frac{1-\beta(1-\delta)}{\beta \delta}\right) \widehat{u}_{t}^{*}-\ldots \\
\widehat{c}_{F} \widehat{r s}_{t}-\left(1+\eta\left(\frac{\psi}{1+(1-\psi) \varphi}\right)\left(\varphi+(1-\psi)\left(\frac{1+\varphi}{\psi}\right)^{2}\right)\right)\left(\widehat{p}_{t}^{F, W *}-\widehat{p}_{t}^{W *}\right)+\ldots \\
\phi_{F}\left(\widehat{p}_{t}^{F, R}-\widehat{p}_{t}^{R}\right)-(1-\psi)\left(\frac{1+\varphi}{\psi}\right)_{t}^{*}-\left(\frac{1+\varphi}{\psi}\right) \widehat{a}_{t}^{*}
\end{array}\right] .
\end{aligned}
$$

We define the log-linearized CPI indexes of both countries as $\widehat{p}_{t} \approx \phi_{H} \widehat{p}_{t}^{H}+\phi_{F} \widehat{p}_{t}^{F}$ and $\widehat{p}_{t}^{*} \approx \phi_{F} \widehat{p}_{t}^{H *}+\phi_{H} \widehat{p}_{t}^{F *}$. In turn, it follows that CPI inflation can be calculated as $\widehat{\pi}_{t} \approx \phi_{H} \widehat{\pi}_{t}^{H}+\phi_{F} \widehat{\pi}_{t}^{F}$ and $\widehat{\pi}_{t}^{*} \approx \phi_{F} \widehat{\pi}_{t}^{H *}+\phi_{H} \widehat{\pi}_{t}^{F *}$. Therefore, it is easy to derive the dynamics of $\widehat{\pi}_{t} \equiv \widehat{p}_{t}-\widehat{p}_{t-1}$ and $\widehat{\pi}_{t}^{*} \equiv \widehat{p}_{t}^{*}-\widehat{p}_{t-1}^{*}$ from the equations above. For domestic inflation, $\widehat{\pi}_{t}$, it follows that,

$$
\begin{aligned}
& \left(\sigma^{-1}+\left(1-\gamma_{x}\right)\left(\frac{\psi}{1+(1-\psi) \varphi}\right)\left(\varphi+(1-\psi)\left(\frac{1+\varphi}{\psi}\right)^{2}\right)\right) \phi_{H} \widehat{c}_{t}^{W}+\ldots \\
& \gamma_{x}\left(\frac{\psi}{1+(1-\psi) \varphi}\right)\left(\varphi+(1-\psi)\left(\frac{1+\varphi}{\psi}\right)^{2}\right) \phi_{H} \widehat{x}_{t}^{W}+\ldots \\
& \widehat{\pi}_{t} \approx \beta \mathbb{E}_{t}\left(\phi_{H} \widehat{\pi}_{t+1}^{H}\right)+\Psi\left[\begin{array}{c}
\gamma_{x}\left(\frac{\psi}{1+(1-\psi) \varphi}\right)\left(\varphi+(1-\psi)\left(\frac{1+\varphi}{\psi}\right)^{2}\right)\left(\frac{1-\beta(1-\delta)}{\beta \delta}\right) \phi_{H} \widehat{u}_{t}+\ldots \\
\phi_{H} \phi_{F} \widehat{r s} t-\left(1+\eta\left(\frac{\psi}{1+(1-\psi) \varphi}\right)\left(\varphi+(1-\psi)\left(\frac{1+\varphi}{\psi}\right)^{2}\right)\right) \phi_{H}\left(\widehat{p}_{t}^{H, W}-\widehat{p}_{t}^{W}\right)-\ldots \\
\phi_{H} \phi_{F}\left(\widehat{p}_{t}^{H, R}-\widehat{p}_{t}^{R}\right)-(1-\psi)\left(\frac{1+\varphi}{\psi}\right) \phi_{H} \widehat{k}_{t}-\left(\frac{1+\varphi}{\psi}\right) \phi_{H} \widehat{a}_{t}
\end{array}\right]+\ldots \\
& \beta \mathbb{E}_{t}\left(\phi_{F} \widehat{\pi}_{t+1}^{F}\right)+\phi_{F}\left(\widehat{s}_{t}-\widehat{s}_{t-1}\right)-\phi_{F} \beta \mathbb{E}_{t}\left(\widehat{s}_{t+1}-\widehat{s}_{t}\right)+\ldots \\
& \Psi\left[\begin{array}{c}
\left(\sigma^{-1}+\left(1-\gamma_{x}\right)\left(\frac{\psi}{1+(1-\psi) \varphi}\right)\left(\varphi+(1-\psi)\left(\frac{1+\varphi}{\psi}\right)^{2}\right)\right) \phi_{F} \widehat{c}_{t}^{W *}+\ldots \\
\gamma_{x}\left(\frac{\psi}{1+(1-\psi) \varphi}\right)\left(\varphi+(1-\psi)\left(\frac{1+\varphi}{\psi}\right)^{2}\right) \phi_{F} \widehat{x}_{t}^{W *}+\ldots \\
\gamma_{x}\left(\frac{\psi}{1+(1-\psi) \varphi}\right)\left(\varphi+(1-\psi)\left(\frac{1+\varphi}{\psi}\right)^{2}\right)\left(\frac{1-\beta(1-\delta)}{\beta \delta}\right) \phi_{F} \widehat{u}_{t}^{*}-\ldots \\
\phi_{F} \phi_{F} \widehat{r s}_{t}-\left(1+\eta\left(\frac{\psi}{1+(1-\psi) \varphi}\right)\left(\varphi+(1-\psi)\left(\frac{1+\varphi}{\psi}\right)^{2}\right)\right) \phi_{F}\left(\widehat{p}_{t}^{F, W *}-\widehat{p}_{t}^{W *}\right)+\ldots \\
\phi_{F} \phi_{F}\left(\widehat{p}_{t}^{F, R}-\widehat{p}_{t}^{R}\right)-(1-\psi)\left(\frac{1+\varphi}{\psi}\right) \phi_{F} \widehat{k}_{t}^{*}-\left(\frac{1+\varphi}{\psi}\right) \phi_{F} \widehat{a}_{t}^{*}
\end{array}\right],
\end{aligned}
$$


and, after further re-arranging, we get that,

$$
\left.\begin{array}{c}
\widehat{\pi}_{t} \approx \beta \mathbb{E}_{t}\left(\widehat{\pi}_{t+1}\right)+\phi_{F}\left(\widehat{s}_{t}-\widehat{s}_{t-1}\right)-\phi_{F} \beta \mathbb{E}_{t}\left(\widehat{s}_{t+1}-\widehat{s}_{t}\right)+\ldots \\
\left(\sigma^{-1}+\left(1-\gamma_{x}\right)\left(\frac{\psi}{1+(1-\psi) \varphi}\right)\left(\varphi+(1-\psi)\left(\frac{1+\varphi}{\psi}\right)^{2}\right)\right)\left[\phi_{H} \widehat{c}_{t}^{W}+\phi_{F} \widehat{c}_{t}^{W *}\right]+\ldots \\
\gamma_{x}\left(\frac{\psi}{1+(1-\psi) \varphi}\right)\left(\varphi+(1-\psi)\left(\frac{1+\varphi}{\psi}\right)^{2}\right)\left[\phi_{H} \widehat{x}_{t}^{W}+\phi_{F} \widehat{x}_{t}^{W *}\right]+\ldots \\
\gamma_{x}\left(\frac{\psi}{1+(1-\psi) \varphi}\right)\left(\varphi+(1-\psi)\left(\frac{1+\varphi}{\psi}\right)^{2}\right)\left(\frac{1-\beta(1-\delta)}{\beta \delta}\right)\left[\phi_{H} \widehat{u}_{t}+\phi_{F} \widehat{u}_{t}^{*}\right]+\left(\phi_{H}-\phi_{F}\right) \phi_{F} \widehat{r s}_{t}-\ldots \\
\left(1+\eta\left(\frac{\psi}{1+(1-\psi) \varphi}\right)\left(\varphi+(1-\psi)\left(\frac{1+\varphi}{\psi}\right)^{2}\right)\right)\left[\phi_{H}\left(\widehat{p}_{t}^{H, W}-\widehat{p}_{t}^{W}\right)+\phi_{F}\left(\widehat{p}_{t}^{F, W *}-\widehat{p}_{t}^{W *}\right)\right]-\ldots \\
\phi_{H} \phi_{F}\left(\widehat{p}_{t}^{H, R}-\widehat{p}_{t}^{R}\right)+\phi_{F} \phi_{F}\left(\widehat{p}_{t}^{F, R}-\widehat{p}_{t}^{R}\right)-(1-\psi)\left(\frac{1+\varphi}{\psi}\right) \widehat{k}_{t}^{W}-\left(\frac{1+\varphi}{\psi}\right)\left[\phi_{H} \widehat{a}_{t}+\phi_{F} \widehat{a}_{t}^{*}\right]
\end{array}\right],
$$

and,

$$
\left.\begin{array}{c}
\widehat{\pi}_{t} \approx \beta \mathbb{E}_{t}\left(\widehat{\pi}_{t+1}\right)+\phi_{F}\left[\left(\widehat{s}_{t}-\widehat{s}_{t-1}\right)-\beta \mathbb{E}_{t}\left(\widehat{s}_{t+1}-\widehat{s}_{t}\right)-\Psi\left(\widehat{r}_{t}-\left(\widehat{p}_{t}^{F, R}-\widehat{p}_{t}^{R}\right)\right)\right]+\ldots \\
\left(\sigma^{-1}+\left(1-\gamma_{x}\right)\left(\frac{\psi}{1+(1-\psi) \varphi}\right)\left(\varphi+(1-\psi)\left(\frac{1+\varphi}{\psi}\right)^{2}\right)\right)\left[\phi_{H} \widehat{c}_{t}^{W}+\phi_{F} \widehat{c}_{t}^{W *}\right]+\ldots \\
\gamma_{x}\left(\frac{\psi}{1+(1-\psi) \varphi}\right)\left(\varphi+(1-\psi)\left(\frac{1+\varphi}{\psi}\right)^{2}\right)\left[\phi_{H} \widehat{x}_{t}^{W}+\phi_{F} \widehat{x}_{t}^{W *}\right]+\ldots \\
\gamma_{x}\left(\frac{\psi}{1+(1-\psi) \varphi}\right)\left(\varphi+(1-\psi)\left(\frac{1+\varphi}{\psi}\right)^{2}\right)\left(\frac{1-\beta(1-\delta)}{\beta \delta}\right)\left[\phi_{H} \widehat{u}_{t}+\phi_{F} \widehat{u}_{t}^{*}\right]+2 \phi_{H} \phi_{F} \widehat{r s}_{t}-\ldots \\
\left(1+\eta\left(\frac{\psi}{1+(1-\psi) \varphi}\right)\left(\varphi+(1-\psi)\left(\frac{1+\varphi}{\psi}\right)^{2}\right)\right)\left[\phi_{H}\left(\widehat{p}_{t}^{H, W}-\widehat{p}_{t}^{W}\right)+\phi_{F}\left(\widehat{p}_{t}^{F, W *}-\widehat{p}_{t}^{W *}\right)\right]-\ldots \\
\phi_{H} \phi_{F}\left[\left(\widehat{p}_{t}^{H, R}-\widehat{p}_{t}^{R}\right)+\left(\widehat{p}_{t}^{F, R}-\widehat{p}_{t}^{R}\right)\right]-(1-\psi)\left(\frac{1+\varphi}{\psi}\right) \widehat{k}_{t}^{W}-\left(\frac{1+\varphi}{\psi}\right)\left[\phi_{H} \widehat{a}_{t}+\phi_{F} \widehat{a}_{t}^{*}\right]
\end{array}\right],
$$

where world weighted capital is defined as $\widehat{k}_{t}^{W} \equiv \phi_{H} \widehat{k}_{t}+\phi_{F} \widehat{k}_{t}^{*}$. For foreign inflation, $\widehat{\pi}_{t}^{*}$, it follows that,

$$
\begin{aligned}
& \widehat{\pi}_{t}^{*} \approx \beta \mathbb{E}_{t}\left(\phi_{F} \widehat{\pi}_{t+1}^{H *}\right)-\phi_{F}\left(\widehat{s}_{t}-\widehat{s}_{t-1}\right)+\phi_{F} \beta \mathbb{E}_{t}\left(\widehat{s}_{t+1}-\widehat{s}_{t}\right)+\ldots \\
& \Psi\left[\begin{array}{c}
\left(\sigma^{-1}+\left(1-\gamma_{x}\right)\left(\frac{\psi}{1+(1-\psi) \varphi}\right)\left(\varphi+(1-\psi)\left(\frac{1+\varphi}{\psi}\right)^{2}\right)\right) \phi_{F} \widehat{c}_{t}^{W}+\ldots \\
\gamma_{x}\left(\frac{\psi}{1+(1-\psi) \varphi}\right)\left(\varphi+(1-\psi)\left(\frac{1+\varphi}{\psi}\right)^{2}\right) \phi_{F} \widehat{x}_{t}^{W}+\ldots \\
\gamma_{x}\left(\frac{\psi}{1+(1-\psi) \varphi}\right)\left(\varphi+(1-\psi)\left(\frac{1+\varphi}{\psi}\right)^{2}\right)\left(\frac{1-\beta(1-\delta)}{\beta \delta}\right) \phi_{F} \widehat{u}_{t}+\ldots \\
\phi_{F} \phi_{F} \widehat{r}_{t}-\left(1+\eta\left(\frac{\psi}{1+(1-\psi) \varphi}\right)\left(\varphi+(1-\psi)\left(\frac{1+\varphi}{\psi}\right)^{2}\right)\right) \phi_{F}\left(\widehat{p}_{t}^{H, W}-\widehat{p}_{t}^{W}\right)-\ldots \\
\phi_{F} \phi_{F}\left(\widehat{p}_{t}^{H, R}-\widehat{p}_{t}^{R}\right)-(1-\psi)\left(\frac{1+\varphi}{\psi}\right) \phi_{F} \widehat{k}_{t}-\left(\frac{1+\varphi}{\psi}\right) \phi_{F} \widehat{a}_{t}
\end{array}\right]+\ldots \\
& \beta \mathbb{E}_{t}\left(\phi_{H} \widehat{\pi}_{t+1}^{F *}\right)+\Psi\left[\begin{array}{c}
\left(\sigma^{-1}+\left(1-\gamma_{x}\right)\left(\frac{\psi}{1+(1-\psi) \varphi}\right)\left(\varphi+(1-\psi)\left(\frac{1+\varphi}{\psi}\right)^{2}\right)\right) \phi_{H} \widehat{c}_{t}^{W *}+\ldots \\
\gamma_{x}\left(\frac{\psi}{1+(1-\psi) \varphi}\right)\left(\varphi+(1-\psi)\left(\frac{1+\varphi}{\psi}\right)^{2}\right) \phi_{H} \widehat{x}_{t}^{W *}+\ldots \\
\gamma_{x}\left(\frac{\psi}{1+(1-\psi) \varphi}\right)\left(\varphi+(1-\psi)\left(\frac{1+\varphi}{\psi}\right)^{2}\right)\left(\frac{1-\beta(1-\delta)}{\beta \delta}\right) \phi_{H} \widehat{u}_{t}^{*}-\ldots \\
\phi_{H} \phi_{F} \widehat{r s s}_{t}-\left(1+\eta\left(\frac{\psi}{1+(1-\psi) \varphi}\right)\left(\varphi+(1-\psi)\left(\frac{1+\varphi}{\psi}\right)^{2}\right)\right) \phi_{H}\left(\widehat{p}_{t}^{F, W *}-\widehat{p}_{t}^{W *}\right)+\ldots \\
\phi_{H} \phi_{F}\left(\widehat{p}_{t}^{F, R}-\widehat{p}_{t}^{R}\right)-(1-\psi)\left(\frac{1+\varphi}{\psi}\right) \phi_{H} \widehat{k}_{t}^{*}-\left(\frac{1+\varphi}{\psi}\right) \phi_{H} \widehat{a}_{t}^{*}
\end{array}\right],
\end{aligned}
$$


and, after a little bit of algebra,

$$
\begin{aligned}
& \widehat{\pi}_{t}^{*} \approx \beta \mathbb{E}_{t}\left(\widehat{\pi}_{t+1}^{*}\right)-\phi_{F}\left(\widehat{s}_{t}-\widehat{s}_{t-1}\right)+\phi_{F} \beta \mathbb{E}_{t}\left(\widehat{s}_{t+1}-\widehat{s}_{t}\right)+\ldots \\
& \Psi\left[\begin{array}{c}
\left(\sigma^{-1}+\left(1-\gamma_{x}\right)\left(\frac{\psi}{1+(1-\psi) \varphi}\right)\left(\varphi+(1-\psi)\left(\frac{1+\varphi}{\psi}\right)^{2}\right)\right) \phi_{F} \widehat{c}_{t}^{W}+\ldots \\
\gamma_{x}\left(\frac{\psi}{1+(1-\psi) \varphi}\right)\left(\varphi+(1-\psi)\left(\frac{1+\varphi}{\psi}\right)^{2}\right) \phi_{F} \widehat{x}_{t}^{W}+\ldots \\
\gamma_{x}\left(\frac{\psi}{1+(1-\psi) \varphi}\right)\left(\varphi+(1-\psi)\left(\frac{1+\varphi}{\psi}\right)^{2}\right)\left(\frac{1-\beta(1-\delta)}{\beta \delta}\right) \phi_{F} \widehat{u}_{t}+\ldots \\
\phi_{F} \phi_{F} \widehat{r s}_{t}-\left(1+\eta\left(\frac{\psi}{1+(1-\psi) \varphi}\right)\left(\varphi+(1-\psi)\left(\frac{1+\varphi}{\psi}\right)^{2}\right)\right) \phi_{F}\left(\widehat{p}_{t}^{H, W}-\widehat{p}_{t}^{W}\right)-\ldots \\
\phi_{F} \phi_{F}\left(\widehat{p}_{t}^{H, R}-\widehat{p}_{t}^{R}\right)-(1-\psi)\left(\frac{1+\varphi}{\psi}\right) \phi_{F} \widehat{k}_{t}-\left(\frac{1+\varphi}{\psi}\right) \phi_{F} \widehat{a}_{t} \\
\left(\sigma^{-1}+\left(1-\gamma_{x}\right)\left(\frac{\psi}{1+(1-\psi) \varphi}\right)\left(\varphi+(1-\psi)\left(\frac{1+\varphi}{\psi}\right)^{2}\right)\right) \phi_{H} \widehat{c}_{t}^{W *}+\ldots \\
\gamma_{x}\left(\frac{\psi}{1+(1-\psi) \varphi}\right)\left(\varphi+(1-\psi)\left(\frac{1+\varphi}{\psi}\right)^{2}\right) \phi_{H} \widehat{x}_{t}^{W *}+\ldots \\
\gamma_{x}\left(\frac{\psi}{1+(1-\psi) \varphi}\right)\left(\varphi+(1-\psi)\left(\frac{1+\varphi}{\psi}\right)^{2}\right)\left(\frac{1-\beta(1-\delta)}{\beta \delta}\right) \phi_{H} \widehat{u}_{t}^{*}-\ldots \\
\phi_{H} \phi_{F} \widehat{r s}_{t}-\left(1+\eta\left(\frac{\psi}{1+(1-\psi) \varphi}\right)\left(\varphi+(1-\psi)\left(\frac{1+\varphi}{\psi}\right)^{2}\right)\right) \phi_{H}\left(\widehat{p}_{t}^{F, W *}-\widehat{p}_{t}^{W *}\right)+\ldots \\
\phi_{H} \phi_{F}\left(\widehat{p}_{t}^{F, R}-\widehat{p}_{t}^{R}\right)-(1-\psi)\left(\frac{1+\varphi}{\psi}\right) \phi_{H} \widehat{k}_{t}^{*}-\left(\frac{1+\varphi}{\psi}\right) \phi_{H} \widehat{a}_{t}^{*}
\end{array}\right]+
\end{aligned}
$$

and,

$$
\left.\begin{array}{c}
\widehat{\pi}_{t}^{*} \approx \beta \mathbb{E}_{t}\left(\widehat{\pi}_{t+1}^{*}\right)-\phi_{F}\left(\widehat{s}_{t}-\widehat{s}_{t-1}\right)+\phi_{F} \beta \mathbb{E}_{t}\left(\widehat{s}_{t+1}-\widehat{s}_{t}\right)+\ldots \\
\left(\sigma^{-1}+\left(1-\gamma_{x}\right)\left(\frac{\psi}{1+(1-\psi) \varphi}\right)\left(\varphi+(1-\psi)\left(\frac{1+\varphi}{\psi}\right)^{2}\right)\right)\left[\phi_{F} \widehat{c}_{t}^{W}+\phi_{H} \widehat{c}_{t}^{W *}\right]+\ldots \\
\gamma_{x}\left(\frac{\psi}{1+(1-\psi) \varphi}\right)\left(\varphi+(1-\psi)\left(\frac{1+\varphi}{\psi}\right)^{2}\right)\left[\phi_{F} \widehat{x}_{t}^{W}+\phi_{H} \widehat{x}_{t}^{W *}\right]+\ldots \\
\gamma_{x}\left(\frac{\psi}{1+(1-\psi) \varphi}\right)\left(\varphi+(1-\psi)\left(\frac{1+\varphi}{\psi}\right)^{2}\right)\left(\frac{1-\beta(1-\delta)}{\beta \delta}\right)\left[\phi_{F} \widehat{u}_{t}+\phi_{H} \widehat{u}_{t}^{*}\right]+\ldots \\
\phi_{F} \phi_{F} \widehat{r s}_{t}-\phi_{H} \phi_{F} \widehat{r s s}_{t}-\ldots \\
\phi_{F} \phi_{F}\left(\widehat{p}_{t}^{H, R}-\widehat{p}_{t}^{R}\right)+\phi_{H} \phi_{F}\left(\widehat{p}_{t}^{F, R}-\widehat{p}_{t}^{R}\right)-(1-\psi)\left(\frac{1+\varphi}{\psi}\right)\left[\phi_{F} \widehat{k}_{t}+\phi_{H} \widehat{k}_{t}^{*}\right]-\left(\frac{1+\varphi}{\psi}\right)\left[\phi_{F} \widehat{a}_{t}+\phi_{H} \widehat{a}_{t}^{*}\right]
\end{array}\right],
$$

and,

$$
\left.\begin{array}{c}
\widehat{\pi}_{t}^{*} \approx \beta \mathbb{E}_{t}\left(\widehat{\pi}_{t+1}^{*}\right)-\phi_{F}\left[\left(\widehat{s}_{t}-\widehat{s}_{t-1}\right)-\beta \mathbb{E}_{t}\left(\widehat{s}_{t+1}-\widehat{s}_{t}\right)-\Psi\left(\widehat{r}_{t}-\left(\widehat{p}_{t}^{H, R}-\widehat{p}_{t}^{R}\right)\right)\right]+\ldots \\
\left(\sigma^{-1}+\left(1-\gamma_{x}\right)\left(\frac{\psi}{1+(1-\psi) \varphi}\right)\left(\varphi+(1-\psi)\left(\frac{1+\varphi}{\psi}\right)^{2}\right)\right)\left[\phi_{F} \widehat{c}_{t}^{W}+\phi_{H} \widehat{c}_{t}^{W *}\right]+\ldots \\
\gamma_{x}\left(\frac{\psi}{1+(1-\psi) \varphi}\right)\left(\varphi+(1-\psi)\left(\frac{1+\varphi}{\psi}\right)^{2}\right)\left[\phi_{F} \widehat{x}_{t}^{W}+\phi_{H} \widehat{x}_{t}^{W *}\right]+\ldots \\
\gamma_{x}\left(\frac{\psi}{1+(1-\psi) \varphi}\right)\left(\varphi+(1-\psi)\left(\frac{1+\varphi}{\psi}\right)^{2}\right)\left(\frac{1-\beta(1-\delta)}{\beta \delta}\right)\left[\phi_{F} \widehat{u}_{t}+\phi_{H} \widehat{u}_{t}^{*}\right]-\ldots \\
2 \phi_{H} \phi_{F} \widehat{r}_{t}-\left(1+\eta\left(\frac{\psi}{1+(1-\psi) \varphi}\right)\left(\varphi+(1-\psi)\left(\frac{1+\varphi}{\psi}\right)^{2}\right)\right)\left[\phi_{F}\left(\widehat{p}_{t}^{H, W}-\widehat{p}_{t}^{W}\right)+\phi_{H}\left(\widehat{p}_{t}^{F, W *}-\widehat{p}_{t}^{W *}\right)\right]+\ldots \\
\phi_{H} \phi_{F}\left[\left(\widehat{p}_{t}^{H, R}-\widehat{p}_{t}^{R}\right)+\left(\widehat{p}_{t}^{F, R}-\widehat{p}_{t}^{R}\right)\right]-(1-\psi)\left(\frac{1+\varphi}{\psi}\right) \widehat{k}_{t}^{W *}-\left(\frac{1+\varphi}{\psi}\right)\left[\phi_{F} \widehat{a}_{t}+\phi_{H} \widehat{a}_{t}^{*}\right]
\end{array}\right],
$$


where world weighted capital is defined as $\widehat{k}_{t}^{W *} \equiv \phi_{F} \widehat{k}_{t}+\phi_{H} \widehat{k}_{t}^{*}$.

In summary, it follows that the domestic and foreign Phillips curves can be expressed as,

$$
\begin{aligned}
& \widehat{\pi}_{t} \approx \beta \mathbb{E}_{t}\left(\widehat{\pi}_{t+1}\right)+\phi_{F}\left[\left(\widehat{s}_{t}-\widehat{s}_{t-1}\right)-\beta \mathbb{E}_{t}\left(\widehat{s}_{t+1}-\widehat{s}_{t}\right)+\Psi\left(\widehat{p}_{t}^{F, R}-\widehat{s}_{t}\right)\right]+\ldots \\
& \Psi\left[\begin{array}{c}
\left(\sigma^{-1}+\left(1-\gamma_{x}\right)\left(\frac{\psi}{1+(1-\psi) \varphi}\right)\left(\varphi+(1-\psi)\left(\frac{1+\varphi}{\psi}\right)^{2}\right)\right)\left[\phi_{H} \widehat{c}_{t}^{W}+\phi_{F} \widehat{c}_{t}^{W *}\right]+\ldots \\
\gamma_{x}\left(\frac{\psi}{1+(1-\psi) \varphi}\right)\left(\varphi+(1-\psi)\left(\frac{1+\varphi}{\psi}\right)^{2}\right)\left[\phi_{H} \widehat{x}_{t}^{W}+\phi_{F} \widehat{x}_{t}^{W *}\right]+\ldots \\
\gamma_{x}\left(\frac{\psi}{1+(1-\psi) \varphi}\right)\left(\varphi+(1-\psi)\left(\frac{1+\varphi}{\psi}\right)^{2}\right)\left(\frac{1-\beta(1-\delta)}{\beta \delta}\right)\left[\phi_{H} \widehat{u}_{t}+\phi_{F} \widehat{u}_{t}^{*}\right]+2 \phi_{H} \phi_{F} \widehat{r s}_{t}-\ldots \\
\left(1+\eta\left(\frac{\psi}{1+(1-\psi) \varphi}\right)\left(\varphi+(1-\psi)\left(\frac{1+\varphi}{\psi}\right)^{2}\right)\right)\left[\phi_{H}\left(\widehat{p}_{t}^{H, W}-\widehat{p}_{t}^{W}\right)+\phi_{F}\left(\widehat{p}_{t}^{F, W *}-\widehat{p}_{t}^{W *}\right)\right]-\ldots \\
\phi_{H} \phi_{F}\left[\left(\widehat{p}_{t}^{H, R}-\widehat{p}_{t}^{R}\right)+\left(\widehat{p}_{t}^{F, R}-\widehat{p}_{t}^{R}\right)\right]-(1-\psi)\left(\frac{1+\varphi}{\psi}\right) \widehat{k}_{t}^{W}-\left(\frac{1+\varphi}{\psi}\right)\left[\phi_{H} \widehat{a}_{t}+\phi_{F} \widehat{a}_{t}^{*}\right]
\end{array}\right], \\
& \widehat{\pi}_{t}^{*} \approx \beta \mathbb{E}_{t}\left(\widehat{\pi}_{t+1}^{*}\right)-\phi_{F}\left[\left(\widehat{s}_{t}-\widehat{s}_{t-1}\right)-\beta \mathbb{E}_{t}\left(\widehat{s}_{t+1}-\widehat{s}_{t}\right)+\Psi\left(\widehat{p}_{t}^{H, R}-\widehat{s}_{t}\right)\right]+\ldots \\
& \Psi\left[\begin{array}{c}
\left(\sigma^{-1}+\left(1-\gamma_{x}\right)\left(\frac{\psi}{1+(1-\psi) \varphi}\right)\left(\varphi+(1-\psi)\left(\frac{1+\varphi}{\psi}\right)^{2}\right)\right)\left[\phi_{F} \widehat{c}_{t}^{W}+\phi_{H} \widehat{c}_{t}^{W *}\right]+\ldots \\
\gamma_{x}\left(\frac{\psi}{1+(1-\psi) \varphi}\right)\left(\varphi+(1-\psi)\left(\frac{1+\varphi}{\psi}\right)^{2}\right)\left[\phi_{F} \widehat{x}_{t}^{W}+\phi_{H} \widehat{x}_{t}^{W *}\right]+\ldots \\
\gamma_{x}\left(\frac{\psi}{1+(1-\psi) \varphi}\right)\left(\varphi+(1-\psi)\left(\frac{1+\varphi}{\psi}\right)^{2}\right)\left(\frac{1-\beta(1-\delta)}{\beta \delta}\right)\left[\phi_{F} \widehat{u}_{t}+\phi_{H} \widehat{u}_{t}^{*}\right]-2 \phi_{H} \phi_{F} \widehat{r r s}_{t}-\ldots \\
\left(1+\eta\left(\frac{\psi}{1+(1-\psi) \varphi}\right)\left(\varphi+(1-\psi)\left(\frac{1+\varphi}{\psi}\right)^{2}\right)\right)\left[\phi_{F}\left(\widehat{p}_{t}^{H, W}-\widehat{p}_{t}^{W}\right)+\phi_{H}\left(\widehat{p}_{t}^{F, W *}-\widehat{p}_{t}^{W *}\right)\right]+\ldots \\
\phi_{H} \phi_{F}\left[\left(\widehat{p}_{t}^{H, R}-\widehat{p}_{t}^{R}\right)+\left(\widehat{p}_{t}^{F, R}-\widehat{p}_{t}^{R}\right)\right]-(1-\psi)\left(\frac{1+\varphi}{\psi}\right) \widehat{k}_{t}^{W *}-\left(\frac{1+\varphi}{\psi}\right)\left[\phi_{F} \widehat{a}_{t}+\phi_{H} \widehat{a}_{t}^{*}\right]
\end{array}\right],
\end{aligned}
$$

where the world weighted capital measures are defined as $\widehat{k}_{t}^{W} \equiv \phi_{H} \widehat{k}_{t}+\phi_{F} \widehat{k}_{t}^{*}$ and $\widehat{k}_{t}^{W *} \equiv \phi_{F} \widehat{k}_{t}+\phi_{H} \widehat{k}_{t}^{*}$. We can also write certain terms inside the brackets of the Phillips curves more compactly as,

$$
\begin{aligned}
- & \left(1+\eta\left(\frac{\psi}{1+(1-\psi) \varphi}\right)\left(\varphi+(1-\psi)\left(\frac{1+\varphi}{\psi}\right)^{2}\right)\right)\left[\phi_{H}\left(\widehat{p}_{t}^{H, W}-\widehat{p}_{t}^{W}\right)+\phi_{F}\left(\widehat{p}_{t}^{F, W *}-\widehat{p}_{t}^{W *}\right)\right]-\ldots \\
& \phi_{H} \phi_{F}\left[\left(\widehat{p}_{t}^{H, R}-\widehat{p}_{t}^{R}\right)+\left(\widehat{p}_{t}^{F, R}-\widehat{p}_{t}^{R}\right)\right] \\
& =\eta\left(\frac{\psi}{1+(1-\psi) \varphi}\right)\left(\varphi+(1-\psi)\left(\frac{1+\varphi}{\psi}\right)^{2}\right)\left[\phi_{H}-\phi_{F}\right]\left(\widehat{p}_{t}^{F, W *}-\widehat{p}_{t}^{W *}\right)-\ldots \\
& \eta\left(\frac{\psi}{1+(1-\psi) \varphi}\right)\left(\varphi+(1-\psi)\left(\frac{1+\varphi}{\psi}\right)^{2}\right) \phi_{H}\left[\left(\widehat{p}_{t}^{H, W}-\widehat{p}_{t}^{W}\right)+\left(\widehat{p}_{t}^{F, W *}-\widehat{p}_{t}^{W *}\right)\right]-\ldots \\
& \phi_{H}\left[\left(\widehat{p}_{t}^{H, W}-\widehat{p}_{t}^{W}\right)+\phi_{F}\left(\widehat{p}_{t}^{H, R}-\widehat{p}_{t}^{R}\right)\right]-\phi_{F}\left[\left(\widehat{p}_{t}^{F, W *}-\widehat{p}_{t}^{W *}\right)+\phi_{H}\left(\widehat{p}_{t}^{F, R}-\widehat{p}_{t}^{R}\right)\right], \\
- & \left(1+\eta\left(\frac{\psi}{1+(1-\psi) \varphi}\right)\left(\varphi+(1-\psi)\left(\frac{1+\varphi}{\psi}\right)^{2}\right)\right)\left[\phi_{F}\left(\widehat{p}_{t}^{H, W}-\widehat{p}_{t}^{W}\right)+\phi_{H}\left(\widehat{p}_{t}^{F, W *}-\widehat{p}_{t}^{W *}\right)\right]+\ldots \\
& \phi_{F} \phi_{H}\left[\left(\widehat{p}_{t}^{H, R}-\widehat{p}_{t}^{R}\right)+\left(\widehat{p}_{t}^{F, R}-\widehat{p}_{t}^{R}\right)\right] \\
& =-\eta\left(\frac{\psi}{1+(1-\psi) \varphi}\right)\left(\varphi+(1-\psi)\left(\frac{1+\varphi}{\psi}\right)^{2}\right)\left[\phi_{H}-\phi_{F}\right]\left(\widehat{p}_{t}^{F, W *}-\widehat{p}_{t}^{W *}\right)-\ldots \\
& \eta\left(\frac{\psi}{1+(1-\psi) \varphi}\right)\left(\varphi+(1-\psi)\left(\frac{1+\varphi}{\psi}\right)^{2}\right) \phi_{F}\left[\left(\widehat{p}_{t}^{H, W}-\widehat{p}_{t}^{W}\right)+\left(\widehat{p}_{t}^{F, W *}-\widehat{p}_{t}^{W *}\right)\right]-\ldots \\
& \phi_{F}\left[\left(\widehat{p}_{t}^{H, W}-\widehat{p}_{t}^{W}\right)-\phi_{H}\left(\widehat{p}_{t}^{H, R}-\widehat{p}_{t}^{R}\right)\right]-\phi_{H}\left[\left(\widehat{p}_{t}^{F, W *}-\widehat{p}_{t}^{W *}\right)-\phi_{F}\left(\widehat{p}_{t}^{F, R}-\widehat{p}_{t}^{R}\right)\right] .
\end{aligned}
$$

Based on our definitions of the world weighted price indexes and sub-indexes, denoted with the superscripts 
$W$ and $W^{*}$, it is possible for us to argue that,

$$
\begin{aligned}
& \phi_{H}\left[\left(\widehat{p}_{t}^{H, W}-\widehat{p}_{t}^{W}\right)+\phi_{F}\left(\widehat{p}_{t}^{H, R}-\widehat{p}_{t}^{R}\right)\right]+\phi_{F}\left[\left(\widehat{p}_{t}^{F, W *}-\widehat{p}_{t}^{W *}\right)+\phi_{H}\left(\widehat{p}_{t}^{F, R}-\widehat{p}_{t}^{R}\right)\right] \\
& \quad=\phi_{H}\left(\widehat{p}_{t}^{H}-\widehat{p}_{t}\right)+\phi_{F}\left(\widehat{p}_{t}^{F}-\widehat{p}_{t}\right)=0, \\
& \phi_{F}\left[\left(\widehat{p}_{t}^{H, W}-\widehat{p}_{t}^{W}\right)-\phi_{H}\left(\widehat{p}_{t}^{H, R}-\widehat{p}_{t}^{R}\right)\right]+\phi_{H}\left[\left(\widehat{p}_{t}^{F, W *}-\widehat{p}_{t}^{W *}\right)-\phi_{F}\left(\widehat{p}_{t}^{F, R}-\widehat{p}_{t}^{R}\right)\right] \\
& \quad=\phi_{F}\left(\widehat{p}_{t}^{H *}-\widehat{p}_{t}^{*}\right)+\phi_{H}\left(\widehat{p}_{t}^{F *}-\widehat{p}_{t}^{*}\right)=0,
\end{aligned}
$$

since $\widehat{p}_{t}=\phi_{H} \widehat{p}_{t}^{H}+\phi_{F} \widehat{p}_{t}^{F}$ and $\widehat{p}_{t}^{*}=\phi_{F} \widehat{p}_{t}^{H *}+\phi_{H} \widehat{p}_{t}^{F *}$. Furthermore, we also know based on those same definitions, that the following result must hold true,

$$
\begin{aligned}
& \left(\widehat{p}_{t}^{H, W}-\widehat{p}_{t}^{W}\right)+\left(\widehat{p}_{t}^{F, W *}-\widehat{p}_{t}^{W *}\right)=\widehat{p}_{t}^{H, W}+\widehat{p}_{t}^{F, W *}-\left(\widehat{p}_{t}^{W}+\widehat{p}_{t}^{W *}\right) \\
& \quad=\phi_{H}\left(\widehat{p}_{t}^{H}-\widehat{p}_{t}\right)+\phi_{F}\left(\widehat{p}_{t}^{H *}-\widehat{p}_{t}^{*}\right)+\phi_{F}\left(\widehat{p}_{t}^{F}-\widehat{p}_{t}\right)+\phi_{H}\left(\widehat{p}_{t}^{F *}-\widehat{p}_{t}^{*}\right) \\
& \quad=\left[\phi_{H}\left(\widehat{p}_{t}^{H}-\widehat{p}_{t}\right)+\phi_{F}\left(\widehat{p}_{t}^{F}-\widehat{p}_{t}\right)\right]+\left[\phi_{F}\left(\widehat{p}_{t}^{H *}-\widehat{p}_{t}^{*}\right)+\phi_{H}\left(\widehat{p}_{t}^{F *}-\widehat{p}_{t}^{*}\right)\right]=0 .
\end{aligned}
$$

Hence, we argue that the terms inside the brackets of the Phillips curves can be simplified as,

$$
\begin{aligned}
& -\left(1+\eta\left(\frac{\psi}{1+(1-\psi) \varphi}\right)\left(\varphi+(1-\psi)\left(\frac{1+\varphi}{\psi}\right)^{2}\right)\right)\left[\phi_{H}\left(\widehat{p}_{t}^{H, W}-\widehat{p}_{t}^{W}\right)+\phi_{F}\left(\widehat{p}_{t}^{F, W *}-\widehat{p}_{t}^{W *}\right)\right]- \\
& \quad-\phi_{H} \phi_{F}\left[\left(\widehat{p}_{t}^{H, R}-\widehat{p}_{t}^{R}\right)+\left(\widehat{p}_{t}^{F, R}-\widehat{p}_{t}^{R}\right)\right]=\eta\left(\frac{\psi}{1+(1-\psi) \varphi}\right)\left(\varphi+(1-\psi)\left(\frac{1+\varphi}{\psi}\right)^{2}\right)\left[\phi_{H}-\phi_{F}\right]\left(\widehat{p}_{t}^{F, W *}-\widehat{p}_{t}^{W *}\right), \\
& -\left(1+\eta\left(\frac{\psi}{1+(1-\psi) \varphi}\right)\left(\varphi+(1-\psi)\left(\frac{1+\varphi}{\psi}\right)^{2}\right)\right)\left[\phi_{F}\left(\widehat{p}_{t}^{H, W}-\widehat{p}_{t}^{W}\right)+\phi_{H}\left(\widehat{p}_{t}^{F, W *}-\widehat{p}_{t}^{W *}\right)\right]+ \\
& \quad+\phi_{F} \phi_{H}\left[\left(\widehat{p}_{t}^{H, R}-\widehat{p}_{t}^{R}\right)+\left(\widehat{p}_{t}^{F, R}-\widehat{p}_{t}^{R}\right)\right]=-\eta\left(\frac{\psi}{1+(1-\psi) \varphi}\right)\left(\varphi+(1-\psi)\left(\frac{1+\varphi}{\psi}\right)^{2}\right)\left[\phi_{H}-\phi_{F}\right]\left(\widehat{p}_{t}^{F, W *}-\widehat{p}_{t}^{W *}\right) .
\end{aligned}
$$

These derivations are true irrespective of whether the law of one price holds or not. Finally, we conclude 
that both Phillips curves in the model take the following form,

$$
\begin{aligned}
& \widehat{\pi}_{t} \approx \beta \mathbb{E}_{t}\left(\widehat{\pi}_{t+1}\right)+\phi_{F}\left[\left(\widehat{s}_{t}-\widehat{s}_{t-1}\right)-\beta \mathbb{E}_{t}\left(\widehat{s}_{t+1}-\widehat{s}_{t}\right)+\Psi\left(\widehat{p}_{t}^{F, R}-\widehat{s}_{t}\right)\right]+\ldots \\
& \Psi\left[\begin{array}{c}
\left(\sigma^{-1}+\left(1-\gamma_{x}\right)\left(\frac{\psi}{1+(1-\psi) \varphi}\right)\left(\varphi+(1-\psi)\left(\frac{1+\varphi}{\psi}\right)^{2}\right)\right)\left[\phi_{H} \widehat{c}_{t}^{W}+\phi_{F} \widehat{c}_{t}^{W *}\right]+\ldots \\
\gamma_{x}\left(\frac{\psi}{1+(1-\psi) \varphi}\right)\left(\varphi+(1-\psi)\left(\frac{1+\varphi}{\psi}\right)^{2}\right)\left[\phi_{H} \widehat{x}_{t}^{W}+\phi_{F} \widehat{x}_{t}^{W *}\right]+\ldots \\
\gamma_{x}\left(\frac{\psi}{1+(1-\psi) \varphi}\right)\left(\varphi+(1-\psi)\left(\frac{1+\varphi}{\psi}\right)^{2}\right)\left(\frac{1-\beta(1-\delta)}{\beta \delta}\right)\left[\phi_{H} \widehat{u}_{t}+\phi_{F} \widehat{u}_{t}^{*}\right]+2 \phi_{H} \phi_{F} \widehat{r s}_{t}+\ldots \\
\left(\phi_{H}-\phi_{F}\right) \eta\left(\frac{\psi}{1+(1-\psi) \varphi}\right)\left(\varphi+(1-\psi)\left(\frac{1+\varphi}{\psi}\right)^{2}\right)\left(\widehat{p}_{t}^{F, W *}-\widehat{p}_{t}^{W *}\right)-\ldots \\
(1-\psi)\left(\frac{1+\varphi}{\psi}\right) \widehat{k}_{t}^{W}-\left(\frac{1+\varphi}{\psi}\right)\left[\phi_{H} \widehat{a}_{t}+\phi_{F} \widehat{a}_{t}^{*}\right]
\end{array}\right], \\
& \widehat{\pi}_{t}^{*} \approx \beta \mathbb{E}_{t}\left(\widehat{\pi}_{t+1}^{*}\right)-\phi_{F}\left[\left(\widehat{s}_{t}-\widehat{s}_{t-1}\right)-\beta \mathbb{E}_{t}\left(\widehat{s}_{t+1}-\widehat{s}_{t}\right)+\Psi\left(\widehat{p}_{t}^{H, R}-\widehat{s}_{t}\right)\right]+\ldots \\
& {\left[\left(\sigma^{-1}+\left(1-\gamma_{x}\right)\left(\frac{\psi}{1+(1-\psi) \varphi}\right)\left(\varphi+(1-\psi)\left(\frac{1+\varphi}{\psi}\right)^{2}\right)\right)\left[\phi_{F} \widehat{c}_{t}^{W}+\phi_{H} \widehat{c}_{t}^{W *}\right]+\ldots\right.} \\
& \gamma_{x}\left(\frac{\psi}{1+(1-\psi) \varphi}\right)\left(\varphi+(1-\psi)\left(\frac{1+\varphi}{\psi}\right)^{2}\right)\left[\phi_{F} \widehat{x}_{t}^{W}+\phi_{H} \widehat{x}_{t}^{W *}\right]+\ldots \\
& \Psi \quad \gamma_{x}\left(\frac{\psi}{1+(1-\psi) \varphi}\right)\left(\varphi+(1-\psi)\left(\frac{1+\varphi}{\psi}\right)^{2}\right)\left(\frac{1-\beta(1-\delta)}{\beta \delta}\right)\left[\phi_{F} \widehat{u}_{t}+\phi_{H} \widehat{u}_{t}^{*}\right]-2 \phi_{F} \phi_{H} \widehat{r s s}_{t}-\ldots, \\
& \left(\phi_{H}-\phi_{F}\right) \eta\left(\frac{\psi}{1+(1-\psi) \varphi}\right)\left(\varphi+(1-\psi)\left(\frac{1+\varphi}{\psi}\right)^{2}\right)\left(\widehat{p}_{t}^{F, W *}-\widehat{p}_{t}^{W *}\right)-\ldots \\
& (1-\psi)\left(\frac{1+\varphi}{\psi}\right) \widehat{k}_{t}^{W *}-\left(\frac{1+\varphi}{\psi}\right)\left[\phi_{F} \widehat{a}_{t}+\phi_{H} \widehat{a}_{t}^{*}\right]
\end{aligned}
$$

or simply,

$$
\begin{aligned}
& \widehat{\pi}_{t} \approx \beta \mathbb{E}_{t}\left(\widehat{\pi}_{t+1}\right)+\phi_{F}\left[\left(\widehat{s}_{t}-\widehat{s}_{t-1}\right)-\beta \mathbb{E}_{t}\left(\widehat{s}_{t+1}-\widehat{s}_{t}\right)+\Psi\left(\widehat{p}_{t}^{F, R}-\widehat{s}_{t}\right)\right]+\ldots \\
& \Psi\left[\begin{array}{c}
\left(\sigma^{-1}+\left(1-\gamma_{x}\right) \varphi\left(\frac{\varphi \psi^{2}+(1-\psi)(1+\varphi)^{2}}{\varphi \psi+(1-\psi) \psi \varphi^{2}}\right)\right)\left[\phi_{H} \widehat{c}_{t}^{W}+\phi_{F} \widehat{c}_{t}^{W *}\right]+\ldots \\
\gamma_{x} \varphi\left(\frac{\varphi \psi^{2}+(1-\psi)(1+\varphi)^{2}}{\varphi \psi+(1-\psi) \psi \varphi^{2}}\right)\left[\phi_{H} \widehat{x}_{t}^{W}+\phi_{F} \widehat{x}_{t}^{W *}\right]+\ldots \\
\gamma_{x} \varphi\left(\frac{\varphi \psi^{2}+(1-\psi)(1+\varphi)^{2}}{\varphi \psi+(1-\psi) \psi \varphi^{2}}\right)\left(\frac{1-\beta(1-\delta)}{\beta \delta}\right)\left[\phi_{H} \widehat{u}_{t}+\phi_{F} \widehat{u}_{t}^{*}\right]+\ldots \\
2 \phi_{H} \phi_{F} \widehat{r}_{t}+\left(\phi_{H}-\phi_{F}\right) \eta \varphi\left(\frac{\varphi \psi^{2}+(1-\psi)(1+\varphi)^{2}}{\varphi \psi+(1-\psi) \psi \varphi^{2}}\right)\left(\widehat{p}_{t}^{F, W *}-\widehat{p}_{t}^{W *}\right)-\ldots \\
\left(\frac{(1-\psi)(1+\varphi)}{\psi}\right) \widehat{k}_{t}^{W}-\left(\frac{1+\varphi}{\psi}\right)\left[\phi_{H} \widehat{a}_{t}+\phi_{F} \widehat{a}_{t}^{*}\right]
\end{array}\right] \\
& \widehat{\pi}_{t}^{*} \approx \beta \mathbb{E}_{t}\left(\widehat{\pi}_{t+1}^{*}\right)-\phi_{F}\left[\left(\widehat{s}_{t}-\widehat{s}_{t-1}\right)-\beta \mathbb{E}_{t}\left(\widehat{s}_{t+1}-\widehat{s}_{t}\right)+\Psi\left(\widehat{p}_{t}^{H, R}-\widehat{s}_{t}\right)\right]+\ldots \\
& \Psi\left[\begin{array}{c}
\left(\sigma^{-1}+\left(1-\gamma_{x}\right) \varphi\left(\frac{\varphi \psi^{2}+(1-\psi)(1+\varphi)^{2}}{\varphi \psi+(1-\psi) \psi \varphi^{2}}\right)\right)\left[\phi_{F} \widehat{c}_{t}^{W}+\phi_{H} \widehat{c}_{t}^{W *}\right]+\ldots \\
\gamma_{x} \varphi\left(\frac{\varphi \psi^{2}+(1-\psi)(1+\varphi)^{2}}{\varphi \psi+(1-\psi) \psi \varphi^{2}}\right)\left[\phi_{F} \widehat{x}_{t}^{W}+\phi_{H} \widehat{x}_{t}^{W *}\right]+\ldots \\
\gamma_{x} \varphi\left(\frac{\varphi \psi^{2}+(1-\psi)(1+\varphi)^{2}}{\varphi \psi+(1-\psi) \psi \varphi^{2}}\right)\left(\frac{1-\beta(1-\delta)}{\beta \delta}\right)\left[\phi_{F} \widehat{u}_{t}+\phi_{H} \widehat{u}_{t}^{*}\right]-\ldots \\
2 \phi_{F} \phi_{H} \widehat{r s}_{t}-\left(\phi_{H}-\phi_{F}\right) \eta \varphi\left(\frac{\varphi \psi^{2}+(1-\psi)(1+\varphi)^{2}}{\varphi \psi+(1-\psi) \psi \varphi^{2}}\right)\left(\widehat{p}_{t}^{F, W *}-\widehat{p}_{t}^{W *}\right)-\ldots \\
\left(\frac{(1-\psi)(1+\varphi)}{\psi}\right) \widehat{k}_{t}^{W *}-\left(\frac{1+\varphi}{\psi}\right)\left[\phi_{F} \widehat{a}_{t}+\phi_{H} \widehat{a}_{t}^{*}\right]
\end{array}\right],
\end{aligned}
$$

which extends the model under PCP pricing by adding capital, investment, and variable capital utilization rates. Let us define $\widehat{t}_{t}^{W} \equiv \widehat{p}_{t}^{F, W *}-\widehat{p}_{t}^{W *}$ as the world measure of terms of trade in the model. Then, the 
inflation dynamics can be re-expressed as,

$$
\begin{aligned}
& \widehat{\pi}_{t} \approx \beta \mathbb{E}_{t}\left(\widehat{\pi}_{t+1}\right)+\phi_{F}\left[\left(\widehat{s}_{t}-\widehat{s}_{t-1}\right)-\beta \mathbb{E}_{t}\left(\widehat{s}_{t+1}-\widehat{s}_{t}\right)+\left(\frac{(1-\alpha)(1-\alpha \beta)}{\alpha}\right)\left(\widehat{p}_{t}^{F, R}-\widehat{s}_{t}\right)\right]+\ldots \\
& \Psi\left[\begin{array}{c}
\left(\sigma^{-1}+\left(1-\gamma_{x}\right) \varphi\left(\frac{\varphi \psi^{2}+(1-\psi)(1+\varphi)^{2}}{\varphi \psi+(1-\psi) \psi \varphi^{2}}\right)\right)\left[\phi_{H} \widehat{c}_{t}^{W}+\phi_{F} \widehat{c}_{t}^{W *}\right]+\ldots \\
\gamma_{x} \varphi\left(\frac{\varphi \psi^{2}+(1-\psi)(1+\varphi)^{2}}{\varphi \psi+(1-\psi) \psi \varphi^{2}}\right)\left[\phi_{H} \widehat{x}_{t}^{W}+\phi_{F} \widehat{x}_{t}^{W *}\right]+\ldots \\
\gamma_{x} \varphi\left(\frac{\varphi \psi^{2}+(1-\psi)(1+\varphi)^{2}}{\varphi \psi+(1-\psi) \psi \varphi^{2}}\right)\left(\frac{1-\beta(1-\delta)}{\beta \delta}\right)\left[\phi_{H} \widehat{u}_{t}+\phi_{F} \widehat{u}_{t}^{*}\right]+\ldots \\
2 \phi_{H} \phi_{F} \widehat{r s}_{t}+\left(\phi_{H}-\phi_{F}\right) \eta \varphi\left(\frac{\varphi \psi^{2}+(1-\psi)(1+\varphi)^{2}}{\varphi \psi+(1-\psi) \psi \varphi^{2}}\right) \widehat{t}_{t}^{W}-\ldots \\
\left(\frac{(1-\psi)(1+\varphi)}{\psi}\right) \widehat{k}_{t}^{W}-\left(\frac{1+\varphi}{\psi}\right)\left[\phi_{H} \widehat{a}_{t}+\phi_{F} \widehat{a}_{t}^{*}\right]
\end{array}\right] \\
& \widehat{\pi}_{t}^{*} \approx \beta \mathbb{E}_{t}\left(\widehat{\pi}_{t+1}^{*}\right)-\phi_{F}\left[\left(\widehat{s}_{t}-\widehat{s}_{t-1}\right)-\beta \mathbb{E}_{t}\left(\widehat{s}_{t+1}-\widehat{s}_{t}\right)+\left(\frac{(1-\alpha)(1-\alpha \beta)}{\alpha}\right)\left(\widehat{p}_{t}^{H, R}-\widehat{s}_{t}\right)\right]+\ldots \\
& \Psi\left[\begin{array}{c}
\left(\sigma^{-1}+\left(1-\gamma_{x}\right) \varphi\left(\frac{\varphi \psi^{2}+(1-\psi)(1+\varphi)^{2}}{\varphi \psi+(1-\psi) \psi \varphi^{2}}\right)\right)\left[\phi_{F} \widehat{c}_{t}^{W}+\phi_{H} \widehat{c}_{t}^{W *}\right]+\ldots \\
\gamma_{x} \varphi\left(\frac{\varphi \psi^{2}+(1-\psi)(1+\varphi)^{2}}{\varphi \psi+(1-\psi) \psi \varphi^{2}}\right)\left[\phi_{F} \widehat{x}_{t}^{W}+\phi_{H} \widehat{x}_{t}^{W *}\right]+\ldots \\
\gamma_{x} \varphi\left(\frac{\varphi \psi^{2}+(1-\psi)(1+\varphi)^{2}}{\varphi \psi+(1-\psi) \psi \varphi^{2}}\right)\left(\frac{1-\beta(1-\delta)}{\beta \delta}\right)\left[\phi_{F} \widehat{u}_{t}+\phi_{H} \widehat{u}_{t}^{*}\right]-\ldots \\
2 \phi_{F} \phi_{H} \widehat{r s}_{t}-\left(\phi_{H}-\phi_{F}\right) \eta \varphi\left(\frac{\varphi \psi^{2}+(1-\psi)(1+\varphi)^{2}}{\varphi \psi+(1-\psi) \psi \varphi^{2}}\right) \widehat{t}_{t}^{W}-\ldots \\
\left(\frac{(1-\psi)(1+\varphi)}{\psi}\right) \widehat{k}_{t}^{W *}-\left(\frac{1+\varphi}{\psi}\right)\left[\phi_{F} \widehat{a}_{t}+\phi_{H} \widehat{a}_{t}^{*}\right]
\end{array}\right]
\end{aligned}
$$

Furthermore, since we already have argued before that because the law of one price holds, then it must be the case that,

$$
\widehat{p}_{t}^{H, R}-\widehat{s}_{t} \approx 0, \widehat{p}_{t}^{F, R}-\widehat{s}_{t} \approx 0,
$$

so the Phillips curves can be further simplified as,

$$
\begin{gathered}
\widehat{\pi}_{t} \approx \beta \mathbb{E}_{t}\left(\widehat{\pi}_{t+1}\right)+\phi_{F}\left[\Delta \widehat{s}_{t}-\beta \mathbb{E}_{t}\left(\Delta \widehat{s}_{t+1}\right)\right]+\ldots \\
\Psi\left[\begin{array}{c}
\left(\sigma^{-1}+\left(1-\gamma_{x}\right) \varphi\left(\frac{\varphi \psi^{2}+(1-\psi)(1+\varphi)^{2}}{\varphi \psi+(1-\psi) \psi \varphi^{2}}\right)\right)\left[\phi_{H} \widehat{c}_{t}^{W}+\phi_{F} \widehat{c}_{t}^{W *}\right]+\ldots \\
\gamma_{x} \varphi\left(\frac{\varphi \psi^{2}+(1-\psi)(1+\varphi)^{2}}{\varphi \psi+(1-\psi) \psi \varphi^{2}}\right)\left[\phi_{H} \widehat{x}_{t}^{W}+\phi_{F} \widehat{x}_{t}^{W *}\right]+\ldots \\
\gamma_{x} \varphi\left(\frac{\varphi \psi^{2}+(1-\psi)(1+\varphi)^{2}}{\varphi \psi+(1-\psi) \psi \varphi^{2}}\right)\left(\frac{1-\beta(1-\delta)}{\beta \delta}\right)\left[\phi_{H} \widehat{u}_{t}+\phi_{F} \widehat{u}_{t}^{*}\right]+\ldots \\
2 \phi_{H} \phi_{F} \widehat{r s}_{t}+\left(\phi_{H}-\phi_{F}\right) \eta \varphi\left(\frac{\varphi \psi^{2}+(1-\psi)(1+\varphi)^{2}}{\varphi \psi+(1-\psi) \psi \varphi^{2}}\right) \widehat{t}_{t}^{W}-\ldots \\
\left(\frac{(1-\psi)(1+\varphi)}{\psi}\right) \widehat{k}_{t}^{W}-\left(\frac{1+\varphi}{\psi}\right)\left[\phi_{H} \widehat{a}_{t}+\phi_{F} \widehat{a}_{t}^{*}\right]
\end{array}\right], \\
\widehat{\pi}_{t}^{*} \approx \beta \mathbb{E}_{t}\left(\widehat{\pi}_{t+1}^{*}\right)-\phi_{F}\left[\Delta \widehat{s}_{t}-\beta \mathbb{E}_{t}\left(\Delta \widehat{s}_{t+1}\right)\right]+\ldots \\
\\
\Psi\left[\begin{array}{c}
\left(\sigma^{-1}+\left(1-\gamma_{x}\right) \varphi\left(\frac{\varphi \psi^{2}+(1-\psi)(1+\varphi)^{2}}{\varphi \psi+(1-\psi) \psi \varphi^{2}}\right)\right)\left[\phi_{F} \widehat{c}_{t}^{W}+\phi_{H} \widehat{c}_{t}^{W *}\right]+\ldots \\
\gamma_{x} \varphi\left(\frac{\varphi \psi^{2}+(1-\psi)(1+\varphi)^{2}}{\varphi \psi+(1-\psi) \psi \varphi^{2}}\right)\left[\phi_{F} \widehat{x}_{t}^{W}+\phi_{H} \widehat{x}_{t}^{W *}\right]+\ldots \\
\gamma_{x} \varphi\left(\frac{\varphi \psi^{2}+(1-\psi)(1+\varphi)^{2}}{\varphi \psi+(1-\psi) \psi \varphi^{2}}\right)\left(\frac{1-\beta(1-\delta)}{\beta \delta}\right)\left[\phi_{F} \widehat{u}_{t}+\phi_{H} \widehat{u}_{t}^{*}\right]-\ldots \\
2 \phi_{F} \phi_{H} \widehat{r s}_{t}-\left(\phi_{H}-\phi_{F}\right) \eta \varphi\left(\frac{\varphi \psi^{2}+(1-\psi)(1+\varphi)^{2}}{\varphi \psi+(1-\psi) \psi \varphi^{2}}\right) \widehat{t}_{t}^{W}-\ldots \\
\left(\frac{(1-\psi)(1+\varphi)}{\psi}\right) \widehat{k}_{t}^{W *}-\left(\frac{1+\varphi}{\psi}\right)\left[\phi_{F} \widehat{a}_{t}+\phi_{H} \widehat{a}_{t}^{*}\right]
\end{array}\right],
\end{gathered}
$$

where we denote the first-difference of the nominal exchange rate as $\Delta \widehat{s}_{t} \equiv \widehat{s}_{t}-\widehat{s}_{t-1}$. Alternatively, we note 
that these Phillips curves can be re-arranged as follows,

$$
\begin{aligned}
& \widehat{\pi}_{t}-\phi_{F} \Delta \widehat{s}_{t} \approx \beta \mathbb{E}_{t}\left(\widehat{\pi}_{t+1}-\phi_{F} \Delta \widehat{s}_{t+1}\right)+\ldots \\
& \Psi\left[\begin{array}{c}
\left(\sigma^{-1}+\left(1-\gamma_{x}\right) \varphi\left(\frac{\varphi \psi^{2}+(1-\psi)(1+\varphi)^{2}}{\varphi \psi+(1-\psi) \psi \varphi^{2}}\right)\right)\left[\phi_{H} \widehat{c}_{t}^{W}+\phi_{F} \widehat{c}_{t}^{W *}\right]+\ldots \\
\gamma_{x} \varphi\left(\frac{\varphi \psi^{2}+(1-\psi)(1+\varphi)^{2}}{\varphi \psi+(1-\psi) \psi \varphi^{2}}\right)\left[\phi_{H} \widehat{x}_{t}^{W}+\phi_{F} \widehat{x}_{t}^{W *}\right]+\ldots \\
\gamma_{x} \varphi\left(\frac{\varphi \psi^{2}+(1-\psi)(1+\varphi)^{2}}{\varphi \psi+(1-\psi) \psi \varphi^{2}}\right)\left(\frac{1-\beta(1-\delta)}{\beta \delta}\right)\left[\phi_{H} \widehat{u}_{t}+\phi_{F} \widehat{u}_{t}^{*}\right]+\ldots \\
2 \phi_{H} \phi_{F} \widehat{r s}_{t}+\left(\phi_{H}-\phi_{F}\right) \eta \varphi\left(\frac{\varphi \psi^{2}+(1-\psi)(1+\varphi)^{2}}{\varphi \psi+(1-\psi) \psi \varphi^{2}}\right) \widehat{t}_{t}^{W}-\ldots \\
\left(\frac{(1-\psi)(1+\varphi)}{\psi}\right) \widehat{k}_{t}^{W}-\left(\frac{1+\varphi}{\psi}\right)\left[\phi_{H} \widehat{a}_{t}+\phi_{F} \widehat{a}_{t}^{*}\right]
\end{array}\right] \\
& \widehat{\pi}_{t}^{*}+\phi_{F} \Delta \widehat{s}_{t} \approx \beta \mathbb{E}_{t}\left(\widehat{\pi}_{t+1}^{*}+\phi_{F} \Delta \widehat{s}_{t+1}\right)+\ldots \\
& \Psi\left[\begin{array}{c}
\left(\sigma^{-1}+\left(1-\gamma_{x}\right) \varphi\left(\frac{\varphi \psi^{2}+(1-\psi)(1+\varphi)^{2}}{\varphi \psi+(1-\psi) \psi \varphi^{2}}\right)\right)\left[\phi_{F} \widehat{c}_{t}^{W}+\phi_{H} \widehat{c}_{t}^{W *}\right]+\ldots \\
\gamma_{x} \varphi\left(\frac{\varphi \psi^{2}+(1-\psi)(1+\varphi)^{2}}{\varphi \psi+(1-\psi) \psi \varphi^{2}}\right)\left[\phi_{F} \widehat{x}_{t}^{W}+\phi_{H} \widehat{x}_{t}^{W *}+\ldots\right. \\
\gamma_{x} \varphi\left(\frac{\varphi \psi^{2}+(1-\psi)(1+\varphi)^{2}}{\varphi \psi+(1-\psi) \psi \varphi^{2}}\right)\left(\frac{1-\beta(1-\delta)}{\beta \delta}\right)\left[\phi_{F} \widehat{u}_{t}+\phi_{H} \widehat{u}_{t}^{*}\right]-\ldots \\
2 \phi_{F} \phi_{H} \widehat{r s}_{t}-\left(\phi_{H}-\phi_{F}\right) \eta \varphi\left(\frac{\varphi \psi^{2}+(1-\psi)(1+\varphi)^{2}}{\varphi \psi+(1-\psi) \psi \varphi^{2}}\right) \widehat{t}_{t}^{W}-\ldots \\
\left(\frac{(1-\psi)(1+\varphi)}{\psi}\right) \widehat{k}_{t}^{W *}-\left(\frac{1+\varphi}{\psi}\right)\left[\phi_{F} \widehat{a}_{t}+\phi_{H} \widehat{a}_{t}^{*}\right]
\end{array}\right],
\end{aligned}
$$

which means that the Phillips curve in each country can be written as the exact same present discounted value as for the LCP pricing model (with variable capital utilization) except for the addition of an extra term that captures the full pass-through of nominal exchange rate movements into import prices. That is, under LCP pricing current domestic and foreign inflation can be expressed as,

$$
\begin{aligned}
\widehat{\pi}_{t} \approx \Psi \sum_{j=0}^{+\infty} \beta^{j} \mathbb{E}_{t}\left[\begin{array}{c}
\left(\sigma^{-1}+\left(1-\gamma_{x}\right) \varphi\left(\frac{\varphi \psi^{2}+(1-\psi)(1+\varphi)^{2}}{\varphi \psi+(1-\psi) \psi \varphi^{2}}\right)\right)\left[\phi_{H} \widehat{c}_{t+j}^{W}+\phi_{F} \widehat{c}_{t+j}^{W *}\right]+\ldots \\
\gamma_{x} \varphi\left(\frac{\varphi \psi^{2}+(1-\psi)(1+\varphi)^{2}}{\varphi \psi+(1-\psi) \psi \varphi^{2}}\right)\left[\phi_{H} \widehat{x}_{t+j}^{W}+\phi_{F} \widehat{x}_{t+j}^{W *}\right]+\ldots \\
\gamma_{x} \varphi\left(\frac{\varphi \psi^{2}+(1-\psi)(1+\varphi)^{2}}{\varphi \psi+(1-\psi) \psi \varphi^{2}}\right)\left(\frac{1-\beta(1-\delta)}{\beta \delta}\right)\left[\phi_{H} \widehat{u}_{t+j}+\phi_{F} \widehat{u}_{t+j}^{*}\right]+\ldots \\
2 \phi_{H} \phi_{F} \widehat{r s}_{t+j}+\left(\phi_{H}-\phi_{F}\right) \eta \varphi\left(\frac{\varphi \psi^{2}+(1-\psi)(1+\varphi)^{2}}{\varphi \psi+(1-\psi) \psi \varphi^{2}}\right) \widehat{t}_{t+j}^{W}-\ldots \\
\left(\frac{(1-\psi)(1+\varphi)}{\psi}\right) \widehat{k}_{t+j}^{W}-\left(\frac{1+\varphi}{\psi}\right)\left[\phi_{H} \widehat{a}_{t+j}+\phi_{F} \widehat{a}_{t+j}^{*}\right]
\end{array}\right], \\
\widehat{\pi}_{t}^{*} \approx \Psi \sum_{j=0}^{+\infty} \beta^{j} \mathbb{E}_{t}\left[\begin{array}{c}
\left(\sigma^{-1}+\left(1-\gamma_{x}\right) \varphi\left(\frac{\varphi \psi^{2}+(1-\psi)(1+\varphi)^{2}}{\varphi \psi+(1-\psi) \psi \varphi^{2}}\right)\right)\left[\phi_{F} \widehat{c}_{t+j}^{W}+\phi_{H} \widehat{c}_{t+j}^{W *}\right]+\ldots \\
\gamma_{x} \varphi\left(\frac{\varphi \psi^{2}+(1-\psi)(1+\varphi)^{2}}{\varphi \psi+(1-\psi) \psi \varphi^{2}}\right)\left[\phi_{F} \widehat{x}_{t+j}^{W}+\phi_{H} \widehat{x}_{t+j}^{W *}\right]+\ldots \\
\gamma_{x} \varphi\left(\frac{\varphi \psi^{2}+(1-\psi)(1+\varphi)^{2}}{\varphi \psi+(1-\psi) \psi \varphi^{2}}\right)\left(\frac{1-\beta(1-\delta)}{\beta \delta}\right)\left[\phi_{F} \widehat{u}_{t+j}+\phi_{H} \widehat{u}_{t+j}^{*}\right]-\ldots \\
2 \phi_{F} \phi_{H} \widehat{r s}_{t+j}-\left(\phi_{H}-\phi_{F}\right) \eta \varphi\left(\frac{\varphi \psi^{2}+(1-\psi)(1+\varphi)^{2}}{\varphi \psi+(1-\psi) \psi \varphi^{2}}\right) \widehat{t}_{t+j}^{W}-\ldots \\
\left(\frac{(1-\psi)(1+\varphi)}{\psi}\right) \widehat{k}_{t+j}^{W *}-\left(\frac{1+\varphi}{\psi}\right)\left[\phi_{F} \widehat{a}_{t+j}+\phi_{H} \widehat{a}_{t+j}^{*}\right]
\end{array}\right] .
\end{aligned}
$$


while under PCP pricing current domestic and foreign inflation become,

$$
\begin{aligned}
\widehat{\pi}_{t} \approx \phi_{F} \Delta \widehat{s}_{t}+\Psi \sum_{j=0}^{+\infty} \beta^{j} \mathbb{E}_{t}\left[\begin{array}{c}
\left(\sigma^{-1}+\left(1-\gamma_{x}\right) \varphi\left(\frac{\varphi \psi^{2}+(1-\psi)(1+\varphi)^{2}}{\varphi \psi+(1-\psi) \psi \varphi^{2}}\right)\right)\left[\phi_{H} \widehat{c}_{t+j}^{W}+\phi_{F} \widehat{c}_{t+j}^{W *}\right]+\ldots \\
\gamma_{x} \varphi\left(\frac{\varphi \psi^{2}+(1-\psi)(1+\varphi)^{2}}{\varphi \psi+(1-\psi) \psi \varphi^{2}}\right)\left[\phi_{H} \widehat{x}_{t+j}^{W}+\phi_{F} \widehat{x}_{t+j}^{W *}\right]+\ldots \\
\gamma_{x} \varphi\left(\frac{\varphi \psi^{2}+(1-\psi)(1+\varphi)^{2}}{\varphi \psi+(1-\psi) \psi \varphi^{2}}\right)\left(\frac{1-\beta(1-\delta)}{\beta \delta}\right)\left[\phi_{H} \widehat{u}_{t+j}+\phi_{F} \widehat{u}_{t+j}^{*}\right]+\ldots \\
2 \phi_{H} \phi_{F} \widehat{r s}_{t+j}+\left(\phi_{H}-\phi_{F}\right) \eta \varphi\left(\frac{\varphi \psi^{2}+(1-\psi)(1+\varphi)^{2}}{\varphi \psi+(1-\psi) \psi \varphi^{2}}\right) \widehat{t}_{t+j}^{W}-\ldots \\
-\left(\frac{(1-\psi)(1+\varphi)}{\psi}\right) \widehat{k}_{t+j}^{W}-\left(\frac{1+\varphi}{\psi}\right)\left[\phi_{H} \widehat{a}_{t+j}+\phi_{F} \widehat{a}_{t+j}^{*}\right]
\end{array}\right], \\
\widehat{\pi}_{t}^{*} \approx-\phi_{F} \Delta \widehat{s}_{t}+\Psi \sum_{j=0}^{+\infty} \beta^{j} \mathbb{E}_{t}\left[\begin{array}{c}
\left(\sigma^{-1}+\left(1-\gamma_{x}\right) \varphi\left(\frac{\varphi \psi^{2}+(1-\psi)(1+\varphi)^{2}}{\varphi \psi+(1-\psi) \psi \varphi^{2}}\right)\right)\left[\phi_{F} \widehat{c}_{t+j}^{W}+\phi_{H} \widehat{c}_{t+j}^{W *}\right]+\ldots \\
\gamma_{x} \varphi\left(\frac{\varphi \psi^{2}+(1-\psi)(1+\varphi)^{2}}{\varphi \psi+(1-\psi) \psi \varphi^{2}}\right)\left[\phi_{F} \widehat{x}_{t+j}^{W}+\phi_{H} \widehat{x}_{t+j}^{W *}\right]+\ldots \\
\gamma_{x} \varphi\left(\frac{\varphi \psi^{2}+(1-\psi)(1+\varphi)^{2}}{\varphi \psi+(1-\psi) \psi \varphi^{2}}\right)\left(\frac{1-\beta(1-\delta)}{\beta \delta}\right)\left[\phi_{F} \widehat{u}_{t+j}+\phi_{H} \widehat{u}_{t+j}^{*}\right]-\ldots \\
2 \phi_{F} \phi_{H} \widehat{r s s}_{t+j}-\left(\phi_{H}-\phi_{F}\right) \eta \varphi\left(\frac{\varphi \psi^{2}+(1-\psi)(1+\varphi)^{2}}{\varphi \psi+(1-\psi) \psi \varphi^{2}}\right) \widehat{t}_{t+j}^{W}-\ldots \\
\left(\frac{(1-\psi)(1+\varphi)}{\psi}\right) \widehat{k}_{t+j}^{W *}-\left(\frac{1+\varphi}{\psi}\right)\left[\phi_{F} \widehat{a}_{t+j}+\phi_{H} \widehat{a}_{t+j}^{*}\right]
\end{array}\right] .
\end{aligned}
$$

These expressions reflect the role played by the LCP and PCP assumptions on the trade-off between nominal and real variables captured by the Phillips curves.

The International Relative Prices. Let us define $\widehat{t}_{t}^{W} \equiv\left(\widehat{p}_{t}^{F, W *}-\widehat{p}_{t}^{W *}\right)$ as the world measure of terms of trade in this model. As in the model under PCP without capital utilization in (176), the only constraint that determines the world terms of trade is given by,

$$
\left(\phi_{H}-\phi_{F}\right)\left(\widehat{p}_{t}^{F, W *}-\widehat{p}_{t}^{W *}\right) \approx \phi_{H} \phi_{F}\left[\left(\widehat{p}_{t}^{H, R}-\widehat{p}_{t}^{R}\right)+\left(\widehat{p}_{t}^{F, R}-\widehat{p}_{t}^{R}\right)\right]
$$

If the model has no home-product bias in consumption, i.e. $\phi_{H}=\phi_{F}$, then $\widehat{t}_{t}^{W} \equiv\left(\widehat{p}_{t}^{F, W *}-\widehat{p}_{t}^{W *}\right)$ only matters because it affects aggregate output and because aggregate output enters into the Taylor feedback rule for monetary policy. Therefore, in this case with no home-product bias it must follow from (295) that $\left(\widehat{p}_{t}^{H, R}-\widehat{p}_{t}^{R}\right)+\left(\widehat{p}_{t}^{F, R}-\widehat{p}_{t}^{R}\right) \approx 0$. Hence, the constraint imposes no restriction on the world terms of trade $\widehat{t}_{t}^{W} \equiv\left(\widehat{p}_{t}^{F, W *}-\widehat{p}_{t}^{W *}\right)$, and we need to keep track of the price sub-indexes in some other way in order to close down the model.

If the model has a home-product bias in consumption, i.e. $\phi_{H} \neq \phi_{F}$, then $\widehat{t}_{t}^{W} \equiv\left(\widehat{p}_{t}^{F, W *}-\widehat{p}_{t}^{W *}\right)$ matters because it affects aggregate output in both countries and it also matters because it affects the real marginal costs of firms. Moreover, we can write the world terms of trade as follows,

$$
\widehat{t}_{t}^{W} \equiv \widehat{p}_{t}^{F, W *}-\widehat{p}_{t}^{W *}=\frac{\phi_{H} \phi_{F}}{\phi_{H}-\phi_{F}}\left[\left(\widehat{p}_{t}^{H, R}-\widehat{p}_{t}^{R}\right)+\left(\widehat{p}_{t}^{F, R}-\widehat{p}_{t}^{R}\right)\right] .
$$

This equality is crucial to derive the dynamics of world terms of trade. We have shown that under PCP it 
must be the case that $\widehat{p}_{t}^{F, R} \approx \widehat{p}_{t}^{H, R} \approx \widehat{s}_{t}$, so it follows that,

$$
\begin{aligned}
\widehat{t}_{t}^{W} & \equiv \widehat{p}_{t}^{F, W *}-\widehat{p}_{t}^{W *}=\frac{\phi_{H} \phi_{F}}{\phi_{H}-\phi_{F}}\left[\left(\widehat{s}_{t}-\widehat{p}_{t}^{R}\right)+\left(\widehat{s}_{t}-\widehat{p}_{t}^{R}\right)\right] \\
& =\frac{\phi_{H} \phi_{F}}{\phi_{H}-\phi_{F}}\left[\left(\widehat{p}_{t}^{R}+\widehat{r s}_{t}-\widehat{p}_{t}^{R}\right)+\left(\widehat{p}_{t}^{R}+\widehat{r s}_{t}-\widehat{p}_{t}^{R}\right)\right] \\
& =\frac{2 \phi_{H} \phi_{F}}{\phi_{H}-\phi_{F}} \widehat{r s}_{t},
\end{aligned}
$$

which is an equation that clearly ties the world terms of trade to the real exchange rate (an endogenous variable already accounted for in the model). This is the same relationship as in (177) without variable capital utilization. This relationship coupled with the definition of the real exchange rate, i.e.

$$
\begin{aligned}
\Delta \widehat{r s}_{t} & =\Delta \widehat{s}_{t}+\widehat{\pi}_{t}^{*}-\widehat{\pi}_{t} \\
\Delta \widehat{r}_{t} & \equiv \widehat{r s}_{t}-\widehat{r s}_{t-1} \\
\Delta \widehat{s}_{t} & \equiv \widehat{s}_{t}-\widehat{s}_{t-1}
\end{aligned}
$$

suffices to close the model in the PCP case.

Given that $\widehat{p}_{t}^{F, W *} \equiv \phi_{F} \widehat{p}_{t}^{F}+\phi_{H} \widehat{p}_{t}^{F *}, \widehat{p}_{t}^{W *} \equiv \phi_{F} \widehat{p}_{t}+\phi_{H} \widehat{p}_{t}^{*}, \widehat{p}_{t} \approx \phi_{H} \widehat{p}_{t}^{H}+\phi_{F} \widehat{p}_{t}^{F}$ and $\widehat{p}_{t}^{*} \approx \phi_{F} \widehat{p}_{t}^{H *}+\phi_{H} \widehat{p}_{t}^{F *}$, the definition of world terms of trade, $\widehat{t}_{t}^{W}$, can be expressed as,

$$
\begin{aligned}
\widehat{t}_{t}^{W} & \equiv \widehat{p}_{t}^{F, W *}-\widehat{p}_{t}^{W *}=\phi_{F} \widehat{p}_{t}^{F}+\phi_{H} \widehat{p}_{t}^{F *}-\phi_{F} \widehat{p}_{t}-\phi_{H} \widehat{p}_{t}^{*} \\
& =\phi_{F}\left(\widehat{p}_{t}^{F}-\widehat{p}_{t}\right)+\phi_{H}\left(\widehat{p}_{t}^{F *}-\widehat{p}_{t}^{*}\right) \\
& \approx \phi_{F}\left(\widehat{p}_{t}^{F}-\phi_{H} \widehat{p}_{t}^{H}-\phi_{F} \widehat{p}_{t}^{F}\right)+\phi_{H}\left(\widehat{p}_{t}^{F *}-\phi_{F} \widehat{p}_{t}^{H *}-\phi_{H} \widehat{p}_{t}^{F *}\right) \\
& =\phi_{F}\left(\left(1-\phi_{F}\right) \widehat{p}_{t}^{F}-\phi_{H} \widehat{p}_{t}^{H}\right)+\phi_{H}\left(\left(1-\phi_{H}\right) \widehat{p}_{t}^{F *}-\phi_{F} \widehat{p}_{t}^{H *}\right) \\
& =\phi_{F} \phi_{H}\left(\widehat{p}_{t}^{F}-\widehat{p}_{t}^{H}\right)+\phi_{F} \phi_{H}\left(\widehat{p}_{t}^{F *}-\widehat{p}_{t}^{H *}\right) .
\end{aligned}
$$

Then, using the implication that the law of one price holds under PCP pricing, i.e. $\widehat{p}_{t}^{H} \approx \widehat{s}_{t}+\widehat{p}_{t}^{H *}$ and $\widehat{p}_{t}^{F} \approx \widehat{s}_{t}+\widehat{p}_{t}^{F *}$, we can immediately obtain the following expression,

$$
\begin{aligned}
\widehat{t}_{t}^{W} & =\phi_{F} \phi_{H}\left(\widehat{p}_{t}^{F}-\widehat{p}_{t}^{H}\right)+\phi_{F} \phi_{H}\left(\widehat{p}_{t}^{F *}-\widehat{p}_{t}^{H *}\right) \\
& =\phi_{F} \phi_{H}\left(\widehat{p}_{t}^{F}-\widehat{p}_{t}^{H}\right)+\phi_{F} \phi_{H}\left(\left(\widehat{p}_{t}^{F}-\widehat{s}_{t}\right)-\left(\widehat{p}_{t}^{H}-\widehat{s}_{t}\right)\right) \\
& =2 \phi_{F} \phi_{H}\left(\widehat{p}_{t}^{F}-\widehat{p}_{t}^{H}\right) .
\end{aligned}
$$

If we put this expression together with the expression that we just derived linking the world terms of trade to the real exchange rate, then it immediately follows that,

$$
\frac{2 \phi_{H} \phi_{F}}{\phi_{H}-\phi_{F}} \widehat{r s}_{t}=\widehat{t}_{t}^{W}=2 \phi_{F} \phi_{H}\left(\widehat{p}_{t}^{F}-\widehat{p}_{t}^{H}\right)
$$

and,

$$
\widehat{r s}_{t}=\left(\phi_{H}-\phi_{F}\right)\left(\widehat{p}_{t}^{F}-\widehat{p}_{t}^{H}\right),
$$

which gives us the conventional that the real exchange rate is proportional to domestic terms of trade, 
$\widehat{\text { tot }}_{t} \equiv\left(\widehat{p}_{t}^{F}-\widehat{p}_{t}^{H}\right)$, in the absence of deviations from the law of one price. Furthermore, if there is no home-product bias, i.e. $\phi_{H}=\phi_{F}$, then the real exchange rate would be invariant over time.

\subsubsection{Other Relationships}

On Aggregate Output and the Efficiency Conditions. Using the definition of world terms of trade we can write aggregate output from equations $(251)-(252)$ as,

$$
\begin{aligned}
& \widehat{y}_{t} \approx \eta \widehat{t}_{t}^{W}+\left(1-\gamma_{x}\right) \widehat{c}_{t}^{W}+\gamma_{x} \widehat{x}_{t}^{W}+\gamma_{x}\left(\frac{1-\beta(1-\delta)}{\beta \delta}\right) \widehat{u}_{t}, \\
& \widehat{y}_{t}^{*} \approx-\eta \widehat{t}_{t}^{W}+\left(1-\gamma_{x}\right) \widehat{c}_{t}^{W *}+\gamma_{x} \widehat{x}_{t}^{W *}+\gamma_{x}\left(\frac{1-\beta(1-\delta)}{\beta \delta}\right) \widehat{u}_{t}^{*} .
\end{aligned}
$$

Using the efficiency conditions in (253) - (254) and, after a little bit of algebra, it follows that the real rental rates on capital can be expressed as,

$$
\begin{aligned}
& \widehat{r}_{t}^{z} \approx\left(\frac{1}{\sigma}+\left(1-\gamma_{x}\right) \frac{1+\varphi}{\psi}\right) \widehat{c}_{t}^{W}+\gamma_{x} \frac{1+\varphi}{\psi} \widehat{x}_{t}^{W}+\gamma_{x} \frac{1+\varphi}{\psi}\left(\frac{1-\beta(1-\delta)}{\beta \delta}\right) \widehat{u}_{t}+\phi_{F} \widehat{r s}_{t}+\eta \frac{1+\varphi}{\psi} \widehat{t}_{t}^{W}-\ldots \\
& \quad\left(\frac{1+(1-\psi) \varphi}{\psi}\right) \widehat{k}_{t}-\frac{1+\varphi}{\psi} \widehat{a}_{t}, \\
& \widehat{r}_{t}^{z *} \approx\left(\frac{1}{\sigma}+\left(1-\gamma_{x}\right) \frac{1+\varphi}{\psi}\right) \widehat{c}_{t}^{W *}+\gamma_{x} \frac{1+\varphi}{\psi} \widehat{x}_{t}^{W *}+\gamma_{x} \frac{1+\varphi}{\psi}\left(\frac{1-\beta(1-\delta)}{\beta \delta}\right) \widehat{u}_{t}^{*}-\phi_{F} \widehat{r s}_{t}-\eta \frac{1+\varphi}{\psi} \widehat{t}_{t}^{W}-\ldots \\
& \quad\left(\frac{1+(1-\psi) \varphi}{\psi}\right) \widehat{k}_{t}^{*}-\frac{1+\varphi}{\psi} \widehat{a}_{t}^{*} .
\end{aligned}
$$

Here, we simply re-write the previous equations replacing out the relative prices with the definition of world terms of trade. However, for the purpose of deriving the Phillips curves of the model suffices to use the expressions obtained in (251) - (252) and (253) - (254), replacing the relative prices with world terms of trade afterward. To posit the model, however, we need to add (302) - (303) in place of (251) - (252).

These expressions do not depend on whether LCP pricing or PCP pricing are assumed. However, with PCP pricing the law of one price holds and we can tie the world terms of trade to the real exchange rate as noted before.

On Aggregate Employment. The aggregate employment can be easily derived from the production functions in (91) and (92) as,

$$
\begin{aligned}
\widehat{y}_{t} & \approx \widehat{a}_{t}+(1-\psi) \widehat{k}_{t}+\psi \widehat{l}_{t}, \\
\widehat{y}_{t}^{*} & \approx \widehat{a}_{t}^{*}+(1-\psi) \widehat{k}_{t}^{*}+\psi \widehat{l}_{t}^{*} .
\end{aligned}
$$

These are the same equations that we obtained in the model without capital utilization. However, $\widehat{k}_{t}$ denotes now capital services rather than physical capital as before.

On Real Exports, Real Imports, and the Net Exports Share. In a two-country model, suffices to determine the net exports share of the domestic country, $\widehat{t b}_{t}$. The net exports share can be easily computed as the difference between domestic aggregate output and domestic aggregate consumption, investment and 
capital utilization costs in real terms (the domestic absorption) (see, e.g., Galí and Monacelli, 2005), i.e.

$$
\widehat{t b}_{t} \equiv \widehat{y}_{t}-\left(1-\gamma_{x}\right) \widehat{c}_{t}-\gamma_{x} \widehat{x}_{t}-\gamma_{x}\left(\frac{1-\beta(1-\delta)}{\beta \delta}\right) \widehat{u}_{t}
$$

Using the formula derived above for aggregate output, we obtain the following expression,

$$
\begin{aligned}
\widehat{t b}_{t} & \approx \eta \widehat{t}_{t}^{W}+\left(1-\gamma_{x}\right)\left[\left(\phi_{H} \widehat{c}_{t}+\phi_{F} \widehat{c}_{t}^{*}\right)-\widehat{c}_{t}\right]+\gamma_{x}\left[\left(\phi_{H} \widehat{x}_{t}+\phi_{F} \widehat{x}_{t}^{*}\right)-\widehat{x}_{t}\right] \\
& =\eta \widehat{t}_{t}^{W}-\left(1-\gamma_{x}\right) \phi_{F}\left[\widehat{c}_{t}-\widehat{c}_{t}^{*}\right]-\gamma_{x} \phi_{F} \widehat{x}_{t}^{R}
\end{aligned}
$$

where $\widehat{c}_{t}^{W} \equiv \phi_{H} \widehat{c}_{t}+\phi_{F} \widehat{c}_{t}^{*}, \widehat{x}_{t}^{W} \equiv \phi_{H} \widehat{x}_{t}+\phi_{F} \widehat{x}_{t}^{*}$, and $\widehat{x}_{t}^{R} \equiv \widehat{x}_{t}-\widehat{x}_{t}^{*}$. Using the perfect international risk-sharing condition in (99), we can express the net exports share as,

$$
\widehat{t b}_{t} \approx \eta \widehat{t}_{t}^{W}-\left(1-\gamma_{x}\right) \phi_{F} \sigma \widehat{r s}_{t}-\gamma_{x} \phi_{F} \widehat{x}_{t}^{R}
$$

In other words, the trade balance is not directly affected by capital utilization costs because aggregate capital cannot be traded across countries (only varieties are tradable). In this environment, therefore, the capital utilization term appears on the domestic aggregate output demand and it also appears on the domestic absorption, so it cancels out. The trade balance can be simplified for the PCP case by recalling that world terms of trade are tied to the real exchange rate whenever the law of one price holds.

The real exports and imports of domestic goods in the model can be inferred from equations (49) - (52) and their foreign counterparts as follows,

$$
\begin{aligned}
E X P_{t} & \equiv \int_{0}^{1}\left[C_{t}^{*}(h)+X_{t}^{*}(h)\right] d h=\phi_{H}^{*} \int_{0}^{1}\left(\frac{P_{t}^{*}(h)}{P_{t}^{H *}}\right)^{-\theta}\left(\frac{P_{t}^{H *}}{P_{t}^{*}}\right)^{-\eta}\left[C_{t}^{*}+X_{t}^{*}\right] d h, \\
I M P_{t} & \equiv \int_{0}^{1}\left[C_{t}(f)+X_{t}(f)\right] d f=\phi_{F} \int_{0}^{1}\left(\frac{P_{t}(f)}{P_{t}^{F}}\right)^{-\theta}\left(\frac{P_{t}^{F}}{P_{t}}\right)^{-\eta}\left[C_{t}+X_{t}\right] d f,
\end{aligned}
$$

where $\phi_{H}^{*}=\phi_{F}$ under our assumption of home-product bias in consumption and investment. A simple log-linearization of both definitions allows us to obtain the following pair of equations,

$$
\begin{aligned}
\widehat{\exp }_{t} & \approx-\theta\left(\int_{0}^{1} \widehat{p}_{t}^{*}(h) d h-\widehat{p}_{t}^{H *}\right)-\eta\left(\widehat{p}_{t}^{H *}-\widehat{p}_{t}^{*}\right)+\left(1-\gamma_{x}\right) \widehat{c}_{t}^{*}+\gamma_{x} \widehat{x}_{t}^{*} \\
\widehat{i m p}_{t} & \approx-\theta\left(\int_{0}^{1} \widehat{p}_{t}(f) d h-\widehat{p}_{t}^{F}\right)-\eta\left(\widehat{p}_{t}^{F}-\widehat{p}_{t}\right)+\left(1-\gamma_{x}\right) \widehat{c}_{t}+\gamma_{x} \widehat{x}_{t} .
\end{aligned}
$$

The log-linearization of the price sub-indexes in (13) - (14) clearly implies that $\int_{0}^{1} \widehat{p}_{t}^{*}(h) d h \approx \widehat{p}_{t}^{H *}$ and $\int_{0}^{1} \widehat{p}_{t}(f) d h \approx \hat{p}_{t}^{F}$. Therefore, the first-order effects of relative price dispersion at the variety level are negligible for real exports and real imports (as they are for aggregate output), and we can re-write the equations above simply as,

$$
\begin{aligned}
\widehat{\exp }_{t} & \approx-\eta\left(\widehat{p}_{t}^{H *}-\widehat{p}_{t}^{*}\right)+\left(1-\gamma_{x}\right) \widehat{c}_{t}^{*}+\gamma_{x} \widehat{x}_{t}^{*} \\
\widehat{i m p}_{t} & \approx-\eta\left(\widehat{p}_{t}^{F}-\widehat{p}_{t}\right)+\left(1-\gamma_{x}\right) \widehat{c}_{t}+\gamma_{x} \widehat{x}_{t} .
\end{aligned}
$$

We have defined the world weighted price sub-indexes as $\widehat{p}_{t}^{H, W} \equiv \phi_{H} \widehat{p}_{t}^{H}+\phi_{F} \widehat{p}_{t}^{H *}$ and $\widehat{p}_{t}^{F, W *} \equiv \phi_{F} \widehat{p}_{t}^{F}+$ 
$\phi_{H} \widehat{p}_{t}^{F *}$, and the relative price sub-indexes as $\widehat{p}_{t}^{H, R} \equiv \widehat{p}_{t}^{H}-\widehat{p}_{t}^{H *}$ and $\widehat{p}_{t}^{F, R} \equiv \widehat{p}_{t}^{F}-\widehat{p}_{t}^{F *}$. Then, naturally, we can write that,

$$
\begin{aligned}
\widehat{p}_{t}^{H} & =\widehat{p}_{t}^{H, W}+\phi_{F} \widehat{p}_{t}^{H, R}, \widehat{p}_{t}^{H *}=\widehat{p}_{t}^{H, W}-\phi_{H} \widehat{p}_{t}^{H, R}, \\
\widehat{p}_{t}^{F} & =\widehat{p}_{t}^{F, W *}+\phi_{H} \widehat{p}_{t}^{F, R}, \widehat{p}_{t}^{F *}=\widehat{p}_{t}^{F, W *}-\phi_{F} \widehat{p}_{t}^{F, R}
\end{aligned}
$$

Analogously, we have defined the world CPI as $\widehat{p}_{t}^{W} \equiv \phi_{H} \widehat{p}_{t}+\phi_{F} \widehat{p}_{t}^{*}$ and $\widehat{p}_{t}^{W *} \equiv \phi_{F} \widehat{p}_{t}+\phi_{H} \widehat{p}_{t}^{*}$, and the relative CPI as $\widehat{p}_{t}^{R} \equiv \widehat{p}_{t}-\widehat{p}_{t}^{*}$. Then, we can write that,

$$
\begin{aligned}
& \widehat{p}_{t}=\widehat{p}_{t}^{W}+\phi_{F} \widehat{p}_{t}^{R}, \widehat{p}_{t}^{*}=\widehat{p}_{t}^{W}-\phi_{H} \widehat{p}_{t}^{R} \\
& \widehat{p}_{t}=\widehat{p}_{t}^{W *}+\phi_{H} \widehat{p}_{t}^{R}, \widehat{p}_{t}^{*}=\widehat{p}_{t}^{W *}-\phi_{F} \widehat{p}_{t}^{R}
\end{aligned}
$$

Using these definitions, it is possible to express the relative prices embedded in the real export and real import equations in the following terms, i.e.

$$
\begin{aligned}
\widehat{p}_{t}^{H *}-\widehat{p}_{t}^{*} & =\left(\widehat{p}_{t}^{H, W}-\phi_{H} \widehat{p}_{t}^{H, R}\right)-\left(\widehat{p}_{t}^{W}-\phi_{H} \widehat{p}_{t}^{R}\right) \\
& =\widehat{p}_{t}^{H, W}-\widehat{p}_{t}^{W}-\phi_{H}\left(\widehat{p}_{t}^{H, R}-\widehat{p}_{t}^{R}\right), \\
\widehat{p}_{t}^{F}-\widehat{p}_{t} & =\widehat{p}_{t}^{F, W *}+\phi_{H} \widehat{p}_{t}^{F, R}-\left(\widehat{p}_{t}^{W *}+\phi_{H} \widehat{p}_{t}^{R}\right) \\
& =\widehat{p}_{t}^{F, W *}-\widehat{p}_{t}^{W *}+\phi_{H}\left(\widehat{p}_{t}^{F, R}-\widehat{p}_{t}^{R}\right),
\end{aligned}
$$

where the world terms of trade is defined as $\widehat{t}_{t}^{W} \equiv\left(\widehat{p}_{t}^{F, W *}-\widehat{p}_{t}^{W *}\right)$. The definition of CPI in both countries, i.e. $\widehat{p}_{t} \approx \phi_{H} \widehat{p}_{t}^{H}+\phi_{F} \widehat{p}_{t}^{F}$ and $\widehat{p}_{t}^{*} \approx \phi_{F} \widehat{p}_{t}^{H *}+\phi_{H} \widehat{p}_{t}^{F *}$, can be written as,

$$
\begin{aligned}
\phi_{H}\left[\widehat{p}_{t}^{H}-\widehat{p}_{t}\right]+\phi_{F}\left[\widehat{p}_{t}^{F}-\widehat{p}_{t}\right] & \approx 0, \\
\phi_{F}\left[\widehat{p}_{t}^{H *}-\widehat{p}_{t}^{*}\right]+\phi_{H}\left[\widehat{p}_{t}^{F *}-\widehat{p}_{t}^{*}\right] & \approx 0 .
\end{aligned}
$$

Then, based on the relationships described before, we can re-write these definitions of the CPI indexes as,

$$
\begin{aligned}
& \phi_{H}\left[\left(\widehat{p}_{t}^{H, W}-\widehat{p}_{t}^{W}\right)+\phi_{F}\left(\widehat{p}_{t}^{H, R}-\widehat{p}_{t}^{R}\right)\right]+\phi_{F}\left[\left(\widehat{p}_{t}^{F, W *}-\widehat{p}_{t}^{W *}\right)+\phi_{H}\left(\widehat{p}_{t}^{F, R}-\widehat{p}_{t}^{R}\right)\right] \approx 0, \\
& \phi_{F}\left[\left(\widehat{p}_{t}^{H, W}-\widehat{p}_{t}^{W}\right)-\phi_{H}\left(\widehat{p}_{t}^{H, R}-\widehat{p}_{t}^{R}\right)\right]+\phi_{H}\left[\left(\widehat{p}_{t}^{F, W *}-\widehat{p}_{t}^{W *}\right)-\phi_{F}\left(\widehat{p}_{t}^{F, R}-\widehat{p}_{t}^{R}\right)\right] \approx 0 .
\end{aligned}
$$

Using the second equality derived above and the definition of the world terms of trade, we can write the relative prices embedded in the definition of real exports and imports in the following terms, i.e.

$$
\begin{aligned}
\widehat{p}_{t}^{H *}-\widehat{p}_{t}^{*} & \approx-\frac{\phi_{H}}{\phi_{F}}\left[\widehat{t}_{t}^{W}-\phi_{F}\left(\widehat{p}_{t}^{F, R}-\widehat{p}_{t}^{R}\right)\right] \\
\widehat{p}_{t}^{F}-\widehat{p}_{t} & \approx \widehat{t}_{t}^{W}+\phi_{H}\left(\widehat{p}_{t}^{F, R}-\widehat{p}_{t}^{R}\right)
\end{aligned}
$$


and,

$$
\begin{aligned}
\phi_{F}\left(\widehat{p}_{t}^{H *}-\widehat{p}_{t}^{*}\right) & \approx-\phi_{H}\left[\widehat{t}_{t}^{W}-\phi_{F}\left(\widehat{p}_{t}^{F, R}-\widehat{p}_{t}^{R}\right)\right], \\
\phi_{F}\left(\widehat{p}_{t}^{F}-\widehat{p}_{t}\right) & \approx \phi_{F}\left[\widehat{t}_{t}^{W}+\phi_{H}\left(\widehat{p}_{t}^{F, R}-\widehat{p}_{t}^{R}\right)\right] .
\end{aligned}
$$

Naturally, under these conditions it follows that,

$$
\begin{aligned}
\phi_{F}\left[\left(\hat{p}_{t}^{H *}-\widehat{p}_{t}^{*}\right)-\left(\widehat{p}_{t}^{F}-\widehat{p}_{t}\right)\right] & \approx-\phi_{H}\left[\widehat{t}_{t}^{W}-\phi_{F}\left(\widehat{p}_{t}^{F, R}-\widehat{p}_{t}^{R}\right)\right]-\phi_{F}\left[\widehat{t}_{t}^{W}+\phi_{H}\left(\widehat{p}_{t}^{F, R}-\widehat{p}_{t}^{R}\right)\right] \\
& =-\left(\phi_{H}+\phi_{F}\right) \widehat{t}_{t}^{W}=-\widehat{t}_{t}^{W} .
\end{aligned}
$$

Therefore, we can compute the real trade balance in this model straight from the definitions of real exports and imports as,

$$
\begin{aligned}
\phi_{F}\left(\widehat{\exp }_{t}-\widehat{i m p} p_{t}\right) & \approx-\eta \phi_{F}\left(\widehat{p}_{t}^{H *}-\widehat{p}_{t}^{*}\right)+\phi_{F}\left[\left(1-\gamma_{x}\right) \widehat{c}_{t}^{*}+\gamma_{x} \widehat{x}_{t}^{*}\right]+\eta \phi_{F}\left(\widehat{p}_{t}^{F}-\widehat{p}_{t}\right)-\phi_{F}\left[\left(1-\gamma_{x}\right) \widehat{c}_{t}+\gamma_{x} \widehat{x}_{t}\right] \\
& =-\eta \phi_{F}\left[\left(\widehat{p}_{t}^{H *}-\widehat{p}_{t}^{*}\right)-\left(\widehat{p}_{t}^{F}-\widehat{p}_{t}\right)\right]+\phi_{F}\left[\left(1-\gamma_{x}\right)\left(\widehat{c}_{t}^{*}-\widehat{c}_{t}\right)+\gamma_{x}\left(\widehat{x}_{t}^{*}-\widehat{x}_{t}\right)\right] \\
& =\eta \widehat{t}_{t}^{W}-\left(1-\gamma_{x}\right) \phi_{F}\left[\widehat{c}_{t}-\widehat{c}_{t}^{*}\right]-\gamma_{x} \phi_{F} \widehat{x}_{t}^{R} \approx \widehat{t b}
\end{aligned}
$$

where relative investment is $\widehat{x}_{t}^{R} \equiv \widehat{x}_{t}-\widehat{x}_{t}^{*}$. In other words, our measure of the trade balance is equivalent to the difference between the log of real exports and real imports (in deviations relative to their respective steady states), scaled by the parameter $\phi_{F}$. In the deterministic steady state of our model, it follows easily that the parameter $\phi_{F}$ denotes the share of domestic imports (and foreign exports) for consumption and investment purposes relative to aggregate output.

Real exports and real imports can be re-written in the following form,

$$
\begin{aligned}
& \widehat{\exp }_{t} \approx \eta \frac{\phi_{H}}{\phi_{F}} \widehat{t}_{t}^{W}-\eta \phi_{H}\left(\widehat{p}_{t}^{F, R}-\widehat{p}_{t}^{R}\right)+\left(1-\gamma_{x}\right) \widehat{c}_{t}^{*}+\gamma_{x} \widehat{x}_{t}^{*}, \\
& \widehat{i m p}_{t} \approx-\eta \widehat{t}_{t}^{W}-\eta \phi_{H}\left(\widehat{p}_{t}^{F, R}-\widehat{p}_{t}^{R}\right)+\left(1-\gamma_{x}\right) \widehat{c}_{t}+\gamma_{x} \widehat{x}_{t},
\end{aligned}
$$

or, simply,

$$
\begin{aligned}
& \widehat{\exp }_{t} \approx \eta \phi_{H}\left[\frac{1}{\phi_{F}} \widehat{t}_{t}^{W}-\left(\widehat{p}_{t}^{F, R}-\widehat{p}_{t}^{R}\right)\right]+\left(1-\gamma_{x}\right) \widehat{c}_{t}^{*}+\gamma_{x} \widehat{x}_{t}^{*} \\
& \widehat{i m p}_{t} \approx-\eta \phi_{H}\left[\frac{1}{\phi_{H}} \widehat{t}_{t}^{W}+\left(\widehat{p}_{t}^{F, R}-\widehat{p}_{t}^{R}\right)\right]+\left(1-\gamma_{x}\right) \widehat{c}_{t}+\gamma_{x} \widehat{x}_{t} .
\end{aligned}
$$

Therefore, we can use equation $(282)$ - which relates $\left(\widehat{p}_{t}^{F, R}-\widehat{p}_{t}^{R}\right)$ to the world terms of trade $\widehat{t}_{t}^{W}$-in order to express the real imports and real exports more compactly as follows,

$$
\begin{aligned}
& \widehat{\exp }_{t} \approx \eta \phi_{H}\left[\frac{1}{\phi_{F}}-\frac{\left(\phi_{H}-\phi_{F}\right)}{2 \phi_{H} \phi_{F}}\right] \widehat{t}_{t}^{W}+\left(1-\gamma_{x}\right) \widehat{c}_{t}^{*}+\gamma_{x} \widehat{x}_{t}^{*} \\
& \widehat{i m p}_{t} \approx-\eta \phi_{H}\left[\frac{1}{\phi_{H}}+\frac{\left(\phi_{H}-\phi_{F}\right)}{2 \phi_{H} \phi_{F}}\right] \widehat{t}_{t}^{W}+\left(1-\gamma_{x}\right) \widehat{c}_{t}+\gamma_{x} \widehat{x}_{t} .
\end{aligned}
$$


or,

$$
\begin{aligned}
& \widehat{\exp }_{t} \approx \eta\left(\frac{1}{2 \phi_{F}}\right) \widehat{t}_{t}^{W}+\left(1-\gamma_{x}\right) \widehat{c}_{t}^{*}+\gamma_{x} \widehat{x}_{t}^{*} \\
& \widehat{i m p}_{t} \approx-\eta\left(\frac{1}{2 \phi_{F}}\right) \widehat{t}_{t}^{W}+\left(1-\gamma_{x}\right) \widehat{c}_{t}+\gamma_{x} \widehat{x}_{t} .
\end{aligned}
$$

These two equations tell us that the strength of the demand for consumption and investment purposes is likely to have a major impact on both exports and imports. However, they also tell us that exports and imports depend on world terms of trade, $\widehat{t}_{t}^{W}$, which is the sole variable that summarizes the impact of international relative prices on both real exports and real imports in the context of our model.

Let us define domestic terms of trade as $\widehat{t o t}_{t} \equiv\left(\widehat{p}_{t}^{F}-\widehat{s}_{t}-\widehat{p}_{t}^{H *}\right)$ in a model under LCP pricing, where deviations of the law of one price can occur. Operating with the definition of world terms of trade, $\widehat{t}_{t}^{W} \equiv$ $\widehat{p}_{t}^{F, W *}-\widehat{p}_{t}^{W *}$, the real exchange rate, $\widehat{r s}_{t}=\widehat{s}_{t}+\widehat{p}_{t}^{*}-\widehat{p}_{t}$, and the CPI for both countries, i.e. $\widehat{p}_{t} \approx \phi_{H} \widehat{p}_{t}^{H}+\phi_{F} \widehat{p}_{t}^{F}$ and $\widehat{p}_{t}^{*} \approx \phi_{F} \widehat{p}_{t}^{H *}+\phi_{H} \widehat{p}_{t}^{F *}$, we can easily see that world terms of trade, $\widehat{t}_{t}^{W}$, are a simple linear function of the domestic terms of trade, $\widehat{\operatorname{tot}}_{t} \equiv\left(\widehat{p}_{t}^{F}-\widehat{s}_{t}-\widehat{p}_{t}^{H *}\right)$, and the real exchange rate, $\widehat{r}_{t}$, expressed in the following terms,

$$
\widehat{t}_{t}^{W} \approx \phi_{F}\left(\widehat{t o t}_{t}+\widehat{r s}_{t}\right) .
$$

The advantage of this transformation is that the world terms of trade can be expressed as a linear function of domestic terms of trade and the real exchange, which are both measurable in the data (unlike world terms of trade itself). This expression is exactly the same one that we found in (191) in a model without variable capital utilization.

We have shown that under PCP it must be the case that $\widehat{p}_{t}^{F, R} \approx \widehat{p}_{t}^{H, R} \approx \widehat{s}_{t}$, so it follows that,

$$
\left(\widehat{p}_{t}^{F, R}-\widehat{p}_{t}^{R}\right) \approx \widehat{s}_{t}+\widehat{p}_{t}^{*}-\widehat{p}_{t}=\widehat{r s}_{t}
$$

We also know that under PCP the world terms of trade are tied to the real exchange rate as well, i.e.

$$
\widehat{t}_{t}^{W}=\frac{2 \phi_{H} \phi_{F}}{\phi_{H}-\phi_{F}} \widehat{r s}_{t}
$$

so it is possible to simplify equations (306) - (307) by replacing $\widehat{t}_{t}^{W}$ and $\left(\widehat{p}_{t}^{F, R}-\widehat{p}_{t}^{R}\right)$ with a linear transformation of the real exchange rate as we have done before. We, then, obtain the following expressions for the 
real exports and real imports under PCP,

$$
\begin{aligned}
\widehat{\exp }_{t} & \approx \eta \phi_{H}\left[\frac{1}{\phi_{F}} \frac{2 \phi_{H} \phi_{F}}{\phi_{H}-\phi_{F}} \widehat{r}_{t}-\widehat{r}_{t}\right]+\left(1-\gamma_{x}\right) \widehat{c}_{t}^{*}+\gamma_{x} \widehat{x}_{t}^{*} \\
& =\eta \phi_{H}\left[\frac{\phi_{H}+\phi_{F}}{\phi_{H}-\phi_{F}}\right] \widehat{r} s_{t}+\left(1-\gamma_{x}\right) \widehat{c}_{t}^{*}+\gamma_{x} \widehat{x}_{t}^{*} \\
& =\eta\left(\frac{\phi_{H}}{\phi_{H}-\phi_{F}}\right) \widehat{r s}_{t}+\left(1-\gamma_{x}\right) \widehat{c}_{t}^{*}+\gamma_{x} \widehat{x}_{t}^{*}, \\
\widehat{i m p}_{t} & \approx-\eta \phi_{H}\left[\frac{1}{\phi_{H}} \frac{2 \phi_{H} \phi_{F}-\phi_{F}}{\phi_{s}}+\widehat{r s}_{t}\right]+\left(1-\gamma_{x}\right) \widehat{c}_{t}+\gamma_{x} \widehat{x}_{t} \\
& =-\eta \phi_{H}\left[\frac{\phi_{F}+\phi_{H}}{\phi_{H}-\phi_{F}}\right] \widehat{r} s_{t}+\left(1-\gamma_{x}\right) \widehat{c}_{t}+\gamma_{x} \widehat{x}_{t} \\
& =-\eta\left(\frac{\phi_{H}}{\phi_{H}-\phi_{F}}\right) \widehat{r s}_{t}+\left(1-\gamma_{x}\right) \widehat{c}_{t}+\gamma_{x} \widehat{x}_{t} .
\end{aligned}
$$

These equations are the same ones that we derived in (192) - (193) under PCP, but without variable capital utilization. Contrary to what happens with the LCP model, we do not need to add a third equation to close the model because all international relative prices that affect the path of real imports and real exports are summarized by the real exchange rate which is already one of the endogenous variables of the model. 


\section{References}

[1] Calvo, Guillermo A. (1983): "Staggered Prices in a Utility-Maximizing Framework." Journal of Monetary Economics, vol. 12 (3), pp. 383-398.

[2] Chari, V. V., Patrick J. Kehoe and Ellen R. McGrattan (2002): "Can Sticky Price Models Generate Volatile and Persistent Real Exchange Rates?" Review of Economic Studies, vol. 69 (3), pp. 533-563.

[3] Christiano, Lawrence J., Martin Eichenbaum and Charles Evans (2005): "Nominal Rigidities and the Dynamic Effects of a Shock to Monetary Policy." Journal of Political Economy, vol. 113 (1), pp. 1-45.

[4] Engel, Charles (forthcoming): "Currency Misalignments and Optimal Monetary Policy: A Reexamination." American Economic Review.

[5] Galí, Jordi and Tommaso Monacelli (2005): "Monetary Policy and Exchange Rate Volatility in a Small Open Economy." Review of Economic Studies, vol. 72 (3), pp. 707-734.

[6] Martínez-García, Enrique and Jens Søndergaard (2008): "The Real Exchange Rate in Sticky Price Models: Does Investment Matter?" GMPI Working Paper No. 16, Federal Reserve Bank of Dallas.

[7] Smets, Frank and Raf Wouters (2003): "An Estimated Dynamic Stochastic General Equilibrium Model of the Euro Area." Journal of the European Economic Association, vol. 1 (5), pp. 1123-1175.

[8] Steinsson, Jón (2008): "The Dynamic Behavior of the Real Exchange Rate in Sticky Price Models." American Economic Review, vol. 98 (1), pp. 519-533.

[9] Taylor, John B. (1993): "Discretion Versus Policy Rules in Practice." Carnegie-Rochester Conference Series, vol. 39, pp. 195-214.

[10] Warnock, Francis E. (2003): "Exchange Rate Dynamics and the Welfare Effects of Monetary Policy in a Two-Country Model with Home-Product Bias." Journal of International Money and Finance, vol. 22 (3), pp. 343-363. 


\section{Appendix}

\section{A The Linearized Equilibrium Conditions with LCP: A Summary}

Here, we report the system of equations derived after log-linearizing the equilibrium conditions of the model with LCP.

\section{A.1 The Model Without Capital}

\begin{tabular}{|c|c|}
\hline & The Households' Problem: \\
\hline IS & $\widehat{c}_{t} \approx \mathbb{E}_{t}\left[\widehat{c}_{t+1}\right]-\sigma\left(\widehat{i}_{t}-\mathbb{E}_{t}\left[\widehat{\pi}_{t+1}\right]\right)$ \\
\hline $\mathbf{I S}^{*}$ & $\widehat{c}_{t}^{*} \approx \mathbb{E}_{t}\left[\widehat{c}_{t+1}^{*}\right]-\sigma\left(\widehat{i}_{t}^{*}-\mathbb{E}_{t}\left[\widehat{\pi}_{t+1}^{*}\right]\right)$ \\
\hline \multirow[t]{2}{*}{$\mathbf{R S}$} & $\widehat{c}_{t}-\widehat{c}_{t}^{*} \approx \sigma \widehat{r s}_{t}$ \\
\hline & The Firms' Problem: \\
\hline AS & $+\left(\frac{(1-\alpha)(1-\alpha \beta)}{\alpha}\right)\left[\begin{array}{c}\widehat{\pi}_{t} \approx \beta \mathbb{E}_{t}\left(\widehat{\pi}_{t+1}\right)+ \\
\left(\sigma^{-1}+\varphi\right)\left[\phi_{H} \widehat{c}_{t}^{W}+\phi_{F} \widehat{c}_{t}^{W *}\right]+2 \phi_{H} \phi_{F} \widehat{r}_{t}+\left(\phi_{H}-\phi_{F}\right) \eta \varphi \widehat{t}_{t}^{W}- \\
-(1+\varphi)\left[\phi_{H} \widehat{a}_{t}+\phi_{F} \widehat{a}_{t}^{*}\right]\end{array}\right]$ \\
\hline $\mathbf{A} \mathbf{S}^{*}$ & $+\left(\frac{(1-\alpha)(1-\alpha \beta)}{\alpha}\right)\left[\begin{array}{c}\left(\sigma^{-1}+\varphi\right)\left[\hat{\pi}_{t}^{*} \approx \beta \mathbb{E}_{t}\left(\widehat{\pi}_{t+1}^{*}\right)+\phi_{H} \widehat{c}_{t}^{W *}\right]-2 \phi_{F} \phi_{H} \widehat{r s s}_{t}-\left(\phi_{H}-\phi_{F}\right) \eta \varphi \widehat{t}_{t}^{W}- \\
-(1+\varphi)\left[\phi_{F} \widehat{a}_{t}+\phi_{H} \widehat{a}_{t}^{*}\right]\end{array}\right]$ \\
\hline \multirow[t]{2}{*}{$\mathrm{T}^{\mathrm{W}}$} & $\Delta \widehat{t}_{t}^{W}-\beta \mathbb{E}_{t}\left(\Delta \widehat{t}_{t+1}^{W}\right)+\left(\frac{(1-\alpha)(1-\alpha \beta)}{\alpha}\right) \widehat{t}_{t}^{W} \approx \frac{\phi_{H} \phi_{F}}{\phi_{H}-\phi_{F}}\left[\left(\frac{(1-\alpha)(1-\alpha \beta)}{\alpha}\right) \widehat{r}_{t}-\widehat{\pi}_{t}^{R}+\beta \mathbb{E}_{t}\left(\widehat{\pi}_{t+1}^{R}\right)\right]$ \\
\hline & Aggregate Output and Employment: \\
\hline $\mathbf{Y}$ & $\widehat{y}_{t} \approx \eta \widehat{t}_{t}^{W}+\widehat{c}_{t}^{W}$ \\
\hline $\mathbf{Y}^{*}$ & $\widehat{y}_{t}^{*} \approx-\eta \widehat{t}_{t}^{W}+\widehat{c}_{t}^{W *}$ \\
\hline $\mathbf{L}$ & $\widehat{l}_{t} \approx \widehat{y}_{t}-\widehat{a}_{t}$ \\
\hline \multirow[t]{2}{*}{$\mathbf{L}^{*}$} & $\widehat{l}_{t}^{*} \approx \widehat{y}_{t}^{*}-\widehat{a}_{t}^{*}$ \\
\hline & The Monetary Policy: \\
\hline MP & $\widehat{i}_{t} \approx \rho_{i} \widehat{i}_{t-1}+\left(1-\rho_{i}\right)\left[\psi_{y} \widehat{y}_{t}+\psi_{\pi} \widehat{\pi}_{t}\right]+\widehat{m}_{t}$ \\
\hline \multirow[t]{3}{*}{$\mathbf{M P}^{*}$} & $\widehat{i}_{t}^{*} \approx \rho_{i} \hat{i}_{t-1}^{*}+\left(1-\rho_{i}\right)\left[\psi_{y} \widehat{y}_{t}^{*}+\psi_{\pi} \widehat{\pi}_{t}^{*}\right]+\widehat{m}_{t}^{*}$ \\
\hline & Other Definitions: \\
\hline & $\begin{array}{c}\widehat{c}_{t}^{W} \equiv \phi_{H} \widehat{c}_{t}+\phi_{F} \widehat{c}_{t}^{*}, \widehat{c}_{t}^{W *} \equiv \phi_{F} \widehat{c}_{t}+\phi_{H} \widehat{c}_{t}^{*} \\
\Delta \widehat{t}_{t}^{W} \equiv \widehat{t}_{t}^{W}-\widehat{t}_{t-1}^{W}, \widehat{\pi}_{t}^{R} \equiv \widehat{\pi}_{t}-\widehat{\pi}_{t}^{*}, \widehat{\pi}_{t}=\widehat{p}_{t}-\widehat{p}_{t-1}, \widehat{\pi}_{t}^{*}=\widehat{p}_{t}^{*}-\widehat{p}_{t-1}^{*}\end{array}$ \\
\hline
\end{tabular}




\section{A.2 The Model With Capital, Without Capital Utilization - NAC}

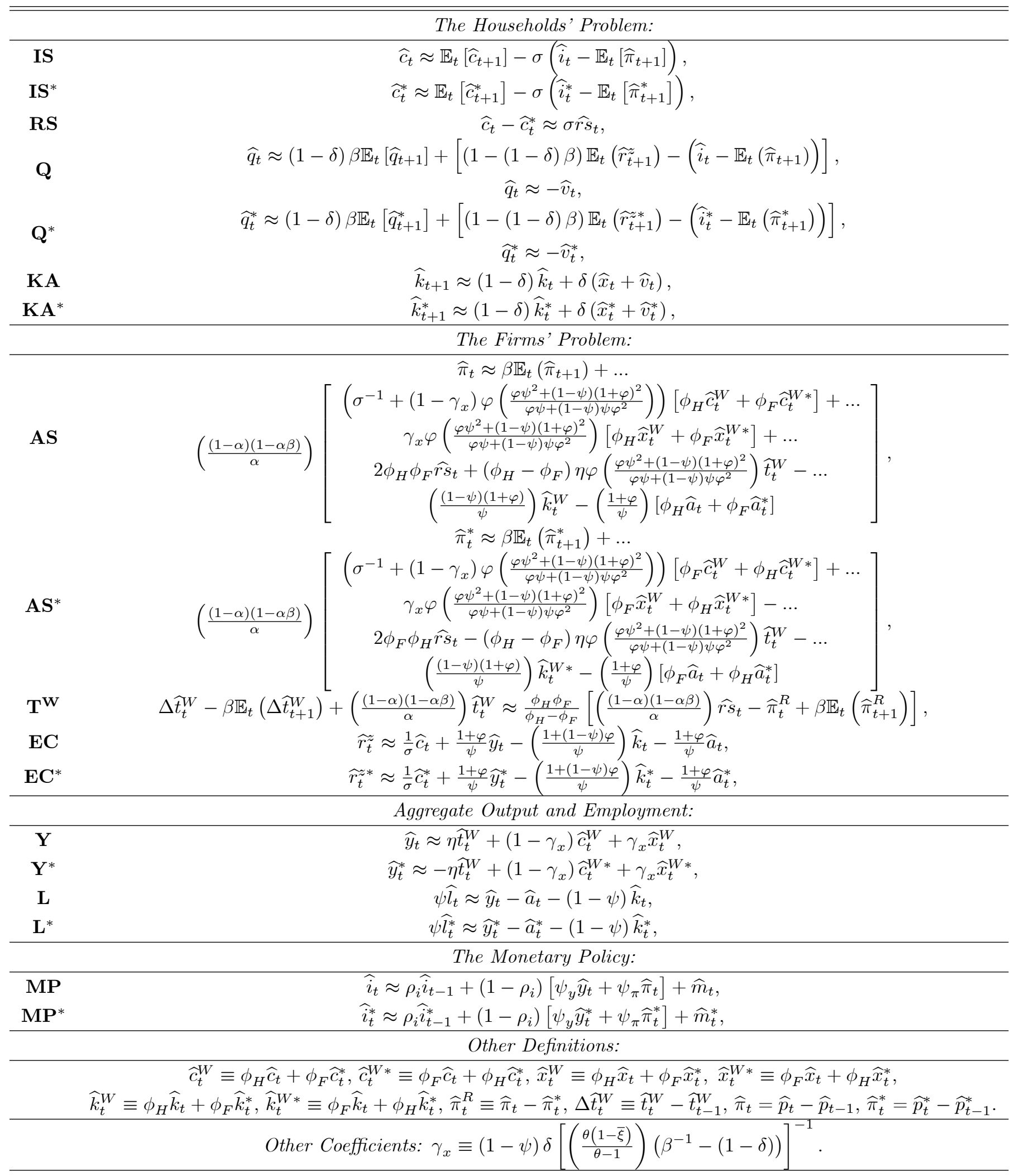




\section{A.3 The Model With Capital, Without Capital Utilization - CAC}

\begin{tabular}{|c|c|}
\hline & The Households' Problem: \\
\hline IS & $\widehat{c}_{t} \approx \mathbb{E}_{t}\left[\widehat{c}_{t+1}\right]-\sigma\left(\widehat{i}_{t}-\mathbb{E}_{t}\left[\widehat{\pi}_{t+1}\right]\right)$, \\
\hline $\mathbf{I S}^{*}$ & $\widehat{c}_{t}^{*} \approx \mathbb{E}_{t}\left[\widehat{c}_{t+1}^{*}\right]-\sigma\left(\widehat{i}_{t}^{*}-\mathbb{E}_{t}\left[\widehat{\pi}_{t+1}^{*}\right]\right)$ \\
\hline $\mathbf{R S}$ & $\widehat{c}_{t}-\widehat{c}_{t}^{*} \approx \sigma \widehat{r s}{ }_{t}$ \\
\hline Q & $\begin{aligned} \widehat{q}_{t} \approx \beta \mathbb{E}_{t}\left[\widehat{q}_{t+1}\right]+[ & \left.(1-(1-\delta) \beta) \mathbb{E}_{t}\left(\widehat{r}_{t+1}^{z}\right)-\left(\widehat{i}_{t}-\mathbb{E}_{t}\left(\widehat{\pi}_{t+1}\right)\right)\right] \\
\widehat{q}_{t} & \approx \chi \delta\left(\widehat{x}_{t}-\widehat{k}_{t}\right)-\widehat{v}_{t}\end{aligned}$ \\
\hline $\mathrm{Q}^{*}$ & $\begin{aligned} & \widehat{q}_{t}^{*} \approx \beta \mathbb{E}_{t}\left[\widehat{q}_{t+1}^{*}\right]+[\left.(1-(1-\delta) \beta) \mathbb{E}_{t}\left(\widehat{r}_{t+1}^{z *}\right)-\left(\widehat{i}_{t}^{*}-\mathbb{E}_{t}\left(\widehat{\pi}_{t+1}^{*}\right)\right)\right] \\
& \widehat{q}_{t}^{*} \approx \chi \delta\left(\widehat{x}_{t}^{*}-\widehat{k}_{t}^{*}\right)-\widehat{v}_{t}^{*}\end{aligned}$ \\
\hline KA & $\widehat{k}_{t+1} \approx(1-\delta) \widehat{k}_{t}+\delta\left(\widehat{x}_{t}+\widehat{v}_{t}\right)$ \\
\hline $\mathbf{K A}^{*}$ & $\widehat{k}_{t+1}^{*} \approx(1-\delta) \widehat{k}_{t}^{*}+\delta\left(\widehat{x}_{t}^{*}+\widehat{v}_{t}^{*}\right)$ \\
\hline & The Firms' Problem: \\
\hline AS & 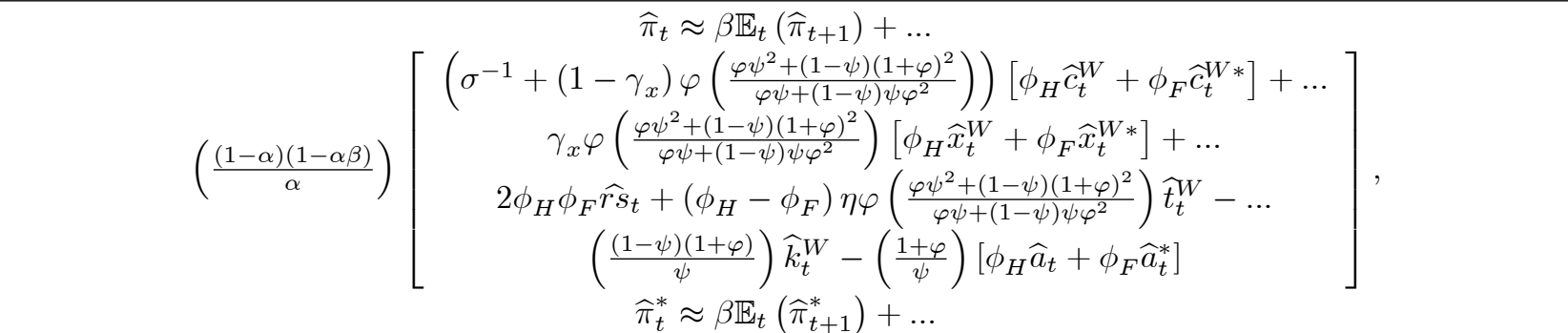 \\
\hline $\mathbf{A S}^{*}$ & $\left(\frac{(1-\alpha)(1-\alpha \beta)}{\alpha}\right)\left[\begin{array}{c}\left(\sigma^{-1}+\left(1-\gamma_{x}\right) \varphi\left(\frac{\varphi \psi^{2}+(1-\psi)(1+\varphi)^{2}}{\varphi \psi+(1-\psi) \psi \varphi^{2}}\right)\right)\left[\phi_{F} \widehat{c}_{t}^{W}+\phi_{H} \widehat{c}_{t}^{W *}\right]+\ldots \\
\gamma_{x} \varphi\left(\frac{\varphi \psi^{2}+(1-\psi)(1+\varphi)^{2}}{\varphi \psi+(1-\psi) \psi \varphi^{2}}\right)\left[\phi_{F} \widehat{x}_{t}^{W}+\phi_{H} \widehat{x}_{t}^{W *}\right]-\ldots \\
2 \phi_{F} \phi_{H} \widehat{r s s}_{t}-\left(\phi_{H}-\phi_{F}\right) \eta \varphi\left(\frac{\varphi \psi^{2}+(1-\psi)(1+\varphi)^{2}}{\varphi \psi+(1-\psi) \psi \varphi^{2}}\right) \widehat{t}_{t}^{W}-\ldots \\
\left(\frac{(1-\psi)(1+\varphi)}{\psi}\right) \widehat{k}_{t}^{W *}-\left(\frac{1+\varphi}{\psi}\right)\left[\phi_{F} \widehat{a}_{t}+\phi_{H} \widehat{a}_{t}^{*}\right]\end{array}\right]$ \\
\hline $\mathbf{T}^{\mathrm{W}}$ & $\Delta \widehat{t}_{t}^{W}-\beta \mathbb{E}_{t}\left(\Delta \widehat{t}_{t+1}^{W}\right)+\left(\frac{(1-\alpha)(1-\alpha \beta)}{\alpha}\right) \widehat{t}_{t}^{W} \approx \frac{\phi_{H} \phi_{F}}{\phi_{H}-\phi_{F}}\left[\left(\frac{(1-\alpha)(1-\alpha \beta)}{\alpha}\right) \widehat{r s}_{t}-\widehat{\pi}_{t}^{R}+\beta \mathbb{E}_{t}\left(\widehat{\pi}_{t+1}^{R}\right)\right]$ \\
\hline EC & $\widehat{r}_{t}^{z} \approx \frac{1}{\sigma} \widehat{c}_{t}+\frac{1+\varphi}{\psi} \widehat{y}_{t}-\left(\frac{1+(1-\psi) \varphi}{\psi}\right) \widehat{k}_{t}-\frac{1+\varphi}{\psi} \widehat{a}_{t}$ \\
\hline $\mathbf{E C}^{*}$ & $\widehat{r}_{t}^{z *} \approx \frac{1}{\sigma} \widehat{c}_{t}^{*}+\frac{1+\varphi}{\psi} \widehat{y}_{t}^{*}-\left(\frac{1+(1-\psi) \varphi}{\psi}\right) \widehat{k}_{t}^{*}-\frac{1+\varphi}{\psi} \widehat{a}_{t}^{*}$ \\
\hline & Aggregate Output and Employment: \\
\hline $\mathbf{Y}$ & $\widehat{y}_{t} \approx \eta \widehat{t}_{t}^{W}+\left(1-\gamma_{x}\right) \widehat{c}_{t}^{W}+\gamma_{x} \widehat{x}_{t}^{W}$ \\
\hline $\mathbf{Y}^{*}$ & $\widehat{y}_{t}^{*} \approx-\eta \widehat{t}_{t}^{W}+\left(1-\gamma_{x}\right) \widehat{c}_{t}^{W *}+\gamma_{x} \widehat{x}_{t}^{W *}$, \\
\hline $\mathbf{L}$ & $\psi \widehat{l}_{t} \approx \widehat{y}_{t}-\widehat{a}_{t}-(1-\psi) \widehat{k}_{t}$ \\
\hline $\mathbf{L}^{*}$ & $\psi \widehat{l}_{t}^{*} \approx \widehat{y}_{t}^{*}-\widehat{a}_{t}^{*}-(1-\psi) \widehat{k}_{t}^{*}$, \\
\hline & The Monetary Policy: \\
\hline MP & $\widehat{i}_{t} \approx \rho_{i} \widehat{i}_{t-1}+\left(1-\rho_{i}\right)\left[\psi_{y} \widehat{y}_{t}+\psi_{\pi} \widehat{\pi}_{t}\right]+\widehat{m}_{t}$ \\
\hline $\mathrm{MP}^{*}$ & $\hat{i}_{t}^{*} \approx \rho_{i} \hat{i}_{t-1}^{*}+\left(1-\rho_{i}\right)\left[\psi_{y} \widehat{y}_{t}^{*}+\psi_{\pi} \widehat{\pi}_{t}^{*}\right]+\widehat{m}_{t}^{*}$, \\
\hline & Other Definitions: \\
\hline & $\begin{array}{c}\widehat{c}_{t}^{W} \equiv \phi_{H} \widehat{c}_{t}+\phi_{F} \widehat{c}_{t}^{*}, \widehat{c}_{t}^{W *} \equiv \phi_{F} \widehat{c}_{t}+\phi_{H} \widehat{c}_{t}^{*}, \widehat{x}_{t}^{W} \equiv \phi_{H} \widehat{x}_{t}+\phi_{F} \widehat{x}_{t}^{*}, \widehat{x}_{t}^{W *} \equiv \phi_{F} \widehat{x}_{t}+\phi_{H} \widehat{x}_{t}^{*}, \\
\widehat{k}_{t}^{W} \equiv \phi_{H} \widehat{k}_{t}+\phi_{F} \widehat{k}_{t}^{*}, \widehat{k}_{t}^{W *} \equiv \phi_{F} \widehat{k}_{t}+\phi_{H} \widehat{k}_{t}^{*}, \widehat{\pi}_{t}^{R} \equiv \widehat{\pi}_{t}-\widehat{\pi}_{t}^{*}, \Delta \widehat{t}_{t}^{W} \equiv \widehat{t}_{t}^{W}-\widehat{t}_{t-1}^{W}, \widehat{\pi}_{t}=\widehat{p}_{t}-\widehat{p}_{t-1}, \widehat{\pi}_{t}^{*}=\widehat{p}_{t}^{*}-\widehat{p}_{t-1}^{*}\end{array}$ \\
\hline & Other Coefficients: $\gamma_{x} \equiv(1-\psi) \delta\left[\left(\frac{\theta(1-\bar{\xi})}{\theta-1}\right)\left(\beta^{-1}-(1-\delta)\right)\right]^{-1}$ \\
\hline
\end{tabular}




\section{A.4 The Model With Capital, Without Capital Utilization - IAC}

\begin{tabular}{|c|c|}
\hline & The Households' Problem: \\
\hline IS & $\widehat{c}_{t} \approx \mathbb{E}_{t}\left[\widehat{c}_{t+1}\right]-\sigma\left(\widehat{i}_{t}-\mathbb{E}_{t}\left[\widehat{\pi}_{t+1}\right]\right)$, \\
\hline $\mathbf{I S}^{*}$ & $\widehat{c}_{t}^{*} \approx \mathbb{E}_{t}\left[\widehat{c}_{t+1}^{*}\right]-\sigma\left(\widehat{i}_{t}^{*}-\mathbb{E}_{t}\left[\widehat{\pi}_{t+1}^{*}\right]\right)$, \\
\hline $\mathbf{R S}$ & $\widehat{c}_{t}-\widehat{c}_{t}^{*} \approx \sigma \widehat{r s}_{t}$ \\
\hline Q & $\begin{array}{c}\widehat{q}_{t} \approx(1-\delta) \beta \mathbb{E}_{t}\left[\widehat{q}_{t+1}\right]+\left[(1-(1-\delta) \beta) \mathbb{E}_{t}\left(\widehat{r}_{t+1}^{z}\right)-\left(\widehat{i}_{t}-\mathbb{E}_{t}\left[\widehat{\pi}_{t+1}\right]\right)\right] \\
\widehat{x}_{t} \approx \frac{1}{1+\beta} \widehat{x}_{t-1}+\frac{\beta}{1+\beta} \mathbb{E}_{t}\left[\widehat{x}_{t+1}\right]+\frac{1}{\kappa(1+\beta)}\left(\widehat{q}_{t}+\widehat{v}_{t}\right)\end{array}$ \\
\hline $\mathrm{Q}^{*}$ & $\begin{array}{c}\widehat{q}_{t}^{*} \approx(1-\delta) \beta \mathbb{E}_{t}\left[\widehat{q}_{t+1}^{*}\right]+\left[(1-(1-\delta) \beta) \mathbb{E}_{t}\left(\widehat{r}_{t+1}^{z *}\right)-\left(\widehat{i}_{t}^{*}-\mathbb{E}_{t}\left[\widehat{\pi}_{t+1}^{*}\right]\right)\right] \\
\widehat{x}_{t}^{*} \approx \frac{1}{1+\beta} \widehat{x}_{t-1}^{*}+\frac{\beta}{1+\beta} \mathbb{E}_{t}\left[\widehat{x}_{t+1}^{*}\right]+\frac{1}{\kappa(1+\beta)}\left(\widehat{q}_{t}^{*}+\widehat{v}_{t}^{*}\right)\end{array}$ \\
\hline KA & $\widehat{k}_{t+1} \approx(1-\delta) \widehat{k}_{t}+\delta\left(\widehat{x}_{t}+\widehat{v}_{t}\right)$ \\
\hline $\mathbf{K A}^{*}$ & $\widehat{k}_{t+1}^{*} \approx(1-\delta) \widehat{k}_{t}^{*}+\delta\left(\widehat{x}_{t}^{*}+\widehat{v}_{t}^{*}\right)$ \\
\hline & The Firms' Problem: \\
\hline AS & 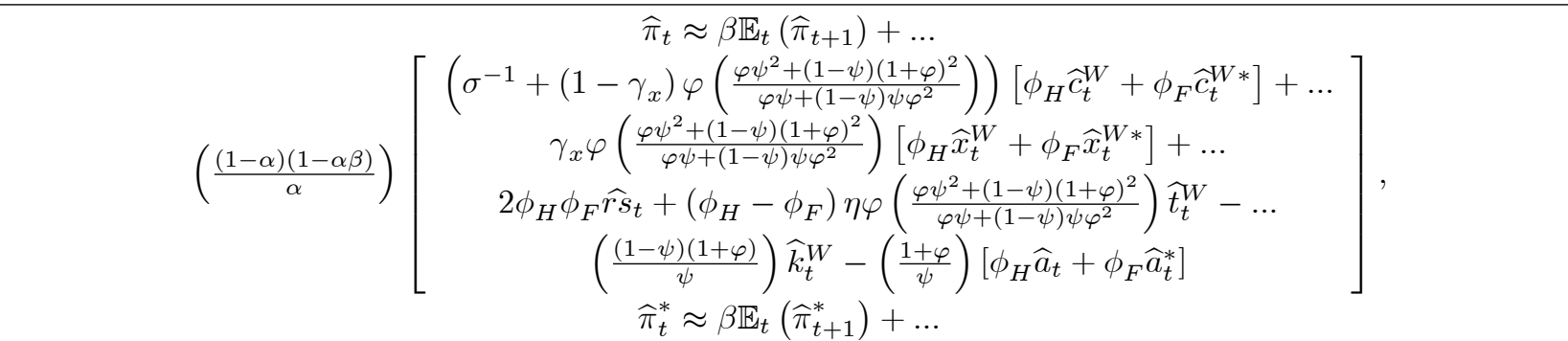 \\
\hline $\mathbf{A S}^{*}$ & $\left(\frac{(1-\alpha)(1-\alpha \beta)}{\alpha}\right)\left[\begin{array}{c}\left(\sigma^{-1}+\left(1-\gamma_{x}\right) \varphi\left(\frac{\varphi \psi^{2}+(1-\psi)(1+\varphi)^{2}}{\varphi \psi+(1-\psi) \psi \varphi^{2}}\right)\right)\left[\phi_{F} \widehat{c}_{t}^{W}+\phi_{H} \widehat{c}_{t}^{W *}\right]+\ldots \\
\gamma_{x} \varphi\left(\frac{\varphi \psi^{2}+(1-\psi)(1+\varphi)^{2}}{\varphi \psi+(1-\psi) \psi \varphi^{2}}\right)\left[\phi_{F} \widehat{x}_{t}^{W}+\phi_{H} \widehat{x}_{t}^{W *}\right]-\ldots \\
2 \phi_{F} \phi_{H} \widehat{r s s}_{t}-\left(\phi_{H}-\phi_{F}\right) \eta \varphi\left(\frac{\varphi \psi^{2}+(1-\psi)(1+\varphi)^{2}}{\varphi \psi+(1-\psi) \psi \varphi^{2}}\right) \widehat{t}_{t}^{W}-\ldots \\
\left(\frac{(1-\psi)(1+\varphi)}{\psi}\right) \widehat{k}_{t}^{W *}-\left(\frac{1+\varphi}{\psi}\right)\left[\phi_{F} \widehat{a}_{t}+\phi_{H} \widehat{a}_{t}^{*}\right]\end{array}\right]$ \\
\hline $\mathrm{T}^{\mathrm{W}}$ & $\Delta \widehat{t}_{t}^{W}-\beta \mathbb{E}_{t}\left(\Delta \widehat{t}_{t+1}^{W}\right)+\left(\frac{(1-\alpha)(1-\alpha \beta)}{\alpha}\right) \widehat{t}_{t}^{W} \approx \frac{\phi_{H} \phi_{F}}{\phi_{H}-\phi_{F}}\left[\left(\frac{(1-\alpha)(1-\alpha \beta)}{\alpha}\right) \widehat{r s}_{t}-\widehat{\pi}_{t}^{R}+\beta \mathbb{E}_{t}\left(\widehat{\pi}_{t+1}^{R}\right)\right]$, \\
\hline EC & $\widehat{r}_{t}^{z} \approx \frac{1}{\sigma} \widehat{c}_{t}+\frac{1+\varphi}{\psi} \widehat{y}_{t}-\left(\frac{1+(1-\psi) \varphi}{\psi}\right) \widehat{k}_{t}-\frac{1+\varphi}{\psi} \widehat{a}_{t}$, \\
\hline $\mathbf{E C}^{*}$ & $\widehat{r}_{t}^{z *} \approx \frac{1}{\sigma} \widehat{c}_{t}^{*}+\frac{1+\varphi}{\psi} \widehat{y}_{t}^{*}-\left(\frac{1+(1-\psi) \varphi}{\psi}\right) \widehat{k}_{t}^{*}-\frac{1+\varphi}{\psi} \widehat{a}_{t}^{*}$ \\
\hline & Aggregate Output and Employment: \\
\hline $\mathbf{Y}$ & $\widehat{y}_{t} \approx \eta \widehat{t}_{t}^{W}+\left(1-\gamma_{x}\right) \widehat{c}_{t}^{W}+\gamma_{x} \widehat{x}_{t}^{W}$ \\
\hline $\mathbf{Y}^{*}$ & $\widehat{y}_{t}^{*} \approx-\eta \widehat{t}_{t}^{W}+\left(1-\gamma_{x}\right) \widehat{c}_{t}^{W *}+\gamma_{x} \widehat{x}_{t}^{W *}$, \\
\hline $\mathbf{L}$ & $\psi \widehat{l}_{t} \approx \widehat{y}_{t}-\widehat{a}_{t}-(1-\psi) \widehat{k}_{t}$ \\
\hline $\mathbf{L}^{*}$ & $\psi \widehat{l}_{t}^{*} \approx \widehat{y}_{t}^{*}-\widehat{a}_{t}^{*}-(1-\psi) \widehat{k}_{t}^{*}$ \\
\hline & The Monetary Policy: \\
\hline MP & $\widehat{i}_{t} \approx \rho_{i} \hat{i}_{t-1}+\left(1-\rho_{i}\right)\left[\psi_{y} \widehat{y}_{t}+\psi_{\pi} \widehat{\pi}_{t}\right]+\widehat{m}_{t}$ \\
\hline $\mathrm{MP}^{*}$ & $\hat{i}_{t}^{*} \approx \rho_{i} \hat{i}_{t-1}^{*}+\left(1-\rho_{i}\right)\left[\psi_{y} \widehat{y}_{t}^{*}+\psi_{\pi} \widehat{\pi}_{t}^{*}\right]+\widehat{m}_{t}^{*}$, \\
\hline & Other Definitions: \\
\hline & $\begin{array}{c}\widehat{c}_{t}^{W} \equiv \phi_{H} \widehat{c}_{t}+\phi_{F} \widehat{c}_{t}^{*}, \widehat{c}_{t}^{W *} \equiv \phi_{F} \widehat{c}_{t}+\phi_{H} \widehat{c}_{t}^{*}, \widehat{x}_{t}^{W} \equiv \phi_{H} \widehat{x}_{t}+\phi_{F} \widehat{x}_{t}^{*}, \widehat{x}_{t}^{W *} \equiv \phi_{F} \widehat{x}_{t}+\phi_{H} \widehat{x}_{t}^{*}, \\
\widehat{k}_{t}^{W} \equiv \phi_{H} \widehat{k}_{t}+\phi_{F} \widehat{k}_{t}^{*}, \widehat{k}_{t}^{W *} \equiv \phi_{F} \widehat{k}_{t}+\phi_{H} \widehat{k}_{t}^{*}, \widehat{\pi}_{t}^{R} \equiv \widehat{\pi}_{t}-\widehat{\pi}_{t}^{*}, \Delta \widehat{t}_{t}^{W} \equiv \widehat{t}_{t}^{W}-\widehat{t}_{t-1}^{W}, \widehat{\pi}_{t}=\widehat{p}_{t}-\widehat{p}_{t-1}, \widehat{\pi}_{t}^{*}=\widehat{p}_{t}^{*}-\widehat{p}_{t-1}^{*}\end{array}$ \\
\hline & Other Coefficients: $\gamma_{x} \equiv(1-\psi) \delta\left[\left(\frac{\theta(1-\bar{\xi})}{\theta-1}\right)\left(\beta^{-1}-(1-\delta)\right)\right]^{-1}$ \\
\hline
\end{tabular}




\section{A.5 The Model With Capital, With Capital Utilization - NAC}

\begin{tabular}{|c|c|}
\hline & The Households' Problem: \\
\hline IS & $\widehat{c}_{t} \approx \mathbb{E}_{t}\left[\widehat{c}_{t+1}\right]-\sigma\left(\widehat{i}_{t}-\mathbb{E}_{t}\left[\widehat{\pi}_{t+1}\right]\right)$ \\
\hline IS $^{*}$ & $\widehat{c}_{t}^{*} \approx \mathbb{E}_{t}\left[\widehat{c}_{t+1}^{*}\right]-\sigma\left(\hat{i}_{t}^{*}-\mathbb{E}_{t}\left[\widehat{\pi}_{t+1}^{*}\right]\right)$, \\
\hline $\mathbf{R S}$ & $\widehat{c}_{t}-\widehat{c}_{t}^{*} \approx \sigma \widehat{r s}_{t}$ \\
\hline Q & $\begin{array}{c}\widehat{q}_{t} \approx(1-\delta) \beta \mathbb{E}_{t}\left[\widehat{q}_{t+1}\right]+\left[(1-\beta(1-\delta)) \mathbb{E}_{t}\left(\widehat{r}_{t+1}^{z}\right)-\left(\widehat{i}_{t}-\mathbb{E}_{t}\left(\widehat{\pi}_{t+1}\right)\right)\right] \\
\widehat{q}_{t} \approx-\widehat{v}_{t}\end{array}$ \\
\hline $\mathrm{Q}^{*}$ & $\begin{array}{c}\widehat{q}_{t}^{*} \approx(1-\delta) \beta \mathbb{E}_{t}\left[\widehat{q}_{t+1}^{*}\right]+\left[(1-\beta(1-\delta)) \mathbb{E}_{t}\left(\widehat{r}_{t+1}^{z *}\right)-\left(\hat{i}_{t}^{*}-\mathbb{E}_{t}\left(\widehat{\pi}_{t+1}^{*}\right)\right)\right] \\
\widehat{q}_{t}^{*} \approx-\widehat{v}_{t}^{*},\end{array}$ \\
\hline KU & $\mathbb{E}_{t}\left[\widehat{r}_{t+1}^{z}\right] \approx \lambda \mathbb{E}_{t}\left[\widehat{u}_{t+1}\right]$ \\
\hline $\mathbf{K U}^{*}$ & $\mathbb{E}_{t}\left[\underset{\approx}{\widehat{r}_{t+1}^{z *}}\right] \approx \lambda \mathbb{E}_{t}\left[\widehat{u}_{t+1}^{*}\right], \quad \quad \widehat{\approx}$ \\
\hline $\mathbf{K A}$ & 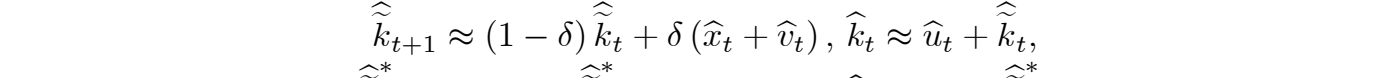 \\
\hline $\mathbf{K A}^{*}$ & $\widehat{\widetilde{k}}_{t+1}^{*} \approx(1-\delta) \widehat{\widetilde{k}}_{t}^{*}+\delta\left(\widehat{x}_{t}^{*}+\widehat{v}_{t}^{*}\right), \widehat{k}_{t}^{*} \approx \widehat{u}_{t}^{*}+\widehat{\widetilde{k}}_{t}^{*}$ \\
\hline & The Firms' Problem: \\
\hline AS & $\left(\frac{(1-\alpha)(1-\alpha \beta)}{\alpha}\right)\left[\begin{array}{c}\widehat{\pi}_{t} \approx \beta \mathbb{E}_{t}\left(\widehat{\pi}_{t+1}\right)+\ldots \\
\left(\sigma^{-1}+\left(1-\gamma_{x}\right) \varphi\left(\frac{\varphi \psi^{2}+(1-\psi)(1+\varphi)^{2}}{\varphi \psi+(1-\psi) \psi \varphi^{2}}\right)\right)\left[\phi_{H} \widehat{c}_{t}^{W}+\phi_{F} \widehat{c}_{t}^{W *}\right]+\ldots \\
\gamma_{x} \varphi\left(\frac{\varphi \psi^{2}+(1-\psi)(1+\varphi)^{2}}{\varphi \psi+(1-\psi) \psi \varphi^{2}}\right)\left[\phi_{H} \widehat{x}_{t}^{W}+\phi_{F} \widehat{x}_{t}^{W *}\right]+\ldots \\
\gamma_{x} \varphi\left(\frac{\varphi \psi^{2}+(1-\psi)(1+\varphi)^{2}}{\varphi \psi+(1-\psi) \psi \varphi^{2}}\right)\left(\frac{1-\beta(1-\delta)}{\beta \delta}\right)\left[\phi_{H} \widehat{u}_{t}+\phi_{F} \widehat{u}_{t}^{*}\right]+\ldots \\
2 \phi_{H} \phi_{F} \widehat{r s}_{t}+\left(\phi_{H}-\phi_{F}\right) \eta \varphi\left(\frac{\varphi \psi^{2}+(1-\psi)(1+\varphi)^{2}}{\varphi \psi+(1-\psi) \psi \varphi^{2}}\right) \widehat{t}_{t}^{W}-\ldots \\
\left(\frac{(1-\psi)(1+\varphi)}{\psi}\right) \widehat{k}_{t}^{W}-\left(\frac{1+\varphi}{\psi}\right)\left[\phi_{H} \widehat{a}_{t}+\phi_{F} \widehat{a}_{t}^{*}\right] \\
\widehat{\pi}_{t}^{*} \approx \beta \mathbb{E}_{t}\left(\widehat{\pi}_{t+1}^{*}\right)+\ldots\end{array}\right.$ \\
\hline $\mathbf{A} \mathbf{S}^{*}$ & $\left(\frac{(1-\alpha)(1-\alpha \beta)}{\alpha}\right)\left[\begin{array}{c}\left(\sigma^{-1}+\left(1-\gamma_{x}\right) \varphi\left(\frac{\varphi \psi^{2}+(1-\psi)(1+\varphi)^{2}}{\varphi \psi+(1-\psi) \psi \varphi^{2}}\right)\right)\left[\phi_{F} \widehat{c}_{t}^{W}+\phi_{H} \widehat{c}_{t}^{W *}\right]+\ldots \\
\gamma_{x} \varphi\left(\frac{\varphi \psi^{2}+(1-\psi)(1+\varphi)^{2}}{\varphi \psi+(1-\psi) \psi \varphi^{2}}\right)\left[\phi_{F} \widehat{x}_{t}^{W}+\phi_{H} \widehat{x}_{t}^{W *}\right]+\ldots \\
\gamma_{x} \varphi\left(\frac{\varphi \psi^{2}+(1-\psi)(1+\varphi)^{2}}{\varphi \psi+(1-\psi) \psi \varphi^{2}}\right)\left(\frac{1-\beta(1-\delta)}{\beta \delta}\right)\left[\phi_{F} \widehat{u}_{t}+\phi_{H} \widehat{u}_{t}^{*}\right]-\ldots \\
2 \phi_{F} \phi_{H} \widehat{r s s}_{t}-\left(\phi_{H}-\phi_{F}\right) \eta \varphi\left(\frac{\varphi \psi^{2}+(1-\psi)(1+\varphi)^{2}}{\varphi \psi+(1-\psi) \psi \varphi^{2}}\right) \widehat{t}_{t}^{W}-\ldots \\
\left(\frac{(1-\psi)(1+\varphi)}{\psi}\right) \widehat{k}_{t}^{W *}-\left(\frac{1+\varphi}{\psi}\right)\left[\phi_{F} \widehat{a}_{t}+\phi_{H} \widehat{a}_{t}^{*}\right]\end{array}\right]$ \\
\hline $\mathrm{T}^{\mathrm{W}}$ & $\Delta \widehat{t}_{t}^{W}-\beta \mathbb{E}_{t}\left(\Delta \widehat{t}_{t+1}^{W}\right)+\left(\frac{(1-\alpha)(1-\alpha \beta)}{\alpha}\right) \widehat{t}_{t}^{W} \approx \frac{\phi_{H} \phi_{F}}{\phi_{H}-\phi_{F}}\left[\left(\frac{(1-\alpha)(1-\alpha \beta)}{\alpha}\right) \widehat{r s}_{t}-\widehat{\pi}_{t}^{R}+\beta \mathbb{E}_{t}\left(\widehat{\pi}_{t+1}^{R}\right)\right]$ \\
\hline EC & $\widehat{r}_{t}^{z} \approx \frac{1}{\sigma} \widehat{c}_{t}+\frac{1+\varphi}{\psi} \widehat{y}_{t}-\left(\frac{1+(1-\psi) \varphi}{\psi}\right) \widehat{k}_{t}-\frac{1+\varphi}{\psi} \widehat{a}_{t}$ \\
\hline $\mathbf{E C}^{*}$ & $\widehat{r}_{t}^{z *} \approx \frac{1}{\sigma} \widehat{c}_{t}^{*}+\frac{1+\varphi}{\psi} \widehat{y}_{t}^{*}-\left(\frac{1+(1-\psi) \varphi}{\psi}\right) \widehat{k}_{t}^{*}-\frac{1+\varphi}{\psi} \widehat{a}_{t}^{*}$ \\
\hline & Aggregate Output and Employment: \\
\hline $\mathbf{Y}$ & $\widehat{y}_{t} \approx \eta \widehat{t}_{t}^{W}+\left(1-\gamma_{x}\right) \widehat{c}_{t}^{W}+\gamma_{x} \widehat{x}_{t}^{W}+\gamma_{x}\left(\frac{1-\beta(1-\delta)}{\beta \delta}\right) \widehat{u}_{t}$ \\
\hline $\mathbf{Y}^{*}$ & $\widehat{y}_{t}^{*} \approx-\eta \widehat{t}_{t}^{W}+\left(1-\gamma_{x}\right) \widehat{c}_{t}^{W *}+\gamma_{x} \widehat{x}_{t}^{W *}+\gamma_{x}\left(\frac{1-\beta(1-\delta)}{\beta \delta}\right) \widehat{u}_{t}^{*}$ \\
\hline $\mathbf{L}$ & $\psi \widehat{\psi} \widehat{l}_{t} \approx \widehat{y}_{t}-\widehat{a}_{t}-(1-\psi) \widehat{k}_{t}$ \\
\hline $\mathbf{L}^{*}$ & $\psi \widehat{l}_{t}^{*} \approx \widehat{y}_{t}^{*}-\widehat{a}_{t}^{*}-(1-\psi) \widehat{k}_{t}^{*}$ \\
\hline & The Monetary Policy: \\
\hline MP & $\widehat{i}_{t} \approx \rho_{i} \widehat{i}_{t-1}+\left(1-\rho_{i}\right)\left[\psi_{y} \widehat{y}_{t}+\psi_{\pi} \widehat{\pi}_{t}\right]+\widehat{m}_{t}$ \\
\hline MP* $^{*}$ & $\widehat{i}_{t}^{*} \approx \rho_{i} \widehat{i}_{t-1}^{*}+\left(1-\rho_{i}\right)\left[\psi_{y} \widehat{y}_{t}^{*}+\psi_{\pi} \widehat{\pi}_{t}^{*}\right]+\widehat{m}_{t}^{*}$ \\
\hline
\end{tabular}

Other Definitions:

$\widehat{c}_{t}^{W} \equiv \phi_{H} \widehat{c}_{t}+\phi_{F} \widehat{c}_{t}^{*}, \widehat{c}_{t}^{W *} \equiv \phi_{F} \widehat{c}_{2} \not t \phi_{H} \widehat{c}_{t}^{*}, \widehat{x}_{t}^{W} \equiv \phi_{H} \widehat{x}_{t}+\phi_{F} \widehat{x}_{t}^{*}, \widehat{x}_{t}^{W *} \equiv \phi_{F} \widehat{x}_{t}+\phi_{H} \widehat{x}_{t}^{*}$,

$\widehat{k}_{t}^{W} \equiv \phi_{H} \widehat{k}_{t}+\phi_{F} \widehat{k}_{t}^{*}, \widehat{k}_{t}^{W *} \equiv \phi_{F} \widehat{k}_{t}+\phi_{H} \widehat{k}_{t}^{*}, \widehat{\pi}_{t}^{R} \equiv \widehat{\pi}_{t}-\widehat{\pi}_{t}^{*}, \Delta \widehat{t}_{t}^{W} \equiv \widehat{t}_{t}^{W}-\widehat{t}_{t-1}^{W}, \widehat{\pi}_{t}=\widehat{p}_{t}-\widehat{p}_{t-1}, \widehat{\pi}_{t}^{*}=\widehat{p}_{t}^{*}-\widehat{p}_{t-1}^{*}$.

Other Coefficients: $\gamma_{x} \equiv(1-\psi) \delta\left[\left(\frac{\theta(1-\bar{\xi})}{\theta-1}\right)\left(\beta^{-1}-(1-\delta)\right)\right]^{-1}$. 


\section{A.6 The Model With Capital, With Capital Utilization - CAC}

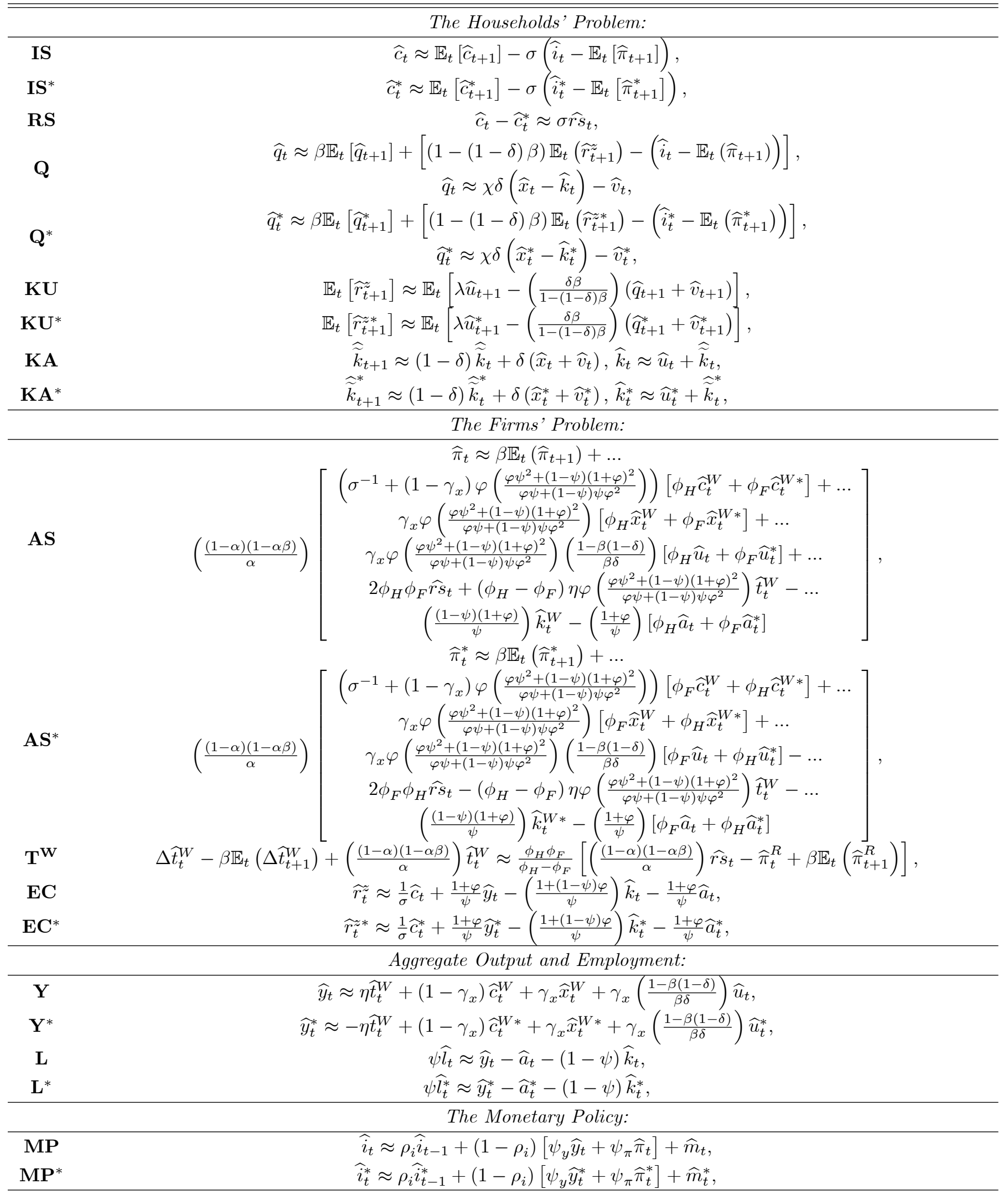

12 Qther Definitions:

$\widehat{c}_{t}^{W} \equiv \phi_{H} \widehat{c}_{t}+\phi_{F} \widehat{c}_{t}^{*}, \widehat{c}_{t}^{W *} \equiv \phi_{F} \widehat{c}_{t}+\phi_{H} \widehat{c}_{t}^{*}, \widehat{x}_{t}^{W} \equiv \phi_{H} \widehat{x}_{t}+\phi_{F} \widehat{x}_{t}^{*}, \widehat{x}_{t}^{W *} \equiv \phi_{F} \widehat{x}_{t}+\phi_{H} \widehat{x}_{t}^{*}$,

$\widehat{k}_{t}^{W} \equiv \phi_{H} \widehat{k}_{t}+\phi_{F} \widehat{k}_{t}^{*}, \widehat{k}_{t}^{W *} \equiv \phi_{F} \widehat{k}_{t}+\phi_{H} \widehat{k}_{t}^{*}, \widehat{\pi}_{t}^{R} \equiv \widehat{\pi}_{t}-\widehat{\pi}_{t}^{*}, \Delta \widehat{t}_{t}^{W} \equiv \widehat{t}_{t}^{W}-\widehat{t}_{t-1}^{W}, \widehat{\pi}_{t}=\widehat{p}_{t}-\widehat{p}_{t-1}, \widehat{\pi}_{t}^{*}=\widehat{p}_{t}^{*}-\widehat{p}_{t-1}^{*}$.

Other Coefficients: $\gamma_{x} \equiv(1-\psi) \delta\left[\left(\frac{\theta(1-\bar{\xi})}{\theta-1}\right)\left(\beta^{-1}-(1-\delta)\right)\right]^{-1}$. 


\section{A.7 The Model With Capital, With Capital Utilization - IAC}

\begin{tabular}{|c|c|}
\hline & The Households' Problem: \\
\hline IS & $\widehat{c}_{t} \approx \mathbb{E}_{t}\left[\widehat{c}_{t+1}\right]-\sigma\left(\widehat{i}_{t}-\mathbb{E}_{t}\left[\widehat{\pi}_{t+1}\right]\right)$ \\
\hline IS $^{*}$ & $\widehat{c}_{t}^{*} \approx \mathbb{E}_{t}\left[\widehat{c}_{t+1}^{*}\right]-\sigma\left(\hat{i}_{t}^{*}-\mathbb{E}_{t}\left[\widehat{\pi}_{t+1}^{*}\right]\right)$ \\
\hline RS & $\widehat{c}_{t}-\widehat{c}_{t}^{*} \approx \sigma \widehat{r s}_{t}$ \\
\hline Q & $\begin{array}{c}\widehat{q}_{t} \approx(1-\delta) \beta \mathbb{E}_{t}\left[\widehat{q}_{t+1}\right]+\left[(1-(1-\delta) \beta) \mathbb{E}_{t}\left(\widehat{r}_{t+1}^{z}\right)-\left(\widehat{i}_{t}-\mathbb{E}_{t}\left[\widehat{\pi}_{t+1}\right]\right)\right] \\
\widehat{x}_{t} \approx \frac{1}{1+\beta} \widehat{x}_{t-1}+\frac{\beta}{1+\beta} \mathbb{E}_{t}\left[\widehat{x}_{t+1}\right]+\frac{1}{\kappa(1+\beta)}\left(\widehat{q}_{t}+\widehat{v}_{t}\right)\end{array}$ \\
\hline $\mathbf{Q}^{*}$ & $\begin{array}{c}\widehat{q}_{t}^{*} \approx(1-\delta) \beta \mathbb{E}_{t}\left[\widehat{q}_{t+1}^{*}\right]+\left[(1-(1-\delta) \beta) \mathbb{E}_{t}\left(\widehat{r}_{t+1}^{z *}\right)-\left(\widehat{i}_{t}^{*}-\mathbb{E}_{t}\left[\widehat{\pi}_{t+1}^{*}\right]\right)\right] \\
\widehat{x}_{t}^{*} \approx \frac{1}{1+\beta} \widehat{x}_{t-1}^{*}+\frac{\beta}{1+\beta} \mathbb{E}_{t}\left[\widehat{x}_{t+1}^{*}\right]+\frac{1}{\kappa(1+\beta)}\left(\widehat{q}_{t}^{*}+\widehat{v}_{t}^{*}\right)\end{array}$ \\
\hline KU & $\mathbb{E}_{t}\left[\widehat{r}_{t+1}^{z}\right] \approx \lambda \mathbb{E}_{t}\left[\widehat{u}_{t+1}\right]$ \\
\hline $\mathbf{K U}^{*}$ & $\mathbb{E}_{t}\left[\widehat{r}_{t+1}^{z *}\right] \approx \lambda \mathbb{E}_{t}\left[\widehat{u}_{t+1}^{*}\right]$ \\
\hline KA & $\widehat{\widetilde{k}}_{t+1} \approx(1-\delta) \widehat{\widetilde{k}}_{t}+\delta\left(\widehat{x}_{t}+\widehat{v}_{t}\right), \widehat{k}_{t} \approx \widehat{u}_{t}+\widehat{\widetilde{k}}_{t}$ \\
\hline $\mathbf{K A}^{*}$ & $\widehat{\widetilde{k}}_{t+1}^{*} \approx(1-\delta) \widehat{\widetilde{k}}_{t}^{*}+\delta\left(\widehat{x}_{t}^{*}+\widehat{v}_{t}^{*}\right), \widehat{k}_{t}^{*} \approx \widehat{u}_{t}^{*}+\widehat{\widetilde{k}}_{t}^{*}$ \\
\hline & The Firms' Problem: \\
\hline AS & $\left(\frac{(1-\alpha)(1-\alpha \beta)}{\alpha}\right)\left[\begin{array}{c}\widehat{\pi}_{t} \approx \beta \mathbb{E}_{t}\left(\widehat{\pi}_{t+1}\right)+\ldots \\
\left(\sigma^{-1}+\left(1-\gamma_{x}\right) \varphi\left(\frac{\varphi \psi^{2}+(1-\psi)(1+\varphi)^{2}}{\varphi \psi+(1-\psi) \psi \varphi^{2}}\right)\right)\left[\phi_{H} \widehat{c}_{t}^{W}+\phi_{F} \widehat{c}_{t}^{W *}\right]+\ldots \\
\gamma_{x} \varphi\left(\frac{\varphi \psi^{2}+(1-\psi)(1+\varphi)^{2}}{\varphi \psi+(1-\psi) \psi \varphi^{2}}\right)\left[\phi_{H} \widehat{x}_{t}^{W}+\phi_{F} \widehat{x}_{t}^{W *}+\ldots\right. \\
\gamma_{x} \varphi\left(\frac{\varphi \psi^{2}+(1-\psi)(1+\varphi)^{2}}{\varphi \psi+(1-\psi) \psi \varphi^{2}}\right)\left(\frac{1-\beta(1-\delta)}{\beta \delta}\right)\left[\phi_{H} \widehat{u}_{t}+\phi_{F} \widehat{u}_{t}^{*}\right]+\ldots \\
2 \phi_{H} \phi_{F} \widehat{r s}_{t}+\left(\phi_{H}-\phi_{F}\right) \eta \varphi\left(\frac{\varphi \psi^{2}+(1-\psi)(1+\varphi)^{2}}{\varphi \psi+(1-\psi) \psi \varphi^{2}}\right) \widehat{t}_{t}^{W}-\ldots \\
\left(\frac{(1-\psi)(1+\varphi)}{\psi}\right) \widehat{k}_{t}^{W}-\left(\frac{1+\varphi}{\psi}\right)\left[\phi_{H} \widehat{a}_{t}+\phi_{F} \widehat{a}_{t}^{*}\right] \\
\widehat{\pi}_{t}^{*} \approx \beta \mathbb{E}_{t}\left(\widehat{\pi}_{t+1}^{*}\right)+\ldots\end{array}\right]$ \\
\hline $\mathbf{A S}^{*}$ & $\left(\frac{(1-\alpha)(1-\alpha \beta)}{\alpha}\right)\left[\begin{array}{c}\left(\sigma^{-1}+\left(1-\gamma_{x}\right) \varphi\left(\frac{\varphi \psi^{2}+(1-\psi)(1+\varphi)^{2}}{\varphi \psi+(1-\psi) \psi \varphi^{2}}\right)\right)\left[\phi_{F} \widehat{c}_{t}^{W}+\phi_{H} \widehat{c}_{t}^{W *}\right]+\ldots \\
\gamma_{x} \varphi\left(\frac{\varphi \psi^{2}+(1-\psi)(1+\varphi)^{2}}{\varphi \psi+(1-\psi) \psi \varphi^{2}}\right)\left[\phi_{F} \widehat{x}_{t}^{W}+\phi_{H} \widehat{x}_{t}^{W *}\right]+\ldots \\
\gamma_{x} \varphi\left(\frac{\varphi \psi^{2}+(1-\psi)(1+\varphi)^{2}}{\varphi \psi+(1-\psi) \psi \varphi^{2}}\right)\left(\frac{1-\beta(1-\delta)}{\beta \delta}\right)\left[\phi_{F} \widehat{u}_{t}+\phi_{H} \widehat{u}_{t}^{*}\right]-\ldots \\
2 \phi_{F} \phi_{H} \widehat{r s}_{t}-\left(\phi_{H}-\phi_{F}\right) \eta \varphi\left(\frac{\varphi \psi^{2}+(1-\psi)(1+\varphi)^{2}}{\varphi \psi+(1-\psi) \psi \varphi^{2}}\right) \widehat{t}_{t}^{W}-\ldots \\
\left(\frac{(1-\psi)(1+\varphi)}{\psi}\right) \widehat{k}_{t}^{W *}-\left(\frac{1+\varphi}{\psi}\right)\left[\phi_{F} \widehat{a}_{t}+\phi_{H} \widehat{a}_{t}^{*}\right]\end{array}\right]$ \\
\hline $\mathrm{T}^{\mathrm{W}}$ & $\Delta \widehat{t}_{t}^{W}-\beta \mathbb{E}_{t}\left(\Delta \widehat{t}_{t+1}^{W}\right)+\left(\frac{(1-\alpha)(1-\alpha \beta)}{\alpha}\right) \widehat{t}_{t}^{W} \approx \frac{\phi_{H} \phi_{F}}{\phi_{H}-\phi_{F}}\left[\left(\frac{(1-\alpha)(1-\alpha \beta)}{\alpha}\right) \widehat{r s}_{t}-\widehat{\pi}_{t}^{R}+\beta \mathbb{E}_{t}\left(\widehat{\pi}_{t+1}^{R}\right)\right]$ \\
\hline EC & $\widehat{r}_{t}^{z} \approx \frac{1}{\sigma} \widehat{c}_{t}+\frac{1+\varphi}{\psi} \widehat{y}_{t}-\left(\frac{1+(1-\psi) \varphi}{\psi}\right) \widehat{k}_{t}-\frac{1+\varphi}{\psi} \widehat{a}_{t}$ \\
\hline $\mathbf{E C}^{*}$ & $\widehat{r}_{t}^{z *} \approx \frac{1}{\sigma} \widehat{c}_{t}^{*}+\frac{1+\varphi}{\psi} \widehat{y}_{t}^{*}-\left(\frac{1+(1-\psi) \varphi}{\psi}\right) \widehat{k}_{t}^{*}-\frac{1+\varphi}{\psi} \widehat{a}_{t}^{*}$ \\
\hline & Aggregate Output and Employment: \\
\hline $\mathbf{Y}$ & $\widehat{y}_{t} \approx \eta \widehat{t}_{t}^{W}+\left(1-\gamma_{x}\right) \widehat{c}_{t}^{W}+\gamma_{x} \widehat{x}_{t}^{W}+\gamma_{x}\left(\frac{1-\beta(1-\delta)}{\beta \delta}\right) \widehat{u}_{t}$ \\
\hline $\mathbf{Y}^{*}$ & $\widehat{y}_{t}^{*} \approx-\eta \widehat{t}_{t}^{W}+\left(1-\gamma_{x}\right) \widehat{c}_{t}^{W *}+\gamma_{x} \widehat{x}_{t}^{W *}+\gamma_{x}\left(\frac{1-\beta(1-\delta)}{\beta \delta}\right) \widehat{u}_{t}^{*}$ \\
\hline $\mathbf{L}$ & $\psi \widehat{l_{t}} \approx \widehat{y}_{t}-\widehat{a}_{t}-(1-\psi) \widehat{k}_{t}$ \\
\hline $\mathbf{L}^{*}$ & $\psi \widehat{l}_{t}^{*} \approx \widehat{y}_{t}^{*}-\widehat{a}_{t}^{*}-(1-\psi) \widehat{k}_{t}^{*}$ \\
\hline & The Monetary Policy: \\
\hline MP & $\widehat{i}_{t} \approx \rho_{i} \widehat{i}_{t-1}+\left(1-\rho_{i}\right)\left[\psi_{y} \widehat{y}_{t}+\psi_{\pi} \widehat{\pi}_{t}\right]+\widehat{m}_{t}$ \\
\hline $\mathrm{MP}^{*}$ & $\hat{i}_{t}^{*} \approx \rho_{i} \hat{i}_{t-1}^{*}+\left(1-\rho_{i}\right)\left[\psi_{y} \widehat{y}_{t}^{*}+\psi_{\pi} \widehat{\pi}_{t}^{*}\right]+\widehat{m}_{t}^{*}$ \\
\hline & Other Definitions: \\
\hline & $\begin{array}{c}\widehat{c}_{t}^{W} \equiv \phi_{H} \widehat{c}_{t}+\phi_{F} \widehat{c}_{t}^{*}, \widehat{c}_{t}^{W *} \equiv \phi_{F} \widehat{c}_{2} 29 \phi_{H} \widehat{c}_{t}^{*}, \widehat{x}_{t}^{W} \equiv \phi_{H} \widehat{x}_{t}+\phi_{F} \widehat{x}_{t}^{*}, \widehat{x}_{t}^{W *} \equiv \phi_{F} \widehat{x}_{t}+\phi_{H} \widehat{x}_{t}^{*}, \\
\widehat{k}_{t}^{W} \equiv \phi_{H} \widehat{k}_{t}+\phi_{F} \widehat{k}_{t}^{*}, \widehat{k}_{t}^{W *} \equiv \phi_{F} \widehat{k}_{t}+\phi_{H} \widehat{k}_{t}^{*}, \widehat{\pi}_{t}^{R} \equiv \widehat{\pi}_{t}-\widehat{\pi}_{t}^{*}, \Delta \widehat{t}_{t}^{W} \equiv \widehat{t}_{t}^{W}-\widehat{t}_{t-1}^{W}, \widehat{\pi}_{t}=\widehat{p}_{t}-\widehat{p}_{t-1}, \widehat{\pi}_{t}^{*}=\widehat{p}_{t}^{*}-\widehat{p}_{t-1}^{*}\end{array}$ \\
\hline & Other Coefficients: $\gamma_{x} \equiv(1-\psi) \delta\left[\left(\frac{\theta(1-\bar{\xi})}{\theta-1}\right)\left(\beta^{-1}-(1-\delta)\right)\right]^{-1}$ \\
\hline
\end{tabular}




\section{A.8 Net Exports, Real Exports and Real Imports}

\begin{tabular}{cc}
\hline \hline TXe Model Without Capital: \\
\hline EXP & $\widehat{t b}_{t} \equiv \eta \widehat{t}_{t}^{W}-\phi_{F} \sigma \widehat{r s}_{t}$, \\
IMP & $\widehat{\exp }_{t} \approx \eta\left(\frac{1}{2 \phi_{F}}\right) \widehat{t}_{t}^{W}+\widehat{c}_{t}^{*}$, \\
\hline & $\widehat{i m p}_{t} \approx-\eta\left(\frac{1}{2 \phi_{F}}\right) \widehat{t}_{t}^{W}+\widehat{c}_{t}$, \\
\hline $\mathbf{N X}$ & The Model With Capital $(N A C$, CAC, IAC), With or Without Capital Utilization: \\
$\mathbf{E X P}$ & $\widehat{t b}_{t} \equiv \eta \widehat{t}_{t}^{W}-\left(1-\gamma_{x}\right) \phi_{F} \sigma \widehat{r s}_{t}-\gamma_{x} \phi_{F}\left(\widehat{x}_{t}-\widehat{x}_{t}^{*}\right)$, \\
$\mathbf{I M P}$ & $\widehat{\exp }_{t} \approx \eta\left(\frac{1}{2 \phi_{F}}\right) \widehat{t}_{t}^{W}+\left(1-\gamma_{x}\right) \widehat{c}_{t}^{*}+\gamma_{x} \widehat{x}_{t}^{*}$, \\
\hline & $\widehat{i m p}_{t} \approx-\eta\left(\frac{1}{2 \phi_{F}}\right) \widehat{t}_{t}^{W}+\left(1-\gamma_{x}\right) \widehat{c}_{t}+\gamma_{x} \widehat{x}_{t}$, \\
\hline & Other Coefficients: \\
\hline
\end{tabular}




\section{B The Linearized Equilibrium Conditions with PCP: A Summary}

Here, we report the system of equations derived after log-linearizing the equilibrium conditions of the model with PCP.

\section{B.1 The Model Without Capital}

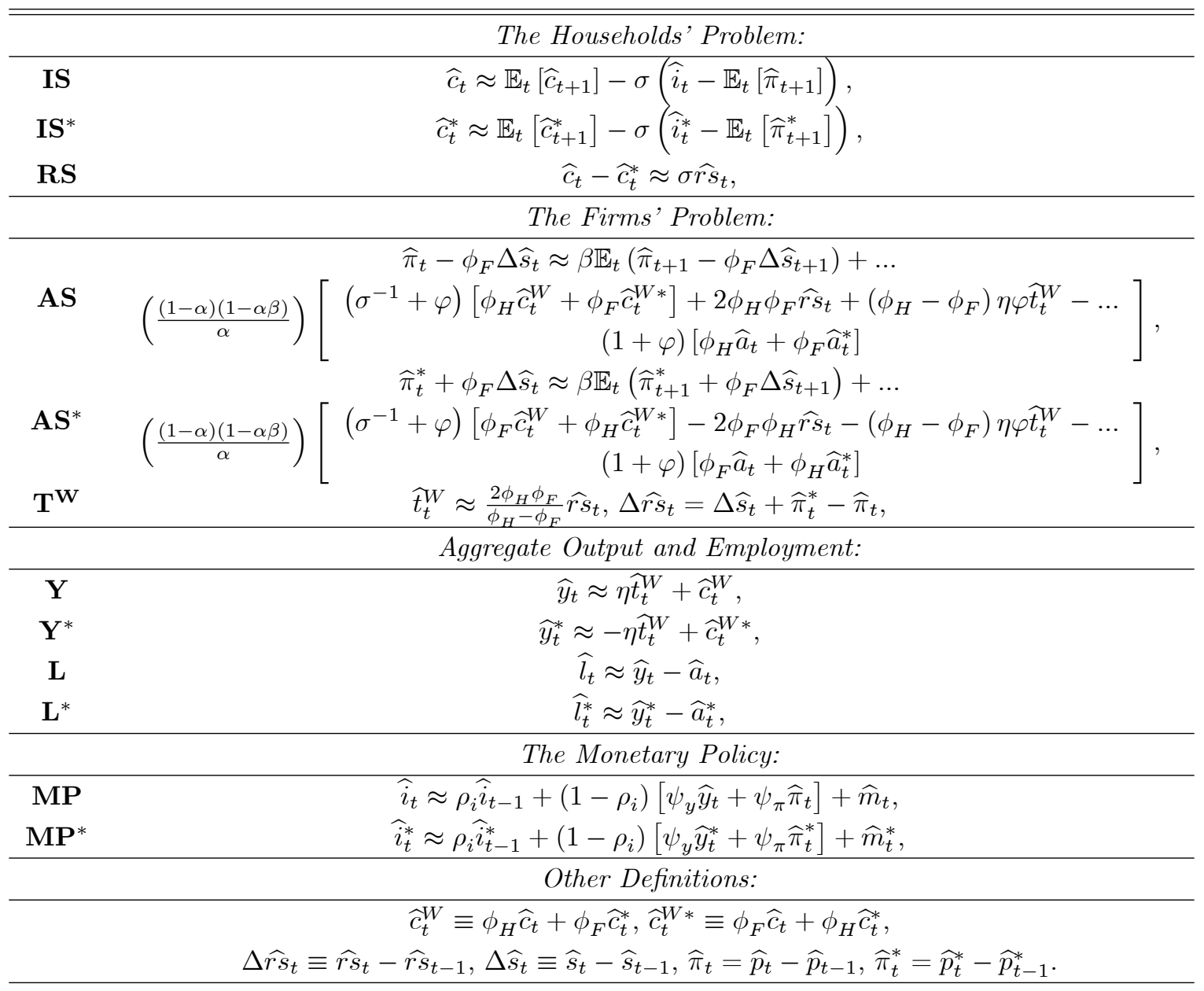




\section{B.2 The Model With Capital, Without Capital Utilization - NAC}

\begin{tabular}{|c|c|}
\hline & The Households' Problem: \\
\hline IS & $\widehat{c}_{t} \approx \mathbb{E}_{t}\left[\widehat{c}_{t+1}\right]-\sigma\left(\widehat{i}_{t}-\mathbb{E}_{t}\left[\widehat{\pi}_{t+1}\right]\right)$ \\
\hline $\mathbf{I S}^{*}$ & $\widehat{c}_{t}^{*} \approx \mathbb{E}_{t}\left[\widehat{c}_{t+1}^{*}\right]-\sigma\left(\widehat{i}_{t}^{*}-\mathbb{E}_{t}\left[\widehat{\pi}_{t+1}^{*}\right]\right)$ \\
\hline $\mathbf{R S}$ & $\widehat{c}_{t}-\widehat{c}_{t}^{*} \approx \sigma \widehat{r s}_{t}$ \\
\hline $\mathbf{Q}$ & $\begin{array}{c}\widehat{q}_{t} \approx(1-\delta) \beta \mathbb{E}_{t}\left[\widehat{q}_{t+1}\right]+\left[(1-(1-\delta) \beta) \mathbb{E}_{t}\left(\widehat{r}_{t+1}^{z}\right)-\left(\widehat{i}_{t}-\mathbb{E}_{t}\left(\widehat{\pi}_{t+1}\right)\right)\right] \\
\widehat{q}_{t} \approx-\widehat{v}_{t}\end{array}$ \\
\hline $\mathrm{Q}^{*}$ & $\begin{array}{c}\widehat{q}_{t}^{*} \approx(1-\delta) \beta \mathbb{E}_{t}\left[\widehat{q}_{t+1}^{*}\right]+\left[(1-(1-\delta) \beta) \mathbb{E}_{t}\left(\widehat{r}_{t+1}^{z *}\right)-\left(\widehat{i}_{t}^{*}-\mathbb{E}_{t}\left(\widehat{\pi}_{t+1}^{*}\right)\right)\right] \\
\widehat{q}_{t}^{*} \approx-\widehat{v}_{t}^{*}\end{array}$ \\
\hline $\mathbf{K A}$ & $\widehat{k}_{t+1} \approx(1-\delta) \widehat{k}_{t}+\delta\left(\widehat{x}_{t}+\widehat{v}_{t}\right)$ \\
\hline $\mathbf{K A}^{*}$ & $\widehat{k}_{t+1}^{*} \approx(1-\delta) \widehat{k}_{t}^{*}+\delta\left(\widehat{x}_{t}^{*}+\widehat{v}_{t}^{*}\right)$ \\
\hline & The Firms' Problem: \\
\hline $\mathbf{A S}$ & $\left(\frac{(1-\alpha)(1-\alpha \beta)}{\alpha}\right)\left[\begin{array}{c}\widehat{\pi}_{t}-\phi_{F} \Delta \widehat{s}_{t} \approx \beta \mathbb{E}_{t}\left(\widehat{\pi}_{t+1}-\phi_{F} \Delta \widehat{s}_{t+1}\right)+\ldots \\
\left(\sigma^{-1}+\left(1-\gamma_{x}\right) \varphi\left(\frac{\varphi \psi^{2}+(1-\psi)(1+\varphi)^{2}}{\varphi \psi+(1-\psi) \psi \varphi^{2}}\right)\right)\left[\phi_{H} \widehat{c}_{t}^{W}+\phi_{F} \widehat{c}_{t}^{W *}\right]+\ldots \\
\gamma_{x} \varphi\left(\frac{\varphi \psi^{2}+(1-\psi)(1+\varphi)^{2}}{\varphi \psi+(1-\psi) \psi \varphi^{2}}\right)\left[\phi_{H} \widehat{x}_{t}^{W}+\phi_{F} \widehat{x}_{t}^{W *}\right]+\ldots \\
2 \phi_{H} \phi_{F} \widehat{r s}_{t}+\left(\phi_{H}-\phi_{F}\right) \eta \varphi\left(\frac{\varphi \psi^{2}+(1-\psi)(1+\varphi)^{2}}{\varphi \psi+(1-\psi) \psi \varphi^{2}}\right) \widehat{t}_{t}^{W}-\ldots \\
\left(\frac{(1-\psi)(1+\varphi)}{\psi}\right) \widehat{k}_{t}^{W}-\left(\frac{1+\varphi}{\psi}\right)\left[\phi_{H} \widehat{a}_{t}+\phi_{F} \widehat{a}_{t}^{*}\right]\end{array}\right.$ \\
\hline $\mathbf{A} \mathbf{S}^{*}$ & $\left(\frac{(1-\alpha)(1-\alpha \beta)}{\alpha}\right)\left[\begin{array}{c}\widehat{\pi}_{t}^{*}+\phi_{F} \Delta \widehat{s}_{t} \approx \beta \mathbb{E}_{t}\left(\widehat{\pi}_{t+1}^{*}+\phi_{F} \Delta \widehat{s}_{t+1}\right)+\ldots \\
\left(\sigma^{-1}+\left(1-\gamma_{x}\right) \varphi\left(\frac{\varphi \psi^{2}+(1-\psi)(1+\varphi)^{2}}{\varphi \psi+(1-\psi) \psi \varphi^{2}}\right)\right)\left[\phi_{F} \widehat{c}_{t}^{W}+\phi_{H} \widehat{c}_{t}^{W *}\right]+\ldots \\
\gamma_{x} \varphi\left(\frac{\varphi \psi^{2}+(1-\psi)(1+\varphi)^{2}}{\varphi \psi+(1-\psi) \psi \varphi^{2}}\right)\left[\phi_{F} \widehat{x}_{t}^{W}+\phi_{H} \widehat{x}_{t}^{W *}\right]-\ldots \\
2 \phi_{F} \phi_{H} \widehat{r s}_{t}-\left(\phi_{H}-\phi_{F}\right) \eta \varphi\left(\frac{\varphi \psi}{\varphi \psi(1-\psi)(1+\varphi)^{2}}\right) \widehat{t}_{t}^{W}-\ldots \\
\left(\frac{(1-\psi)(1+\varphi)}{\psi}\right) \widehat{k}_{t}^{W *}-\left(\frac{1+\varphi}{\psi}\right)\left[\phi_{F} \widehat{a}_{t}+\phi_{H} \widehat{a}_{t}^{*}\right]\end{array}\right]$ \\
\hline $\begin{array}{l}\mathbf{T}^{\mathbf{W}} \\
\mathbf{E C}\end{array}$ & $\begin{aligned} \hat{t}_{t}^{W} & \approx \frac{2 \phi_{H} \phi_{F}}{\phi_{H}-\phi_{F}} \widehat{r s}_{t}, \Delta \widehat{r s}_{t}=\Delta \widehat{s}_{t}+\widehat{\pi}_{t}^{*}-\widehat{\pi}_{t} \\
\widehat{r}_{t}^{z} & \approx \frac{1}{\sigma} \widehat{c}_{t}+\frac{1+\varphi}{\psi} \widehat{y}_{t}-\left(\frac{1+(1-\psi) \varphi}{\psi}\right) \widehat{k}_{t}-\frac{1+\varphi}{\psi} \widehat{a}_{t}\end{aligned}$ \\
\hline $\mathbf{E C}^{*}$ & $\widehat{r}_{t}^{z *} \approx \frac{1}{\sigma} \widehat{c}_{t}^{*}+\frac{1+\varphi}{\psi} \widehat{y}_{t}^{*}-\left(\frac{1+(1-\psi) \varphi}{\psi}\right) \widehat{k}_{t}^{*}-\frac{1+\varphi}{\psi} \widehat{a}_{t}^{*}$ \\
\hline & Aggregate Output and Employment: \\
\hline $\mathbf{Y}$ & $\widehat{y}_{t} \approx \eta \widehat{t}_{t}^{W}+\left(1-\gamma_{x}\right) \widehat{c}_{t}^{W}+\gamma_{x} \widehat{x}_{t}^{W}$ \\
\hline $\mathbf{Y}^{*}$ & $\widehat{y}_{t}^{*} \approx-\eta \widehat{t}_{t}^{W}+\left(1-\gamma_{x}\right) \widehat{c}_{t}^{W *}+\gamma_{x} \widehat{x}_{t}^{W *}$ \\
\hline $\mathbf{L}$ & $\psi \widehat{l}_{t} \approx \widehat{y}_{t}-\widehat{a}_{t}-(1-\psi) \widehat{k}_{t}$ \\
\hline $\mathbf{L}^{*}$ & $\psi \widehat{l}_{t}^{*} \approx \widehat{y}_{t}^{*}-\widehat{a}_{t}^{*}-(1-\psi) \widehat{k}_{t}^{*}$ \\
\hline & The Monetary Policy: \\
\hline MP & $\widehat{i}_{t} \approx \rho_{i} \widehat{i}_{t-1}+\left(1-\rho_{i}\right)\left[\psi_{y} \widehat{y}_{t}+\psi_{\pi} \widehat{\pi}_{t}\right]+\widehat{m}_{t}$ \\
\hline $\mathbf{M P}^{*}$ & $\hat{i}_{t}^{*} \approx \rho_{i} \widehat{i}_{t-1}^{*}+\left(1-\rho_{i}\right)\left[\psi_{y} \widehat{y}_{t}^{*}+\psi_{\pi} \widehat{\pi}_{t}^{*}\right]+\widehat{m}_{t}^{*}$ \\
\hline & Other Definitions: \\
\hline & $\begin{array}{c}\widehat{c}_{t}^{W} \equiv \phi_{H} \widehat{c}_{t}+\phi_{F} \widehat{c}_{t}^{*}, \widehat{c}_{t}^{W *} \equiv \phi_{F} \widehat{c}_{t}+\phi_{H} \widehat{c}_{t}^{*}, \widehat{x}_{t}^{W} \equiv \phi_{H} \widehat{x}_{t}+\phi_{F} \widehat{x}_{t}^{*}, \widehat{x}_{t}^{W *} \equiv \phi_{F} \widehat{x}_{t}+\phi_{H} \widehat{x}_{t}^{*} \\
\widehat{k}_{t}^{W} \equiv \phi_{H} \widehat{k}_{t}+\phi_{F} \widehat{k}_{t}^{*}, \widehat{k}_{t}^{W *} \equiv \phi_{F} \widehat{k}_{t}+\phi_{H} \widehat{k}_{t}^{*}, \Delta \widehat{r s}_{t} \equiv \widehat{r s} t-\widehat{r s}_{t-1}, \Delta \widehat{s}_{t} \equiv \widehat{s}_{t}-\widehat{s}_{t-1}, \widehat{\pi}_{t}=\widehat{p}_{t}-\widehat{p}_{t-1}, \widehat{\pi}_{t}^{*}=\widehat{p}_{t}^{*}-\widehat{p}_{t-1}^{*}\end{array}$ \\
\hline & Other Coefficients: $\gamma_{x} \equiv(1-\psi) \delta\left[\left(\frac{\theta(1-\bar{\xi})}{\theta-1}\right)\left(\beta^{-1}-(1-\delta)\right)\right]^{-1}$ \\
\hline
\end{tabular}




\section{B.3 The Model With Capital, Without Capital Utilization - CAC}

\begin{tabular}{|c|c|}
\hline & The Households' Problem: \\
\hline IS & $\widehat{c}_{t} \approx \mathbb{E}_{t}\left[\widehat{c}_{t+1}\right]-\sigma\left(\widehat{i}_{t}-\mathbb{E}_{t}\left[\widehat{\pi}_{t+1}\right]\right)$ \\
\hline $\mathbf{I S}^{*}$ & $\widehat{c}_{t}^{*} \approx \mathbb{E}_{t}\left[\widehat{c}_{t+1}^{*}\right]-\sigma\left(\widehat{i}_{t}^{*}-\mathbb{E}_{t}\left[\widehat{\pi}_{t+1}^{*}\right]\right)$ \\
\hline $\mathbf{R S}$ & $\widehat{c}_{t}-\widehat{c}_{t}^{*} \approx \sigma \widehat{r s}_{t}$ \\
\hline Q & $\begin{array}{c}\widehat{q}_{t} \approx \beta \mathbb{E}_{t}\left[\widehat{q}_{t+1}\right]+\left[(1-(1-\delta) \beta) \mathbb{E}_{t}\left(\widehat{r}_{t+1}^{z}\right)-\left(\widehat{i}_{t}-\mathbb{E}_{t}\left(\widehat{\pi}_{t+1}\right)\right)\right] \\
\widehat{q}_{t} \approx \chi \delta\left(\widehat{x}_{t}-\widehat{k}_{t}\right)-\widehat{v}_{t}\end{array}$ \\
\hline $\mathrm{Q}^{*}$ & $\begin{aligned} & \widehat{q}_{t}^{*} \approx \beta \mathbb{E}_{t}\left[\widehat{q}_{t+1}^{*}\right]+ {\left[(1-(1-\delta) \beta) \mathbb{E}_{t}\left(\widehat{r}_{t+1}^{z *}\right)-\left(\widehat{i}_{t}^{*}-\mathbb{E}_{t}\left(\widehat{\pi}_{t+1}^{*}\right)\right)\right] } \\
& \widehat{q}_{t}^{*} \approx \chi \delta\left(\widehat{x}_{t}^{*}-\widehat{k}_{t}^{*}\right)-\widehat{v}_{t}^{*}\end{aligned}$ \\
\hline KA & $\widehat{k}_{t+1} \approx(1-\delta) \widehat{k}_{t}+\delta\left(\widehat{x}_{t}+\widehat{v}_{t}\right)$ \\
\hline $\mathbf{K A}^{*}$ & $\widehat{k}_{t+1}^{*} \approx(1-\delta) \widehat{k}_{t}^{*}+\delta\left(\widehat{x}_{t}^{*}+\widehat{v}_{t}^{*}\right)$, \\
\hline & The Firms' Problem: \\
\hline AS & $\left(\frac{(1-\alpha)(1-\alpha \beta)}{\alpha}\right)\left[\begin{array}{c}\widehat{\pi}_{t}-\phi_{F} \Delta \widehat{s}_{t} \approx \beta \mathbb{E}_{t}\left(\widehat{\pi}_{t+1}-\phi_{F} \Delta \widehat{s}_{t+1}\right)+\ldots \\
\left(\sigma^{-1}+\left(1-\gamma_{x}\right) \varphi\left(\frac{\varphi \psi^{2}+(1-\psi)(1+\varphi)^{2}}{\varphi \psi+(1-\psi) \psi \varphi^{2}}\right)\right)\left[\phi_{H} \widehat{c}_{t}^{W}+\phi_{F} \widehat{c}_{t}^{W *}\right]+\ldots \\
\gamma_{x} \varphi\left(\frac{\varphi \psi^{2}+(1-\psi)(1+\varphi)^{2}}{\varphi \psi+(1-\psi) \psi \varphi^{2}}\right)\left[\phi_{H} \widehat{x}_{t}^{W}+\phi_{F} \widehat{x}_{t}^{W *}\right]+\ldots \\
2 \phi_{H} \phi_{F} \widehat{r s}_{t}+\left(\phi_{H}-\phi_{F}\right) \eta \varphi\left(\frac{\varphi \psi^{2}+(1-\psi)(1+\varphi)^{2}}{\varphi \psi+(1-\psi) \psi \varphi^{2}}\right) \hat{t}_{t}^{W}-\ldots \\
\left(\frac{(1-\psi)(1+\varphi)}{\psi}\right) \widehat{k}_{t}^{W}-\left(\frac{1+\varphi}{\psi}\right)\left[\phi_{H} \widehat{a}_{t}+\phi_{F} \widehat{a}_{t}^{*}\right]\end{array}\right]$ \\
\hline $\mathbf{A S} \mathbf{S}^{*}$ & $\left(\frac{(1-\alpha)(1-\alpha \beta)}{\alpha}\right)\left[\begin{array}{c}\widehat{\pi}_{t}^{*}+\phi_{F} \Delta \widehat{s}_{t} \approx \beta \mathbb{E}_{t}\left(\widehat{\pi}_{t+1}^{*}+\phi_{F} \Delta \widehat{s}_{t+1}\right)+\ldots \\
\left(\sigma^{-1}+\left(1-\gamma_{x}\right) \varphi\left(\frac{\varphi \psi^{2}+(1-\psi)(1+\varphi)^{2}}{\varphi \psi+(1-\psi) \psi \varphi^{2}}\right)\right)\left[\phi_{F} \widehat{c}_{t}^{W}+\phi_{H} \widehat{c}_{t}^{W *}\right]+\ldots \\
\gamma_{x} \varphi\left(\frac{\varphi \psi^{2}+(1-\psi)(1+\varphi)^{2}}{\varphi \psi+(1-\psi) \psi \varphi^{2}}\right)\left[\phi_{F} \widehat{x}_{t}^{W}+\phi_{H} \widehat{x}_{t}^{W *}\right]-\ldots \\
2 \phi_{F} \phi_{H} \widehat{r s}_{t}-\left(\phi_{H}-\phi_{F}\right) \eta \varphi\left(\frac{\varphi \psi^{2}+(1-\psi)(1+\varphi)^{2}}{\varphi \psi+(1-\psi) \psi \varphi^{2}}\right) \widehat{t}_{t}^{W}-\ldots \\
\left(\frac{(1-\psi)(1+\varphi)}{\psi}\right) \widehat{k}_{t}^{W *}-\left(\frac{1+\varphi}{\psi}\right)\left[\phi_{F} \widehat{a}_{t}+\phi_{H} \widehat{a}_{t}^{*}\right]\end{array}\right]$, \\
\hline $\begin{array}{l}\mathbf{T}^{\mathbf{W}} \\
\mathbf{E C}\end{array}$ & $\begin{array}{c}\widehat{t}_{t}^{W} \approx \frac{2 \phi_{H} \phi_{F}}{\phi_{H}-\phi_{F}}, \Delta \widehat{r s}_{t}=\Delta \widehat{s}_{t}+\widehat{\pi}_{t}^{*}-\widehat{\pi}_{t}, \\
\widehat{r}_{t}^{z} \approx \frac{1}{\sigma} \widehat{c}_{t}+\frac{1+\varphi}{\psi} \widehat{y}_{t}-\left(\frac{1+(1-\psi) \varphi}{\psi}\right) \widehat{k}_{t}-\frac{1+\varphi}{\psi} \widehat{a}_{t},\end{array}$ \\
\hline $\mathbf{E C}^{*}$ & $\widehat{r}_{t}^{z *} \approx \frac{1}{\sigma} \widehat{c}_{t}^{*}+\frac{1+\varphi}{\psi} \widehat{y}_{t}^{*}-\left(\frac{1+(1-\psi) \varphi}{\psi}\right) \widehat{k}_{t}^{*}-\frac{1+\varphi}{\psi} \widehat{a}_{t}^{*}$ \\
\hline & Aggregate Output and Employment: \\
\hline $\mathbf{Y}$ & $\widehat{y}_{t} \approx \eta \widehat{t}_{t}^{W}+\left(1-\gamma_{x}\right) \widehat{c}_{t}^{W}+\gamma_{x} \widehat{x}_{t}^{W}$ \\
\hline $\mathbf{Y}^{*}$ & $\widehat{y}_{t}^{*} \approx-\eta \widehat{t}_{t}^{W}+\left(1-\gamma_{x}\right) \widehat{c}_{t}^{W *}+\gamma_{x} \widehat{x}_{t}^{W *}$ \\
\hline $\mathbf{L}$ & $\psi \widehat{l}_{t} \approx \widehat{y}_{t}-\widehat{a}_{t}-(1-\psi) \widehat{k}_{t}$ \\
\hline $\mathbf{L}^{*}$ & $\psi \widehat{l}_{t}^{*} \approx \widehat{y}_{t}^{*}-\widehat{a}_{t}^{*}-(1-\psi) \widehat{k}_{t}^{*}$ \\
\hline & The Monetary Policy: \\
\hline MP & $\widehat{i}_{t} \approx \rho_{i} \widehat{i}_{t-1}+\left(1-\rho_{i}\right)\left[\psi_{y} \widehat{y}_{t}+\psi_{\pi} \widehat{\pi}_{t}\right]+\widehat{m}_{t}$ \\
\hline $\mathbf{M P}^{*}$ & $\widehat{i}_{t}^{*} \approx \rho_{i} \widehat{i}_{t-1}^{*}+\left(1-\rho_{i}\right)\left[\psi_{y} \widehat{y}_{t}^{*}+\psi_{\pi} \widehat{\pi}_{t}^{*}\right]+\widehat{m}_{t}^{*}$ \\
\hline & Other Definitions: \\
\hline & $\begin{array}{c}\widehat{c}_{t}^{W} \equiv \phi_{H} \widehat{c}_{t}+\phi_{F} \widehat{c}_{t}^{*}, \widehat{c}_{t}^{W *} \equiv \phi_{F} \widehat{c}_{t}+\phi_{H} \widehat{c}_{t}^{*}, \widehat{x}_{t}^{W} \equiv \phi_{H} \widehat{x}_{t}+\phi_{F} \widehat{x}_{t}^{*}, \widehat{x}_{t}^{W *} \equiv \phi_{F} \widehat{x}_{t}+\phi_{H} \widehat{x}_{t}^{*} \\
\widehat{k}_{t}^{W} \equiv \phi_{H} \widehat{k}_{t}+\phi_{F} \widehat{k}_{t}^{*}, \widehat{k}_{t}^{W *} \equiv \phi_{F} \widehat{k}_{t}+\phi_{H} \widehat{k}_{t}^{*}, \Delta \widehat{r s}_{t} \equiv \widehat{r s}_{t}-\widehat{r s}_{t-1}, \Delta \widehat{s}_{t} \equiv \widehat{s}_{t}-\widehat{s}_{t-1}, \widehat{\pi}_{t}=\widehat{p}_{t}-\widehat{p}_{t-1}, \widehat{\pi}_{t}^{*}=\widehat{p}_{t}^{*}-\widehat{p}_{t-1}^{*}\end{array}$ \\
\hline & Other Coefficients: $\gamma_{x} \equiv(1-\psi) \delta\left[\left(\frac{\theta(1-\bar{\xi})}{\theta-1}\right)\left(\beta^{-1}-(1-\delta)\right)\right]^{-1}$ \\
\hline
\end{tabular}




\section{B.4 The Model With Capital, Without Capital Utilization - IAC}

\begin{tabular}{|c|c|}
\hline & The Households' Problem: \\
\hline IS & $\widehat{c}_{t} \approx \mathbb{E}_{t}\left[\widehat{c}_{t+1}\right]-\sigma\left(\widehat{i}_{t}-\mathbb{E}_{t}\left[\widehat{\pi}_{t+1}\right]\right)$ \\
\hline $\mathbf{I S}^{*}$ & $\widehat{c}_{t}^{*} \approx \mathbb{E}_{t}\left[\widehat{c}_{t+1}^{*}\right]-\sigma\left(\hat{i}_{t}^{*}-\mathbb{E}_{t}\left[\widehat{\pi}_{t+1}^{*}\right]\right)$ \\
\hline $\mathbf{R S}$ & $\widehat{c}_{t}-\widehat{c}_{t}^{*} \approx \sigma \widehat{r s} t$ \\
\hline Q & $\begin{array}{c}\widehat{q}_{t} \approx(1-\delta) \beta \mathbb{E}_{t}\left[\widehat{q}_{t+1}\right]+\left[(1-(1-\delta) \beta) \mathbb{E}_{t}\left(\widehat{r}_{t+1}^{z}\right)-\left(\widehat{i}_{t}-\mathbb{E}_{t}\left[\widehat{\pi}_{t+1}\right]\right)\right] \\
\widehat{x}_{t} \approx \frac{1}{1+\beta} \widehat{x}_{t-1}+\frac{\beta}{1+\beta} \mathbb{E}_{t}\left[\widehat{x}_{t+1}\right]+\frac{1}{\kappa(1+\beta)}\left(\widehat{q}_{t}+\widehat{v}_{t}\right)\end{array}$ \\
\hline $\mathbf{Q}^{*}$ & $\begin{array}{c}\widehat{q}_{t}^{*} \approx(1-\delta) \beta \mathbb{E}_{t}\left[\widehat{q}_{t+1}^{*}\right]+\left[(1-(1-\delta) \beta) \mathbb{E}_{t}\left(\widehat{r}_{t+1}^{z *}\right)-\left(\widehat{i}_{t}^{*}-\mathbb{E}_{t}\left[\widehat{\pi}_{t+1}^{*}\right]\right)\right] \\
\widehat{x}_{t}^{*} \approx \frac{1}{1+\beta} \widehat{x}_{t-1}^{*}+\frac{\beta}{1+\beta} \mathbb{E}_{t}\left[\widehat{x}_{t+1}^{*}\right]+\frac{1}{\kappa(1+\beta)}\left(\widehat{q}_{t}^{*}+\widehat{v}_{t}^{*}\right)\end{array}$ \\
\hline KA & $\widehat{k}_{t+1} \approx(1-\delta) \widehat{k}_{t}+\delta\left(\widehat{x}_{t}+\widehat{v}_{t}\right)$ \\
\hline $\mathbf{K A}^{*}$ & $\widehat{k}_{t+1}^{*} \approx(1-\delta) \widehat{k}_{t}^{*}+\delta\left(\widehat{x}_{t}^{*}+\widehat{v}_{t}^{*}\right)$ \\
\hline & The Firms' Problem: \\
\hline $\mathbf{A S}$ & $\left(\frac{(1-\alpha)(1-\alpha \beta)}{\alpha}\right)\left[\begin{array}{c}\widehat{\pi}_{t}-\phi_{F} \Delta \widehat{s}_{t} \approx \beta \mathbb{E}_{t}\left(\widehat{\pi}_{t+1}-\phi_{F} \Delta \widehat{s}_{t+1}\right)+\ldots \\
\left(\sigma^{-1}+\left(1-\gamma_{x}\right) \varphi\left(\frac{\varphi \psi^{2}+(1-\psi)(1+\varphi)^{2}}{\varphi \psi+(1-\psi) \psi \varphi^{2}}\right)\right)\left[\phi_{H} \widehat{c}_{t}^{W}+\phi_{F} \widehat{c}_{t}^{W *}\right]+\ldots \\
\gamma_{x} \varphi\left(\frac{\varphi \psi^{2}+(1-\psi)(1+\varphi)^{2}}{\varphi \psi+(1-\psi) \psi \varphi^{2}}\right)\left[\phi_{H} \widehat{x}_{t}^{W}+\phi_{F} \widehat{x}_{t}^{W *}+\ldots\right. \\
2 \phi_{H} \phi_{F} \widehat{r s}_{t}+\left(\phi_{H}-\phi_{F}\right) \eta \varphi\left(\frac{\varphi \psi^{2}+(1-\psi)(1+\varphi)^{2}}{\varphi \psi+(1-\psi) \psi \varphi^{2}}\right) \widehat{t}_{t}^{W}-\ldots \\
\left(\frac{(1-\psi)(1+\varphi)}{\psi}\right) \widehat{k}_{t}^{W}-\left(\frac{1+\varphi}{\psi}\right)\left[\phi_{H} \widehat{a}_{t}+\phi_{F} \widehat{a}_{t}^{*}\right]\end{array}\right]$ \\
\hline $\mathbf{A S}^{*}$ & $\left(\frac{(1-\alpha)(1-\alpha \beta)}{\alpha}\right)\left[\begin{array}{c}\widehat{\pi}_{t}^{*}+\phi_{F} \Delta \widehat{s}_{t} \approx \beta \mathbb{E}_{t}\left(\widehat{\pi}_{t+1}^{*}+\phi_{F} \Delta \widehat{s}_{t+1}\right)+\ldots \\
\left.\sigma^{-1}+\left(1-\gamma_{x}\right) \varphi\left(\frac{\varphi \psi^{2}+(1-\psi)(1+\varphi)^{2}}{\varphi \psi+(1-\psi) \psi \varphi^{2}}\right)\right)\left[\phi_{F} \widehat{c}_{t}^{W}+\phi_{H} \widehat{c}_{t}^{W *}\right]+\ldots \\
2 \phi_{F} \varphi\left(\frac{\varphi \psi^{2}+(1-\psi)(1+\varphi)^{2}}{\varphi \psi+(1-\psi) \psi \varphi^{2}}\right)\left[\phi_{F} \widehat{x}_{t}^{W}+\phi_{H} \widehat{x}_{t}^{W *}-\ldots\right. \\
\left(\frac{(1-\psi)(1+\varphi)}{\psi}\right) \widehat{k}_{t}^{W *}-\left(\frac{1+\varphi}{\psi}\right)\left[\phi_{F} \widehat{a}_{t}+\phi_{H} \widehat{a}_{t}^{*}\right]\end{array}\right]$ \\
\hline $\begin{array}{l}\mathbf{T}^{\mathbf{W}} \\
\mathbf{E C}\end{array}$ & $\begin{array}{r}\widehat{t}_{t}^{W} \approx \frac{2 \phi_{H} \phi_{F}}{\phi_{H}-\phi_{F}} \widehat{r s}_{t}, \Delta \widehat{r s}_{t}=\Delta \widehat{s}_{t}+\widehat{\pi}_{t}^{*}-\widehat{\pi}_{t} \\
\widehat{r}_{t}^{z} \approx \frac{1}{\sigma} \widehat{c}_{t}+\frac{1+\varphi}{\psi} \widehat{y}_{t}-\left(\frac{1+(1-\psi) \varphi}{\psi}\right) \widehat{k}_{t}-\frac{1+\varphi}{\psi} \widehat{a}_{t}\end{array}$ \\
\hline $\mathbf{E C}^{*}$ & $\widehat{r}_{t}^{z *} \approx \frac{1}{\sigma} \widehat{c}_{t}^{*}+\frac{1+\varphi}{\psi} \widehat{y}_{t}^{*}-\left(\frac{1+(1-\psi) \varphi}{\psi}\right) \widehat{k}_{t}^{*}-\frac{1+\varphi}{\psi} \widehat{a}_{t}^{*}$ \\
\hline & Aggregate Output and Employment: \\
\hline $\mathbf{Y}$ & $\widehat{y}_{t} \approx \eta \widehat{t}_{t}^{W}+\left(1-\gamma_{x}\right) \widehat{c}_{t}^{W}+\gamma_{x} \widehat{x}_{t}^{W}$ \\
\hline $\mathbf{Y}^{*}$ & $\widehat{y}_{t}^{*} \approx-\eta \widehat{t}_{t}^{W}+\left(1-\gamma_{x}\right) \widehat{c}_{t}^{W *}+\gamma_{x} \widehat{x}_{t}^{W *}$ \\
\hline $\mathbf{L}$ & $\psi \widehat{l}_{t} \approx \widehat{y}_{t}-\widehat{a}_{t}-(1-\psi) \widehat{k}_{t}$ \\
\hline $\mathbf{L}^{*}$ & $\psi \widehat{l}_{t}^{*} \approx \widehat{y}_{t}^{*}-\widehat{a}_{t}^{*}-(1-\psi) \widehat{k}_{t}^{*}$ \\
\hline & The Monetary Policy: \\
\hline MP & $\widehat{i}_{t} \approx \rho_{i} \widehat{i}_{t-1}+\left(1-\rho_{i}\right)\left[\psi_{y} \widehat{y}_{t}+\psi_{\pi} \widehat{\pi}_{t}\right]+\widehat{m}_{t}$ \\
\hline $\mathbf{M P}^{*}$ & $\widehat{i}_{t}^{*} \approx \rho_{i} \widehat{i}_{t-1}^{*}+\left(1-\rho_{i}\right)\left[\psi_{y} \widehat{y}_{t}^{*}+\psi_{\pi} \widehat{\pi}_{t}^{*}\right]+\widehat{m}_{t}^{*}$ \\
\hline & Other Definitions: \\
\hline & $\begin{array}{c}\widehat{c}_{t}^{W} \equiv \phi_{H} \widehat{c}_{t}+\phi_{F} \widehat{c}_{t}^{*}, \widehat{c}_{t}^{W *} \equiv \phi_{F} \widehat{c}_{t}+\phi_{H} \widehat{c}_{t}^{*}, \widehat{x}_{t}^{W} \equiv \phi_{H} \widehat{x}_{t}+\phi_{F} \widehat{x}_{t}^{*}, \widehat{x}_{t}^{W *} \equiv \phi_{F} \widehat{x}_{t}+\phi_{H} \widehat{x}_{t}^{*} \\
\widehat{k}_{t}^{W} \equiv \phi_{H} \widehat{k}_{t}+\phi_{F} \widehat{k}_{t}^{*}, \widehat{k}_{t}^{W *} \equiv \phi_{F} \widehat{k}_{t}+\phi_{H} \widehat{k}_{t}^{*}, \Delta \widehat{r s}_{t} \equiv \widehat{r s} t-\widehat{r s}_{t-1}, \Delta \widehat{s}_{t} \equiv \widehat{s}_{t}-\widehat{s}_{t-1}, \widehat{\pi}_{t}=\widehat{p}_{t}-\widehat{p}_{t-1}, \widehat{\pi}_{t}^{*}=\widehat{p}_{t}^{*}-\widehat{p}_{t-1}^{*}\end{array}$ \\
\hline & Other Coefficients: $\gamma_{x} \equiv(1-\psi) \delta\left[\left(\frac{\theta(1-\bar{\xi})}{\theta-1}\right)\left(\beta^{-1}-(1-\delta)\right)\right]^{-1}$ \\
\hline
\end{tabular}




\section{B.5 The Model With Capital, With Capital Utilization - NAC}

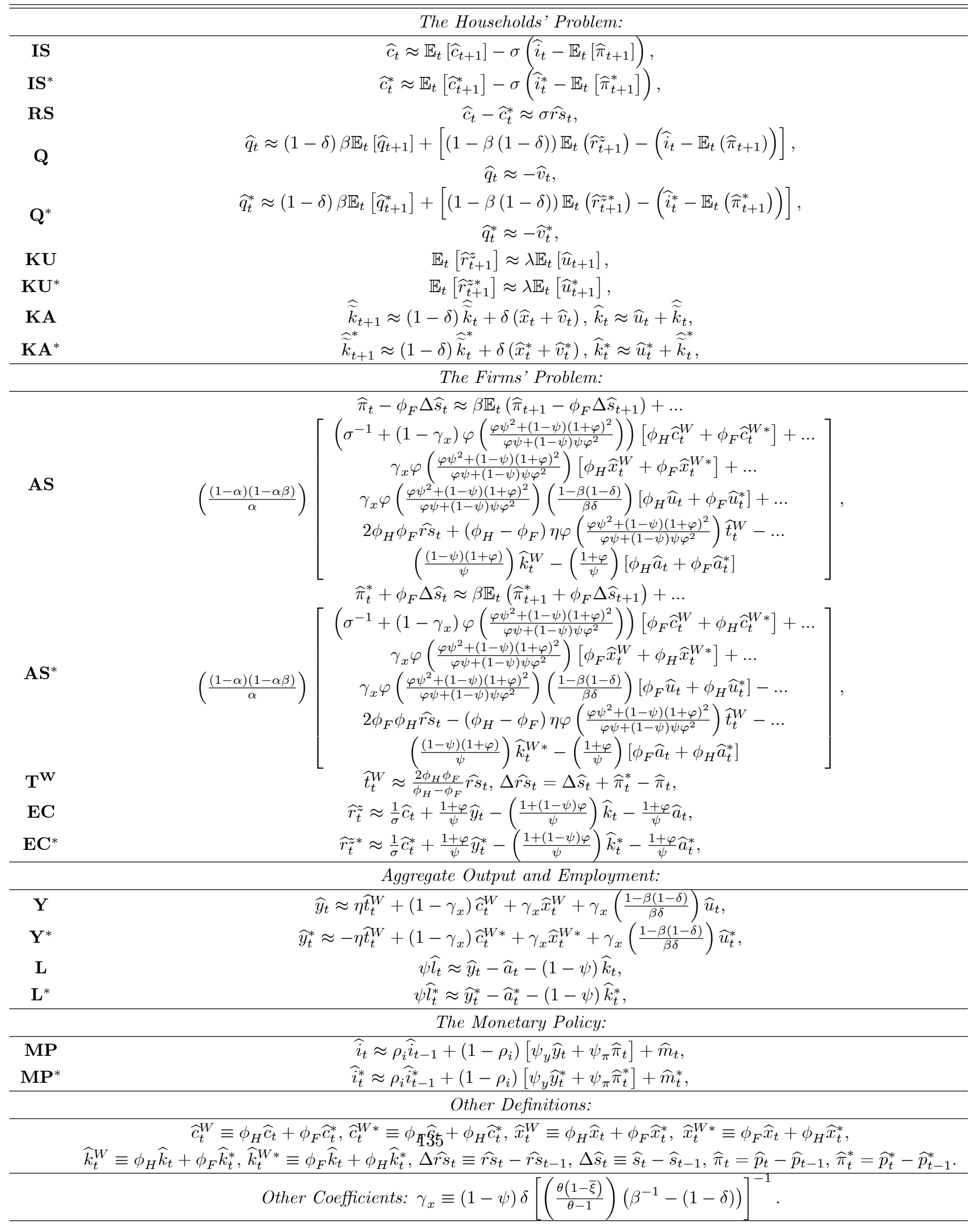




\section{B.6 The Model With Capital, With Capital Utilization - CAC}

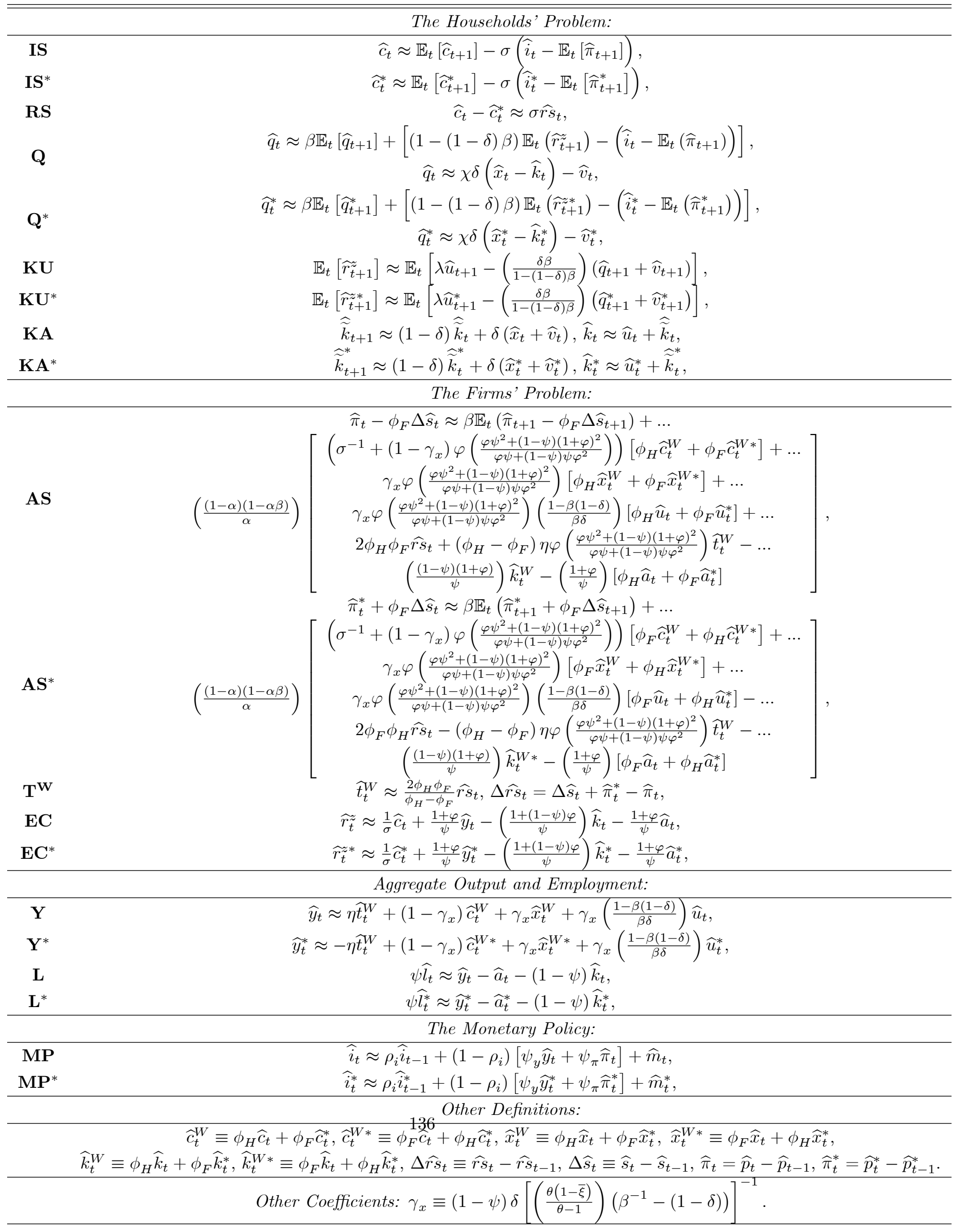




\section{B.7 The Model With Capital, With Capital Utilization - IAC}

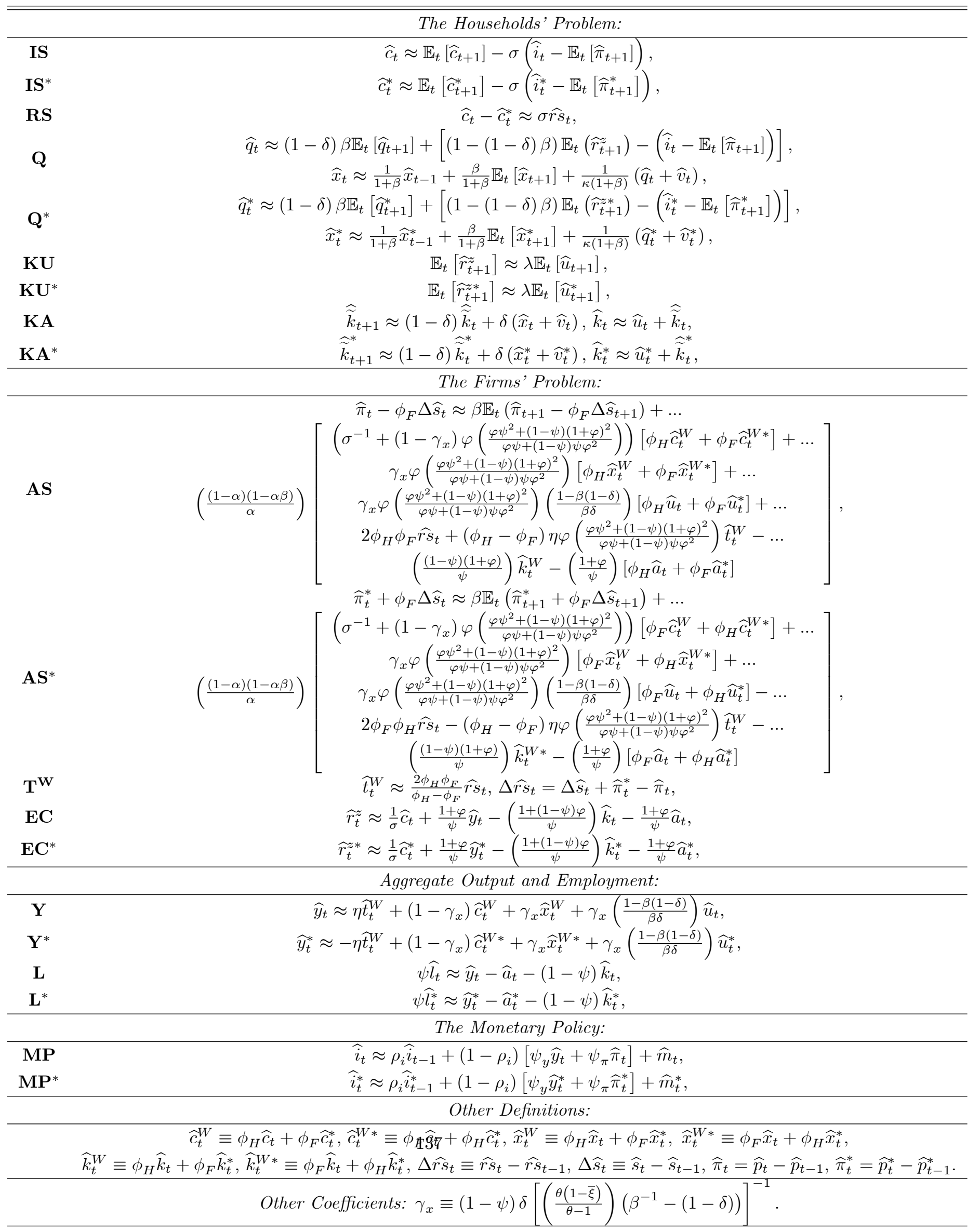




\section{B.8 Net Exports, Real Exports and Real Imports}

\begin{tabular}{lc}
\hline \hline & The Model Without Capital: \\
\hline NX & $\widehat{t b}_{t} \equiv \eta \widehat{t}_{t}^{W}-\phi_{F} \sigma \widehat{r s}_{t}$, \\
EXP & $\widehat{e x p}_{t} \approx \eta\left(\frac{\phi_{H}}{\phi_{H}-\phi_{F}}\right) \widehat{r s}_{t}+\widehat{c}_{t}^{*}$, \\
IMP & $\widehat{i m p}_{t} \approx-\eta\left(\frac{\phi_{H}}{\phi_{H}-\phi_{F}}\right) \widehat{r s}_{t}+\widehat{c}_{t}$, \\
\hline & The Model With Capital $(N A C$, CAC, IAC), With or Without Capital Utilization: \\
\hline NX & $\widehat{t b}_{t} \equiv \eta \widehat{t}_{t}^{W}-\left(1-\gamma_{x}\right) \phi_{F} \sigma \widehat{r s}_{t}-\gamma_{x} \phi_{F}\left(\widehat{x}_{t}-\widehat{x}_{t}^{*}\right)$, \\
EXP & $\widehat{\exp }_{t} \approx \eta\left(\frac{\phi_{H}}{\phi_{H}-\phi_{F}}\right) \widehat{r s}_{t}+\left(1-\gamma_{x}\right) \widehat{c}_{t}^{*}+\gamma_{x} \widehat{x}_{t}^{*}$, \\
IMP & $\widehat{i m p}_{t} \approx-\eta\left(\frac{\phi_{H}}{\phi_{H}-\phi_{F}}\right) \widehat{r s}_{t}+\left(1-\gamma_{x}\right) \widehat{c}_{t}+\gamma_{x} \widehat{x}_{t}$, \\
\hline & Other Coefficients: \\
\hline & $\gamma_{x} \equiv(1-\psi) \delta\left[\left(\frac{\theta(1-\bar{\xi})}{\theta-1}\right)\left(\beta^{-1}-(1-\delta)\right)\right]^{-1}$. \\
\hline
\end{tabular}

